\title{
HOLOMORPHIC EXTENSION OF CR FUNCTIONS, ENVELOPES OF HOLOMORPHY, AND REMOVABLE SINGULARITIES
}

\author{
JOËL MERKER AND EGMONT PORTEN
}

Département de Mathématiques et Applications, UMR 8553 du CNRS, École Normale Supérieure, 45 rue d'Ulm, F-75230 Paris Cedex 05, France.

merker@dma.univ-mrs.fr http://www.cmi.univ-mrs.fr/ merker/index.html

Department of Engineering, Physics and Mathematics, Mid Sweden University, Campus Sundsvall, S-85170

Sundsvall, Sweden Egmont.Porten@miun.se

Table of contents (7 main parts)

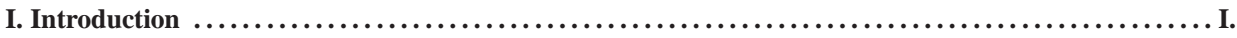

II. Analytic vector field systems, formal CR mappings and local CR automorphism groups .......... II.

III. Sussman's orbit theorem, locally integrable systems of vector fields and CR functions ...........III.

IV. Hilbert transform and Bishop's equation in Hölder spaces $\ldots \ldots \ldots \ldots \ldots \ldots \ldots \ldots \ldots \ldots \ldots \ldots \ldots$ IV.

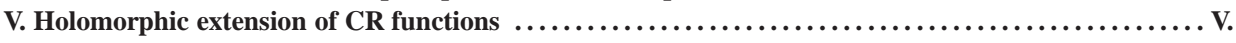

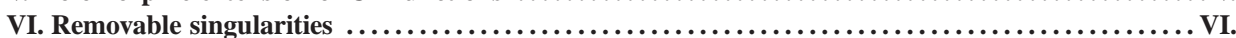

$[0+3+7+1+19+7$ diagrams $]$

\begin{tabular}{|c|}
\hline IMRS International Mathematics Research Surveys \\
\hline Volume 2006, Article ID 28295, 287 pages \\
\hline www.hindawi.com/journals/imrs
\end{tabular}

Date: 2007-1-19.

2000 Mathematics Subject Classification. Primary: 32-01, 32-02. Secondary: 32Bxx, 32Dxx, 32D10, 32D15, 32D20, 32D26, 32H12, 32F40, 32T15, 32T25, 32T27, 32Vxx, 32V05, 32V10, 32V15, 32V25, 32V35, 32V40. 


\section{I: Introduction}

1.1. CR extension theory. In the past decades, remarkable progress has been accomplished towards the understanding of compulsory extendability of holomorphic functions, of CR functions and of differential forms. These phenomena, whose exploration is still active in current research, originate from the seminal Hartogs-Bochner extension theorem.

In local CR extension theory, the most satisfactory achievement was the discovery that, on a smooth embedded generic submanifold $M \subset \mathbb{C}^{n}$, there is a precise correspondence between $C R$ orbits of $M$ and families of small Bishop discs attached to $M$. Such discs cover a substantial part of the polynomial hull of $M$, and in most cases, this part may be shown to constitute a global one-sided neighborhood $\mathcal{V}^{ \pm}(M)$ of $M$, if $M$ is a hypersurface, or else a wedgelike domain $\mathcal{W}$ attached to $M$, if $M$ has codimension $\geqslant 2$. A local polynomial approximation theorem, or a CR version of the Kontinuitätssatz (continuity principle) assures that CR functions automatically extend holomorphically to such domains $\mathcal{W}$, which are in addition contained in the envelope of holomorphy of arbitrarily thin neighborhoods of $M$ in $\mathbb{C}^{n}$.

Trépreau in the hypersurface (1986) case and slightly after Tumanov in arbitrary codimension (1988) established a nowadays celebrated extension theorem: if $M \subset \mathbb{C}^{n}$ is a sufficiently smooth $\left(\mathcal{C}^{2}\right.$ or $\mathcal{C}^{2, \alpha}$ suffices) generic submanifold, then at every point $p \in M$ whose local $C R$ orbit $\mathcal{O}_{C R}^{\text {loc }}(M, p)$ has maximal dimension equal to $\operatorname{dim} M$, there exists a local wedge $\mathcal{W}_{p}$ of edge $M$ at $p$ to which continuous $C R$ functions extend holomorphically. Several reconstructions and applications of this groundbreaking result, together with surveys about the local Bishop equation have already appeared in the literature.

Propagational aspects of CR extension theory are less known by contemporary experts of several complex variables, but they lie deeper in the theory. Using FBI transform and concepts of microlocal analysis, Trépreau showed in 1990 that holomorphic extension to a wedge propagates along curves whose velocity vector is complex-tangential to $M$. His conjecture that extension to a wedge should hold at every point of a generic submanifold $M \subset \mathbb{C}^{n}$ consisting of a single global CR orbit has been answered independently by Jöricke and by the first author in 1994, using tools introduced previously by Tumanov. To the knowledge of the two authors, there is no survey of these global aspects in the literature.

The first main objective of the present survey is to expose the techniques underlying these results in a comprehensive and unified way, emphasizing 
propagational aspects of embedded CR geometry and discussing optimal smoothness assumptions. Thus, topics that are necessary to build the theory from scratch will be selected and accompanied with thorough proofs, whereas other results that are nevertheless central in CR geometry will be presented in concise survey style, without any proof.

The theory of CR extension by means of analytic discs combines various concepts emanating mainly from three (wide) mathematical areas: Harmonic analysis, Partial differential equations and Complex analysis in several variables. As the project evolved, we felt the necessity of being conceptional, extensive and systematic in the restitution of (semi)known results, so that various contributions to the subject would recover a certain coherence and a certain unity. With the objective of adressing to a younger audience, we decided to adopt a style accessible to doctoral candidates working on a dissertation. Parts III, IV and V present elementarily general CR extension theory. Also, most sections of the text may be read independently by experts, as quanta of mathematical information.

1.2. Concise presentation of the contents. The survey text is organized in six main parts. Actually, the present brief introduction constitutes the first and shortest one. Although the reader will find a "conceptional summaryintroduction" at the beginning of each part, a few descriptive words explaining some of our options governing the reconstruction of CR extension theory (Parts III, IV and V) are welcome.

The next Part II is independent of the others and can be skipped in a first reading. It opens the text, because it is concerned with propagational aspects of analytic CR structures, better understood than the smooth ones.

- In Part III, exclusively concerned with the smooth category, Sussmann's orbit theorem and its consequences are first explained in length. Involutive structures and embedded CR manifolds, together with their elementary properties, are introduced. Structural properties of finite type structures, of $\mathrm{CR}$ orbits and of CR functions are presented without proofs. As a collection of background material, this part should be consulted first.

- In Part IV, fundamental results about singular integral operators in the complex plane are first surveyed. Explicit estimates of the norms of the Cauchy, of the Schwarz and of the Hilbert transforms in the Hölder spaces $\mathcal{C}^{\kappa, \alpha}$ are provided. They are useful to reconstruct the main Theorem 3.7(IV), due to Tumanov, which asserts the existence of unique solutions to a parametrized Bishop-type equation with an optimal loss of smoothness with respect to parameters. Following Bishop's constructive philosophy, the smallness of the constants insuring existence is precised 
explicitly, thanks to sharp norm inequalities in Hölder spaces. This part is meant to introduce interested readers to further reading of Tumanov's recent works about extremal (pseudoholomorphic) discs in higher codimension.

- In Part V, CR extension theory is first discussed in the hypersurface case. A simplified proof of wedge extendability that treats both locally minimal and globally minimal generic submanifolds on the same footing constitutes the main Theorem 4.12(V): If $M$ is a globally minimal $\mathcal{C}^{2, \alpha}(0<\alpha<$ 1) generic submanifold of $\mathbb{C}^{n}$ of codimension $\geqslant 1$ and of $C R$ dimension $\geqslant 1$, there exists a wedgelike domain $\mathcal{W}$ attached to $M$ such that every continuous $C R$ function $f \in \mathcal{C}_{C R}^{0}(M)$ possesses a holomorphic extension $F \in \mathcal{O}(\mathcal{W}) \cap \mathcal{C}^{0}(M \cup \mathcal{W})$ with $\left.F\right|_{M}=f$. The figures are intended to share the geometric insight of experts in higher codimensional geometry.

In fact, throughout the text, diagrams (33 in sum) facilitating readability (especially of Part V) are included. Selected open questions and open problems (16 in sum) are formulated. They are systematically inserted in the right place of the architecture. The sign " $[*]$ " added after one or several bibliographical references in a statement (Problem, Definition, Theorem, Proposition, Lemma, Corollary, Example, Open question and Open problem, e.g. Theorem 1.11(I)) indicates that, compared to the existing literature, a slight modification or a slight improvement has been brought by the two authors. Statements containing no bibliographical reference are original and appear here for the first time.

We apologize for having not treated some central topics of CR geometry that also involve propagation of holomorphicity, exempli gratia the geometric reflection principle, in the sense of Pinchuk, Webster, Diederich, Fornæss, Shafikov and Verma. By lack of space, embeddability of abstract CR structures, polynomial hulls, Bishop discs growing at elliptic complex tangencies, filling by Levi-flat surfaces, Riemann-Hilbert boundary value problems, complex Plateau problem in Kähler manifolds, partial indices of analytic discs, pseudoholomorphic discs, etc. are not reviewed either. Certainly, better experts will fill this gap in the near future.

To conclude this introductory presentation, we believe that, although uneasy to build, surveys and syntheses play a decisive rôle in the evolution of mathematical subjects. For instance, in the last decades, the remarkable development of $\bar{\partial}$ techniques and of $L^{2}$ estimates has been regularly accompanied by monographs and panoramas, some of which became landmarks in the field. Certainly, the (local) method of analytic discs deserves to be 
known by a wider audience; in fact, its main contributors have brought it to the degree of achievement that opened the way to the present survey.

1.3. Further readings. Using the tools exposed and reconstructed in this survey, the research article [MP2006] studies removable singularities on $\mathrm{CR}$ manifolds of CR dimension equal to 1 and solves a delicate remaining open problem in the field (see the Introduction there for motivations). Recently also, the authors built in [MP2006] a new, rigorous proof of the classical Hartogs extension theorem which relies only on the basic local Levi argument along analytic discs, hence avoids both multidimensional integral representation formulas and the Serre-Ehrenpreis argument about vanishing of $\bar{\partial}$ cohomology with compact support. 


\title{
II: Analytic vector field systems and formal CR mappings
}

\author{
Table of contents
}

1. Analytic vector field systems and Nagano's theorem 7.

2. Analytic CR manifolds, Segre chains and minimality .................

3. Formal CR mappings, jets of Segre varieties and CR reflection mapping ... 28.

[3 diagrams]

According to the theorem of Frobenius, a system $\mathbb{L}$ of local vector fields having real or complex analytic coefficients enjoys the integral manifolds property, provided it is closed under Lie bracket. If the Lie brackets exceed $\mathbb{L}$, considering the smallest analytic system $\mathbb{L}^{\text {lie }}$ containing $\mathbb{L}$ which is closed under Lie bracket, Nagano showed that through every point, there passes a submanifold whose tangent space is spanned by $\mathbb{L}^{\text {lie }}$. Without considering Lie brackets, these submanifolds may also be constructed by means of compositions of local flows of elements of $\mathbb{L}$. Such a construction has applications in real analytic Cauchy-Riemann geometry, in the reflection principle, in formal CR mappings, in analytic hypoellipticity theorems and in the problem of local solvability and of local uniqueness for systems of first order linear partial differential operators (Part III).

For a generic set of $r \geqslant 2$ vector fields having analytic coefficients, $\mathbb{L}^{\text {lie }}$ has maximal rank equal to the dimension of the ambient space.

The extrinsic complexification $\mathcal{M}$ of a real algebraic or analytic CauchyRiemann submanifold $M$ of $\mathbb{C}^{n}$ carries two pairs of intrinsic foliations, obtained by complexifying the classical Segre varieties together with their conjugates. The Nagano leaves of this pair of foliations coincide with the extrinsic complexifications of local CR orbits. If $M$ is (Nash) algebraic, its CR orbits are algebraic too, because they are projections of complexified algebraic Nagano leaves.

A complexified formal CR mapping between two complexified generic submanifolds must respect the two pairs of intrinsic foliations that lie in the source and in the target. This constraint imposes strong rigidity properties, as for instance: convergence, analyticity or algebraicity of the formal CR mapping, according to the smoothness of the target and of the source. There is a combinatorics of various nondegeneracy conditions that entail versions of the so-called analytic reflection principle. The concept of $C R$ reflection mapping provides a unified synthesis of recent results of the literature. 


\section{$\S 1$. AnAlytic Vector Field Systems and NaGANo's THEOREM}

1.1. Formal, analytic and (Nash) algebraic power series. Let $n \in \mathbb{N}$ with $n \geqslant 1$ and let $\mathrm{x}=\left(\mathrm{x}_{1}, \ldots, \mathrm{x}_{n}\right) \in \mathbb{K}^{n}$, where $\mathbb{K}=\mathbb{R}$ or $\mathbb{C}$. Let $\mathbb{K} \llbracket \mathrm{x} \rrbracket$ be the ring of formal power series in $\left(\mathrm{x}_{1}, \ldots, \mathrm{x}_{n}\right)$. An element $\varphi(\mathrm{x}) \in \mathbb{K} \llbracket \mathrm{x} \rrbracket$ writes $\varphi(\mathrm{x})=\sum_{\alpha \in \mathbb{N}^{n}} \varphi_{\alpha} \mathrm{x}^{\alpha}$, with $\mathrm{x}^{\alpha}:=\mathrm{x}_{1}^{\alpha_{1}} \cdots \mathrm{x}_{n}^{\alpha_{n}}$ and with $\varphi_{\alpha} \in \mathbb{K}$, for every multiindex $\alpha:=\left(\alpha_{1}, \ldots, \alpha_{n}\right) \in \mathbb{N}^{n}$. We put $|\alpha|:=\alpha_{1}+\cdots+\alpha_{n}$.

On the vector space $\mathbb{K}^{n}$, we choose once for all the maximum norm $|x|:=\max _{1 \leqslant i \leqslant n}\left|x_{i}\right|$ and, for any "radius" $\rho_{1}$ satisfying $0<\rho_{1} \leqslant \infty$, we define the open cube

$$
\square_{\rho_{1}}^{n}:=\left\{\mathrm{x} \in \mathbb{K}^{n}:|x|<\rho_{1}\right\}
$$

as a fundamental, concrete open set. For $\rho_{1}=\infty$, we identify of course $\square_{\infty}^{n}$ with $\mathbb{K}^{n}$.

If the coefficients $\varphi_{\alpha}$ satisfy a Cauchy estimate of the form $\left|\varphi_{\alpha}\right| \leqslant$ $C \rho_{2}^{-|\alpha|}, C>0$, for every $\rho_{2}$ satisfying $0<\rho_{2}<\rho_{1}$, the formal power series is $\mathbb{K}$-analytic $\left(\mathcal{C}^{\omega}\right)$ in $\square_{\rho_{1}}^{n}$. It then defines a true point map $\varphi: \square_{\rho_{1}}^{n} \rightarrow \mathbb{K}$. Such a $\mathbb{K}$-analytic function $\varphi$ is called (Nash) $\mathbb{K}$-algebraic if there exists a nonzero polynomial $P(\mathrm{X}, \Phi) \in \mathbb{K}[\mathrm{X}, \Phi]$ in $(n+1)$ variables such that the relation $P(\mathrm{x}, \varphi(\mathrm{x})) \equiv 0$ holds in $\mathbb{K} \llbracket \mathrm{x} \rrbracket$, hence for all $\mathrm{x}$ in $\square_{\rho_{1}}^{n}$. The category of $\mathbb{K}$-algebraic functions and maps is stable under elementary algebraic operations, under differentiation and under composition. Implicit solutions of $\mathbb{K}$-algebraic equations are $\mathbb{K}$-algebraic ([BER1999]).

\subsection{Analytic vector field systems and their integral manifolds. Let}

$$
\mathbb{L}^{0}:=\left\{L_{a}\right\}_{1 \leqslant a \leqslant r}, \quad r \in \mathbb{N}, \quad r \geqslant 1,
$$

be a finite set of vector fields $L_{a}=\sum_{i=1}^{n} \varphi_{a, i}(\mathrm{x}) \frac{\partial}{\partial \mathrm{x}_{i}}$, whose coefficients $\varphi_{a, i}$ are algebraic or analytic in $\square_{\rho_{1}}^{n}$. Let $\mathbb{A}_{\rho_{1}}$ denote the ring of algebraic or analytic functions in $\square_{\rho_{1}}^{n}$. The set of linear combinations of elements of $\mathbb{L}^{0}$ with coefficients in $\mathbb{A}_{\rho_{1}}$ will be denoted by $\mathbb{L}\left(\right.$ or $\mathbb{L}^{1}$ ) and will be called the $\mathbb{A}_{\rho_{1}}$-linear hull of $\mathbb{L}^{0}$.

If $p$ is a point of $\square_{\rho_{1}}^{n}$, denote by $L_{a}(p)$ the vector $\left.\sum_{i=1}^{n} \varphi_{a, i}(p) \frac{\partial}{\partial x_{i}}\right|_{p}$. It is an element of $T_{p} \square_{\rho_{1}}^{n} \simeq \mathbb{K}^{n}$. Define the linear subspace

$$
\mathbb{L}(p):=\operatorname{Span}_{\mathbb{K}}\left\{L_{a}(p): 1 \leqslant a \leqslant r\right\}=\{L(p): L \in \mathbb{L}\} .
$$

No constancy of dimension, no linear independency assumption are made.

Problem 1.3. Find local submanifolds $\Lambda$ passing through the origin satisfying $T_{q} \Lambda \supset \mathbb{L}(q)$ for every $q \in \Lambda$. 
By the theorem of Frobenius ([Stk2000]; original article: [Fr1877]), if the $L_{a}$ are linearly independent at every point of $\square_{\rho_{1}}^{n}$ and if the Lie brackets $\left[L_{a}, L_{a^{\prime}}\right]$ belong to $\mathbb{L}$, for all $a, a^{\prime}=1, \ldots, r$, then $\square_{\rho_{1}}^{n}$ is foliated by $r$ dimensional submanifolds $N$ satisfying $T_{q} N=\mathbb{L}(q)$ for every $q \in N$.

Lemma 1.4. If there exists a local submanifold $\Lambda$ passing through the origin and satisfying $T_{q} \Lambda \supset \mathbb{L}(q)$ for every $q \in \Lambda$, then for every two vector fields $L, L^{\prime} \in \mathbb{L}$, the restriction to $\Lambda$ of the Lie bracket $\left[L, L^{\prime}\right]$ is tangent to $\Lambda$.

Accordingly, set $\mathbb{L}^{1}:=\mathbb{L}$ and for $k \geqslant 2$, define $\mathbb{L}^{k}$ to be the $\mathbb{A}_{\rho_{1}}$ linear hull of $\mathbb{L}^{k-1}+\left[\mathbb{L}^{1}, \mathbb{L}^{k-1}\right]$. Concretely, $\mathbb{L}^{k}$ is generated by $\mathbb{A}_{\rho_{1}}$-linear combinations of iterated Lie brackets $\left[L_{1},\left[L_{2}, \ldots,\left[L_{k-1}, L_{k}\right] \ldots\right]\right]$, where $L_{1}, L_{2}, \ldots, L_{k-1}, L_{k} \in \mathbb{L}^{1}$. The Jacobi identity insures (by induction) that $\left[\mathbb{L}^{k_{1}}, \mathbb{L}^{k_{2}}\right] \subset \mathbb{L}^{k_{1}+k_{2}}$. Define then $\mathbb{L}^{\text {lie }}:=\cup_{k \geqslant 1} \mathbb{L}^{k}$. Clearly, $\left[L, L^{\prime}\right] \in \mathbb{L}^{\text {lie }}$, for every two vector fields $L, L^{\prime} \in \mathbb{L}^{\text {lie }}$.

Theorem 1.5. (NAGANo [Na1966, Trv1992, BER1999, BCH2005]) There exists a unique local $\mathbb{K}$-analytic submanifold $\Lambda$ of $\mathbb{K}^{n}$ passing through the origin which satisfies $\mathbb{L}(q) \subset T_{q} \Lambda=\mathbb{L}^{\text {lie }}(q)$, for every $q \in \Lambda$.

A discussion about what happens in the algebraic category is postponed to $\S 1.12$. In Frobenius' theorem, $\mathbb{L}^{\text {lie }}=\mathbb{L}$ and the dimension of $\mathbb{L}^{\text {lie }}(p)$ is constant. In the above theorem, the dimension of $\mathbb{L}^{\text {lie }}(q)$ is constant for $q$ belonging to $\Lambda$, but in general, not constant for $p \in \square_{\rho_{1}}^{n}$, the function $p \mapsto \operatorname{dim}_{\mathbb{K}} \mathbb{L}(p)$ being lower semi-continuous.

Nagano's theorem is stated at the origin; it also holds at every point $p \in \square_{\rho_{1}}^{n}$. The associated local submanifold $\Lambda_{p}$ passing through $p$ with the property that $T_{q} \Lambda=\mathbb{L}^{\text {lie }}(q)$ for every $q \in \Lambda_{p}$ is called a (local) Nagano leaf.

In the $\mathcal{C}^{\infty}$ category, the consideration of $\mathbb{L}^{\text {lie }}$ is insufficient. Part III handles smooth vector field systems, providing a different answer to the search of similar submanifolds $\Lambda_{p}$.

Example 1.6. In $\mathbb{R}^{2}$, take $\mathbb{L}^{0}=\left\{L_{1}, L_{2}\right\}$, where $L_{1}=\partial_{x_{1}}$ and $L_{2}=$ $e^{-1 / \times_{1}^{2}} \partial_{x_{2}}$. Then $\mathbb{L}^{\text {lie }}(0)$ is the line $\left.\mathbb{R} \partial_{\mathrm{x}_{1}}\right|_{0}$, while $\mathbb{L}^{\text {lie }}(p)=\left.\mathbb{R} \partial_{\mathrm{x}_{1}}\right|_{p}+\left.\mathbb{R} \partial_{\mathrm{x}_{2}}\right|_{p}$ at every point $p \notin \mathbb{R} \times\{0\}$. Hence, there cannot exist a $\mathcal{C}^{\infty}$ curve $\Lambda$ passing through 0 with $T_{0} \Lambda=\left.\mathbb{R} \partial_{\mathrm{x}_{1}}\right|_{0}$ and $T_{q} \Lambda=\mathbb{L}^{\text {lie }}(q)$ for every $q \in \Lambda$.

Proof of Theorem 1.5. (May be skipped in a first reading.) If $n=1$, the statement is clear, depending on whether or not all vector fields in $\mathbb{L}^{\text {lie }}$ vanish at the origin. Let $n \geqslant 2$. Since $\mathbb{L}(q) \subset \mathbb{L}^{\text {lie }}(q)$, the condition 
$T_{q} \Lambda=\mathbb{L}^{\text {lie }}(q)$ implies the inclusion $\mathbb{L}(q) \subset T_{q} \Lambda$. Replacing $\mathbb{L}$ by $\mathbb{L}^{\text {lie }}$ if necessary, we may therefore assume that $\mathbb{L}^{\text {lie }}=\mathbb{L}$ and we then have to prove the existence of $\Lambda$ with $T_{q} \Lambda=\mathbb{L}^{\text {lie }}(q)=\mathbb{L}(q)$, for every $q \in \Lambda$.

We reason by induction, supposing that, in dimension $(n-1)$, for every $\mathbb{A}_{\rho_{1}}$-linear system $\mathbb{L}^{\prime}=\left(\mathbb{L}^{\prime}\right)^{\text {lie }}$ of vector fields locally defined in a neighborhood of the origin in $\mathbb{K}^{n-1}$, there exists a local $\mathbb{K}$-analytic submanifold $\Lambda^{\prime}$ passing through the origin and satisfying $T_{q^{\prime}} \Lambda^{\prime}=\mathbb{L}^{\prime}\left(q^{\prime}\right)$, for every $q^{\prime} \in \Lambda^{\prime}$.

If all vector fields in $\mathbb{L}=\mathbb{L}^{\text {lie }}$ vanish at 0 , we are done, trivially. Thus, assume there exists $L_{1} \in \mathbb{L}$ with $L_{1}(0) \neq 0$. After local straightening, $L_{1}=\partial_{\mathrm{x}_{1}}$. Every $L \in \mathbb{L}$ writes uniquely $L=a(\mathrm{x}) \partial_{\mathrm{x}_{1}}+\widetilde{L}$, for some $a(\mathrm{x}) \in \mathbb{K}\{\mathrm{x}\}$, with $\widetilde{L}=\sum_{2 \leqslant i \leqslant n} a_{i}(\mathrm{x}) \partial_{\mathrm{x}_{i}}$. Introduce the space $\widetilde{\mathbb{L}}:=\{\widetilde{L}$ : $L \in \mathbb{L}\}$ of such vector fields. As $\partial_{x_{1}}$ belongs to $\mathbb{L}$ and as $\mathbb{L}$ is $\mathbb{A}_{\rho_{1}}$-linear, $\widetilde{L}=L-a(\mathbf{x}) \partial_{\mathrm{x}_{1}}$ belongs to $\mathbb{L}$. Since $[\mathbb{L}, \mathbb{L}] \subset \mathbb{L}$, we have $[\widetilde{\mathbb{L}}, \widetilde{\mathbb{L}}] \subset \mathbb{L}$. On the other hand, we observe that the Lie bracket between two elements of $\widetilde{\mathbb{L}}$ does not involve $\partial_{x_{1}}$ :

$$
\begin{aligned}
{\left[\widetilde{L}_{1}, \widetilde{L}_{2}\right] } & =\left[\sum_{2 \leqslant i_{2} \leqslant n} a_{i_{2}}^{1} \partial_{x_{i_{2}}}, \sum_{2 \leqslant i_{1} \leqslant n} a_{i_{1}}^{2} \partial_{x_{i_{1}}}\right] \\
& =\sum_{2 \leqslant i_{1} \leqslant n}\left(\sum_{2 \leqslant i_{2} \leqslant n}\left[a_{i_{2}}^{1} \frac{\partial a_{i_{1}}^{2}}{\partial x_{i_{2}}}-a_{i_{2}}^{2} \frac{\partial a_{i_{1}}^{1}}{\partial x_{i_{2}}}\right]\right) \partial_{x_{i_{1}}} .
\end{aligned}
$$

We deduce that $[\tilde{\mathbb{L}}, \tilde{\mathbb{L}}] \subset \widetilde{\mathbb{L}}$. In other words, $\widetilde{\mathbb{L}}^{\text {lie }}=\widetilde{\mathbb{L}}$. Next, we define the restriction

$$
\mathbb{L}^{\prime}:=\left\{L^{\prime}=\left.\widetilde{L}\right|_{\left\{x_{1}=0\right\}}: \widetilde{L} \in \widetilde{\mathbb{L}}\right\},
$$

and we claim that $\left(\mathbb{L}^{\prime}\right)^{\text {lie }}=\mathbb{L}^{\prime}$ also holds true. Indeed, restricting (1.7) above to $\left\{\mathrm{x}_{1}=0\right\}$, we observe that

$$
\left[\left.\widetilde{L}_{1}\right|_{\left\{\mathrm{x}_{1}=0\right\}},\left.\widetilde{L}_{2}\right|_{\left\{\mathrm{x}_{1}=0\right\}}\right]=\left.\left[\widetilde{L}_{1}, \widetilde{L}_{2}\right]\right|_{\left\{\mathrm{x}_{1}=0\right\}},
$$

since neither $\widetilde{L}_{1}$ nor $\widetilde{L}_{2}$ involves $\partial_{x_{1}}$. This shows that $\left[\mathbb{L}^{\prime}, \mathbb{L}^{\prime}\right] \subset \mathbb{L}^{\prime}$, as claimed.

Since $\left(\mathbb{L}^{\prime}\right)^{\text {lie }}=\mathbb{L}^{\prime}$, the induction assumption applies: there exists a local $\mathbb{K}$-analytic submanifold $\Lambda^{\prime}$ of $\mathbb{K}^{n-1}$ passing through the origin such that $T_{q^{\prime}} \Lambda^{\prime}=\mathbb{L}^{\prime}\left(q^{\prime}\right)$, for every point $q^{\prime} \in \Lambda^{\prime}$. Let $d$ denote its codimension. If $d=0$, i.e. if $\Lambda^{\prime}$ coincides with an open neighborhood of the origin in $\mathbb{K}^{n-1}$, it suffices to chose for $\Lambda$ an open neighborhood of the origin in $\mathbb{K}^{n}$. Assuming $d \geqslant 1$, we split the coordinates $\mathrm{x}=\left(\mathrm{x}_{1}, \mathrm{x}^{\prime}\right) \in \mathbb{K} \times \mathbb{K}^{n-1}$ and we let $\rho_{j}\left(\mathrm{x}^{\prime}\right)=0, j=1, \ldots, d$, denote local $\mathbb{K}$-analytic defining equations for 
$\Lambda^{\prime}$. We claim that it suffices to choose for $\Lambda$ the local submanifold of $\mathbb{K}^{n}$ with the same equations, hence having the same codimension.

Indeed, since these equations are independent of $x_{1}$, it is first of all clear that the vector field $\partial_{\mathrm{x}_{1}} \in \mathbb{L}$ is tangent to $\Lambda$. To conclude that every $L=$ $a \partial_{x_{1}}+\widetilde{L} \in \mathbb{L}$ is tangent to $\Lambda$, we thus have to prove that every $\widetilde{L} \in \widetilde{\mathbb{L}}$ is tangent to $\Lambda$.

Let $\widetilde{L}=\sum_{2 \leqslant i \leqslant n} a_{i}\left(\mathrm{x}, \mathrm{x}^{\prime}\right) \partial_{\mathrm{x}_{i}} \in \widetilde{\mathbb{L}}$. As a preliminary observation:

$$
\left(\operatorname{ad} \partial_{\mathrm{x}_{1}}\right) \widetilde{L}:=\left[\partial_{\mathrm{x}_{1}}, \widetilde{L}\right]=\sum_{2 \leqslant i \leqslant n} \frac{\partial a_{i}}{\partial \mathrm{x}_{1}}\left(\mathrm{x}_{1}, \mathrm{x}^{\prime}\right) \frac{\partial}{\partial \mathrm{x}_{i}},
$$

and more generally, for $\ell \in \mathbb{N}$ arbitrary:

$$
\left(\operatorname{ad} \partial_{\mathrm{x}_{1}}\right)^{\ell} \widetilde{L}=\sum_{2 \leqslant i \leqslant n} \frac{\partial^{\ell} a_{i}}{\partial \mathrm{x}_{1}^{\ell}}\left(\mathrm{x}_{1}, \mathrm{x}^{\prime}\right) \frac{\partial}{\partial \mathrm{x}_{i}} .
$$

Since $\mathbb{L}$ is a Lie algebra, we have $\left(\operatorname{ad} \partial_{x_{1}}\right)^{\ell} \widetilde{L} \in \mathbb{L}$. Since $\left(\operatorname{ad} \partial_{x_{1}}\right)^{\ell} \widetilde{L}$ does not involve $\partial_{x_{1}}$, according to its expression above, it belongs in fact to $\widetilde{\mathbb{L}}$. Also, after restriction $\left.\left(\operatorname{ad} \partial_{x_{1}}\right)^{\ell} \widetilde{L}\right|_{x_{1}=0} \in \mathbb{L}^{\prime}$. By assumption, $\mathbb{L}^{\prime}$ is tangent to $\Lambda^{\prime}$. We deduce that, for every $\ell \in \mathbb{N}$, the vector field

$$
L_{\ell}^{\prime}:=\left.\left(\operatorname{ad} \partial_{\mathrm{x}_{1}}\right)^{\ell} \widetilde{L}\right|_{\mathrm{x}_{1}=0}=\sum_{2 \leqslant i \leqslant n} \frac{\partial^{\ell} a_{i}}{\partial \mathrm{x}_{1}^{\ell}}\left(0, \mathrm{x}^{\prime}\right) \frac{\partial}{\partial \mathrm{x}_{i}}
$$

is tangent to $\Lambda^{\prime}$. Equivalently, $\left[L_{\ell}^{\prime} \rho_{j}\right]\left(\mathrm{x}^{\prime}\right)=0$ for every $\mathrm{x}^{\prime} \in \Lambda^{\prime}$. Letting $\left(\mathrm{x}_{1}, \mathrm{x}^{\prime}\right) \in \Lambda$, whence $\mathrm{x}^{\prime} \in \Lambda^{\prime}$, we compute:

$$
\begin{aligned}
{\left[\widetilde{L} \rho_{j}\right]\left(\mathrm{x}_{1}, \mathrm{x}^{\prime}\right) } & =\sum_{2 \leqslant i \leqslant n} a_{i}\left(\mathrm{x}_{1}, \mathrm{x}^{\prime}\right) \frac{\partial \rho_{j}}{\partial \mathrm{x}_{i}}\left(\mathrm{x}^{\prime}\right) \\
& =\sum_{2 \leqslant i \leqslant n} \sum_{\ell=0}^{\infty} \frac{\mathrm{x}_{1}^{\ell}}{\ell !} \frac{\partial^{\ell} a_{i}}{\partial \mathrm{x}_{1}^{\ell}}\left(0, \mathrm{x}^{\prime}\right) \frac{\partial \rho_{j}}{\partial \mathrm{x}_{i}}\left(\mathrm{x}^{\prime}\right) \quad \text { [Taylor development] } \\
& =\sum_{\ell=0}^{\infty} \frac{\mathrm{x}_{1}^{\ell}}{\ell !}\left[L_{\ell}^{\prime} \rho_{j}\right]\left(\mathrm{x}^{\prime}\right)=0
\end{aligned}
$$

so $\widetilde{L}$ is tangent to $\Lambda$. Finally, the property $T_{\mathrm{x}_{1}, \mathrm{x}^{\prime}} \Lambda=\mathbb{L}\left(\mathrm{x}_{1}, \mathrm{x}^{\prime}\right)$ follows immediately from $T_{x^{\prime}} \Lambda^{\prime}=\mathbb{L}^{\prime}\left(x^{\prime}\right)$ and the proof is complete (the Taylor development argument above was crucially used, and this enlightens why the theorem does not hold in the $\mathcal{C}^{\infty}$ category). 
1.8. Free Lie algebras and generic sets of $\mathbb{K}$-analytic vector fields. For a generic set of $r \geqslant 2$ vector fields $\mathbb{L}^{0}=\left\{L_{a}\right\}_{1 \leqslant a \leqslant r}$, or after slightly perturbing any given set, one expects that $\mathbb{L}^{\text {lie }}(0)=T_{0} \mathbb{K}^{n}$. Then the Nagano leaf $\Lambda$ passing through 0 is just an open neighborhood of 0 in $\mathbb{K}^{n}$. Also, one expects that the dimensions of the intermediate spaces $\mathbb{L}^{k}(0)$ be maximal.

To realize this intuition, one has to count the maximal number of iterated Lie brackets that are linearly independent in $\mathbb{L}^{k}$, for $k=1,2,3, \ldots$, modulo antisymmetry and Jacobi identity.

Let $r \geqslant 2$ and let $h_{1}, h_{2}, \ldots, h_{r}$ be $r$ linearly independent elements of a vector space over $\mathbb{K}$. The free Lie algebra $\mathrm{F}(r)$ of rank $r$ is the smallest (non-commutative, non-associative) $\mathbb{K}$-algebra ([Re1993]) having $h_{1}, h_{2}, \ldots, h_{r}$ as elements, with multiplication $\left(h, h^{\prime}\right) \mapsto h h^{\prime}$ satisfying antisymmetry $0=h h^{\prime}+h^{\prime} h$ and Jacobi identity $0=h\left(h^{\prime} h^{\prime \prime}\right)+$ $h^{\prime \prime}\left(h h^{\prime}\right)+h^{\prime}\left(h^{\prime \prime} h\right)$. It is unique up to isomorphism. The case $r=1$ yields only $\mathrm{F}(1)=\mathbb{K}$. The multiplication in $\mathrm{F}(r)$ plays the role of the Lie bracket in $\mathbb{L}^{\text {lie }}$. Importantly, no linear relation exists between iterated multiplications, i.e. between iterated Lie brackets, except those generated by antisymmetry and Jacobi identity. Thus, $\mathrm{F}(r)$ is infinite-dimensional. Every finite-dimensional Lie $\mathbb{K}$-algebra having $r$ generators embeds as a subalgebra of $\mathrm{F}(r)$, see [Re1993].

Since the bracket multiplication is not associative, one must carefully write some parentheses, for instance in $\left(h_{1} h_{2}\right) h_{3}$, or in $h_{1}\left(h_{2}\left(h_{1} h_{2}\right)\right)$, or in $\left(h_{1} h_{2}\right)\left(h_{3}\left(h_{5} h_{1}\right)\right)$. Writing all such words only with the alphabet $\left\{h_{1}, h_{2}, \ldots, h_{r}\right\}$, we define the length of a word $\mathbf{h}$ to be the number of elements $h_{i_{\alpha}}$ in it. For $\ell \in \mathbb{N}$ with $\ell \geqslant 1$, let $\mathrm{W}_{r}^{\ell}$ be the set of words of length equal to $\ell$ and let $\mathrm{W}_{r}=\bigcup_{\ell \geqslant 1} \mathrm{~W}_{r}^{\ell}$ be the set of all words.

Define $\mathrm{F}_{1}(r)$ to be the vector space generated by $h_{1}, h_{2}, \ldots, h_{r}$ and for $\ell \geqslant 2$, define $\mathrm{F}_{\ell}(r)$ to be the vector space generated by words of length $\leqslant \ell$. This corresponds to $\mathbb{L}^{\ell}$, except that in $\mathbb{L}^{\ell}$, there might exist special linear relations that are absent in the abstract case. Thus, $\mathrm{F}(r)$ is a graded Lie algebra. The Jacobi identity insures (by induction) that $\mathrm{F}_{\ell_{1}}(r) \mathrm{F}_{\ell_{2}}(r) \subset$ $\mathrm{F}_{\ell_{1}+\ell_{2}}(r)$, a property similar to $\left[\mathbb{L}^{k_{1}}, \mathbb{L}^{k_{2}}\right] \subset \mathbb{L}^{k_{1}+k_{2}}$. It follows that $\mathrm{F}_{\ell}(r)$ is generated by words of the form

$$
h_{i_{1}}\left(h_{i_{2}}\left(\ldots\left(h_{i_{\ell^{\prime}-1}} h_{i_{\ell^{\prime}}}\right) \ldots\right)\right)
$$

where $\ell^{\prime} \leqslant \ell$ and where $1 \leqslant i_{1}, i_{2}, \ldots, i_{\ell^{\prime}-1}, i_{\ell^{\prime}} \leqslant r$. For instance, $\left(h_{1} h_{2}\right)\left(h_{3}\left(h_{5} h_{1}\right)\right)$ may be written as a linear combination of such simple 
words whose length is $\leqslant 5$. Let us denote by

$$
\mathrm{SW}_{r}=\bigcup_{\ell \geqslant 1} \mathrm{SW}_{r}^{\ell}
$$

the set of these simple words, where $\mathrm{SW}_{r}^{\ell}$ denotes the set of simple words of length $\ell$. Although it generates $\mathrm{F}(r)$ as a vector space over $\mathbb{K}$, we point out that it is not a basis of $\mathrm{F}(r)$ : for instance, we have $h_{1}\left(h_{2}\left(h_{1} h_{2}\right)\right)=h_{2}\left(h_{1}\left(h_{1} h_{2}\right)\right)$, because of an obvious Jacobi identity in which $\left(h_{1} h_{2}\right)\left(h_{1} h_{2}\right)=0$ disappears. In fact, one verifies that this is the only Jacobi relation between simple words of length 4 , that simple words of length 5 have no Jacobi relation, hence a basis of $F_{5}(2)$ is

$$
\begin{aligned}
& h_{1}, \quad h_{2}, \quad h_{1} h_{2}, \\
& h_{1}\left(h_{1} h_{2}\right), \quad h_{2}\left(h_{1} h_{2}\right), \\
& h_{1}\left(h_{1}\left(h_{1} h_{2}\right)\right), \quad h_{1}\left(h_{2}\left(h_{1} h_{2}\right)\right), \quad h_{2}\left(h_{2}\left(h_{2} h_{1}\right)\right), \\
& h_{1}\left(h_{1}\left(h_{1}\left(h_{1} h_{2}\right)\right)\right), \quad h_{1}\left(h_{1}\left(h_{2}\left(h_{1} h_{2}\right)\right)\right), \quad h_{1}\left(h_{2}\left(h_{2}\left(h_{2} h_{1}\right)\right)\right), \\
& \qquad h_{2}\left(h_{1}\left(h_{1}\left(h_{1} h_{2}\right)\right)\right), \quad h_{2}\left(h_{2}\left(h_{1}\left(h_{2} h_{1}\right)\right)\right), \quad h_{2}\left(h_{2}\left(h_{2}\left(h_{2} h_{1}\right)\right)\right) .
\end{aligned}
$$

In general, what are the dimensions of the $\mathrm{F}_{\ell}(r)$ ? How to find bases for them, when considered as vector spaces?

Definition 1.9. A Hall-Witt basis of $\mathrm{F}(r)$ is a linearly ordered (infinite) subset $\mathrm{HW}_{r}=\bigcup_{\ell \geqslant 1} \mathrm{HW}_{r}^{\ell}$ of the set of simple words $\mathrm{SW}_{r}$ such that:

- if two simple words $\mathbf{h}$ and $\mathbf{h}^{\prime}$ satisfy length $(\mathbf{h})<\operatorname{length}\left(\mathbf{h}^{\prime}\right)$, then $\mathbf{h}<\mathbf{h}^{\prime}$;

- $\mathrm{HW}_{r}^{1}=\left\{h_{1}, h_{2}, \ldots, h_{r}\right\}$;

- $\mathrm{HW}_{r}^{2}=\left\{h_{i_{1}} h_{i_{2}}: 1 \leqslant i_{1}<i_{2} \leqslant r\right\}$;

- $\mathrm{HW}_{r} \backslash\left(\mathrm{HW}_{r}^{1} \cup \mathrm{HW}_{r}^{2}\right)=\left\{\mathbf{h}\left(\mathbf{h}^{\prime} \mathbf{h}^{\prime \prime}\right): \mathbf{h}, \mathbf{h}^{\prime}, \mathbf{h}^{\prime \prime} \in \mathrm{HW}_{r}, \mathbf{h}^{\prime}<\right.$ $\mathbf{h}^{\prime \prime}$ and $\left.\mathbf{h}^{\prime} \leqslant \mathbf{h}<\mathbf{h}^{\prime} \mathbf{h}^{\prime \prime}\right\}$.

A Hall-Witt basis essentially consists of the choice, for every $\ell \geqslant 1$, of some (among many possible) finite subset $\mathrm{HW}_{r}^{\ell}$ of $\mathrm{SW}_{r}^{\ell}$ that generates the finite-dimensional quotient vector space $\mathrm{F}_{\ell}(r) / \mathrm{F}_{\ell-1}(r)$. To fix ideas, an arbitrary linear ordering is added among the elements of the chosen basis $\mathrm{HW}_{r}^{\ell}$ of the vector space $\mathrm{F}_{\ell}(r) / \mathrm{F}_{\ell-1}(r)$. The last condition of the definition takes account of the Jacobi identity.

Theorem 1.10. ([Bo1972, Re1993] ) Hall-Witt bases exist and are bases of the free Lie algebra $\mathrm{F}(r)$ of rank $r$, when considered as a vector space. The 
dimensions $\mathrm{n}_{\ell}(r)-\mathrm{n}_{\ell-1}(r)$ of $\mathrm{F}_{\ell}(r) / \mathrm{F}_{\ell-1}(r)$, or equivalently the cardinals of $\mathrm{HW}_{r}^{\ell}$, satisfy the induction relation

$$
\mathrm{n}_{\ell}(r)-\mathrm{n}_{\ell-1}(r)=\frac{1}{\ell} \sum_{d \text { divides } \ell} \mu(d) r^{\ell / d}
$$

where $\mu$ is the Möbius function.

Remind that $\mu(d)=\left\{\begin{array}{l}1, \text { if } d=1 \\ 0, \text { if } d \text { contains square integer factors; } \\ (-1)^{\nu}, \text { if } d=p_{1} \cdots p_{\nu} \text { is the product of } \nu \text { distinct prime numbers. }\end{array}\right.$

Now, we come back to the system $\mathbb{L}^{0}=\left\{L_{a}\right\}_{1 \leqslant a \leqslant r}$ of local $\mathbb{K}$-analytic vector fields of $\S 1.1$, where $L_{a}=\sum_{i=1}^{n} \varphi_{a, i}(\mathrm{x}) \frac{\partial}{\partial \mathrm{x}_{i}}$. If the vector space $\mathbb{L}(0)$ has dimension $<r$, a slight perturbation of the coefficients $\varphi_{a, i}(\mathrm{x})$ of the $L_{a}$ yields a system $\mathbb{L}^{\prime 0}$ with $\mathbb{L}^{\prime}(0)$ of dimension $=r$. By an elementary computation with Lie brackets, one sees that a further slight perturbation yields a system $\mathbb{L}^{\prime \prime}$ with $\mathbb{L}^{\prime \prime}(0)$ of dimension $r+\frac{r(r-1)}{2}=\mathrm{n}_{2}(r)$.

To pursue, any simple iterated Lie bracket $\left[L_{a_{1}},\left[L_{a_{2}}, \ldots\left[L_{a_{\ell-1}}, L_{a_{\ell}}\right] \ldots\right]\right]$ of length $\ell$ is a vector field $\sum_{i=1}^{n} A_{a_{1}, a_{2}, \ldots, a_{\ell-1}, a_{\ell}}^{i} \frac{\partial}{\partial x_{i}}$ having coefficients $A_{a_{1}, a_{2}, \ldots, a_{\ell-1}, a_{\ell}}^{i}$ that are universal polynomials in the jets

$$
J_{\times}^{\ell-1} \varphi(\mathrm{x}):=\left(\partial_{\mathrm{x}}^{\alpha} \varphi_{a, i}(\mathrm{x})\right)_{1 \leqslant a \leqslant r, 1 \leqslant i \leqslant n}^{\alpha \in \mathbb{N}^{n},|\alpha| \leqslant \ell-1} \in \mathbb{K}^{N_{r n, n, \ell-1}}
$$

of order $(\ell-1)$ of the coefficients of $L_{1}, L_{2}, \ldots, L_{r}$. Here, $N_{r n, n, \ell-1}=$ $r n \frac{(n+\ell-1) !}{n !(\ell-1) !}$ denotes the number of such independent partial derivatives. A careful inspection of the polynomials $A_{a_{1}, a_{2}, \ldots, a_{\ell-1}, a_{\ell}}^{i}$ enables to get the following genericity statement, whose proof will appear elsewhere. It says in a precise way that $\mathbb{L}^{\text {lie }}(0)=T_{0} \mathbb{K}^{n}$ with the maximal freedom, for generic sets of vector fields.

Theorem 1.11. ([GV1987, Ge1988], [*]) If $\ell_{0}$ denotes the smallest length $\ell$ such that $\mathrm{n}_{\ell}(r) \geqslant n$, there exists a proper $\mathbb{K}$-algebraic subset $\Sigma$ of the jet space $J_{0}^{\ell_{0}-1} \varphi=\mathbb{K}^{N_{r n, n, \ell_{0}-1}}$ such that for every collection $\mathbb{L}^{0}=\left\{L_{a}\right\}_{1 \leqslant a \leqslant r}$ of $r$ vector fields $L_{a}=\sum_{i=1}^{n} \varphi_{a, i}(\mathrm{x}) \frac{\partial}{\partial \mathrm{x}_{i}}$ such that $J_{0}^{\ell_{0}-1} \varphi(0)$ does not belong to $\Sigma$, the following two properties hold:

- $\operatorname{dim} \mathbb{L}^{\ell}(0)=\mathrm{n}_{\ell}(r)$, for every $\ell \leqslant \ell_{0}-1$,

- $\operatorname{dim} \mathbb{L}^{\ell_{0}}(0)=n$, hence $\mathbb{L}^{\text {lie }}(0)=T_{0} \mathbb{K}^{n}$. 
The number of divisors of $\ell$ being an $\mathrm{O}\left(\frac{\log \ell}{\log 2}\right)$, one verifies that $\mathrm{n}_{\ell}(r)-$ $\mathrm{n}_{\ell-1}(r)=\frac{1}{\ell} r^{\ell}+\mathrm{O}\left(r^{\ell / 2} \frac{\log \ell}{\log 2}\right)$. It follows that, for $r$ fixed, the integer $\ell_{0}$ of the theorem is equivalent to $\frac{\log n}{\log r}$ as $n \rightarrow \infty$.

1.12. Local orbits of $\mathbb{K}$-analytic and of (Nash) $\mathbb{K}$-algebraic systems. We now describe a second, more concrete, simple and useful approach to the local Nagano Theorem 1.5. It is inspired by Sussmann's Theorem 1.21(III) and does not involve the consideration of any Lie bracket. Theorem 1.13 below will be applied in $\S 2.11$.

As above, consider a finite set

$$
\mathbb{L}^{0}:=\left\{L_{a}\right\}_{1 \leqslant a \leqslant r}, \quad r \in \mathbb{N}, \quad r \geqslant 1,
$$

of nonzero vector fields defined in the cube $\square_{\rho_{1}}^{n}$ and having $\mathbb{K}$-analytic coefficients. We shall neither consider its $\mathbb{A}_{\rho_{1}}$-linear hull $\mathbb{L}$, nor $\mathbb{L}^{\text {lie }}$. We will reconstruct the Nagano leaf passing through the origin only by means of the flows of $L_{1}, L_{2}, \ldots, L_{r}$.

Referring the reader to $\S 1.3$ (III) for background, we denote the flow map of a vector field $L \in \mathbb{L}^{0}$ shortly by $(\mathrm{t}, \mathrm{x}) \mapsto L_{\mathrm{t}}(\mathrm{x})=\exp (\mathrm{t} L)(\mathrm{x})$. It is $\mathbb{K}$ analytic. What happens in the algebraic category?

So, assume that the coefficients of all vector fields $L \in \mathbb{L}^{0}$ are $\mathbb{K}$ algebraic. Unfortunately, algebraicity fails to be preserved under integration, so the flows are only $\mathbb{K}$-analytic, in general. To get algebraicity of Nagano leaves, there is nothing else than supposing that the flows are algebraic, which we will do (second phrase of (5) below).

Choose now $\rho_{2}$ with $0<\rho_{2}<\rho_{1}$. Let $k \in \mathbb{N}$ with $k \geqslant 1$, let $L=$ $\left(L^{1}, \ldots, L^{k}\right) \in\left(\mathbb{L}^{0}\right)^{k}$, let $\mathrm{t}=\left(\mathrm{t}_{1}, \ldots, \mathrm{t}_{k}\right) \in \mathbb{K}^{k}$ with $|\mathrm{t}|<\rho_{2}$, i.e. $\mathrm{t} \in \square_{\rho_{2}}^{k}$, and let $\mathrm{x} \in \square_{\rho_{2}}^{n}$. We shall adopt the contracted notation

$$
L_{\mathrm{t}}(\mathrm{x}):=L_{\mathrm{t}_{k}}^{k}\left(\cdots\left(L_{\mathrm{t}_{1}}^{1}(\mathrm{x})\right) \cdots\right)
$$

for the composition of flow maps, whenever it is defined. In fact, since $L_{0}(0)=\exp (0 L)(0)=0$, it is clear that if we bound the length $k \leqslant 2 n$, then there exists $\rho_{2}>0$ sufficiently small such that all maps $(\mathrm{t}, \mathrm{x}) \mapsto L_{\mathrm{t}}(\mathrm{x})$ are well-defined, with $L_{\mathrm{t}}(\mathrm{x}) \in \square_{\rho_{1}}^{n}$, at least for all $\mathrm{t} \in \square_{\rho_{2}}^{k}$ and all $\mathrm{x} \in \square_{\rho_{2}}^{n}$. The reason why we may restrict to consider only compositions of length $k \leqslant 2 n$ will appear a posteriori in the proof of the theorem below. We shall be concerned with rank properties of $(\mathrm{t}, \mathrm{x}) \mapsto L_{\mathrm{t}}(\mathrm{x})$.

Let $n \geqslant 1, m \geqslant 1, \rho_{1}>0, \sigma_{1}>0$ and let $f: \square_{\rho_{1}}^{n} \rightarrow \square_{\sigma_{1}}^{m}, \mathrm{x} \mapsto f(\mathrm{x})$, be a $\mathbb{K}$-algebraic or $\mathbb{K}$-analytic map between two open cubes. Denote its Jacobian matrix by $\operatorname{Jac}(f)=\left(\frac{\partial f_{j}}{\partial \mathrm{x}_{i}}(\mathrm{x})\right)_{\substack{1 \leqslant j \leqslant n \\ 1 \leqslant i \leqslant n}}$. At a point $\mathrm{x} \in \square_{\rho_{1}}^{n}$, the map 
$f$ has rank $r$ if and only if Jac $f$ has rank $r$ at $\mathrm{x}$. Equivalently, by linear algebra, there is a $r \times r$ minor that does not vanish at $\mathrm{x}$ but all $s \times s$ minors with $r+1 \leqslant s \leqslant n$ do vanish at $\mathrm{x}$.

For every $s \in \mathbb{N}$ with $1 \leqslant s \leqslant \min (n, m)$, compute all the possible $s \times s$ minors $\Delta_{1}^{s \times s}, \ldots, \Delta_{N(s)}^{s \times s}$ of Jac $(f)$. They are universal (homogeneous of degree $s$ ) polynomials in the partial derivatives of $f$, hence are all $\mathbb{K}$ algebraic or $\mathbb{K}$-analytic functions. Let $e$ with $0 \leqslant e \leqslant \min (n, m)$ be the maximal integer $s$ with the property that there exists a minor $\Delta_{\mu}^{s \times s}(\mathrm{x})$, $1 \leqslant \mu \leqslant N(s)$, not vanishing identically. Then the set

$$
\mathcal{R}_{f}:=\left\{\mathrm{x} \in \square_{\rho_{1}}^{n}: \Delta_{\mu}^{s \times s}(\mathrm{x})=0, \mu=1, \ldots, N(s)\right\}
$$

is a proper $\mathbb{K}$-algebraic or analytic subset of $\square_{\rho_{1}}^{n}$. The principle of analytic continuation insures that $\square_{\rho_{1}}^{n} \backslash \mathcal{R}_{f}$ is open and dense.

The integer $e$ is called the generic rank of $f$. For every open, connected and nonempty subset $\Omega \subset \square_{\rho_{1}}^{n}$ the restriction $\left.f\right|_{\Omega}$ has the same generic rank $e$.

Theorem 1.13. ([Me1999, Me2001a, Me2004a] $]$ There exists an integer $e$ with $1 \leqslant e \leqslant n$ and an e-tuple of vector fields $L^{*}=\left(L^{* 1}, \ldots, L^{* e}\right) \in$ $\left(\mathbb{L}^{0}\right)^{e}$ such that the following six properties hold true.

(1) For every $k=1, \ldots, e$, the map $\left(\mathrm{t}_{1}, \ldots, \mathrm{t}_{k}\right) \mapsto$ $L_{\mathrm{t}_{k}}^{* k}\left(\cdots\left(L_{\mathrm{t}_{1}}^{* 1}(0)\right) \cdots\right)$ is of generic rank equal to $k$.

(2) For every arbitrary element $L^{\prime} \in \mathbb{L}^{0}$, the map $\left(t_{1}, \ldots, t_{e}, t^{\prime}\right) \mapsto$ $L_{\mathrm{t}^{\prime}}^{\prime}\left(L_{\mathrm{t}_{e}}^{* e}\left(\cdots\left(L_{\mathrm{t}_{1}}^{* 1}(0)\right) \cdots\right)\right)$ is of generic rank e, hence $e$ is the maximal possible generic rank.

(3) There exists an element $\mathrm{t}^{*} \in \square_{\rho_{2}}^{e}$ arbitrarily close to the origin which is of the special form $\left(\mathrm{t}_{1}^{*}, \ldots, \mathrm{t}_{e-1}^{*}, 0\right)$, namely with $\mathrm{t}_{e}^{*}=0$, and there exists an open connected neighborhood $\omega^{*}$ of $\mathrm{t}_{*}$ in $\square_{\rho_{2}}^{e}$ such that the map $\mathrm{t} \mapsto L_{\mathrm{t}_{e}}^{* e}\left(\cdots\left(L_{\mathrm{t}_{1}}^{* 1}(0)\right) \cdots\right)$ is of constant rank $e$ in $\omega^{*}$.

(4) Setting $L^{*}:=\left(L^{* 1}, \ldots, L^{* e}\right), K^{*}:=\left(L^{* e-1}, \ldots, L^{* 1}\right)$ and $s^{*}:=$ $\left(-\mathrm{t}_{e-1}^{*}, \ldots,-\mathrm{t}_{1}^{*}\right)$, we have $K_{s^{*}}^{*} \circ L_{\mathrm{t}^{*}}^{*}(0)=0$ and the map $\psi: \omega^{*} \rightarrow$ $\square_{\rho_{1}}^{n}$ defined by $\psi: \mathrm{t} \mapsto K_{s^{*}}^{*} \circ L_{\mathrm{t}}^{*}(0)$ is also of constant rank equal to $e$ in $\omega^{*}$.

(5) The image $\Lambda:=\psi\left(\omega^{*}\right)$ is a piece of $\mathbb{K}$-analytic submanifold passing through the origin enjoying the most important property that every vector field $L^{\prime} \in \mathbb{L}^{0}$ is tangent to $\Lambda$. If the flows of all elements of $\mathbb{L}^{0}$ are algebraic, $\Lambda$ is $\mathbb{K}$-algebraic. 
(6) Every local $\mathbb{K}$-algebraic or $\mathbb{K}$-analytic submanifold $\Lambda^{\prime}$ passing trough the origin to which all vector fields $L^{\prime} \in \mathbb{L}^{0}$ are tangent must contain $\Lambda$ in a neighborhood of 0 .

In conclusion, the dimension e of $\Lambda$ is characterized by the generic rank properties (1) and (2).

Previously, $\Lambda$ was called Nagano leaf. Since the above statement is superseded by Sussmann's Theorem 1.21(III), we prefer to call it the local $\mathbb{L}$-orbit of 0 , introducing in advance the terminology of Part III and denoting it by $\mathcal{O}_{\mathbb{L}^{0}}^{\text {loc }}\left(\square_{\rho_{1}}^{n}, 0\right)$. The integer $e$ of the theorem is $\leqslant n$, just because the target of the maps $\left(\mathrm{t}_{1}, \ldots, \mathrm{t}_{k}\right) \mapsto L_{\mathrm{t}_{k}}^{* k}\left(\cdots\left(L_{\mathrm{t}_{1}}^{* 1}(0)\right) \cdots\right)$ is $\mathbb{K}^{n}$. It follows that in (4) and (5) we need $2 e-1 \leqslant 2 n-1$ compositions of flows to cover $\Lambda$.

We quickly mention an application about separate algebraicity. In [BM1949], it is shown that a local $\mathbb{K}$-analytic function $g: \square_{\rho_{1}}^{n} \rightarrow \mathbb{K}$ is $\mathbb{K}$-algebraic if and only if its restriction to every affine coordinate segment is $\mathbb{K}$-algebraic. Call the system $\mathbb{L}^{0}$ minimal at the origin if $\mathcal{O}_{\mathbb{L}^{0}}^{l o c}\left(\square_{\rho_{1}}^{n}, 0\right)$ contains a neighborhood of the origin. Equivalently, the integer $e$ of Theorem 1.13 equals $n$.

Theorem 1.14. ([Me2001a]) If $\mathbb{L}^{0}=\left\{L_{a}\right\}_{1 \leqslant a \leqslant r}$ is minimal at 0 , a local $\mathbb{K}$ analytic function $g: \square_{\rho_{1}}^{n} \rightarrow \mathbb{K}$ is $\mathbb{K}$-algebraic if and only it its restriction to every integral curve of every $L_{a} \in \mathbb{L}^{0}$ is $\mathbb{K}$-algebraic.

Proof of Theorem 1.13. (May be skipped in a first reading.) If all vector fields of $\mathbb{L}^{0}$ vanish at the origin, $\Lambda=\{0\}$. We now exclude this possibility. Choose a vector field $L^{* 1} \in \mathbb{L}^{0}$ which does not vanish at 0 . The map $\mathrm{t}_{1} \mapsto L_{\mathrm{t}_{1}}^{* 1}(0)$ is of (generic) rank one at every $\mathrm{t}_{1} \in \square_{\rho_{2}}^{1}$. If there exists $L^{\prime} \in \mathbb{L}^{0}$ such that the map $\left(\mathrm{t}_{1}, \mathrm{t}^{\prime}\right) \mapsto L_{\mathrm{t}^{\prime}}^{\prime}\left(L_{\mathrm{t}_{1}}^{* 1}(0)\right)$ is of generic rank two, we choose one such $L^{\prime}$ and we denote it by $L^{* 2}$. Continuing in this way, we get vector fields $L^{* 1}, \ldots, L^{* e}$ satisfying properties (1) and (2), with $e \leqslant n$.

Since the generic rank of the map $\left(\mathrm{t}_{1}, \ldots, \mathrm{t}_{e}\right) \mapsto L_{\mathrm{t}_{e}}^{* e}\left(\cdots\left(L_{\mathrm{t}_{1}}^{* 1}(0)\right) \cdots\right)$ equals $e$, and since this map is $\mathbb{K}$-analytic, there exists a $t^{*} \in \square_{\rho_{2}}^{e}$ arbitrarily close to the origin at which its rank equals $e$. We claim that we can moreover choose $\mathrm{t}^{*}$ to be of the special form $\left(\mathrm{t}_{1}^{*}, \ldots, \mathrm{t}_{e-1}^{*}, 0\right)$, i.e. with $t_{e}^{*}=0$. It suffices to apply the following lemma to $\varphi(\mathrm{t}):=L_{\mathrm{t}_{e-1}}^{* e-1}\left(\cdots\left(L_{\mathrm{t}_{1}}^{* 1}(0)\right) \cdots\right)$ and to $L^{\prime}:=L^{* e}$.

Lemma 1.15. Let $n \in \mathbb{N}, n \geqslant 1$, let $e \in \mathbb{N}, 1 \leqslant e \leqslant n$, let $\mathrm{t} \in \square_{\rho_{2}}^{e-1}$ and let

$$
\square_{\rho_{2}}^{e-1} \ni \mathrm{t} \mapsto \varphi(\mathrm{t})=\left(\varphi_{1}(\mathrm{t}), \ldots, \varphi_{n}(\mathrm{t})\right) \in \square_{\rho_{1}}^{n}
$$


be a $\mathbb{K}$-analytic map whose generic rank equals $(e-1)$. Let $L^{\prime}$ be a $\mathbb{K}$ analytic vector field and assume that the map $\psi:\left(\mathrm{t}, \mathrm{t}^{\prime}\right) \mapsto L_{\mathrm{t}^{\prime}}^{\prime}(\varphi(\mathrm{t}))$ has generic rank $e$. Then there exists a point $\left(\mathrm{t}^{*}, 0\right)$ arbitrarily close to the origin at which the rank of $\psi$ is equal to $e$.

Proof. Choose $\mathrm{t}^{\sharp} \in \square_{\rho_{2}}^{e-1}$ arbitrarily close to zero at which $\varphi$ has maximal rank, equal to $(e-1)$. Since the rank is lower semi-continuous, there exists a connected neighborhood $\omega^{\sharp}$ of $t^{\sharp}$ in $\square_{\rho_{2}}^{e-1}$ such that $\varphi$ has rank $(e-1)$ at every point of $\omega^{\sharp}$. By the constant rank theorem, $\Pi:=\varphi\left(\omega^{\sharp}\right)$ is then a local $\mathbb{K}$-analytic submanifold of $\square_{\rho_{1}}^{n}$ passing through the point $\varphi\left(\mathrm{t}^{\sharp}\right)$. To complete the proof, we claim that there exists $t^{*} \in \omega^{\sharp}$ arbitrarily close to $\mathrm{t}^{\sharp}$ such that the map $\left(\mathrm{t}, \mathrm{t}^{\prime}\right) \mapsto L_{\mathrm{t}^{\prime}}^{\prime}(\varphi(\mathrm{t}))$ has rank $e$ at $\left(\mathrm{t}^{*}, 0\right)$.

Let us reason by contradiction, supposing that at all points of the form $\left(\mathrm{t}^{*}, 0\right)$, for $\mathrm{t}^{*} \in \omega^{\sharp}$, the map $\psi:\left(\mathrm{t}, \mathrm{t}^{\prime}\right) \mapsto L_{\mathrm{t}^{\prime}}^{\prime}(\varphi(\mathrm{t}))$ has rank equal to $(e-1)$. Pick an arbitrary $\mathrm{t}^{*} \in \omega^{\sharp}$. Reminding that when $\mathrm{t}^{\prime}=0$, we have $L_{\mathrm{t}^{\prime}}^{\prime}=L_{0}^{\prime}=\mathrm{Id}$, we observe that $\psi(\mathrm{t}, 0) \equiv \varphi(\mathrm{t})$. Consequently, the partial derivatives of $\psi$ with respect to the variables $\mathrm{t}_{i}, i=1, \ldots, e-1$ at an arbitrary point $\left(\mathrm{t}^{*}, 0\right)$, with $\mathrm{t}^{*} \in \omega^{\sharp}$, coincide with the $(e-1)$ linearly independent vectors $\frac{\partial \varphi}{\partial \mathrm{t}_{i}}\left(\mathrm{t}^{*}\right) \in \mathbb{K}^{n}, i=1, \ldots, e-1$. In fact, the tangent space to $\Pi$ at the point $\psi\left(\mathrm{t}^{*}, 0\right)=\varphi\left(\mathrm{t}^{*}\right)$ is generated by these $(e-1)$ vectors.

Reminding the fundamental property $\left.\frac{\partial}{\partial \mathrm{t}^{\prime}} L_{\mathrm{t}^{\prime}}^{\prime}(\mathrm{x})\right|_{\mathrm{t}^{\prime}=0}=L^{\prime}(\mathrm{x})$, we deduce [from our assumption that the map $\left(\mathrm{t}, \mathrm{t}^{\prime}\right) \mapsto L_{\mathrm{t}^{\prime}}^{\prime}(\varphi(\mathrm{t}))$ has rank equal to $(e-1)]$ that the vector

$$
\left.\frac{\partial}{\partial \mathrm{t}^{\prime}} L_{\mathrm{t}^{\prime}}^{\prime}(\varphi(\mathrm{t}))\right|_{\mathrm{t}^{\prime}=0}=L^{\prime}(\varphi(\mathrm{t}))
$$

must be linearly dependent with the $(e-1)$ vectors $\frac{\partial \varphi}{\partial \mathrm{t}_{i}}(\mathrm{t}), i=1, \ldots, e-$ 1 , for every $\mathrm{t} \in \omega^{\sharp}$. Equivalently, the vector field $L^{\prime}$ is tangent to the submanifold $\Pi$. It follows that the local flow of $L^{\prime}$ necessarily stabilizes $\Pi$ : if $\mathrm{x}=\varphi(\mathrm{t}) \in \Pi, \mathrm{t} \in \omega^{\sharp}$, then $L_{\mathrm{t}^{\prime}}^{\prime}(\mathrm{x}) \in \Pi$, for all $\mathrm{t}^{\prime} \in \square_{\rho(\mathrm{t})}^{1}$, where $\rho(\mathrm{t})>0$ is sufficiently small. Set $\Omega^{\sharp}:=\left\{\left(\mathrm{t}, \mathrm{t}^{\prime}\right): \mathrm{t} \in \omega^{\sharp}, \mathrm{t}^{\prime} \in \square_{\rho(\mathrm{t})^{1}}\right\}$. It is a nonempty connected open subset of $\square_{\rho_{2}}^{e}$. We have thus deduced that $\psi\left(\Omega^{\sharp}\right)$ is contained in the $(e-1)$-dimensional submanifold $\Pi$. This constraint entails that $\psi$ is of rank $\leqslant e-1$ at every point of $\Omega^{\sharp}$. However, $\left.\psi\right|_{\Omega^{\sharp}}$ being $\mathbb{K}$-analytic and of generic rank equal to $e$, by assumption, it should be of rank $e$ at every point of an open dense subset of $\Omega^{\sharp}$. This is the desired contradiction which proves the lemma. 
Hence, there exists $\mathrm{t}^{*}=\left(\mathrm{t}_{1}^{*}, \ldots, \mathrm{t}_{e-1}^{*}, 0\right) \in \square_{\rho_{2}}^{e}$ arbitrarily close to the origin at which the rank of $\mathrm{t} \mapsto L_{\mathrm{t}_{e}}^{* e}\left(\cdots\left(L_{\mathrm{t}_{1}}^{* 1}(0)\right) \cdots\right)$ is maximal (hence locally constant) equal to $e$, so we get the constant rank property (3), for a sufficiently small neighborhood $\omega^{*}$ of $t^{*}$.

In (4), the property $K_{s^{*}}^{*} \circ L_{t^{*}}^{*}(0)=0$ is obvious, using $\mathrm{x} \equiv L_{0}(\mathrm{x}) \equiv$ $L_{-\mathrm{t}} \circ L_{\mathrm{t}}(\mathrm{x})$ :

$$
L_{-\mathrm{t}_{1}^{*}}^{* 1} \circ \cdots \circ L_{-\mathrm{t}_{e-1}^{*}}^{*} \circ L_{0}^{* e} \circ L_{\mathrm{t}_{e-1}^{*}}^{*} \circ \cdots \circ L_{\mathrm{t}_{1}^{*}}^{*}(\mathrm{x}) \equiv \mathrm{x} .
$$

Since the map $\mathrm{x} \mapsto K_{s^{*}}^{*}(\mathrm{x})$ is a local diffeomorphism, it is clear that the map $\psi: \mathrm{t} \mapsto K_{\mathrm{s}^{*}}^{*} \circ L_{\mathrm{t}}^{*}(0)$ is also of constant rank $e$ in $\omega^{*}$. Thus, we obtain (4), and moreover, by the constant rank theorem, the image $\Lambda:=$ $\psi\left(\omega^{*}\right)$ constitutes a local $\mathbb{K}$-analytic submanifold of $\mathbb{K}^{n}$ passing through the origin. If the flows of elements of $\mathbb{L}^{0}$ are all $\mathbb{K}$-algebraic, clearly $\psi$ and $\Lambda$ are also $\mathbb{K}$-algebraic.

It remains to check that every vector field $L^{\prime} \in \mathbb{L}^{0}$ is tangent to $\Lambda$. As a preliminary, denote by $L_{\mathrm{t}^{\prime}}^{\prime}(\varphi(\mathrm{t})), \mathrm{t} \in \square_{\rho_{2}}^{e}, \mathrm{t}^{\prime} \in \square_{\rho_{2}}^{1}$, the map appearing in (2), where $L^{\prime} \in \mathbb{L}^{0}$ is arbitrary. Reasoning as in the lemma above, we see that $L^{\prime}$ is necessarily tangent to some local submanifold $\Pi$ obtained as the local image of an open connected set where $\varphi$ has maximal locally constant rank. It follows that the flows and the multiple flows of elements of $\mathbb{L}^{0}$ stabilize this submanifold. We deduce a generalization of (2): for $k \leqslant 2 n$, for $L^{\prime} \in\left(\mathbb{L}^{0}\right)^{k}$, for $\mathrm{t}^{\prime} \in \square_{\rho_{2}}^{k}$, the map $\left(\mathrm{t}, \mathrm{t}^{\prime}\right) \longmapsto L_{\mathrm{t}^{\prime}}^{\prime}\left(L_{\mathrm{t}_{e}}^{* e}\left(\cdots\left(L_{\mathrm{t}_{1}}^{* 1}(0)\right) \cdots\right)\right)$ is of generic rank $e$.

In particular, for every $L^{\prime} \in \mathbb{L}^{0}$, the map $\left(\mathrm{t}^{\prime}, \mathrm{s}, \mathrm{t}\right) \longmapsto L_{\mathrm{t}^{\prime}}^{\prime} \circ K_{\mathrm{s}}^{*} \circ L_{\mathrm{t}}^{*}(0)$ is of generic rank $e$. In fact, the restriction $\psi: \mathrm{t} \mapsto K_{\mathrm{s}^{*}}^{*} \circ L_{\mathrm{t}}^{*}(0)$ of this map to the open set $\left\{\left(0, s^{*}, t\right): t \in \omega^{*}\right\}$ is already of rank $e$ at every point and its image is the local submanifold $\Lambda$, by the above construction. So the map $\left(\mathrm{t}^{\prime}, \mathrm{t}\right) \longmapsto L_{\mathrm{t}^{\prime}}^{\prime} \circ K_{\mathrm{s}^{*}}^{*} \circ L_{\mathrm{t}}^{*}(0)$ must be of rank $e$ at every point. In particular, the vector

$$
\left.\frac{\partial}{\partial \mathrm{t}^{\prime}} L_{\mathrm{t}^{\prime}}^{\prime} \circ K_{\mathrm{s}^{*}}^{*} \circ L_{\mathrm{t}}^{*}(0)\right|_{\mathrm{t}^{\prime}=0}=L^{\prime}\left(K_{\mathrm{s}^{*}}^{*} \circ L_{\mathrm{t}}^{*}(0)\right)
$$

must necessarily be tangent to $\Lambda$ at the point $K_{\mathrm{s}^{*}}^{*} \circ L_{\mathrm{t}}^{*}(0) \in \Lambda$. Thus, (5) is proved.

Take $\Lambda^{\prime}$ as in (6). The local flows of all vector $L^{\prime} \in \mathbb{L}^{0}$ stabilize $\Lambda^{\prime}$. Shrinking $\rho_{2}$ if necessary, all the maps $(\mathrm{t}, \mathrm{x}) \longmapsto L_{\mathrm{t}}(\mathrm{x})$ have range in $\Lambda^{\prime}$. So $\Lambda \subset \Lambda^{\prime}$, proving (6). 


\section{$\S 2$. ANALytiC CR MANifolds, SEgRE CHAins AND Minimality}

2.1. Local Cauchy-Riemann submanifolds of $\mathbb{C}^{n}$. Let $\left(z_{1}, \ldots, z_{n}\right)=$ $\left(x_{1}+i y_{1}, \ldots, x_{n}+i y_{n}\right)$ denote the canonical coordinates on $\mathbb{C}^{n}$. As before, we use the maximum norms $|x|=\max _{1 \leqslant k \leqslant n}\left|x_{k}\right|,|y|=\max _{1 \leqslant k \leqslant n}\left|y_{k}\right|$ and $|z|=\max _{1 \leqslant k \leqslant n}\left|z_{k}\right|$, where $\left|z_{k}\right|=\left(x_{k}^{2}+y_{k}^{2}\right)^{1 / 2}$. If $\rho>0$, we denote by $\Delta_{\rho}^{n}=\left\{z \in \mathbb{C}^{n}:|z|<\rho\right\}$ the open polydisc of radius $\rho$ centered at the origin, not to be confused with $\square_{\rho}^{2 n}=\left\{x+i y \in \mathbb{C}^{n}:|x|,|y|<\rho\right\}$.

Let $J$ denote the complex structure of $T \mathbb{C}^{n}$, acting on real vectors as if it were multiplication by $\sqrt{-1}$. Precisely, if $p$ is any point, $T_{p} \mathbb{C}^{n}$ is spanned by the $2 n$ vectors $\left.\frac{\partial}{\partial x_{k}}\right|_{p},\left.\frac{\partial}{\partial y_{k}}\right|_{p}, k=1, \ldots, n$, and $J$ acts as follows: $\left.J \frac{\partial}{\partial x_{k}}\right|_{p}=\left.\frac{\partial}{\partial y_{k}}\right|_{p} ;\left.J \frac{\partial}{\partial y_{k}}\right|_{p}=-\left.\frac{\partial}{\partial x_{k}}\right|_{p}$.

Choose the origin as a center point and consider a real $d$-codimensional local submanifold $M$ of $\mathbb{C}^{n} \simeq \mathbb{R}^{2 n}$ passing through the origin, defined by $d$ Cartesian equations $r_{1}(x, y)=\cdots=r_{d}(x, y)=0$, where the differentials $d r_{1}, \ldots, d r_{d}$ are linearly independent at the origin. The functions $r_{j}$ are assumed to be of class $^{1} \mathcal{C}^{\mathcal{R}}$, where $\mathcal{R}=(\kappa, \alpha), \kappa \geqslant 1,0 \leqslant \alpha \leqslant 1$, $\mathcal{R}=\infty, \mathcal{R}=\omega$ or $\mathcal{R}=\mathcal{A l g}$. Accordingly, $M$ is said to be of class $\mathcal{C}^{\mathcal{A l g}}$ (real algebraic), $\mathcal{C}^{\omega}$ (real analytic), $\mathcal{C}^{\infty}$ or $\mathcal{C}^{\kappa, \alpha}$.

For $p \in M$, the smallest $J$-invariant subspace of the tangent space $T_{p} M$ is given by $T_{p}^{c} M:=T_{p} M \cap J T_{p} M$ and is called the complex tangent space to $M$ at $p$.

Definition 2.2. The submanifold $M$ is called:

- holomorphic if $T_{p}^{c} M=T_{p} M$ at every point $p \in M$;

- totally real if $T_{p}^{c} M=\{0\}$ at every point $p \in M$;

- generic if $T_{p} M+J T_{p} M=T_{p} \mathbb{C}^{n}$ at every point $p \in M$;

- Cauchy-Riemann (CR for short) if the dimension of $T_{p}^{c} M$ is equal to a fixed constant at every point $p \in M$.

For fundamentals about Cauchy-Riemann (CR for short) structures, we refer the reader to [Ch1989, Ja1990, Ch1991, Bo1991, BER1999, Me2004a. Here, we only summarize some elementary useful properties. The two $J$-invariant spaces $T_{p} M \cap J T_{p} M$ and $T_{p} M+J T_{p} M$ are of even real dimension. We denote by $m_{p}$ the integer $\frac{1}{2} \operatorname{dim}_{\mathbb{R}}\left(T_{p} M \cap J T_{p} M\right)$ and call it the $C R$ dimension of $M$ at $p$. If $M$ is CR, $m_{p} \equiv m$ is constant. Holomorphic, totally real and generic submanifolds are CR, with $m=n-\frac{1}{2} d$, $m=0$ and $m=n-d$ respectively. If $M$ is totally real and generic,

\footnotetext{
${ }^{1}$ Background about Hölder classes appears in Section 1(IV).
} 
$\operatorname{dim}_{\mathbb{R}} M=n$ and $M$ is called maximally real. We denote by $c_{p}$ the integer $n-\frac{1}{2} \operatorname{dim}_{\mathbb{R}}\left(T_{p} M+J T_{p} M\right)$ and call it the holomorphic codimension of $M$ at $p$. It is constant if and only if $M$ is CR. Holomorphic, totally real, generic and Cauchy-Riemann submanifolds are all CR and have constant holomorphic codimensions $c=\frac{1}{2} d, c=d-n, c=0$ and $c=d-n+m$ respectively. Submanifolds of class $\mathcal{C}^{\kappa, \alpha}$ or $\mathcal{C}^{\infty}$ will be studied in Part III.

Let $M$ or be a real algebraic $\left(\mathcal{C}^{\mathcal{A l g}}\right)$ or analytic $\left(\mathcal{C}^{\omega}\right)$ submanifold of $\mathbb{C}^{n}$ of (real) codimension $d$ and let $p_{0} \in M$. There exist complex algebraic or analytic coordinates centered at $p_{0}$ and $\rho_{1}>0$ such that $M$ is locally represented as follows.

Theorem 2.3. ([Ch1989, Bo1991, BER1999, Me2004a])

- If $M$ is holomorphic, letting $m=n-\frac{1}{2} d \geqslant 0$ and $c:=\frac{1}{2} d$, then $m+c=n$ and $M=\left\{\left(z, w_{1}\right) \in \Delta_{\rho_{1}}^{m} \times \Delta_{\rho_{1}}^{c}: w_{1}=0\right\}$.

- If $M$ is totally real, letting $d_{1}=2 n-d \geqslant 0$ and $c=d-n \geqslant 0$, then $d_{1}+c=n$ and $M=$ $\left\{\left(w_{1}, w_{2}\right) \in \square_{\rho_{1}}^{2 d_{1}} \times \Delta_{\rho_{1}}^{c}: \operatorname{Im} w_{1}=0, w_{2}=0\right\}$.

- If $M$ is generic, letting $m=d-n$, then $m+d=n$ and $M=\left\{(z, w) \in \Delta_{\rho_{1}}^{m} \times\left(\square_{\rho_{1}}^{d}+i \mathbb{R}^{d}\right): \operatorname{Im} w=\varphi(z, \bar{z}, \operatorname{Re} w)\right\}$, for some $\mathbb{R}^{d}$-valued algebraic or analytic map $\varphi$ satisfying $\varphi(0)=$ 0 whose power series converges normally in $\Delta_{2 \rho_{1}}^{m} \times \Delta_{2 \rho_{1}}^{m} \times \square_{2 \rho_{1}}^{d}$.

- If $M$ is Cauchy-Riemann, letting $m=\operatorname{CRdim} M, c=d-n+m \geqslant$ 0 , and $d_{1}=2 n-2 m-d \geqslant 0$, then $m+d_{1}+c=n$ and

$$
\begin{array}{r}
M=\left\{\left(z, w_{1}, w_{2}\right) \in \Delta_{\rho_{1}}^{m} \times\left(\square_{\rho_{1}}^{d_{1}}+i \mathbb{R}^{d_{1}}\right) \times \Delta_{\rho_{1}}^{c}:\right. \\
\left.\operatorname{Im} w_{1}=\varphi_{1}\left(z, \bar{z}, \operatorname{Re} w_{1}\right), w_{2}=0\right\},
\end{array}
$$

for some $\mathbb{R}^{d_{1}}$-valued algebraic or analytic map $\varphi_{1}$ satisfying $\varphi_{1}(0)=0$ whose power series converges normally in $\Delta_{2 \rho_{1}}^{m} \times \Delta_{2 \rho_{1}}^{m} \times$ $\square_{2 \rho_{1}}^{d_{1}}$.

A further linear change of coordinates may yield $d \varphi(0)=0$ and $d \varphi_{1}(0)=0$.

A CR algebraic or analytic manifold being generic in some local complex manifold of (smaller) dimension $n-c$, called its intrinsic complexification, in most occasions, questions, results and articles, one deals with generic manifolds. In this chapter, all generic submanifolds will be of positive codimension $d \geqslant 1$ and of positive CR dimension $m \geqslant 1$. 
2.4. Algebraic and analytic generic submanifolds and their extrinsic complexification. Let $M$ be generic, represented by $\operatorname{Im} w=\varphi(z, \bar{z}, \operatorname{Re} w)$. The implicit function theorem applied to the vectorial equation $\frac{w-\bar{w}}{2 i}=\varphi\left(z, \bar{z}, \frac{w+\bar{w}}{2}\right)$, enables to solve the variables $\bar{w} \in \mathbb{C}^{d}$, or the variables $w \in \mathbb{C}^{d}$. This yields the so-called complex defining equations for $M$, most useful in applications, as stated just below. Recall that, given a power series $\Phi(t)=\sum_{\gamma \in \mathbb{N}^{n}} \Phi_{\gamma} t^{\gamma}, t \in \mathbb{C}^{n}, \Phi_{\gamma} \in \mathbb{C}$, $\gamma \in \mathbb{N}^{n}$, one defines the series $\bar{\Phi}(t):=\sum_{\gamma \in \mathbb{N}^{n}} \bar{\Phi}_{\gamma} t^{\gamma}$ by conjugating only its complex coefficients. Then $\overline{\Phi(t)} \equiv \bar{\Phi}(\bar{t})$, a frequently used property.

Theorem 2.5. ([BER1999, Me2004a]]) A local generic real algebraic or analytic d-codimensional generic submanifold $M \cap \Delta_{\rho_{1}}^{n}$ may be represented by $\bar{w}=\Theta(\bar{z}, z, w)$, or equivalently by $w=\bar{\Theta}(z, \bar{z}, \bar{w})$, for some complex algebraic or analytic $\mathbb{C}^{d}$-valued map $\Theta$ whose power series converges normally in $\Delta_{2 \rho_{1}}^{m} \times \Delta_{2 \rho_{1}}^{m} \times \Delta_{2 \rho_{1}}^{d}$, with $\rho_{1}>0$. Here, $\Theta$ and $\bar{\Theta}$ satisfy the two (equivalent by conjugation) vectorial functional equations:

$$
\left\{\begin{array}{l}
\bar{w} \equiv \Theta(\bar{z}, z, \bar{\Theta}(z, \bar{z}, \bar{w})), \\
w \equiv \bar{\Theta}(z, \bar{z}, \Theta(\bar{z}, z, w)) .
\end{array}\right.
$$

Conversely, if such a $\mathbb{C}^{d}$-valued map $\Theta$ satisfies the above, the set $M:=$ $\left\{(z, w) \in \Delta_{\rho_{1}}^{n}: \bar{w}=\Theta(\bar{z}, z, w)\right\}$ is a real local generic submanifold of codimension d.

The coordinates $(z, w) \in \mathbb{C}^{m} \times \mathbb{C}^{d}$ will also be denoted by $t \in \mathbb{C}^{n}$. Let $\tau=(\zeta, \xi) \in \mathbb{C}^{m} \times \mathbb{C}^{d}$ be new independent complex variables. Define the extrinsic complexification $\mathcal{M}=(M)^{c}$ of $M$ to be the complex algebraic or analytic $d$-codimensional submanifold of $\mathbb{C}^{n} \times \mathbb{C}^{n}$ defined by the vectorial equation $\xi-\Theta(\zeta, t)=0$ (the map $\Theta$ being analytic, we may indeed substitute $\zeta$ for $\bar{z}$ in its power series). We also write $\tau=(\bar{t})^{c}$. Observe that $M$ identifies with the intersection $\mathcal{M} \cap\{\tau=\bar{t}\}$.

Lemma 2.6. ([Me2004a, Me2005] $]$ ) There exists an invertible $d \times d$ matrix $a(t, \tau)$ of algebraic or analytic power series converging normally in $\Delta_{2 \rho_{1}}^{n} \times$ $\Delta_{2 \rho_{1}}^{n}$ such that $w-\bar{\Theta}(z, \tau) \equiv a(t, \tau)[\xi-\Theta(\zeta, t)]$.

Thus, $\mathcal{M}$ is equivalently defined by $w-\bar{\Theta}(z, \tau)=0$.

2.7. Complexified Segre varieties and complexified $C R$ vector fields. Let $\tau_{p}, t_{p} \in \Delta_{\rho_{1}}^{n}$ be fixed and define the complexified Segre varieties $\mathcal{S}_{\tau_{p}}$ 
and the complexified conjugate Segre varieties $\underline{\mathcal{S}}_{t_{p}}$ by:

$$
\left\{\begin{array}{l}
\mathcal{S}_{\tau_{p}}:=\left\{(t, \tau) \in \Delta_{\rho_{1}}^{n} \times \Delta_{\rho_{1}}^{n}: \tau=\tau_{p}, w=\bar{\Theta}\left(z, \tau_{p}\right)\right\} \quad \text { and } \\
\underline{\mathcal{S}}_{t_{p}}:=\left\{(t, \tau) \in \Delta_{\rho_{1}}^{n} \times \Delta_{\rho_{1}}^{n}: t=t_{p}, \xi=\Theta\left(\zeta, t_{p}\right)\right\} .
\end{array}\right.
$$

Geometrically, $\mathcal{S}_{\tau_{p}}=\mathcal{M} \cap\left\{\tau=\tau_{p}\right\}$ and $\underline{\mathcal{S}}_{t_{p}}=\mathcal{M} \cap\left\{t=t_{p}\right\}$. We draw a diagram.

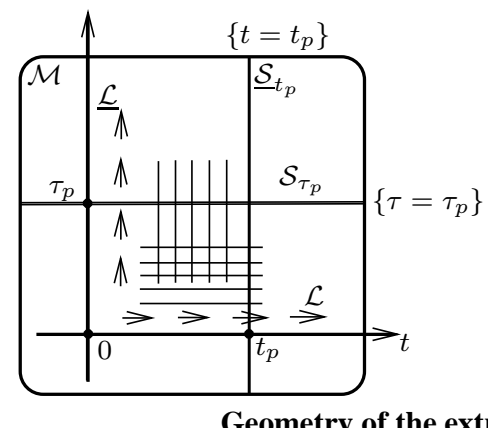

The complexification of a real analytic generic submanifold carries a pair of invariant foliations which are the integral submanifolds of the complexified $(1,0)$ and $(0,1)$ vector fields and which identify also with the complexified Segre varieties

We warn the reader that

$$
\operatorname{dim}_{\mathbb{C}} \mathcal{M}-\operatorname{dim}_{\mathbb{C}} \mathcal{S}_{\tau_{p}}-\operatorname{dim}_{\mathbb{C}} \underline{\mathcal{S}}_{t_{p}}=d \geqslant 1,
$$

so that the ambient codimension $d$ of the unions of $\mathcal{S}_{\tau_{p}}$ and of $\underline{\mathcal{S}}_{t_{p}}$ is invisible in this picture; one should imagine for instance that $\mathcal{M}$ is the threedimensional physical space equipped with a pair of foliations by horizontal orthogonal real lines.

Next, define two collections of complex vector fields:

$$
\left\{\begin{array}{l}
\mathcal{L}_{k}:=\frac{\partial}{\partial z_{k}}+\sum_{j=1}^{d} \frac{\partial \bar{\Theta}_{j}}{\partial z_{k}}(z, \zeta, \xi) \frac{\partial}{\partial w_{j}}, \quad k=1, \ldots, m, \quad \text { and } \\
\underline{\mathcal{L}}_{k}:=\frac{\partial}{\partial \zeta_{k}}+\sum_{j=1}^{d} \frac{\partial \Theta_{j}}{\partial \zeta_{k}}(\zeta, z, w) \frac{\partial}{\partial \xi_{j}}, \quad k=1, \ldots, m .
\end{array}\right.
$$

One verifies that $\mathcal{L}_{k}\left(w_{j}-\bar{\Theta}_{j}(z, \zeta, \xi)\right) \equiv 0$, which shows that the $\mathcal{L}_{k}$ are tangent to $\mathcal{M}$. Similarly, $\underline{\mathcal{L}}_{k}\left(\xi_{j}-\Theta_{j}(\zeta, z, w)\right) \equiv 0$, so the $\underline{\mathcal{L}}_{k}$ are also tangent to $\mathcal{M}$. In addition, $\left[\mathcal{L}_{k}, \mathcal{L}_{k^{\prime}}\right]=0$ and $\left[\underline{\mathcal{L}}_{k}, \underline{\mathcal{L}}_{k^{\prime}}\right]=0$ for $k, k^{\prime}=$ $1, \ldots, m$, so the theorem of Frobenius applies. In fact, the $m$-dimensional integral submanifolds of the two collections $\left\{\mathcal{L}_{k}\right\}_{1 \leqslant k \leqslant m}$ and $\left\{\underline{\mathcal{L}}_{k}\right\}_{1 \leqslant k \leqslant m}$ are the $\mathcal{S}_{\tau_{p}}$ and the $\underline{\mathcal{S}}_{t_{p}}$. In summary, $\mathcal{M}$ carries a fundamental pair of foliations. 
Observe that the vector fields $\mathcal{L}_{k}$ are the complexifications of the vector fields $L_{k}:=\frac{\partial}{\partial z_{k}}+\sum_{j=1}^{d} \frac{\partial \bar{\Theta}_{j}}{\partial z_{k}}(z, \bar{z}, \bar{w}) \frac{\partial}{\partial w_{j}}, k=1, \ldots, m$, that generate the holomorphic tangent bundle $T^{1,0} M$. A similar observation applies to the vector fields $\underline{\mathcal{L}}_{k}$.

In general (unless $M$ is Levi-flat), the total collection $\left\{\mathcal{L}_{k}, \underline{\mathcal{L}}_{k}\right\}_{1 \leqslant k \leqslant m}$ does not enjoy the Frobenius property. In fact, the noncommutativity of this system of $2 m$ vector fields is at the very core of Cauchy-Riemann geometry.

To apply Theorem 1.13, introduce the "multiple" flows of the two collections $\left\{\mathcal{L}_{k}\right\}_{1 \leqslant k \leqslant m}$ and $\left\{\underline{\mathcal{L}}_{k}\right\}_{1 \leqslant k \leqslant m}$. If $p \in \mathcal{M}$ has coordinates $\left(z_{p}, w_{p}, \zeta_{p}, \xi_{p}\right) \in \Delta_{\rho_{1}}^{m} \times \Delta_{\rho_{1}}^{d} \times \Delta_{\rho_{1}}^{m} \times \Delta_{\rho_{1}}^{d}$ satisfying $w_{p}=\bar{\Theta}\left(z_{p}, \zeta_{p}, \xi_{p}\right)$ and $\xi_{p}=\Theta\left(\zeta_{p}, z_{p}, w_{p}\right)$ and if $z_{1}:=\left(z_{1,1}, \ldots, z_{1, m}\right) \in \mathbb{C}^{m}$ is a small "multitime" parameter, define the "multiple" flow of $\mathcal{L}$ by:

$$
\begin{aligned}
\mathcal{L}_{z_{1}}\left(z_{p}, w_{p}, \zeta_{p}, \xi_{p}\right) & :=\exp \left(z_{1} \mathcal{L}\right)(p) \\
& :=\exp \left(z_{1,1} \mathcal{L}_{1}\left(\cdots\left(\exp \left(z_{1, m} \mathcal{L}_{m}(p)\right)\right) \cdots\right)\right) \\
& :=\left(z_{p}+z_{1}, \bar{\Theta}\left(z_{p}+z_{1}, \zeta_{p}, \xi_{p}\right), \zeta_{p}, \xi_{p}\right)
\end{aligned}
$$

Of course, $\mathcal{L}_{z_{1}}(p) \in \mathcal{M}$. Similarly, for $p \in \mathcal{M}$ and $\zeta_{1} \in \mathbb{C}^{m}$, defining:

$$
\underline{\mathcal{L}}_{\zeta_{1}}\left(z_{p}, w_{p}, \zeta_{p}, \xi_{p}\right):=\left(z_{p}, w_{p}, \zeta_{p}+\zeta_{1}, \Theta\left(\zeta_{p}+\zeta_{1}, z_{p}, w_{p}\right)\right)
$$

we have $\underline{\mathcal{L}}_{\zeta_{1}}(p) \in \mathcal{M}$. Clearly, $\left(p, z_{1}\right) \mapsto \mathcal{L}_{z_{1}}(p)$ and $\left(p, \zeta_{1}\right) \mapsto \underline{\mathcal{L}}_{\zeta_{1}}(p)$ are complex algebraic or analytic local maps.

2.10. Segre chains. Let us start from $p=0$ being the origin and move vertically along the complexified conjugate Segre variety $\underline{\mathcal{S}}_{0}$ of a height $z_{1} \in \mathbb{C}^{m}$, namely let us consider the point $\underline{\mathcal{L}}_{z_{1}}(0)$, which we shall also denote by $\underline{\Gamma}_{1}\left(z_{1}\right)$. We have $\underline{\Gamma}_{1}(0)=0$. Let $z_{2} \in \mathbb{C}^{m}$. Starting from the point $\underline{\Gamma}_{1}\left(z_{1}\right)$, let us move horizontally along the complexified Segre variety of a length $z_{2} \in \mathbb{C}^{m}$, namely let us consider the point

$$
\underline{\Gamma}_{2}\left(z_{1}, z_{2}\right):=\mathcal{L}_{z_{2}}\left(\underline{\mathcal{L}}_{z_{1}}(0)\right)
$$

Next, define $\underline{\Gamma}_{3}\left(z_{1}, z_{2}, z_{3}\right):=\underline{\mathcal{L}}_{z_{3}}\left(\mathcal{L}_{z_{2}}\left(\underline{\mathcal{L}}_{z_{1}}(0)\right)\right)$, and then

$$
\underline{\Gamma}_{4}\left(z_{1}, z_{2}, z_{3}, z_{4}\right):=\mathcal{L}_{z_{4}}\left(\underline{\mathcal{L}}_{z_{3}}\left(\mathcal{L}_{z_{2}}\left(\underline{\mathcal{L}}_{z_{1}}(0)\right)\right)\right),
$$

and so on. We draw a diagram: 


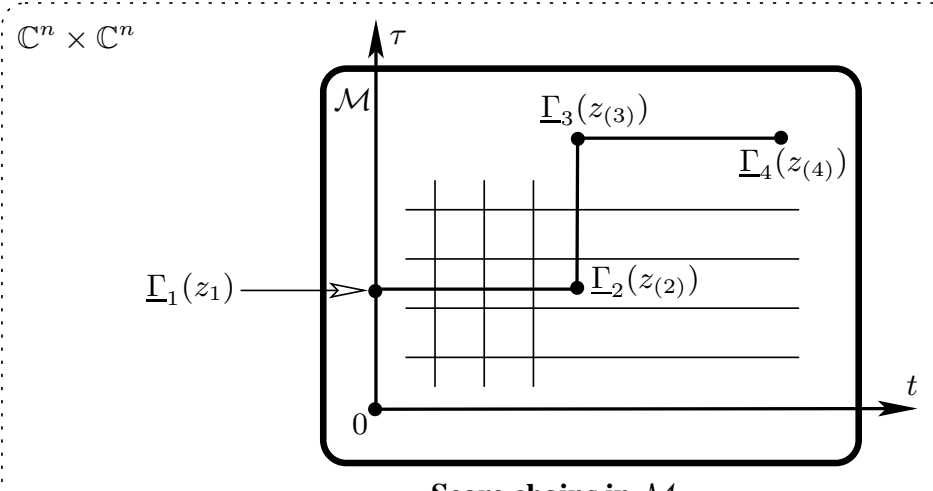

Segre chains in $\mathcal{M}$

By induction, for every $k \in \mathbb{N}, k \geqslant 1$, we obtain a local complex algebraic or analytic map $\underline{\Gamma}_{k}\left(z_{1}, \ldots, z_{k}\right)$, valued in $\mathcal{M}$, defined for sufficiently small $z_{1}, \ldots, z_{k} \in \mathbb{C}^{m}$ which satisfies $\underline{\Gamma}_{k}(0, \ldots, 0)=0$. The abbreviated notation $z_{(k)}:=\left(z_{1}, \ldots, z_{k}\right) \in \mathbb{C}^{m k}$ will be used. The map $\underline{\Gamma}_{k}$ is called the $k$-th conjugate Segre chain ([Me2004a, Me2005]).

If we had conducted this procedure by starting with $\mathcal{L}$ instead of starting with $\underline{\mathcal{L}}$, we would have obtained maps $\Gamma_{1}\left(z_{1}\right):=\mathcal{L}_{z_{1}}(0), \Gamma_{2}\left(z_{(2)}\right):=$ $\underline{\mathcal{L}}_{z_{2}}\left(\mathcal{L}_{z_{1}}(0)\right)$, etc., and generally $\Gamma_{k}\left(z_{(k)}\right)$. The map $\Gamma_{k}$ is called the $k$-th Segre chain.

There is a symmetry relation between $\Gamma_{k}$ and $\underline{\Gamma}_{k}$. Indeed, let $\bar{\sigma}$ be the antiholomorphic involution of $\mathbb{C}^{n} \times \mathbb{C}^{n}$ defined by $\bar{\sigma}(t, \tau):=(\bar{\tau}, \bar{t})$. Since we have $w=\bar{\Theta}(z, \zeta, \xi)$ if and only if $\xi=\Theta(\zeta, z, w)$, this involution is a bijection of $\mathcal{M}$. Applying $\bar{\sigma}$ to the definitions (2.8) and (2.9) of the flows of $\mathcal{L}$ and of $\underline{\mathcal{L}}$, one may verify that $\bar{\sigma}\left(\mathcal{L}_{z_{1}}(p)\right)=\underline{\mathcal{L}}_{\bar{z}_{1}}(\bar{\sigma}(p))$. It follows the general symmetry relation $\bar{\sigma}\left(\Gamma_{k}\left(z_{(k)}\right)\right)=\underline{\Gamma}_{k}\left(\overline{z_{(k)}}\right)$. Thus, $\Gamma_{k}$ and $\underline{\Gamma}_{k}$ have the same behavior.

2.11. Minimality of $\mathcal{M}$ at the origin and complexified local CR orbits. Since $\Gamma_{k}(0)=\underline{\Gamma}_{k}(0)=0$, for every integer $k \geqslant 1$, there exists $\delta_{k}>0$ sufficiently small such that $\Gamma_{k}\left(z_{(k)}\right)$ and $\underline{\Gamma}_{k}\left(z_{(k)}\right)$ are well defined and belong to $\mathcal{M}$, at least for all $z_{(k)} \in \Delta_{\delta_{k}}^{m k}$. To fiw ideas, it will be convenient to consider that $\Delta_{\delta_{k}}^{m k}$ is the precise domain of definition of $\Gamma_{k}$ and of $\underline{\Gamma}_{k}$. We aim to apply the procedure of Theorem 1.13 to the system $\mathbb{L}^{0}:=\left\{\mathcal{L}_{1}, \ldots, \mathcal{L}_{m}, \underline{\mathcal{L}}_{1}, \ldots, \underline{\mathcal{L}}_{m}\right\}$.

However, there is a slight (innocuous) difference: each multitime $t=$ $\left(\mathrm{t}_{1}, \ldots, \mathrm{t}\right) \in \mathbb{K}^{k}$ had scalar components $\mathrm{t}_{i} \in \mathbb{K}$, whereas now each $z_{(k)}=\left(z_{1}, \ldots, z_{k}\right) \in \mathbb{C}^{m k}$ has vectorial components $z_{i} \in \mathbb{C}^{m}$. It is easy to see that both $\Gamma_{1}$ and $\underline{\Gamma}_{1}$ are of constant rank $m$. Also, both $\Gamma_{2}$ and $\underline{\Gamma}_{2}$ 
are of constant rank $2 m$, since $\mathcal{L}_{1}, \ldots, \mathcal{L}_{m}, \underline{\mathcal{L}}_{1}, \ldots, \underline{\mathcal{L}}_{m}$ are linearly independent at the origin. However, when passing to (conjugate) Segre chains of length $\geqslant 3$, it is necessary to speak of generic ranks and to introduce some combinatorial integers $e_{k} \geqslant 1$. Justifying examples may be found in [Me1999, Me2004a].

Theorem 2.12. ([BER1996, BER1999, Me1999, Me2001a, Me2004a]) There exists an integer $\nu_{0}$ with $1 \leqslant \nu_{0} \leqslant d$ and, for $k=3, \ldots, \nu_{0}+1$, integers $e_{k}$ with $1 \leqslant e_{k} \leqslant m$ such that the following nine properties hold true.

(1) For every $k=3, \ldots, \nu_{0}+1$, the two maps $\Gamma_{k}$ and $\underline{\Gamma}_{k}$ are of generic rank equal to $2 m+e_{3}+\cdots+e_{k}$. In the special case $\nu_{0}=1$, the $e_{k}$ are inexistent ${ }^{2}$ and nothing is stated.

(2) For every $k \geqslant \nu_{0}+1$, both $\Gamma_{k}$ and $\underline{\Gamma}_{k}$ are of fixed, stabilized generic rank equal to $2 m+e$, where

$$
e:=e_{3}+\cdots+e_{\nu_{0}} \leqslant d .
$$

(3) Setting $\mu_{0}:=2 \nu_{0}+1$, there exist two points $z_{\left(\mu_{0}\right)}^{*} \in \Delta_{\delta_{\mu_{0}}}^{m \mu_{0}}$ and $\underline{z}_{\left(\mu_{0}\right)}^{*} \in \Delta_{\delta_{\mu_{0}}}^{m \mu_{0}}$ satisfying $\Gamma_{\mu_{0}}\left(z_{\left(\mu_{0}\right)}^{*}\right)=0$ and $\underline{\Gamma}_{\mu_{0}}\left(\underline{z}_{\left(\mu_{0}\right)}^{*}\right)=0$ which are arbitrarily close to the origin in $\Delta_{\delta_{\mu_{0}}}^{m \mu_{0}}$ such that $\Gamma_{\mu_{0}}$ and $\underline{\Gamma}_{\mu_{0}}$ are of constant rank $2 m+e$ in neighborhoods $\omega^{*}$ and $\underline{\omega}^{*}$ of $z_{\left(\mu_{0}\right)}^{*}$ and of $\underline{z}_{\left(\mu_{0}\right)}^{*}$. The images $\Gamma_{\mu_{0}}\left(\omega^{*}\right)$ and $\underline{\Gamma}_{\mu_{0}}\left(\underline{\omega}^{*}\right)$ then constitute two pieces of local $\mathbb{K}$-algebraic or analytic submanifold of dimension $2 m+e$ contained in $\mathcal{M}$.

(4) Both $\Gamma_{\mu_{0}}\left(\omega^{*}\right)$ and $\underline{\Gamma}_{\mu_{0}}\left(\underline{\omega}^{*}\right)$ enjoy the most important property that all vector fields $\mathcal{L}_{1}, \ldots, \mathcal{L}_{m}, \underline{\mathcal{L}}_{1}, \ldots, \underline{\mathcal{L}}_{m}$ are tangent to $\Gamma_{\mu_{0}}\left(\omega^{*}\right)$ and to $\underline{\Gamma}_{\mu_{0}}\left(\underline{\omega}^{*}\right)$.

(5) $\Gamma_{\mu_{0}}\left(\omega^{*}\right)$ and $\underline{\Gamma}_{\mu_{0}}\left(\underline{\omega}^{*}\right)$ coincide together in a neighborhood of 0 in $\mathcal{M}$.

(6) Denoting by

$$
\mathcal{O}_{\mathcal{L}, \underline{\mathcal{L}}}(\mathcal{M}, 0)
$$

this common local piece of complex analytic submanifold of $\mathcal{M}$, it is algebraic provided that the flows of $\left\{\mathcal{L}_{1}, \ldots, \mathcal{L}_{m}, \underline{\mathcal{L}}_{1}, \ldots, \underline{\mathcal{L}}_{m}\right\}$ are themselves algebraic.

\footnotetext{
${ }^{2}$ One may set $e_{1}:=m$ and $e_{2}:=m$ in any case.
} 
(7) Every local complex analytic or algebraic submanifold $\mathcal{N} \subset \mathcal{M}$ passing through the origin to which $\mathcal{L}_{1}, \ldots, \mathcal{L}_{m}, \underline{\mathcal{L}}_{1}, \ldots, \underline{\mathcal{L}}_{m}$ are all tangent must contain $\mathcal{O}_{\mathcal{L}, \mathcal{L}}(\mathcal{M}, 0)$ in a neighborhood of the origin.

(8) The integers $\nu_{0}, e_{3}, \ldots, e_{\mu_{0}}$ and e are biholomorphic invariants of $\mathcal{M}$.

(9) $\Gamma_{\mu_{0}}\left(\omega^{*}\right)$ and $\underline{\Gamma}_{\mu_{0}}\left(\underline{\omega}^{*}\right)$ also coincide (in a neighborhood of the origin) with the Nagano leaf of the system $\left\{\mathcal{L}_{1}, \ldots, \mathcal{L}_{m}, \underline{\mathcal{L}}_{1}, \ldots, \underline{\mathcal{L}}_{m}\right\}$, as it was constructed in Theorem 1.5.

As in [Me2004a, Me2005] (with different notations), the integer $\nu_{0}$ will be called the Segre type of $M$.

The "orbit notation" $\mathcal{O}_{\mathcal{L}, \mathcal{L}}(\mathcal{M}, 0)$ anticipates the presentation and the notation of Section 1(III). We will abandon Lie brackets and Nagano leaves.

The complex vector fields $L_{k}:=\frac{\partial}{\partial z_{k}}+\sum_{j=1}^{d} \frac{\partial \bar{\Theta}_{j}}{\partial z_{k}}(z, \bar{z}, \bar{w}) \frac{\partial}{\partial w_{j}}, k=$ $1, \ldots, m$, are tangent to $M$ of equations $w_{j}=\bar{\Theta}_{j}(z, \bar{z}, \bar{w}), j=1, \ldots, d$; their conjugates $\bar{L}_{k}$ are also tangent to $M$; it follows that the real and imaginary parts $\operatorname{Re} L_{k}$ and $\operatorname{Im} L_{k}$ are also tangent to $M$. We may then apply Theorem 1.13 to the system $\left\{\operatorname{Re} L_{k}, \operatorname{Im} L_{k}\right\}_{1 \leqslant k \leqslant m}$, getting a certain real analytic local submanifold $\mathcal{O}_{L, \bar{L}}(M, 0)$ of $M$ passing through the origin. It will be called the local $C R$ orbit of the origin in $M$ (terminology of Part III).

The relation between $\mathcal{O}_{\mathcal{L}, \underline{\mathcal{L}}}(\mathcal{M}, 0)$ and $\mathcal{O}_{L, \bar{L}}(M, 0)$ is as follows ([BER1996, Me1999, Me2001a, Me2004a]). Let $\pi_{t}(t, \tau):=t$ and $\pi_{\tau}(t, \tau):=\tau$ denote the two canonical projections associated to the product $\Delta_{\rho_{1}}^{n} \times \Delta_{\rho_{1}}^{n}$. Let $\underline{A}:=\left\{(t, \tau) \in \Delta_{\rho_{1}}^{n} \times \Delta_{\rho_{1}}^{n}: \tau=\bar{t}\right\}$ be the antiholomorphic diagonal. Observe that $\pi_{t}(\underline{A} \cap \mathcal{M})=M$.

- The extrinsic complexification $\left[\mathcal{O}_{L, L}(M, 0)\right]^{c}=\mathcal{O}_{\mathcal{L}, \underline{L}}(\mathcal{M}, 0)$.

- The projection $\pi_{t}\left(\underline{A} \cap \mathcal{O}_{\mathcal{L}, \underline{\mathcal{L}}}(\mathcal{M}, 0)\right)=\mathcal{O}_{L, \bar{L}}(M, 0)$.

Concerning smoothness, a striking subtelty happens: if $M$ is real algebraic, although the local multiple flows of $\mathcal{L}$ and of $\mathcal{L}$ are complex algebraic (thanks to their definitions (2.8) and (2.9)), the flows of Re $L_{k}$ and of $\operatorname{Im} L_{k}$ are only real analytic in general.

Example 2.13. ([Me2004a] $)$ For the real algebraic hypersurface of $\mathbb{C}^{2}$ defined by $\operatorname{Im} w=\sqrt{1+z \bar{z}}-1$, the vector field $L:=\frac{\partial}{\partial z}+i \bar{z} \sqrt{1+z \bar{z}} \frac{\partial}{\partial w}$ generates $T^{1,0} M$ and the flow of $2 \operatorname{Re} L$ involves the transcendent function Arcsh. 
Theorem 2.14. ([BER1996, Me2001a]]) The local $C R$ orbit $\mathcal{O}_{L, \bar{L}}(M, 0)$ is real algebraic if $M$ is.

For the proof, assuming $M$ to be real algebraic, it is impossible, because of the example, to apply the second phrase of Theorem 1.13 (5) to the system $\left\{\operatorname{Re} L_{k}, \operatorname{Im} L_{k}\right\}_{1 \leqslant k \leqslant m}$. Fortunately, this phrase applies to the complexified system $\left\{\mathcal{L}_{k}, \underline{\mathcal{L}}_{k}\right\}_{1 \leqslant k \leqslant m}$, whence $\mathcal{O}_{\mathcal{L}, \underline{\mathcal{L}}}(\mathcal{M}, 0)$ is algebraic, and then the local CR orbit $\mathcal{O}_{L, \bar{L}}(M, 0)=\pi_{t}\left(\underline{A} \cap \mathcal{O}_{\mathcal{L}, \underline{\mathcal{L}}}(\mathcal{M}, 0)\right)$ is real algebraic.

Definition 2.15. The generic submanifold $M$ or its extrinsic complexification $\mathcal{M}$ is said to be minimal at the origin if $\mathcal{O}_{L, \bar{L}}(M, 0)$ contains a neighborhood of 0 in $M$, or equivalently if $\mathcal{O}_{\mathcal{L}, \underline{\mathcal{L}}}(\mathcal{M}, 0)$ contains a neighborhood of 0 in $\mathcal{M}$.

The minimality at the origin of the algebraic or analytic complexified local generic submanifold $\mathcal{M}=(M)^{c}$ is a biholomorphically invariant property; it neither depends on the choice of defining equations nor on the choice of a conjugate pair of systems of complex vector fields $\left\{\mathcal{L}_{k}\right\}_{1 \leqslant k \leqslant m}$ and $\left\{\underline{\mathcal{L}}_{k}\right\}_{1 \leqslant k \leqslant m}$ spanning the tangent space to the two foliations.

Minimality at 0 reads $e=d$ in Theorem 2.12. For a hypersurface $M$, namely with $d=1$, minimality at 0 is equivalent to $\nu_{0}=2$.

2.16. Projections of the submersions $\Gamma_{\mu_{0}}$ and $\underline{\Gamma}_{\mu_{0}}$. Let $\mu_{0}=2 \nu_{0}+1$ as in Theorem 2.12. If $\mathcal{M}$ is minimal at the origin, the two local holomorphic maps

$$
\Gamma_{\mu_{0}} \text { and } \underline{\Gamma}_{\mu_{0}}: \Delta_{\delta_{\mu_{0}}}^{m \mu_{0}} \longrightarrow \mathcal{M}
$$

satisfy $\Gamma_{\mu_{0}}\left(z_{\left(\mu_{0}\right)}^{*}\right)=0$ and $\underline{\Gamma}_{\mu_{0}}\left(\underline{z}_{\left(\mu_{0}\right)}^{*}\right)=0$ and they are submersive at $z_{\left(\mu_{0}\right)}^{*}$ and at $\underline{z}_{\left(\mu_{0}\right)}^{*}$.

Consider the two projections $\pi_{t}(t, \tau):=t$ and $\pi_{\tau}(t, \tau):=\tau$ and four compositions $\pi_{t}\left(\Gamma_{\mu_{0}}\left(z_{\left(\mu_{0}\right)}\right)\right), \pi_{t}\left(\underline{\Gamma}_{\mu_{0}}\left(z_{\left(\mu_{0}\right)}\right)\right)$ and $\pi_{\tau}\left(\Gamma_{\mu_{0}}\left(z_{\left(\mu_{0}\right)}\right)\right)$, $\pi_{\tau}\left(\underline{\Gamma}_{\mu_{0}}\left(z_{\left(\mu_{0}\right)}\right)\right)$. Since $\mu_{0}=2 \nu_{0}+1$ is odd, observe that the composition $\underline{\Gamma}_{2 \nu_{0}+1}=\underline{\mathcal{L}}(\cdots)$ ends with a $\underline{\mathcal{L}}$ and that $\Gamma_{2 \nu_{0}+1}=\mathcal{L}(\cdots)$ ends with a $\mathcal{L}$. According to the two definitions of the flow maps, the coordinates $\left(\zeta_{p}, \xi_{p}\right)$ are untouched in (2.8) and the coordinates $\left(z_{p}, w_{p}\right)$ are untouched in (2.9). It follows that

$$
\left\{\begin{aligned}
\pi_{t}\left(\underline{\Gamma}_{2 \nu_{0}+1}\left(z_{\left(2 \nu_{0}+1\right)}\right)\right) & \equiv \pi_{t}\left(\underline{\Gamma}_{2 \nu_{0}}\left(z_{\left(2 \nu_{0}\right)}\right)\right) \\
\pi_{\tau}\left(\Gamma_{2 \nu_{0}+1}\left(z_{\left(2 \nu_{0}+1\right)}\right)\right) & \equiv \pi_{\tau}\left(\Gamma_{2 \nu_{0}}\left(z_{\left(2 \nu_{0}\right)}\right)\right) .
\end{aligned}\right. \text { and }
$$

Corollary 2.17. ([Me1999, BER1999, Me2004a] $]$ ) If $M$ is minimal at the origin, there exists a integer $\nu_{0} \leqslant d+1$ (the Segre type of $M$ at the origin) 
and there exist points $\underline{z}_{\left(2 \nu_{0}\right)}^{*} \in \mathbb{C}^{2 m \nu_{0}}$ and $z_{\left(2 \nu_{0}\right)}^{*} \in \mathbb{C}^{2 m \nu_{0}}$ arbitrarily close to the origin, such that the two maps

$$
\left\{\begin{array}{l}
\Delta_{\delta_{2 \nu_{0}}}^{m 2 \nu_{0}} \ni z_{\left(2 \nu_{0}\right)} \longmapsto \pi_{t}\left(\underline{\Gamma}_{2 \nu_{0}}\left(z_{\left(2 \nu_{0}\right)}\right)\right) \in \mathbb{C}^{n} \quad \text { and } \\
\Delta_{\delta_{2 \nu_{0}}}^{m 2 \nu_{0}} \ni z_{\left(2 \nu_{0}\right)} \longmapsto \pi_{\tau}\left(\Gamma_{2 \nu_{0}}\left(z_{\left(2 \nu_{0}\right)}\right)\right) \in \mathbb{C}^{n}
\end{array}\right.
$$

are of rank $n$ and send $\underline{z}_{\left(2 \nu_{0}\right)}^{*}$ and $z_{\left(2 \nu_{0}\right)}^{*}$ to the origin.

\section{§3. Formal CR MAPPINGS, JETS OF SEGRE VARIETIES AND CR REFLECTION MAPPING}

3.1. Complexified CR mappings respect pairs of foliations. Let $n^{\prime} \in \mathbb{N}$ with $n^{\prime} \geqslant 1$ and let $M^{\prime} \subset \mathbb{C}^{n^{\prime}}$ be a second algebraic or analytic generic submanifold of codimension $d^{\prime} \geqslant 1$ and of CR dimension $m^{\prime}=n^{\prime}-d^{\prime} \geqslant 1$. Let $p^{\prime} \in M^{\prime}$. There exist local coordinates $t^{\prime}=\left(z^{\prime}, w^{\prime}\right) \in \mathbb{C}^{m^{\prime}} \times \mathbb{C}^{d^{\prime}}$ centered at $p^{\prime}$ in which $M^{\prime}$ is represented by $\bar{w}^{\prime}=\Theta^{\prime}\left(\bar{z}^{\prime}, t^{\prime}\right)$, or equivalently by $w^{\prime}=\bar{\Theta}^{\prime}\left(z^{\prime}, \bar{t}^{\prime}\right)$. If $\left(\bar{t}^{\prime}\right)^{c}=\tau^{\prime}=\left(\zeta^{\prime}, \xi^{\prime}\right) \in \mathbb{C}^{m^{\prime}} \times \mathbb{C}^{d^{\prime}}$, the extrinsic complexification is represented by $\xi^{\prime}=\Theta^{\prime}\left(\zeta^{\prime}, t^{\prime}\right)$, or equivalently by $w^{\prime}=$ $\bar{\Theta}^{\prime}\left(z^{\prime}, \tau^{\prime}\right)$. We shall denote by $0^{\prime}$ the origin of $\mathbb{C}^{n^{\prime}}$.

Let $t \in \mathbb{C}^{n}$ and let $h(t)=\left(h_{1}(t), \ldots, h_{n^{\prime}}(t)\right) \in \mathbb{C} \llbracket t \rrbracket^{n^{\prime}}$ be a formal power series mapping with no constant term, i.e. $h(0)=0^{\prime}$; it may also be holomorphic namely $h(t) \in \mathbb{C}\{t\}^{n^{\prime}}$, or even (Nash) algebraic. We have $(\overline{h(t)})^{c}=\bar{h}\left((\bar{t})^{c}\right)=\bar{h}(\tau)$. Define $h^{c}(t, \tau):=(h(t), \bar{h}(\tau))$.

Set $r(t, \tau):=\xi-\Theta(\zeta, t)$, set $\bar{r}(\tau, t):=w-\bar{\Theta}(z, \tau)$, set $r^{\prime}\left(t^{\prime}, \tau^{\prime}\right):=$ $\xi^{\prime}-\Theta^{\prime}\left(\zeta^{\prime}, t^{\prime}\right)$ and set $\bar{r}^{\prime}\left(\tau^{\prime}, t^{\prime}\right):=w^{\prime}-\bar{\Theta}^{\prime}\left(z^{\prime}, \tau^{\prime}\right)$. We say that the power series mapping $h$ is a formal $C R$ mapping from $(M, 0)$ to $\left(M^{\prime}, 0^{\prime}\right)$ if there exists a $d^{\prime} \times d$ matrix of formal power series $b(t, \bar{t})$ such that

$$
r^{\prime}(h(t), \bar{h}(\bar{t})) \equiv b(t, \bar{t}) r(t, \bar{t})
$$

in $\mathbb{C} \llbracket t, \bar{t} \rrbracket^{d^{\prime}}$. By complexification, it follows that $r^{\prime}(h(t), \bar{h}(\tau)) \equiv$ $b(t, \tau) r(t, \tau)$ in $\mathbb{C} \llbracket t, \tau \rrbracket^{d^{\prime}}$, namely $h^{c}(t, \tau)=(h(t), \bar{h}(\tau))$ maps $(\mathcal{M}, 0)$ formally to $\left(\mathcal{M}^{\prime}, 0^{\prime}\right)$. By Lemma 2.6 , there exist two complex analytic invertible matrices $a(t, \tau)$ and $a^{\prime}\left(t^{\prime}, \tau^{\prime}\right)$ satisfying :

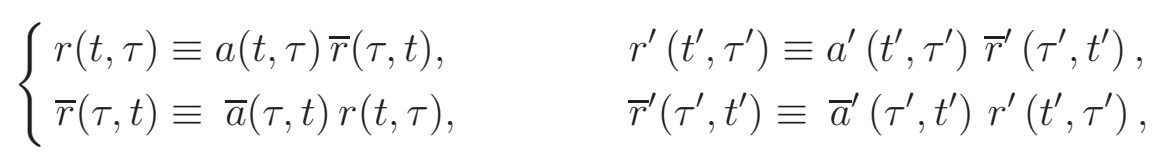

in $\mathbb{C} \llbracket t, \tau \rrbracket^{d}$ and in $\mathbb{C} \llbracket t^{\prime}, \tau^{\prime} \rrbracket^{d^{\prime}}$. So, to define a complexified formal CR mapping $h^{c}:(\mathcal{M}, 0) \mapsto \mathcal{F}\left(\mathcal{M}^{\prime}, 0^{\prime}\right)$, we get four vectorial formal identities, 
each one implying the remaining three:

$$
\begin{cases}r^{\prime}(h(t), \bar{h}(\tau)) \equiv b(t, \tau) r(t, \tau), & r^{\prime}(h(t), \bar{h}(\tau)) \equiv \bar{c}(\tau, t) \bar{r}(\tau, t), \\ \bar{r}^{\prime}(\bar{h}(\tau), h(t)) \equiv \bar{b}(\tau, t) \bar{r}(\tau, t), & \bar{r}^{\prime}(\bar{h}(\tau), h(t)) \equiv c(t, \tau) r(t, \tau) .\end{cases}
$$

Here, we have set $c(t, \tau):=\bar{b}(\tau, t) a(t, \tau)$.

These identities are independent of the choice of local coordinates and of local complex defining equations for $(M, 0)$ and for $\left(M^{\prime}, 0^{\prime}\right)$. Since $h$ is not a true point-map, we write $h:(M, 0) \rightarrow_{\mathcal{F}}\left(M^{\prime}, 0^{\prime}\right)$, the index $\mathcal{F}$ being the initial of Formal. If $h$ is convergent, it is a true point-map from a neighborhood of 0 in $M$ to a neighborhood of $0^{\prime}$ in $M^{\prime}$.

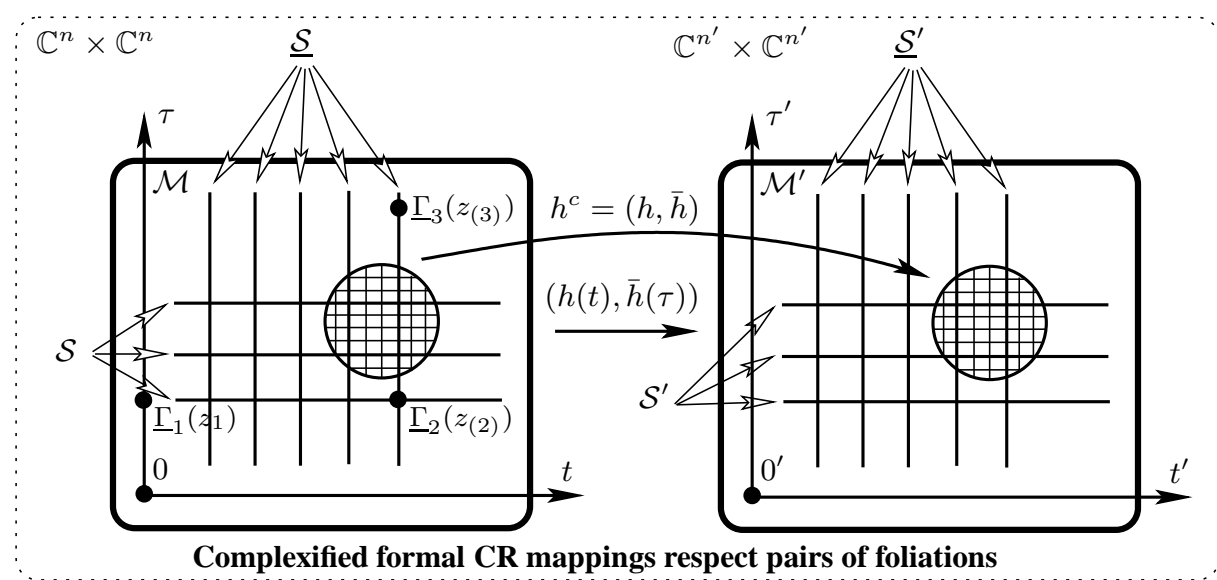

If $h$ is holomorphic in a polydisc $\Delta_{\rho_{1}}^{n}, \rho_{1}>0$, its extrinsic complexification $h^{c}$ sends both the $n$-dimensional coordinate spaces $\{t=$ cst. $\}$ and $\{\tau=$ cst. $\}$ to the $n^{\prime}$-dimensional coordinate spaces $\left\{t^{\prime}=\right.$ cst. $\}$ and $\left\{\tau^{\prime}=\right.$ cst. $\}$.

Equivalently, $h^{c}$ maps complexified (conjugate) Segre varieties of the source to complexified (conjugate) Segre varieties of the target. Some strong rigidity properties are due to the fact that $h^{c}=(h, \bar{h})$ must respect the two pairs of Segre foliations.

The most important rigidity feature, called the reflection principle ${ }^{3}$, says that the smoothness of $M, M^{\prime}$ governs the smoothness of $h$ :

- suppose that $M$ and $M^{\prime}$ are real analytic and that $h(t) \in \mathbb{C} \llbracket t \rrbracket^{n^{\prime}}$ is only formal; statement: under suitable assumptions, $h(t) \in \mathbb{C}\{t\}^{n^{\prime}}$ is in fact convergent.

\footnotetext{
${ }^{3}$ Other rigidity phenomena are: parametrization of CR automorphism groups by a jet of finite order, finiteness of their dimension, genericity of nonalgebraizable CR submanifolds, genericity of CR submanifolds having no infinitesimal CR automorphisms, etc.
} 
- suppose that $M$ and $M^{\prime}$ are real algebraic and that $h(t) \in \mathbb{C} \llbracket t \rrbracket^{n^{\prime}}$ is only formal; statement: under suitable assumptions, $h(t)$ is complex algebraic.

After a mathematical phenomenon has been observed in a special, well understood situation, the research has to focus attention on the finest, the most adequate, the necessary and sufficient conditions insuring it to hold true.

In this section, we aim to expose various possible assumptions for the reflection principle to hold. Our goal is to provide a synthesis by gathering various nondegeneracy assumptions which imply reflection. For more about history, for other results, for complements and for different points of view we refer to [Pi1975, Le1977, We1977, We1978, Pi1978, DF1978, DW1980, DF1988, BR1988, BR1990, DP1993, DP1995, DP1998, BER1999, Sh2000, BER2000, Me2001a, Me2002, Hu2001, Sh2003, DP2003, Ro2003, MMZ2003b, ER2004, Me2005].

The main theorems will be presented in $\S 3.19$ and in $\S 3.22$ below, after a long preliminary. In these results, $M$ will always be assumed to be minimal at the origin. Corollary 2.17 says already how to use concretely this assumption: to show the convergence or the algebraicity of a formal CR mapping $h:(M, 0) \mapsto_{\mathcal{F}}\left(M^{\prime}, 0^{\prime}\right)$, it suffices to establish that for every $k \in \mathbb{N}$, the formal maps $z_{(k)} \longmapsto \mathcal{F} h\left(\pi_{t}\left(\underline{\Gamma}_{k}\left(z_{(k)}\right)\right)\right)$ are convergent or algebraic.

Before surveying recent results about the reflection principle (without any indication of proof), we have to analyze thoroughly the geometry of the target $\mathcal{M}^{\prime}$ and to present the nondegeneracy conditions both on $\mathcal{M}^{\prime}$ and on $h$. Of course, everything will also be meaningful for sufficiently smooth $\left(\mathcal{C}^{\infty}\right.$ or $\left.\mathcal{C}^{\kappa}\right)$ local CR mappings, by considering Taylor series.

These conditions are classical in local analytic geometry and they may already be illustrated here with a plain formal map $h(t) \in \mathbb{C} \llbracket t \rrbracket^{n^{\prime}}$, not necessarily being CR.

Definition 3.2. A formal power series mapping $h:\left(\mathbb{C}^{n}, 0\right) \mapsto_{\mathcal{F}}\left(\mathbb{C}^{n^{\prime}}, 0^{\prime}\right)$ with components $h_{i^{\prime}}(t) \in \mathbb{C} \llbracket t \rrbracket, i^{\prime}=1, \ldots, n^{\prime}$, is called

(1) invertible if $n^{\prime}=n$ and $\operatorname{det}\left(\left[\partial h_{i_{1}} / \partial t_{i_{2}}\right](0)\right)_{1 \leqslant i_{1}, i_{2} \leqslant n} \neq 0$;

(2) submersive if $n \geqslant n^{\prime}$ and there exist integers $1 \leqslant i(1)<\cdots<$ $i\left(n^{\prime}\right) \leqslant n$ such that $\operatorname{det}\left(\left[\partial h_{i_{1}^{\prime}} / \partial t_{i\left(i_{2}^{\prime}\right)}\right](0)\right)_{1 \leqslant i_{1}^{\prime}, i_{2}^{\prime} \leqslant n^{\prime}} \neq 0$;

(3) finite if the ideal generated by the components $h_{1}(t), \ldots, h_{n^{\prime}}(t)$ is of finite codimension in $\mathbb{C} \llbracket t \rrbracket$; this implies $n^{\prime} \geqslant n$; 
(4) dominating if $n \geqslant n^{\prime}$ and there exist integers $1 \leqslant i(1)<\cdots<$ $i\left(n^{\prime}\right) \leqslant n$ such that $\operatorname{det}\left(\left[\partial h_{i_{1}^{\prime}} / \partial t_{i\left(i_{2}^{\prime}\right)}\right](t)\right)_{1 \leqslant i_{1}^{\prime}, i_{2}^{\prime} \leqslant n^{\prime}} \not \equiv 0$ in $\mathbb{C} \llbracket t \rrbracket ;$

(5) transversal if there does not exist a nonzero power series $G\left(t_{1}^{\prime}, \ldots, t_{n^{\prime}}^{\prime}\right) \in \mathbb{C} \llbracket t_{1}^{\prime}, \ldots, t_{n^{\prime}}^{\prime} \rrbracket$ such that $G\left(h_{1}(t), \ldots, h_{n^{\prime}}(t)\right) \equiv 0$ in $\mathbb{C} \llbracket t \rrbracket$.

It is elementary to see that invertibility implies submersiveness which implies domination. Furthermore, if a formal power series is either invertible, submersive or dominating, then it is transversal. Philosophically, the "distance" between finite and dominating or transversal is large, whereas the "distance" between invertible and submersive or finite is "small".

3.3. Jets of Segre varieties and Segre mapping. The target $M^{\prime}$ concentrates all geometric conditions that are central for the reflection principle. With respect to $\mathcal{M}^{\prime}$, the complexified conjugate Segre variety associated to a fixed $t^{\prime}$ is $\underline{\mathcal{S}}_{t^{\prime}}^{\prime}:=\left\{\left(\zeta^{\prime}, \xi^{\prime}\right) \in \mathbb{C}^{n^{\prime}}: \xi^{\prime}=\Theta^{\prime}\left(\zeta^{\prime}, t^{\prime}\right)\right\}$. Here, $\zeta^{\prime}$ is a parametrizing variable. For $k^{\prime} \in \mathbb{N}$, define the morphism of $k^{\prime}$-th jets of complexified conjugate Segre varieties by:

$$
\varphi_{k^{\prime}}^{\prime}\left(\zeta^{\prime}, t^{\prime}\right):=J_{\tau^{\prime}}^{k^{\prime}} \underline{\mathcal{S}}_{t^{\prime}}^{\prime}:=\left(\zeta^{\prime},\left(\frac{1}{\beta^{\prime} !} \partial_{\zeta^{\prime}}^{\beta^{\prime}} \Theta_{j^{\prime}}^{\prime}\left(\zeta^{\prime}, t^{\prime}\right)\right)_{1 \leqslant j^{\prime} \leqslant d^{\prime}, \beta^{\prime} \in \mathbb{N}^{m^{\prime}},\left|\beta^{\prime}\right| \leqslant k^{\prime}}\right) .
$$

It takes values in $\mathbb{C}^{m^{\prime}+N_{d^{\prime}, m^{\prime}, k^{\prime}}}$, with $N_{d^{\prime}, m^{\prime}, k^{\prime}}:=d^{\prime} \frac{\left(m^{\prime}+k^{\prime}\right) !}{m^{\prime} ! k^{\prime} !}$. If $k_{1}^{\prime} \leqslant k_{2}^{\prime}$, we have of course $\pi_{k_{2}^{\prime}, k_{1}^{\prime}} \circ \varphi_{k_{2}^{\prime}}^{\prime}=\varphi_{k_{1}^{\prime}}^{\prime}$.

As observed in [DW1980], the properties of this morphism govern the various reflection principles. We shall say ([Me2004a, Me2005]) that $M^{\prime}$ (or equivalently $\mathcal{M}^{\prime}$ ) is:

(nd1) Levi non-degenerate at the origin if $\varphi_{1}^{\prime}$ is of rank $m^{\prime}+n^{\prime}$ at $\left(\zeta^{\prime}, t^{\prime}\right)=$ $\left(0^{\prime}, 0^{\prime}\right)$;

(nd2) finitely nondegenerate at the origin if there exists an integer $\ell_{0}^{\prime}$ such that $\varphi_{k^{\prime}}^{\prime}$ is of rank $n^{\prime}+m^{\prime}$ at $\left(\zeta^{\prime}, t^{\prime}\right)=\left(0^{\prime}, 0^{\prime}\right)$, for $k^{\prime}=\ell_{0}^{\prime}$, hence for all $k^{\prime} \geqslant \ell_{0}^{\prime}$

(nd3) essentially finite at the origin if there exists an integer $\ell_{0}^{\prime}$ such that $\varphi_{k^{\prime}}^{\prime}$ is a finite holomorphic map at $\left(\zeta^{\prime}, t^{\prime}\right)=\left(0^{\prime}, 0^{\prime}\right)$, for $k^{\prime}=\ell_{0}^{\prime}$, hence for all $k^{\prime} \geqslant \ell_{0}^{\prime}$;

(nd4) Segre nondegenerate at the origin if there exists an integer $\ell_{0}^{\prime}$ such that the restriction of $\varphi_{k^{\prime}}^{\prime}$ to the complexified Segre variety $\mathcal{S}_{0}^{\prime}$ (of complex dimension $m^{\prime}$ ) is of generic rank $m^{\prime}$, for $k^{\prime}=\ell_{0}^{\prime}$, hence for all $k^{\prime} \geqslant \ell_{0}^{\prime}$; 
(nd5) holomorphically nondegenerate if there exists an integer $\ell_{0}^{\prime}$ such that the map $\varphi_{k^{\prime}}^{\prime}$ is of maximal possible generic rank, equal to $m^{\prime}+$ $n^{\prime}$, for $k^{\prime}=\ell_{0}^{\prime}$, hence for all $k^{\prime} \geqslant \ell_{0}^{\prime}$.

Theorem 3.4. ([Me2004a $]$ ) These five conditions are biholomorphically invariant and: (nd1) $\Rightarrow$ (nd2) $\Rightarrow$ (nd3) $\Rightarrow$ (nd4) $\Rightarrow$ (nd5).

Being not punctual, the last condition (nd5) is the finest: as every condition of maximal generic rank, it propagates from any small open subet to big connected open sets, thanks to the principle of analytic continuation. Notably, if a connected real analytic $M^{\prime}$ is holomorphically nondegenerate "at" a point, it is automatically holomorphically nondegenerate "at" every point ([St1996, BER1999, Me2004a]).

To explain the (crucial) biholomorphic invariance of the jet map $\varphi_{k^{\prime}}^{\prime}$, consider a local biholomorphism $t^{\prime} \mapsto h^{\prime}\left(t^{\prime}\right)=t^{\prime \prime}$, where $t^{\prime}, t^{\prime \prime} \in \mathbb{C}^{n^{\prime}}$, that fixes the origin, $h_{i^{\prime}}^{\prime}\left(t^{\prime}\right) \in \mathbb{C}\left\{t^{\prime}\right\}, h_{i^{\prime}}^{\prime}\left(0^{\prime}\right)=0^{\prime}$, for $i^{\prime}=1, \ldots, n^{\prime}$. Splitting the coordinates $t^{\prime \prime}=\left(z^{\prime \prime}, w^{\prime \prime}\right) \in \mathbb{C}^{m^{\prime}} \times \mathbb{C}^{d^{\prime}}$, the image $M^{\prime \prime}$ may be similarly represented by $\bar{w}^{\prime \prime}=\Theta^{\prime \prime}\left(\bar{z}^{\prime \prime}, t^{\prime \prime}\right)$ and there exists a $d^{\prime} \times d^{\prime}$ matrix $b^{\prime}\left(t^{\prime}, \tau^{\prime}\right)$ of local holomorphic functions such that

$$
r^{\prime \prime}\left(h^{\prime}\left(t^{\prime}\right), \bar{h}^{\prime}\left(\tau^{\prime}\right)\right) \equiv b^{\prime}\left(t^{\prime}, \tau^{\prime}\right) r^{\prime}\left(t^{\prime}, \tau^{\prime}\right)
$$

in $\mathbb{C}\left\{t^{\prime}, \tau^{\prime}\right\}^{d^{\prime}}$, where $r_{j^{\prime}}^{\prime}\left(t^{\prime}, \tau^{\prime}\right):=\xi_{j^{\prime}}^{\prime}-\Theta_{j^{\prime}}^{\prime}\left(\zeta^{\prime}, t^{\prime}\right)$ and $r_{j^{\prime}}^{\prime \prime}\left(t^{\prime \prime}, \tau^{\prime \prime}\right):=\xi_{j^{\prime}}^{\prime \prime}-$ $\Theta_{j^{\prime}}^{\prime \prime}\left(\zeta^{\prime \prime}, t^{\prime \prime}\right)$, for $j^{\prime}=1, \ldots, d^{\prime}$. Setting $h^{\prime}\left(t^{\prime}\right):=\left(f^{\prime}\left(t^{\prime}\right), g^{\prime}\left(t^{\prime}\right)\right) \in \mathbb{C}\left\{t^{\prime}\right\}^{m^{\prime}} \times$ $\mathbb{C}\left\{t^{\prime}\right\}^{d^{\prime}}$ and replacing $\xi^{\prime}$ by $\Theta^{\prime}\left(\zeta^{\prime}, t^{\prime}\right)$ in the above equation, the right hand side vanishes identically (since $r^{\prime}\left(t^{\prime}, \tau^{\prime}\right)=\xi^{\prime}-\Theta^{\prime}\left(\zeta^{\prime}, t^{\prime}\right)$ by definition) and we obtain the following formal identity in $\mathbb{C}\left\{\zeta^{\prime}, t^{\prime}\right\}^{d^{\prime}}$ :

$$
\bar{g}^{\prime}\left(\zeta^{\prime}, \Theta^{\prime}\left(\zeta^{\prime}, t^{\prime}\right)\right) \equiv \Theta^{\prime \prime}\left(\bar{f}^{\prime}\left(\zeta^{\prime}, \Theta^{\prime}\left(\zeta^{\prime}, t^{\prime}\right)\right), h^{\prime}\left(t^{\prime}\right)\right) .
$$

Some algebraic manipulations conduct to the following.

Lemma 3.5. ([Me2004a, Me2005]]) For every $j^{\prime}=1, \ldots, d^{\prime}$ and every $\beta^{\prime} \in \mathbb{N}^{m^{\prime}}$, there exists a universal rational map $Q_{j^{\prime}, \beta^{\prime}}^{\prime}$ whose expression depends neither on $\mathcal{M}^{\prime}$, nor on $h^{\prime}$, nor on $\mathcal{M}^{\prime \prime}$, such that the following identities in $\mathbb{C}\left\{\zeta^{\prime}, t^{\prime}\right\}$ hold true:

$$
\begin{aligned}
& \frac{1}{\beta^{\prime} !} \frac{\partial^{\left|\beta^{\prime}\right|} \Theta_{j^{\prime}}^{\prime \prime}}{\partial\left(\zeta^{\prime \prime}\right)^{\beta^{\prime}}}\left(\bar{f}^{\prime}\left(\zeta^{\prime}, \Theta^{\prime}\left(\zeta^{\prime}, t^{\prime}\right)\right), h^{\prime}\left(t^{\prime}\right)\right) \equiv \\
& \equiv Q_{j^{\prime}, \beta^{\prime}}^{\prime}\left(\left(\partial_{\zeta^{\prime}}^{\beta_{1}^{\prime}} \Theta_{j_{1}^{\prime}}^{\prime}\left(\zeta^{\prime}, t^{\prime}\right)\right)_{1 \leqslant j_{1}^{\prime} \leqslant d^{\prime},\left|\beta_{1}^{\prime}\right| \leqslant\left|\beta^{\prime}\right|},\left(\partial_{\tau^{\prime}}^{\alpha_{1}^{\prime}} \bar{h}_{i_{1}^{\prime}}^{\prime}\left(\zeta^{\prime}, \Theta^{\prime}\left(\zeta^{\prime}, t^{\prime}\right)\right)\right)_{1 \leqslant i_{1}^{\prime} \leqslant n^{\prime},\left|\alpha_{1}^{\prime}\right| \leqslant\left|\beta^{\prime}\right|}\right) \\
& =: R_{j^{\prime}, \beta^{\prime}}^{\prime}\left(\zeta^{\prime},\left(\partial_{\zeta^{\prime}}^{\beta_{1}^{\prime}} \Theta_{j_{1}^{\prime}}\left(\zeta^{\prime}, t^{\prime}\right)\right)_{1 \leqslant j_{1}^{\prime} \leqslant d^{\prime},\left|\beta_{1}^{\prime}\right| \leqslant\left|\beta^{\prime}\right|}\right)
\end{aligned}
$$


where the last line defines $R_{j^{\prime}, \beta^{\prime}}^{\prime}$ by forgetting the jets of $\bar{h}^{\prime}$. Here, the $Q_{j^{\prime}, \beta^{\prime}}^{\prime}$ are holomorphic in a neighborhood of the constant jet

$$
\left(\left(\partial_{\zeta^{\prime}}^{\beta_{1}^{\prime}} \Theta_{j_{1}^{\prime}}^{\prime}(0,0)\right)_{1 \leqslant j_{1}^{\prime} \leqslant d^{\prime},\left|\beta_{1}^{\prime}\right| \leqslant\left|\beta^{\prime}\right|},\left(\partial_{\tau^{\prime}}^{\alpha_{1}^{\prime}} \bar{h}_{i_{1}^{\prime}}^{\prime}(0,0)\right)_{1 \leqslant i_{1}^{\prime} \leqslant n,\left|\alpha_{1}^{\prime}\right| \leqslant\left|\beta^{\prime}\right|}\right) .
$$

Some symmetric relations hold after replacing $\Theta^{\prime}, \Theta^{\prime \prime}, \zeta^{\prime}, t^{\prime}, \bar{f}^{\prime}, h^{\prime}$ by $\bar{\Theta}^{\prime}$, $\bar{\Theta}^{\prime \prime}, z^{\prime}, \tau^{\prime}, f^{\prime}, \bar{h}^{\prime}$.

The existence of $R_{j^{\prime}, \beta^{\prime}}^{\prime}$ says that the following diagram is commutative :

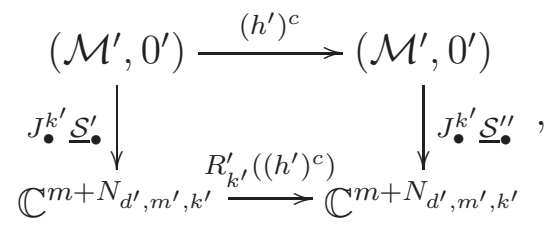

where the biholomorphic map $R_{k^{\prime}}^{\prime}\left(\left(h^{\prime}\right)^{c}\right)$, which depends on $\left(h^{\prime}\right)^{c}$, is defined by its components $R_{j^{\prime}, \beta^{\prime}}^{\prime}$ for $j^{\prime}=1, \ldots, d^{\prime}$ and $\left|\beta^{\prime}\right| \leqslant k^{\prime}$. Thanks to the invertibility of $h^{\prime}$, the map $R_{k^{\prime}}^{\prime}\left(\left(h^{\prime}\right)^{c}\right)$ is also checked to be invertible, and then the invariance of the five nondegeneracy conditions (nd1), (nd2), (nd3), (nd4) and (nd5) is easily established ("Me2004a]).

We now present the Segre mapping of $M^{\prime}$. By developing the series $\Theta_{j^{\prime}}^{\prime}\left(\zeta^{\prime}, t^{\prime}\right)$ in powers of $\zeta^{\prime}$, we may write the equations of $\mathcal{M}^{\prime}$ under the form $\xi_{j^{\prime}}^{\prime}=\sum_{\gamma^{\prime} \in \mathbb{N}^{m^{\prime}}}\left(\zeta^{\prime}\right)^{\gamma^{\prime}} \Theta_{j^{\prime}, \gamma^{\prime}}^{\prime}\left(t^{\prime}\right)$ for $j^{\prime}=1, \ldots, d^{\prime}$. In terms of such a development, the infinite Segre mapping of $M^{\prime}$ is defined to be the mapping

$$
\mathcal{Q}_{\infty}^{\prime}: \quad \mathbb{C}^{n^{\prime}} \ni t^{\prime} \longmapsto\left(\Theta_{j^{\prime}, \gamma^{\prime}}^{\prime}\left(t^{\prime}\right)\right)_{1 \leqslant j^{\prime} \leqslant d^{\prime}, \gamma^{\prime} \in \mathbb{N}^{m^{\prime}}} \in \mathbb{C}^{\infty} .
$$

Let $k^{\prime} \in \mathbb{N}$. For finiteness reasons, it is convenient to truncate this infinite collection and to define the $k^{\prime}$-th Segre mapping of $M^{\prime}$ by

$$
\mathcal{Q}_{k^{\prime}}^{\prime}: \quad \mathbb{C}^{n^{\prime}} \ni t^{\prime} \longmapsto\left(\Theta_{j^{\prime}, \gamma^{\prime}}^{\prime}\left(t^{\prime}\right)\right)_{1 \leqslant j^{\prime} \leqslant d^{\prime},\left|\gamma^{\prime}\right| \leqslant k^{\prime}} \in \mathbb{C}^{N_{d^{\prime}, n^{\prime}, k^{\prime}}},
$$

where $N_{d^{\prime}, n^{\prime}, k^{\prime}}=d^{\prime} \frac{\left(n^{\prime}+k^{\prime}\right) !}{n^{\prime} ! k^{\prime} !}$. If $k_{2}^{\prime} \geqslant k_{1}^{\prime}$, we have $\pi_{k_{2}^{\prime}, k_{1}^{\prime}}\left[\mathcal{Q}_{k_{2}^{\prime}}^{\prime}\left(t^{\prime}\right)\right]=\mathcal{Q}_{k_{1}^{\prime}}^{\prime}\left(t^{\prime}\right)$. One verifies ([Me2004a ]) the following characterizations.

(nd1) $M^{\prime}$ is Levi non-degenerate at the origin if and only if $\mathcal{Q}_{1}^{\prime}$ is of rank $n^{\prime}$ at $t^{\prime}=0^{\prime}$.

(nd2) $M^{\prime}$ is finitely nondegenerate at the origin if and only if there exists an integer $\ell_{0}^{\prime}$ such that $\mathcal{Q}_{k^{\prime}}^{\prime}$ is of rank $n^{\prime}$ at $t^{\prime}=0^{\prime}$, for all $k^{\prime} \geqslant \ell_{0}^{\prime}$.

(nd3) $M^{\prime}$ is essentially finite at the origin if there exists an integer $\ell_{0}^{\prime}$ such that $\mathcal{Q}_{k^{\prime}}^{\prime}$ is a finite holomorphic map at $t^{\prime}=0^{\prime}$, for all $k^{\prime} \geqslant \ell_{0}^{\prime}$. 
(nd4) $M^{\prime}$ is Segre nondegenerate at the origin if there exists an integer $\ell_{0}^{\prime}$ such that the restriction of $\mathcal{Q}_{k^{\prime}}^{\prime}$ to the complexified Segre variety $\mathcal{S}_{0^{\prime}}^{\prime}$ (of complex dimension $m^{\prime}$ ) is of generic rank $m^{\prime}$, for all $k^{\prime} \geqslant \ell_{0}^{\prime}$.

(nd5) $M^{\prime}$ is holomorphically nondegenerate if there exists an integer $\ell_{0}^{\prime}$ such that the map $\mathcal{Q}_{k^{\prime}}^{\prime}$ is of maximal possible generic rank, equal to $n^{\prime}$, for all $k^{\prime} \geqslant \ell_{0}^{\prime}$.

3.6. Essential holomorphic dimension and Levi multitype. Assume now that $M^{\prime}$ is not nececessarily local, but connected. Denote by $\ell_{M^{\prime}}^{\prime}$ the smallest integer $k^{\prime}$ such that the generic rank of the jet mappings $\left(t^{\prime}, \tau^{\prime}\right) \mapsto$ $J_{\tau^{\prime}}^{k^{\prime}} \underline{\mathcal{S}}_{t^{\prime}}^{\prime}$ does not increase after $k^{\prime}$ and denote by $m^{\prime}+n_{M^{\prime}}^{\prime} \leqslant m^{\prime}+n^{\prime}$ the (maximal) generic rank of $\left(t^{\prime}, \tau^{\prime}\right) \mapsto J_{\tau^{\prime}}^{\ell^{\prime}} \underline{\mathcal{S}}_{t^{\prime}}^{\prime}$. Since $w^{\prime} \mapsto \Theta^{\prime}\left(\zeta^{\prime}, z^{\prime}, w^{\prime}\right)$ is of rank $d^{\prime}$ according to Theorem 2.5, the (generic) rank of the zero-th order jet map satisfies

$$
\operatorname{genrk}_{\mathbb{C}}\left(\left(t^{\prime}, \tau^{\prime}\right) \mapsto J_{\tau^{\prime}}^{0} \underline{\mathcal{S}}_{t^{\prime}}^{\prime}=\left(\zeta^{\prime}, \Theta^{\prime}\left(\zeta^{\prime}, z^{\prime}, w^{\prime}\right)\right)\right)=m^{\prime}+d^{\prime}=n^{\prime} .
$$

Thus, $d^{\prime} \leqslant n_{M^{\prime}}^{\prime} \leqslant n^{\prime}$. It is natural to call $n_{M^{\prime}}^{\prime}$ the essential holomorphic dimension of $M^{\prime}$ because of the following.

Proposition 3.7. (Me2001a, Me2004a] ) Locally in a neighborhood of a Zariski-generic point $p^{\prime} \in M^{\prime}$, the generic submanifold $M^{\prime}$ is biholomorphically equivalent to the product $\underline{M}_{p^{\prime}}^{\prime} \times \Delta^{n^{\prime}-n_{M^{\prime}}^{\prime}}$, of a generic submanifold $\underline{M}_{p^{\prime}}^{\prime}$ of codimension $d^{\prime}$ in $\mathbb{C}_{M^{\prime}}^{n^{\prime}}$ by a complex polydisc $\Delta^{n^{\prime}-n_{M^{\prime}}^{\prime}}$.

Generally speaking, we may define $\lambda_{0, M^{\prime}}^{\prime}:=\operatorname{genrk}_{\mathbb{C}}\left(\left(t^{\prime}, \tau^{\prime}\right) \mapsto J_{\tau^{\prime}}^{0} \underline{\mathcal{S}}_{t^{\prime}}^{\prime}\right)-$ $m^{\prime}=d^{\prime}$ and for every $k^{\prime}=1, \ldots, \ell_{M^{\prime}}^{\prime}$,

$$
\lambda_{k^{\prime}, M^{\prime}}^{\prime}:=\operatorname{genrk}_{\mathbb{C}}\left(\left(t^{\prime}, \tau^{\prime}\right) \mapsto J_{\tau^{\prime}}^{k^{\prime}} \underline{\mathcal{S}}_{t^{\prime}}^{\prime}\right)-\operatorname{genrk}_{\mathbb{C}}\left(\left(t^{\prime}, \tau^{\prime}\right) \mapsto J_{\tau^{\prime}}^{k^{\prime}-1} \underline{\mathcal{S}}_{t^{\prime}}^{\prime}\right) .
$$

One verifies ([Me2004a] $]$ ) that $\lambda_{1, M^{\prime}}^{\prime} \geqslant 1, \ldots, \lambda_{\ell_{M^{\prime}}^{\prime}, M^{\prime}}^{\prime} \geqslant 1$. With these definitions, we have the relations

$$
\operatorname{genrk}_{\mathbb{C}}\left(\left(t^{\prime}, \tau^{\prime}\right) \mapsto J_{\tau^{\prime}}^{k^{\prime}} \underline{\mathcal{S}}_{t^{\prime}}^{\prime}\right)=m^{\prime}+\lambda_{0, M^{\prime}}^{\prime}+\lambda_{1, M^{\prime}}^{\prime}+\cdots+\lambda_{k^{\prime}, M^{\prime}}^{\prime},
$$

for $k^{\prime}=0,1, \ldots, \ell_{M^{\prime}}^{\prime}$ and

$\operatorname{genrk}_{\mathbb{C}}\left(\left(t^{\prime}, \tau^{\prime}\right) \mapsto J_{\tau^{\prime}}^{k^{\prime}} \underline{\mathcal{S}}_{t^{\prime}}^{\prime}\right)=m^{\prime}+d^{\prime}+\lambda_{1, M^{\prime}}^{\prime}+\cdots+\lambda_{\ell_{M^{\prime}}^{\prime}, M^{\prime}}^{\prime}=m^{\prime}+n_{M^{\prime}}^{\prime}$,

for all $k^{\prime} \geqslant \ell_{M^{\prime}}^{\prime}$. It follows that

$$
\ell_{M^{\prime}}^{\prime} \leqslant \lambda_{1, M^{\prime}}^{\prime}+\cdots+\lambda_{\ell_{M^{\prime}}^{\prime}, M^{\prime}}^{\prime}=n_{M^{\prime}}^{\prime}-d^{\prime} \leqslant m^{\prime} .
$$


Theorem 3.8. ([Me2004a]) Let $M^{\prime}$ be a connected real algebraic or analytic generic submanifold in $\mathbb{C}^{n^{\prime}}$ of codimension $d^{\prime} \geqslant 1$ and of $C R$ dimension $m^{\prime}=n^{\prime}-d^{\prime} \geqslant 1$. Then there exist well defined integers $n_{M^{\prime}}^{\prime} \geqslant d^{\prime}$, $\ell_{M^{\prime}}^{\prime} \geqslant 0, \lambda_{0, M^{\prime}}^{\prime} \geqslant 1, \lambda_{1, M^{\prime}}^{\prime} \geqslant 1, \ldots, \lambda_{\ell_{M^{\prime}}^{\prime}, M^{\prime}}^{\prime} \geqslant 1$ and a proper real algebraic or analytic subvariety $E^{\prime}$ of $M^{\prime}$ such that for every point $p^{\prime} \in M^{\prime} \backslash E^{\prime}$ and for every system of coordinates $\left(z^{\prime}, w^{\prime}\right)$ vanishing at $p^{\prime}$ in which $M^{\prime}$ is represented by defining equations $\bar{w}_{j^{\prime}}=\Theta_{j^{\prime}}^{\prime}\left(\bar{z}^{\prime}, t^{\prime}\right), j^{\prime}=1, \ldots, d^{\prime}$, then the following four properties hold:

- $\lambda_{0, M^{\prime}}^{\prime}=d^{\prime}, d^{\prime} \leqslant n_{M^{\prime}}^{\prime} \leqslant n^{\prime}$ and $\ell_{M^{\prime}}^{\prime} \leqslant n_{M^{\prime}}^{\prime}-d^{\prime}$.

- For every $k^{\prime}=0,1, \ldots, \ell_{M^{\prime}}^{\prime}$, the mapping of $k^{\prime}$-th order jets of the conjugate complexified Segre varieties $\left(t^{\prime}, \tau^{\prime}\right) \mapsto J_{\tau^{\prime}}^{k^{\prime}} \underline{\mathcal{S}}_{t^{\prime}}^{\prime}$ is of rank equal to $m^{\prime}+\lambda_{0, M^{\prime}}^{\prime}+\cdots+\lambda_{k^{\prime}, M^{\prime}}^{\prime}$ at $\left(t_{p^{\prime}}^{\prime}, \bar{t}_{p^{\prime}}^{\prime}\right)=\left(0^{\prime}, 0^{\prime}\right)$.

- $n_{M^{\prime}}^{\prime}=d^{\prime}+\lambda_{1, M^{\prime}}^{\prime}+\cdots+\lambda_{\ell_{M^{\prime}}^{\prime}, M^{\prime}}^{\prime}$ and for every $k^{\prime} \geqslant \ell_{M^{\prime}}^{\prime}$, the mapping of $k^{\prime}$-th order jets of the conjugate complexified Segre varieties $\left(t^{\prime}, \tau^{\prime}\right) \mapsto J_{\tau^{\prime}}^{k^{\prime}} \underline{\mathcal{S}}_{t^{\prime}}^{\prime}$ is of rank equal to $n_{M^{\prime}}^{\prime}$ at $\left(0^{\prime}, 0^{\prime}\right)$.

- There exists a local complex algebraic or analytic change of coordinates $t^{\prime \prime}=h^{\prime}\left(t^{\prime}\right)$ fixing $p^{\prime}$ such that the image $M_{p^{\prime}}^{\prime \prime}:=h^{\prime}\left(M^{\prime}\right)$ is locally in a neighborhood of $p^{\prime}$ the product $\underline{M}_{p^{\prime}}^{\prime \prime} \times \Delta^{n^{\prime}-n_{M^{\prime}}^{\prime}}$ of a real algebraic or analytic generic submanifold of codimension $d^{\prime}$ in $\mathbb{C}_{M^{\prime}}^{n^{\prime}}$ by a complex polydisc $\Delta^{n^{\prime}-n_{M^{\prime}}^{\prime}}$. Furthermore, at the central point $\underline{p}^{\prime} \in \underline{M}_{p^{\prime}}^{\prime \prime} \subset \mathbb{C}_{M^{\prime}}^{n^{\prime}}$, the generic submanifold $\underline{M}_{p^{\prime}}^{\prime \prime}$ is $\ell_{M^{\prime}}^{\prime}$-finitely nondegenerate, hence in particular its essential holomorphic dimension $n_{\underline{M}_{p^{\prime}}^{\prime \prime}}^{\prime}$ coincides with $n_{M^{\prime}}^{\prime}$.

In particular, $M^{\prime}$ is holomorphically nondegenerate if and only if $n_{M^{\prime}}^{\prime}=$ $n^{\prime}$ and in this case, $M^{\prime}$ is finitely nondegenerate at every point of the Zariski-open subset $M^{\prime} \backslash E^{\prime}$.

3.9. CR-horizontal nondegeneracy conditions. As in $\S 3.1$, let $h=$ $h(t) \in \mathbb{C} \llbracket t \rrbracket^{n^{\prime}}$ be a formal CR mapping $(M, 0) \rightarrow_{\mathcal{F}}\left(M^{\prime}, 0^{\prime}\right)$. Decompose $h(t)=(f(t), g(t)) \in \mathbb{C} \llbracket t \rrbracket^{m^{\prime}} \times \mathbb{C} \llbracket t \rrbracket^{d^{\prime}}$, as in the splitting $t^{\prime}=\left(z^{\prime}, w^{\prime}\right) \in \mathbb{C}^{m^{\prime}} \times \mathbb{C}^{d^{\prime}}$. Replacing $w$ by $\bar{\Theta}(z, \tau)$ in the fundamental identity $\bar{r}^{\prime}(\bar{h}(\tau), h(t)) \equiv \bar{b}(\tau, t) \bar{r}(\tau, t)$, the right hand side vanishes identically (since $\bar{r}(\tau, t)=w-\bar{\Theta}(z, \tau)$ by definition), and we get a formal identity in $\mathbb{C} \llbracket z, \tau \rrbracket^{d^{\prime}}$ :

$$
g(z, \bar{\Theta}(z, \tau)) \equiv \bar{\Theta}^{\prime}(f(z, \bar{\Theta}(z, \tau)), \bar{h}(\tau)) .
$$


Setting $\tau:=0$, we get $g(z, \bar{\Theta}(z, 0)) \equiv \bar{\Theta}^{\prime}(f(z, \bar{\Theta}(z, 0)), 0)$. In other words, $\left.h\right|_{\mathcal{S}_{0}}$ maps $\mathcal{S}_{0}$ formally to $\mathcal{S}_{0^{\prime}}^{\prime}$. The restriction $\left.h\right|_{\mathcal{S}_{0}}$ coincides with the formal map:

$$
\mathbb{C}^{m} \ni z \longmapsto \mathcal{F}\left(f(z, \bar{\Theta}(z, 0)), \bar{\Theta}^{\prime}(f(z, \bar{\Theta}(z, 0)), 0)\right) \in \mathbb{C}^{m^{\prime}} \times \mathbb{C}^{d^{\prime}} .
$$

The rank properties of this formal map are the same as those of its $C R$ horizontal part:

$$
\mathbb{C}^{m} \ni z \longmapsto \mathcal{F} f(z, \bar{\Theta}(z, 0)) \in \mathbb{C}^{m^{\prime}} .
$$

The formal CR mapping $h$ is said ([Me2004a] $]$ ) to be:

(cr1) $C R$-invertible at the origin if $m^{\prime}=m$ and if its CR-horizontal part is a formal equivalence at $z=0$;

(cr2) $C R$-submersive at the origin if $m^{\prime} \leqslant m$ and if its CR-horizontal part is a formal submersion at $z=0$;

(cr3) $C R$-finite at the origin if $m^{\prime}=m$ and if its CR-horizontal part is a finite formal map at $z=0$, namely the quotient ring $\mathbb{C} \llbracket z \rrbracket /\left(f_{k^{\prime}}(z, \bar{\Theta}(z, 0))_{1 \leqslant k^{\prime} \leqslant m^{\prime}}\right)$ is finite-dimensional (the requirement $m^{\prime}=m$ is necessary for the reflection principle below);

(cr4) CR-dominating at the origin if $m^{\prime} \leqslant m$ and if there exist integers $1 \leqslant k(1)<\cdots<k\left(m^{\prime}\right) \leqslant m$ such that the determinant $\operatorname{det}\left(\left[\partial \phi_{k_{1}^{\prime}} / \partial z_{k\left(k_{2}^{\prime}\right)}\right](z)\right)_{1 \leqslant k_{1}^{\prime}, k_{2}^{\prime} \leqslant m^{\prime}} \not \equiv 0$ does not vanish identically in $\mathbb{C} \llbracket z \rrbracket$, where $\phi_{k^{\prime}}(z):=f_{k^{\prime}}(z, \bar{\Theta}(z, 0))$;

(cr5) $C R$-transversal at the origin if there does not exist a nonzero formal power series $F^{\prime}\left(f_{1}, \ldots, f_{m^{\prime}}\right) \in \mathbb{C} \llbracket f_{1}, \ldots, f_{m^{\prime}} \rrbracket$ such that $F^{\prime}\left(\phi_{1}(z), \ldots, \phi_{m^{\prime}}(z)\right) \equiv 0$ in $\mathbb{C} \llbracket z \rrbracket$, where $\phi_{k^{\prime}}(z):=f_{k^{\prime}}(z, \bar{\Theta}(z, 0))$.

One verifies ([Me2004a $]$ ) biholomorphic invariance and the four implications:

$$
\text { (cr1) } \Rightarrow(\text { cr2 }) \Rightarrow(\text { cr3) } \Rightarrow \text { (cr4) } \Rightarrow \text { (cr5), }
$$

provided that $m^{\prime}=m$ in the second and in the third. By far, CRtransversality is the most general nondegeneracy condition.

3.10. Nondegeneracy conditions for CR mappings. This subsection explains how to synthetize the combinatorics of various formal reflection principles published in the last decade.

As in $\S 3.1$, let $h^{c}:(\mathcal{M}, 0) \rightarrow_{\mathcal{F}}\left(\mathcal{M}^{\prime}, 0\right)$ be a complexified formal CR mapping between two formal, analytic or algebraic complexified generic submanifolds of equations $0=r(t, \tau):=\xi-\Theta(\zeta, t)$ and $0=r^{\prime}\left(t^{\prime}, \tau^{\prime}\right):=$ 
$\xi^{\prime}-\Theta^{\prime}\left(\zeta^{\prime}, t^{\prime}\right)$. By hypothesis, $r^{\prime}(h(t), \bar{h}(\tau)) \equiv b(t, \tau) r(t, \tau)$. Denoting $h=(f, g) \in \mathbb{C}^{m^{\prime}} \times \mathbb{C}^{d^{\prime}}$, replacing $\xi$ by $\Theta(\zeta, t)$ in $r^{\prime}(h(t), \bar{h}(\tau)) \equiv$ $b(t, \tau) r(t, \tau)$ and developing $\Theta^{\prime}(\bar{f}, h)=\sum_{\gamma^{\prime} \in \mathbb{N}^{m^{\prime}}} \bar{f}^{\gamma^{\prime}} \Theta_{\gamma^{\prime}}^{\prime}(h)$, we start with the following fundamental power series identity in $\mathbb{C} \llbracket \zeta, t \rrbracket^{d^{\prime}}$ :

$$
\begin{aligned}
\bar{g}(\zeta, \Theta(\zeta, t)) & \equiv \Theta^{\prime}(\bar{f}(\zeta, \Theta(\zeta, t)), h(t)) \\
& \equiv \sum_{\gamma^{\prime} \in \mathbb{N}^{m^{\prime}}} \bar{f}(\zeta, \Theta(\zeta, t))^{\gamma^{\prime}} \Theta_{\gamma^{\prime}}^{\prime}(h(t)) .
\end{aligned}
$$

Consider the $m$ complex vector fields $\underline{\mathcal{L}}_{1}, \ldots, \underline{\mathcal{L}}_{m}$ tangent to $\mathcal{M}$ that were defined in $\S 2.7$. For every $\beta=\left(\beta_{1}, \ldots, \beta_{m}\right) \in \mathbb{N}^{m}$, define the multiple derivation $\underline{\mathcal{L}}^{\beta}=\underline{\mathcal{L}}_{1}^{\beta_{1}} \cdots \underline{\mathcal{L}}_{m}^{\beta_{m}}$. Applying them to the above $d^{\prime}$ scalar equations, observing that they do not differentiate the variables $t=(z, w)$, we get, without writing the arguments:

$$
\underline{\mathcal{L}}^{\beta} \bar{g}_{j^{\prime}}-\sum_{\gamma^{\prime} \in \mathbb{N}^{m^{\prime}}} \underline{\mathcal{L}}^{\beta}\left(\bar{f}^{\gamma^{\prime}}\right) \Theta_{j^{\prime}, \gamma^{\prime}}^{\prime}(h) \equiv 0,
$$

for all $\beta \in \mathbb{N}^{m}$, all $j^{\prime}=1, \ldots, d^{\prime}$ and all $(t, \tau) \in \mathcal{M}$.

Lemma 3.11. ([Me2004a, Me2005]) For every $i^{\prime}=1, \ldots, n^{\prime}$ and every $\beta \in \mathbb{N}^{m}$, there exists a polynomial $P_{i^{\prime}, \beta}$ in the jet $J_{\tau}^{|\beta|} \bar{h}(\tau)$ with coefficients being power series in $(t, \tau)$ which depend only on the defining functions $\xi_{j}-\Theta_{j}(\zeta, t)$ of $\mathcal{M}$ and which can be computed by means of some combinatorial formula, such that

$$
\underline{\mathcal{L}}^{\beta} \bar{h}_{i^{\prime}}(\tau) \equiv P_{i^{\prime}, \beta}\left(t, \tau, J_{\tau}^{|\beta|} \bar{h}(\tau)\right) .
$$

Convention 3.12. Let $k, l \in \mathbb{N}$. On the complexification $\mathcal{M}$, equipped with either the coordinates $(z, \tau)$ or $(\zeta, t)$, which correspond to either replacing $w$ by $\bar{\Theta}(z, \tau)$ or $\xi$ by $\Theta(\zeta, t)$, we shall identify (notationally) a power series written under the complete form

$$
R\left(t, \tau, J^{k} h(t), J^{l} \bar{h}(\tau)\right),
$$

with a power series written under one of the following four forms:

- $R\left(t, \zeta, \Theta(\zeta, t), J^{k} h(t), J^{l} \bar{h}(\zeta, \Theta(\zeta, t))\right)$,

- $R\left(t, \zeta, J^{k} h(t), J^{l} \bar{h}(\zeta, \Theta(\zeta, t))\right)$,

- $R\left(z, \bar{\Theta}(z, \tau), \tau, J^{k} h(z, \bar{\Theta}(z, \tau)), J^{l} \bar{h}(\tau)\right)$,

- $R\left(z, \tau, J^{k} h(z, \bar{\Theta}(z, \tau)), J^{l} \bar{h}(\tau)\right)$. 
Thanks to the lemma and to the convention, we may therefore write:

$$
\underline{\mathcal{L}}^{\beta}\left[\bar{g}_{j^{\prime}}(\tau)-\Theta_{j^{\prime}}^{\prime}(\bar{f}(\tau), h(t))\right]=: R_{j^{\prime}, \beta}^{\prime}\left(t, \tau, J_{\tau}^{|\beta|} \bar{h}(\tau): h(t)\right) \equiv 0,
$$

for $j^{\prime}=1, \ldots, d^{\prime}$. Remind that $h(t)$ is not differentiated, since the derivations $\underline{\mathcal{L}}^{\beta}$ involve only $\frac{\partial}{\partial \tau_{i}}, i=1, \ldots, n$. This is why we write $h(t)$ after “:". Furthmerore, the identities " $\equiv 0$ " are understood "on $\mathcal{M}$ ", namely as formal power series identities in $\mathbb{C} \llbracket \zeta, t \rrbracket$ after replacing $\xi$ by $\Theta(\zeta, t)$ or equivalently, as a formal power series identities in $\mathbb{C} \llbracket z, \tau \rrbracket$ after replacing $w$ by $\bar{\Theta}(z, \tau)$.

To understand the reflection principle, it is important to observe immediately that the smoothness of the power series $R_{j^{\prime}, \beta}^{\prime}$ is the minimum of the two smoothnesses of $M$ and of $M^{\prime}$. For instance, the power series $R_{j^{\prime}, \beta}^{\prime}$ are all complex analytic if $M$ is real analytic and if $M^{\prime}$ is real algebraic, even if the power series CR mapping $h(t)$ was assumed to be purely formal and nonconvergent. By a careful inspection of the application of the chain rule in the development of the above equations (3.13) ( $c f$. Lemma 3.11), we even see that each $R_{j^{\prime}, \beta}^{\prime}$ is relatively polynomial with respect to the derivatives of positive order $\left(\partial_{\tau}^{\alpha} \bar{h}(\tau)\right)_{1 \leqslant|\alpha| \leqslant|\beta|}$.

3.14. Nondegeneracy conditions for formal CR mappings. In the equations (3.13), we replace $h(t)$ by a new independent variable $t^{\prime} \in \mathbb{C}^{n^{\prime}}$, we set $(t, \tau)=(0,0)$, and we define the following collection of power series

$$
\Psi_{j^{\prime}, \beta}^{\prime}\left(t^{\prime}\right):=\left[\underline{\mathcal{L}}^{\beta} \bar{g}_{j^{\prime}}-\sum_{\gamma^{\prime} \in \mathbb{N}^{\prime}{ }^{\prime}} \underline{\mathcal{L}}^{\beta}\left(\bar{f}^{\gamma^{\prime}}\right) \Theta_{j^{\prime}, \gamma^{\prime}}^{\prime}\left(t^{\prime}\right)\right]_{t=\tau=0},
$$

for $j^{\prime}=1, \ldots, d^{\prime}$ and $\beta \in \mathbb{N}^{m}$. Here, if $\beta=0$, we mean that $\Psi_{j^{\prime}, 0}^{\prime}\left(t^{\prime}\right)=$ $-\Theta_{j^{\prime}}^{\prime}\left(0, t^{\prime}\right)$. According to (3.13), an equivalent definition is:

$$
\Psi_{j^{\prime}, \beta}^{\prime}\left(t^{\prime}\right):=R_{j^{\prime}, \beta}^{\prime}\left(0,0, J_{\tau}^{|\beta|} \bar{h}(0): t^{\prime}\right) .
$$

Now, just before introducing five new nondegeneracy conditions, we make a crucial heuristic remark. When $n=n^{\prime}, m=m^{\prime}, M=M^{\prime}$ and $h=$ Id, writing $T^{\prime}$ instead of $t^{\prime}$ the special variable above in order to avoid confusion, we get for $j^{\prime}=1, \ldots, d^{\prime}$ and $\beta^{\prime} \in \mathbb{N}^{m^{\prime}}$ :

$$
\begin{aligned}
\Psi_{j^{\prime}, \beta^{\prime}}^{\prime}\left(T^{\prime}\right) & =\left[\underline{\mathcal{L}}^{\prime \beta^{\prime}} \xi_{j^{\prime}}^{\prime}-\sum_{\gamma^{\prime} \in \mathbb{N}^{m^{\prime}}}{\underline{\mathcal{L}^{\prime}}}^{\beta^{\prime}}\left(\zeta^{\prime}\right)^{\gamma^{\prime}} \Theta_{j^{\prime}, \gamma^{\prime}}^{\prime}\left(T^{\prime}\right)\right]_{t^{\prime}=\tau^{\prime}=0^{\prime}} \\
& =\left[\underline{\mathcal{L}}^{\prime \beta^{\prime}} \Theta_{j^{\prime}}^{\prime}\left(\zeta^{\prime}, t^{\prime}\right)-\beta^{\prime} ! \Theta_{j^{\prime}, \beta^{\prime}}^{\prime}\left(T^{\prime}\right)\right]_{t^{\prime}=\tau^{\prime}=0^{\prime}} \\
& =\beta^{\prime} !\left(\Theta_{j^{\prime}, \beta^{\prime}}^{\prime}\left(0^{\prime}\right)-\Theta_{j^{\prime}, \beta^{\prime}}^{\prime}\left(T^{\prime}\right)\right) .
\end{aligned}
$$


Consequently, up to a translation by a constant, we recover with $\Psi_{j^{\prime}, \beta^{\prime}}^{\prime}\left(T^{\prime}\right)$ the components of the infinite Segre mapping $\mathcal{Q}_{\infty}^{\prime}$ of $M^{\prime}$. Hence the next definition generalizes the concepts introduced before.

Definition 3.15. The formal CR mapping $h:(M, 0) \rightarrow_{\mathcal{F}}\left(M^{\prime}, 0^{\prime}\right)$ is called

(h1) Levi-nondegenerate at the origin if the mapping

$$
t^{\prime} \mapsto\left(R_{j^{\prime}, \beta}^{\prime}\left(0,0, J_{\tau}^{|\beta|} \bar{h}(0): t^{\prime}\right)\right)_{1 \leqslant j^{\prime} \leqslant d^{\prime},|\beta| \leqslant 1}
$$

is of rank $n^{\prime}$ at $t^{\prime}=0^{\prime}$;

(h2) finitely nondegenerate at the origin if there exists an integer $\ell_{1}$ such that the mapping

$$
t^{\prime} \mapsto\left(R_{j^{\prime}, \beta}^{\prime}\left(0,0, J_{\tau}^{|\beta|} \bar{h}(0): t^{\prime}\right)\right)_{1 \leqslant j^{\prime} \leqslant d^{\prime},|\beta| \leqslant k}
$$

is of rank $n^{\prime}$ at $t^{\prime}=0^{\prime}$, for $k=\ell_{1}$, hence for every $k \geqslant \ell_{1}$;

(h3) essentially finite at the origin if there exists an integer $\ell_{1}$ such that the mapping

$$
t^{\prime} \mapsto\left(R_{j^{\prime}, \beta}^{\prime}\left(0,0, J_{\tau}^{|\beta|} \bar{h}(0): t^{\prime}\right)\right)_{1 \leqslant j^{\prime} \leqslant d^{\prime},|\beta| \leqslant k}
$$

is locally finite at $t^{\prime}=0^{\prime}$, for $k=\ell_{1}$, hence for every $k \geqslant \ell_{1}$;

(h4) Segre nondegenerate at the origin if there exist an integer $\ell_{1}$, integers $j_{*}^{\prime 1}, \ldots, j_{*}^{\prime n^{\prime}}$ with $1 \leqslant j_{*}^{\prime i^{\prime}} \leqslant d^{\prime}$ for $i^{\prime}=1, \ldots, n^{\prime}$ and multiindices $\beta_{*}^{1}, \ldots, \beta_{*}^{n^{\prime}}$ with $\left|\beta_{*}^{i^{\prime}}\right| \leqslant \ell_{1}$ for $i^{\prime}=1, \ldots, n^{\prime}$, such that the determinant

$\operatorname{det}\left(\frac{\partial R_{j_{*}^{\prime} i_{1}^{\prime}, \beta_{*}^{i_{1}^{\prime}}}}{\partial t_{i_{2}^{\prime}}^{\prime}}\left(z, \bar{\Theta}(z, 0), 0,0, J^{\left|\beta_{*}^{i_{1}^{\prime}}\right|} \bar{h}(0): h(z, \bar{\Theta}(z, 0))\right)\right)_{1 \leqslant i_{1}^{\prime}, i_{2}^{\prime} \leqslant n^{\prime}}$

does not vanish identically in $\mathbb{C} \llbracket z \rrbracket$;

(h5) holomorphically nondegenerate at the origin if there exists an integer $\ell_{1}$, integers $j_{*}^{\prime 1}, \ldots, j_{*}^{\prime n^{\prime}}$ with $1 \leqslant j_{*}^{\prime i^{\prime}} \leqslant d^{\prime}$ for $i^{\prime}=1, \ldots, n^{\prime}$ and multiindices $\beta_{*}^{1}, \ldots, \beta_{*}^{n^{\prime}}$ with $\left|\beta_{*}^{i^{\prime}}\right| \leqslant \ell_{1}$ for $i^{\prime}=1, \ldots, n^{\prime}$, such that the determinant

$$
\operatorname{det}\left(\frac{\partial R_{j_{*}^{\prime} i_{1}^{\prime}, \beta_{*}^{i_{1}^{\prime}}}^{\prime}}{\partial t_{i_{2}^{\prime}}^{\prime}}\left(0,0,0,0, J^{\left|\beta_{*}^{i_{1}^{\prime}}\right|} \bar{h}(0): h(t)\right)\right)_{1 \leqslant i_{1}^{\prime}, i_{2}^{\prime} \leqslant n^{\prime}}
$$

does not vanish identically in $\mathbb{C} \llbracket t \rrbracket$. 
The nondegeneracy of the formal mapping $h$ requires the same nondegeneracy on the target $\left(M^{\prime}, 0^{\prime}\right)$.

Lemma 3.16. ([Me2004a]) Let $h:(M, 0) \rightarrow_{\mathcal{F}}\left(M^{\prime}, 0^{\prime}\right)$ be a formal $C R$ mapping.

(1) If $h$ is Levi-nondegenerate at 0 , then $M^{\prime}$ is necessarily Levinondegenerate at $0^{\prime}$.

(2) If $h$ is finitely nondegenerate at 0 , then $M^{\prime}$ is necessarily finitely nondegenerate at $0^{\prime}$.

(3) If $h$ is essentially finite at 0 , then $M^{\prime}$ is necessarily essentially finite at $0^{\prime}$.

(4) If $h$ is Segre nondegenerate at 0 , then $M^{\prime}$ is necessarily Segre nondegenerate at 0 '.

(5) If $h$ is holomorphically nondegenerate at 0 , then $M^{\prime}$ is necessarily holomorphically nondegenerate at 0 '.

We now show that CR-transversality of the mapping $h$ insures that it enjoys exactly the same nondegeneracy condition as the target $\left(M^{\prime}, 0^{\prime}\right)$.

Theorem 3.17. ([Me2004a]) Assume that the formal CR mapping $h$ : $(M, 0) \rightarrow_{\mathcal{F}}\left(M^{\prime}, 0^{\prime}\right)$ is CR-transversal at 0 . Then the following five implications hold:

(1) If $M^{\prime}$ is Levi nondegenerate at $0^{\prime}$, then $h$ is finitely nondegenerate at 0 .

(2) If $M^{\prime}$ is finitely nondegenerate at $0^{\prime}$, then $h$ is finitely nondegenerate at 0.

(3) If $M^{\prime}$ is essentially finite at $0^{\prime}$, then $h$ is essentially finite at 0.

(4) If $M^{\prime}$ is Segre nondegenerate at $0^{\prime}$, then $h$ is Segre nondegenerate at 0 .

(5) If $M^{\prime}$ is holomorphically nondegenerate, and if moreover $h$ is transversal at 0 , then $h$ is holomorphically nondegenerate at 0 .

The above five implications also hold under the assumption that $h$ is either CR-invertible, or CR-submersive, or CR-finite with $m=m^{\prime}$ or CRdominating: this provides at least 20 more (less refined) versions of the theorem, some of which appear in the literature.

Other relations hold true between the nondegeneracy conditions on $h$ and on the generic submanifolds $(M, 0)$ and $\left(M^{\prime}, 0^{\prime}\right)$. We mention some, concisely. As above, assume that $h:(M, 0) \mapsto_{\mathcal{F}}\left(M^{\prime}, 0\right)$ is a formal CR 
mapping. Since $d h_{0}\left(T_{0}^{c} M\right) \subset T_{0}^{c} M^{\prime}$, a linear map $d h_{0}^{\operatorname{trv}}: T_{0} M / T_{0}^{c} M \rightarrow$ $T_{0} M^{\prime} / T_{0}^{c} M^{\prime}$ is induced. Assume $d^{\prime}=d$ and $m^{\prime}=m$. The next statement may be interpreted as a kind of Hopf Lemma for CR mappings.

Theorem 3.18. ([BR1990, ER2004]) If $M$ is minimal at 0 and if $h$ is $C R$ dominating at 0 , then $d h_{0}^{\operatorname{trv}}: T_{0} M / T_{0}^{c} M \rightarrow T_{0} M^{\prime} / T_{0}^{c} M^{\prime}$ is an isomorphim.

An open question is to determine whether the condition that the jacobian determinant $\operatorname{det}\left(\frac{\partial h_{i}}{\partial t_{j}}(t)\right)_{1 \leqslant i, j \leqslant n}$ does not vanish identically in $\mathbb{C} \llbracket t \rrbracket$ is sufficient to insure that $d h_{0}^{\text {trv }}: T_{0} M / T_{0}^{c} M \rightarrow T_{0} M^{\prime} / T_{0}^{c} M^{\prime}$ is an isomorphism. A deeper understanding of the constraints between various nondegeneracy conditions on $h, M$ and $M^{\prime}$ would be desirable.

3.19. Classical versions of the reflection principle. Let $h:(M, 0) \rightarrow \mathcal{F}$ $\left(M^{\prime}, 0\right)$ be a formal power series CR mapping between two generic submanifolds. Assume that $M$ is minimal at 0 .

Theorem 3.20. ([BER1999, Me2004a, Me2005]) If $M$ and $M^{\prime}$ are real analytic, if $h$ is either Levi nondegenerate, or finitely nondegenerate, or essentially finite, or Segre nondegenerate at the origin, then $h(t)$ is convergent, namely $h(t) \in \mathbb{C}\{t\}^{n^{\prime}}$. If moreover, $M$ and $M^{\prime}$ are algebraic, then $h$ is algebraic.

If one puts separate nondegeneracy conditions on $h$ and on $M^{\prime}$, as in Theorem 3.17, one obtains a combinatorics of possible statements, some of which appear in the literature.

If $h$ is finitely nondegenerate (level (2)), the (paradigmatic) proof yields more information.

Theorem 3.21. ([BER1999, Me2005]) As above, let $h:(M, 0) \rightarrow$ $\left(M^{\prime}, 0^{\prime}\right)$ be a formal power series $C R$ mapping. Assume that $M$ is minimal at 0 and let $\nu_{0}$ be the integer of Corollary 2.17. Assume also that $h$ is $\ell_{1}$-finitely nondegenerate at 0 . Then there exists a $\mathbb{C}^{n^{\prime}}$-valued power series mapping $H\left(t, J^{2 \nu_{0} \ell_{1}}\right)$ which is constructed algorithmically by means of the defining equations of $(M, 0)$ and of $\left(M^{\prime}, 0^{\prime}\right)$, such that the power series identity

$$
h(t) \equiv H\left(t, J^{2 \nu_{0} \ell_{1}} h(0)\right)
$$

holds in $\mathbb{C} \llbracket t \rrbracket^{n^{\prime}}$. If $M$ and $M^{\prime}$ are real analytic (resp. algebraic), $H$ is holomorphic (resp. complex algebraic) in a neighborhood of $0 \times J^{2 \nu_{0} \ell_{1}} h(0)$. 
In [BER1999, GM2004], the above formula $h(t) \equiv H\left(t, J^{2 \nu_{0} \ell_{1}} h(0)\right)$ is studied horoughly in the case where $M^{\prime}=M$ and $h$ is a local holomorphic automorphism of $(M, 0)$ close to the identity.

At level (5), namely with a holomorphically nondegenerate target $\left(M^{\prime}, 0^{\prime}\right)$, the reflection principle is much more delicate. It requires the introduction of a new object, whose regularity properties hold in fact without any nondegeneracy assumption on the target $\left(M^{\prime}, 0^{\prime}\right)$.

3.22. Convergence of the reflection mapping. The reflection mapping associated to $h$ and to the system of coordinates $\left(z^{\prime}, w^{\prime}\right)$ is :

$$
\mathcal{R}_{h}^{\prime}\left(\tau^{\prime}, t\right):=\xi^{\prime}-\Theta^{\prime}\left(\zeta^{\prime}, h(t)\right) \in \mathbb{C} \llbracket \tau^{\prime}, t \rrbracket^{d^{\prime}} .
$$

Since $h$ is formal, it is only a formal power series mapping. As argued in the introduction of [Me2005], it is the most fundamental object in the analytic reflection principle. In the case of CR mappings between essentially finite hypersurfaces, the analytic regularity of the reflection mapping is equivalent to the extension of CR mappings as correspondences, as studied in [DP1995, Sh2000, Sh2003, DP2003]. Without nondegeneracy assumption on $\left(M^{\prime}, 0^{\prime}\right)$, the reflection mapping enjoys regularity properties from which all analytic reflection principles may be deduced. Here is the very main theorem of this Section 3.

Theorem 3.23. ([Me2001b, BMR2002, Me2005]) If $M$ is minimal at the origin and if $h$ is either CR-invertible, or CR-submersive, or CR-finite, or $C R$-dominating, or CR-transversal, then for every system of coordinates $\left(z^{\prime}, w^{\prime}\right) \in \mathbb{C}^{m^{\prime}} \times \mathbb{C}^{d^{\prime}}$ in which the extrinsic complexification $\mathcal{M}^{\prime}$ is represented by $\xi^{\prime}=\Theta^{\prime}\left(\zeta^{\prime}, t^{\prime}\right)$, the associated CR-reflection mapping is convergent, namely $\mathcal{R}_{h}^{\prime}\left(\tau^{\prime}, t\right) \in \mathbb{C}\left\{\tau^{\prime}, t\right\}{ }^{d^{\prime}}$.

If the convergence property holds in one such system of coordinates, it holds in all systems of coordinates ([Me2005]; Proposition 3.26 below). Further, if we develope $\Theta^{\prime}\left(\zeta^{\prime}, t^{\prime}\right)=\sum_{\gamma^{\prime} \in \mathbb{N}^{m^{\prime}}}\left(\zeta^{\prime}\right)^{\gamma^{\prime}} \Theta_{\gamma^{\prime}}^{\prime}\left(t^{\prime}\right)$, the convergence of $\mathcal{R}_{h}^{\prime}\left(\tau^{\prime}, t\right)$ has a concrete signification.

Corollary 3.24. All the components $\Theta_{\gamma^{\prime}}^{\prime}(h(t))$ of the reflection mapping are convergent, namely $\Theta_{\gamma^{\prime}}^{\prime}(h(t)) \in \mathbb{C}\{t\}^{d^{\prime}}$ for every $\gamma^{\prime} \in \mathbb{N}^{m^{\prime}}$.

Conversely ([Me2001b, Me2005]), if $\Theta_{\gamma^{\prime}}^{\prime}(h(t)) \in \mathbb{C}\{t\}^{d^{\prime}}$ for every $\gamma^{\prime} \in$ $\mathbb{N}^{m^{\prime}}$, an elementary application of the Artin approximation Theorem 3.28 (below) yields Cauchy estimates: there exist $\rho>0, \sigma>0$ and $C>0$ so that $\left|\Theta_{\gamma^{\prime}}^{\prime}(h(t))\right|<C(\rho)^{-\left|\gamma^{\prime}\right|}$, for every $t \in \mathbb{C}^{n}$ with $|t|<\sigma$. It follows that $\mathcal{R}_{h}^{\prime}\left(\tau^{\prime}, t\right) \in \mathbb{C}\left\{\tau^{\prime}, t\right\}^{d^{\prime}}$. 
Taking account of the nondegeneracy conditions (ndi) and (crj), several corollaries may be deduced from the theorem. Most of them are already expressed by Theorem 3.20, except notably the delicate case where $\left(M^{\prime}, 0^{\prime}\right)$ is holomorphically nondegenerate.

Corollary 3.25. ([Me2001b, Me2005]) If $M$ is minimal at the origin, if $\left(M^{\prime}, 0^{\prime}\right)$ is holomorphically nondegenerate and if $h$ is either $C R$-invertible and invertible, or CR-submersive and submersive, or CR-finite and finite with $m^{\prime}=m$, or CR-dominating and dominating, or CR-transversal and transversal, then $h(t) \in \mathbb{C}\{t\}^{n^{\prime}}$ is convergent.

It is known ([St1996]) that $\left(M^{\prime}, 0^{\prime}\right)$ is holomorphically degenerate if and only if there exists a nonzero $(1,0)$ vector field $X^{\prime}=\sum_{i^{\prime}=1}^{n^{\prime}} a_{i^{\prime}}^{\prime}\left(t^{\prime}\right) \frac{\partial}{\partial t_{i^{\prime}}^{\prime}}$ having holomorphic coefficients which is tangent to $\left(M^{\prime}, 0^{\prime}\right)$. In the corollary above, holomorphic nondegeneracy is optimal for the convergence of a formal equivalence: if $M^{\prime}$ is holomorphically degenerate, if $\left(s^{\prime}, t^{\prime}\right) \longmapsto \exp \left(s^{\prime} X^{\prime}\right)\left(t^{\prime}\right)$ denotes the local flow of $X^{\prime}$, where $s^{\prime} \in \mathbb{C}$, $t^{\prime} \in \mathbb{C}^{n^{\prime}}$, there indeed exist ([BER1999, Me2005]) nonconvergent power series $\varpi^{\prime}\left(t^{\prime}\right) \in \mathbb{C} \llbracket t^{\prime} \rrbracket$ such that $t^{\prime} \mapsto_{\mathcal{F}} \exp \left(\varpi^{\prime}\left(t^{\prime}\right) X^{\prime}\right)\left(t^{\prime}\right)$ is a nonconvergent formal equivalence of $M^{\prime}$.

The invariance of the reflection mapping is crucial.

Proposition 3.26. ([Me2002, Me2004a, Me2005]) The convergence of the reflection mapping is a biholomorphically invariant property. More precisely, if $t^{\prime \prime}=\phi^{\prime}\left(t^{\prime}\right)$ is a local biholomorphism fixing $0^{\prime}$ and transforming $\left(M^{\prime}, 0^{\prime}\right)$ into a generic submanifold $\left(M^{\prime \prime}, 0^{\prime}\right)$ of equations $\bar{w}_{j^{\prime}}^{\prime \prime}=$ $\Theta_{j^{\prime}}^{\prime \prime}\left(\bar{z}^{\prime \prime}, t^{\prime \prime}\right), j^{\prime}=1, \ldots, d^{\prime}$, the composed reflection mapping of $\phi^{\prime} \circ h$ : $(M, 0) \rightarrow_{\mathcal{F}}\left(M^{\prime \prime}, 0^{\prime}\right)$ defined by

$$
\begin{aligned}
\mathcal{R}_{\phi^{\prime} \circ h}^{\prime \prime}\left(\tau^{\prime \prime}, t\right) & :=\xi^{\prime \prime}-\Theta^{\prime \prime}\left(\zeta^{\prime \prime}, \phi^{\prime}(h(t))\right) \\
& =\xi^{\prime \prime}-\sum_{\gamma^{\prime} \in \mathbb{N}^{m^{\prime}}}\left(\zeta^{\prime \prime}\right)^{\gamma^{\prime}} \Theta_{\gamma^{\prime}}^{\prime \prime}\left(\phi^{\prime}(h(t))\right)
\end{aligned}
$$

has components $\Theta_{\gamma^{\prime}}^{\prime \prime}\left(\phi^{\prime}(h(t))\right)$ given by formulas

$$
\Theta_{\gamma^{\prime}}^{\prime \prime}\left(\phi^{\prime}(h(t))\right) \equiv S_{\gamma^{\prime}}^{\prime}\left(\left(\Theta_{\gamma_{1}^{\prime}}^{\prime}(h(t))\right)_{\gamma_{1}^{\prime} \in \mathbb{N}^{m^{\prime}}}\right),
$$

where the local holomorphic functions $S_{\gamma^{\prime}}^{\prime}$ depend only on the biholomorphism $t^{\prime \prime}=\phi^{\prime}\left(t^{\prime}\right)$ (they have an infinite number of variables, but the necessary Cauchy estimates insuring convergence are automatically satisfied). 
A few words about the proof of the main Theorem 3.23. Although the classical reflection principle deals only with the "reflection identities" (3.13), to get the most adequate version of the reflection principle, it is unavoidable to understand the symmetry between the variables $t$ and the variables $\tau=(\bar{t})^{c}$.

The assumption that $h^{c}$ maps formally $(\mathcal{M}, 0)$ to $\left(\mathcal{M}^{\prime}, 0^{\prime}\right)$ is equivalent to each one of the following two formal identities:

$$
\left\{\begin{array}{l}
\bar{g}(\tau)=\sum_{\gamma^{\prime} \in \mathbb{N}^{m^{\prime}}} \bar{f}(\tau)^{\gamma^{\prime}} \Theta_{\gamma^{\prime}}^{\prime}(h(t)), \\
g(t)=\sum_{\gamma^{\prime} \in \mathbb{N}^{m^{\prime}}} f(t)^{\gamma^{\prime}} \bar{\Theta}_{\gamma^{\prime}}^{\prime}(\bar{h}(\tau)),
\end{array}\right.
$$

on $\mathcal{M}$, namely after replacing either $w$ by $\bar{\Theta}(z, \tau)$ or $\xi$ by $\Theta(\zeta, t)$. The symmetry may be pursued by considering the two families of derivations:

$$
\left\{\begin{array}{l}
\mathcal{L}^{\beta}:=\left(\mathcal{L}_{1}\right)^{\beta_{1}}\left(\mathcal{L}_{1}\right)^{\beta_{2}} \cdots\left(\mathcal{L}_{m}\right)^{\beta_{m}} \quad \text { and } \\
\underline{\mathcal{L}}^{\beta}:=\left(\underline{\mathcal{L}}_{1}\right)^{\beta_{1}}\left(\underline{\mathcal{L}}_{1}\right)^{\beta_{2}} \cdots\left(\underline{\mathcal{L}}_{m}\right)^{\beta_{m}},
\end{array}\right.
$$

where $\beta=\left(\beta_{1}, \beta_{2}, \ldots, \beta_{m}\right) \in \mathbb{N}^{m}$. Applying them to the two formal identities above, if we respect the completeness of the combinatorics, we will get four families of reflection identities. The first pair is obtained by applying $\underline{\mathcal{L}}^{\beta}$ to the two formal identities above:

$$
\left\{\begin{aligned}
\underline{\mathcal{L}}^{\beta} \bar{g}(\tau) & =\sum_{\gamma^{\prime} \in \mathbb{N}^{m^{\prime}}} \underline{\mathcal{L}}^{\beta}\left[\bar{f}(\tau)^{\gamma^{\prime}}\right] \Theta_{\gamma^{\prime}}^{\prime}(h(t)), \\
0 & =\sum_{\gamma^{\prime} \in \mathbb{N}^{m^{\prime}}} f(t)^{\gamma^{\prime}} \underline{\mathcal{L}}^{\beta}\left[\bar{\Theta}_{\gamma^{\prime}}^{\prime}(\bar{h}(\tau))\right] .
\end{aligned}\right.
$$

The second pair is obtained by applying $\mathcal{L}^{\beta}$, permuting the two lines:

$$
\left\{\begin{aligned}
\mathcal{L}^{\beta} g(t) & =\sum_{\gamma^{\prime} \in \mathbb{N}^{m^{\prime}}} \mathcal{L}^{\beta}\left[f(t)^{\left.\gamma^{\prime}\right]} \bar{\Theta}_{\gamma^{\prime}}^{\prime}(\bar{h}(\tau)),\right. \\
0 & =\sum_{\gamma^{\prime} \in \mathbb{N}^{m^{\prime}}} \bar{f}(\tau)^{\gamma^{\prime}} \mathcal{L}^{\beta}\left[\Theta_{\gamma^{\prime}}^{\prime}(h(t))\right] .
\end{aligned}\right.
$$

We immediately see that these two pairs are conjugate line by line. In each pair, we notice a crucial difference between the first and the second line: whereas it is $\bar{g}$ and the power $\bar{f}^{\gamma^{\prime}}$ (or $g$ and $f^{\gamma^{\prime}}$ ) that are differentiated in each first line, in each second line, only the components $\bar{\Theta}_{\gamma^{\prime}}^{\prime}(\bar{h})$ (or $\left.\Theta_{\gamma^{\prime}}^{\prime}(h)\right)$ of the reflection mapping, which are the right invariant functions, 
are differentiated. In a certain sense, it is forbidden to differentiate $\bar{g}$ and $\bar{f}^{\gamma^{\prime}}$ (or $g$ and $f^{\gamma^{\prime}}$ ), because the components $(f, g)$ of $h$ need not enjoy a reflection principle. In fact, in the proof of the main Theorem 3.23, one has to play constantly with the four reflection identities above.

Since we cannot summarize here the long and refined proof, we only formulate the main technical proposition. Denote by $J_{t}^{\ell} \psi$ the $\ell$-th jet of a power series $\psi(t) \in \mathbb{C} \llbracket t \rrbracket^{d^{\prime}}$, for instance $J_{t}^{\ell} \Theta_{\gamma^{\prime}}^{\prime}(h)$ for some $\gamma^{\prime} \in \mathbb{N}^{m^{\prime}}$. Remind that $\underline{\Gamma}_{k}$ and $\Gamma_{k}$ are (conjugate) Segre chains. Let $N_{d^{\prime}, n, \ell}:=d^{\prime} \frac{(n+\ell) !}{n ! \ell !}$.

Proposition 3.27. ([Me2005]) For every $k \in \mathbb{N}$ and every $\ell \in \mathbb{N}$, the following two properties hold:

- if $k$ is odd, for every $\gamma^{\prime} \in \mathbb{N}^{m^{\prime}}$ :

$$
\left[J_{t}^{\ell} \Theta_{\gamma^{\prime}}^{\prime}(h)\right]\left(\Gamma_{k}\left(z_{(k)}\right)\right) \in \mathbb{C}\left\{z_{(k)}\right\}^{N_{d^{\prime}, n, \ell}}
$$

- if $k$ is even, for every $\gamma^{\prime} \in \mathbb{N}^{m^{\prime}}$ :

$$
\left[J_{\tau}^{\ell} \bar{\Theta}_{\gamma^{\prime}}^{\prime}(\bar{h})\right]\left(\Gamma_{k}\left(z_{(k)}\right)\right) \in \mathbb{C}\left\{z_{(k)}\right\}^{N_{d^{\prime}, n, \ell}}
$$

With $\ell=0$ and $k=2 \nu_{0}$, thanks to Corollary 2.17, we deduce from this main proposition that $\Theta_{\gamma^{\prime}}^{\prime}(h(t)) \in \mathbb{C}\{t\}^{\gamma^{\prime}}$ for every $\gamma^{\prime} \in \mathbb{N}^{m^{\prime}}$. This yields Theorem 3.23.

The main tool in the proof of this proposition is an approximation theorem saying that a formal power series mapping that is a solution of some analytic equations may be corrected so as to become convergent and still a solution.

Theorem 3.28. (ARTIN [Ar1968, JoPf2000]) Let $\mathbb{K}=\mathbb{R}$ or $\mathbb{C}$, let $n \in \mathbb{N}$ with $n \geqslant 1$, let $\mathrm{x}=\left(\mathrm{x}_{1}, \ldots, \mathrm{x}_{n}\right) \in \mathbb{K}^{n}$, let $m \in \mathbb{N}$, with $m \geqslant 1$, let $\mathrm{y}=$ $\left(\mathrm{y}_{1}, \ldots, \mathrm{y}_{m}\right) \in \mathbb{K}^{n}$, let $d \in \mathbb{N}$ with $d \geqslant 1$ and let $R_{1}(\mathrm{x}, \mathrm{y}), \ldots, R_{d}(\mathrm{x}, \mathrm{y})$ be an arbitrary collection of formal power series in $\mathbb{K}\{\mathrm{x}, \mathrm{y}\}$ that vanish at the origin, namely $R_{j}(0,0)=0, j=1, \ldots, d$. Assume that there exists a formal mapping $h(\mathrm{x})=\left(h_{1}(\mathrm{x}), \ldots, h_{m}(\mathrm{x})\right) \in \mathbb{K} \llbracket \mathrm{x} \rrbracket^{m}$ with $h(0)=0$ such that

$$
R_{j}(\mathrm{x}, h(\mathrm{x})) \equiv 0 \text { in } \mathbb{K} \llbracket \mathrm{x} \rrbracket, \quad \text { for } j=1, \ldots, d .
$$

Let $\mathfrak{m}(\mathrm{x}):=\mathrm{x}_{1} \mathbb{K} \llbracket \mathrm{x} \rrbracket+\cdots+\mathrm{x}_{n} \mathbb{K} \llbracket \mathrm{x} \rrbracket$ be the maximal ideal of $\mathbb{K} \llbracket \mathrm{x} \rrbracket$. For every integer $N \geqslant 1$, there exists a convergent power series mapping $h^{N}(\mathrm{x}) \in$ $\mathbb{K}\{\mathrm{x}\}^{m}$ such that

$$
R_{j}\left(\mathrm{x}, h^{N}(\mathrm{x})\right) \equiv 0 \text { in } \mathbb{K} \llbracket \mathrm{x} \rrbracket, \quad \text { for } j=1, \ldots, d,
$$


that approximates $h(\mathrm{x})$ to order $N-1$ :

$$
h^{N}(\mathbf{x}) \equiv h(\mathbf{x}) \bmod \left(\mathfrak{m}(x)^{N}\right) .
$$

As an application of the main Theorem 3.23, an approximation property for formal CR mappings holds.

Theorem 3.29. ([Me2005]) Under the assumptions of Theorem 3.23, for every integer $N \geqslant 1$, there exists a convergent power series mapping $\mathrm{H}^{N}(t) \in \mathbb{C}\{t\}^{n^{\prime}}$ with $\mathrm{H}^{N}(t) \equiv h(t) \bmod (\mathfrak{m}(t))^{N}$ (whence $\left.H(0)=0\right)$, that induces a local holomorphic map from $(M, 0)$ to $\left(M^{\prime}, 0^{\prime}\right)$.

Corollary 3.30. ([Me2001b, Me2005]) Assume that $n^{\prime}=n$, that $d^{\prime}=d$, that $M$ is minimal at the origin, and that $h:(M, 0) \rightarrow \mathcal{F}\left(M^{\prime}, 0^{\prime}\right)$ is a formal (invertible) equivalence. Then $M$ and $M^{\prime}$ are biholomorphically equivalent.

It is known ([St1996, BER1999, GM2004]) that a minimal holomorphically nondegenerate real analytic generic submanifold of $\mathbb{C}^{n}$ has finitedimensional local holomorphic automorphism group. Unique determination by a jet of finite order follows from a representation formula, as in Theorem 3.21. More generally:

Corollary 3.31. ([Me2001b, BMR2002, Me2005] $]$ ) Assume that $m^{\prime}=m$ and $d^{\prime}=d$, that $(M, 0)$ is minimal at the origin and that $\left(M^{\prime}, 0\right)$ is holomorphically nondegenerate. There exists an integer $\kappa=\kappa(m, d)$ such that, if two local biholomorphisms $h^{1}, h^{2}:(M, 0) \rightarrow\left(M^{\prime}, 0\right)$ have the same $\kappa^{-}$ th jet at the origin, then $h^{1}=h^{2}$.

From an inspection of the proof, Theorem 3.29 holds without the assumption that $(M, 0)$ is minimal, but with the assumption that its $\mathrm{CR}$ orbits have constant dimension in a neighborhood of 0 . However, the case where $\mathrm{CR}$ orbits have arbitrary dimension is delicate.

Open question 3.32. Does formal equivalence coincide with biholomorphic equivalence in the category of real analytic generic local submanifolds of $\mathbb{C}^{n}$ whose $C R$ orbits have non-constant dimension?

3.33. Algebraicity of the reflection mapping. We will assume that both $M$ and $M^{\prime}$ are algebraic. Remind that Theorem 3.20 shows the algebraicity of $h$ under some hypotheses. A much finer result is as follows. It synthetizes all existing results ([We1977, SS1996, CMS1999, BER1999, Za1999]) about algebraicity of local holomorphic mappings. 
Theorem 3.34. ([Me2001a]]) If $h$ is a local holomorphic map $(M, 0) \rightarrow$ $\left(M^{\prime}, 0^{\prime}\right)$, if $M$ and $M^{\prime}$ are algebraic, if $M$ is minimal at the origin and if $M^{\prime}$ is the smallest (for inclusion) local real algebraic manifold containing $h(M)$, then the reflection mapping $\mathcal{R}_{h}^{\prime}\left(\tau^{\prime}, t\right)$ is algebraic.

Trivial examples ([Me2001a] ) show that the algebraicity of $\mathcal{R}_{h}^{\prime}$ need not hold if $M^{\prime}$ is not the smallest one.

In fact, Theorem 3.34 also holds (with the same proof) if one assumes only that the source $M$ is minimal at a Zariski-generic point: it suffices to shrink $M$ and the domain of definition of $h$ around such points, getting local algebraicity of $\mathcal{R}_{h}^{\prime}$ there, and since algebraicity is a global property, $\mathcal{R}_{h}^{\prime}$ is algebraic everywhere.

An equivalent formulation of Theorem 3.34 uses the concept of transcendence degree, studied in [Pu1990, CMS1999, Me2001a. With $n_{M^{\prime}}^{\prime}$ being the essential holomorphic dimension of $\left(M^{\prime}, 0^{\prime}\right)$ defined in $\S 3.6$, set $\kappa_{M^{\prime}}^{\prime}:=n^{\prime}-n_{M^{\prime}}^{\prime}$. Observe that $\left(M^{\prime}, 0^{\prime}\right)$ is holomorphically nondegenerate precisely when $\kappa_{M^{\prime}}^{\prime}=0$. Denote by $\mathbb{C}[t]$ the ring of complex polynomials of the variable $t \in \mathbb{C}^{n}$ and by $\mathbb{C}(t)$ its quotient field. Let $t^{\prime}=h(t)$ be a local holomorphic mapping as in Theorem 3.34. and let $\mathbb{C}(t)\left(h_{1}(t), \ldots, h_{n^{\prime}}(t)\right)$ be the field generated by the components of $h$.

Theorem 3.35. ([Me2001a]) With the same assumptions as in Theorem 3.34, the transcendence degree of the field extension $\mathbb{C}(t) \rightarrow$ $\mathbb{C}(t)(h(t))$ is less than or equal to $\kappa_{M^{\prime}}^{\prime}$

Corollary 3.36. ([CMS1999, Za1999, Me2001a]) If $M$ is minimal at a Zariski-generic point and if the real algebraic target $M^{\prime}$ does not contain any complex algebraic curve, then the local holomorphic mapping $h$ is algebraic.

However, in case $h$ is only a formal CR mapping, it is impossible to shift the central point to a nearby minimal point. Putting the simplest rank assumption (invertibility) on $h$, we may thus formulate delicate problems for the future.

Open question 3.37. Let $h$ be a formal equivalence between two real analytic generic submanifolds of $\mathbb{C}^{n}$ which are minimal at a Zariski-generic point.

- Is the reflection mapping convergent?

- Is h uniquely determined by a jet of finite order when the target is holomorphically nondegenerate? 
- Is h convergent under the assumption that the real analytic target $M^{\prime}$ does not contain any complex analytic curve?

For $M^{\prime}$ algebraic containing no complex algebraic curve and $M$ minimal at 0, the third question has been settled in [MMZ2003b]. However, the assumption of algebraicity of $M^{\prime}$ is strongly used there, because these authors deal with the transcendence degree of the field extension $\mathbb{C}(t) \rightarrow \mathbb{C}(t)(h(t))$, a concept which is meaningless if $M^{\prime}$ is real analytic. For further (secondary) results and open questions, we refer to [BMR2002, Ro2003]. This closes up our survey of the for$\mathrm{mal} /$ algebraic/analytic reflection principle.

A generic submanifold $M \subset \mathbb{C}^{n}$ is called locally algebraizable at one of its points $p$ if there exist local holomorphic coordinates centered at $p$ in which it is Nash algebraic. Unlike partial results, the following question remains up to now unsolved.

Open problem 3.38. ([Hu2001, HJY2001, एi2002, GM2004, Fo2004]) Formulate a necessary and sufficient condition for the local algebraizability of a real analytic hypersurface $M \subset \mathbb{C}^{n}$ in terms of a basis of the (differential) algebra of its Cartan-Hachtroudi-Chern invariants.

To conclude, we would like to mention that the complete theory of CR mappings may be transferred to systems of partial differential equations having finite-dimensional Lie symmetry group. This aspect will be treated in subsequent publications ([Me2006a, Me2006]]). 


\section{III: Systems of vector fields and CR functions}

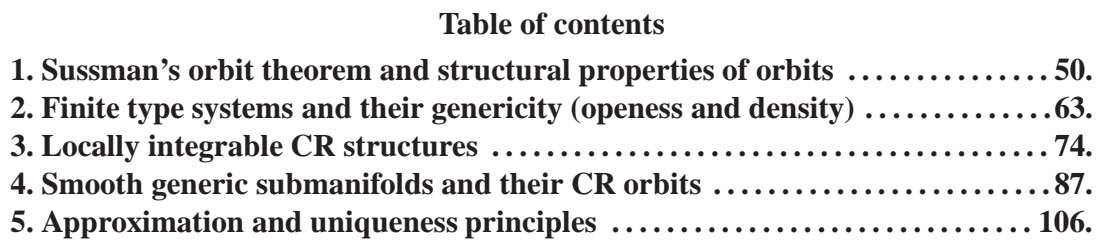

[7 diagrams]

Beyond the theorems of Frobenius and of Nagano, Sussmann's theorem provides a means, valid in the smooth category, to construct all the integral manifolds of an arbitrary system of vector fields, as orbits of the pseudo-group actions of global flows. The fundamental properties of such orbits: lower semi-continuity of dimension, local flow box structure, propagation of embeddedness, intersection with a transversal curve in the one-codimensional case, are essentially analogous, but different from the ones known in foliation theory. Orbits possess wide applications in Control Theory, in sub-Riemannian Geometry, in the Analysis of Linear Partial Differential Equations and in Cauchy-Riemann geometry.

Let $L_{j} f=g_{j}, j=1, \ldots, \lambda$, be a linear PDE system with unknown $f$, where $g$ is smooth and where $\left\{L_{k}\right\}_{1 \leqslant k \leqslant r}$ is an involutive (in the sense of Frobenius) system of smooth vector fields on $\mathbb{R}^{n}$ having complex-valued coefficients. Since Lewy's celebrated discovery of an example of a single equation $L f=g$ in $\mathbb{R}^{3}$ without any solution, a major problem in the Analysis of PDE's is to find adequate criterions for the existence of local solutions. Condition (P) of NirenbergTreves has appeared to be necessary and sufficient to insure local integrability of a single equation of principal type having simple characteristics. The problem of characterizing systems of several linear first order PDE's having maximal space of solutions is not yet solved in full generality; several fine questions remain open.

Following Treves, to abstract the notion of systems involving several equations, an involutive structure on a smooth $\mu$-dimensional real manifold $M$ is a $\lambda$-dimensional complex subbundle $\mathcal{L}$ of $\mathbb{C} \otimes T M$ satisfying $[\mathcal{L}, \mathcal{L}] \subset \mathcal{L}$. The automatic integrability of smooth almost complex structures (those with $\mathcal{L} \oplus \overline{\mathcal{L}}=\mathbb{C} \otimes T M$ ) and the classical (non)integrability theorems for smooth abstract CR structures (those with $\mathcal{L} \cap \overline{\mathcal{L}}=\{0\}$ ) are inserted in this general framework.

Beyond such problematics, it is of interest to study the analysis and the geometry of subbundles $\mathcal{L}$ whose space of solutions is maximal, viz the preceding question is assumed to be solved, optimally: in a neighborhood of every point of $M$, there exist $(\mu-\lambda)$ local complex valued functions $z_{1}, \ldots, z_{\mu-\lambda}$ having linearly 
independent differentials which are solutions of $\mathcal{L} z_{k}=0$. Such involutive structures are called locally integrable. Some representative examples are provided by the bundle of anti-holomorphic vector fields tangent to various embedded generic submanifolds of $\mathbb{C}^{n}$. According to a theorem due to Baouendi-Treves, every local solution of $\mathcal{L} f=0$ may be approximated sharply by polynomials in a set of fundamental solutions $z_{1}, \ldots, z_{\mu-\lambda}$, in the topology of functional spaces as $\mathcal{C}^{\kappa, \alpha}$, $L_{l o c}^{\mathrm{p}}$, or $\mathcal{D}^{\prime}$.

In a locally integrable structure, the Sussmann orbits of the vector fields $\operatorname{Re} L_{k}, \operatorname{Im} L_{k}$ are then of central importance in analytic and in geometrical questions. They show up propagational aspects, as for instance: the support of a function or distribution solution $f$ of $\mathcal{L} f=0$ is a union of orbits. The approximation theorem also yields an elegant proof of uniqueness in the Cauchy problem. Further propagational aspects will be studied in the next chapters, using the method of analytic discs. Sections 3, 4 and 5 of this chapter and the remainder of the memoir are focused on embedded generic submanifolds.

\section{$\S 1$. SUSSMANN'S THEOREM AND STRUCTURAL PROPERTIES OF ORBITS}

1.1. Integral manifolds of a system of vector fields. Ordinary differential equations in the modern sense emerged in the seventieth century, concomitantly with the infinitesimal calculus. Nowadays, in contemporary mathematics, the abstract study of vector fields is inserted in several broad areas of research, among which we perceive the following.

- Control Theory: controllability of vector fields on $\mathcal{C}^{\infty}$ and real analytic manifolds; nonholonomic systems; sub-Riemannian geometry ([GV1987, Bel1996]).

- Dynamical systems: singularities of real or complex vector fields and foliations; normal forms and classification; phase diagrams; Lyapunov theory; Poincaré-Bendixson theory; theory of limit cycles of polynomial and analytic vector fields; small divisors ([Ar1978, Ar1988]).

- Lie-Cartan theory: infinitesimal symmetries of differential equations; classification of local Lie group actions; Lie algebras of vector fields; representations of Lie algebras; exterior differential systems; Cartan-Vessiot-Kähler theorem; Janet-Riquier theory; Cartan's method of equivalence ([O11995, Stk2000]).

- Numerical analysis: systems of (non)linear ordinary differential equations; methods of: Euler, Newton-Cotes, Newton-Raphson, Runge-Kutta, Adams-Bashforth, Adams-Moulton ([De1996]). 
- PDE theory: Local solvability of linear partial differential equations; uniqueness in the Cauchy problem; propagation of singularities; FBI transform and control of wave front set ([ES1993, Trv1992]).

To motivate the present Part III, let us expose informally two dual questions about systems of vector fields. Consider a set $\mathbb{L}$ of local vector fields defined on a domain of $\mathbb{R}^{n}$. Frobenius' theorem provides local foliations by submanifolds to which every element of $\mathbb{L}$ is tangent, provided $\mathbb{L}$ is closed under Lie brackets. However, for a generic set $\mathbb{L}$, the condition $[\mathbb{L}, \mathbb{L}] \subset \mathbb{L}$ fails and in addition, the tangent spaces spanned by elements of $\mathbb{L}$ are of varying dimension. To surmount these imperfections, two inverse options present themselves:

Sub: find the subsystems $\mathbb{L}^{\prime} \subset \mathbb{L}$ which satisfy Frobenius' condition $\left[\mathbb{L}^{\prime}, \mathbb{L}^{\prime}\right] \subset \mathbb{L}^{\prime}$ and which are maximal in an appropriate sense;

Sup: find the supsystems $\mathbb{L}^{\prime} \supset \mathbb{L}$ which have integral manifolds and which are minimal in an appropriate sense.

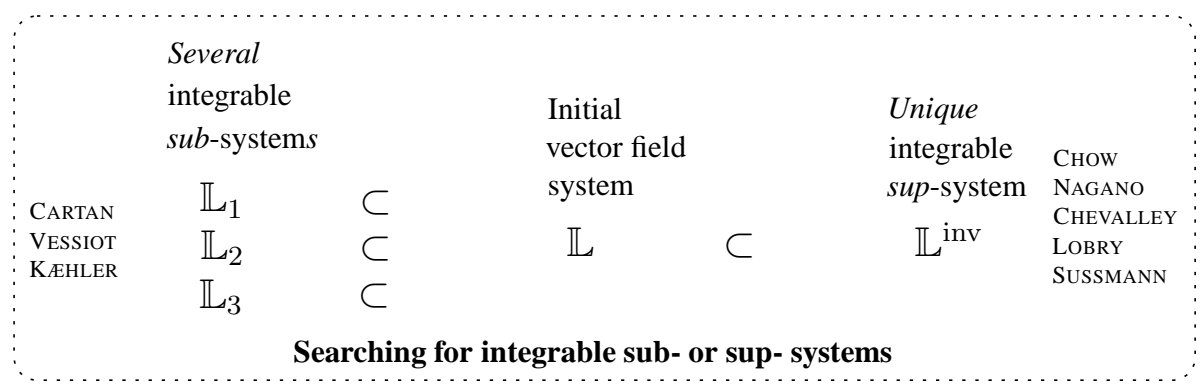

The first problem Sub is answered by the Cartan-Vessiot-Kähler theorem, thanks to an algorithm which provides all the minimal Frobeniusintegrable subsystems $\mathbb{L}^{\prime}$ of $\mathbb{L}$ (we recommend [Stk2000] for a presentation). Generically, there are infinitely many solutions and their cardinality is described by means of a sequence of integers together with the so-called Cartan character of $\mathbb{L}$. In the course of the proof, the CauchyKowalevskaya integrability theorem, valid only in the analytic category, is heavily used. It was not a serious restriction at the time of É. Cartan, but, in the second half of the twentieth century, the progress of the Analysis of PDE showed deep new phenomena in the differentiable category. Hence, one may raise the:

Open problem 1.2. Find versions of the Cartan-Vessiot-Kähler theorem for systems of vector fields having smooth non-analytic coefficients. 
The Cauchy characteristic subsystem of $\mathbb{L}$ ([Stk2000] $)$ is always involutive, hence the smooth Frobenius theorem applies to it ${ }^{4}$. However, for intermediate systems, the question is wide open. Possibly, this question is related to some theorems about local solvability of smooth partial differential equations ( $c f$. Section 3 ) that were established to understand the Hans Lewy counterexample ( $(3.1)$.

The second problem Sup is already answered by Nagano's theorem (Part II), though only in the analytic category, with a unique integrable minimal supsystem $\mathbb{L}^{\text {lie }} \supset \mathbb{L}$. In the general smooth category, the stronger Chevalley-Lobry-Stefan-Sussmann theorem, dealing with flows of vector fields instead of Lie brackets, shows again that there is a unique integrable sup-system of $\mathbb{L}$ which has integral manifolds. As this theorem will be central in this memoir, it will be exposed thoroughly in the present Section 1.

1.3. Flows of vector fields and their regularity. Let $\mathbb{K}=\mathbb{R}$ or $\mathbb{C}$. Let $D$ be a open connected subset of $\mathbb{K}^{n}$. Let $\mathrm{x}=\left(\mathrm{x}_{1}, \ldots, \mathrm{x}_{n}\right) \in D$. Let $L=\sum_{i=1}^{n} a_{i}(\mathrm{x}) \frac{\partial}{\partial \mathrm{x}_{i}}$ be a vector field defined over $D$. Throughout this section, we shall assume that its coefficients $a_{i}$ are either $\mathbb{K}$-analytic (of class $\mathcal{C}^{\omega}$ ), of class $\mathcal{C}^{\infty}$, or of class $\mathcal{C}^{\kappa, \alpha}$, where $\kappa \geqslant 1$ and $0 \leqslant \alpha \leqslant 1$ (see Section 1(IV) for background about Hölder classes).

By the classical Cauchy-Lipschitz theorem, through each point $\mathrm{x}_{0} \in D$, there passes a unique local integral curve of the vector field $L$, namely a local solution $\mathrm{x}(\mathrm{t})=\left(\mathrm{x}_{1}(\mathrm{t}), \ldots, \mathrm{x}_{n}(\mathrm{t})\right)$ of the system of ordinary differential equations:

$$
d \mathrm{x}_{1}(\mathrm{t}) / d \mathrm{t}=a_{1}(\mathrm{x}(\mathrm{t})), \ldots \ldots, d \mathrm{x}_{n}(\mathrm{t}) / d \mathrm{t}=a_{n}(\mathrm{x}(\mathrm{t})),
$$

which satisfies the initial condition $x(0)=x_{0}$. This solution is defined at least for small $\mathrm{t} \in \mathbb{K}$ and is classically denoted by $\mathrm{t} \mapsto \exp (\mathrm{t} L)\left(\mathrm{x}_{0}\right)$, because it has the local pseudogroup property

$$
\exp \left(\mathrm{t}^{\prime} L\right)\left(\exp (\mathrm{t} L)\left(\mathrm{x}_{0}\right)\right)=\exp \left(\left(\mathrm{t}+\mathrm{t}^{\prime}\right) L\right)\left(\mathrm{x}_{0}\right),
$$

whenever the composition is defined. Denote by $\Omega_{\mathrm{x}_{0}}$ the largest connected open set containing the origin in $\mathbb{K}$ in which $\exp (\mathrm{t} L)\left(\mathrm{x}_{0}\right)$ is defined. One shows that the union of various $\Omega_{\mathrm{x}_{0}}$, for $\mathrm{x}_{0}$ running in $D$, is an open connected set $\Omega_{L}$ of $\mathbb{K} \times \mathbb{K}^{n}$ which contains $\{0\} \times D$. Some regularity with respect to both variables $t$ and $x_{0}$ is got automatically.

Theorem 1.4. ([[La1983], [*]) The global flow $\Omega_{L} \ni\left(\mathrm{t}, \mathrm{x}_{0}\right) \mapsto$ $\exp (\mathrm{t} L)\left(\mathrm{x}_{0}\right) \in D$ of a vector field $L=\sum_{i=1}^{n} a_{i}(\mathrm{x}) \partial_{\mathrm{x}_{i}}$ defined in the

\footnotetext{
${ }^{4} \mathrm{We}$ are grateful to Stormark for pointing out this observation
} 
domain D has exactly the same smoothness as L, namely it is $\mathcal{C}^{\omega}, \mathcal{C}^{\infty}$ or $\mathcal{C}^{\kappa, \alpha}$.

As a classical corollary, a local straightening property holds : in a neighborhood of a point at which $L$ does not vanish, there exists a $\mathcal{C}^{\omega}, \mathcal{C}^{\infty}$ or $\mathcal{C}^{\kappa, \alpha}$ change of coordinates $x^{\prime}=x^{\prime}(x)$ in which the transformed vector field is the unit positive vector field directed by the $x_{1}^{\prime}$ lines, viz $L^{\prime}=\partial / \partial x_{1}^{\prime}$.

Up to the end of this Section 1, we will work with $\mathbb{K}=\mathbb{R}$.

1.5. Searching integral manifolds of a system of vector fields. Let $M$ be a smooth paracompact real manifold, which is $\mathcal{C}^{\omega}, \mathcal{C}^{\infty}$ or $\mathcal{C}^{\kappa+1, \alpha}$, where $\kappa \geqslant 1,0 \leqslant \alpha \leqslant 1$. Let $\mathbb{L}:=\left\{L_{a}\right\}_{a \in A}$ be a collection of vector fields defined on open subsets $D_{a}$ of $M$ and having $\mathcal{C}^{\omega}, \mathcal{C}^{\infty}$ or $\mathcal{C}^{\kappa, \alpha}$ coefficients, where $A$ is an arbitrary set. It is no restriction to assume that $\cup_{a \in A} D_{a}=$ $M$, since otherwise, it suffices to shrink $M$. Call $\mathbb{L}$ a system of vector fields on $M$.

Problem 1.6. Find submanifolds $N$ of $M$ such that each element of $\mathbb{L}$ is tangent to $N$.

To analyze this (still imprecise) problem, let $\mathbb{F}_{M}$ denote the collection of all $\mathcal{C}^{\omega}, \mathcal{C}^{\infty}$ or $\mathcal{C}^{\kappa, \alpha}$ functions defined on open subsets of $M$, and call the system $\mathbb{L}$ of vector fields $\mathbb{F}_{M}$-linear if every combined vector field $f K+g L$ belongs to $\mathbb{L}$, whenever $f, g \in \mathbb{F}_{M}$ and $K, L \in \mathbb{L}$. Here, $f K+g L$ is defined in the intersection of the domains of definition of $f, g, K$ and $L$. To study the problem, it is obviously no restriction to assume that $\mathbb{L}$ is $\mathbb{F}_{M}$-linear.

For $p \in M$ arbitrary, define

$$
\mathbb{L}(p):=\{L(p): L \in \mathbb{L}\} .
$$

Since $\mathbb{L}$ is $\mathbb{F}_{M}$-linear, this is a linear subspace of $T_{p} M$. So Problem 1.6 is to find submanifolds $N$ satisfying $T_{p} N \supset \mathbb{L}(p)$, for every $p \in N$. Notice that an appropriate answer should enable one to find all such submanifolds. Also, suppose that $N_{1}$ and $N_{2}$ are two solutions with $N_{2} \subset N_{1}$. Then the problem with the pair $(M, N)$ is exactly the same as the problem with the pair $\left(N_{1}, N_{2}\right)$. Hence a better formulation.

Problem 1.6'. Find all the submanifolds $N \subset M$ of smallest dimension that satisfy $T_{p} N \supset \mathbb{L}(p)$, for every $p \in N$.

The classical Frobenius theorem ([Fr1877, Sp1970, BER1999, Bo1991, Ch1991, Stk2000, Trv1992]) provides an answer in the (for us simplest) case where $\mathbb{L}$ is closed under Lie brackets and is of constant dimension: every point $p \in M$ admits an open neighborhood foliated by submanifolds 
$N$ satisfying $T_{q} N=\mathbb{L}(q)$, for every $q \in N$. The global properties of these submanifolds were not much studied until C. Ehresmann and G. Reeb endeavoured to understand them (birth of foliation theory). A line with irrational slope in the 2 -torus $(\mathbb{R} / \mathbb{Z})^{2}$ shows that it is necessary to admit submanifolds $N$ of $M$ which are not closed. Let $\mathcal{A}_{M}$ denote the manifold structure of $M$.

Definition 1.7. An immersed submanifold of $\left(M, \mathcal{A}_{M}\right)$ is a subset of $N$ of $M$ equipped with its own smooth manifold structure $\mathcal{A}_{N}$, such that the inclusion map $i:\left(N, \mathcal{A}_{N}\right) \rightarrow\left(M, \mathcal{A}_{M}\right)$ is smooth, immersive and injective.

Thus, to keep maximally open Problem 1.6', one should seek immersed submanifolds and make no assumption about closedness under Lie brackets. For later use, recall that an immersed submanifold $N$ of $M$ is embedded if its own manifold structure coincides with the manifold inherited from the inclusion $N \subset M$. It is well known ([CLN1985]) that an immersed submanifold $N$ is embedded if and only if for every point $p \in N$, there exists a neighborhood $U_{p}$ of $p$ in $M$ such that the pair $\left(U_{p}, N \cap U_{p}\right)$ is diffeomorphic to $\left(\mathbb{R}^{\operatorname{dim} M}, \mathbb{R}^{\operatorname{dim} N}\right)$.

1.8. Maximal strong integral manifolds property. In order to understand Problem 1.6', for heuristic reasons, it will be clever to discuss the differences between the two possibilities $\mathbb{L}(p)=T_{p} N$ and $\mathbb{L}(p) \varsubsetneqq T_{p} N$. Consider an arbitrary $\mathbb{F}_{M}$-linear system of vector fields $\widehat{\mathbb{L}}$ containing $\mathbb{L}$, for instance $\mathbb{L}$ itself. Let $p \in M$ and define the linear subspace $\widehat{\mathbb{L}}(p):=$ $\{\widehat{L}(p): \widehat{L} \in \widehat{\mathbb{L}}\}$.

Definition 1.9. An immersed submanifold $N$ of $M$ is said to be:

- a strong $\widehat{\mathbb{L}}$-integral manifold if $T_{q} N=\widehat{\mathbb{L}}(q)$, at every point $q \in N$;

- a weak $\mathbb{L}$-integral manifold if $T_{q} N \supset \widehat{\mathbb{L}}(q)$, at every point $q \in N$.

In advance, the answer (Theorem 1.21 below) to Problem 1.6' states that it is possible to construct a unique system of vector fields $\widehat{\mathbb{L}}$ containing $\mathbb{L}$, whose strong integral manifolds coincide with the smallest weak $\mathbb{L}$ integral manifolds $N$. Further definitions are needed.

A system of vector fields $\widehat{\mathbb{L}}$ is said to have the strong integral manifolds property if for every point $p \in M$, there exists a strong $\widehat{\mathbb{L}}$-integral submanifold $N$ passing through $p$. A maximal strong $\widehat{\mathbb{L}}$-integral manifold $N$ is an immersed $\widehat{\mathbb{L}}$-integral manifold with the property that every connected strong $\widehat{\mathbb{L}}$-integral manifold which intersects $N$ is an open submanifold of 
$N$. Thus, through a point $p \in M$, there passes at most one maximal strong $\widehat{\mathbb{L}}$-integral submanifold. Finally, the system $\widehat{\mathbb{L}}$ has the maximal strong integral manifolds property if, through every point $p \in M$, there passes a maximal strong $\widehat{\mathbb{L}}$-integral manifold. The $\mathbb{F}_{M}$-linear systems $\widehat{\mathbb{L}}$ containing $\mathbb{L}$ are ordered by inclusion. We then admit that Problem 1.6' is essentially reduced to:

Problem 1.6". How to construct the (a posteriori unique) smallest (for inclusion) $\mathbb{F}_{M}$-linear system of vector fields $\widehat{\mathbb{L}}$ containing $\mathbb{L}$ which has the maximal strong integral manifolds property?

1.10. Taking account of the Lie brackets. Here is a basic geometric observation inspired by Frobenius' and Nagano's theorems.

Lemma 1.11. Assume the $\mathbb{F}_{M}$-linear system $\widehat{\mathbb{L}}$ has the strong integral manifolds property. Then for every two vector fields $\widehat{L}, \widehat{L^{\prime}} \in \widehat{\mathbb{L}}$ and for every $p$ in the intersection of their domains, the Lie bracket $\left[\widehat{L}, \widehat{L}^{\prime}\right](p)$ belongs to $\widehat{\mathbb{L}}(p)$.

Proof. Indeed, let $N$ be a strong $\widehat{\mathbb{L}}$-integral manifold, namely satisfying $T N=\left.\widehat{\mathbb{L}}\right|_{N}$. If $\widehat{L}, \widehat{L} \widehat{L}^{\prime} \in \widehat{\mathbb{L}}$, the two restrictions $\left.\widehat{L}\right|_{N}$ and $\left.\widehat{L}^{\prime}\right|_{N}$ are tangent to $N$. Hence the restriction to $N$ of the Lie bracket $\left[\widehat{L}, \widehat{L}^{\prime}\right]$ is also tangent to $N$. In conclusion, at every $p \in N$, we have $\left[\widehat{L}, \widehat{L}^{\prime}\right](p) \in T_{p} N=\widehat{\mathbb{L}}(p)$.

So it is a temptation to believe that the smallest system $\mathbb{L}^{\text {lie }}$ of vector fields containing $\mathbb{L}$ which is closed under Lie brackets does enjoy the maximal integral manifolds property. However, just after the statement of Nagano's theorem (Part II), we have already learnt by means of Example 1.6(II) that in the $\mathcal{C}^{\infty}$ and $\mathcal{C}^{\kappa, \alpha}$ categories, the consideration of $\mathbb{L}^{\text {lie }}$ is inappropriate.

1.12. Transport of a vector field by the flow of another vector field. To understand why $\mathbb{L}^{\text {lie }}$ is insufficient, it will be clever to recall one of the classical definitions of the Lie bracket between two vector fields. Let $p \in M$ and let $K$ be a vector field defined in a neighborhood of $p$. Denote by $K(q)$ the value of $K$ at a point $q$ (this is a vector in $T_{q} M$ ), by $g_{*}(K)$ the push-forward of $K$ by a local diffeomorphism $g$, and by $q \mapsto K_{\mathrm{s}}(q)$ [instead of $q \mapsto \exp (\mathrm{s} K)(q)$ ] the local diffeomorphism at time $\mathrm{s}$ induced by the flow of $K$. If $L$ is a second vector field defined in a neighborhood 
of $p$, the Lie bracket between $K$ and $L$ at $p$ is defined by:

$$
[K, L](p):=\lim _{\mathrm{s} \rightarrow 0}\left(\frac{L(p)-\left(K_{\mathrm{s}}\right)_{*}\left(L\left(K_{-\mathrm{s}}(p)\right)\right)}{\mathrm{s}}\right) .
$$

Observe that for every fixed $\mathrm{s} \neq 0$, the two vectors $L(p)$ and $\left(K_{\mathbf{s}}\right)_{*}\left(L\left(K_{-\mathbf{s}}(p)\right)\right)$ belong $T_{p} M$.

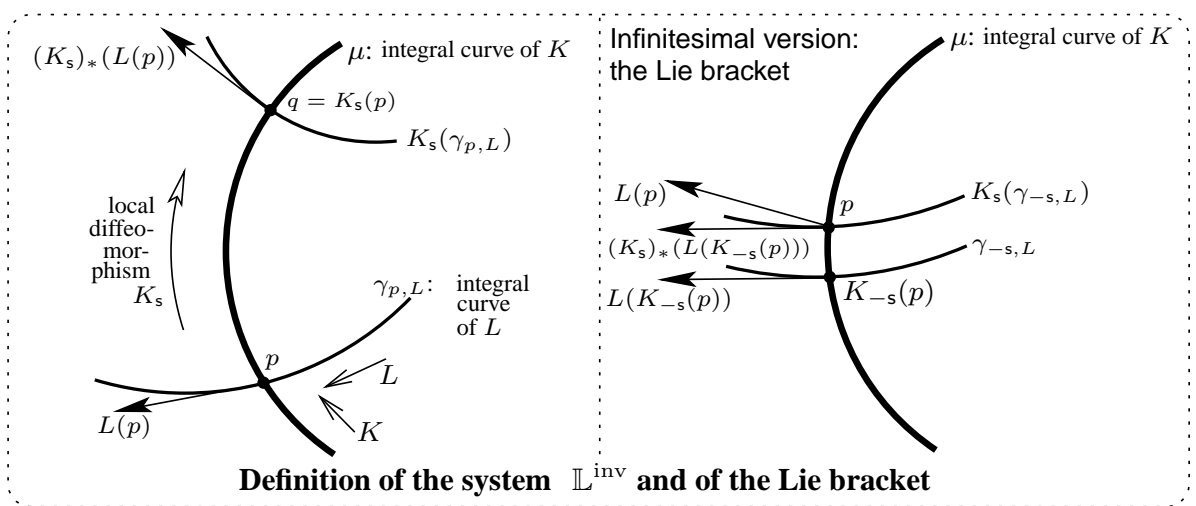

We explain how to read the right hand side of the diagram. In it: the integral curve of $K$ passing through $p$ is denoted by $\mu$; the integral curve of $L$ passing through the point $K_{-\mathrm{s}}(p)$ for $\mathrm{s}$ very small is denoted by $\gamma_{-\mathrm{s}, L}$; its image by the local diffeomorphism $K_{\mathrm{s}}$ is denoted by $K_{\mathrm{s}}\left(\gamma_{-\mathrm{s}, L}\right)$; the vector $L\left(K_{-\mathrm{s}}(p)\right)$ is tangent to $\gamma_{-\mathrm{s}, L}$ at the point $K_{-\mathrm{s}}(p)$; the vector $\left(K_{\mathrm{s}}\right)_{*}\left(L\left(K_{-\mathrm{s}}(p)\right)\right)$, transported by the differerential of $K_{\mathrm{s}}$, is in general distinct from the vector $L(p)$; in fact, the difference $L(p)-\left(K_{\mathrm{s}}\right)_{*}\left(L\left(K_{-\mathrm{s}}(p)\right)\right)$ divided by $\mathrm{s}$, tends to $[K, L](p)$ as $\mathrm{s} \rightarrow 0$.

Essentially, $\mathbb{L}^{\text {lie }}$ collects all vector fields obtained by taking infinitesimal differences (1.13) between vectors $L(p)$ and transported vectors $\left(K_{\mathrm{s}}\right)_{*}\left(L\left(K_{-\mathrm{s}}(p)\right)\right)$, and then iterating this processus to absorb all multiple Lie brackets.

As suggested in the left hand side of the diagram, instead of taking the infinitesimal differences, it is more general to collect all the vectors of the form $\left(K_{\mathrm{s}}\right)_{*}(L(p))$. This is the clue of Sussmann's theorem. In fact, the system $\widehat{\mathbb{L}}$ which is sought for in Problem 1.6 " should not only contain $\mathbb{L}^{\text {lie, }}$, but should also collect all the vector fields of the form $\left(K_{\mathrm{s}}\right)_{*}(L)$, where $\mathrm{s}$ is not an infinitesimal.

Lemma 1.14. Let $\widehat{\mathbb{L}}$ be a $\mathbb{F}_{M}$-linear system of vector fields containing $\mathbb{L}$ which has the strong integral manifolds property. Let $p \in M$, let $K, L \in \mathbb{L}$ be two arbitrary vector fields defined in a neighborhood of $p$ and let $q=$ $K_{\mathrm{s}}(p)$ be a point in the integral curve of $K$ issued from $p$, with $\mathrm{s} \in \mathbb{R}$ small. 
Then the linear subspace $\widehat{\mathbb{L}}(q)$ necessarily contains the transported vector $\left(K_{\mathrm{s}}\right)_{*}(L(p))$.

Proof. Let $N$ be a strong $\widehat{\mathbb{L}}$-integral manifold passing through $p$. As $\widehat{\mathbb{L}}(r)=T_{r} N$ at every point $r \in N$, and as $\mathbb{L}$ is contained in $\widehat{\mathbb{L}}$, it follows that the restricted vector field $\left.K\right|_{N}$ is tangent to $N$. Consequently, the integral curve of $K$ issued from $p$ is locally contained in $N$, hence the point $q=K_{\mathrm{s}}(p)$ belongs to $N$.

Moreover, as $\mathbb{L}$ is contained in $\widehat{\mathbb{L}}$, the vector $L(p)$ is tangent to $N$ at $p$. The differential $\left(K_{\mathrm{s}}\right)_{*}$ being a linear isomorphism between $T_{p} N$ and $T_{q} N$, it follows that the vector $\left(K_{\mathrm{s}}\right)_{*}(L(p))$ belongs to the tangent space $T_{q} N$, which coincides with $\widehat{\mathbb{L}}(q)$ by assumption.

1.15. The smallest $\mathbb{L}$-invariant system of vector fields $\mathbb{L}^{\text {inv }}$. Based on this crucial observation, we may introduce the smallest $\mathbb{F}_{M}$-linear system of vector fields $\mathbb{L}^{\text {inv }}$ ("inv" abbreviates "invariant") containing $\mathbb{L}$ which contains all vectors of the form $\left(K_{\mathrm{s}}\right)_{*}(L)$, whenever $K, L \in \mathbb{L}$ and $\mathrm{s} \in \mathbb{R}$. It follows that $\left(K_{\mathrm{s}}\right)_{*}\left(\mathbb{L}^{\text {inv }}(p)\right)=\mathbb{L}^{\text {inv }}\left(K_{\mathrm{s}}(p)\right)$ : the distribution of linear subspaces $p \mapsto \mathbb{L}^{\text {inv }}(p) \subset T_{p} M$ is invariant under the local flow maps.

In [Su1973], it is shown that $\mathbb{L}^{\text {inv }}$ is concretely and finitely generated as stated in Lemma 1.16 below. At first, some more notation is needed to denote the composition of several local diffeomorphisms of the form $K_{\mathrm{s}}$. Let $\mathbb{X}$ denote the system of all tangent vector fields to $M$, defined on open subsets of $M$. Let $k \in \mathbb{N}$ with $k \geqslant 1$ and let $K=\left(K^{1}, \ldots, K^{k}\right) \in \mathbb{X}^{k}$ be a $k$-tuple of vector fields defined in their domains of definition. If $\mathrm{s}=$ $\left(\mathrm{s}_{1}, \ldots, \mathrm{s}_{k}\right) \in \mathbb{R}^{k}$ is a $k$-tuple of "time" parameters, we will denote by $K_{\mathrm{s}}(p)$ the point

$$
K_{\mathrm{s}_{1}}^{1}\left(\cdots\left(K_{\mathrm{s}_{k}}^{k}(p)\right) \cdots\right):=\exp \left(\mathrm{s}_{1} K^{1}\left(\cdots\left(\exp \left(\mathrm{s}_{k} K^{k}(p)\right)\right) \cdots\right)\right),
$$

whenever the composition is defined. The $k$-tuple $\left(\mathrm{s}_{1}, \ldots, \mathrm{s}_{k}\right)$ will also be called a multitime parameter. For s fixed, the map $p \mapsto K_{\mathrm{s}}(p)$ is a local diffeomorphism between two open subsets of $M$. Its local inverse is the map $p \mapsto \widetilde{K}_{-\widetilde{\mathbf{s}}}(p)$, where $\widetilde{K}:=\left(K^{k}, \ldots, K^{1}\right) \in \mathbb{L}^{k}$ and $\widetilde{\mathrm{s}}:=\left(\mathrm{s}_{k}, \ldots, \mathrm{s}_{1}\right)$. Moreover, if we define $\left(\mathrm{s}, \mathrm{s}^{\prime}\right):=\left(\mathrm{s}_{1}, \ldots, \mathrm{s}_{k}, \mathrm{~s}_{1}^{\prime}, \ldots, \mathrm{s}_{k^{\prime}}^{\prime}\right)$ for general $\mathrm{s}=\left(\mathrm{s}_{1}, \ldots, \mathrm{s}_{k}\right) \in \mathbb{R}^{k}$ and $\mathrm{s}^{\prime}=\left(\mathrm{s}_{1}^{\prime}, \ldots, \mathrm{s}_{k^{\prime}}^{\prime}\right) \in \mathbb{R}^{k^{\prime}}$, we have $K_{\mathrm{s}^{\prime}}^{\prime} \circ K_{\mathrm{s}}=\left(K^{\prime}, K\right)_{\left(\mathrm{s}^{\prime}, \mathrm{s}\right)}$.

After shrinking the domains of definition, the composition of local diffeomorphisms $K_{\mathrm{s}}$ is clearly associative, where it is defined. It follows that the set of local diffeomorphisms $K_{\mathrm{s}}$ constitutes a pseudogroup of local 
diffeomorphisms. Here, the term "pseudo" stems from the fact that the domains of definitions have to be adjusted; not all compositions are allowed.

Lemma 1.16. ([Su1973]) The system $\mathbb{L}^{\text {inv }}$ is generated by the $\mathbb{F}_{M}$-linear combinations of all vector fields of the form $\left(K_{\mathrm{s}}\right)_{*}(L)$, for all $L \in \mathbb{L}$, all $k$-tuples $K=\left(K^{1}, \ldots, K^{k}\right) \in \mathbb{L}^{k}$ of elements of $\mathbb{L}$ and all multitime parameters $\mathrm{s}=\left(\mathrm{s}_{1}, \ldots, \mathrm{s}_{k}\right) \in \mathbb{R}^{k}$.

The definitions and the above reasonings show that the $\mathbb{F}_{M}$-linear system $\mathbb{L}^{\text {lie }}$ is a subsystem of the $\mathbb{F}_{M}$-linear system $\mathbb{L}^{\text {inv }}$ (of course, every system is contained in $\mathbb{X}$ ):

$$
\mathbb{L} \subset \mathbb{L}^{\text {lie }} \subset \mathbb{L}^{\text {inv }} \subset \mathbb{X} \text {. }
$$

In general, at a fixed point $p \in M$, the inclusions $\mathbb{L}(p) \subset \mathbb{L}^{\text {lie }}(p) \subset$ $\mathbb{L}^{\text {inv }}(p) \subset \mathbb{X}(p)=T_{p} M$ may be all strict.

Example 1.17. On $\mathbb{R}^{4}$, consider the system $\mathbb{L}$ generated by the three vector fields

Then it may be checked that

$$
\frac{\partial}{\partial \mathbf{x}_{1}}, \quad \mathbf{x}_{1} \frac{\partial}{\partial \mathbf{x}_{2}}, \quad e^{-1 / \mathrm{x}_{1}^{2}} \frac{\partial}{\partial \mathrm{x}_{3}}
$$

$$
\left\{\begin{aligned}
\mathbb{L}(0) & =\mathbb{R} \partial_{\mathrm{x}_{1}}, \\
\mathbb{L}^{\text {lie }}(0) & =\mathbb{R} \partial_{\mathrm{x}_{1}} \oplus \mathbb{R} \partial_{\mathrm{x}_{2}}, \\
\mathbb{L}^{\text {inv }}(0) & =\mathbb{R} \partial_{\mathrm{x}_{1}} \oplus \mathbb{R} \partial_{\mathrm{x}_{2}} \oplus \mathbb{R} \partial_{\mathrm{x}_{3}}, \\
\mathbb{X}(0) & =\mathbb{R} \partial_{\mathrm{x}_{1}} \oplus \mathbb{R} \partial_{\mathrm{x}_{2}} \oplus \mathbb{R} \partial_{\mathrm{x}_{3}} \oplus \mathbb{R} \partial_{\mathrm{x}_{4}} .
\end{aligned}\right.
$$

Theorem 1.18. ([Na1966, Su1973]) In the $\mathcal{C}^{\omega}, \mathcal{C}^{\infty}$ and $\mathcal{C}^{\kappa, \alpha}$ categories, the system $\mathbb{L}^{\text {inv }}$ is the smallest one containing $\mathbb{L}$ that has the maximal strong integral manifolds property. In the $\mathcal{C}^{\omega}$ category, $\mathbb{L}^{\text {inv }}=\mathbb{L}^{\text {lie }}$.

Further structural properties remain to be explained.

1.19. $\mathbb{L}$-orbits. The maximal strong integral manifolds of $\mathbb{L}^{\text {inv }}$ may be defined directly by means of $\mathbb{L}$, without refering to $\mathbb{L}^{\text {inv }}$, as follows. Two points $p, q \in M$ are said to be $\mathbb{L}$-equivalent if there exists a local diffeomorphism of the form $K_{\mathrm{s}}, K=\left(K^{1}, \ldots, K^{k}\right), \mathrm{s}=\left(\mathrm{s}_{1}, \ldots, \mathrm{s}_{k}\right), k \in \mathbb{N}$, with $K_{\mathrm{s}}(p)=q$. This clearly defines an equivalence relation on $M$. The equivalence classes are called the $\mathbb{L}$-orbits of $M$ and will be denoted either by $\mathcal{O}_{\mathbb{L}}(p)$ or shortly by $\mathcal{O}_{\mathbb{L}}$, when the reference to one point of the orbit is superfluous.

Concretely, two points $p, q \in M$ belong to the same $\mathbb{L}$-orbit if and only if there exist a continuous curve $\gamma:[0,1] \rightarrow M$ with $\gamma(0)=p$ and $\gamma(1)=q$ 
together with a partition of the interval $[0,1]$ by numbers $0=\mathrm{s}_{0}<\mathrm{s}_{1}<$ $\mathrm{s}_{2}<\cdots<\mathrm{s}_{k}=1$ and vector fields $K^{1}, \ldots, K^{k} \in \mathbb{L}$ such that for each $i=1, \ldots, k$, the restriction of $\gamma$ to the subinterval $\left[\mathbf{s}_{i-1}, \mathbf{s}_{i}\right]$ is an integral curve of $K^{i}$. Such a curve will be called a piecewise integral curve of $\mathbb{L}$.

Let $p \in M$. Then its $\mathbb{L}_{\text {-orbit }} \mathcal{O}_{\mathbb{L}}(p)$ may be equipped with the finest topology which makes all the maps $\mathrm{s} \mapsto K_{\mathrm{s}}(p)$ continuous, for all $k \geqslant 1$, all $K=\left(K^{1}, \ldots, K^{k}\right) \in \mathbb{L}^{k}$ and all multitime parameters $\mathrm{s}=\left(\mathrm{s}_{1}, \ldots, \mathrm{s}_{k}\right)$. This topology is independent of the choice of a central point $p$ inside a given orbit ([Su1973]). Since the maps $\mathbb{R}^{k} \ni \mathrm{s} \mapsto K_{\mathrm{s}}(p) \in M$ are already continuous, the topology of $\mathcal{O}_{\mathbb{L}}(p)$ is always finer than the topology induced by the inclusion $\mathcal{O}_{\mathbb{L}}(p) \subset M$. It follows that the inclusion map from $\mathcal{O}_{\mathbb{L}}(p)$ into $M$ is continuous. In particular, $\mathcal{O}_{\mathbb{L}}(p)$ is Hausdorff.

1.20. Precise statement of the orbit theorem. We now state in length the fundamental theorem of Sussmann, based on preliminary versions due to Hermann ([ [He1963] $)$, to Nagano ([Na1966]) and to Lobry ([ [Lo1970] $)$. It describes $\mathbb{L}$-orbits as immersed submanifolds (1), (2) enjoying the everywhere accessibility conditions (3), (4), together with a local flow-box property (5), useful in applications.

Theorem 1.21. (Sussmann [Su1973, Trv1992, BM1997, BER1999, BCH2005], [*]) The following five properties hold true.

(1) Every $\mathbb{L}$-orbit $\mathcal{O}_{\mathbb{L}}$, equipped with the finest topology which makes all the maps $\mathrm{s} \mapsto K_{\mathrm{s}}(p)$ continuous, admits $a$ unique differentiable structure with the property that $\mathcal{O}_{\mathbb{L}}$ is an immersed submanifold of $M$, of $\operatorname{class} \mathcal{C}^{\omega}, \mathcal{C}^{\infty}$ or $\mathcal{C}^{\kappa, \alpha}$.

(2) With this topology, each $\mathbb{L}$-orbit $\mathcal{O}_{\mathbb{L}}$ is simultaneously a (connected) maximal weak integral manifold of $\mathbb{L}$ and a (connected) maximal strong integral manifold of the $\mathbb{L}$-invariant $\mathbb{F}_{M}$-linear system $\mathbb{L}^{\text {inv }}$; thus, for every point $p \in M$, it holds $T_{p} \mathcal{O}_{\mathbb{L}}(p)=\mathbb{L}^{\text {inv }}(p)$, whence in particular $\operatorname{dim} \mathbb{L}^{\operatorname{inv}}(q)=\operatorname{dim} \mathcal{O}_{\mathbb{L}}(p)$ is constant for all $q$ belonging to a given $\mathbb{L}$-orbit $\mathcal{O}_{\mathbb{L}}(p)$.

(3) For every $p \in M, k \geqslant 1, K \in \mathbb{L}^{k}, \mathrm{~s} \in \mathbb{R}^{k}$ such that $K_{\mathrm{s}}(p)$ is defined, the differential map $\left(K_{\mathrm{s}}\right)_{*}$ makes a linear isomorphism from $T_{p} \mathcal{O}_{\mathbb{L}}(p)=\mathbb{L}^{\text {inv }}(p)$ onto $T_{K_{\mathbf{s}}(p)} \mathcal{O}_{\mathbb{L}}(p)=\mathbb{L}^{\text {inv }}\left(K_{\mathbf{s}}(p)\right)$.

(4) For every $p, q \in M$ belonging to the same $\mathbb{L}$-orbit, there exists an integer $k \geqslant 1$, there exists a $k$-tuple of vector fields $K=\left(K^{1}, \ldots, K^{k}\right) \in \mathbb{L}^{k}$ and there exists a multitime $\mathrm{s}^{*}=$ 
$\left(\mathrm{s}_{1}^{*}, \ldots, \mathrm{s}_{k}^{*}\right) \in \mathbb{R}^{k}$ such that $p=K_{\mathrm{s}^{*}}(q)$ and such that the differential at $\mathrm{s}^{*}$ of the map

$$
\mathbb{R}^{k} \ni \mathrm{s} \mapsto K_{\mathrm{s}}(q) \in \mathcal{O}_{\mathbb{L}}(p)
$$

is of rank equal to $\operatorname{dim} \mathcal{O}_{\mathbb{L}}(p)$.

(5) For every $p \in M$, there exists an open connected neighborhood $V_{p}$ of $p$ in $M$ and there exists a $\mathcal{C}^{\omega}, \mathcal{C}^{\infty}$ or $\mathcal{C}^{\kappa, \alpha}$ diffeomorphism

$$
\square^{e} \times \square^{n-e} \ni(\mathrm{s}, \mathrm{r}) \longmapsto \varphi(\mathrm{s}, \mathrm{r}) \in V_{p},
$$

where $e=\operatorname{dim} \mathcal{O}_{\mathbb{L}}(p)$, where $\square=\{\mathrm{x} \in \mathbb{R}:|\mathrm{x}|<1\}$, such that:

- $\varphi(0,0)=p$;

- the plaque $\varphi\left(\square^{e} \times\{0\}\right)$ is an open piece of the $\mathbb{L}$-orbit of $p$;

- each plaque $\varphi\left(\square^{e} \times\{r\}\right)$ is contained in a single $\mathbb{L}$-orbit; and:

- the set of $r \in \square^{n-e}$ such that $\varphi\left(\square^{e} \times\{r\}\right)$ is contained in the same $\mathbb{L}$-orbit $\mathcal{O}_{\mathbb{L}}(p)$ is either finite or countable.

In general, for $r \neq 0$, the $e$-dimensional plaques $\varphi\left(\square^{e} \times\{r\}\right)$ have positive codimension in the nearby orbits. We draw a diagram, in which $e=\operatorname{dim} \mathcal{O}_{\mathbb{L}}(p)=1$, with the nearby $\mathbb{L}$-orbits $\mathcal{O}_{2}, \mathcal{O}_{2}^{\prime}, \mathcal{O}_{3}$ and $\mathcal{O}_{3}^{\prime}$ having dimensions $2,2,3$ and 3 .

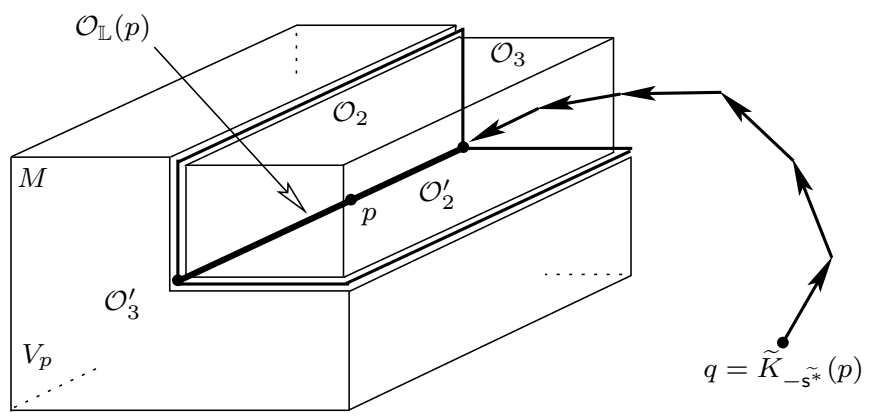

Local orbit flow box theorem

Property (4) is crucial: the maps (1.22) of $\operatorname{rank} \operatorname{dim} \mathcal{O}_{\mathbb{L}}(p)$ are used to define the differentiable structure on $\mathcal{O}_{\mathbb{L}}(p)$; they are also used to obtain the local orbit flow box property (5), as follows.

Let $p \in M$ and choose $q \in \mathcal{O}_{\mathbb{L}}(p)$ with $q \neq p$, to fit with the diagrams ( $q=p$ would also do). Assuming that (4) holds, set $e:=\operatorname{dim} \mathcal{O}_{\mathbb{L}}(p)$, introduce an open subset $T_{e}$ in some $e$-dimensional affine subspace passing through $\mathrm{s}^{*}$ in $\mathbb{R}^{k}$ so that the restriction of the map (1.23) to $T_{e}$ still has rank 
$e$ at $\mathbf{s}=\mathrm{s}^{*}$. Introduce also an $(n-e)$-dimensional local submanifold $\Lambda_{p}$ passing through $p$ with $T_{p} \Lambda_{p} \oplus T_{p} \mathcal{O}_{\mathbb{L}}(p)=T_{p} M$ and set $\Lambda_{q}:=\widetilde{K}_{-\widetilde{\mathbf{s}}}\left(\Lambda_{p}\right)$. Notice that $T_{q} \Lambda_{q} \oplus T_{q} \mathcal{O}_{\mathbb{L}}(p)=T_{q} M$, since the multiple flow map $K_{\mathrm{s}}(\cdot)$ stabilizes $\mathcal{O}_{\mathbb{L}}(p)$. Then, as one of the possible maps $\varphi$ whose existence is claimed in (5), we may choose a suitable restriction of:

$$
T_{e} \times \Lambda_{q} \ni(\mathrm{s}, \mathrm{r}) \longmapsto K_{\mathrm{s}}(\mathrm{r}) \in M .
$$

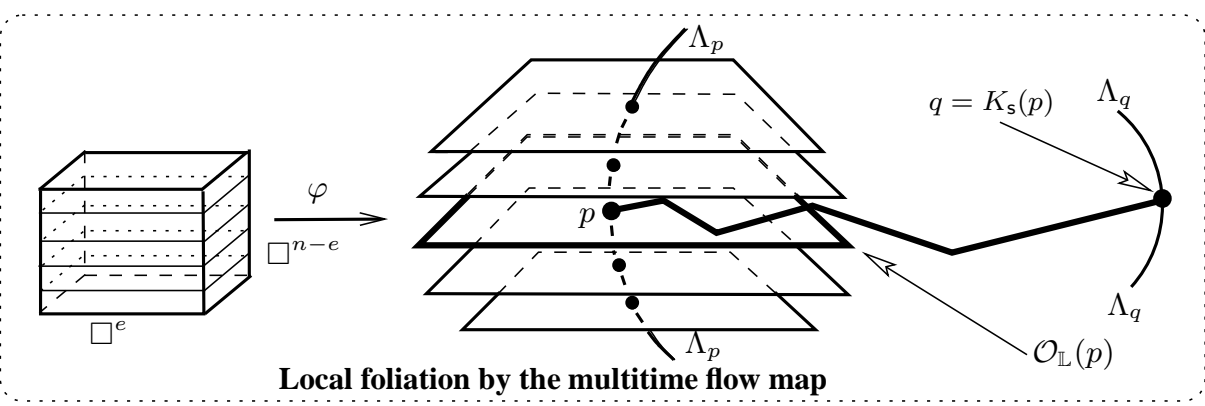

1.24. Characterization of embedded $\mathbb{L}$-orbits. A smooth manifold $N$ together with an immersion $i: N \rightarrow M$ is called weakly embedded if for every manifold $P$, every smooth map $\psi: P \rightarrow M$ with $\psi(P) \subset N$, then $\psi: P \rightarrow N$ is in fact smooth ([Sp1970]; the diagram is also borrowed).

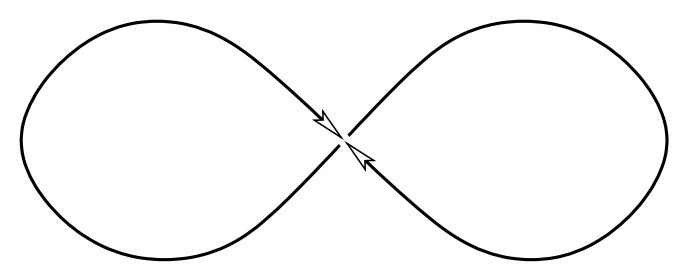

An immersion of the real line in $\mathbb{R}^{2}$ that is not weakly embedded

Proposition 1.25. ([Bel1996, BM1997, BCH2005]) Each $\mathbb{L}$-orbit is countable at infinity (second countable) and weakly embedded in $M$.

As the multiple flows are diffeomorphisms, embeddability propagates.

Proposition 1.26. ([Bel1996, BM1997, BCH2005]) Let $\mathcal{O}_{\mathbb{L}}$ be an $\mathbb{L}$-orbit in $M$ and let $e:=\operatorname{dim} \mathcal{O}_{\mathbb{L}}$. The following three conditions are equivalent:

- $\mathcal{O}_{\mathbb{L}}$ is an embedded submanifold of $M$;

- for every point $p \in \mathcal{O}_{\mathbb{L}}$, there exists a straightening map $\varphi$ as in (1.23) with $\mathcal{O}_{\mathbb{L}} \cap \varphi\left(\square^{e} \times \square^{n-e}\right)=\varphi\left(\square^{e} \times\{0\}\right)$;

- there exists at least one point at which the preceding property holds. 
Conversely, $\mathcal{O}_{\mathbb{L}}$ is not embedded in $M$ if and only if for every $p \in \mathcal{O}_{\mathbb{L}}$ and for every local straightening map $\varphi$ centered at $p$ as in (1.23), the set of $\mathrm{r} \in \square^{n-e}$ such that $\varphi\left(\square^{n-e} \times\{\mathrm{r}\}\right)$ is contained in $\mathcal{O}_{\mathbb{L}}=\mathcal{O}_{\mathbb{L}}(p)$ is infinite (nonetheless countable).

1.27. Local $\mathbb{L}$-orbits and their smoothness. For $U$ running in the collection of all nonempty open connected subsets of $M$ containing $p$, consider the localized $\left.\mathbb{L}\right|_{U}$-orbit of $p$ in $U$, denoted by $\mathcal{O}_{\mathbb{L}}(U, p)$. If $p \in U_{2} \subset U_{1}$, then $\mathcal{O}_{\mathbb{L}}\left(U_{2}, p\right) \subset \mathcal{O}_{\mathbb{L}}\left(U_{1}, p\right) \cap U_{2}$, so the dimension of $\mathcal{O}_{\mathbb{L}}(U, p)$ decreases as $U$ shrinks. Consequently, the localized $\mathbb{L}$-orbit $\mathcal{O}_{\mathbb{L}}(U, p)$ stabilizes and defines a unique piece of local ${ }^{5} \mathbb{L}$-integral submanifold through $p_{0}$, called the local $\mathbb{L}$ orbit of $p_{0}$ and denoted by $\mathcal{O}_{\mathbb{L}}^{\text {loc }}(p)$. In the CR context, this concept will be of interest in Parts V and VI. Sometimes, $\mathbb{L}$-orbits (in $M$ ) are called global, to distinguish them and to emphasize their nonlocal, nonpointwise nature.

From the flow regularity Theorem 1.4 and from Theorem 1.21, it follows:

Lemma 1.28. Global and local $\mathbb{L}$-orbits are as smooth as $\mathbb{L}$, i.e. $\mathcal{C}^{\omega}, \mathcal{C}^{\infty}$ or $\mathcal{C}^{\kappa, \alpha}$. Furthermore,

$$
T_{p} \mathcal{O}_{\mathbb{L}}^{\text {loc }}(p) \subset T_{p} \mathcal{O}_{\mathbb{L}}(M, p)=\mathbb{L}^{\text {inv }}(p),
$$

for every $p \in M$. This inclusion may be strict in the smooth categories $\mathcal{C}^{\infty}$ and $\mathcal{C}^{\kappa, \alpha}$, whereas, in the $\mathcal{C}^{\omega}$ category, local and global $C R$ orbits have the same dimension.

In the $\mathcal{C}^{\kappa, \alpha}$ category, the maximal integral curve of an arbitrary element of $\mathbb{L}$ is $\mathcal{C}^{\kappa+1, \alpha}$, trivially because the right hand sides of the equations $d \mathrm{x}_{k}(\mathrm{t}) / d \mathrm{t}=a_{k}(\mathrm{x}(\mathrm{t})), k=1, \ldots, n$, are $\mathcal{C}^{\kappa, \alpha}$. May it be induced that general $\mathbb{L}$-orbits are $\mathcal{C}^{\kappa+1, \alpha}$ ? Trivially yes if $\operatorname{dim} \mathbb{L}=1$ at every point.

Another instance is as follows. Let $r \in \mathbb{N}$ with $1 \leqslant r \leqslant n-1$ and let $\mathbb{L}^{0}=\left\{L_{a}\right\}_{1 \leqslant a \leqslant r}$ be a system of $\mathcal{C}^{\kappa, \alpha}$ vector fields defined in a neighborhood of the origin in $\mathbb{R}^{n}$ that are linearly independent there. Consider the system $\mathbb{L}$ generated by linear combinations of elements of $\mathbb{L}^{0}$. Achieving Gaussian elimination and a linear change of coordinates, we may assume that $r$ generators of $\mathbb{L}$, still denoted by $L_{1}, \ldots, L_{r}$, take the form $L_{i}=\frac{\partial}{\partial \mathrm{x}_{i}}+\sum_{j=1}^{n-r} a_{i j}(\mathrm{x}, \mathrm{y}) \frac{\partial}{\partial \mathrm{y}_{j}}, i=1, \ldots, r$, with $(\mathrm{x}, \mathrm{y})=$

\footnotetext{
${ }^{5}$ In certain references, local $\mathbb{L}$-orbits are considered as germs. Knowing by experience that the language of germs becomes misleading when several quantifiers are involved in complex statements, we will always prefer to speak of local submanifolds of a certain small size.
} 
$\left(\mathrm{x}_{1}, \ldots, \mathrm{x}_{r}, \mathrm{y}_{1}, \ldots, \mathrm{y}_{n-r}\right)$ and with $a_{i j}(\mathrm{x}, \mathrm{y})$ of class $\mathcal{C}^{\kappa, \alpha}$ in a neighborhood of the origin.

We claim that if $\mathcal{O}_{\mathbb{L}}(0)$ has (minimal possible) dimension $r$, then it is $\mathcal{C}^{\kappa+1, \alpha}$. This happens in particular if $\mathbb{L}$ is Frobenius-integrable.

Indeed, the local graphed equations of $\mathcal{O}_{\mathbb{L}}(0)$ must then be of the form $\mathrm{y}_{j}=h_{j}(\mathrm{x}), j=1, \ldots, n-r$, with the $h_{j}$ of class at least $\mathcal{C}^{\kappa, \alpha}$, thanks to the lemma above. Observe that the $L_{i}$ are tangent to this submanifold if and only if the $h_{j}$ satisfy the complete system of partial differential equations $\frac{\partial h_{j}}{\partial \mathrm{x}_{i}}(\mathrm{x})=a_{i j}(\mathrm{x}, h(\mathrm{x}))$, for $i=1, \ldots, r, j=1, \ldots, n-r$, implying directly that the $h_{j}$ are $\mathcal{C}^{\kappa+1, \alpha}$. In general, this argument shows that if $\operatorname{dim} \mathcal{O}_{\mathbb{L}}(p)$ coincides with $\operatorname{dim} \mathbb{L}(p)$, the orbit is $\mathcal{C}^{\kappa+1, \alpha}$ at $p$.

Example 1.29. However, this improvement of smoothness is untrue when $\operatorname{dim} \mathbb{L}(p)+1 \leqslant \operatorname{dim} \mathcal{O}_{\mathbb{L}}(p) \leqslant n-1$.

Indeed, pick the function $\chi_{\kappa, \alpha}=\chi_{\kappa, \alpha}(\mathrm{z})$ of $\mathrm{z} \in \mathbb{R}$ equal to zero for $z \leqslant 0$ and, for $z \geqslant 0$, defined by:

$$
\chi_{\kappa, \alpha}(z)= \begin{cases}z^{\kappa+\alpha}, & \text { if } 0<\alpha \leqslant 1, \\ z^{\kappa} / \log z, & \text { if } \alpha=0 .\end{cases}
$$

This function is $\mathcal{C}^{\kappa, \alpha}$ on $\mathbb{R}$, but for $(\lambda, \beta)>(\kappa, \alpha)$, it is not $\mathcal{C}^{\lambda, \beta}$ in any neighborhood of the origin. Then in $\mathbb{R}^{4}$ equipped with coordinates $(\mathrm{x}, \mathrm{y}, \mathrm{z}, \mathrm{t})$, consider the hypersurface $\Sigma$ of equation:

$$
0=\mathrm{t}-\chi_{\kappa+1, \alpha}(\mathrm{y}) \chi_{\kappa, \alpha}(\mathrm{z})
$$

Then $\Sigma$ is $\mathcal{C}^{\kappa, \alpha}$, not better. The two vector fields $L_{1}:=\frac{\partial}{\partial x}$ and $L_{2}:=$ $\frac{\partial}{\partial y}+\left[\mathrm{x} \chi_{\kappa, \alpha}(-\mathrm{y})\right] \frac{\partial}{\partial z}+\left[\chi_{\kappa+1, \alpha}^{\prime}(\mathrm{y}) \chi_{\kappa, \alpha}(\mathrm{z})\right] \frac{\partial}{\partial \mathrm{t}}$ have $\mathcal{C}^{\kappa, \alpha}$ coefficients and are tangent to $\Sigma$. We claim that $\Sigma$ is the local $\left\{L_{1}, L_{2}\right\}$-orbit of the origin.

Otherwise, there would exist a local two-dimensional submanifold $\{\mathrm{z}=g(\mathrm{x}, \mathrm{y}), \mathrm{t}=h(\mathrm{x}, \mathrm{y})\}$ with $L_{1}$ and $L_{2}$ tangent to it. Then $\left[L_{1}, L_{2}\right]=\chi_{\kappa, \alpha}(-\mathrm{y}) \frac{\partial}{\partial \mathrm{z}}$ should also be tangent. However, at points $(0, \mathrm{y}, g(0, \mathrm{y}), h(0, \mathrm{y}))$, with y negative and arbitrarily small, $L_{1}, L_{2}$ and $L_{3}$ are equal to the three linearly independent vectors $\frac{\partial}{\partial x}, \frac{\partial}{\partial y}$ and $\chi_{\kappa, \alpha}(-y) \frac{\partial}{\partial z}$.

\section{§2. FINITE TYPE SYSTEM AND THEIR GENERICITY (OPENESS AND DENSITY)}

2.1. Systems of vector fields satisfying $\mathbb{L}^{\text {lie }}=\mathbb{L}$. Let $M$ be a $\mathcal{C}^{\kappa}(1 \leqslant \kappa \leqslant$ $\infty$ ) connected manifold of dimension $n \geqslant 1$. By $\mathbb{X}$, denote the system of 
all vector fields defined on open subsets of $M$ (it is a sheaf). Let

$$
\mathbb{L}^{0}=\left\{L_{a}\right\}_{1 \leqslant a \leqslant r}, \quad r \geqslant 1,
$$

be a finite collection of $\mathcal{C}^{\kappa-1}$ vector fields defined on $M$, namely $L_{a} \in$ $\mathbb{X}(M)$. Unlike in the $\mathcal{C}^{\omega}$ category, in the $\mathcal{C}^{\kappa}$ category, $\mathbb{X}(M)$ is always nonempty and quite large, thanks to partitions of unity. For this reason, we shall not work in the real analytic category, except in some specific local situations. The set of linear combinations of elements of $\mathbb{L}^{0}$ with coefficients in $\mathcal{C}^{\kappa-1}(M, \mathbb{R})$ will be denoted by $\mathbb{L}\left(\right.$ or $\mathbb{L}^{1}$ ) and called the $\mathcal{C}^{\kappa-1}(M)$-linear hull of $\mathbb{L}^{0}$.

Definition 2.2. A $\mathcal{C}^{\kappa-1}(M)$-linear system $\mathbb{L} \subset \mathbb{X}$ is said to be of finite type at a point $p \in M$ if $\mathbb{L}^{\text {lie }}(p)=T_{p} M$.

If $\mathbb{L}^{\text {lie }}$ is of finite type at every point, then $\mathbb{L}^{\text {lie }}=\mathbb{L}^{\text {inv }}=\mathbb{X}$ and there is just one maximal $\mathbb{L}$-integral manifold in the sense of Sussmann: $M$ itself.

In 1939, Chow had already shown that the equality $\mathbb{L}^{\text {lie }}=\mathbb{X}$ implies the everywhere accessibility condition: every two points of $M$ may be joined by integral curves of $\mathbb{L}$. In 1967, Hörmander established that every second order partial differential operator $P:=L_{1}^{2}+\cdots+L_{r}^{2}+R_{1}+R_{0}$ on a domain $\Omega \subset \mathbb{R}^{n}$ whose top order part is a sum of squares of $\mathcal{C}^{\infty}$ vector fields $L_{a}, 1 \leqslant a \leqslant r$, such that $\mathbb{L}^{\text {lie }}=\mathbb{X}$ is $\mathcal{C}^{\infty}$-hypoelliptic, namely Pf $\in \mathcal{C}^{\infty}$ implies $f \in \mathcal{C}^{\infty}$. Vector field systems satisfying $\mathbb{L}^{\text {lie }}=\mathbb{X}$ have been further studied by workers in hypoelliptic partial differential equations and in nilpotent Lie algebras: Métivier, Stein, Mitchell, Stefan, Lobry and others.

In the next Parts V and VI, we will focus on propagational aspects that are enjoyed by the (more general) smooth systems $\mathbb{L}$ that satisfy $\mathbb{L}^{\text {inv }}=\mathbb{X}$, but possibly $\mathbb{L}^{\text {lie }}(p) \neq \mathbb{X}(p)$ at every $p \in M$. Nevertheless, for completeness, we shall survey in the present section some classical geometric properties of finite type systems.

2.3. Lie bracket flags, weights, privilegied coordinates and distance estimate. Define $\mathbb{L}^{1}:=\mathbb{L}$ and by induction, for $s \in \mathbb{N}$ with $2 \leqslant s \leqslant \kappa$, define $\mathbb{L}^{s}$ to be the $\mathcal{C}^{\kappa-s}$-linear hull of $\mathbb{L}^{s-1}+\left[\mathbb{L}^{1}, \mathbb{L}^{s-1}\right]$. Concretely, $\mathbb{L}^{s}$ is generated over $\mathcal{C}^{\kappa-s}$ by iterated Lie brackets of length $\leqslant s$ of the form:

$$
L_{\alpha}=\left[L_{\alpha_{1}},\left[L_{\alpha_{2}}, \ldots,\left[L_{\alpha_{\ell-1}}, L_{\alpha_{\ell}}\right] \ldots\right]\right], \quad 1 \leqslant \ell \leqslant s .
$$

Jacobi's identity insures that $\left[\mathbb{L}^{s_{1}}, \mathbb{L}^{s_{2}}\right] \subset \mathbb{L}^{s_{1}+s_{2}}$.

Denote $\mathbb{L}^{s}(p):=\operatorname{Vect}_{\mathbb{R}}\left\{L(p): L \in \mathbb{L}^{s}\right\}$. Clearly, $\mathbb{L}$ is of finite type at $p \in M$ if and only it there exists an integer $d(p) \leqslant \kappa$ with $\mathbb{L}^{d(p)}(p)=T_{p} M$. The smallest $d(p)$ is sometimes called the degree of non-holonomy of $\mathbb{L}$ at 
$p$. Other authors call it the type of $\mathbb{L}$ at $p$, which we will do. The function $p \mapsto d(p) \in[1, \kappa] \cup\{\infty\}$ is upper-semi-continuous: $d(q) \leqslant d(p)$ for $q$ near $p$.

Combinatorially, at a finite type point, it is of interest to introduce the Lie bracket flag:

$$
\{0\} \subset \mathbb{L}^{1}(p) \subset \mathbb{L}^{2}(p) \subset \cdots \subset \mathbb{L}^{s}(p) \subset \cdots \subset \mathbb{L}^{d(p)}(p)=T_{p} M .
$$

Then a finite type point $p$ is called regular if the integers $n_{s}(q):=$ $\operatorname{dim} \mathbb{L}^{s}(q)$ remain constant in some neighborhood of $p$. It is elementary to verify ([Bel1996] $]$ ) that, at such a regular point, the dimensions are strictly increasing:

$$
0<n_{1}(p)<n_{2}(p)<\cdots<n_{d(p)}(p)=n .
$$

Fix $p$, not necessarily regular. A local coordinate system $\left(x_{1}, x_{2}, \ldots, x_{n}\right)$ centered at $p$ is linearly adapted at $p$ if:

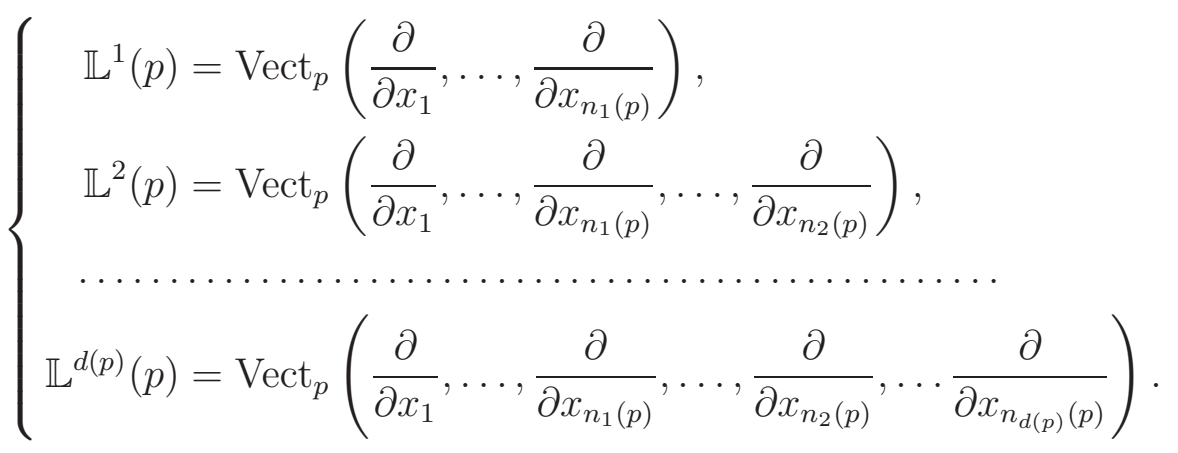

Let us assign weights $w_{i}$ to such linearly adapted coordinates $x_{i}$ as follows: the first group $\left(x_{1}, \ldots, x_{n_{1}(p)}\right)$ being linked to $\mathbb{L}^{1}(p)$, their weights will all be equal to one: $w_{1}=\cdots=w_{n_{1}(p)}=1$. The second group $\left(x_{n_{1}(p)+1}, \ldots, x_{n_{2}(p)}\right)$, linked to the quotient $\mathbb{L}^{2}(p) / \mathbb{L}^{1}(p)$, will be assigned uniform weight two: $w_{n_{1}(p)+1}=\cdots=w_{n_{2}(p)}=2$, and so on, until $w_{n_{d(p)-1}(p)+1}=\cdots=w_{n_{d(p)}(p)}=d(p)$.

Provided $\mathbb{L}$ is of finite type at every point, we claim that the original finite collection $\mathbb{L}^{0}$ produces what is called a sub-Riemannian metric; then by means of weights, the topology associated to this metric may be compared to the manifold topology of $M$ in a highly precise way.

Indeed, let us define the (infinitesimal) sub-Riemannian length of a vector $v_{p} \in \mathbb{L}^{1}(p)$ by:

$$
\left\|v_{p}\right\|_{\mathbb{L}^{0}}:=\inf \left\{\left(u_{1}^{2}+\cdots+u_{m}^{2}\right)^{1 / 2}: v_{p}=u_{1} L_{1}(p)+\cdots+u_{r} L_{r}(p)\right\} .
$$


For $v_{p} \notin \mathbb{L}^{1}(p)$, we set $\left\|v_{p}\right\|_{\mathbb{L}^{0}}=\infty$. The length of a piecewise $\mathcal{C}^{1}$ curve $\gamma(t), t \in[0,1]$, will be the integral:

$$
\operatorname{length}_{\mathbb{L}^{0}}(\gamma):=\int_{0}^{1}\|d \gamma(t) / d t\|_{\mathbb{L}^{0}} d t
$$

Finally, the distance associated to the finite collection $\mathbb{L}^{0}$ is:

$$
d_{\mathbb{L}^{0}}(p, q):=\inf _{\gamma: p \rightarrow q} \operatorname{length}_{\mathbb{L}^{0}}(\gamma) .
$$

Assume for instance $d(p)=2$, so that $n_{2}(p)=n$. If the coordinates are linearly adapted, the tangent space $T_{p} M$ then splits in the "horizontal" space, the $\left(x_{1}, \ldots, x_{n_{1}(p)}\right)$-plane, together with a (not unique) "vertical" space generated $e . g$. by the remaining coordinates. It is then classical that the distance from $p$ to a point of coordinates $\left(x_{1}, \ldots, x_{n}\right)$ close to $p$ enjoys the estimate:

$d_{\mathbb{L}^{0}}\left(p,\left(x_{1}, \ldots, x_{n}\right)\right) \asymp\left|x_{1}\right|+\cdots+\left|x_{n_{1}(p)}\right|+\left|x_{n_{1}(p)+1}\right|^{1 / 2}+\cdots+\left|x_{n}\right|^{1 / 2}$.

Here, the abbreviation $\Phi \asymp \Psi$ means that there exists $C>1$ with $C^{-1} \Psi<\Phi<C \Psi$. Notice that the successive exponents coincide with the weights $w_{1}, \ldots, w_{n_{1}(p)}, w_{n_{1}(p)+1}, \ldots, w_{n}$. In particular, to reach a point of coordinates $(0, \ldots, 0, \varepsilon, \ldots, \varepsilon)$, it is necessary to flow along $\mathbb{L}^{0}$ during a time $\sim \operatorname{cst} . \varepsilon^{1 / 2}$. Observe that $\left|x_{1}\right|+\cdots+\left|x_{n}\right|$ is equivalent to the distance from $p$ to $x$ induced by any Riemannian metric. Thus, the modified distance $d_{\mathbb{L}^{0}}$ is just obtained by replacing each $\left|x_{i}\right|$ by $\left|x_{i}\right|^{1 / w_{i}}$, up to a multiplicative constant.

To generalize such a quantitative comparison between the $d_{\mathbb{L}^{0}}$-distance and the underlying topology of $M$, linearly adapted coordinates appear to be insufficient. For $\beta=\left(\beta_{1}, \ldots, \beta_{r}\right) \in \mathbb{N}^{r}$, denote by $L^{\beta}$ the $|\beta|$-th order derivation $L_{1}^{\beta_{1}} L_{2}^{\beta_{2}} \cdots L_{r}^{\beta_{r}}$. Beyond linearly adapted coordinates, one must introduce privileged coordinates, whose existence is assured by the following.

Theorem 2.4. ([Bel1996]) There exist local coordinates $\left(x_{1}, \ldots, x_{n}\right)$ centered at $p$ that are privileged in the sense that each $x_{i}$ is of order exactly equal to $w_{i}$ with respect to $\mathbb{L}^{0}$-derivations, namely, for $i=1, \ldots, n$ :

$$
\begin{gathered}
\left.L^{\gamma} x_{i}\right|_{p}=0, \quad \text { for all } \gamma \text { with }|\gamma| \leqslant w_{i}-1, \\
\left.L^{\beta_{i}^{*}} x_{i}\right|_{p} \neq 0, \quad \text { for some } \beta_{i}^{*} \text { with }\left|\beta_{i}^{*}\right|=w_{i} .
\end{gathered}
$$

Only if $d(p)=2$, linearly adapted coordinates are automatically privileged ([Bel1996]). As soon as $d(p) \geqslant 3$, privileged systems are unavoidable. 
Theorem 2.5. ([Bel1996]) For $x$ in a neighborhood of $p$, the estimate:

$$
d_{\mathbb{L}^{0}}\left(p,\left(x_{1}, \ldots, x_{n}\right)\right) \asymp\left|x_{1}\right|^{1 / w_{1}}+\cdots+\left|x_{n}\right|^{1 / w_{n}}
$$

holds if and only if the coordinates are privileged.

For $\varepsilon>0$ small, define the anisotropic ball $B_{\mathbb{L}^{0}}(p, \varepsilon):=\{x$ : $\left.d_{\mathbb{L}^{0}}(p, x)<\varepsilon\right\}$.

Corollary 2.6. (Bel1996]) There exist $C>1$ such that

$$
\frac{1}{C} \prod_{i=1}^{n}\left[-\varepsilon^{w_{i}}, \varepsilon^{w_{i}}\right] \subset B_{\mathbb{L}^{0}}(p, \varepsilon) \subset C \prod_{i=1}^{n}\left[-\varepsilon^{w_{i}}, \varepsilon^{w_{i}}\right] .
$$

2.7. Local basis. At a non-regular point, the integers $n_{k}(p)$, $k=1, \ldots, d(p)$ are not necessarily strictly increasing. Thus, it is necessary to express the combinatorics of the Lie bracket flag with more precision, in terms of what is sometimes called Hörmander numbers $m_{i}$, $\ell_{i}$. From now on, we shall assume that the $r$ vector fields $L_{a}, 1 \leqslant a \leqslant r$, are linearly independent at $p$ and have $\mathcal{C}^{\infty}$ or $\mathcal{C}^{\omega}$ coefficients. In both cases, the formal Taylor series of every coefficient exists.

In the flag

$$
\{0\} \subset \mathbb{L}^{1}(p) \subset \mathbb{L}^{2}(p) \subset \cdots \subset \mathbb{L}^{s}(p) \subset \cdots \subset \mathbb{L}^{d(p)}(p)=T_{p} M .
$$

let $m_{1}$ denote the smallest $k \geqslant 2$ such that the dimension of $\mathbb{L}^{k}(p)$ is larger than the dimension of $\mathbb{L}^{1}(p)$ (at a regular point, $m_{1}=2$ ) and set $\ell_{1}:=\operatorname{dim} \mathbb{L}^{m_{1}}(p)-r \geqslant 1$. Similarly, let $m_{2}$ denote the smallest $k \geqslant 1+m_{1}$ such that the dimension of $\mathbb{L}^{k}(p)$ is larger than the dimension of $\mathbb{L}^{m_{1}}(p)$ (at a regular point, $m_{2}=3$ ) and set $\ell_{2}:=\operatorname{dim} \mathbb{L}^{m_{2}}(p)-\operatorname{dim} \mathbb{L}^{m_{1}}(p)$. By induction, let $m_{j+1}$ denote the smallest $k \geqslant 1+m_{j}$ such that the dimension of $\mathbb{L}^{k}(p)$ is larger than the dimension of $\mathbb{L}^{m_{j}}(p)$ and set $\ell_{j+1}:=$ $\operatorname{dim} \mathbb{L}^{m_{j+1}}(p)-\operatorname{dim} \mathbb{L}^{m_{j}}(p)$.

Since $p$ is a point of finite type, the process terminates until $m_{h}=d(p)$ reaches the degree of non-holonomy at $p$, for a certain integer $h \geqslant 1$. We thus have extracted the interesting information, namely the strict flag of linear spaces:

$$
\mathbb{L}^{1}(p) \subset \mathbb{L}^{m_{1}}(p) \subset \mathbb{L}^{m_{2}}(p) \subset \cdots \subset \mathbb{L}^{m_{h}}(p)=T_{p} M,
$$

with Lie bracket orders $1<m_{1}<m_{2}<\cdots<m_{h}$, whose successive dimensions may be listed parallelly:

$$
r<r+\ell_{1}<r+\ell_{1}+\ell_{2}<\cdots<r+\sum_{1 \leqslant j \leqslant h} \ell_{j} .
$$


Next, let $x=\left(x_{1}, \ldots, x_{n}\right)$ be linearly adapted coordinates, vanishing at $p$. We shall denote them by $\left(y, s_{1}, s_{2}, \ldots, s_{h}\right)$, where $y \in \mathbb{R}^{r}$, $s_{1} \in \mathbb{R}^{\ell_{1}}, s_{2} \in \mathbb{R}^{\ell_{2}}, \ldots, s_{h} \in \mathbb{R}^{\ell_{h}}$. As in the preceding paragraph, we assign weight 1 to the $y$-coordinates, weight $m_{1}$ to the $s_{1}$-coordinates, weight $m_{2}$ to the $s_{2}$-coordinates, $\ldots$, weight $m_{h}$ to the $s_{h}$-coordinates. The weight of a monomial $x^{\alpha}=y^{\beta} s_{1}^{\gamma_{1}} s_{2}^{\gamma_{2}} \cdots s_{h}^{\gamma_{h}}$ is obviously defined as $|\beta|+m_{1}\left|\gamma_{1}\right|+m_{2}\left|\gamma_{2}\right|+\cdots+m_{h}\left|\gamma_{h}\right|$. We say that a formal power series $a(x)=a\left(y, s_{1}, \ldots, s_{h}\right)$ is an $\mathrm{O}(\kappa)$ if all its monomials have weight $\geqslant \kappa$. Also, $a(x)$ is called weighted homogeneous of degree $\kappa$ if

$$
a\left(t y, t^{m_{1}} s_{1}, t^{m_{2}} s_{2}, \ldots, t^{m_{h}} s_{h}\right)=t^{\kappa} a\left(y, s_{1}, s_{2}, \ldots, s_{h}\right),
$$

for all $t \in \mathbb{R}$. As in the case of $\mathbb{R} \llbracket z_{1}, \ldots, z_{n} \rrbracket$ with all weights equal to 1 , every formal series $a\left(y, s_{1}, s_{2}, \ldots, s_{h}\right)$ may be decomposed as a countable sum of weighted homogeneous polynomials of increasing degree.

Dually, we also assign weights to all the basic vector fields: $\frac{\partial}{\partial y_{a}}$ will have weight -1 , whereas for $j=1, \ldots, m_{h}$, the $\frac{\partial}{\partial s_{j l}}, l=1, \ldots, \ell_{j}$, will have weight $-m_{j}$. The weight of a monomial vector field $x^{\alpha} \frac{\partial}{\partial x_{i}}$ is defined to be the sum the weights of $x^{\alpha}$ with the weight of $\frac{\partial}{\partial x_{i}}$. Every vector field having formal power series coefficients may be decomposed as a countable sum of weighted homogeneous vector fields having polynomial coefficients.

Theorem 2.8. ([Bel1996, BER1999]) Assume the local vector fields $L_{a}$, $a=1, \ldots, r$, have $\mathcal{C}^{\infty}$ or $\mathcal{C}^{\omega}$ coefficients and are linearly independend at $p$. If the $\mathcal{C}^{\infty}$ or $\mathcal{C}^{\omega}$ coordinates $x=\left(y, s_{1}, s_{2}, \ldots, s_{h}\right)$ centered at $p$ are priveleged, then each $L_{a}$ may be developed as:

$$
L_{a}=\widehat{L}_{a}+\mathrm{O}(0)
$$

where each vector field:

$$
\widehat{L}_{a}:=\frac{\partial}{\partial y_{a}}+\sum_{1 \leqslant j \leqslant 1} \sum_{1 \leqslant l \leqslant \ell_{j}} p_{a, j, l}\left(y, s_{1}, \ldots, s_{j-1}\right) \frac{\partial}{\partial s_{j, l}},
$$

is homogeneous of degree -1 and has as its coefficients some polynomials $p_{a, j, l}=p_{a, j, l}\left(y, s_{1}, \ldots, s_{j-1}\right)$ that are independent of $s_{j}$ and are homogeneous of degree $m_{j}-1$.

A crucial algebraic information is missing in this statement: what are the nondegeneracy conditions on the $p_{a, j, l}$ that insure that the system is indeed of finite type at $p$ with the combinatorial invariants $m_{j}$ and $\ell_{j}$ ? The real problem is to classify vector field systems that are of finite type, up to local changes of coordinates. At least, the following may be verified. 
Theorem 2.9. ([Bel1996, BER1999]) The vector fields $\widehat{L}_{a}, a=1, \ldots, r$, form a finite type system $\mathbb{L}^{0}$ at $p$ having the same combinatorial invariants $m_{j}$ and $\ell_{j}$ and satisfying the same distance estimate as $d_{\mathbb{L}^{0}}$ in Theorem 2.5. Moreover, the linear hull of $\widehat{\mathbb{L}}^{0}$ generates a Lie algebra $\widehat{\mathbb{L}}^{\text {lie }}$ with the nilpotency property that all Lie brackets of length $\geqslant m_{h}+1$ all vanish.

2.10. Finite-typisation of smooth systems of vector fields. As previously, let $\mathbb{L}^{0}=\left\{L_{a}\right\}_{1 \leqslant a \leqslant r}$ be a finite collection of $\mathcal{C}^{\kappa-1}$ vector fields globally defined on a connected manifold $M$ of class $\mathcal{C}^{\kappa}(1 \leqslant \kappa \leqslant \infty)$ and of dimension $n \geqslant 1$. Let $\mathbb{L}$ be its $\mathcal{C}^{\kappa-1}(M)$-linear hull. If $r=1$, then $\mathbb{L}^{\text {lie }}=\mathbb{L}$, hence $\mathbb{L}$ cannot be of finite type, unless $n=1$. So we assume $n \geqslant 2$ and $r \geqslant 2$. We want to perturb $\mathbb{L}$ slightly to $\widetilde{\mathbb{L}}$ so as to get finite-typeness at every point: $\widetilde{\mathbb{L}}^{l i e}(p)=T_{p} M$ at every $p \in M$. Since the composition of Lie brackets of length $\ell$ requires coefficients of vector fields to be at least $\mathcal{C}^{\ell}$, if $\kappa<\infty$, then necessarily $\widetilde{\mathbb{L}^{l i e}}=\widetilde{\mathbb{L}}^{\kappa}$ stops at length $\kappa$.

At a central point, say the origin in $\mathbb{K}^{n}$, and for $\mathbb{K}$-analytic vector field systems, the already presented Theorem 1.11(II) yields small perturbations that are of finite type at 0 . Of course, the same local result holds true for collections of vector fields that are $\mathcal{C}^{\infty}$, or even $\mathcal{C}^{\kappa-1}$ with $\kappa$ large enough. Now, we want a global theorem.

What does it mean for $\widetilde{\mathbb{L}}$ to be close to $\mathbb{L}$ ? A vector field $L \in \mathbb{X}(M)$ may be interpreted as a section of the tangent bundle, in particular a $\mathcal{C}^{\kappa-1}$ map $M \rightarrow T M$. The most useful topology on the set $\mathcal{C}^{\lambda}(M, N)$ of all $\mathcal{C}^{\lambda}$ maps from a manifold $M$ to another manifold $N$ (e.g. $N=T M$ with $\lambda=\kappa-1$ ) is the strong Whitney topology; it controls better than the socalled weak topology the behaviour of maps at infinity in the noncompact case. Essentially, $f, g \in \mathcal{C}^{\lambda}(M, N)$ are (strongly) close to each other if all their partial derivatives of order $\leqslant \lambda$, computed in a countable collection of charts $\varphi_{\nu}: U_{\nu} \rightarrow \mathbb{R}^{n}$ and $\psi_{\nu}: V_{\nu} \rightarrow \mathbb{R}^{m}$ covering $M$ and $N, \nu \in \mathbb{N}$, are $\varepsilon_{\nu}$-close, the smallness of $\varepsilon_{\nu}>0$ depending on the pair of charts $\left(\varphi_{\nu}, \psi_{\nu}\right)$. Precise definitions may be found in the monograph [Hi1976]. We then topologize this way the finite product $\mathbb{X}(M)^{r}$.

Already two vector fields may well be of finite type on a manifold of arbitrary dimension, e.g. $\frac{\partial}{\partial x_{1}}$ and $\sum_{i=2}^{n} x_{1}^{i-1} \frac{\partial}{\partial x_{i}}$ on $\mathbb{R}^{n}$.

Theorem 2.11. ([Lo1970]) If the connected manifold $M$ of dimension $n \geqslant$ 2 is $\mathcal{C}^{n+n^{2}}$, then the set of pairs of vector fields $\mathbb{L}^{0}:=(K, L) \in \mathbb{X}(M)^{2}$ on $M$ whose $\mathcal{C}^{n^{2}+n-1}$-linear hull $\mathbb{L}$ satisfies $\mathbb{L}^{n^{2}+n}=\mathbb{L}$, is open and dense in the strong Whitney topology. 
According to [Su1976], the smoothness $M \in \mathcal{C}^{n+n^{2}}$ in [Lo1970] was improved to $M \in \mathcal{C}^{2 n}$ in Lobry's thesis (unpublished). We will summarize the demonstration in the case $M \in \mathcal{C}^{2 n}$. However, since neither $\mathcal{C}^{2 n}$ nor $\mathcal{C}^{n+n^{2}}$ are optimal, we will improve this result afterwards (Theorem 2.16 below).

Proof. Openness is no mystery. For denseness, we need some preliminary. If $M$ and $N$ are two $\mathcal{C}^{\lambda}$ manifolds, we denote by $J^{\lambda}(M, N)$ the bundle of $\lambda$-th jets of $\mathcal{C}^{\lambda}$ maps from $M$ to $N$. We recall that, to a $\mathcal{C}^{\lambda}$ map $f: M \rightarrow$ $N$ is associated the $\lambda$-th jet map $j^{\lambda} f: M \rightarrow J^{\lambda}(M, N)$, a continuous map that may be considered as a kind of intrinsic collection of all partial derivatives of $f$ up to order $\lambda$. Let $\pi: J^{\lambda}(M, N) \rightarrow M$ be the canonical projection, sending a jet to its base point. For $p \in M$, the fiber $\pi^{-1}(p)$ may be identified with $\mathbb{R}^{N_{m, n, \lambda}}$, where $N_{m, n, \lambda}:=m \frac{(n+\lambda) !}{n ! \lambda !}$ counts the number of partial derivatives of order $\leqslant \lambda$ of maps $\mathbb{R}^{n} \rightarrow \mathbb{R}^{m}$.

We will state a lemma which constitutes a special case of the jet transversality theorem. This particular statement (Lemma 2.12 below) generalizes the intuitively obvious statement that any $\mathcal{C}^{0}$ curve graphed over $\mathbb{R} \times\{0\}^{2}$ in $\mathbb{R}^{3}$ may always be slightly perturbed to avoid a given fixed $\mathcal{C}^{1}$ curve $\Sigma$.

Call a subset $\Sigma \subset J^{\lambda}(M, N)$ algebraic in the jet variables if in every pair of local charts, it possesses defining equations that are polynomials in the jet variables $f_{j, \alpha}, 1 \leqslant j \leqslant m, \alpha \in \mathbb{N}^{n},|\alpha| \leqslant \lambda$, whose coefficients are independent of the coordinates $x \in M$. Of course, after a local diffeomorphism $x \mapsto \bar{x}(x)$ of $M$, a general polynomial in the jet variables which is independent of $x$ almost never remains independent of $\bar{x}$ in the new coordinates. Nevertheless, in the sequel, we shall only encounter special sets $\Sigma \subset J^{\lambda}(M, N)$ which, in any coordinate system, may be defined as zero sets of such special polynomials.

For instance, taking $\left(x_{k}, y_{j}, y_{j, k}\right)$ as coordinates on $J^{1}(M, N)$, where $1 \leqslant k \leqslant n=\operatorname{dim} M$ and $1 \leqslant j \leqslant m=\operatorname{dim} N$, a change of coordinates $x \mapsto \bar{x}(x)$ induces $\left(x_{k}, y_{j}, y_{j, k}\right) \longmapsto\left(\bar{x}_{k}, \bar{y}_{j}, \bar{y}_{j, k}\right)$, where $\bar{y}_{j}=y_{j}$ is unchanged but the new jet variables $y_{j, k}=\sum_{l=1}^{n} \bar{y}_{j, l} \frac{\partial \bar{x}_{l}}{\partial x_{k}}$ involve the variables $x$ (or $\bar{x}$ ). Nevertheless, the equations $\left\{y_{j, k}=0,1 \leqslant j \leqslant m, 1 \leqslant k \leqslant n\right\}$ saying that the first (pure) jet vanishes are equivalent to $\left\{\bar{y}_{j, k}=0,1 \leqslant j \leqslant\right.$ $m, 1 \leqslant k \leqslant n\}$, since the invertible Jacobian matrix $\left(\frac{\partial \bar{x}_{l}}{\partial x_{k}}\right)$ may be erased: vanishing properties in a jet bundle are intrinsic !

A theorem due to Whitney states that real algebraic sets are stratified, i.e. are finite unions of geometrically smooth real algebraic manifolds. The codimension of $\Sigma$ is thus well-defined. 
Lemma 2.12. ([Hi1976]) Assume $\Sigma \subset J^{\lambda}(M, N)$ is algebraic in the jet variables and of codimension $\geqslant 1+\operatorname{dim} M$. Then the set of maps $f \in$ $\mathcal{C}^{\lambda}(M, N)$ whose $\lambda$-th prolongation $j^{\lambda} f: M \rightarrow J^{\lambda}(M, N)$ does not meet $\Sigma$ at any point is open and dense in the strong Whitney topology.

Although $j^{\lambda} f$ is only continuous, the fact that the bad set $\Sigma$ is algebraic enables to apply the appropriate version of Sard's theorem that is used in the jet transversality theorem.

We shall apply the lemma by defining a certain bad set $\Sigma$ which, if avoided, means that a pair of vector fields on $M$ is of finite type at every point.

Assume $M \in \mathcal{C}^{2 n}$ and let $(K, L) \in \mathbb{X}(M)^{2}$. Both vector fields have $\mathcal{C}^{2 n-1}$ coefficients. With $\lambda:=2 n-1$, denote by $J^{2 n-1}\left(\mathbb{X}(M)^{2}\right)$ the fiber bundle of the $(2 n-1)$-th jets of these pairs. In some coordinates provided by a local chart $U \ni q \mapsto\left(x^{1}(q), \ldots, x^{n}(q)\right) \in \mathbb{R}^{n}$, with $U \subset M$ open, we may write $K=\sum_{1 \leqslant i \leqslant n} K_{i}(x) \frac{\partial}{\partial x^{i}}$ and $L=\sum_{1 \leqslant i \leqslant n} L_{i}(x) \frac{\partial}{\partial x^{i}}$. In such a chart, the $(2 n-1)$-th jet map $j^{2 n-1}(K, L): U \longrightarrow J^{2 n-1}\left(\left.\mathbb{X}^{2}(M)\right|_{U}\right)$ is concretely given by:

$$
U \ni x \longmapsto\left(\partial_{x}^{\alpha} K_{i}(x), \partial_{x}^{\alpha} L_{i}(x)\right)_{\alpha \in \mathbb{N}^{n},|\alpha| \leqslant 2 n-1,1 \leqslant i \leqslant n} .
$$

We denote by $K_{i, \alpha}$ and $L_{i, \alpha}$ the corresponding jet variables. A $\mathcal{C}^{2 n}$ local diffeomorphism $x \mapsto \bar{x}=\bar{x}(x)$ induces a triangular transformation involving the chain rule between these jets variables, with coefficients depending on the $2 n$-th jet of $\bar{x}(x)$, some of which are only $\mathcal{C}^{0}$, which might be unpleasant. Fortunately, our bad set $\Sigma$ will be shown to be algebraic with respect to the jet variables $K_{i, \alpha}$ and $L_{i, \alpha}$ in any system of coordinates.

Let $(K, L) \in \mathbb{X}(M)^{2}$. To write shortly iterated Lie brackets, we denote $\operatorname{ad}(K) L:=[K, L]$, so that $\operatorname{ad}(K)^{2} L=[K,[K, L]], \operatorname{ad}(K)^{3} L=$ $[K,[K,[K, L]]]$ and so on. Also, we set $\operatorname{ad}^{0}(K) L:=L$. Define a subset $\Sigma \subset J^{2 n-1}\left(\mathbb{X}^{2}(M)\right)$ as a union $\Sigma=\Sigma^{\prime} \cup \Sigma^{\prime \prime} \cup \Sigma^{\prime \prime \prime}$, where:

- firstly $\Sigma^{\prime}$ is defined by the $2 n$ equations $K_{i, 0}=L_{i, 0}=0$;

- secondly, $\Sigma^{\prime \prime}$ is defined by requiring that all the $n \times n$ minors of the following $n \times(2 n)$ matrix

$$
\left(\operatorname{ad}^{0}(K) L \operatorname{ad}^{1}(K) L \cdots \cdots \operatorname{ad}^{2 n-1}(K) L\right)
$$

vanish;

- thirdly, $\Sigma^{\prime \prime \prime}$ is defined similarly, after exchanging $K$ with $L$. 
Lemma 2.13. In the vector space of real $n \times(2 n)$ matrices, isomorphic to $\mathbb{R}^{2 n^{2}}$, the subset of matrices of rank $\leqslant(n-1)$ is a real algebraic subset of codimension equal to $(n+1)$.

Without obtaining a complete explicit expression, it is easily verified that $\operatorname{ad}^{j}(K)(L), 0 \leqslant j \leqslant 2 n-1$, is a universal polynomial in the jet variables $K_{i, \alpha}$ and $L_{i, \alpha}$. Under a local change of coordinates $x \mapsto \bar{x}(x)$, if the two vector fields $K$ and $L$ transform to $\bar{K}$ and to $\bar{L}$ (push-forward), all the multiple Lie brackets $\operatorname{ad}^{j}(K) L$ then transform to $\operatorname{ad}^{j}(\bar{K}) \bar{L}$, thanks to the invariance of Lie brackets. Geometrically, the vanishing of each of the $n \times n$ minors defining $\Sigma^{\prime \prime}$ and $\Sigma^{\prime \prime \prime}$ means the linear dependence of a system of $n$ vectors, thus it is an intrinsic condition. Consequently, although the jet variables $K_{i, \alpha}$ and $L_{i, \alpha}$ are transformed in an unpleasant way through diffeomorphisms, the sets $\Sigma^{\prime}, \Sigma^{\prime \prime}$ and $\Sigma^{\prime \prime \prime}$ may be defined by universal polynomials in the jet variables $K_{i, \alpha}$ and $L_{i, \alpha}$, that are the same in any system of local coordinates.

The lemma above and an inspection of a part of the complete expression of the $\operatorname{ad}^{j}(K)(L), 0 \leqslant j \leqslant 2 n-1$ provides the following information. Details will be skipped.

Lemma 2.14. The two subsets $\Sigma^{\prime \prime}$ and $\Sigma^{\prime \prime \prime}$ of $J^{2 n-1}\left(\mathbb{X}(M)^{2}\right)$ are both algebraic in the jet variables and of codimension $(n+1)$ outside $\Sigma^{\prime}$.

To conclude the proof of the theorem, we have to show that arbitrarily close to $(K, L)$, there are pairs of finite type. Since $\Sigma^{\prime}$ has codimension $2 n>\operatorname{dim} M$, a first application of the avoidance Lemma 2.12 yields a perturbed pair, still denoted by $(K, L)$, with the property that at every point $p \in M$, either $K(p) \neq 0$ or $L(p) \neq 0$. Since $\Sigma^{\prime \prime}$ and $\Sigma^{\prime \prime \prime}$ both have codimension $n+1>\operatorname{dim} M$, a second application of the avoidance Lemma 2.12 yields a perturbed pair such that the two collections of $2 n$ vector fields $\operatorname{ad}^{j}(K) L, 0 \leqslant j \leqslant 2 n-1$, and $\operatorname{ad}^{j}(L) K, 0 \leqslant j \leqslant 2 n-1$, generate $T M$ at every point $p \in M$. The proof is complete.

To improve this theorem, let $r \geqslant 2$ and consider the set $\mathbb{X}(M)^{r}$ of collections of $r$ vector fields globally defined on $M$ that are $\mathcal{C}^{\kappa-1}$ for some $\kappa \geqslant 2$ to be chosen later. If $\mathbb{L}^{0}=\left\{L_{1}, L_{2}, \ldots, L_{r}\right\}$, is such a collection, its elements may be expressed in a local chart $\left(x_{1}, \ldots, x_{n}\right)$ as $L_{a}=\sum_{i=1}^{n} \varphi_{a, i}(x) \frac{\partial}{\partial x_{i}}$, for $a=1, \ldots, r$. Since the coefficients are $\mathcal{C}^{\kappa-1}$, it is possible to speak of $\mathbb{L}^{\lambda}$ only for $\lambda \leqslant \kappa$. We want to determine the smallest regularity $\kappa$ such that the set of $r$-tuples $\mathbb{L}^{0} \in \mathbb{X}(M)^{r}$ that are of finite type at every point of $M$ is open and dense in $\mathbb{X}(M)^{r}$ for the strong Whitney topology. 
As in $\S 1.8(\mathrm{II})$, let $\mathrm{n}_{\kappa}(r)$ denote the dimension of the subspace $\mathrm{F}_{\kappa}(r)$ of the free Lie algebra $\mathrm{F}(r)$ that is generated as a real vector space by simple words (abstract Lie brackets) of length $\leqslant \kappa$. Then $\mathrm{n}_{\kappa}(r)$ is the maximal possible dimension of $\mathbb{L}^{\kappa}(p)$ at a point $p \in M$. We know that $\mathbb{L}^{\kappa}$ is generated by simple iterated Lie brackets of the form

$$
\left[L_{a_{1}},\left[L_{a_{2}}, \ldots,\left[L_{a_{\kappa-1}}, L_{a_{\lambda}}\right] \ldots\right]\right],
$$

for all $\lambda \leqslant \kappa$ and for certain (not all) $a_{i}$ with $1 \leqslant a_{1}, a_{2}, \ldots, a_{\lambda-1}, a_{\lambda} \leqslant r$ that depend on the choice of a Hall-Witt basis (Definition 1.9(II)) of $F_{\kappa}(r)$.

We choose $\kappa$ minimal so that $\mathrm{n}_{\kappa}(r) \geqslant 2 \operatorname{dim} M=2 n$. This fixes the smoothness of $M$. For $b=r+1, \ldots, \mathrm{n}_{\kappa}(r)$, we order linearly as $L_{b}=\sum_{i=1}^{n} \psi_{b, i}(x) \frac{\partial}{\partial x_{i}}$ the chosen collection of iterated Lie brackets that generate $\mathbb{L}^{\kappa}$. If $\lambda=\lambda(b)$ denotes the length of $L_{b}$, namely $L_{b} \in \mathbb{L}^{\lambda(b)}$ of the form $L_{b}=\left[L_{a_{1}}, \ldots,\left[L_{a_{\lambda(b)-1}}, L_{a_{\lambda(b)}}\right] \ldots\right]$, there are universal differential polynomials $A_{a_{1}, \ldots, a_{\lambda(b)}}^{i}$ in the $(\lambda(b)-1)$-th jet of the coefficients $\varphi_{a, i}$ such that $\psi_{b, i}(x)=A_{a_{1}, \ldots, a_{\lambda(b)}}^{i}\left(J_{x}^{\lambda(b)-1} \varphi(x)\right)$. Also, in a fixed local system of coordinates, we form the $n \times(2 n)$ matrix

$$
\left(\varphi_{1, i} \ldots \varphi_{r, i} \psi_{r+1, i} \ldots \psi_{2 n, i}\right)_{1 \leqslant i \leqslant n} .
$$

Similarly as in the proof of the previous theorem, we define a "bad" subset $\Sigma$ of $J^{\kappa-1}\left(\mathbb{X}(M)^{r}\right)$ by requiring that the dimension of $\mathbb{L}^{\kappa}(p)$ is $\leqslant(n-1)$ at every point $p \in M$. This geometric condition is intrinsic and neither depends on the choice of local coordinates nor on the choice of a Hall-Witt basis. Concretely, in a local system of coordinates, $\Sigma$ is described as the zero-set of all $n \times n$ minors of the above matrix. Thanks to Lemma 2.13 and to an inspection of a portion of the explicit expressions of the jet polynomials $A_{a_{1}, \ldots, a_{\lambda(b)}}^{i}\left(J_{x}^{\lambda(b)-1} \varphi(x)\right)$, we may establish the following assertion.

Lemma 2.15. The so defined subset $\Sigma=\left\{\operatorname{dim} \mathbb{L}^{\kappa}(p) \leqslant n-1, \forall p \in\right.$ $M\}$ of $J^{\kappa-1}\left(\mathbb{X}(M)^{r}\right)$ is algebraic in the jet variables and of codimension $(n+1)$.

Then an application of the avoidance Lemma 2.12 yields that, after an arbitrarily small perturbation of $\mathbb{L}^{0}$, still denoted by $\mathbb{L}^{0}$, we have $\mathbb{L}^{\kappa}(p)=$ $T_{p} M$ for every $p \in M$. Equivalently, the type $d(p)$ of $p$ is finite at every point and satisfies $d(p) \leqslant \kappa$.

Theorem 2.16. Let $r \geqslant 2$ be an integer and assume that the connected $n$-dimensional abstract manifold $M$ is $\mathcal{C}^{\kappa}$, where $\kappa$ is minimal with the property that the dimension $\mathrm{n}_{\kappa}(r)$ of the vector subspace $\mathrm{F}_{\kappa}(r)$, of the free 
Lie algebra $\mathrm{F}(r)$ having $r$ generators, that is generated by all brackets of length $\leqslant \kappa$, satisfies

$$
\mathrm{n}_{\kappa}(r) \geqslant 2 \operatorname{dim} M=2 n \text {. }
$$

Then the set of collections of $r$ vector fields $\mathbb{L}^{0} \in \mathbb{X}(M)^{r}$ that are of type $\leqslant \kappa$ at every point is open and dense in $\mathbb{X}(M)^{r}$ for the strong Whitney topology.

A more general problem about finite-typisation of vector field structures is concerned with general substructures of a given finite type structure.

Open question 2.17. Given a finite type collection $\mathbb{K}^{0}=\left\{K_{b}\right\}_{1 \leqslant b \leqslant s}, s \geqslant$ 3 , of $\mathcal{C}^{\kappa-1}$ vector fields on $M$ of class $\mathcal{C}^{\kappa}$ with the property that $\mathbb{K}^{\kappa}(p)=$ $T_{p} M$ at every point and given a $\mathcal{C}^{\kappa-1}$ subsystem $\mathbb{L}^{0}=\left\{L_{a}\right\}_{1 \leqslant a \leqslant r}, 2 \leqslant r \leqslant$ $s-1$, of the form $L_{a}=\sum_{1 \leqslant b \leqslant s} \psi_{a, b} K_{b}$, is it always possible to perturb slightly the functions $\psi_{a, b}: M \rightarrow \mathbb{R}$ so as to render $\mathbb{L}^{0}$ of finite type at every point? If so, what is the smallest regularity $\kappa$, in terms of $r, s$ and the highest type of $\mathbb{K}^{0}$ at points of $M$ ?

Finally, we mention a result similar to Theorem 2.16 that is valid in the $\mathcal{C}^{2}$ category and does not use any Lie bracket. It is based on Sussmann's orbit Theorem 1.21. The reference [Su1976] deals with several other genericity properties, motivated by Control Theory.

Theorem 2.18. ([Su1976]) Assume $r \geqslant 2$ and $\kappa \geqslant 2$. The set of collections $\mathbb{L}^{0}=\left\{L_{a}\right\}_{1 \leqslant a \leqslant r}$ of $r$ vector fields on a connected $\mathcal{C}^{\kappa}$ manifold $M$ so that $M$ consists of a single $\mathbb{L}$-orbit, is open and dense in $\mathbb{X}(M)^{r}$ equipped with the strong Whitney $\mathcal{C}^{\kappa-1}$ topology.

2.19. Transition. The next Section 3 exposes the point of view of Analysis, where vector field systems are considered as partial differential operators, until we come back to the applications of the notion of orbits to CR geometry in Section 4.

\section{§3. LOCALLY INTEGRABLE CR STRUCTURES}

3.1. Local insolvability of partial differential equations. Until the 1950 's, among analysts, it was believed and expected that all linear partial differential equations having smooth coefficients had local solutions ([Trv2000]). In fact, elliptic, parabolic, hyperbolic and constant coefficient equations were known to be locally solvable. Although his thesis subject was to confirm this expectation in full generality, in 1957, Hans 
Lewy ([Lew1957]) exhibited a striking and now classical counterexample of a $\mathcal{C}^{\infty}$ function $g$ in a neighborhood of the origin of $\mathbb{R}^{3}$, such that $\bar{L} f=g$ has no local solution at all. Here, $\bar{L}=\frac{\partial}{\partial \bar{z}}+z \frac{\partial}{\partial v}$ is the generator of the Cauchy-Riemann anti-holomorphic bundle tangential to the Heisenberg sphere of equation $v=z \bar{z}$ in $\mathbb{C}^{2}$, equipped with coordinates $(z, w)=(x+i y, u+i v)$.

From the side of Analysis, almost absent in the two grounding works Po1907] and [Ca1932] of Henri Poincaré and of Élie Cartan, Lewy's discovery constituted the birth of smooth linear PDE theory and of smoooth Cauchy-Riemann geometry. Later, in 1971, the simpler two-variables Mizohata equation $\frac{\partial f}{\partial x}-i x^{k} \frac{\partial f}{\partial y}=g$ was shown by Grushin to be non-solvable, if $k$ is odd, for certain $g$. One may verify that the set of smooth functions $g$ for which Lewy's or Grushin's equation is insolvable, even in the distributional sense, is generic in the sense of Baire. For $k=1$, the Mizohata vector field $\frac{\partial}{\partial x}-i x \frac{\partial}{\partial y}$ intermixes the holomorphic and antiholomorphic structures, depending on the sign of $x$.

In 1973 answering a question of Lewy, Nirenberg ([Ni1973]) exhibited a perturbation $\frac{\partial}{\partial x}-i x(1+\varphi(x, y)) \frac{\partial}{\partial y}$ of the Mizohata vector field, where $\varphi$ is $\mathcal{C}^{\infty}$ and null for $x \leqslant 0$, such that the only local solutions of $L f=0$ are the constants. A year later, in [Ni1974], he exhibited a perturbation of the Lewy vector field having the same property. A refined version is as follows.

Let $\Omega$ be a domain in $\mathbb{R}^{3}$, exhausted by a countable family of compact sets $K_{j}, j=1,2, \ldots$ with $K_{j} \subset \operatorname{Int} K_{j+1}$. If $f \in \mathcal{C}^{\infty}(\Omega, \mathbb{C})$, define the Fréchet semi-norms $\rho_{j}(f):=\max _{x \in K_{j},|\alpha| \leqslant j}\left|\partial_{x}^{\alpha} f(x)\right|$ and topologize $\mathcal{C}^{\infty}(\Omega, \mathbb{C})$ by means of the metric $d(f, g):=\sum_{j=1}^{\infty} \frac{\rho_{j}(f-g)}{1+\rho_{j}(f-g)}$. Consider the set

$$
\widehat{\mathbf{L}}:=\left\{L=\sum_{j=1}^{3} a_{j}(x) \frac{\partial}{\partial x_{j}}: a_{j} \in \mathcal{C}^{\infty}(\Omega, \mathbb{C})\right\},
$$

equipped with this topology for each coefficient $a_{j}$.

Theorem 3.2. ([[]T1982, ]a1990]) The set of $L \in \widehat{\mathbf{L}}$ for which the solutions $u \in \mathcal{C}^{1}(\Omega, \mathbb{C})$ of $L u=0$ are the constants only, is dense in $\widehat{\mathbf{L}}$.

These phenomena and others were not suspected at the time of Lie, of Poincaré, of É. Cartan, of Vessiot and of Janet, when PDE theory was focused on the algebraic complexity of systems of differential equations having analytic coefficients. In 1959, Hörmander explained the behavior of the Lewy counter-example, as follows. The references [Trv1970, Trv1986, 
ES1993, Trv2000] provide further survey informations about operators of principal type, operators with multiple characteristics, pseudodifferential operators, hypoelliptic operators, microlocal analysis, etc.

Let $P=P\left(x, \partial_{x}\right)=\sum_{\alpha \in \mathbb{N}^{n},|\alpha| \leqslant m} a_{\alpha}(x) \partial_{x}^{\alpha}$ be a linear partial differential operator of degree $m$ having $\mathcal{C}^{\infty}$ complex-valued coefficients $a_{\alpha}: \Omega \rightarrow \mathbb{C}$ defined in a domain $\Omega \subset \mathbb{R}^{n}$. Its symbol $P(x, \xi):=$ $\sum_{|\alpha| \leqslant m} a_{\alpha}(x)(i \xi)^{\alpha}$ is a function from the cotangent $T^{*} \Omega \equiv \Omega \times \mathbb{R}^{n}$ to $\mathbb{C}$. Its principal symbol is the homogeneous degree $m$ part $P_{m}(x, \xi):=$ $\sum_{|\alpha|=m} a_{\alpha}(x)(i \xi)^{\alpha}$. The cone of points $(x, \xi) \in \Omega \times\left(\mathbb{R}^{n} \backslash\{0\}\right)$ such that $P_{m}(x, \xi)=0$ is the characteristic set of $P$, the locus of the obstructions to existence as well as to regularity of solutions $f$ of $P\left(x, \partial_{x}\right) f=g$.

The real characteristics of $P$ are called simple if, at every characteristic point $\left(x_{0}, \xi_{0}\right)$ with $\xi_{0} \neq 0$, the differential $d_{\xi} P_{m}=\sum_{k=1}^{n} \frac{\partial P_{m}}{\partial \xi_{k}} d \xi_{k}$ with respect to $\xi$ is nonzero. It follows from homogeneity and from Euler's identity that the zeros of $P$ are simple, so the characteristic set is a regular hypersurface of $\Omega \times\left(\mathbb{R}^{n} \backslash\{0\}\right)$. One can show that this assumption entails that the behaviour of $P$ is the same as that of $P_{m}$ : in a certain rigorous sense, lower order terms may be neglected. In his thesis (1955), Hörmander called such operators of principal type, a label that has stuck ([Trv1970]).

Call $P$ solvable at a point $x_{0} \in \Omega$ if there exists a neighborhood $U$ of $x_{0}$ such that for every $g \in \mathcal{C}^{\infty}(U)$, there exists a distribution $f$ supported in $U$ that satisfies $P f=g$ in $U$. In 1955, Hörmander had shown that a principal type partial differential operator $P$ is locally solvable if all the coefficients $a_{\alpha}(x),|\alpha|=m$, of its principal part $P_{m}$ are real-valued. On the contrary, if they are complex-valued, in 1959, he showed:

Theorem 3.3. ([Hö1963]) If the quantity

$$
\sum_{k=1}^{n} \frac{\overline{\partial P_{m}(x, \xi)}}{\partial \xi_{k}} \frac{\partial P_{m}(x, \xi)}{\partial x_{k}}
$$

is nonzero at a characteristic point $\left(x_{0}, \xi_{0}\right) \in T^{*} \Omega$, for some $\xi_{0} \neq 0$, then $P$ is insolvable at $x_{0}$.

With $\bar{P}_{m}\left(x, \partial_{x}\right):=\sum_{|\alpha|=m} \overline{a_{\alpha}(x)} \partial_{x}^{\alpha}$, denote by $C_{2 m-1}(x, \xi)$ the principal symbol of the commutator $\left[P_{m}\left(x, \partial_{x}\right), \bar{P}_{m}\left(x, \partial_{x}\right)\right]$, obviously zero if $P_{m}$ has real coefficients. The above necessary condition for local solvability may be rephrased as: if $P$ is locally solvable at $x_{0}$, then for all $\xi \in \mathbb{R}^{n} \backslash\{0\}:$

$$
P_{m}(x, \xi)=0 \Longrightarrow C_{2 m-1}(x, \xi)=0 .
$$


This condition explained the non-solvability of the Lewy operator appropriately.

3.4. Condition (P) of Nirenberg-Treves and local solvability. The geometric content of the above necessary condition was explored and generalized by Nirenberg-Treves ([NT1963, NT1970, Trv1970]). Recall that the Hamiltonian vector field associated to a function $f=f(x, \xi) \in \mathcal{C}^{1}\left(\Omega \times \mathbb{R}^{n}\right)$ is $H_{f}:=\sum_{k=1}^{n}\left(\frac{\partial f}{\partial \xi_{k}} \frac{\partial}{\partial x_{k}}-\frac{\partial f}{\partial x_{k}} \frac{\partial}{\partial \xi_{k}}\right)$. A bicharacteristic of the real part $A(x, \xi)$ of $P_{m}(x, \xi)$ is an integral curve of $H_{A}$, namely:

$$
\frac{d x}{d t}=\operatorname{grad}_{\xi} A(x, \xi), \quad \frac{d \xi}{d t}=-\operatorname{grad}_{x} A(x, \xi) .
$$

It follows at once that the function $A(x, \xi)$ must be constant along its bicharacteristics. When the constant is zero, a bicharacteristic is called a null bicharacteristic. In particular, null bicharacteristics are contained in the characteristic set, which explains the terminology.

Then Hörmander's necessary condition may be interpreted as follows. Let $B(x, \xi)$ be the imaginary part of $P_{m}(x, \xi)$. An immediate computation shows that the principal symbol of $\left[A\left(x, \partial_{x}\right), B\left(x, \partial_{x}\right)\right]$ is given by:

$$
C_{1}(x, \xi)=\sum_{k=1}^{n}\left\{\frac{\partial A}{\partial \xi_{k}}(x, \xi) \frac{\partial B}{\partial x_{k}}(x, \xi)-\frac{\partial B}{\partial \xi_{k}}(x, \xi) \frac{\partial A}{\partial x_{k}}(x, \xi)\right\} .
$$

Equivalently,

$$
C_{1}(x, \xi)=(d B / d t)(x, \xi) .
$$

Theorem 3.3 says that the nonvanishing of $C_{1}$ at a characteristic point entails insolvability. In fact, Nirenberg-Treves observed that if the order of vanishing of $B$ along the null characteristic of $A$ is odd then insolvability holds. Beyond finite order of vanishing, what appeared to matter is only the change of sign. Since the equation $P f=g$ has the same solvability properties as $z P f=g$, for all $z \in \mathbb{C} \backslash\{0\}$, this led to the following:

Definition 3.5. ([NT1963, NT1970]) A differential operator $P$ of principal type is said to satisfy condition (P) if, for every $z \in \mathbb{C} \backslash\{0\}$, the function $\operatorname{Im}\left(z P_{m}\right)$ does not change sign along the null bicharacteristic of $\operatorname{Re}\left(z P_{m}\right)$.

The next theorem has been shown for $P$ having $\mathcal{C}^{\omega}$ coefficients and in certain cases for $P$ having $\mathcal{C}^{\infty}$ coefficients by Nirenberg-Treves, and finally, in the general $\mathcal{C}^{\infty}$ category by Beals-Fefferman (sufficiency) and by Moyer (necessity). 
Theorem 3.6. ([NT1963, Trv1970, NT1970, BeFe1973, Trv1986]) Condition (P) is necessary and sufficient for the local solvability in $L^{2}$ of a principal type linear partial differential equation $\mathrm{P} f=g$.

Except for complex and strongly pseudoconvex structures, little is known about solvability of $\mathcal{C}^{\infty}$ systems of PDE's, especially overdetermined ones ([[Trv2000]). In the sequel, only vector field systems (order $m=1$ ), studied for themselves, will be considered.

3.7. Involutive and CR structures. Following [Trv1981, Trv1992, BCH2005, let $M$ be a $\mathcal{C}^{\omega}, \mathcal{C}^{\infty}$ or $\mathcal{C}^{\kappa, \alpha}(\kappa \geqslant 2,0<\alpha<1)$ paracompact Hausdorff second countable abstract real manifold of dimension $\mu \geqslant 1$ and let $\mathcal{L}$ be a $\mathcal{C}^{\omega}, \mathcal{C}^{\infty}$ or $\mathcal{C}^{\kappa-1, \alpha}$ complex vector subbundle of $\mathbb{C} T M:=\mathbb{C} \otimes T M$ of rank $\lambda$, with $1 \leqslant \lambda \leqslant \mu$. Denote by $\mathcal{L}_{p}$ its fiber at a point $p \in M$. Denote by $\mathcal{T}$ the orthogonal of $\mathcal{L}$ for the duality between differential forms and vector fields. It is a vector subbundle of $\mathbb{C} T^{*} M$, whose fiber at a point $p \in M$ is $\mathcal{L}_{p}^{\perp}=\left\{\varpi \in \mathbb{C} T_{p}^{*} M: \varpi=0\right.$ on $\left.\mathcal{L}_{p}\right\}$. The characteristic set $\mathcal{C}:=\mathcal{T} \cap T^{*} M$ (real $T^{*} M$ ) is in general not a vector bundle: the dimension of $\mathcal{C}_{p}^{0}$ may vary with $p$, as shown for instance by the bundle generated over $\mathbb{R}^{2}$ by the Mizohata operator $\partial_{x}-i x \partial_{y}$.

From now on, we shall assume that the bundle $\mathcal{L}$ is formally integrable, i.e. that $[\mathcal{L}, \mathcal{L}] \subset \mathcal{L}$. Then $\mathcal{L}$ defines:

- an elliptic structure if $\mathcal{C}_{p}=0$ for all $p \in M$;

- a complex structure of $\mathcal{L}_{p} \oplus \overline{\mathcal{L}_{p}}=\mathbb{C} T_{p} M$ for all $p \in M$;

- a Cauchy-Riemann (CR for short) structure if $\mathcal{L}_{p} \cap \overline{\mathcal{L}_{p}}=\{0\}$ for all $p \in M$;

- an essentially real structure if $\mathcal{L}_{p}=\overline{\mathcal{L}_{p}}$, for all $p \in M$.

In general, $\mathcal{L}$ will be called an involutive structure if $[\mathcal{L}, \mathcal{L}]=\mathcal{L}$. Let us summarize basic linear algebra properties ([Trv1981, Trv1992, BCH2005]). Every essentially real structure is locally generated by real vector fields. Every complex structure is elliptic. If $\mathcal{L}$ is a $\mathrm{CR}$ structure (often called abstract), the characteristic set $\mathcal{C}$ is in fact a vector subbundle of $T^{*} M$ of rank $\mu-2 \lambda$; this integer is the codimension of the CR structure. A CR structure is of hypersurface type if its codimension equals 1.

3.8. Local integrability and generic submanifolds of $\mathbb{C}^{n}$. The bundle $\mathcal{L}$ is locally integrable if every $p \in M$ has a neighborhood $U_{p}$ in which there exist $\tau:=\mu-\lambda$ functions $z_{1}, \ldots, z_{\tau}: U_{p} \rightarrow \mathbb{C}$ of class $\mathcal{C}^{\omega}, \mathcal{C}^{\infty}$ or $\mathcal{C}^{\kappa, \alpha}$ whose differentials $d z_{1}, \ldots, d z_{\tau}$ are linearly independent and span 
$\left.\mathcal{T}\right|_{U_{p}}$ (or equivalently, are annihilated by sections of $\mathcal{L}$ ). In other words, the homogeneous PDE system $\mathcal{L} f=0$ has the best possible space of solutions.

Here is a canonical example of locally integrable structure. Consider a generic submanifold $M$ of $\mathbb{C}^{n}$ of class $\mathcal{C}^{\omega}, \mathcal{C}^{\infty}$ or $\mathcal{C}^{\kappa, \alpha}, \kappa \geqslant 1,0 \leqslant \alpha \leqslant 1$, as defined in $\S 2.1(\mathrm{II})$ and in $\S 4.1$ below. Let $d \geqslant 0$ be its codimension and let $m=n-d \geqslant 0$ be its CR dimension. Let $T^{c} M=T M \cap J T M$ (a real vector bundle) and let $\mathbb{C} T M=\mathbb{C} \otimes T M$. Define the two complex subbundles $T^{1,0} M$ and $T^{0,1} M=\overline{T^{1,0} M}$ of $\mathbb{C} T M$ whose fibers at a point $p \in M$ are:

$$
\left\{\begin{array}{l}
T_{p}^{1,0} M=\left\{X_{p}+i J X_{p}: X_{p} \in T_{p}^{c} M\right\}=\left\{Z_{p} \in \mathbb{C} T_{p} M: J Z_{p}=-i Z_{p}\right\}, \\
T_{p}^{0,1} M=\left\{X_{p}-i J X_{p}: X_{p} \in T_{p}^{c} M\right\}=\left\{Z_{p} \in \mathbb{C} T_{p} M: J Z_{p}=i Z_{p}\right\} .
\end{array}\right.
$$

Geometrically, $T^{1,0} M$ and $T^{0,1} M$ are just the traces on $M$ of the holomorphic and anti-holomorphic bundles $T^{1,0} \mathbb{C}^{n}$ and $T^{0,1} \mathbb{C}^{n}$, whose fibers at a point $p$ are $\left.\sum_{k=1}^{n} a_{k} \frac{\partial}{\partial z_{k}}\right|_{p}$ and $\left.\sum_{k=1}^{n} b_{k} \frac{\partial}{\partial \bar{z}_{k}}\right|_{p}$. They satisfy the Frobenius involutivity conditions $\left[T^{1,0} M, T^{1,0} M\right] \subset T^{1,0} M$ and $\left[T^{0,1} M, T^{0,1} M\right] \subset$ $T^{0,1} M$. More detailed background information may be found in [Ch1991, Bo1991, Trv1992, BER1999.

On such an embedded generic submanifold $M$, choose as structure bundle $\mathcal{L}$ just $T^{0,1} M \subset \mathbb{C} T M$. Then clearly, the $n$ holomorphic coordinate functions $z_{1}, \ldots, z_{n}$ are annihilated by the anti-holomorphic local sections $\sum_{k=1}^{n} b_{k} \frac{\partial}{\partial \bar{z}_{k}}$ of $T^{0,1} M$ and they have linearly independent differential, at every point of $M$. A generic submanifold embedded in $\mathbb{C}^{n}$ carries a locally integrable involutive structure. Conversely:

Lemma 3.9. Every locally integrable CR structure is locally realizable as the anti-holomorphic structure induced on a generic submanifold embed$\operatorname{ded} \mathbb{C}^{n}$.

Proof. Indeed, if a real $\mu$-dimensional $\mathcal{C}^{\omega}, \mathcal{C}^{\infty}$ or $\mathcal{C}^{\kappa, \alpha}$ manifold $M$ bears a locally integrable $\mathrm{CR}$ structure, the map $Z=\left(z_{1}, \ldots, z_{\tau}\right)$ produces an embedding of the open set $U_{p}$ as a local generic submanifold $M:=Z\left(U_{p}\right)$ of $\mathbb{C}^{\tau}$, with $Z_{*}(\mathcal{L})=T^{0,1} M$.

A locally integrable $\mathrm{CR}$ structure is sometimes called locally realizable or locally embeddable.

3.10. Levi form. Let $\mathcal{L}$ be an involutive structure, not necessarily locally integrable and let $\mathrm{c}_{p} \in \mathcal{C}_{p} \subset T_{p}^{*} M$ be a nonzero characteristic covector at $p$. 
Definition 3.11. The Levi form at $p$ in the characteristic codirection $\mathrm{c}_{p} \in$ $\mathcal{C}_{p} \backslash\{0\} \subset T_{p}^{*} M \backslash\{0\}$ is the Hermitian form acting on two vectors $X_{p}, Y_{p} \in$ $\mathcal{L}(p)$ as:

$$
\mathfrak{L}_{p, c_{p}}\left(X_{p}, \bar{Y}_{p}\right):=\frac{1}{2 i} \mathrm{c}_{p}([X, \bar{Y}]),
$$

where $X, Y$ are any two sections of $\mathcal{L}$ defined in a neighborhood of $p$ and satisfying $X(p)=X_{p}, Y(p)=Y_{p}$. The resulting number is independent of the choice of such extensions $X, Y$.

For the study of realizability of CR structures of codimension one, nondegeneracy of the Levi form, especially positivity or negativity, is of crucial importance. An abstract CR structure of hypersurface type whose Levi form has a definite signe is said to be strongly pseudoconvex, since, after a possible rescaling of sign of a nonzero characteristic covector, all the eigenvalues of its Levi form are positive.

3.12. Nonembeddable CR structures. After Lewy's discovery, the first example of a smooth strictly pseudoconvex CR structure in real dimension 3 which is not locally embeddable was produced by Nirenberg in 1973 (Ni1973]), $c f$. Theorem 3.3 above. For CR structures of hypersurface type, Nirenberg's work has been generalized in higher dimension under the assumption that the Levi form is neither positive nor negative, in any characteristic codirection. Let $n \geqslant 2$ and let $\varepsilon_{1}=1, \varepsilon_{k}=-2, k=2, \ldots, n$.

Theorem 3.13. ([JT1982, BCH2005]) There exists a $\mathcal{C}^{\infty}$ complex-valued function $g=g(x, y, s)$ defined in a neighborhood of the origin in $\mathbb{C}^{n} \times \mathbb{R}$ and vanishing to infinite order along $\left\{x_{1}=y_{1}=0\right\}$ such that the vector fields:

$$
\widehat{L}_{j}:=\frac{\partial}{\partial \bar{z}_{j}}-i \varepsilon_{j} z_{j}(1+g(x, y, s)) \frac{\partial}{\partial s},
$$

are pairwise commuting and such that every $\mathcal{C}^{1}$ solution $h$ of $\widehat{L}_{j} h=0, j=$ $1,2, \ldots, n$ defined in a neighborhood of the origin must satisfy $\frac{\partial h}{\partial s}(0)=0$.

This entails that the involutive structure spanned by the $\widehat{L}_{j}$ is not locally integrable at 0 . One may establish that the set of such $g$ is generic. Crucially, the Levi-form is of signature $(n-1)$.

Open problem 3.14. Find versions of generic non-embeddability for $C R$ structures of codimension 1 having degenerate Levi-form. Find higher codimensional versions of generic non-embeddability. 
3.15. Integrability of complex structures and embeddability of strongly pseudoconvex CR structures. Let us now expose positive results. Every formally integrable essentially real structure $\mathcal{L}=\operatorname{Re} \mathcal{L}$ is locally integrable, thanks to Frobenius' theorem; however the condition $[\mathcal{L}, \mathcal{L}] \subset \mathcal{L}$ entails the involutivity of $\operatorname{Re} \mathcal{L}$ only in this special case. Also, every analytic formally integrable $\mathrm{CR}$ structure is locally integrable: it suffices to complexify the coefficients of a generating set of vector fields and to apply the holomorphic version of Frobenius' theorem. For complex structure, the proof is due to Libermann (1950) and to Eckman-Frölicher (1951), see [AH1972a, Trv1981, Trv1992].

Theorem 3.16. Smooth complex structures are locally integrable.

This deeper fact has a long history, which we shall review concisely. On real analytic surfaces, isothermal coordinates where discovered by Gauss in 1825-26, before he published his Disquisitiones generales circa superficies curvas. In the 1910's, by a nontrivial advance, Korn and Lichtenstein transferred this theorem to Hölder continuous metrics.

Theorem 3.17. Let $d s^{2}=E d u^{2}+2 F d u d v+G d v^{2}$ be a $\mathcal{C}^{0, \alpha}(0<\alpha<1)$ Gaussian metric defined in some neighborhood of 0 in $\mathbb{R}^{2}$. Then there exists a $\mathcal{C}^{1, \alpha}$ change of coordinates $(u, v) \mapsto(\tilde{u}, \tilde{v})$ fixing 0 and a $\mathcal{C}^{0, \alpha}$ function $\tilde{\lambda}=\tilde{\lambda}(\tilde{u}, \tilde{v})$ such that:

$$
\tilde{\lambda}\left(d \tilde{u}^{2}+d \tilde{v}^{2}\right)=E d u^{2}+2 F d u d v+G d v^{2} .
$$

A modern proof of this theorem based on the complex notation and on the $\bar{\partial}$ formalism was provided by Bers ([Be1957]) and by Chern ([Ch1955]). In the monograph [Ve1962], deeper weakenings of smoothness assumptions are provided.

As a consequence of this theorem, complex structures of class $\mathcal{C}^{0, \alpha}$ on surfaces may be shown to be locally integrable. Let us explain in length this corollary.

At first, remind that an almost complex structure on $2 n$-dimensional manifold $M$ is a smoothly varying field $J=\left(J_{p}\right)_{p \in M}$ of endomorphisms of $T_{p} M$ satisfying $J_{p} \circ J_{p}=-\mathrm{Id}$. Thanks to $J$, as in the standard complex case, one may define $T_{p}^{0,1} M:=\left\{X_{p}+i J_{p} X_{p}: X_{p} \in T_{p} M\right\}$ and then the bundle $\mathcal{L}:=T^{0,1} M$ is a complex structure in the PDE sense of $\S 3.7$ above. Conversely, given a complex structure $\mathcal{L} \subset \mathbb{C} T M$, then locally in some neighborhood $U_{p}$ of an arbitrary point $p \in M$, there exist local coordinates $\left(x_{1}, \ldots, x_{n}, y_{1}, \ldots, y_{n}\right)$ vanishing at $p$ so that $n$ complex vector fields of 
the form:

$$
Z_{j}:=\sum_{k=1}^{n} a_{k, j} \partial_{x_{k}}+i \sum_{k=1}^{n} b_{k, j} \partial_{y_{k}},
$$

with $a_{k, j}(0)=\delta_{k, j}=b_{k, j}(0)$, span $\left.\mathcal{L}\right|_{U_{p}}$. The associated almost complex structure is obtained by declaring that, at a point of coordinates $(x, y)$, one has:

$$
J\left(\sum_{k=1}^{n} a_{k, j} \partial_{x_{k}}\right)=\sum_{k=1}^{n} b_{k, j} \partial_{y_{k}} \text { and } J\left(\sum_{k=1}^{n} b_{k, j} \partial_{y_{k}}\right)=-\sum_{k=1}^{n} a_{k, j} \partial_{x_{k}} .
$$

Lemma 3.18. The bundle $\mathcal{L} \subset \mathbb{C} T M$ satisfies $[\mathcal{L}, \mathcal{L}] \subset \mathcal{L}$ if and only if, for every two vector fields $X$ and $Y$ on $M$, the Nijenhuis expression:

$$
N(X, Y):=[J X, J Y]-J[X, J Y]-J[J X, Y]-[X, Y]
$$

vanishes identically.

The proof is abstract nonsense. Also, one verifies that $N(f X, g Y)=$ $f g N(X, Y)$ for every two smooth local function $f$ and $g$ : the expression $N$ is of tensorial character. In symplectic and in almost complex geometry ([MS1995]), the following is settled.

Definition 3.19. The almost complex structure is called integrable if, in some neighborhood $U_{p}$ of every point $p \in M$ there exist $n$ complex-valued functions $z_{1}, \ldots, z_{n}: U_{p} \rightarrow \mathbb{C}$ of class at least $\mathcal{C}^{1}$ and having linearly independent differentials such that $d z_{k} \circ J=i \circ d z_{k}$, for $k=1, \ldots, n$.

One verifies that it is equivalent to require $\mathcal{L} z_{k}=0, k=1, \ldots, n$ : integrability of an almost complex structure coincides with local integrability of $\mathcal{L}=T^{0,1} M$.

Now, we may come back to the integrability Theorem 3.16. To an arbitrary Gaussian metric $g=d s^{2}$ as in Theorem 3.17, with $E>0, G>0$ and $E G-F^{2}>0$, are associated both a volume form and an almost complex structure:

$$
d \operatorname{vol}_{g}:=\sqrt{E G-F^{2}} d u \wedge d v \text { and } J_{g}:=\frac{1}{\sqrt{E G-F^{2}}}\left(\begin{array}{cc}
-F & -G \\
E & F
\end{array}\right) .
$$

Conversely, given a volume form and an almost complex structure $J$ on a surface, an associated Riemannian metric is provided by:

$$
g(\cdot, \cdot):=d \operatorname{vol}(\cdot, J \cdot)
$$

According to Korn's and Lichtenstein's theorem, there exist coordinates in which the metric is conformally flat, equal to $\lambda\left(d u^{2}+d v^{2}\right)$. In these 
coordinates, the associated complex structure is obviously the standard one: $J \partial_{u}=\partial_{v}$ and $J \partial_{v}=-\partial_{u}$. In fact, any local change of coordinates $(u, v) \mapsto(\tilde{u}, \tilde{v})$ which respects orthogonality of the curvilinear coordinates, i.e. transforms the Gaussian isothermal metric to a similar one $\tilde{\lambda}\left(d \tilde{u}^{2}+d \tilde{v}^{2}\right)$, commutes with $J$, so that the map $u+i v \mapsto \tilde{u}+i \tilde{v}$ is holomorphic. In conclusion:

Theorem 3.20. $\mathcal{C}^{0, \alpha}(0<\alpha<1)$ complex structures are locally integrable.

The generalization to several variables of the theorem of Korn and Lichtenstein is due to Newlander-Nirenberg, who solved a question raised by Chern. The proof was modified and the smoothness assumption was perfected by several mathematicians: Nijenhuis-Woolf ([NW1963]), Malgrange, Kohn, Hörmander, Nirenberg, Treves ([Trv1992]) and finally Webster ([We1989g] $)$ who used the Leray-Koppelman $\bar{\partial}$ homotopy formula together with the Nash-Moser rapidly convergent iteration scheme for solving nonlinear functional equations.

Theorem 3.21. $\left(\mathcal{C}^{2 n, \alpha}, 0<\alpha<1\right.$ : [NN1957]; $\mathcal{C}^{1, \alpha}, 0<\alpha<1$ : [NW1963, We1989d; $\mathcal{C}^{\infty}$ : [Trv1992]) Suppose that on the real manifold $M$ of dimension $2 n \geqslant 4$, the formally integrable complex structure $\mathcal{L}$ is $\mathcal{C}^{\infty}$ or $\mathcal{C}^{\kappa-1, \alpha}, \kappa \geqslant 2,0<\alpha<1$. Then there exist local complex-valued coordinates $\left(z_{1}, \ldots, z_{n}\right)$ annihilated by $\mathcal{L}$ which are $\mathcal{C}^{\infty}$ or $\mathcal{C}^{\kappa, \alpha}$.

Finally, an elementary linear algebra argument ([Trv1981, Trv1992, BCH2005]) enables to deduce local integrability of $\mathcal{C}^{\infty}$ or $\mathcal{C}^{\kappa-1, \alpha}$ elliptic structures from the above theorem. In fact, elliptic structures are shown to be locally isomorphic to $\mathbb{C}^{\tau} \times \mathbb{R}^{\lambda-\tau}$, equipped with $\frac{\partial}{\partial \bar{z}_{i}}, \frac{\partial}{\partial t_{j}}$.

Problem 3.22. Is a formally integrable involutive structure having positive-dimensional characteristic set locally integrable?

Again the history is rich. Integrability results are known only for strongly pseudoconvex CR structures of hypersurface type. Solving a question raised by Kohn in 1965, Kuranishi ([Ku1982]) showed in 1982 that $\mathcal{C}^{\infty}$ strongly pseudoconvex abstract CR structures of dimension $\geqslant 9$ are locally realizable. His delicate proof involved a study of the Neumann operator in $L^{2}$ spaces, for solving the tangential Cauchy-Riemann equations, together with the Nash-Moser argument. In 1987, Akahori ([Ak1987]) modified the technique of Kuranishi and included the case of dimension 7. 
In 1989, to solve an associated linearized problem, instead of the Neumann operator, Webster used the totally explicit integral operators of Henkin.

Theorem 3.23. ([We1989a, We1989b] Let $M$ be a strongly pseudoconvex $(2 n-1)$-dimensional CR manifold of class $\mathcal{C}^{\mu}$. Then $M$ admit, locally near each point, a holomorphic embedding of class $\mathcal{C}^{\kappa}$, provided

$$
n \geqslant 4, \quad \kappa \geqslant 21, \quad \mu \geqslant 6 \kappa+5 n-3 .
$$

The main new ingredient in his proof was Henkin' local homotopy operator $\bar{\partial}_{M}$ on a hypersurface $M \subset \mathbb{C}^{n}$ :

$$
f=\bar{\partial}_{M} P(f)+Q\left(\bar{\partial}_{M} f\right), \quad f \text { a }(0,1)-\text { form, }
$$

known to hold for $n \geqslant 4$. For this reason, Webster suspected the existence of refinements based on an insider knowledge of $\bar{\partial}$ techniques. In 1994, using a modified homotopy formula yielding better $\mathcal{C}^{\kappa}$-estimates, Ma-Michel [MM1994] improved smoothness:

$$
\kappa \geqslant 18, \quad \mu \geqslant \kappa+13 .
$$

Up to now, the five dimensional remains open. In fact, the solvability of $\bar{\partial}_{M} f=g$ for a $(0,1)$-form on a hypersurface of $\mathbb{C}^{3}$ requires a special trick which does not lead to a homotopy formula. Nagel-Rosay [NR1989] showed the nonexistence of a homotopy formula in the 5-dimensional case, emphasizing an obstacle.

Open problem 3.24. Find generalizations of the Kuranishi-AkahoriWebster-Ma-Michel theorem to higher codimension, using the integral formulas for solving the $\bar{\partial}_{M}$ due to Ayrapetian-Henkin. Replace the assumption of strong pseudoconvexity by finer nondegeneracy conditions, e.g. weak pseudoconvexity and finite type in the sense of Kohn.

3.25. Local generators of locally integrable structures. Abandoning these deep problems of local solvability and of local realizability, let us survey basic properties of locally integrable structures. Thus, let $\mathcal{L}$ be a $\mathcal{C}^{\infty}$ or $\mathcal{C}^{\kappa-1, \alpha}$ locally integrable structure of rank $\lambda$ on a $\mathcal{C}^{\kappa, \alpha}$ or $\mathcal{C}^{\infty}$ manifold $M$ of dimension $\mu$. Denote by $\tau=\mu-\lambda$ the dimension of $\mathcal{T}=\mathcal{L}^{\perp}$. Let $p \in M$ and let $\delta_{p}$ denote the dimension of $\mathcal{C}_{p}=\mathcal{T} \cap T_{p}^{*} M$. Notice that $\left(\tau-\delta_{p}\right)+\left(\tau-\delta_{p}\right)+\delta_{p}+\left(\lambda-\tau+\delta_{p}\right)=\tau+\lambda=\mu$ just below.

Theorem 3.26. ([Trv1981, Trv1992, BCH2005]]) There exist real coordinates:

$$
\left(x_{1}, \ldots, x_{\tau-\delta_{p}}, y_{1}, \ldots, y_{\tau-\delta_{p}}, u_{1}, \ldots, u_{\delta_{p}}, s_{1}, \ldots, s_{\lambda-\tau+\delta_{p}}\right),
$$


defined in a neighborhood $U_{p}$ of $p$ and vanishing at $p$, and there exist $\mathcal{C}^{\infty}$ or $\mathcal{C}^{\kappa, \alpha}$ functions $\varphi_{j}=\varphi_{j}(x, y, u, s)$ with $\varphi_{j}(0)=0, d \varphi_{j}(0)=0, j=$ $1, \ldots, \delta_{p}$, such that the differentials of the $\tau$ functions:

$$
\left\{\begin{aligned}
z_{k}:=x_{k}+i y_{k}, \quad k=1, \ldots, \tau-\delta_{p}, \\
w_{j}:=u_{j}+i \varphi_{j}(x, y, u, s), \quad j=1, \ldots, \delta_{p}
\end{aligned}\right.
$$

$\left.\operatorname{span} \mathcal{T}\right|_{U_{p}}$

Since $d \varphi_{j}(0)=0$, there exist unique coefficients $b_{l, j}=b_{l, j}(x, y, u, s)$ such that the vector fields:

$$
K_{j}:=\sum_{l=1}^{\delta_{p}} b_{l, j} \frac{\partial}{\partial u_{l}}, \quad k=1, \ldots, \delta_{p},
$$

satisfy $K_{j_{1}}\left(w_{j_{2}}\right)=\delta_{j_{1}, j_{2}}$, for $j_{1}, j_{2}=1, \ldots, \delta_{p}$. Define then the $\lambda$ vector fields:

$$
\left\{\begin{array}{rlrl}
\bar{L}_{k}:=\frac{\partial}{\partial \bar{z}_{k}}-i \sum_{l=1}^{\delta_{p}} \frac{\partial \varphi_{l}}{\partial \bar{z}_{k}} K_{l}, & & k=1, \ldots, \tau-\delta_{p}, \\
L_{j}^{\prime}:=\frac{\partial}{\partial s_{j}}-i \sum_{l=1}^{\delta_{p}} \frac{\partial \varphi_{l}}{\partial s_{j}} K_{l}, & j=1, \ldots, \lambda-\tau+\delta_{p} .
\end{array}\right.
$$

Clearly, $0=\bar{L}_{k_{1}}\left(z_{k_{2}}\right)=\bar{L}_{k}\left(w_{j}\right)=L_{j}^{\prime}\left(z_{k}\right)=L_{j_{1}}^{\prime}\left(w_{j_{2}}\right)$, hence the structure bundle $\left.\mathcal{L}\right|_{U_{p}}$ is spanned by the $L_{k}, L_{j}^{\prime}$. One may verify the commutation relations ([[Trv1981, Trv1992, BCH2005]):

$$
\begin{aligned}
& 0=\left[\bar{L}_{k_{1}}, \bar{L}_{k_{2}}\right]=\left[\bar{L}_{k}, L_{j}^{\prime}\right]=\left[L_{j_{1}}^{\prime}, L_{j_{2}}^{\prime}\right], \\
& 0=\left[\bar{L}_{k}, K_{j}\right]=\left[L_{j_{1}}^{\prime}, K_{j_{2}}\right]=\left[K_{j_{1}}, K_{j_{2}}\right] .
\end{aligned}
$$

Remind that if an involutive structure $\mathcal{L}$ is $\mathrm{CR}$, then $\delta_{p}$ is independent of $p$ and equal to the codimension $\mu-2 \lambda=: \delta$. It follows that $\tau-\delta=\tau-\mu+$ $2 \lambda=\lambda$, or $\lambda-\tau+\delta=0$ : this means that the variables $\left(s_{1}, \ldots, s_{\lambda-\tau+\delta_{p}}\right)$ disappear.

Corollary 3.27. In the case of a CR structure of codimension $\delta$, the local integrals are:

$$
\left\{\begin{array}{c}
z_{k}:=x_{k}+i y_{k}, \quad k=1, \ldots, \lambda \\
w_{j}:=u_{j}+i \varphi_{j}(x, y, u), \quad j=1, \ldots, \delta
\end{array}\right.
$$


and a local basis for the structure bundle $\left.\mathcal{L}\right|_{U_{p}}$ is:

$$
\bar{L}_{k}:=\frac{\partial}{\partial \bar{z}_{k}}-i \sum_{l=1}^{\delta} \frac{\partial \varphi_{l}}{\partial \bar{z}_{k}} K_{l}, \quad k=1, \ldots, \lambda .
$$

We recover a generic submanifold embedded in $\mathbb{C}^{\tau}$ which is graphed by the equations $v_{j}=\varphi_{j}(x, y, u)$, as in Theorem 2.3(II), or as in Theorem 4.2 below.

3.28. Local embedding into a CR structure. But in general, the coordinates $\left(s_{1}, \ldots, s_{\lambda-\tau+\delta_{p}}\right)$ are present. A trick ([Ma1992]) is to introduce extra coordinates $\left(t_{1}, \ldots, t_{\lambda-\tau+\delta_{p}}\right)$ and to define a new structure on the product $U_{p} \times \mathbb{R}^{\lambda-\tau+\delta_{p}}$ generated by the following local integrals:

$$
\left\{\begin{aligned}
\tilde{z}_{k}:=z_{k}, & & k=1, \ldots, \tau-\delta_{p}, \\
\tilde{z}_{k}:=s_{k-\tau+\delta_{p}}+i t_{k-\tau+\delta_{p}}, & & k=\tau-\delta_{p}+1, \ldots, \lambda, \\
\tilde{w}_{j}:=w_{j}, & & j=1, \ldots, \delta_{p} .
\end{aligned}\right.
$$

The associated structure bundle $\widetilde{\mathcal{L}}$ is spanned by:

$$
\left\{\begin{array}{l}
\overline{\widetilde{L}}_{k}:=\bar{L}_{k}, \quad k=1, \ldots, \tau-\delta_{p}, \\
\overrightarrow{\widetilde{L}}_{j}^{\prime}:=\frac{1}{2} L_{j}^{\prime}+\frac{i}{2} \frac{\partial}{\partial t_{j}}, \quad j=1, \ldots, \lambda-\tau+\delta_{p} .
\end{array}\right.
$$

It is a CR structure of codimension $\delta_{p}$ on $U_{p} \times \mathbb{R}^{\lambda-\tau+\delta_{p}}$. All analyticogeometric objects defined in $U_{p}$ can be lifted to $U_{p} \times \mathbb{R}^{\lambda-\tau+\delta_{p}}$, just by declaring them to be independent of the extra variables $\left(t_{1}, \ldots, t_{\lambda-\tau+\delta_{p}}\right)$.

Such an embedding enables one to transfer elementarily several theorems valid in embedded Cauchy-Riemann Geometry, to the more general setting of locally integrable structures. For instance, this is true of most of the theorems about holomorphic or CR extension of CR functions presented Part V. In addition, most of the results stated in $\S 3, \S 4$ and $\S 5$ below hold in locally integrable structures.

3.29. Transition. However, for reasons of space and because the possible generalizations which we could state by applying this embedding trick would require dry technical details, we will content ourselves to just mention these virtual generalizations, as was done in [MP1999]. For further study of locally integrable structures, we refer mainly to [Trv1992, BCH2005] and to the references therein.

In summary, in this Section 3, we wanted to show how our approach is inserted into a broad architecture of questions about solvability of partial 
differential equations, about the problem of realizability of abstract CR structures, as well as into hypo-analytic structures. Thus, even if some of the subsequent surveyed results (exempli gratia: the celebrated BaouendiTreves approximation Theorem 5.2) were originally stated in the context of locally integrable structures, even though we could as well state them in this context or at least in the context of locally embeddable CR structures (as was done in [MP1999]), we shall content ourselves to state them in the context of embedded Cauchy-Riemann geometry, just because the very core of the present memoir is concerned by Several Complex Variables topics: analytic discs, envelopes of holomorphy, removable singularities, etc.

\section{$\S 4$. SMOOTH GENERIC SUBMANIFOLDS AND THEIR CR ORBITS}

4.1. Definitions of CR submanifolds and local graphing equations. We begin by some coordinate-invariant geometric definitions. Some implicit lemmas are involved (the reader is referred to [Ch1989, Ch199], Bo199], BER1999). Let $J$ denote the complex structure of $T \mathbb{C}^{n}$ (see $\left.\S 2.1(\mathrm{II})\right)$. A real connected submanifold $M \subset \mathbb{C}^{n}$ of class at least $\mathcal{C}^{1}$ is called:

- Totally real if $T_{p} M \cap J T_{p} M=\{0\}$ for every $p \in M$. Then $M$ has codimension $d \geqslant n$ and is called maximally real if $d=n$. The complex vector subspace $H_{p}:=T_{p} M+J T_{p} M$ of $T_{p} \mathbb{C}^{n}$ has complex dimension $2 n-d$. If $\operatorname{proj}_{H_{p}}(\cdot)$ denotes any $\mathbb{C}$-linear projection of $T_{p} \mathbb{C}^{n}$ onto $H_{p}$ and if $\mathcal{U}_{p}$ is a small neighborhood of $p$ in $\mathbb{C}^{n}$, then $\operatorname{proj}_{H_{p}}\left(M \cap \mathcal{U}_{p}\right)$ is maximally real in $H_{p}$.

- Generic if $T_{p} M+J T_{p} M=T_{p} \mathbb{C}^{n}$ for every $p \in M$. Then $M$ has codimension $d \leqslant n$ and is maximally real only if $d=n$. Then $T_{p} M \cap J T_{p} M$ is the maximal $\mathbb{C}$-linear subspace of $T_{p} M$ and has complex dimension equal to the integer $m:=n-d$, called the $C R$ dimension of $M$. It is obviously constant, as $p$ runs in $M$.

- Cauchy-Riemann (CR for short) if the maximal $\mathbb{C}$-linear subspace $T_{p} M \cap J T_{p} M$ of $T_{p} M$ has constant dimension $m$ (necessarily $\leqslant n$ ) for $p$ running in $M$. If $M$ has codimension $d$, the integer $c:=$ $d-n+m$ is called the holomorphic codimension of $M$. Then for $p$ running in $M$, the complex vector subspaces $H_{p}:=T_{p} M+J T_{p} M$ of $T_{p} \mathbb{C}^{n}$ have constant complex codimension $c$, which justifies the terminology. If $\operatorname{proj}_{H_{p}}(\cdot)$ denotes any $\mathbb{C}$-linear projection of $T_{p} \mathbb{C}^{n}$ onto $H_{p}$, and if $\mathcal{U}_{p}$ is a small open neighborhood of $p$ in $\mathbb{C}^{n}$, then

$$
\widetilde{M}_{p}:=\operatorname{proj}_{H_{p}}\left(M \cap \mathcal{U}_{p}\right)
$$


is a generic submanifold of $\mathbb{C}^{n-c}$.

In $\S 2.1($ II), we have graphed totally real, generic and, generally, CauchyRiemann local submanifolds $M \subset \mathbb{C}^{n}$, but only in the algebraic and in the analytic category. In the smooth category, the intrinsic complexification $\left\{w_{2}=0\right\}$ disappears, but $H_{p}=T_{p} M+J T_{p} M$ still exists, so that further graphing functions are needed.

Theorem 4.2. ([Ch1989, Ch1991, Bo1991, BER1999, Me2004a]) Let $M \subset \mathbb{C}^{n}$ be a real submanifold of codimension d and let $p \in M$. There exist complex algebraic or analytic coordinates centered at $p$ and $\rho_{1}>0$ such that $M$, supposed to be $\mathcal{C}^{\mathcal{R}}$, where $\mathcal{R}=\infty$ or where $\mathcal{R}=(\kappa, \alpha)$, $\kappa \geqslant 1,0 \leqslant \alpha \leqslant 1$, is locally represented as follows:

- If $M$ is totally real, letting $d_{1}=2 n-d \geqslant 0$ and $c=d-n \geqslant 0$, then $d_{1}+c=n$ and

$$
\begin{aligned}
M=\left\{\left(w_{1}, w_{2}\right) \in\right. & \left(\square_{\rho_{1}}^{d_{1}} \times i \mathbb{R}^{d_{1}}\right) \times \mathbb{C}^{c}: \\
& \left.\operatorname{Im} w_{1}=\varphi_{1}\left(\operatorname{Re} w_{1}\right), w_{2}=\psi_{2}\left(\operatorname{Re} w_{1}\right)\right\},
\end{aligned}
$$

for some $\mathbb{R}^{d_{1}}$-valued $\mathcal{C}^{\mathcal{R}}$ map $\varphi_{1}$ and some $\mathbb{C}^{c}$-valued $\mathcal{C}^{\mathcal{R}}$ map $\psi_{2}$ satisfying $\varphi_{1}(0)=0$ and $\psi_{2}(0)=0$.

- If $M$ is generic, letting $m=d-n$, then $m+d=n$ and

$$
M=\left\{(z, w) \in \Delta_{\rho_{1}}^{m} \times\left(\square_{\rho_{1}}^{d} \times i \mathbb{R}^{d}\right): \operatorname{Im} w=\varphi(\operatorname{Re} z, \operatorname{Im} z, \operatorname{Re} w)\right\},
$$$$
\text { for some } \mathbb{R}^{d} \text {-valued } \mathcal{C}^{\mathcal{R}} \text { map } \varphi \text { satisfying } \varphi(0)=0 \text {. }
$$

- If $M$ is Cauchy-Riemann, letting $m=\operatorname{CRdim} M, c=d-n+m \geqslant$ 0 , and $d_{1}=2 n-2 m-d \geqslant 0$, then $m+d_{1}+c=n$ and

$$
\begin{aligned}
M= & \left\{\left(z, w_{1}, w_{2}\right) \in \Delta_{\rho_{1}}^{m} \times\left(\square_{\rho_{1}}^{d_{1}} \times i \mathbb{R}^{d_{1}}\right) \times \mathbb{C}^{c}:\right. \\
& \left.\operatorname{Im} w_{1}=\varphi_{1}\left(\operatorname{Re} z, \operatorname{Im} z, \operatorname{Re} w_{1}\right), w_{2}=\psi_{2}\left(\operatorname{Re} z, \operatorname{Im} z, \operatorname{Re} w_{1}\right)\right\}, \\
& \text { for some } \mathbb{R}^{d_{1}} \text {-valued } \mathcal{C}^{\mathcal{R}} \text { map } \varphi_{1} \text { with } \varphi_{1}(0)=0 \text { and some } \\
& \mathbb{C}^{c} \text {-valued } \mathcal{C}^{\mathcal{R}} \text { map } \psi_{2} \text { with } \psi_{2}(0)=0 \text { which is } C R \text { (defini- } \\
& \text { tion in } \S 4.25 \text { below) on the local generic submanifold } M^{1}:= \\
& \left\{\left(z, w_{1}\right) \in \Delta_{\rho_{1}}^{m} \times\left(\square_{\rho_{1}}^{d_{1}} \times i \mathbb{R}^{d_{1}}\right): \operatorname{Im} w_{1}=\varphi_{1}\left(\operatorname{Re} z, \operatorname{Im} z, \operatorname{Re} w_{1}\right)\right\} .
\end{aligned}
$$

An adapted linear change of coordinates insures that the differentials at the origin of the graphing maps all vanish.

A CR manifold $M$ being always locally graphed above a generic submanifold of $\mathbb{C}^{n-c}$, the remainder of this memoir will mostly be devoted to the study of $\mathcal{C}^{\infty}$ or $\mathcal{C}^{\kappa, \alpha}$ generic submanifolds of $\mathbb{C}^{n}$. The above local representation of a generic $M$ will be constantly used. 
4.3. CR vector fields. Let $M$ be generic, of class at least $\mathcal{C}^{1}$, represented by $v=\varphi(x, y, u)$ as above, in coordinates $(z, w)=(x+i y, u+i v)$. Sometimes, we shall also write $v=\varphi(z, u)$, being it clear that $\varphi$ is not holomorphic with respect to $z$. Here, we provide a description in coordinates of three useful families of vector fields.

There exist $m$ anti-holomorphic vector fields defined in $\Delta_{\rho_{1}}^{m} \times\left(\square_{\rho_{1}}^{d} \times i \mathbb{R}^{d}\right)$ of the form:

$$
\bar{L}_{k}^{\prime}=\frac{\partial}{\partial \bar{z}_{k}}+\sum_{j=1}^{d} a_{j, k}^{\prime}(x, y, u) \frac{\partial}{\partial \bar{w}_{j}},
$$

whose restrictions to $M$ span $T^{0,1} M$. To compute the coefficients $a_{j, k}^{\prime}$, the conditions $0 \equiv \bar{L}_{k}^{\prime}\left(\varphi_{j}(x, y, u)-v_{j}\right)$ yield:

$$
2 \varphi_{j, \bar{z}_{k}}=\sum_{l=1}^{d}\left(i \delta_{j, l}-\varphi_{j, u_{l}}\right) a_{l, k}^{\prime} .
$$

In matrix notation, the solution is: $a^{\prime}=2\left(i I_{d \times d}-\varphi_{u}\right)^{-1} \cdot \varphi_{\bar{z}}$, with $a^{\prime}=\left(a_{j, k}^{\prime}\right)_{1 \leqslant j \leqslant d}^{1 \leqslant k \leqslant m}$ and $\varphi_{\bar{z}}=\left(\varphi_{j, \bar{z}_{k}}\right)_{1 \leqslant j \leqslant d}^{1 \leqslant k \leqslant m}$ both of size $d \times m$. Since $\left[T^{0,1} M, T^{0,1} M\right] \subset T^{0,1} M$, the $\bar{L}_{k}^{\prime}$ commute. They are extrinsic.

Also, there exist $m$ intrinsic sections of $\mathbb{C} T M$ of the form:

$$
\bar{L}_{k}:=\frac{\partial}{\partial \bar{z}_{k}}+\sum_{j=1}^{d} a_{j, k}(x, y, u) \frac{\partial}{\partial u_{j}},
$$

written in the coordinates $(x, y, u)$ of $M$, which span the structure bundle $T^{0,1} M \subset \mathbb{C} T M$. Since $(x, y, u)$ are coordinates on $M$, restricting the $\left.\bar{L}_{k}^{\prime}\right|_{M}$ to $M$ amounts to just drop the terms $\frac{i}{2} \frac{\partial}{\partial v_{j}}$ in each $\frac{\partial}{\partial \bar{w}_{j}}$ appearing in $\bar{L}_{k}^{\prime}$. Hence:

Lemma 4.4. One has $a_{j, k}=\frac{1}{2} a_{j, k}^{\prime}$.

Another argument is to first introduce the $d$ vector fields:

$$
K_{j}=\sum_{l=1}^{d} b_{l, j}(x, y, u) \frac{\partial}{\partial u_{l}},
$$

that are uniquely determined by the conditions

$$
\delta_{j_{1}, j_{2}}=K_{j_{1}}\left(\left.w_{j_{2}}\right|_{M}\right)=K_{j_{1}}\left(u_{j_{2}}+i \varphi_{j_{2}}(x, y, u)\right) .
$$


Equivalently, the coefficients $b_{l, j}$ satisfy:

$$
\delta_{j_{1}, j_{2}}=\sum_{l=1}^{d}\left(\delta_{j_{2}, l}+i \varphi_{j_{2}, u_{l}}\right) b_{l, j_{1}},
$$

whence, in matrix notation: $b=\left(I_{d \times d}+i \varphi_{u}\right)^{-1}$. Here, $b=\left(b_{l, j}\right)_{1 \leqslant l \leqslant d}^{1 \leqslant j \leqslant d}$ and $\varphi_{u}=\left(\varphi_{j, u_{l}}\right)_{1 \leqslant j \leqslant d}^{1 \leqslant l \leqslant d}$ both are $d \times d$ matrices.

Similarly as in Corollary 3.27, the $\bar{L}_{k}$ defined above span the structure bundle $\mathcal{L}=T^{0,1} M$ having the local integrals $z_{1}, \ldots, z_{m},\left.w_{1}\right|_{M}, \ldots,\left.w_{d}\right|_{M}$, if and only if they satisfy $0=\bar{L}_{k}\left(\left.w_{j}\right|_{M}\right)=\bar{L}_{k}\left[u_{j}+i \varphi_{j}(x, y, u)\right]$. Seeking the $\bar{L}_{k}$ under the form $\bar{L}_{k}=\frac{\partial}{\partial \bar{z}_{k}}+\sum_{l=1}^{d} c_{l, k}(x, y, u) K_{l}$, it follows from $\delta_{j_{1}, j_{2}}=K_{j_{1}}\left(u_{j_{2}}+i \varphi_{j_{2}}(x, y, u)\right)$ that $c_{j, k}=-i \varphi_{j, \bar{z}_{k}}$. Reexpressing explicitly the $K_{l}$ in terms of the $\frac{\partial}{\partial u_{j}}$ as achieved above, we finally get in matrix notation $a=\left(i I_{d \times d}-\varphi_{u}\right)^{-1} \cdot \varphi_{\bar{z}}$. This yields a second, more intrinsic computation of the coefficients $a_{j, k}$ and a second proof of $a_{j, k}=\frac{1}{2} a_{j, k}^{\prime}$.

If $\chi$ is a $\mathcal{C}^{\infty}$ or $\mathcal{C}^{\kappa, \alpha}$ function on $M$, its differential may be computed as

$$
d \chi=\sum_{k=1}^{m} L_{k}(\chi) d z_{k}+\sum_{k=1}^{m} \bar{L}_{k}(\chi) d \bar{z}_{k}+\left.\sum_{j=1}^{d} K_{j}(\chi) d w_{j}\right|_{M} .
$$

Lemma 4.5. ([[Trv1981, BR1987, Trv1992, BCH2005]) The following relations hold:

$$
\left\{\begin{array}{l}
L_{k_{1}}\left(z_{k_{2}}\right)=\delta_{k_{1}, k_{2}}, L_{k}\left(w_{j}\right)=0, \quad K_{j}\left(z_{k}\right)=0, \quad K_{j_{1}}\left(\left.w_{j_{2}}\right|_{M}\right)=\delta_{j_{1}, j_{2}}, \\
{\left[L_{k_{1}}, L_{k_{2}}\right]=\left[L_{k}, K_{j}\right]=\left[K_{j_{1}}, K_{j_{2}}\right]=0 .}
\end{array}\right.
$$

4.6. Vector-valued Levi form. Let $p \in M$ and denote by $\pi_{p}$ the projection $\mathbb{C} T_{p} M \longrightarrow \mathbb{C} T_{p} M /\left(T_{p}^{1,0} M \oplus T_{p}^{0,1} M\right)$.

Definition 4.7. The Levi map at $p$ is the Hermitian $\mathbb{C}^{d}$-valued form acting on two vectors $X_{p}, Y_{p} \in T_{p}^{1,0} M$ as:

$$
\left[\begin{array}{ll}
\mathfrak{L}_{p}: & T_{p}^{1,0} M \times T_{p}^{1,0} M \longrightarrow \mathbb{C} T_{p} M /\left(T_{p}^{1,0} M \oplus T_{p}^{0,1} M\right) \\
& \mathfrak{L}_{p}\left(X_{p}, Y_{p}\right):=\frac{1}{2 i} \pi_{p}([X, \bar{Y}](p)),
\end{array}\right.
$$

where $X, Y$ are any two sections of $T^{1,0} M$ defined in a neighborhood of $p$ satisfying $X(p)=X_{p}, Y(p)=Y_{p}$. The resulting number is independent of the choice of such extensions $X, Y$.

As $p$ varies, this yields a smooth bundle map. The Levi map $\mathfrak{L}_{p}$ is nondegenerate at $p$ if its kernel is null: $\mathfrak{L}_{p}\left(X_{p}, Y_{p}\right)=0$ for every $Y_{p}$ implies 
$X_{p}=0$. On the opposite, $M$ is Levi-flat if the kernel of $\mathfrak{L}_{p}$ equals $T_{p}^{1,0} M$ at every $p$. If $M$ is a hypersurface $(d=1)$, one calls $\mathfrak{L}_{p}$ the Levi form at $p$. Then $M$ is strongly pseudoconvex at $p$ if the Levi form $\mathfrak{L}_{p}$ is definite, positive or negative. These definitions agree with the ones formulated in $\S 3.10$ for abstract CR structures.

Theorem 4.8. ([[Fr1977, Ch1991]) In a neighborhood $U_{p}$ of a point $p \in M$ in which the kernel of the Levi map is of constant rank and defines a $\mathcal{C}^{\omega}$, $\mathcal{C}^{\infty}$ or $\mathcal{C}^{\kappa-1, \alpha}(\kappa \geqslant 2,0 \leqslant \alpha \leqslant 1)$ distribution of $m_{1}$-dimensional complex planes $P_{q} \subset T_{q} M, \forall q \in U_{p}$, the distribution is Frobenius-integrable, hence $M$ is $\mathcal{C}^{\omega}, \mathcal{C}^{\infty}$ or $\mathcal{C}^{\kappa-1, \alpha}$ foliated by complex manifolds of dimension $m_{1}$.

In particular, a Levi-flat generic submanifold of CR dimension $m$ is foliated by $m$-dimensional complex manifolds. These observations go back to Sommer (1959). In [Ch1991], one founds a systematic study of foliations by complex and by CR manifolds.

4.9. CR orbits. Let $M \subset \mathbb{C}^{n}$ be generic and consider the system $\mathbb{L}$ of sections of $T^{c} M$. To apply the orbit Theorem 1.21, we need $M$ to be at least $\mathcal{C}^{2}$, in order that the flows are at least $\mathcal{C}^{1}$. By definition, a weak $T^{c} M$ integral submanifold $S \subset M$ satisfies $T_{p} S \supset T_{p}^{c} M$, at every point $p \in S$. Equivalently, $S$ has the same CR dimension as $M$ at every point.

In the theory of holomorphic extension exposed in Part V, local and global CR orbits will appear to be adequate objects of study. They constitute one of the main topics of this memoir.

Proposition 4.10. ([Trp1990, Tu1990, Tu1994a, Me1994, Jö1996, MP1999, MP2002]) Let $M \subset \mathbb{C}^{n}$ be generic of class $\mathcal{C}^{\omega}, \mathcal{C}^{\infty}$ or $\mathcal{C}^{\kappa, \alpha}$ with $\kappa \geqslant 2$ and $0 \leqslant \alpha \leqslant 1$.

(a) The (global) $T^{c} M$-orbits are called $\mathrm{CR}$ orbits. They are denoted by $\mathcal{O}_{C R}$ or by $\mathcal{O}_{C R}(M, p)$, if the reference to one of point $p \in \mathcal{O}_{C R}$ is needed.

(b) The local CR orbit of a point $p \in M$ is denoted by $\mathcal{O}_{C R}^{\text {loc }}(M, p)$. It is a local submanifold embedded in $M$, closed in a sufficiently small neighborhood of $p$ in $M$.

(c) Local and global $C R$ orbits are $\mathcal{C}^{\omega}, \mathcal{C}^{\infty}$ or $\mathcal{C}^{\kappa, \beta}$, for every $\beta$ with $0<\beta<\alpha$.

(d) $M$ is partitioned in global CR orbits. Each global CR orbit is injectively immersed and weakly embedded in $M$, is a CR submanifold of $\mathbb{C}^{n}$ contained in $M$ and has the same CR dimension as $M$. 
(e) Every (immersed) $C R$ submanifold $S \subset M$ having the same $C R$ dimension as $M$ contains the local $C R$ orbit of each of its points

(f) $C R$ orbits of the smallest possible real dimension $2 m=$ $2 \mathrm{CR} \operatorname{dim} M$ satisfy $T_{p} \mathcal{O}_{C R}=T_{p}^{c} \mathcal{O}_{C R}$ at every point, hence are complex m-dimensional submanifolds.

According to Example 1.29, CR orbits should be $\mathcal{C}^{\kappa-1, \alpha}$, not smoother. But in generic submanifolds, they also can be described as boundaries of small attached analytic discs ([Tu1990, Tu1994a, Me1994]) and the $\mathcal{C}^{\kappa, \alpha-0}=\bigcap_{\beta<\alpha} \mathcal{C}^{\kappa, \beta}$ smoothness of the solution in Theorem 3.7(IV) explains (c).

Let us summarize some structural properties of CR orbits, useful in applications. A specialization of Theorem 1.21(4) yields the following.

Proposition 4.11. For every $p \in M$, there exist $k \in \mathbb{N}$, sections $L^{1}, \ldots, L^{k}$ of $T^{c} M$ and parameters $\mathbf{s}_{1}^{*}, \ldots, \mathbf{s}_{k}^{*} \in \mathbb{R}$ such that $L_{\mathbf{s}_{k}^{*}}^{k}\left(\cdots\left(L_{\mathbf{s}_{1}^{*}}^{1}(p)\right) \cdots\right)=p$ and the map $\left(\mathrm{s}_{1}, \ldots, \mathrm{s}_{k}\right) \longmapsto L_{\mathrm{s}_{k}}^{k}\left(\cdots\left(L_{\mathrm{s}_{1}}^{1}(p)\right) \cdots\right)$ is of rank $\operatorname{dim} \mathcal{O}_{C R}(M, p)$ at $\left(\mathrm{s}_{1}^{*}, \ldots, \mathrm{s}_{k}^{*}\right)$.

The dimension of any $\mathcal{O}_{C R}$ is equal to $2 m+e$, for some $e \in \mathbb{N}$ with $0 \leqslant e \leqslant d$. Denote:

- $\mathcal{O}_{2 m+e} \subset M$ the union of CR orbits of dimension $=2 m+e$;

- $\mathcal{O}_{2 m+e}^{\geqslant} \subset M$ the union of CR orbits of dimension $\geqslant 2 m+e$;

- $\mathcal{O}_{2 m+e}^{\leqslant} \subset M$ the union of CR orbits of dimension $\leqslant 2 m+e$.

The function $p \mapsto \operatorname{dim} \mathcal{O}_{C R}(M, p)$ is lower semicontinuous. It follows that $\mathcal{O}_{2 m+e}^{\geqslant}$is open in $M$ and that $\mathcal{O}_{2 m+e}^{\leqslant}$is closed in $M$.

Let $p \in M$, let $\mathcal{O}_{p}$ be a small piece of $\mathcal{O}_{C R}(M, p)$ passing through $p$, of dimension $2 m+e_{p}$, for some integer $e_{p}$ with $0 \leqslant e_{p} \leqslant d$, and let $H_{p}$ be a local $\mathcal{C}^{\infty}$ or $\mathcal{C}^{\kappa, \alpha}$ submanifold of $M$ passing through $p$ and satisfying $T_{p} H_{p} \oplus T_{p} \mathcal{O}_{p}=T_{p} M$. Call $H_{p}$ a local orbit-transversal. Implicitly, $H_{p}=\emptyset$ if $e_{p}=d$. Then, in a sufficiently small neighborhood of $p$ :

- lower semi-continuity: $H_{p} \cap \mathcal{O}_{2 m+e_{p}+1}^{\geqslant}=\emptyset$;

- equivalently: $H_{p} \cap \mathcal{O}_{2 m+e_{p}}^{\geqslant}=H_{p} \cap \mathcal{O}_{2 m+e_{p}}$;

- $H_{p} \cap \mathcal{O}_{2 m+e_{p}}^{\leqslant}$is closed.

Proposition 4.12. If $M$ is $\mathcal{C}^{\omega}$, then at every point $p \in M$, for every orbittransversal $H_{p}$ passing through $p$, the closed set $H_{p} \cap \mathcal{O}_{2 m+e_{p}}^{\leqslant}$is a local real analytic subset of $H_{p}$ containing $p$. 
A $C R$-invariant subset of $M$ is a union of $\mathrm{CR}$ orbits. A closed (for the topology of $M$ ) CR-invariant subset is minimal if it does not contain any proper subset which is also a closed CR-invariant subset.

Problem 4.13. Describe the possible structure of the decomposition of $M$ in CR orbits.

There are differences between embedded and locally embeddable generic submanifolds, which we shall not discuss, assuming that $M$ is embedded in $\mathbb{C}^{n}$ or in $P_{n}(\mathbb{C})$. Also, the $\mathcal{C}^{\omega}$ category enjoys special features.

Indeed, if $M$ is a connected real analytic hypersurface, Proposition 4.12 entails that each minimal closed invariant subset of $M$ is either an embedded complex hypersurface or an open orbit; also if $M$ contains at least one CR orbit of maximal dimension $(2 n-1)$ (hence an open subset of $M$ ), all its CR orbits of codimension one are complex $(n-1)$-dimensional embedded submanifold of $M$ (a real analytic subset of codimension one in $\mathbb{R}$ consists of isolated points). In the smooth category things are different.

So, let $M$ be a connected $\mathcal{C}^{\infty}$ or $\mathcal{C}^{\kappa, \alpha}(\kappa \geqslant 2,0 \leqslant \alpha \leqslant 1)$ hypersurface of $\mathbb{C}^{n}$. Its CR-orbits are either $(2 n-1)$-dimensional, i.e. open in $M$, or $(2 n-2)$-dimensional and $T^{c} M$-integral, hence complex $(n-1)$ dimensional hypersurfaces immersed in $M$.

Proposition 4.14. ([ए̈1999a]) In the smooth category, the structure of every minimal closed CR-invariant subset $C$ of $M$ has one of the following types:

(i) $C=M$ consists of a single embedded open CR orbit;

(ii) $C=\bigcup_{a \in A} \mathcal{O}_{C R, a}=M$ is a union of complex hypersurfaces, each being dense in $C$, with $\operatorname{Card} A=\operatorname{Card} \mathbb{R}$;

(iii) $C=\bigcup_{a \in A} \mathcal{O}_{C R, a}$ has empty interior in $M$ and is a union union of complex hypersurfaces, each being dense in $C$, with Card $A=$ Card $\mathbb{R}$;

(iv) $C$ consists of a single complex hypersurface embedded in $M$.

Furthermore, the closure, with respect to the topology of $M$, of every CR orbit of dimension $(2 n-2)$ is a minimal closed CR-invariant subset of $M$.

These four options are known in foliation theory ([HH1983, CLN1985]). One has to remind that each CR orbit contained in $C$ is dense in $C$. In the first two cases, the trace of $C$ on any orbit-transversal is a full open segment; in the third, it is a Cantor set; in the last, it is an isolated point. In the 
third case, impossible if $M$ is real analytic, $C$ will be called an exceptional minimal CR-invariant subset, similarly as in foliation theory. We shall see below that compactness of $M \subset \mathbb{C}^{n}$ imposes a strong restriction on the possible $C$ 's.

We mention that an analog of Proposition 4.14 holds for connected generic submanifolds of codimension $d \geqslant 2$, provided one puts the restrictive assumption that all its CR orbits are of codimension $\leqslant 1$, the only difference being that $\mathrm{CR}$ orbits of codimension 1 are not complex manifolds in this case.

The presence of $\mathrm{CR}$ orbits of codimension $\geqslant 2$ in $M$ may produce minimal closed CR-invariant subsets with complicated transversal structure, even in the real analytic category ([BM1997]). Also, in a bounded strongly pseudoconvex boundary (see $\S 1.15(\mathrm{~V})$ for background), there may exist a $\mathrm{CR}$ orbit of codimension one whose closure constitutes an exceptional minimal CR-invariant subset.

Theorem 4.15. ([ए̈̈1999a]) There exists a bounded strongly pseudoconvex domain $\Omega \subset \mathbb{C}^{3}$ with $\mathcal{C}^{\infty}$ boundary and a compact $\mathcal{C}^{\infty}$ submanifold $M \subset$ $\partial \Omega$ of dimension 4 which is generic in $\mathbb{C}^{3}$ such that:

- $M$ is $\mathcal{C}^{\infty}$ foliated by $C R$ orbits of dimension 3;

- $M$ contains a compact exceptional minimal CR-invariant set, but no compact $C R$ orbit.

Summarized proof. The main idea is to start with an example due to Sacksteder, known in foliation theory, of a compact real analytic 3-dimensional manifold $\mathcal{N}$ equipped with a $\mathcal{C}^{\omega}$ foliation $\mathcal{F}$ of codimension one which carries a compact exceptional minimal set, but no compact leaf. According to [HH1983, there exists such a pair $(\mathcal{N}, \mathcal{F})$, together with a $\mathcal{C}^{\infty}$ diffeomorphism $\varphi_{1}: \mathcal{N} \rightarrow B \times S^{1}$, where $B$ is some compact orientable $\mathcal{C}^{\infty}$ surface of genus 2 embedded in $\mathbb{R}^{3}$, and where $S^{1}$ is the unit circle. Let $B \ni b \mapsto \mathbf{n}(b) \in T_{b} \mathbb{R}^{3}$ denote the $\mathcal{C}^{\infty}$ unit outward normal vector field to such a $B \subset \mathbb{R}^{3}$, and consider $\mathbb{R}^{3}$ to be embedded in $\mathbb{C}^{3}$. For $\varepsilon>0$ small, the map

$$
\varphi_{2}: B \times S^{1} \ni(b, \zeta) \longmapsto b+\mathbf{n}(b) \cdot \varepsilon \zeta \in \mathbb{C}^{3}
$$

may be seen to be a totally real $\mathcal{C}^{\infty}$ embedding. By results of BruhatWhitney and Grauert, one may approximate the $\mathcal{C}^{\infty}$ totally real embedding $\varphi_{2} \circ \varphi_{1}$ by a $\mathcal{C}^{\omega}$ embedding $\varphi: \mathcal{N} \rightarrow \mathbb{C}^{3}$ which is arbitrarily close to $\varphi_{2} \circ \varphi_{1}$ in $\mathcal{C}^{1}$ norm, hence totally real. Denote $N:=\varphi(\mathcal{N})$. The transported foliation $F:=\varphi_{*}(\mathcal{F})$ being real analytic, one may then proceed to an intrinsic complexification of all its totally real 2-dimensional leaves, 
getting some 5-dimensional real analytic hypersurface $N^{i_{c}}$ containing $N$, equipped with a foliation $F^{i_{c}}$ of $N^{i_{c}}$ by 2-dimensional complex manifolds, with $F^{i_{c}} \cap N=F$. This foliation $F^{i_{c}}$ is closed in some tubular neighborhood $\Omega$ of $N$ in $\mathbb{C}^{3}$, say $\Omega:=\left\{z \in \mathbb{C}^{3}: \operatorname{dist}(z, N)<\delta\right\}$, with $\delta>0$ small. Since $N$ is totally real, the boundary $\partial \Omega$ is strongly pseudoconvex (Grauert) and is $\mathcal{C}^{\infty}$. Clearly, the intersection $M:=N^{i_{c}} \cap \partial \Omega$ is a 4-dimensional $\mathcal{C}^{\infty}$ submanifold. The intersections of the 2-dimensional complex leaves of $F^{i_{c}}$ with $\partial \Omega$ show that $M$ is foliated by strongly pseudoconvex 3-dimensional boundaries, which obviously consist of a single CR orbit. Thus a CR orbit of $M$ is just the intersection of a global leaf of $F^{i_{c}}$ with $\partial \Omega$. In conclusion, letting $\operatorname{Exc}_{\mathcal{F}}$ be the minimal exceptional set of Sacksteder's example, $M$ contains the exceptional minimal CR-invariant set $\left[\varphi_{*}\left(\operatorname{Exc}_{\mathcal{F}}\right)\right]^{i_{c}} \cap \partial \Omega$ and no compact CR orbit.

4.16. Global minimality and laminations by complex manifolds. The $\mathrm{CR}$ orbits being essentially independent bricks, it is natural to define the class of CR manifolds which consist of only one brick.

Definition 4.17. A $\mathcal{C}^{\omega}, \mathcal{C}^{\infty}$ or $\mathcal{C}^{\kappa, \alpha} \mathrm{CR}$ manifold $M$ is called globally minimal if if consists of a single CR orbit.

Each CR orbit of a CR manifold is a globally minimal immersed CR submanifold of $\mathbb{C}^{n}$. To simplify the overall presentation and not to expose superficial corollaries, almost all the theorems of Parts V and VI in this memoir will be formulated with a global minimality $M$.

Lemma 4.18. (Gr1968, Øö1995, BCH2005]) A compact connected $\mathcal{C}^{2}$ hypersurface in $\mathbb{C}^{n}$ is necessarily globally minimal.

Proof. Otherwise, the closure of a CR-orbit of codimension one in $M$ would produce a compact CR-invariant subset $C$ which is a union of immersed complex hypersurfaces, each dense in $C$. Looking at a point of $C$ where the pluriharmonic function $r_{i}:=\operatorname{Re} z_{i}$ (or $s_{i}:=\operatorname{Im} z_{i}$ ) is maximal, the maximum principle entails that $r_{i}$ (or $s_{i}$ ) is constant on $C$, for $i=1, \ldots, n$, hence $C=\{p t$.$\} , contradiction.$

More generally, the same simple argument yields:

Corollary 4.19. Any Stein manifold cannot contain a compact set which is laminated by complex manifolds

In the projective space $P_{n}(\mathbb{C})$, one expects compact orientable connected $\mathcal{C}^{2}$ hypersurfaces $M$ to be still globally minimal, but arguments are far to be simple. In fact, there are infinitely many compact projective 
algebraic complex hypersurfaces $\Sigma$ in $P_{n}(\mathbb{C})$. However, they cannot be contained in such an $M \subset P_{n}(\mathbb{C})$ since, otherwise, their complex normal bundle $\left.T P_{n}(\mathbb{C})\right|_{\Sigma} / T \Sigma$, known to be never trivial, but equal to the complexification of the trvial bundle $\left.T M\right|_{\Sigma} / T \Sigma$, would be trivialized.

Thus, according to Proposition 4.14 above, the very question is about nonexistence of closed CR-invariant sets $C \subset M$ laminated by complex hypersurfaces which either coincide with $M$ or are transversally Cantor sets. If $M \subset P_{n}(\mathbb{C})$ is real analytic, it might only be Levi-flat.

Nonexistence of orientable Levi-flat hypersurfaces in $P_{n}(\mathbb{C})$ was expected, because they would divide the projective space in two smoothly bounded pseudoconvex domains. In the real analytic case, non-existence was verified by Lins-Neto for $n \geqslant 3$ and by Ohsawa for $n=2$; in the smooth (harder) case, see [Si2000].

Open question 4.20. Is any compact orientable connected $\mathcal{C}^{2}$ hypersurface of $P_{n}(\mathbb{C})$ globally minimal?

So, the expected answer is yes. In fact, the question is a particular case of a deep conjecture stemming from Hilbert's sixteen problem about phase diagrams of vector fields having polynomial coefficients on the twodimensional projective space. This conjecture is inspired by the PoincaréBendixson theory valid over the real numbers, according to which the closure of each leaf of such a foliation over $P_{2}(\mathbb{R})$ contains either a compact leaf or a singularity. In its most general form, it says that $P_{n}(\mathbb{C})$ cannot contain a compact set laminated by $(n-1)$-dimensional complex manifold, unless it is a trivial lamination, viz just a compact complex projective algebraic hypersurface; however, nontrivial laminations by $(n-2)$-dimensional complex manifolds may be shown to exist.

A related general open question is to find topologico-geometrical criteria on open subsets of $P_{n}(\mathbb{C})$ insuring the existence of nonconstant holomorphic functions there.

4.21. Finite-typisation of generic submanifolds. Let $M$ be a connected $\mathcal{C}^{\kappa}(2 \leqslant \kappa \leqslant \infty)$ generic submanifold of $\mathbb{C}^{n}$ of codimension $d \geqslant 1$ and of $\mathrm{CR}$ dimension $m=n-d \geqslant 1$. The distribution of subspaces $p \mapsto T_{p}^{c} M$ of $T M$ is of constant rank $2 \mathrm{~m}$. We apply to the complex tangential bundle $T^{c} M$ the concept of finite-type.

Definition 4.22. A point $p \in M$ is said to be of finite type if the system $\mathbb{L}$ of local sections of $T^{c} M$ defined in a neighborhood of $p$ satisfies $\mathbb{L}^{\kappa}(p)=$ $T_{p} M$. The smallest integer $d(p) \leqslant \kappa$ with $\mathbb{L}^{d(p)}(p)=T_{p} M$ is called the type of $M$ at $p$. 
We want now to figure out how, in general, a generic submanifold of $\mathbb{C}^{n}$ must be globally minimal and in fact, of finite type at every point. We equip with the strong Whitney topology the $\operatorname{set}{ }^{\kappa} \mathcal{G}_{d, m}^{n}$ of $\mathcal{C}^{\kappa}(2 \leqslant \kappa \leqslant \infty)$ connected generic submanifolds $M \subset \mathbb{C}^{n}$ of codimension $d \geqslant 1$ and of $\mathrm{CR}$ dimension $m=n-d \geqslant 1$. No restriction is made on the global topology.

As a model case, let $\kappa \geqslant 2$ and consider $M$ to be rigid algebraic represented by

$$
w_{j}=\bar{w}_{j}+i P_{j}(z, \bar{z})=\bar{w}_{j}+i \sum_{|\alpha|+|\beta| \leqslant \kappa} p_{j, \alpha, \beta} z^{\alpha} \bar{z}^{\beta},
$$

where $\alpha, \beta \in \mathbb{N}^{m}$, where the polynomials $P_{j}$ are real, $p_{j, \alpha, \beta}=\bar{p}_{j, \beta, \alpha}$ and have no pluriharmonic term, namely $0 \equiv P_{j}(z, 0) \equiv P_{j}(0, \bar{z})$, for $j=$ $1, \ldots, d$. A basis of $(1,0)$ and of $(0,1)$ vector fields is given by

$$
L_{k}:=\frac{\partial}{\partial z_{k}}+i \sum_{j=1}^{d} P_{j, z_{k}} \frac{\partial}{\partial w_{j}} \quad \text { and } \quad \bar{L}_{k}:=\frac{\partial}{\partial \bar{z}_{k}}-i \sum_{j=1}^{d} \bar{P}_{j, \bar{z}_{k}} \frac{\partial}{\partial \bar{w}_{j}},
$$

for $k=1, \ldots, n$. The Lie algebra $\mathbb{L}^{\kappa}$ generated by all Lie brackets of length $\leqslant \kappa$ of the system $\mathbb{L}:=\left\{L_{k}, \bar{L}_{k}\right\}_{1 \leqslant \kappa \leqslant m}$ contains the subalgebra $\mathbb{L}_{C R \text {, rigid }}^{\kappa}$ generated by the only brackets of the form

$$
\left[L_{\lambda_{1}}, \ldots,\left[L_{\lambda_{a}},\left[\bar{L}_{\mu_{1}}, \ldots,\left[\bar{L}_{\mu_{b}},\left[L_{k_{1}}, \bar{L}_{k_{2}}\right]\right] \ldots\right]\right] \ldots\right]
$$

where $2+a+b \leqslant \kappa$ and where $1 \leqslant \lambda_{1}, \ldots, \lambda_{a}, \mu_{1}, \ldots, \mu_{b}, k_{1}, k_{2} \leqslant m$. One computes

$$
\left[L_{k_{1}}, \bar{L}_{k_{2}}\right]=-i\left(\sum_{j=1}^{d} P_{j, z_{k_{1}} \bar{z}_{k_{2}}} \frac{\partial}{\partial w_{j}}+\sum_{j=1}^{d} \bar{P}_{j, z_{k_{1}} \bar{z}_{k_{2}}} \frac{\partial}{\partial \bar{w}_{j}}\right),
$$

hence the above iterated Lie brackets are equal to

$$
-i\left(\sum_{j=1}^{d} P_{j, z_{\lambda_{1}} \ldots z_{\lambda_{a}} \bar{z}_{\mu_{1}} \ldots \bar{z}_{\mu_{b}} z_{k_{1}} \bar{z}_{k_{2}}} \frac{\partial}{\partial w_{j}}+\sum_{j=1}^{d} \bar{P}_{j, z_{\lambda_{1}} \ldots z_{\lambda_{a}} \bar{z}_{\mu_{1}} \ldots \bar{z}_{\mu_{b}} z_{k_{1}} \bar{z}_{k_{2}}} \frac{\partial}{\partial \bar{w}_{j}}\right) .
$$

This shows that all these brackets are linearly independent as functions of the jets of the $P_{j}$. In fact, the number of such brackets is exactly equal to the dimension of the space of polynomials $P(z, \bar{z})$ of degree $\leqslant \kappa$ having no pluriharmonic term, namely equal to

$$
\frac{(2 m+\kappa) !}{2 m ! \kappa !}-2 \frac{(m+\kappa) !}{m ! \kappa !}+1 .
$$


For a general $\mathcal{C}^{\kappa}$ submanifold $M$ (not necessarily rigid), one verifies that the same collection of brackets is independent in terms of the jets of the defining equation of $M$. Generalizing slightly Lemma 2.13 , we see that in the vector space of $d \times(d+e)$ (real or complex) matrices, the subset of matrices of rank $\leqslant d-1$ is a real algebraic set of codimension equal to $(e+1)$. If we choose $\kappa$ large enough so that the dimension of $\mathbb{L}_{C R \text {, rigid }}^{\kappa}$ is $\geqslant d+\operatorname{dim} M=2(m+d)=2 n$ (applying the previous assertion with $e:=\operatorname{dim} M)$, if we form the $d \times\left(d+e^{\prime}\right), e^{\prime} \geqslant e$, matrix consisting of the coordinates of the $\frac{\partial}{\partial w}$-part of brackets of length $\leqslant \kappa$ as above, then the set where this matrix is of rank $\leqslant d-1$ is of codimension $\geqslant \operatorname{dim} M+1$ in the space of $\kappa$-th jets of the defining equations of $M$. Consequently, the jet transversality theorem applies.

Theorem 4.23. Let $n \geqslant 1, m \geqslant 1$ and $d \geqslant 1$ be integers satisfying $m+d=n$ and let $\kappa$ be the minimal integer having the property that

$$
\frac{(2 m+\kappa) !}{2 m ! \kappa !}-2 \frac{(m+\kappa) !}{m ! \kappa !}+1 \geqslant 2(m+d)=2 n .
$$

Then the set of $\mathcal{C}^{\kappa}$ connected generic submanifolds $M \subset \mathbb{C}^{n}$ of codimension $d$ and of $C R$ dimension $m$ that are of finite type $\leqslant \kappa$ at every point is open and dense in the set ${ }^{\kappa} \mathcal{G}_{d, m}^{n}$ of all generic submanifolds.

In particular, a connected $\mathcal{C}^{4}$ (resp. $\mathcal{C}^{3}$, resp. $\left.\mathcal{C}^{2}\right)$ hypersurface in $\mathbb{C}^{2}$ (resp. in $\mathbb{C}^{3}$, resp. in $\mathbb{C}^{n}$ for $n \geqslant 4$ ) is of finite type 4 (resp. 3, resp. 2 , or equivalently, is not Levi-flat) at every point after an arbitrarily small perturbation.

Similarly, if instead of the subalgebra $\mathbb{L}_{\mathrm{CR} \text {,rigid }}$, one would have considered the (smaller) subalgebra consisting of only the brackets $\left[L_{\lambda_{1}}, \ldots,\left[L_{\lambda_{a}},\left[L_{k_{1}}, \bar{L}_{k_{2}}\right]\right] \ldots\right]$, where $2+a \leqslant \kappa$ and where $1 \leqslant \lambda_{1}, \ldots, \lambda_{a}, k_{1}, k_{2} \leqslant \kappa$, one would have obtained finite type $\leqslant \kappa$, for $\kappa$ minimal satisfying $2 m \frac{(m+\kappa-1) !}{m !(\kappa-1) !} \geqslant m^{2}+2(2 m+d)$. We also mention that the same technique enables one to prove that, after an arbitrarily small perturbation, $M$ is finitely nondegenerate at every point and of finite nondegeneracy type $\leqslant \ell$, with $\ell$ minimal satisfying $2 d \frac{(\ell+m) !}{\ell ! m !} \geqslant 4 n-1$. In particular, $\ell=3$ when $m=d=1$ while $\ell=2$ suffices when $m=1$ for all $d \geqslant 2$. Details are left to the reader.

To conclude, we state the analog of Open question 2.17 for induced CR structures.

Open question 4.24. ([JS2004], [*]) Given a fixed generic submanifold $M$ of class $\mathcal{C}^{\kappa}$ that is of finite type at every point, is it always possible 
to perturb slightly a $\mathcal{C}^{\kappa}$ submanifold $M_{1}$ of $M$ that is generic in $\mathbb{C}^{n}$, of codimension $d_{1} \geqslant 1$ and of $C R$ dimension $m_{1}=n-d_{1} \geqslant 1$, as a $\mathcal{C}^{\kappa}$ submanifold $\widetilde{M}_{1}$ of $M$ that is of finite type at every point? If so, what is the smallest regularity $\kappa$ in terms of $d, m, d_{1}, m_{1}$ and of the highest type at points of $M$ ?

4.25. Spaces of $\mathbf{C R}$ functions and of $\mathbf{C R}$ distributions. A $\mathcal{C}^{1}$ function $f: M \rightarrow \mathbb{C}$ is called Cauchy-Riemann ( $C R$ briefly) if it is annihilated by every section of $T^{0,1} M$. Equivalently:

- $d f$ is $\mathbb{C}$-linear on $T^{c} M$;

- $\left.d f \wedge d t_{1} \wedge \cdots \wedge d t_{n}\right|_{M}=0$;

- $\int_{M} f \bar{\partial} \omega=0$, for every $\mathcal{C}^{1}$ form $\omega$ of type $(n, m-1)$ in $\mathbb{C}^{n}$ having compact support.

(Remind the local expression of $(r, s)$ forms: $\sum_{I, J} a_{I, J} d t^{I} \wedge d \bar{t}^{J}$, where $I=\left(i_{1}, \ldots, i_{r}\right)$ and $J=\left(j_{1}, \ldots, j_{s}\right)$.) A (only) continuous function $f: M \rightarrow \mathbb{C}$ is $C R$ if the last condition $\int_{M} f \bar{\partial} \omega=0$ holds. Further, Lebesgue-integrable CR functions, CR measures, CR distributions and CR currents may be defined as follows ([Trv1981, Trv1992, HM1998, Trp1996, Jö1999b).

Thanks to graphing functions, one may equip locally $M$ with some (in fact many) volume form, or equivalently, some deformation of the canonical $\operatorname{dim} M$-dimensional Legesgue measure defined on tangent spaces. Let $\mathrm{p}$ be a real number with $1 \leqslant \mathrm{p} \leqslant \infty$. Since two such measures are multiple of each other, it makes sense to speak of $L_{l o c}^{\mathrm{p}}$ functions $M \rightarrow \mathbb{C}$. In this setting, a $L_{l o c}^{\mathrm{p}}(M)$ function $f$ is $\mathrm{CR}$ if $\int_{M} f \bar{\partial} \omega=0$, for every $\mathcal{C}^{1}$ form $\omega$ of type $(n, m-1)$ in $\mathbb{C}^{n}$ having compact support.

A distribution $\mathrm{T}$ on $M$ is $C R$ if for every section $\bar{L}$ of $T^{0,1} M$ defined in an open subset $U \subset M$ and every $\chi \in \mathcal{C}_{c}^{\infty}(U, \mathbb{C})$, one has $\langle\mathrm{T}, \bar{L}(\chi)\rangle=0$.

A CR distribution of order zero on $M$ is called a $C R$ measure. Equivalently, a CR measure is a continuous linear map $\omega^{\prime} \mapsto \mu\left(\omega^{\prime}\right)$ from compactly supported forms on $M$ of maximal degree $2 m+d$ to $\mathbb{C}$, that is $\mathrm{CR}$ in the weak sense, namely $\mu(\bar{\partial} \omega)=0$, for every $\mathcal{C}^{1}$ form $\omega$ of type $(n, m-1)$ having compact support. Once a volume form $\operatorname{dvol}_{M}$ is fixed on $M$, the quantity $\mu \mathrm{dvol}_{M}$ is a CR (Borel) measure on $M$.

4.26. Traces of $\mathbf{C R}$ functions on $\mathbf{C R}$ orbits. A $\mathcal{C}^{1}$ function $f: M \rightarrow$ $\mathbb{C}$ is $\mathrm{CR}$ on $M$ if and only if its restriction to every CR orbit of $M$ is $\mathrm{CR}$ (obvious). If $f$ is $\mathcal{C}^{0}$ or $L_{l o c}^{\mathrm{p}}$, a similar but nontrivial statement holds. 
By "almost every CR orbit", we shall mean "except a union of CR orbits whose $\operatorname{dim} M$-dimensional measure vanishes".

Theorem 4.27. $(d=1$ : [Ö1999b; $d \geqslant 1$ : [Po1997, MP1999]) Assume that $M$ is at least $\mathcal{C}^{3}$ and let $f$ be a function in $L_{\text {loc }}^{\mathrm{p}}(M)$ with $1 \leqslant \mathrm{p} \leqslant$ $\infty$. Then the restriction $\left.f\right|_{\mathcal{O}_{C R}}$ is in $L_{l o c}^{\mathrm{p}}$ on $\mathcal{O}_{C R}$, for almost every $\mathcal{O}_{C R}$. Furthermore, $f$ is $C R$ if and only if, for almost every $C R$ orbit $\mathcal{O}_{C R}$ of $M$, its restriction $\left.f\right|_{\mathcal{O}_{C R}}$ is $C R$.

The theorem also holds for $f$ continuous, with $\left.f\right|_{\mathcal{O}_{C R}}$ being CR for every $\mathrm{CR}$ orbit. Here, $\mathcal{C}^{3}$-smoothness is needed. Property (5) of Sussman's orbit Theorem 1.21 together with a topological reasoning yields a covering by orbit-chart which is used in the proof.

Proposition 4.28. ([ए̈1999a, Po1997, MP1999]) Assume $M$ is $\mathcal{C}^{\infty}$ or $\mathcal{C}^{\kappa, \alpha}$, with $\kappa \geqslant 2,0 \leqslant \alpha \leqslant 1$ and let $\square:=\{x \in \mathbb{R}:|x|<1\}$. There exists $a$ countable covering $\bigcup_{k \in \mathbb{N}} U_{k}=M$ such that for each $k$, there exist $e_{k} \in \mathbb{N}$ with $0 \leqslant e_{k} \leqslant d$ and $a \mathcal{C}^{\kappa-1, \alpha}$ diffeomorphism:

$$
\varphi_{k}:\left(s_{k}, t_{k}\right) \ni \square^{2 m+e_{k}} \times \square^{d-e_{k}} \longmapsto \varphi_{k}\left(s_{k}, t_{k}\right) \in U_{k},
$$

such that:

- $\varphi_{k}\left(\square^{2 m+e_{k}} \times\left\{t_{k}^{*}\right\}\right)$ is contained in a single CR orbit, for every fixed $t_{k}^{*} \in \square^{d-e_{k}}$;

- for each $p \in M$, there exists $k=k_{p} \in \mathbb{N}$ with $p \in U_{k_{p}}$, viz there exist $s_{k_{p}, p}$ and $t_{k_{p}, p}$ with $\varphi_{k_{p}}\left(s_{k_{p}, p}, t_{k_{p}, p}\right)=p$, such that $\varphi_{k_{p}}\left(\square^{2 m+e_{k_{p}}} \times\left\{t_{k_{p}, p}\right\}\right)$ is an open piece of the CR orbit of $p$, i.e. $\operatorname{dim} \mathcal{O}_{C R}(M, p)=2 m+e_{k_{p}}$.

In the proof of the theorem, $\mathcal{C}^{2}$-smoothness of the maps $\varphi_{k}$ (hence $\mathcal{C}^{3}$ smoothness of $M)$ is required to insure that the pull-back $\varphi_{k}^{*}\left(\left.T^{c} M\right|_{U_{k}}\right)$ is $\mathcal{C}^{1}$. However, we would like to mention that if $M$ is $\mathcal{C}^{2, \alpha}$ with $0<\alpha<1$ results of [Tu1990, Tu1994a, Tu1996] and Theorem 3.7(IV) insuring the $\mathcal{C}^{2, \beta}$-smoothness of local and global CR orbits, for every $\beta<\alpha$, this would yield orbit-charts $\varphi_{k}$ of class $\mathcal{C}^{2, \beta}$, and then the above theorem holds true with $M$ of class $\mathcal{C}^{2, \alpha}$.

4.29. Boundary values of holomorphic functions for functional spaces $\mathcal{C}^{\kappa, \alpha}, \mathcal{D}^{\prime}, L_{l o c}^{\mathrm{p}}$. Let $M$ be a generic submanifold of $\mathbb{C}^{n}$ of codimention $d \geqslant$ 1 and of nonnegative $\mathrm{CR}$ dimension $m \geqslant 0$ (we admit $m=0$ ). Assume $M$ 
is at least $\mathcal{C}^{1}$. In appropriate coordinates $t=(z, w)=(x+i y, u+i v) \in$ $\mathbb{C}^{n} \times \mathbb{C}^{m}$ centered at one of its points $p$ :

$$
M=\left\{(z, w) \in \Delta_{\rho_{1}}^{m} \times\left(\square_{\rho_{1}}^{d} \times i \mathbb{R}^{d}\right): v=\varphi(x, y, u)\right\},
$$

for some $\rho_{1}>0$, with $\varphi(0)=0$ and $d \varphi(0)=0$. Let $\rho$ be a real number with $0 \leqslant \rho \leqslant \rho_{1}$. The height function:

$$
\sigma(\rho):=\max _{|x|,|y|,|u| \leqslant \rho}|\varphi(x, y, u)|
$$

is continuous and tends to 0 , as $\rho$ tends to 0 . For every $\rho \leqslant \rho_{1}$ and every $\sigma>\sigma(\rho)$, the boundary of $M \cap\left[\Delta_{\rho}^{m} \times\left(\square_{\rho}^{d} \times i \square_{\sigma}^{d}\right)\right]$ is contained in the boundary $\partial\left(\Delta_{\rho}^{d} \times \square_{\rho}^{d}\right)$ of the horizontal space, times the vertical space $i \square_{\sigma}^{d}$.

Let $C$ be an open convex cone in $\mathbb{R}^{d}$ having vertex 0 . We shall assume it to be salient, namely contained in one side of some hyperplane passing through the origin. Equivalently, its intersection $C \cap S^{d-1}$ with the unit sphere of $\mathbb{R}^{d}$ is open, contained in some open hemisphere and convex in the sense of spherical geometry.

A local wedge of edge $M$ at $p$ directed by $C$ is an open set of the form:

$$
\begin{gathered}
\mathcal{W}=\mathcal{W}(\rho, \sigma, C):=\left\{(x+i y, u+i v) \in \Delta_{\rho}^{m} \times \square_{\rho}^{d} \times i \square_{\sigma}^{d}:\right. \\
v-\varphi(x, y, u) \in C\},
\end{gathered}
$$

for some $\rho, \sigma>0$ satisfying $\rho \leqslant \rho_{1}$ and $\sigma>\sigma(\rho)$. This type of open set is independent of the choice of local coordinates and of local defining functions; in codimension $d \geqslant 2$, it generalizes the notion of local side of a hypersurface. Notice that $\mathcal{W}$ is connected.

If there exists a function $F$ that is holomorphic in $\mathcal{W}$ and that extends continuously up to the edge

$$
M_{\rho}:=M \cap\left[\Delta_{\rho}^{m} \times\left(\square_{\rho}^{d} \times i \mathbb{R}^{d}\right)\right]
$$

of the wedge $\mathcal{W}$, then the limiting values of $F$ define a continuous $\mathrm{CR}$ function on $M_{\rho}$.

A more general phenomenon holds. A function $F$, holomorphic in the wedge $\mathcal{W}$, has slow growth up to $M$, if there exist $k \in \mathbb{N}$ and $C>0$ such that

$$
|F(t)| \leqslant C|v-\varphi(x, y, u)|^{-k}, \quad t=(x+i y, u+i v) \in \mathcal{W} .
$$

Equivalently, $|F(t)| \leqslant C[\operatorname{dist}(t, M)]^{-k}$, with the same $k$ but a possibly different $C$. As in the cited references, we shall assume $M$ to be $\mathcal{C}^{\infty}$. 
Theorem 4.31. ([BCT1983, Hö1985, BR1987, BER1999]) If $F \in \mathcal{O}(\mathcal{W}(\rho, \sigma, C))$ has slow growth up to $M$, it possesses a boundary value $b_{M} F$ which is a CR distribution on the edge $M \cap\left[\Delta_{\rho}^{m} \times\left(\square_{\rho}^{d} \times i \square_{\sigma}^{d}\right)\right]$ precisely defined by:

$$
\begin{gathered}
\left\langle\mathrm{b}_{M} F, \chi\right\rangle:=\lim _{C \ni \theta \rightarrow 0} \int_{\Delta_{\rho}^{m} \times \square_{\rho}^{d}} F(x+i y, u+i \varphi(x, y, u)+i \theta) . \\
\cdot \chi(x, y, u) d x d y d u,
\end{gathered}
$$

where $\chi=\chi(x, y, u)$ is a $\mathcal{C}^{\infty}$ function having compact support in $\Delta_{\rho}^{m} \times \square_{\rho}^{d}$.

(i) The limit is independent of the way how $\theta \in C$ approaches $0 \in \mathbb{R}^{d}$.

(ii) If $\mathrm{b}_{M} F$ is $\mathcal{C}^{\lambda, \beta}, \lambda \geqslant 0,0 \leqslant \beta \leqslant 1$, then $F$ extends as a $\mathcal{C}^{\lambda, \beta}$ function on $\mathcal{W}^{\prime} \cup\left(M \cap\left[\Delta_{\rho}^{m} \times\left(\square_{\rho}^{d} \times i \square_{\sigma^{\prime}}^{d}\right)\right]\right)$, for every wedge $\mathcal{W}^{\prime}=\mathcal{W}^{\prime}\left(\rho, \sigma^{\prime}, C^{\prime}\right)$ with $\sigma(\rho)<\sigma^{\prime} \leqslant \sigma$ and with $C^{\prime} \cap S^{d-1} \subset \subset$ $C \cap S^{d-1}$.

(iii) Finally, $F$ vanishes identically in the wege $\mathcal{W}$ if and only if $\mathrm{b}_{M} F$ vanishes on some nonempty open subset of the edge $M_{\rho}$.

The integration is performed on the translation $M_{\rho}^{\theta}:=M_{\rho}+(0, i \theta)$, drawn as follows.

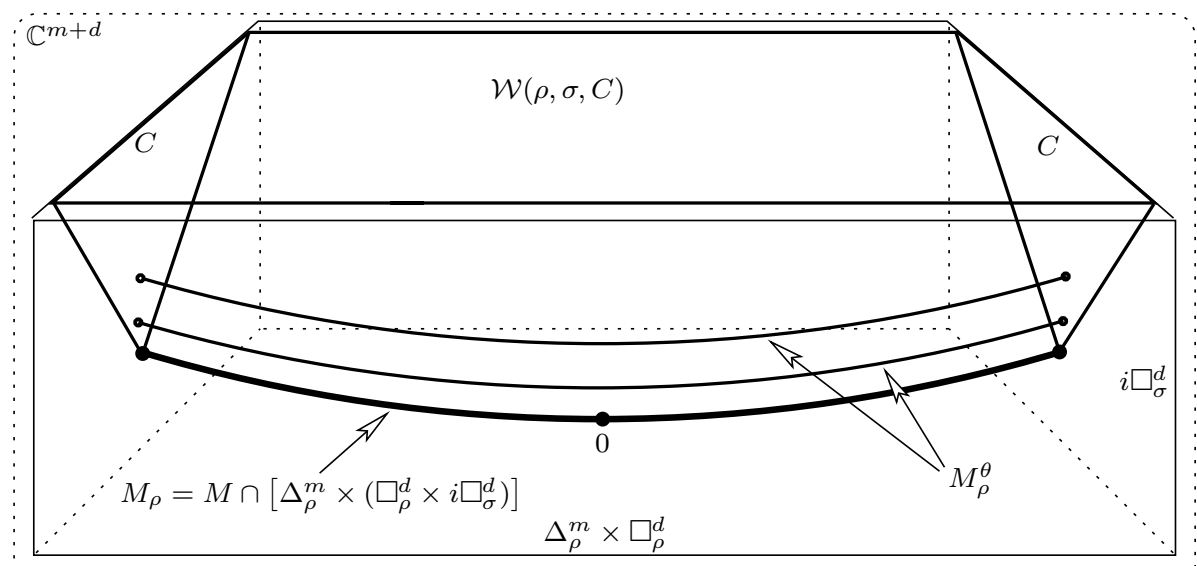

Boundary values of functions holomorphic in a wedge

The proof is standard for $M \equiv \mathbb{R}^{n}$ ([Hö1985] $)$, the main argument going back to Hadamard's finite parts. With technical adaptations in the case of a general generic $M$, several integrations by part are performed on a thin ( $\operatorname{dim} M+1)$-dimensional cycle delimited by $M_{\rho}^{0}$ and $M_{\rho}^{\theta}$, taking advantage of Cauchy's classical formula, until the rate of explosion of $F$ up to the edge is dominated. The uniqueness property (iii) requires analytic disc methods (Part V). 
Boundary values in the $L^{\mathrm{p}}$ sense requires special attention. At first, remind that a function $F$ holomorphic in the unit disc $\Delta$ belongs to the Hardy class $H^{\mathrm{p}}(\Delta)$ if the supremum:

$$
\|F\|_{H^{\mathrm{p}}(\Delta)}:=\sup _{0<r<1}\left(\int_{-\pi}^{\pi}\left|F\left(r e^{i t}\right)\right|^{\mathrm{p}}\right)^{1 / \mathrm{p}}<\infty
$$

is finite. According to Fatou and Privalov, such a function $F$ has radial boundary values $f\left(e^{i t}\right):=\lim _{r \rightarrow 1} F\left(r e^{i t}\right)$, for almost every $t \in[-\pi, \pi]$, so that the boundary value $f$ belongs to $L^{\mathrm{p}}([-\pi, \pi])$. Furthermore, if $1 \leqslant$ $p<\infty$ :

$$
\lim _{r \rightarrow 1} \int_{-\pi}^{\pi}\left|F\left(r e^{i t}\right)-f\left(e^{i t}\right)\right|^{\mathrm{p}}=0 .
$$

Consider a bounded domain $D \subset \mathbb{C}^{n}$ having boundary of class at least $\mathcal{C}^{2}$, defined by $D=\left\{z \in \mathbb{C}^{n}: \rho(z)<0\right\}$, with $\rho \in \mathcal{C}^{2}$ satisfying $d \rho \neq 0$ on $\partial D$. For $\varepsilon>0$ small, let $D_{\varepsilon}:=\{z \in D: \rho(z)<-\varepsilon\}$. The induced Euclidean measure on $\partial D_{\varepsilon}$ (resp. $\partial D$ ) is denoted by $d \sigma_{\varepsilon}$ (resp. $d \sigma$ ). Then the Hardy space $H^{\mathrm{p}}(D)$ consists of holomorphic functions $F \in \mathcal{O}(D)$ having the property that the supremum:

$$
\|F\|_{H^{\mathfrak{p}}(D)}:=\sup _{\varepsilon>0}\left(\int_{\partial D_{\varepsilon}}|F(z)|^{\mathfrak{p}} d \sigma_{\varepsilon}(z)\right)^{1 / \mathrm{p}}<\infty
$$

is finite. The resulting space does not depend on the choice of a defining function $\rho$ ([St1972] $]$. Let $\mathbf{n}_{z}$ be the outward-pointing normal to the boundary at $z \in \partial D$.

Theorem 4.32. ([St1972]]) If $F \in H^{\mathrm{p}}(D)$, for almost all $z \in \partial D$, the normal boundary value $f(z):=\lim _{\varepsilon \rightarrow 0} F\left(z-\varepsilon \mathbf{n}_{z}\right)$ exists and defines $a$ function $f$ which belongs to $L^{\mathrm{p}}(\partial D)$. Furthermore, if $1 \leqslant \mathrm{p}<\infty$ :

$$
\lim _{\varepsilon \rightarrow 0} \int_{\partial D}\left|F\left(z-\varepsilon \mathbf{n}_{z}\right)-f(z)\right|^{\mathrm{p}} d \sigma(z)=0 .
$$

In arbitrary codimension, the notion of $L^{\mathrm{p}}$ boundary values may be defined in the local sense as follows. Let $M$ be generic, let $p \in M$ and let $\mathcal{W}=\mathcal{W}(\rho, \sigma, C)$ be a local wedge of edge $M$ at $p$, as defined by (4.30). A holomorphic function $F \in \mathcal{O}(\mathcal{W})$ belongs to the Hardy space $H_{\text {loc }}^{\mathrm{p}}(\mathcal{W})$ if for every cone $C^{\prime} \subset \mathbb{R}^{d}$ with $C^{\prime} \cap S^{d-1} \subset \subset C \cap S^{d-1}$ and every $\rho^{\prime}<\rho$, the supremum:

$$
\sup _{\theta^{\prime} \in C^{\prime}} \int_{\Delta_{\rho^{\prime}}^{m} \times \square_{\rho^{\prime}}^{d}}\left|F\left(x+i y, u+i \varphi(x, y, u)+i \theta^{\prime}\right)\right|^{\mathrm{p}} d x \wedge d y \wedge d u<\infty
$$


is finite. Up to shrinking cubes, polydiscs and cones, the resulting space neither depends on local coordinates nor on the choice of local defining equations.

Theorem 4.33. $(d=1$ : [St1972, Jö1999b]; $d \geqslant 2$ : [Po1997]) If $F \in$ $H_{\text {loc }}^{\mathrm{p}}(\mathcal{W})$, for almost $(x, y, u+i \varphi(x, y, u)) \in M_{\rho}$ and for every cone $C^{\prime}$ with $C^{\prime} \cap S^{d-1} \subset \subset C \cap S^{d-1}$, the boundary value:

$$
f(x, y, u):=\lim _{C^{\prime} \ni \theta^{\prime} \rightarrow 0} F\left(x+i y, u+i \varphi(x, y, y)+i \theta^{\prime}\right)
$$

exists and defines a function $f$ which belongs to $L_{l o c, C R}^{\mathrm{p}}\left(M_{\rho}\right)$. Furthermore, if $1 \leqslant \mathrm{p}<\infty$, for every $\rho^{\prime}<\rho$ :

$$
\begin{aligned}
\lim _{C^{\prime} \ni \theta^{\prime} \rightarrow 0} \int_{\Delta_{\rho^{\prime}}^{m} \times \square_{\rho^{\prime}}^{d}} \mid F\left(x+i y, u+i \varphi(x, y, y)+i \theta^{\prime}\right)- & \\
& -\left.f(x, y, u)\right|^{\mathrm{p}} d x \wedge d y \wedge d u=0 .
\end{aligned}
$$

4.34. Holomorphic extendability of $\mathbf{C R}$ functions in $\mathcal{C}^{\kappa, \alpha}, \mathcal{D}^{\prime}, L_{l o c}^{\mathrm{p}}$. In Part V, we will study sufficient conditions for the existence of wedges to which CR functions and distributions extend holomorphically.

Definition 4.35. A CR function of class $\mathcal{C}^{\kappa, \alpha}$ or $L_{l o c}^{\mathrm{p}}(1 \leqslant \mathrm{p}<\infty)$ or a CR distribution $f$ defined on $M$ is holomorphically extendable if there exists a local wedge $\mathcal{W}=\mathcal{W}(\rho, \sigma, C)$ at $p$ and a holomorphic function $F \in \mathcal{O}(\mathcal{W})$ whose boundary value $\mathrm{b}_{M} F$ equals $f$ on $M_{\rho}$ in the $\mathcal{C}^{\kappa, \alpha}, L^{\mathrm{p}}$ or $\mathcal{D}^{\prime}$ sense.

4.36. Local CR distributions supported by a local CR orbit. Assume now that $M$, of class $\mathcal{C}^{\infty}$ and represented as in $\S 4.3$, is not locally minimal at $p$. Equivalently, $\mathcal{O}_{C R}^{\text {loc }}(M, p)$ is of dimension $2 m+e \leqslant 2 m+d-1$. In a small neighborhood, $S:=\mathcal{O}_{C R}^{\text {loc }}(M, p)$ is a closed connected CR submanifold of $M$ passing through $p$ and having the same CR dimension as $M$. There exist local holomorphic coordinates $(z, w)=\left(z, w_{1}, w_{2}\right) \in \mathbb{C}^{m} \times$ $\mathbb{C}^{e} \times \mathbb{C}^{d-e}$ vanishing at $p$ in which $M$ is represented by $v=\varphi(x, y, u)$ and $S$ is represented by the supplementary (scalar) equation(s) $u_{2}=\lambda_{2}\left(x, y, u_{1}\right)$, with $\varphi$ and $\lambda_{2}$ of class $\mathcal{C}^{\infty}$ satisfying $\varphi(0)=0, d \varphi(0)=0, \lambda_{2}(0)=0$ and $d \lambda_{2}(0)=0$. According to Theorem 4.2, the assumption that $S$ is CR and has the same CR dimension as $M$ may be expressed as follows.

Proposition 4.37. Decomposing $\varphi=\left(\varphi_{1}, \varphi_{2}\right)$ and defining:

$$
\begin{aligned}
& v_{1}=\varphi_{1}\left(x, y, u_{1}, \lambda_{2}\left(x, y, u_{1}\right)\right)=: \mu_{1}\left(x, y, u_{1}\right), \\
& v_{2}=\varphi_{2}\left(x, y, u_{1}, \lambda_{2}\left(x, y, u_{1}\right)\right)=: \mu_{2}\left(x, y, u_{1}\right) .
\end{aligned}
$$

the map:

$$
\psi_{2}\left(x, y, u_{1}\right):=\lambda_{2}\left(x, y, u_{1}\right)+i \mu_{2}\left(x, y, u_{1}\right)
$$


is $C R$ on the generic submanifold $v_{1}=\mu_{1}\left(x, y, u_{1}\right)$ of $\mathbb{C}^{m} \times \mathbb{C}^{e}$.

In a small neighborhood $U$ of $p$, the restrictions

$$
\left.d z_{1}\right|_{S}, \ldots,\left.d z_{m}\right|_{S},\left.d w_{1}\right|_{S}, \ldots,\left.d w_{e}\right|_{S}
$$

span an $(m+e)$-dimensional subbundle of $\mathbb{C} T^{*} S$. Denoting $d z:=d z_{1} \wedge$ $\cdots \wedge d z_{m}, d \bar{z}:=d \bar{z}_{1} \wedge \cdots \wedge d \bar{z}_{m}$ and $d w^{\prime}:=d w_{1} \wedge \cdots \wedge d w_{e}$, for $\chi \in$ $\mathcal{C}_{c}^{\infty}(U, \mathbb{C})$, consider the (localized) distribution defined by:

$$
\langle[\mathrm{S}], \chi\rangle:=\int_{U \cap S} \chi \cdot d z \wedge d w^{\prime} \wedge d \bar{z}
$$

Proposition 4.38. ([[Trv1992, HT1993]) Then [S] is a nonzero local CR measure supported by $S \cap U$.

Proof. It is clear that $[\mathrm{S}]$ is supported by $S \cap U$ and is of order zero. Let the $(0,1)$ vector fields $\bar{L}_{k}$ and the complex-transversal ones $K_{j}$ be as in $\S 4.3$. Reminding $d \chi=\sum_{k=1}^{m} L_{k}(\chi) d z_{k}+\sum_{k=1}^{m} \bar{L}_{k}(\chi) d \bar{z}_{k}+$ $\left.\sum_{j=1}^{d} K_{j}(\chi) d w_{j}\right|_{M}$, we observe:

$\bar{L}_{k}(\chi) d z \wedge d w^{\prime} \wedge d \bar{z}= \pm d\left(\chi \cdot d z \wedge d w^{\prime} \wedge d \bar{z}_{1} \wedge \cdots \wedge \widehat{d \bar{z}}_{k} \wedge \cdots \wedge d \bar{z}_{m}\right)$.

Replacing this volume form in the integrand:

$$
\begin{aligned}
\left\langle\bar{L}_{k}[\mathrm{~S}], \chi\right\rangle & :=-\left\langle[\mathrm{S}], \bar{L}_{k}(\chi)\right\rangle=-\int_{S \cap U} \bar{L}_{k}(\chi) d z \wedge d w^{\prime} \wedge d \bar{z} \\
& = \pm \int_{S \cap U} d\left(\chi \cdot d z \wedge d w^{\prime} \wedge d \bar{z}_{1} \wedge \cdots \wedge \widehat{d \bar{z}}_{k} \wedge \cdots \wedge d \bar{z}_{m}\right)
\end{aligned}
$$

and applying Stokes' theorem, we deduce $\left\langle\bar{L}_{k}[\mathrm{~S}], \chi\right\rangle=0$, i.e. $[\mathrm{S}]$ is CR.

The last assertion of Theorem 4.31 and the vanishing of $[S]$ on the dense open set $U \backslash(S \cap U)$ entails the following.

Corollary 4.39. ([Trv1992, HT1993]) The nonzero local CR measure [S] does not extend holomorphically to any local wedge of edge $M$ at $p$.

By means of this wedge nonextendable CR measure, one may construct non-extendable CR functions of arbitrary smoothness. Indeed, let $M$ be a local generic submanifold with central point $p$, as represented in $\S 4.3$ and let $K_{j}$ be the complex-transversal vector fields satisfying $K_{j_{1}}\left(w_{j_{2}}\right)=\delta_{j_{1}, j_{2}}$ and $\left[K_{j_{1}}, K_{j_{2}}\right]=0$. 
Proposition 4.40. ([BT1981, Trv1981, BR1990, Trv1992, HT1996, BER1999]) For every $C R$ distribution $\mathrm{T}$ on $M$ and every $\kappa \in \mathbb{N}$, there exist an integer $\mu \in \mathbb{N}$ and a local $C R$ function $f$ of class $\mathcal{C}^{\kappa}$ defined in some neighborhood of $p$ such that:

$$
\mathrm{T}=\left(K_{1}^{2}+\cdots+K_{d}^{2}\right)^{\mu} f .
$$

Then with $\mathrm{T}:=[\mathrm{S}]$ and for $\kappa \in \mathbb{N}$, an associated CR function $f$ of class $\mathcal{C}^{\kappa}$ is also shown to be not holomorphically extendable to any local wedge of edge $M$ at $p$. A Baire category argument ([BR1990]) enables to treat the $\mathcal{C}^{\infty}$ case.

Theorem 4.41. ([BR1990, BER1999]) If $M$ is not locally minimal at $p$, then for every $\kappa=0,1,2, \ldots, \infty$, there exists a CR function $h$ of class $\mathcal{C}^{\kappa}$ defined in a neighborhood of $p$ which does not extend holomorphically to any local wedge of edge $M$ at $p$.

Open problem 4.42. Find criteria for the existence of $C R$ distributions or functions supported by a global CR orbit.

In [BM1997], this question is dealt with in the case of CR orbits of hypersurfaces which are immersed or embedded complex manifolds.

To conclude this section, we give the general form of a CR distribution supported by a local CR orbit $S=\mathcal{O}_{C R}^{\text {loc }}(M, p)$. After restriction to $S$, the collection $K_{S}:=\left(K_{e+1}, \ldots, K_{d}\right)$ of vector fields spans the normal bundle to $S$ in $M$, in a neighborhood of $p$. Let T be a local CR distribution defined on $M$ that is supported by $S$.

Theorem 4.43. ([Trv1992, $\mathrm{BCH} 2005])$ ) There exist an integer $\kappa \in \mathbb{N}$, and for all $\beta \in \mathbb{N}^{d-e}$ with $|\beta| \leqslant \kappa$, local CR distributions $T_{\beta}^{S}$ defined on $S$ such that:

$$
\langle\mathrm{T}, \chi\rangle=\sum_{\beta \in \mathbb{N}^{d-e},|\beta| \leqslant \kappa}\left\langle\mathrm{T}_{\beta}^{S},\left.\left(K_{S}\right)^{\beta}(\chi)\right|_{S}\right\rangle .
$$

\section{$\S 5$. APPROXIMATION AND UNIQUENESS PRINCIPLES}

5.1. Approximation of $\mathbf{C R}$ functions and of $\mathbf{C R}$ distributions. Let $M$ be a generic submanifold of $\mathbb{C}^{n}$. The following approximation theorem has appeared to be a fundamental tool in extending CR functions holomorphically (Part V) and in removing their singularities (Part VI). It is also used naturally in the proof of Theorem 4.43 just above as well as in the Cauchy uniqueness principle Corollary 5.4 below. The statement is valid in the general context of locally integrable structures $\mathcal{L}$, but, as explained 
in the end of Section 3, we decided to focus our attention on embedded Cauchy-Riemann geometry.

Theorem 5.2. ([BT1981, HM1998, 『ö1999b, BCH2005]) For every $p \in$ $M$, there exists a neighborhood $U_{p}$ of $p$ in $M$ such that for every function $f$ or distribution $\mathrm{T}$ as defined below, there exists a sequence of holomorphic polynomials $\left(P_{k}(z)\right)_{k \in \mathbb{N}}$ with:

- if $M$ is $\mathcal{C}^{\kappa+2, \alpha}$, with $\kappa \geqslant 0,0 \leqslant \alpha \leqslant 1$, if $f$ is a CR function of class $\mathcal{C}^{\kappa, \alpha}$ on $M$, then $\lim _{k \rightarrow \infty}\left\|P_{k}-f\right\|_{\mathcal{C}^{\kappa, \alpha}\left(U_{p}\right)} \rightarrow 0$; in particular, continuous $C R$ functions on a $\mathcal{C}^{2}$ generic submanifold are approximable sharply by holomorphic polynomials;

- if $M$ is at least $\mathcal{C}^{2}$, if $f$ is a $L_{\text {loc }}^{\mathrm{p}}$ CR function $(1 \leqslant \mathrm{p}<\infty)$, then $\lim _{k \rightarrow \infty}\left\|P_{k}-f\right\|_{L_{l o c}^{\mathrm{p}}\left(U_{p}\right)} \rightarrow 0$;

- if $M$ is $\mathcal{C}^{\kappa+2}$, if $\mathrm{T}$ is a CR distribution of order $\leqslant \kappa$ on $M$, then $\lim _{k \rightarrow \infty}\left\langle P_{k}, \chi\right\rangle=\langle\mathrm{T}, \chi\rangle$ for every $\chi \in \mathcal{C}_{c}^{\infty}\left(U_{p}\right)$.

In [HM1998, BCH2005], convergence in Besov-Sobolev spaces $L_{s, l o c}^{\mathrm{p}}$ and in Hardy spaces $h^{\mathrm{p}}$, frequently used as substitutes for the $L^{\mathrm{p}}$ spaces when $0<\mathrm{p}<1$, is also considered, in the context of locally integrable structure.

Proof. Let us describe some ideas of the proof, assuming for simplicity that $M$ is $\mathcal{C}^{2}$ and $f$ is $\mathcal{C}^{1}$. In coordinates $\left(t_{1}, \ldots, t_{n}\right)$ vanishing at $p$, choose a local maximally real $\mathcal{C}^{2}$ submanifold $\Lambda_{0}$ contained in $M$, passing through $p$ and satisfying $T_{p} \Lambda_{0}=\{\operatorname{Re} t=0\}$. Let $V_{p}$ be a small neighborhood of $p$, whose projection to $T_{p} M$ is a $(2 m+d)$-dimensional open ball. We may assume that $\Lambda_{0}$ is contained in $V_{p}$ with boundary $B_{0}:=\overline{\Lambda_{0}} \cap \partial V_{p}$ being diffeomorphic to the $(n-1)$-dimensional sphere. Consider a parameter $u \in \mathbb{R}^{d}$ satisfying $|u|<\delta$, with $\delta>0$ small. We may include $\Lambda_{0}$ in a family $\left(\Lambda_{u}\right)_{|u|<\delta}$ of maximally real $\mathcal{C}^{2}$ submanifolds of $U_{p}$ with $\left.\Lambda_{u}\right|_{u=0}=$ $\Lambda_{0}$, whose boundary is fixed: $\partial \Lambda_{u} \equiv \partial \Lambda_{0}=B_{0}$, such that the $\Lambda_{u}$ foliates a small neighborhood $U_{p}$ of $p$ in $M$. For $t \in U_{p}$, there exists a $u=u(t)$ such that $t$ belongs to $\Lambda_{u(t)}$. We then introduce the entire functions:

$$
F_{k}(t):=\left(\frac{k}{\pi}\right)^{n / 2} \int_{\Lambda_{u(t)}} e^{-k(t-\tau)^{2}} f(\tau) d \tau_{1} \wedge \cdots \wedge d \tau_{n},
$$

where $(t-\tau)^{2}:=\sum_{j=1}^{n}\left(t_{j}-\tau_{j}\right)^{2}$ and where $k \in \mathbb{N}$. Shrinking $V_{p}$ and $U_{p}$ if necessary, we may assume that $|\operatorname{Im}(t-\tau)| \leqslant \frac{1}{2}|\operatorname{Re}(t-\tau)|$ for all $t, \tau \in \Lambda_{u} \cap U_{p}$ and all $|u|<\delta$. Here, the $\mathcal{C}^{2}$-smoothness assumption is 
used. With this inequality, the above multivariate Gaussian kernel is easily seen to be an approximation of the Dirac distribution at $\tau=t$ on $\Lambda_{u(t)}$. Consequently $F_{k}(t)$ tends to $f(t)$ as $k \rightarrow \infty$. Moreover, the convergence is uniform and holds in $\mathcal{C}^{0}\left(U_{p}\right)$.

We claim that the assumption that $f$ is $\mathrm{CR}$ insures that $F_{k}(t)$ has the same value if the integration is performed on $\Lambda_{0}$ :

$$
F_{k}(t)=\left(\frac{k}{\pi}\right)^{n / 2} \int_{\Lambda_{0}} e^{-k(t-\tau)^{2}} f(\tau) d \tau_{1} \wedge \cdots \wedge d \tau_{n} .
$$

Indeed, $\Lambda_{u(t)}$ and $\Lambda_{0}$ bound a $(n+1)$-dimensional submanifold $\Pi_{t}$ contained in $V_{p}$ with $\partial \prod_{t}=\Lambda_{u(t)}-\Lambda_{0}$. Since $e^{-k(t-\tau)^{2}}$ is holomorphic with respect to $\tau$ and since $\left.d f(\tau) \wedge d \tau_{1} \wedge \cdots \wedge d \tau_{n}\right|_{M}=0$, because $f$ is $\mathcal{C}^{1}$ and CR, the $(n, 0)$ form $\omega:=e^{-k(t-\tau)^{2}} f(\tau) d \tau=0$ is closed: $d \omega=0$. By an application of Stokes' theorem, it follows that $0=\int_{\Pi_{t}} d \omega=\int_{\Lambda_{u(t)}} \omega-\int_{\Lambda_{0}} \omega$, which proves the claim.

Finally, to approximate $f$ by polynomials on $U_{p}$ in the $\mathcal{C}^{0}$ topology, in the above integral (5.3) that is performed on the fixed maximally real submanifold $\Lambda_{0}$, it suffices to develop the exponential in Taylor series and to integrate term by term. In other functional spaces, the arguments have to be adapted.

As a consequence, uniqueness in the Cauchy problem holds. It may be shown ([Trv1981, Trv1992]) that the trace of a CR distribution on a maximally real submanifold always exists, in the distributional sense.

Corollary 5.4. ([[Trv1981, Trv1992]]) If a CR function or distribution vanishes on a maximally real submanifold $\Lambda$ of $M$, there exists an open neighborhood $U_{\Lambda}$ of $\Lambda$ in $M$ in which it vanishes identically.

Since every submanifold $H$ of $M$ which is generic in $\mathbb{C}^{n}$ contains small maximally real sumanifolds passing through every of its points, the corollary also holds with $\Lambda$ replaced by such a $H$.

Proof. It suffices to localize the above construction in a neighborhood of an arbitrary point $p \in \Lambda$ and to take for $\Lambda_{0}$ a neighborhood of $p$ in $\Lambda$. The integral (5.3) then vanishes identically.

Corollary 5.5. ([[Trv1981, Trv1992]]) The support of a CR function or distribution on $M$ is a closed CR-invariant subset of $M$.

Proof. By contraposition, if a CR function or distribution vanishes in a neighborhood $U_{p}$ of a point $p$ in $M$, it vanishes identically in the $C R$ invariant hull of $U_{p}$, viz the union of CR orbits of all points $q \in U_{p}$. The 
$\mathrm{CR}$ orbits being covered by concatenations of $\mathrm{CR}$ vector fields, neglecting some technicalities, the main step is to establish:

Lemma 5.6. Let $p \in M$, let $L$ be a section of $T^{c} M$ and let $q^{*}=$ $\exp \left(\mathrm{s}^{*} L\right)(p)$ for some $\mathrm{s}^{*} \in \mathbb{R}$. If a CR function or distribution vanishes in a neighborhood of $p$, it vanishes also in a neighborhood of $q$.

Indeed, we may construct a one-parameter family $\left(H_{\mathrm{s}}\right)_{0 \leqslant s \leqslant s^{*}}$ of $\mathcal{C}^{2}$ hypersurfaces of $M$ with $q^{*} \in H_{\mathrm{s}^{*}}$ and with $H_{0}$ contained in a small neighborhood of $p$ at which the CR function of distribution vanishes already. As illustrated by the following diagram, we can insure that at every point $q_{\mathrm{s}}=\exp (\mathrm{s} L)(p)$, the vector $L\left(q_{\mathrm{s}}\right)$ is nontangent to $H_{\mathrm{s}}$.

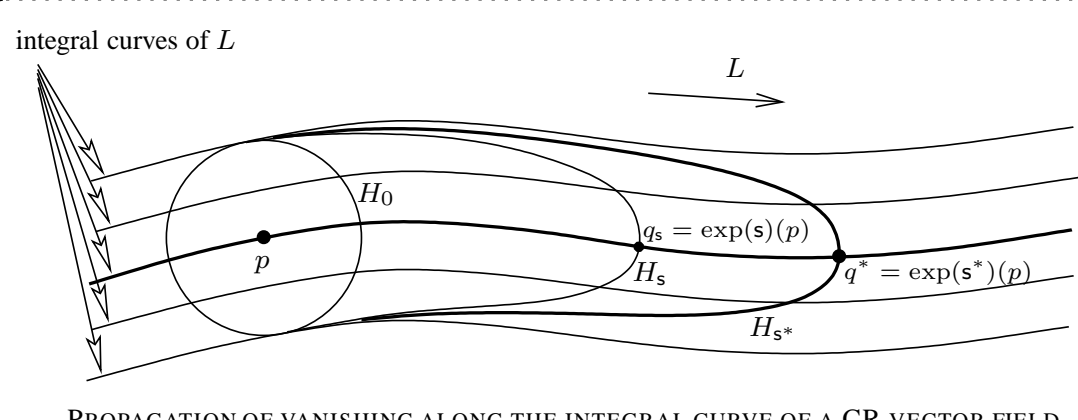

It follows that the hypersurfaces $H_{\mathrm{s}}$ are generic in $\mathbb{C}^{n}$, for every s. Then the phrase after Corollary 5.4 applies to each $H_{\mathrm{s}}$ from $H_{0}$ up to $H_{\mathrm{s}^{*}}$, showing the propagation of vanishing.

5.7. Unique continuation principles. At least three unique continuation properties are known to be enjoyed by holomorphic functions $h$ of several complex variables defined in a domain $D \subset \mathbb{C}^{n}$. Indeed, we have $h \equiv 0$ in either of the following three cases:

(ucp1) the restriction of $h$ to some nonempty open subset of $D$ vanishes identically;

(ucp2) the restriction of $h$ to some generic local submanifold $\Lambda$ of $D$ vanishes identically;

(ucp3) there exists a point $p \in D$ at which the infinite jet of $h$ vanishes.

In Complex Analysis and Geometry, the (ucpi) have deep influence on the whole structure of the theory. Finer principles involving tools from Harmonic Analysis appear in [MP2006b. 
Problem 5.8. Find generalizations of the (ucpi) to the category of embedded generic submanifolds $M$.

Since a domain $D$ of $\mathbb{C}^{n}$ trivially consists of a single CR orbit, it is natural to assume that the given generic manifold $M$ is globally minimal (although some meaningful questions arise without this assumption, we prefer not to enter such technicalities). In this setting, Corollaries 5.4 and 5.5 provide a complete generalization of (ucp1) and of (ucp2).

A version of (ucp3) with the point $p$ in the boundary $\partial D$ does not hold, even in complex dimension one. Indeed, the function $\exp \left(e^{i 5 \pi / 4} / \sqrt{w}\right)$ is holomorphic in $\mathbb{H}^{+}:=\{w \in \mathbb{C}: \operatorname{Re} w>0\}$, of class $\mathcal{C}^{\infty}$ on $\overline{\mathbb{H}}^{+}$and flat at $w=0$. The restriction of this function to the Heisenberg sphere $\operatorname{Re} w=z \bar{z}$ of $\mathbb{C}^{2}$ provides a CR example.

To generalize rightly (ucp3), let $M$ be a $\mathcal{C}^{1}$ generic submanifold of codimension $d \geqslant 1$ and of CR dimension $m \geqslant 1$ in $\mathbb{C}^{n}$, with $n=m+d$. Let $\Sigma$ be a $\mathcal{C}^{1}$ submanifold of $M$ satisfying:

$$
T_{q}^{c} M \oplus T_{q} \Sigma=T_{q} M, \quad q \in \Sigma .
$$

Here, $\Sigma$ plays the rôle of the point $p$ in (ucp3). Denote by $\mathcal{O}_{C R}^{\Sigma}$ the union of CR orbits of points of $\Sigma$, i.e. the $C R$-invariant hull of $\Sigma$. It is an open subset of $M$. We say that a $\mathrm{CR}$ function $f: M \rightarrow \mathbb{C}$ of class $\mathcal{C}^{1}$ vanishes to infinite order along $\Sigma$ if for every $p \in \Sigma$, there exists an open neighborhood $U_{p}$ of $p$ in $M$ such that for every $\nu \in \mathbb{N}$, there exists a constant $C>0$ with

$$
|h(t)| \leqslant C[\operatorname{dist}(t, \Sigma)]^{\nu}, \quad t \in U_{p} .
$$

Theorem 5.9. ([Ro1986b, BT1988], [*]) Assume that $\Sigma$ is the intersection with $M$ of some d-dimensional holomorphic submanifold of $\mathbb{C}^{n}$. If a $C R$ function of class $\mathcal{C}^{1}$ vanishes to infinite order along $\Sigma$, then it vanishes identically on the globally minimal generic submanifold $M$.

Assuming that $\Sigma$ is only a conic $d$-codimensional holomorphic submanifold entering a wedge to which all CR functions of $M$ extend holomorphically (Theorem 3.8(V)), the proof of this theorem may be easily generalized.

Open question 5.10. ([Ro1986b, BT1988]) Is the above unique continuation true for $\Sigma$ merely $\mathcal{C}^{1}$ ?

To attack this question, one should start with $M$ being unit sphere $S^{3} \subset$ $\mathbb{C}^{2}$ and $\Sigma \subset S^{3}$ being any $T^{c} S^{3}$-transversal real segment which is nowhere locally the boundary of a complex curve lying inside the ball. 


\title{
IV: Hilbert transform and Bishop's equation in Hölder spaces
}

\author{
Table of contents
}

1. Hölder spaces: basic properties 112.

2. Cauchy integral, Sokhotskĭ̌-Plemelj formulas and Hilbert transform .....114. 3. Solving a local parametrized Bishop equation with optimal loss of smoothness 129.

4. Appendix: proofs of some lemmas

[ 1 diagram]

In complex and harmonic analysis, the spaces $\mathcal{C}^{\kappa, \alpha}$ of fractionally differentiable maps, called Hölder spaces, are very flexible to generate inequalities and they yield rather satisfactory norm estimates for almost all the classical singular integral operators, especially when $0<\alpha<1$. For instance, the Cauchy integral of a $\mathcal{C}^{\kappa, \alpha}$ function $f: \Gamma \rightarrow \mathbb{C}$ defined on a $\mathcal{C}^{\kappa+1, \alpha}$ Jordan curve $\Gamma$ of the complex plane produces a sectionally holomorphic function, whose boundary values from one or the either side are $\mathcal{C}^{\kappa, \alpha}$ on the curve. The Sokhotskii-Plemelj formulas show that the arithmetic mean of the two (in general different) boundary values at a point of the curve is given by the principal value of the Cauchy integral at that point.

Harmonic and Fourier analysis on the unit disc $\Delta$ is of particular interest for geometric applications in Cauchy-Riemann geometry. According to a theorem due to Privalov, the Hilbert transform $\mathrm{T}$ is a bounded linear endomorphism of $\mathcal{C}^{\kappa, \alpha}(\partial \Delta, \mathbb{R})$ with norm $\|\mathrm{T}\|_{\kappa, \alpha}$ equivalent to $\frac{C}{\alpha(1-\alpha)}$, for some absolute constant $C>0$. This operator produces the harmonic conjugate $\mathrm{T} u$ of any real-valued function $u: \partial \Delta \rightarrow \mathbb{R}$ on the unit circle, so that $u+i \mathrm{~T} u$ always extends holomorphically to $\Delta$. Bishop (1965), Hill-Taiani (1978), Boggess-Pitts (1985) and Tumanov (1990) formulated and solved a functional equation involving $T$ in order to find small analytic discs with boundaries contained in a generic submanifold $M$ of codimension $d$ in $\mathbb{C}^{n}$.

In a general setting, this Bishop-type equation is of the form:

$$
U\left(e^{i \theta}\right)=U_{0}-\mathrm{T}[\Phi(U(\cdot), \cdot s)]\left(e^{i \theta}\right),
$$

where $U_{0} \in \mathbb{R}^{d}$ is a constant vector, where $\Phi=\Phi\left(u, e^{i \theta}, s\right)$ is an $\mathbb{R}^{d}$-valued $\mathcal{C}^{\kappa, \alpha}$ map, with $\kappa \geqslant 1$ and $0<\alpha<1$, where $u \in \mathbb{R}^{d}$, where $e^{i \theta} \in \partial \Delta$ and where $s \in \mathbb{R}^{b}$ is an additional parameter which is useful in geometric applications. Under some explicit assumptions of smallness of $U_{0}$ and of the first order jet of $\Phi$, the general solution $U=U\left(e^{i \theta}, s, U_{0}\right)$ is of class $\mathcal{C}^{\kappa, \alpha}$ with respect to $e^{i \theta}$ and 
in addition, for every $\beta$ with $0<\beta<\alpha$, it is of class $\mathcal{C}^{\kappa, \beta}$ with respect to all the variables $\left(e^{i \theta}, s, U_{0}\right)$. These smoothness properties are optimal.

\section{HÖLDER SPACES: BASIC PROPERTIES}

1.1. Background on Hölder spaces. Let $n \in \mathbb{N}$ with $n \geqslant 1$ and let $\mathrm{x}=$ $\left(\mathrm{x}_{1}, \ldots, \mathrm{x}_{n}\right) \in \mathbb{R}^{n}$. On the vector space $\mathbb{R}^{n}$, we choose once for all the maximum norm $|x|:=\max _{1 \leqslant i \leqslant n}\left|x_{i}\right|$ and, for any "radius" $\rho$ satisfying $0<\rho \leqslant \infty$, we define the open cube $\square_{\rho}^{n}:=\left\{\mathrm{x} \in \mathbb{R}^{n}:|x|<\rho\right\}$ as a fundamental, concrete open set. For $\rho=\infty$, we identify $\square_{\infty}^{n}$ with $\mathbb{R}^{n}$.

Let $\kappa \in \mathbb{N}$ and let $\alpha \in \mathbb{R}$ with $0 \leqslant \alpha \leqslant 1$. If $\mathbb{K}=\mathbb{R}$ or $\mathbb{C}$, a scalar function $f: \square_{\rho}^{n} \rightarrow \mathbb{K}$ belongs to the Hölder class $\mathcal{C}^{\kappa, \alpha}\left(\square_{\rho}^{n}, \mathbb{K}\right)$ if, for every multiindex $\delta=$ $\left(\delta_{1}, \ldots, \delta_{n}\right) \in \mathbb{N}^{n}$ of length $|\delta| \leqslant \kappa$, the partial derivative $f_{\mathrm{x}^{\delta}}(\mathrm{x}):=\frac{\partial^{|\delta|} f}{\partial \mathrm{x}^{\delta^{1} \ldots \partial \mathrm{x}^{\delta_{n}}}}$ is continuous in $\square_{\rho}^{n}$ and if, moreover, the quantity:

$$
\|f\|_{\kappa, \alpha}:=\sum_{0 \leqslant|\delta| \leqslant \kappa} \sup _{\mathrm{x} \in \square_{\rho}^{n}}\left|f_{\mathrm{x}^{\delta}}(\mathrm{x})\right|+\sum_{|\delta|=\kappa} \sup _{\mathrm{x}^{\prime \prime} \neq \mathrm{x}^{\prime} \in \square_{\rho}^{n}} \frac{\left|f_{\mathrm{x}^{\delta}}\left(\mathrm{x}^{\prime \prime}\right)-f_{\mathrm{x}^{\delta}}\left(\mathrm{x}^{\prime}\right)\right|}{\left|\mathrm{x}^{\prime \prime}-\mathrm{x}^{\prime}\right|^{\alpha}}
$$

is finite (if $\alpha=0$, it is understood that the second sum is absent). In case $f=$ $\left(f_{1}, \ldots, f_{m}\right)$ is a $\mathbb{K}^{m}$-valued mapping, with $m \geqslant 1$, we simply define $\|f\|_{\kappa, \alpha}:=$ $\max _{1 \leqslant j \leqslant m}\left\|f_{j}\right\|_{\kappa, \alpha}$. This is coherent with the choice of the maximum norm $|\mathrm{y}|:=$ $\max _{1 \leqslant i \leqslant n}\left|\mathrm{y}_{i}\right|$ on $\mathbb{K}^{m}$. For short, such a map will be said to be $\mathcal{C}^{\kappa, \alpha}$-smooth or of class $\mathcal{C}^{\kappa, \alpha}$ and we write $f \in \mathcal{C}^{\kappa, \alpha}$. One may verify $\left\|f_{1} f_{2}\right\|_{\kappa, \alpha} \leqslant\left\|f_{1}\right\|_{\kappa, \alpha} \cdot\left\|f_{2}\right\|_{\kappa, \alpha}$ and of course $\left\|\lambda_{1} f_{1}+\lambda_{2} f_{2}\right\|_{\kappa, \alpha} \leqslant\left|\lambda_{1}\right|\left\|f_{1}\right\|_{\kappa, \alpha}+\left|\lambda_{2}\right|\left\|f_{2}\right\|_{\kappa, \alpha}$. If $\kappa=0$ and $\alpha=1$, the map $f$ is called Lipschitzian. The condition $\left|f\left(e^{i \theta^{\prime \prime}}\right)-f\left(e^{i \theta^{\prime}}\right)\right| \leqslant C \cdot\left|\theta^{\prime \prime}-\theta^{\prime}\right|$ on the unit circle was first introduced by Lipschitz in 1864 as sufficient for the pointwise convergence of Fourier series.

Thanks to a uniform convergence argument, the space $\mathcal{C}^{\kappa, \alpha}\left(\square_{\rho}^{n}, \mathbb{K}\right)$ is shown to be complete, hence it constitutes a Banach algebra. The space of functions defined on the closure $\overline{\square_{\rho}^{n}}$ also constitutes a Banach algebra. If $\alpha$ is positive, thanks to a prolongation argument, one may verify that $\mathcal{C}^{\kappa, \alpha}\left(\square_{\rho}^{n}, \mathbb{K}\right)$ identifies with the restriction $\left.\mathcal{C}^{\kappa, \alpha}\left(\overline{\square_{\rho}^{n}}, \mathbb{K}\right)\right|_{\square_{\rho}^{n}}$.

Hölder spaces may also be defined on arbitrary convex open subsets. More generally, on an arbitrary subset $\Omega \subset \mathbb{R}^{n}$, it is reasonable to define the Hölder norms $\|\cdot\|_{\kappa, \alpha}, 0<\alpha \leqslant 1$, only if $\operatorname{dist}_{\Omega}\left(\mathrm{x}^{\prime \prime}, \mathrm{x}^{\prime}\right) \leqslant C \cdot\left|\mathrm{x}^{\prime \prime}-\mathrm{x}^{\prime}\right|$ for every two points $x^{\prime \prime}, x^{\prime} \in \Omega$. This is the case for instance if $\Omega$ is a domain in $\mathbb{R}^{n}$ having piecewise $\mathcal{C}^{1,0}$ boundary.

Introducing the total order $\left(\kappa_{1}, \alpha_{1}\right) \leqslant(\kappa, \alpha)$ defined by: $\kappa_{1}<\kappa$, or: $\kappa_{1}=\kappa$ and $\alpha_{1} \leqslant \alpha$, we verify that $\mathcal{C}^{\kappa, \alpha}$ is contained in $\mathcal{C}^{\kappa_{1}, \alpha_{1}}$ and that:

- $\|f\|_{\kappa, 0} \leqslant\|f\|_{\kappa, \alpha}$ for all $\alpha$ with $0<\alpha \leqslant 1$ and for all $\kappa \in \mathbb{N}$;

- $\|f\|_{\kappa, \alpha_{1}} \leqslant 3\|f\|_{\kappa, \alpha_{2}}$ for all $\alpha_{1}, \alpha_{2}$ with $0<\alpha_{1}<\alpha_{2} \leqslant 1$ and for all $\kappa \in \mathbb{N}$; 
- $\|f\|_{\kappa, 1} \leqslant\|f\|_{\kappa+1,0}$, for all $\kappa \in \mathbb{N}$.

The first inequality above is trivial while the third follows from (1.3) below. We explain the factor 3 in the second inequality. Since $\left|x^{\prime \prime}-x^{\prime}\right|^{-\alpha_{1}} \leqslant\left|x^{\prime \prime}-x^{\prime}\right|^{-\alpha_{2}}$ only if $\left|x^{\prime \prime}-x^{\prime}\right| \leqslant 1$, we may estimate:

$$
\sup _{0<\left|x^{\prime \prime}-x^{\prime}\right| \leqslant 1} \frac{\left|f\left(x^{\prime \prime}\right)-f\left(x^{\prime}\right)\right|}{\left|x^{\prime \prime}-x^{\prime}\right|^{\alpha_{1}}} \leqslant \sup _{0<\left|x^{\prime \prime}-x^{\prime}\right| \leqslant 1} \frac{\left|f\left(x^{\prime \prime}\right)-f\left(x^{\prime}\right)\right|}{\left|x^{\prime \prime}-x^{\prime}\right|^{\alpha_{2}}} \leqslant\|f\|_{0, \alpha_{2}} .
$$

On the other hand, if $\left|x^{\prime \prime}-x^{\prime}\right|>1$, we simply apply the (not fine) inequalities:

$$
\frac{\left|f\left(\mathrm{x}^{\prime \prime}\right)-f\left(\mathrm{x}^{\prime}\right)\right|}{\left|\mathrm{x}^{\prime \prime}-\mathrm{x}^{\prime}\right|^{\alpha_{1}}} \leqslant\left|f\left(\mathrm{x}^{\prime \prime}\right)-f\left(\mathrm{x}^{\prime}\right)\right| \leqslant 2\|f\|_{0,0} \leqslant 2\|f\|_{0, \alpha_{2}} .
$$

Consequently:

$$
\|f\|_{0, \alpha_{1}}=\|f\|_{0,0}+\sup _{\mathrm{x}^{\prime \prime} \neq \mathrm{x}^{\prime}} \frac{\left|f\left(\mathrm{x}^{\prime \prime}\right)-f\left(\mathrm{x}^{\prime}\right)\right|}{\left|\mathrm{x}^{\prime \prime}-\mathrm{x}^{\prime}\right|^{\alpha_{1}}} \leqslant 3\|f\|_{0, \alpha_{2}},
$$

with a factor 3 . For general $\kappa \geqslant 1$, the desired inequality follows:

$$
\begin{aligned}
\|f\|_{\kappa, \alpha_{1}}=\|f\|_{\kappa-1,0}+\sum_{|\delta|=\kappa}\left\|f_{\chi^{\delta}}\right\|_{0, \alpha_{1}} & \leqslant\|f\|_{\kappa-1,0}+3 \sum_{|\delta|=\kappa}\left\|f_{\chi^{\delta}}\right\|_{0, \alpha_{2}} \\
& \leqslant 3\|f\|_{\kappa, \alpha_{2}} .
\end{aligned}
$$

In the sequel, sometimes, we might abbreviate $\mathcal{C}^{\kappa, 0}$ by $\mathcal{C}^{\kappa}$, a standard notation. However, we shall never abbreviate $\mathcal{C}^{0, \alpha}$ by $\mathcal{C}^{\alpha}$, in order to avoid the unpleasant ambiguity $\mathcal{C}^{1,0} \equiv \mathcal{C}^{1} \equiv \mathcal{C}^{0,1}$. Without providing proofs, let us state some fundamental structural properties of Hölder spaces. Some of them are in Kr1983.

- The inclusions $\mathcal{C}^{\lambda, \beta} \subset \mathcal{C}^{\kappa, \alpha}$ for $(\lambda, \beta)>(\kappa, \alpha)$ are all strict. For instance, on $\mathbb{R}$, the function $\chi_{\kappa, \alpha}=\chi_{\kappa, \alpha}(\mathrm{x})$ equal to zero for $\mathrm{x} \leqslant 0$ and, for $\mathrm{x} \geqslant 0$ :

$$
\chi_{\kappa, \alpha}(\mathrm{x})= \begin{cases}\mathrm{x}^{\kappa+\alpha}, & \text { if } 0<\alpha \leqslant 1, \\ \mathrm{x}^{\kappa} / \log \mathrm{x}, & \text { if } \alpha=0,\end{cases}
$$

is $\mathcal{C}^{\kappa, \alpha}$ in any neighborhood of the origin, not better.

- If $0<\alpha_{1}<\alpha$, any uniformly bounded set of functions in $\mathcal{C}^{\kappa, \alpha}$ contains a sequence of functions that converges in $\mathcal{C}^{\kappa, \alpha_{1}}$-norm to a function in $\mathcal{C}^{\kappa, \alpha_{1}}$. This is a Hölder-space version of the Arzelà-Ascoli lemma.

- For $0<\alpha \leqslant 1$, define the Hölder semi-norm (notice the wide hat):

$$
\|f\|_{\widehat{0, \alpha}}:=\sup _{\mathrm{x}^{\prime \prime} \neq \mathrm{x}^{\prime} \in \square_{\rho}^{n}} \frac{\left|f\left(\mathrm{x}^{\prime \prime}\right)-f\left(\mathrm{x}^{\prime}\right)\right|}{\left|\mathrm{x}^{\prime \prime}-\mathrm{x}^{\prime}\right|^{\alpha}} .
$$

The constants satisfy $\|c\|_{\widehat{0, \alpha}}=0$ and, of course, we have $\|f\|_{0, \alpha} \equiv\|f\|_{0,0}+\|f\|_{\widehat{0, \alpha}}$. As a function of $\alpha$, the semi-norm is logarithmically convex:

$$
\|f\|_{0, t \alpha_{1}+(1-t) \alpha_{2}}=\left(\|f\|_{\widehat{0, \alpha_{1}}}\right)^{t} \cdot\left(\|f\|_{\widehat{0, \alpha_{2}}}\right)^{1-t} .
$$

Here, $0<\alpha_{1}<\alpha_{2} \leqslant 1$ and $0 \leqslant t \leqslant 1$. 
- Importantly, if $f$ is $\mathbb{K}^{m}$-valued, if $1 \leqslant l \leqslant m$, from the Taylor integral formula:

$$
f_{l}\left(\mathrm{x}^{\prime \prime}\right)-f_{l}\left(\mathrm{x}^{\prime}\right)=\int_{0}^{1} \sum_{i=1}^{n} \frac{\partial f_{l}}{\partial \mathrm{x}_{i}}\left(\mathrm{x}^{\prime}+\mathrm{s}\left(\mathrm{x}^{\prime \prime}-\mathrm{x}^{\prime}\right)\right)\left[\mathrm{x}_{i}^{\prime \prime}-\mathrm{x}_{i}^{\prime}\right] d \mathrm{~s},
$$

follows the mean value inequality:

$$
\begin{aligned}
\left|f\left(\mathrm{x}^{\prime \prime}\right)-f\left(\mathrm{x}^{\prime}\right)\right| & =\max _{1 \leqslant l \leqslant m}\left|f_{l}\left(\mathrm{x}^{\prime \prime}\right)-f_{l}\left(\mathrm{x}^{\prime}\right)\right| \\
& \leqslant\|f\|_{\widehat{1,0}} \cdot\left|\mathrm{x}^{\prime \prime}-\mathrm{x}^{\prime}\right|,
\end{aligned}
$$

where $\mathrm{x}^{\prime \prime}, \mathrm{x}^{\prime} \in \square_{\rho}^{n}$ are arbitrary, and where

$$
\|f\|_{\widehat{1,0}}:=\max _{1 \leqslant l \leqslant m} \sum_{k=1}^{n} \sup _{|\times|<\rho}\left|f_{l, \times_{k}}(\mathrm{x})\right| .
$$

This useful inequality also holds (by definition) if $f$ is merely Lipschitzian, with $\|f\|_{\widehat{1,0}}$ replaced by $\|f\|_{\widehat{0,1}}$.

- If a function $f$ is $\mathcal{C}^{\kappa, 0}$, then for every multiindex $\delta \in \mathbb{N}^{n}$ of length $|\delta| \leqslant \kappa$, the partial derivative $f_{\mathrm{x}^{\delta}}$ is $\mathcal{C}^{\kappa-|\delta|, 0}$ and $\left\|f_{\mathrm{X}^{\delta}}\right\|_{\kappa-|\delta|, 0} \leqslant\|f\|_{\kappa, 0}$.

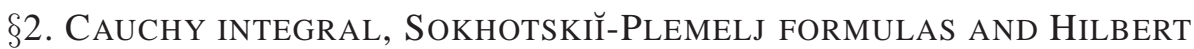 TRANSFORM}

2.1. Boundary behaviour of the Cauchy integral. Let $\Omega$ be a domain in $\mathbb{C}$, let $z \in \Omega$ and let $\Gamma$ be a $\mathcal{C}^{1}$-smooth simple closed curve surrounding $z$ and oriented counterclockwise. Assume that its interior domain (to which $z$ belongs) is entirely contained in $\Omega$. In case $\Gamma$ is a circle, Cauchy (Ca1831]) established in 1831 the celebrated representation formula:

$$
f(z)=\frac{1}{2 \pi i} \int_{\Gamma} \frac{f(\zeta) d \zeta}{\zeta-z}
$$

valid for all functions $f \in \mathcal{O}(\Omega)$ holomorphic in $\Omega$. Remarkably, $\Gamma$ may be modified and deformed without altering the value $f(z)$ of the integral.

The best proof of this formula is to derive it from the more general CauchyGreen-Pompeiu formula, itself being an elementary consequence of the GreenStokes formula, which is valid for functions $f$ of class only $\mathcal{C}^{1}$ defined on the closure of a domain $\Omega \subset \mathbb{C}$ having $\mathcal{C}^{1}$-smooth oriented boundary $\partial \Omega$ ([Ḧ1973]):

$$
f(z)=\frac{1}{2 \pi i} \int_{\partial \Omega} \frac{f(\zeta) d \zeta}{\zeta-z}+\frac{1}{2 \pi i} \iint_{\Omega} \frac{\partial f / \partial \bar{\zeta}}{\zeta-z} d \zeta \wedge d \bar{\zeta}
$$

Indeed, for holomorphic $f$, one clearly sees that the "remainder" double integral disappears. 
The holomorphicity of the kernel $\frac{1}{\zeta-z}$ enables then to build concisely the fundamental properties of holomorphic functions from Cauchy's formula: local convergence of Taylor series, residue theorem, Cauchy uniform convergence theorem, maximum principle, etc. ([Hö1973]). Studying the Cauchy integral for itself appeared therefore to be of interest and became a thoroughly investigated subject in the years 1910-1960, under the influence of Privalov.

If $z \in \Omega$ belongs to the exterior of $\Gamma$, i.e. to the unbounded component of $\mathbb{C} \backslash \Gamma$, by a fundamental theorem also due to Cauchy, the integral vanishes: $0=$ $\frac{1}{2 \pi i} \int_{\Gamma} \frac{f(\zeta) d \zeta}{\zeta-z}$. Thus, fixing the countour $\Gamma$, as $z$ moves toward $\Gamma$, the Cauchy integral is constant, either equal to $f(z)$ or to 0 . What happens when $z$ hits the curve $\Gamma$ ?

Denote by $\zeta_{0}$ a point of $\Gamma$ and by $\Delta\left(\zeta_{0}, \varepsilon\right)$ the open disc of radius $\varepsilon>0$ centered at $\zeta_{0}$. If $\Gamma_{\varepsilon}$ denotes the complement $\Gamma \backslash \Delta\left(\zeta_{0}, \varepsilon\right)$, introducing an arc of small circle contained in $\partial \Delta\left(\zeta_{0}, \varepsilon\right)$ to join the two extreme points of $\Gamma_{\varepsilon}$, it may be verified that

$$
\frac{1}{2} f\left(\zeta_{0}\right)=\lim _{\varepsilon \rightarrow 0} \frac{1}{2 \pi i} \int_{\Gamma_{\varepsilon}} \frac{f(\zeta) d \zeta}{\zeta-\zeta_{0}}
$$

Geometrically speaking, essentially one half of the circle $\partial \Delta\left(\zeta_{0}, \varepsilon\right)$ of radius $\varepsilon$ centered at $\zeta_{0}$ is contained in the domain $\Omega$. Consequently, the "correct value" of the Cauchy integral at a point $\zeta_{0}$ of the curve $\Gamma$ is equal to the arithmetic mean:

$$
\frac{1}{2}\left(\lim _{z \rightarrow \zeta_{0}, z \text { inside }}+\lim _{z \rightarrow \zeta_{0}, z \text { outside }}\right)=\frac{1}{2}\left(f\left(\zeta_{0}\right)+0\right) .
$$

Let us recall briefly why the excision of an $\varepsilon$-neighborhood of $\zeta_{0}$ in the domain of integration is necessary to provide this "correct" average value. Parametrizing $\Gamma$ by a real number, the problem of giving a sense to the singular integral $\int_{\Gamma} \frac{f(\zeta) d \zeta}{\zeta-\zeta 0}$ amounts to the following classical definition of the notion of principal value (Mu1953, Ga1966, EK2000]).

2.3. Principal value integrals. Let $a, b \in \mathbb{R}$ with $a<b$ and let $f$ be a $\mathcal{C}^{1}$ smooth real-valued function defined on the open segment $(a, b)$. Pick $x \in \mathbb{R}$ with $a<\mathrm{x}<b$ and consider the integral $\int_{a}^{b} \frac{d \mathrm{y}}{\mathrm{y}-\mathrm{x}}$ whose integrand is singular. The two integrals avoiding the singularity from the left and from the right, namely:

$$
\begin{aligned}
& \int_{a}^{\mathrm{x}-\varepsilon_{1}} \frac{d \mathrm{y}}{\mathrm{y}-\mathrm{x}}=\log \left(\varepsilon_{1}\right)-\log (\mathrm{x}-a) \quad \text { and } \\
& \int_{\mathrm{x}+\varepsilon_{2}}^{b} \frac{d \mathrm{y}}{\mathrm{y}-\mathrm{x}}=\log (b-\mathrm{x})-\log \left(\varepsilon_{2}\right)
\end{aligned}
$$

tend to $-\infty$, as $\varepsilon_{1} \rightarrow 0^{+}$, and to $+\infty$ as $\varepsilon_{2} \rightarrow 0^{+}$. Clearly, if $\varepsilon_{2}=\varepsilon_{1}$ (or more generally, if $\varepsilon_{1}$ and $\varepsilon_{2}$ both depend continuously on an auxiliary parameter $\varepsilon>0$ 
with $\left.1=\lim _{\varepsilon \rightarrow 0^{+}} \frac{\varepsilon_{2}(\varepsilon)}{\varepsilon_{1}(\varepsilon)}\right)$, the positive and the negative parts compensate, so that the principal value:

$$
\text { p.v. } \int_{a}^{b} \frac{d y}{y-x}:=\lim _{\varepsilon \rightarrow 0^{+}}\left(\int_{a}^{x-\varepsilon}+\int_{x+\varepsilon}^{b}\right)=\log \frac{b-x}{x-a}
$$

exists. Briefly, there is a key cancellation of infinite parts, thanks to the fact that the singular kernel $\frac{1}{y}$ is odd. This is why in (2.2) above, the integration was performed over the excised curve $\Gamma_{\varepsilon}$.

Generally, if $g:[a, b] \rightarrow \mathbb{R}$ is a real-valued function the principal value integral, defined by:

$$
\begin{aligned}
\text { p.v. } \int_{a}^{b} \frac{g(\mathrm{y}) d \mathrm{y}}{\mathrm{y}-\mathrm{x}} & :=\lim _{\varepsilon \rightarrow 0^{+}}\left(\int_{a}^{\mathrm{x}-\varepsilon}+\int_{\mathrm{x}+\varepsilon}^{b}\right) \\
& =\int_{a}^{b} \frac{g(\mathrm{y})-g(\mathrm{x})}{\mathrm{y}-\mathrm{x}} d \mathrm{y}+g(\mathrm{x}) \text { p.v. } \int_{a}^{b} \frac{d \mathrm{y}}{\mathrm{y}-\mathrm{x}} d \mathrm{y} \\
& =\int_{a}^{b} \frac{g(\mathrm{y})-g(\mathrm{x})}{\mathrm{y}-\mathrm{x}} d \mathrm{y}+g(\mathrm{x}) \log \frac{b-\mathrm{x}}{\mathrm{x}-a}
\end{aligned}
$$

exists whenever the quotient $\frac{g(\mathrm{y})-g(\mathrm{x})}{\mathrm{y}-\mathrm{x}}$ is integrable. This is the case for instance if $g$ is of class $\mathcal{C}^{1,0}$ or of class $\mathcal{C}^{0, \alpha}$, with $\alpha>0$, since $\int_{0}^{1} \mathrm{y}^{\alpha-1} d \mathrm{y}<\infty$. More is true.

Theorem 2.4. ("Mu1953, Ve1962, Dy1991, SME1988, EK2000], [*]) Let $g$ : $[a, b] \rightarrow \mathbb{R}$ be $\mathcal{C}^{\kappa, \alpha}$-smooth, with $\kappa \geqslant 0$ and $0<\alpha<1$. Then for every $\mathrm{x} \in(a, b)$, the principal value integral

$$
G(\mathrm{x}):=\text { p.v. } \int_{a}^{b} \frac{g(\mathrm{y}) d \mathrm{y}}{\mathrm{y}-\mathrm{x}}
$$

exists. In every closed segment $\left[a^{\prime}, b^{\prime}\right]$ contained in $(a, b)$, the function $G(\mathrm{x})$ becomes $\mathcal{C}^{\kappa, \alpha}$-smooth and enjoys the norm inequality $\|G\|_{\mathcal{C}^{\kappa, \alpha}\left[a^{\prime}, b^{\prime}\right]} \leqslant \frac{C}{\alpha(1-\alpha)}\|g\|_{\mathcal{C}^{\kappa, \alpha}[a, b]}$, for some constant $C=C\left(\kappa, a, b, a^{\prime}, b^{\prime}\right)$. If $g$ together with its derivatives up to order $\kappa$ vanish at the two extreme points a and $b$, the function $G(\mathrm{x})$ is $\mathcal{C}^{\kappa, \alpha}$-smooth over $[a, b]$ and enjoys the norm inequality $\|G\|_{\mathcal{C}^{\kappa, \alpha}[a, b]} \leqslant \frac{C}{\alpha(1-\alpha)}\|g\|_{\mathcal{C}^{\kappa, \alpha}[a, b]}$, for some constant $C=C(\kappa, a, b)$.

Notice the presence of the (nonremovable) factor $\frac{1}{\alpha(1-\alpha)}$.

2.5. General Cauchy integral. Beginning with works of Sokhotskiı̌ [So1873], of Harnack [Ha1885] and of Morera [Mo1889], the Cauchy integral transform:

$$
F(z):=\frac{1}{2 \pi i} \int_{\Gamma} \frac{f(\zeta) d \zeta}{\zeta-z}
$$

has been studied for itself, in the more general case where $\Gamma$ is an arbitrary closed or non-closed curve in $\mathbb{C}$ and $f$ is an arbitrary smooth complex-valued function 
defined on $\Gamma$, not necessarily holomorphic in a neighborhood of $\Gamma$ (precise rigorous assumptions will follow; historical account may be found in [Ga1966]). In Sokhotskiı's and in Harnack's works, the study of the boundary behaviour of the Cauchy integral was motivated by physical problems; its boundary properties find applications to mechanics, to hydrodynamics and to elasticity theory. Let us restitute briefly the connection to the notion of logarithmic potential ([Mu1953]).

Assuming $\Gamma$ and $f: \Gamma \rightarrow \mathbb{R}$ to be real-valued and of class at least $\mathcal{C}^{1,0}$, parametrize $\Gamma$ by arc-length $\zeta=\zeta(s)$, denote $\mathbf{r}(s):=\zeta(s)-z$ the radial vector from $z$ to $\zeta(s)$, denote $r=r(s)=|\mathbf{r}(s)|$ its euclidean norm, denote $\mathbf{t}(s):=\frac{d \mathbf{r}}{d s}$ the unit tangent vector field to $\Gamma$ and denote $\mathbf{n}(s):=\frac{d \mathbf{r}}{d s} /\left|\frac{d \mathbf{r}}{d s}\right|$ the unit normal vector field to $\Gamma$. Puting $z=x+i y$ and decomposing the Cauchy transform $F(z)=U(x, y)+i V(x, y)$ in real and imaginary parts, the two functions $U$ and $V$ are harmonic in $\mathbb{C} \backslash \Gamma$, since $F$ is clearly holomorphic there. After elementary computations, one shows that $U$ may be expressed under the form:

$$
U(x, y)=\frac{1}{2 \pi} \int_{\Gamma} f \frac{\cos (\mathbf{r}, \mathbf{n})}{r} d s,
$$

which, physically, represents the potential of a double layer with moment-density $\frac{f}{2 \pi}$. Also, $V$ may be expressed under the form:

$$
V(x, y)=\frac{1}{2 \pi} \int_{\Gamma} \frac{d f}{d s} \log r d s,
$$

which, in the case where $\Gamma$ consists of a finite number of closed Jordan curves, represents the potential of a single layer with moment-density $-\frac{1}{2 \pi} \frac{d f}{d s}$.

2.6. The Sokhotskiir-Plemelj formulas. Coming back to the mathematical study of the Cauchy integral, we shall assume that the curve $\Gamma$ over which the integration is performed is a connected curve of finite length parametrized by arc length

$$
[a, b] \ni s \longmapsto \zeta(s) \in \Gamma,
$$

where $a<b$, where $\zeta(s)$ is of class $\mathcal{C}^{\kappa+1, \alpha}$ over the closed segment $[a, b]$, and where $\kappa \geqslant 0,0<\alpha<1$. Topologically, we shall assume that $\Gamma=\zeta[a, b]$ is either:

- a Closed Jordan arc, namely $\zeta:[a, b] \rightarrow \mathbb{C}$ is an embedding;

- or a Jordan contour, namely $\zeta:(a, b) \rightarrow \mathbb{C}$ is an embedding, $\zeta(a)=$ $\zeta(b), \zeta$ extends as a $\mathcal{C}^{\kappa+1, \alpha}$-smooth map on the quotient $[a, b] /(a \sim b)$ and $\Gamma=\zeta[a, b]$ is diffeomorphic to a circle.

Various more general assumptions can be made: $\Gamma$ consists of a finite number of connected pieces, $\Gamma$ is piecewise smooth (corners appear), $\Gamma$ possesses certain cusps, $\Gamma$ is only Lipschitz, the length of $\Gamma$ is not finite, $f$ is $L^{\mathrm{P}}$-integrable, $f$ is $L_{\alpha}^{\mathrm{p}}$, i.e. $f \in L^{\mathrm{P}}(\Gamma)$ and $\int_{\Gamma}|f(s+h)-f(s)|^{\mathrm{P}} \leqslant$ Cte $|h|^{\alpha}, f$ belongs to certain Sobolev spaces, $f(\zeta) d \zeta$ is replaced by a measure $d \mu(\zeta)$, etc., but we shall not review the theory (see [Mu1953, Ve1962, Ga1966] and especially [Dy1991]). 
The natural orientation of the segment $[a, b]$ induced by the order relation on $\mathbb{R}$ enables to orient the two semi-local sides of $\Gamma$ in $\mathbb{C}$ : the region on the left to $\Gamma$ will be called the positive side ("+"), while the region to the right will be called negative ("-"). In the case where $\Gamma$ is Jordan contour, we assume that $\Gamma$ is oriented counterclockwise, so that the positive region coincides with the bounded component of $\mathbb{C} \backslash \Gamma$.

Theorem 2.7. ([Mu1953, Ve1962, Ga1966, Dy1991, SME1988, EK2000], [*]) Let $\Gamma$ be a $\mathcal{C}^{\kappa+1, \alpha}$-smooth closed Jordan arc or Jordan contour in $\mathbb{C}$ and let $f$ : $\Gamma \rightarrow \mathbb{C}$ be a $\mathcal{C}^{\kappa, \alpha}$-smooth complex-valued function.

(a) If $\Gamma^{\prime}$ is any closed portion of $\Gamma$ having no ends in common with those of $\Gamma$, then for every $\zeta_{1} \in \Gamma^{\prime}$, the Cauchy transform $F(z):=\frac{1}{2 \pi i} \int_{\Gamma} \frac{f(\zeta) d \zeta}{\zeta-z}$ possesses (a priori distinct) limits $F^{+}\left(\zeta_{1}\right)$ and $F^{-}\left(\zeta_{1}\right)$, when $z$ tends to $\zeta_{1}$ from the positive or from the negative side.

(b) These two limits $F^{+}$and $F^{-}$are of class $\mathcal{C}^{\kappa, \alpha}$ on $\Gamma^{\prime}$ with a norm estimate $\left\|F^{ \pm}\right\|_{\mathcal{C}^{\kappa, \alpha}\left(\Gamma^{\prime}\right)} \leqslant \frac{C\left(\kappa, \Gamma^{\prime}, \Gamma\right)}{\alpha(1-\alpha)}\|f\|_{\mathcal{C}^{\kappa, \alpha}(\Gamma)}$, for some positive constant where $C\left(\kappa, \Gamma^{\prime}, \Gamma\right)$.

(c) Furthermore, if $\omega_{+}^{\prime}$ and $\omega_{-}^{\prime}$ denote an upper and a lower open one-sided neighborhood $\Gamma^{\prime}$ in $\mathbb{C}$, the two functions $F^{ \pm}: \omega_{ \pm}^{\prime} \rightarrow \mathbb{C}$ defined by:

$$
\begin{cases}F^{ \pm}(z):=F(z) & \text { if } z \in \omega_{ \pm}^{\prime}, \\ F^{ \pm}(z):=F^{ \pm}\left(\zeta_{1}\right) & \text { if } z=\zeta_{1} \in \Gamma^{\prime},\end{cases}
$$

are of class $\mathcal{C}^{\kappa, \alpha}$ in $\omega_{ \pm}^{\prime} \cup \Gamma^{\prime}$, with a similar norm estimate $\left\|F^{ \pm}\right\|_{\mathcal{C}^{\kappa, \alpha}\left(\omega_{ \pm}^{\prime} \cup \Gamma^{\prime}\right)} \leqslant \frac{C_{1}\left(\kappa, \Gamma^{\prime}, \Gamma\right)}{\alpha(1-\alpha)}\|f\|_{\mathcal{C}^{\kappa, \alpha}(\Gamma)}$.

(d) Finally, at every point $\zeta_{0}$ of the curve $\Gamma$ not coinciding with its ends, $\mathrm{F}^{+}$ and $F^{-}$satisfy the two Sokhotskiri-Plemelj formulas:

$$
\left\{\begin{aligned}
F^{+}\left(\zeta_{0}\right)-F^{-}\left(\zeta_{0}\right) & =f\left(\zeta_{0}\right), \\
\frac{1}{2}\left[F^{+}\left(\zeta_{0}\right)+F^{-}\left(\zeta_{0}\right)\right] & =\text { p.v. } \frac{1}{2 \pi i} \int_{\Gamma} \frac{f(\zeta)}{\zeta-\zeta_{0}} d \zeta
\end{aligned}\right.
$$




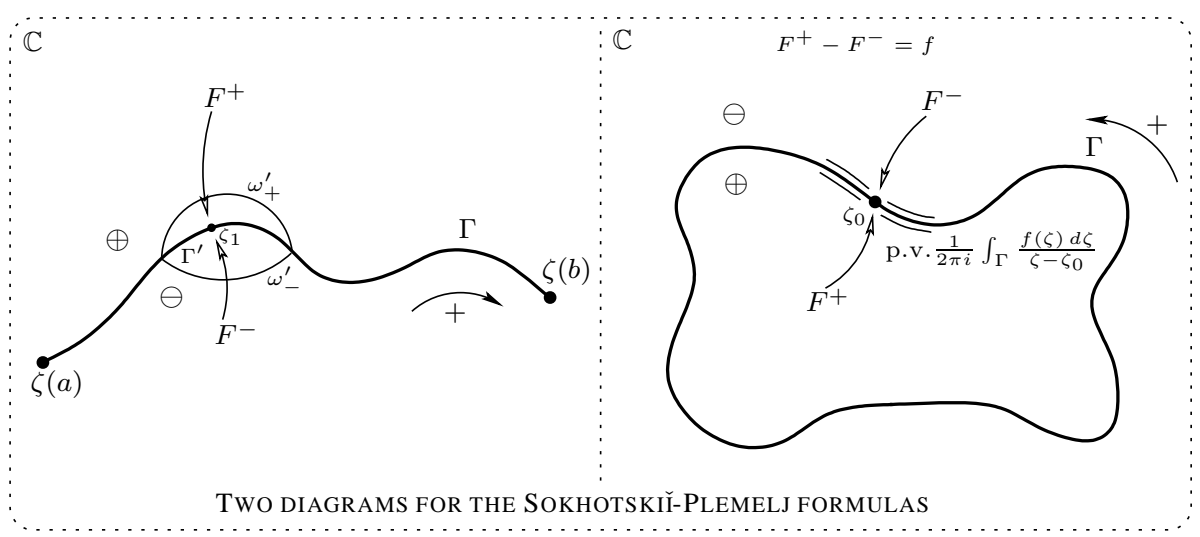

Sometimes, $F$ is called sectionnally holomorphic, as it is discontinuous across $\Gamma$. Its jump across $\Gamma$ is provided by the first formula above, while the arithmetic mean $\frac{F^{+}+F^{-}}{2}$ is given by the value of the Cauchy (singular) integral at $\zeta_{0} \in \Gamma$. Morera's classical theorem ([Mo1889] $]$ ) states that if $F^{+}$and $F^{-}$match up on the interior of $\Gamma$, then the Cauchy integral is holomorphic in $\mathbb{C}$ minus the endpoints of $\Gamma$. As is known ([Sh1990]), this theorem is also true for an arbitrary holomorphic function $F \in \mathcal{O}(\mathbb{C} \backslash \Gamma)$ which is not necessarily defined by a Cauchy integral.

2.8. Less regular boundaries. The boundary behaviour of the Cauchy transform at the two extreme points $\gamma(a)$ and $\gamma(b)$ of a Jordan arc is studied in [Mu1953]. We refer to [Dy1991] for a survey presentation of the finest condition on $\Gamma$ (namely, it to be a Carleson curve) which insures that the Cauchy integral exists and that the Sokhotskiū-Plemelj formulas hold true, almost everywhere. Let us just mention what happens with the Cauchy integral $F(z)$ in the limit case $\alpha=0$.

If $\Gamma$ is (only) $\mathcal{C}^{1,0}$, if $f$ is (only) $\mathcal{C}^{0,0}$, then for $\zeta_{1}$ in the interior of $\Gamma$, the limit $F^{-}\left(\zeta_{1}\right)$ exists if and only if the limit $F^{+}\left(\zeta_{1}\right)$ exists (Mu1953). However, generically, none limit exists.

A more useful statement, valid in the case $\alpha=0$, is as follows. Assume $\Gamma$ to be $\mathcal{C}^{\kappa+1,0}$ with $\kappa \geqslant 0$ and let $\Gamma^{\prime}$ be a closed portion of the interior of $\Gamma$. Parametrize $\Gamma^{\prime}$ by a $\mathcal{C}^{\kappa+1,0}$ map $\zeta^{\prime}:\left[a^{\prime}, b^{\prime}\right] \rightarrow \Gamma^{\prime}$. Extend $\zeta^{\prime}=\zeta^{\prime}(s)$ as a a $\mathcal{C}^{\kappa+1,0}$ embedding $\zeta^{\prime}(s, \varepsilon)$ defined on $\left[a^{\prime}, b^{\prime}\right] \times\left(-\varepsilon_{0}, \varepsilon_{0}\right)$, where $\varepsilon_{0}>0$, with $\zeta^{\prime}(s, 0) \equiv \zeta^{\prime}(s)$ and with $\zeta^{\prime}(s, \varepsilon)$ in the positive side of $\Gamma^{\prime}$ for $\varepsilon>0$. The family of curves $\Gamma_{\varepsilon}^{\prime}:=\zeta^{\prime}\left(\left[a^{\prime}, b^{\prime}\right] \times\{\varepsilon\}\right)$ foliates a strip thickening of $\Gamma^{\prime}$.

Theorem 2.9. (Mu1953]) For every choice of a $\mathcal{C}^{\kappa+1,0}$ extension $\zeta^{\prime}(s, \varepsilon)$, and every $f \in \mathcal{C}^{\kappa, 0}(\Gamma, \mathbb{C})$, the difference from either side of the Cauchy transform $\left.F\right|_{\Gamma_{\varepsilon}^{\prime}} ^{\prime^{\prime}}-\left.F\right|_{\Gamma_{-\varepsilon^{\prime}}^{\prime}}$ tends to $\left.f\right|_{\Gamma^{\prime}}$ in $\mathcal{C}^{\kappa, 0}$ norm as $\varepsilon \rightarrow 0$ :

$$
\lim _{\varepsilon \rightarrow 0} \sup _{s \in\left[a^{\prime}, b^{\prime}\right]}\left\|F\left(\zeta^{\prime}(s, \varepsilon)\right)-F\left(\zeta^{\prime}(s,-\varepsilon)\right)-f\left(\zeta^{\prime}(s)\right)\right\|_{\kappa, 0}=0 .
$$

To conclude, we state a criterion, due to Hardy-Littlewood, which insures $\mathcal{C}^{\kappa, \alpha}{ }_{-}$ smoothness of holomorphic functions up to the boundary. 
Theorem 2.10. ([Mu1953, Ga1966], [*]) Let $\Gamma$ be a $\mathcal{C}^{\kappa+1, \alpha}$-smooth Jordan contour, divinding the complex plane in two components $\Omega^{+}$(bounded) and $\Omega^{-}$(unbounded). If $f \in \mathcal{O}\left(\Omega^{ \pm}\right)$satisfies the estimate $\left|\partial_{z}^{\kappa} f(z)\right| \leqslant C(1-|z|)^{1-\alpha}$, for some $\kappa \in \mathbb{N}$, some $\alpha$ with $0<\alpha<1$, and some positive constant $C>0$, then $f$ is of class $\mathcal{C}^{\kappa, \alpha}$ in the closure $\overline{\Omega^{ \pm}}=\Omega^{ \pm} \cup \Gamma$.

2.11. Functions and maps defined on the unit circle. In the sequel, $\Omega$ will be the unit disc $\Delta:=\{\zeta \in \mathbb{C}:|\zeta|<1\}$ having as boundary the unit circle $\partial \Delta:=$ $\{\zeta \in \mathbb{C}:|\zeta|=1\}$. Consider a function $f: \partial \Delta \rightarrow \mathbb{K}$, where $\mathbb{K}=\mathbb{R}$ or $\mathbb{C}$. Parametrizing $\partial \Delta$ by $\zeta=e^{i \theta}$ with $\theta \in \mathbb{R}$, such an $f$ will be considered as the function

$$
\mathbb{R} \ni \theta \longmapsto f\left(e^{i \theta}\right) \in \mathbb{K} .
$$

For $j \in \mathbb{N}$, we shall write $f_{\theta^{j}}:=\frac{d^{j} f}{d \theta^{j}}$.

Let $\alpha$ satisfy $0<\alpha \leqslant 1$ and assume that $f \in \mathcal{C}^{0, \alpha}$. We define its $\mathcal{C}^{0, \alpha}$ seminorm (notice the wide hat) precisely by:

$$
\|f\|_{\widehat{0, \alpha}}:=\sup _{\theta^{\prime \prime} \neq \theta^{\prime}} \frac{\left|f\left(e^{i \theta^{\prime \prime}}\right)-f\left(e^{i \theta^{\prime}}\right)\right|}{\left|\theta^{\prime \prime}-\theta^{\prime}\right|^{\alpha}} .
$$

Thanks to $2 \pi$-periodicity, $\sup _{\theta^{\prime \prime} \neq \theta^{\prime}}$ may be replaced by $\sup _{0<\left|\theta^{\prime \prime}-\theta^{\prime}\right| \leqslant \pi}$. According to the definition of $\S 1.1$, the function $f$ is $\mathcal{C}^{\kappa, \alpha}$ if the quantity

$$
\|f\|_{\kappa, \alpha}:=\sum_{0 \leqslant j \leqslant \kappa}\left\|f_{\theta^{j}}\right\|_{0,0}+\left\|f_{\theta^{\kappa}}\right\|_{\widehat{0, \alpha}}<\infty
$$

is finite. Besides Hölder spaces, we shall also consider the Lebesgue spaces $L^{\mathrm{p}}(\partial \Delta)$, with $\mathrm{p} \in \mathbb{R}$ satisfying $1 \leqslant \mathrm{p} \leqslant \infty$. As $\partial \Delta$ is compact, the Hölder inequality entails the (strict) inclusions $L^{\infty}(\partial \Delta) \subset L^{\mathrm{p}^{\prime}}(\partial \Delta) \subset L^{\mathrm{p}}(\partial \Delta) \subset$ $L^{1}(\partial \Delta)$, for $1<\mathrm{p}<\mathrm{p}^{\prime}<\infty$.

2.12. Fourier series of Hölder continuous functions. If $f$ is at least of class $L^{1}$ on $\partial \Delta$, let

$$
\widehat{f}_{k}:=\frac{1}{2 \pi i} \int_{\partial \Delta} \zeta^{-k} f(\zeta) \frac{d \zeta}{\zeta}
$$

denote the $k$-th Fourier coefficient of $f$, where $k \in \mathbb{Z}$. Given $n \in \mathbb{N}$, consider the $n$-th partial sum of the Fourier series of $f$ :

$$
\mathrm{F}_{n} f\left(e^{i \theta}\right):=\sum_{-n \leqslant k \leqslant n} \widehat{f}_{k} e^{i k \theta} .
$$

We remind that Dini's (elementary) criterion:

$$
\int_{0}^{\pi} \frac{\left|f\left(e^{i(\theta+t)}\right)+f\left(e^{i(\theta-t)}\right)-2 f\left(e^{i \theta}\right)\right|}{t} d t<\infty
$$


insures the pointwise convergence $\lim _{n \rightarrow \infty} \mathrm{F}_{n} f\left(e^{i \theta}\right)=f\left(e^{i \theta}\right)$. If $f$ is $\mathcal{C}^{0, \alpha}$ on $\partial \Delta$, with $0<\alpha \leqslant 1$, the above integral obviously converges at every $e^{i \theta} \in \partial \Delta$, so that we may identify $f$ with its (complete) Fourier series:

$$
f\left(e^{i \theta}\right)=\mathrm{F} f\left(e^{i \theta}\right):=\sum_{k \in \mathbb{Z}} \widehat{f}_{k} e^{i k \theta} .
$$

In fact ([Zy1959]), if $f \in \mathcal{C}^{\kappa, \alpha}$ with $\kappa \in \mathbb{N}$ and $0 \leqslant \alpha \leqslant 1$, then $\left|\widehat{f}_{k}\right| \leqslant$ $\frac{\pi^{1+\alpha}}{|k|^{\kappa+\alpha}}\|f\|_{\widehat{\kappa, \alpha}}$ for all $k \in \mathbb{Z} \backslash\{0\}$. Also, if $f \in \mathcal{C}^{0, \alpha}$, then $\sum_{k \in \mathbb{Z}}\left|\widehat{f}_{k}\right|^{c}$ converges for $c>\frac{2}{2 \alpha+1}$. In 1913, Berster̆n proved absolute convergence of $\sum_{k \in \mathbb{Z}}\left|\widehat{f}_{k}\right|$ for $\alpha>1 / 2$.

2.13. Three Cauchy transforms in the unit disc. In the case $\Omega=\Delta$, our goal is to formulate Theorem 2.7 with more precision about the constant $C(\kappa, \partial \Omega)$. For $\eta \in \partial \Delta$ in the unit circle and $f \in \mathcal{C}^{\kappa, \alpha}(\partial \Delta, \mathbb{C})$ with $\kappa \geqslant 0,0<\alpha<1$, as in $\S 2.6$, we define:

$$
\begin{aligned}
C^{+} f(\eta) & :=\lim _{r \rightarrow 1^{-}} \frac{1}{2 \pi i} \int_{\partial \Delta} \frac{f(\zeta)}{\zeta-r \eta} d \zeta, \\
C^{0} f(\eta) & :=\text { p.v. } \frac{1}{2 \pi i} \int_{\partial \Delta} \frac{f(\zeta)}{\zeta-\eta} d \zeta, \\
C^{-} f(\eta) & :=\lim _{r \rightarrow 1^{+}} \frac{1}{2 \pi i} \int_{\partial \Delta} \frac{f(\zeta)}{\zeta-r \eta} d \zeta .
\end{aligned}
$$

The Sokhotskiī-Plemelj formulas hold: $f(\eta)=\mathrm{C}^{+} f(\eta)-\mathrm{C}^{-} f(\eta)$ and $\mathrm{C}^{0} f(\eta)=$ $\frac{1}{2}\left[\mathrm{C}^{+} f(\eta)+\mathrm{C}^{-} f(\eta)\right]$. A theorem due to Aleksandrov ${ }^{6}$ enables to obtain a precise estimate of the $\mathcal{C}^{\kappa, \alpha}$ norms of these Cauchy operators. To describe it, define:

$$
\mathcal{M}_{0}^{\alpha}:=\left\{f \in \mathcal{C}^{0, \alpha}(\partial \Delta, \mathbb{C}): \widehat{f}_{0}=0\right\} .
$$

Then $\|\cdot\|_{\widehat{0, \alpha}}$ is a norm on $\mathcal{M}_{0}^{\alpha}$, since only the constants $c$ satisfy $\|c\|_{\widehat{0, \alpha}}=0$. For $p, q \in \mathbb{R}$ with $0<p, q<1$, recall the definition $B(p, q):=\int_{0}^{1} x^{p-1}(1-$ $x)^{q-1} d x$ of the Euler beta function.

Theorem 2.14. ([Al1975]) The operator $C^{0} f(\eta):=$ p.v. $\frac{1}{2 \pi i} \int_{\partial \Delta} \frac{f(\zeta)}{\zeta-\eta} d \zeta$ is a bounded linear endomorphism of $\mathcal{M}_{0}^{\alpha}$ having norm:

$$
\left\|\mathrm{C}^{0}\right\|_{\widehat{0, \alpha}}=\frac{1}{2 \pi} B\left(\frac{\alpha}{2}, \frac{1-\alpha}{2}\right) .
$$

One may easily verify the two equivalences $B\left(\frac{\alpha}{2}, \frac{1-\alpha}{2}\right) \sim \frac{2}{\alpha}$ as $\alpha \rightarrow 0$ and $B\left(\frac{\alpha}{2}, \frac{1-\alpha}{2}\right) \sim \frac{2}{1-\alpha}$ as $\alpha \rightarrow 1$ as well as the two inequalities:

$$
\frac{1}{\alpha(1-\alpha)} \leqslant B\left(\frac{\alpha}{2}, \frac{1-\alpha}{2}\right) \leqslant \frac{4}{\alpha(1-\alpha)} .
$$

${ }^{6}$ We are grateful to Burglind Jöricke who provided the reference [Al1975]. 
Thus, the nonremovable factor $\frac{1}{\alpha(1-\alpha)}$ shows what is the precise rate of explosion of the norm $\left\|C^{0}\right\|_{\widehat{0, \alpha}}$ as $\alpha \rightarrow 0$ or as $\alpha \rightarrow 1$.

Further, if $f \in \mathcal{C}^{0, \alpha}$ does not necessarily belong to $\mathcal{M}_{0}^{\alpha}$, it is elementary to check that $\left\|C^{0} f\right\|_{0,0} \leqslant \frac{C}{\alpha}\|f\|_{0, \alpha}$, for some absolute constant $C>0$. It follows that the (complete) operator norm $\left\|C^{0}\right\|_{0, \alpha}$ behaves like $\frac{C}{\alpha(1-\alpha)}$.

In conclusion, thanks to the Sokhotski1-Plemelj formulas $\mathrm{C}^{+} f=\frac{1}{2}\left(\mathrm{C}^{0} f+f\right)$ and $\mathrm{C}^{-} f=\frac{1}{2}\left(\mathrm{C}^{0} f-f\right)$, we deduce that there exists an absolute constant $C_{1}>1$ such that:

$$
\frac{1 / C_{1}}{\alpha(1-\alpha)} \leqslant\left\|C^{\mathrm{b}}\right\|_{0, \alpha} \leqslant \frac{C_{1}}{\alpha(1-\alpha)},
$$

where $0<\alpha<1$ and where $\mathrm{b}=-, 0,+$.

Next, what happens with $f \in \mathcal{C}^{\kappa, \alpha}$, for $\kappa \in \mathbb{N}$ arbitrary ? For $\mathrm{b}=-, 0,+$, the $\mathrm{C}^{\mathrm{b}}$ are bounded linear endomorphisms of $\mathcal{C}^{\kappa, \alpha}$ and similarly:

Theorem 2.15. There exists an absolute constant $C_{1}>1$ such that if $\kappa \in \mathbb{N}$ and $0<\alpha<1$, for $\mathrm{b}=-, 0,+$ :

$$
\frac{1 / C_{1}}{\alpha(1-\alpha)} \leqslant\left\|\mathrm{C}^{\mathrm{b}}\right\|_{\kappa, \alpha} \leqslant \frac{C_{1}}{\alpha(1-\alpha)} .
$$

In other words, the constant $C_{1}$ is independent of $\kappa$. To deduce this theorem from the estimates with $\kappa=0$ (with different absolute constant $C_{1}$ ) we proceed as follows, without exposing all the rigorous details.

Inserting the Fourier series $\mathrm{F}\left(f, e^{i \theta}\right)$ in the integrals defining $C^{-}, C^{0}, C^{+}$and integrating termwise (an operation which may be justified), we get:

$$
\left\{\begin{array}{l}
\mathrm{C}^{-} f\left(e^{i \theta}\right)=-\sum_{k<0} \widehat{f}_{k} e^{i k \theta} \\
\mathrm{C}^{0} f\left(e^{i \theta}\right)=-\frac{1}{2} \sum_{k<0} \widehat{f}_{k} e^{i k \theta}+\frac{1}{2} \widehat{f}_{0}+\frac{1}{2} \sum_{k>0} \widehat{f}_{k} e^{i k \theta} \\
\mathrm{C}^{+} f\left(e^{i \theta}\right)=\widehat{f}_{0}+\sum_{k>0} \widehat{f}_{k} e^{i k \theta}
\end{array}\right.
$$

If $\kappa \geqslant 1$, by differentiating termwise with respect to $\theta$ these three Fourier representations of the $C^{b}$, we see that these operators commute with differentiation.

Lemma 2.16. For every $j \in \mathbb{N}$ with $0 \leqslant j \leqslant \kappa$ and for $\mathrm{b}=-, 0,+$, we have:

$$
\mathrm{C}^{\mathrm{b}}\left(f_{\theta^{j}}\right)=\left(\mathrm{C}^{\mathrm{b}} f\right)_{\theta^{j}} \text {. }
$$

Dealing directly with the principal value definition of $C^{0} f$, another proof of this lemma for $\mathrm{C}^{0}$ would consist in integrating by parts, deducing afterwards that $\mathrm{C}^{-}$and $\mathrm{C}^{+}$enjoy the same property, thanks to the Sokhotskiı-Plemelj formulas. 
To establish Theorem 2.15, we introduce another auxiliary $\mathcal{C}^{\kappa, \alpha}$ norm:

$$
\|f\|_{\kappa, \alpha}^{\sim}:=\sum_{0 \leqslant j \leqslant \kappa}\left\|f_{\theta^{j}}\right\|_{0, \alpha}=\|f\|_{\kappa, \alpha}+\sum_{0 \leqslant j \leqslant \kappa-1}\left\|f_{\theta^{j}}\right\|_{\widehat{0, \alpha}},
$$

which is equivalent to $\|\cdot\|_{\kappa, \alpha}$, thanks to the elementary inequalities ([*]):

$$
\|f\|_{\kappa, \alpha} \leqslant\|f\|_{\kappa, \alpha}^{\sim} \leqslant(1+\pi)\|f\|_{\kappa, \alpha} .
$$

Notice that $\|\cdot\|_{0, \alpha}^{\sim}=\|\cdot\|_{0, \alpha}$. The next lemma applies to $\mathrm{L}=\mathrm{C}^{-}, \mathrm{C}^{0}, \mathrm{C}^{+}$and to $\mathrm{L}=\mathrm{T}$, the Hilbert conjugation operator defined below.

Lemma 2.17. ([*]) Let $\mathrm{L}$ be a bounded linear endomorphism of all the spaces $\mathcal{C}^{\kappa, \alpha}(\partial \Delta, \mathbb{C})$ with $\kappa \in \mathbb{N}, 0<\alpha<1$, which commutes with differentiations, namely $\mathrm{L}\left(f_{\theta^{j}}\right)=(\mathrm{L} f)_{\theta^{j}}$, for $j \in \mathbb{N}$. Assume that there exist a contant $C_{1}(\alpha)>1$ depending on $\alpha$ such that $C_{1}(\alpha)^{-1} \leqslant\|\mathrm{~L}\|_{0, \alpha} \leqslant C_{1}(\alpha)$. Then for every $\kappa \in \mathbb{N}$ :

$$
\left\{\begin{aligned}
C_{1}(\alpha)^{-1} & \leqslant\|\mathrm{~L}\|_{\kappa, \alpha}^{\sim} \leqslant C_{1}(\alpha), \\
(1+\pi)^{-1} C_{1}(\alpha)^{-1} & \leqslant\|\mathrm{~L}\|_{\kappa, \alpha} \leqslant(1+\pi) C_{1}(\alpha) .
\end{aligned}\right.
$$

Proof. Indeed, if $f \in \mathcal{C}^{\kappa, \alpha}$, we develope a chain of (in)equalities:

$$
\begin{aligned}
\|\mathrm{L} f\|_{\kappa, \alpha} & \leqslant\|\mathrm{L} f\|_{\kappa, \alpha}^{\sim}=\sum_{0 \leqslant j \leqslant \kappa}\left\|(\mathrm{L} f)_{\theta^{j}}\right\|_{0, \alpha}=\sum_{0 \leqslant j \leqslant \kappa}\left\|\mathrm{L}\left(f_{\theta^{j}}\right)\right\|_{0, \alpha} \\
& \leqslant C_{1}(\alpha) \sum_{0 \leqslant j \leqslant \kappa}\left\|f_{\theta^{j}}\right\|_{0, \alpha}=C_{1}(\alpha)\|f\|_{\kappa, \alpha}^{\sim} \\
& \leqslant(1+\pi) C_{1}(\alpha)\|f\|_{\kappa, \alpha} .
\end{aligned}
$$

This yields the two majorations. Minorations are obtained similarly.

To conclude this paragraph, we state a Toplitz type theorem about $\mathrm{C}^{+}$, which will be crucial in solving Bishop's equation with optimal loss of smoothness, as we will see in Section 3. A similar one holds about $\mathbf{C}^{-}$, assuming $\phi \in H^{\infty}(\overline{\mathbb{C}} \backslash \bar{\Delta})$ instead, where $\overline{\mathbb{C}}$ is the Riemann sphere.

Theorem 2.18. ([Tu1994b], [*]) There exists an absolute constant $C_{1}>1$ such that for all $f \in \mathcal{C}^{\kappa, \alpha}, \kappa \in \mathbb{N}, 0<\alpha<1$, and all $\phi \in \mathrm{H}^{\infty}(\Delta):=\mathcal{O}(\Delta) \cap L^{\infty}(\Delta)$ :

$$
\left\|\mathrm{C}^{+}(f \bar{\phi})\right\|_{\kappa, \alpha} \leqslant \frac{C_{1}}{\alpha(1-\alpha)}\|f\|_{\kappa, \alpha}\|\phi\|_{L^{\infty}} .
$$

Closely related to the Cauchy transform are the Schwarz and the Hilbert transforms.

2.19. Schwarz transform on the unit disc. Let $u \in L^{1}(\partial \Delta, \mathbb{R})$ be real-valued. The Schwarz transform of $u$ is the function of $z \in \Delta$ defined by:

$$
\mathrm{S} u(z):=\frac{1}{2 \pi i} \int_{\partial \Delta} u(\zeta)\left(\frac{\zeta+z}{\zeta-z}\right) \frac{d \zeta}{\zeta} .
$$


Thanks to the holomorphicity of the kernel, $\mathrm{S} u(z)$ is a holomorphic function of $z \in \Delta$. Decomposing it in real and imaginary parts:

$$
\mathrm{S} u(z)=\mathrm{P} u(z, \bar{z})+i \mathrm{~T} u(z, \bar{z})
$$

we get the Poisson transform of $u$ :

$$
\mathrm{P} u(z, \bar{z}):=\frac{1}{2 \pi i} \int_{\partial \Delta} u(\zeta) \operatorname{Re}\left(\frac{\zeta+z}{\zeta-z}\right) \frac{d \zeta}{\zeta},
$$

together with the Hilbert transform of $u$ :

$$
\mathrm{T} u(z, \bar{z}):=\frac{1}{2 \pi i} \int_{\partial \Delta} u(\zeta) \operatorname{Im}\left(\frac{\zeta+z}{\zeta-z}\right) \frac{d \zeta}{\zeta} .
$$

Thanks to the harmonicity of the two kernels, $\mathrm{P} u$ and $\mathrm{T} u$ are harmonic in $\Delta$. The power series of $\mathrm{C} u$, of $\mathrm{P} u$ and of $\mathrm{T} u$ are given by:

$$
\left\{\begin{aligned}
\mathrm{S} u(z) & =\widehat{u}_{0}+2 \sum_{k>0} \widehat{u}_{k} z^{k}, \\
\mathrm{P} u(z, \bar{z}) & =\sum_{k<0} \widehat{u}_{k} \bar{z}^{k}+\widehat{u}_{0}+\sum_{k>0} \widehat{u}_{k} z^{k}, \\
\mathrm{~T} u(z, \bar{z}) & =\frac{1}{i}\left(-\sum_{k<0} \widehat{u}_{k} \bar{z}^{k}+\sum_{k>0} \widehat{u}_{k} z^{k}\right),
\end{aligned}\right.
$$

where $\widehat{u}_{k}$ is the $k$-th Fourier coefficient of $u$. These three series converge normally on compact subsets of $\Delta$.

2.20. Poisson transform on the unit disc. Let us first summarize the basic properties of the Poisson transform ([Ka1968, DR2002] $]$ ). Setting $z=r e^{i \theta}$ with $0 \leqslant r<1$ and $\zeta=e^{i t}$, computing $\operatorname{Re}\left(\frac{\zeta+z}{\zeta-z}\right)$ and switching the convolution integral, we obtain:

$$
\mathrm{P} u\left(r e^{i \theta}\right)=\frac{1}{2 \pi} \int_{-\pi}^{\pi} P_{r}(t) u\left(e^{i(\theta-t)}\right) d t=P_{r} * u\left(e^{i \theta}\right),
$$

where

$$
P_{r}(t):=\frac{1-r^{2}}{1-2 r \cos t+r^{2}}
$$

is the Poisson summability kernel. It has three nice properties:

- $P_{r}>0$ on $\partial \Delta$ for $0 \leqslant r<1$,

- $\frac{1}{2 \pi} \int_{-\pi}^{\pi} P_{r}(t) d t=1$ for $0 \leqslant r<1$, and:

- $\lim _{r \rightarrow 1^{-}} P_{r}(t)=0$ for every $t \in[-\pi, \pi] \backslash\{0\}$.

Consequently, $P_{r}$ is an approximation of the Dirac measure $\delta_{1}$ at $1 \in \partial \Delta$. For this reason, the Poisson convolution integral possesses excellent boundary value properties. 
Lemma 2.21. ([Ka1968, DR2002]]) Convergence in norm holds:

(i) If $u \in L^{\mathrm{p}}$ with $1 \leqslant \mathrm{p}<\infty$ or $p=\infty$ and $u$ is continuous, then $\lim _{r \rightarrow 1^{-}}\left\|P_{r} * u-u\right\|_{L^{\mathrm{p}}}=0$.

(ii) If $u \in \mathcal{C}^{\kappa, \alpha}$ with $\kappa \in \mathbb{N}$ and $0 \leqslant \alpha \leqslant 1$, including $\alpha=0$ and $\alpha=1$, then $\lim _{r \rightarrow 1^{-}}\left\|P_{r} * u-u\right\|_{\kappa, \alpha}=0$.

In $\mathcal{C}^{\kappa, \alpha}$, the pointwise convergence $\lim _{r \rightarrow 1^{-}} P_{r} * u\left(e^{i \theta}\right) \rightarrow u\left(e^{i \theta}\right)$ follows obviously. However, in $L^{\mathrm{p}}$, from convergence in norm one may only deduce pointwise convergence almost everywhere for some sequence $r_{k} \rightarrow 1$ which depends on the function. In $L^{\mathrm{p}}$, almost everywhere pointwise convergence was proved by Fatou in 1906.

Theorem 2.22. ([Fa1906, Ka1968, DR2002]) If $u \in L^{\mathrm{p}}$ with $1 \leqslant \mathrm{p} \leqslant \infty$, then for almost every $e^{i \theta} \in \partial \Delta$, we have:

$$
\lim _{r \rightarrow 1^{-}} P_{r} * u\left(e^{i \theta}\right)=u\left(e^{i \theta}\right)
$$

In summary, the Poisson transform $\mathrm{P} u$ yields a harmonic extension to $\Delta$ of any function $u \in L^{\mathrm{P}}(\partial \Delta, \mathbb{R})$ or $u \in \mathcal{C}^{\kappa, \alpha}(\partial \Delta, \mathbb{R})$, with expected boundary value $\mathrm{b}_{\partial \Delta}(\mathrm{P} u)=u$ on $\partial \Delta$.

2.23. Hilbert transform on the unit disc. Next, we survey the fundamental properties of the Hilbert transform. Again, $u$ is real-valued on $\partial \Delta$. Setting $z=r e^{i \theta}$ with $0 \leqslant r<1$ and $\zeta=e^{i t}$, computing $\operatorname{Im}\left(\frac{\zeta+z}{\zeta-z}\right)$ and switching the convolution integral, we obtain:

$$
\mathrm{T} u\left(r e^{i \theta}\right)=\frac{1}{2 \pi} \int_{-\pi}^{\pi} T_{r}(t) u\left(e^{i(\theta-t)}\right) d t
$$

where

$$
T_{r}(t):=\frac{2 r \sin t}{1-2 r \cos t+r^{2}}
$$

is the Hilbert kernel. It is not a summability kernel, being positive and negative with $L^{1}$ norm tending to $\infty$ as $r \rightarrow 1^{-}$; for this reason, the Hilbert transform does not enjoy the same nice boundary value properties as the Poisson transform: Hölder classes are needed.

Setting $r=1$, the Poisson kernel $\mathrm{P}_{1}(t)$ vanishes identically and the Hilbert kernel tends to $\frac{2 \sin t}{2-2 \cos t}=\frac{\cos t / 2}{\sin t / 2}$. Near $t=0$, the function $\cot (t / 2)$ behaves like the function $2 / t$, having infinite $L^{1}$ norm. For $u \in \mathcal{C}^{0, \alpha}(\partial \Delta, \mathbb{R})$, it may be verified that, as $z \rightarrow e^{i \theta} \in \partial \Delta$, the Hilbert transform $\mathrm{T} u(z)$ tends to

$$
\begin{aligned}
\mathrm{T} u\left(e^{i \theta}\right) & :=\text { p.v. } \frac{1}{2 \pi} \int_{-\pi}^{\pi} \frac{u\left(e^{i(\theta-t)}\right)}{\tan (t / 2)} d t \\
& =\text { p.v. } \frac{1}{2 \pi i} \int_{-\pi}^{\pi} u(\zeta) \operatorname{Im}\left(\frac{\zeta+e^{i \theta}}{\zeta-e^{i \theta}}\right) \frac{d \zeta}{\zeta} .
\end{aligned}
$$


Since $\operatorname{Re}\left(\frac{\zeta+e^{i \theta}}{\zeta-e^{i \theta}}\right) \equiv 0$ for $\zeta=e^{i t} \in \partial \Delta$, we get $\operatorname{Im}\left(\frac{\zeta+e^{i \theta}}{\zeta-e^{i \theta}}\right)=\frac{1}{i} \frac{\zeta+e^{i \theta}}{\zeta-e^{i \theta}}$ so that we may rewrite

$$
i \mathrm{~T} u\left(e^{i \theta}\right)=\mathrm{p} . \mathrm{v} \cdot \frac{1}{2 \pi i} \int_{-\pi}^{\pi} u(\zeta) \frac{\zeta+e^{i \theta}}{\zeta-e^{i \theta}} \frac{d \zeta}{\zeta} .
$$

Setting $\mathrm{P}_{0} u:=\frac{1}{2 \pi i} \int_{\partial \Delta} u(\zeta) \frac{d \zeta}{\zeta}=\widehat{u}_{0}$, the algebraic relation $\frac{2}{\zeta-e^{i \theta}}-\frac{1}{\zeta}=\frac{\zeta+e^{i \theta}}{\zeta-e^{i \theta}} \frac{1}{\zeta}$ gives a fundamental relation between $\mathrm{C}^{0}$ and $\mathrm{T}$ :

$$
2 \mathrm{C}^{0}-\mathrm{P}_{0}=i \mathrm{~T} \text {. }
$$

From Theorem 2.15, we deduce ( $\mathrm{P}_{0}$ is innocuous):

Theorem 2.24. ([Pri1916, HiTa1978, Bo1991, BER1999], [*]) There exist an absolute constant $C_{1}>1$ such that if $\kappa \in \mathbb{N}$ and $0<\alpha<1$ :

$$
\frac{1 / C_{1}}{\alpha(1-\alpha)} \leqslant\|\mathrm{T}\|_{\kappa, \alpha} \leqslant \frac{C_{1}}{\alpha(1-\alpha)} .
$$

It follows that at the level of Fourier series, $\mathrm{T}$ transforms $u\left(e^{i \theta}\right)=\mathrm{F} u\left(e^{i \theta}\right)=$ $\sum_{k \in \mathbb{Z}} \widehat{u}_{k} e^{i k \theta}$ to

$$
\mathrm{T} u\left(e^{i \theta}\right):=\frac{1}{i}\left(-\sum_{k<0} \widehat{u}_{k} e^{i k \theta}+\sum_{k>0} \widehat{u}_{k} e^{i k \theta}\right) .
$$

Notice that $(\widehat{\mathrm{T} u})_{0}=0$. In fact, this formula coincides with the series $\frac{1}{i}\left(-\sum_{k<0} \widehat{u}_{k} \bar{z}^{k}+\sum_{k>0} \widehat{u}_{k} z^{k}\right)$, written for $z \rightarrow e^{i \theta}$, the limit existing provided $0<\alpha<1$.

By termwise differentiation of the above formula, $\mathrm{T}\left(u_{\theta^{j}}\right)=(\mathrm{T} u)_{\theta^{j}}$ for $0 \leqslant$ $j \leqslant \kappa$, if $u \in \mathcal{C}^{\kappa, \alpha}$ (some integrations by parts in the singular integral defining $\mathrm{T} u$ would yield a second proof of this property).

The Poisson transform $\mathrm{P} u$ of $u \in \mathcal{C}^{0, \alpha}$ having boundary value $\mathrm{b}_{\partial \Delta}(\mathrm{P} u)=u$ and the Schwarz transform being holomorphic in $\Delta$, we see that the function $u+i \mathrm{~T} u$ on $\partial \Delta$ extends holomorphically to $\Delta$ as $\mathrm{S} u(z)$. So $\mathrm{T} u$ on $\Delta$ is one of the Harmonic conjugates of $u$. In general, these conjugates are defined up to a constant. The property $(\widehat{\mathrm{T} u})_{0}=0$ means that $\mathrm{T} u(0)=0$.

Lemma 2.25. The Hilbert transform $\mathrm{T} u$ on $\partial \Delta$ is the boundary value on $\partial \Delta$ of the unique harmonic conjugate in $\Delta$ of the harmonic Poisson extension $\mathrm{P} u$, that vanishes at $0 \in \Delta$.

$$
\text { For } u \in \mathcal{C}^{\kappa, \alpha}(\partial \Delta, \mathbb{R}), u+i \text { T } u \text { extends holomorphically to } \Delta \text {. }
$$

Furthermore, $\mathrm{T}(\mathrm{T} u)=-u+\widehat{u}_{0}$. 
2.26. Hilbert transform in $L^{\mathrm{p}}$ spaces. It is elementary to show that the study of the principal value integral p.v. $\frac{1}{2 \pi} \int_{-\pi}^{\pi} \frac{u\left(e^{i(\theta-t)}\right)}{\tan (t / 2)} d t$ is equivalent to the study of the same singular convolution operator, in which $\cot (t / 2)$ is replaced by $2 / t$. Similarly, one may define the Hilbert transform on the real line:

$$
\mathrm{H} f(\mathrm{x}):=\text { p.v. } \int_{\mathbb{R}} \frac{f(\mathrm{y})}{\mathrm{y}-\mathrm{x}} d \mathrm{y} .
$$

If $f$ is $\mathcal{C}^{1,0}$ on $\mathbb{R}$ and has compact support or satisfies $\int_{\mathbb{R}}|f|<\infty$, replacing $f(\mathrm{y})$ in the numerator by $[f(\mathrm{y})-f(\mathrm{x})]+f(\mathrm{x})$ and reasoning as in $\S 2.3$, one straightforwardly shows the existence of the above principal value.

Privalov showed that $\mathrm{H} f(\mathrm{x})$ exists for almost every $\mathrm{x} \in \mathbb{R}$ if $f \in L^{1}(\mathbb{R})$. A theorem due to M. Riesz states that the two Hilbert transforms $\mathrm{H}$ on the real line and $\mathrm{T}$ on the unit circle are bounded endomorphisms of $L^{\mathrm{p}}$, for $1<\mathrm{p}<\infty$, namely if $f \in L^{\mathrm{P}}(\mathbb{R})$ and $u \in L^{\mathrm{p}}(\partial \Delta)$, then:

$$
\|\mathrm{H} f\|_{L^{\mathrm{p}}(\mathbb{R})} \leqslant C_{\mathrm{p}}\|f\|_{L^{\mathrm{p}}(\mathbb{R})} \quad \text { and } \quad\|\mathrm{T} u\|_{L^{\mathrm{p}}(\partial \Delta)} \leqslant C_{\mathrm{p}}\|u\|_{L^{\mathrm{p}}(\partial \Delta)},
$$

whith the same constant $C_{\mathrm{p}}$ ([Zy1959], Chapters VII and XVI). In [Pi1972], Zygmund's doctoral student Pichorides obtained the best value of the constant $C_{\mathrm{p}}$ : for $1<\mathrm{p} \leqslant 2, C_{\mathrm{p}}=\tan \frac{\pi}{2 \mathrm{p}}$, while, by a duality argument, $C_{\mathrm{p}}=\cot \frac{\pi}{2 \mathrm{p}}$ for $2 \leqslant \mathrm{p}<\infty$. The two elementary bounds $\tan \frac{\pi}{2 \mathrm{p}} \leqslant \frac{\mathrm{p}}{\mathrm{p}-1}$ for $1<\mathrm{p} \leqslant 2$ and $\cot \frac{\pi}{2 \mathrm{p}} \leqslant \mathrm{p}$ for $2 \leqslant \mathrm{p}<\infty$, yield:

$$
\|\mathrm{H} f\|_{L^{\mathrm{p}}(\mathbb{R})} \leqslant \frac{\mathrm{p}^{2}}{\mathrm{p}-1}\|f\|_{L^{\mathrm{p}}(\mathbb{R})} \quad \text { and } \quad\|\mathrm{T} f\|_{L^{\mathrm{p}}(\partial \Delta)} \leqslant \frac{\mathrm{p}^{2}}{\mathrm{p}-1}\|f\|_{L^{\mathrm{p}}(\partial \Delta)} \text {, }
$$

for $1<\mathrm{p}<\infty$. In $L^{1}$, the Hilbert transform is unbounded but, according to a theorem due to Kolmogorov ([Dy1991, DR2002]), it sastisfies a weak inequality:

$$
\mathfrak{m}\{\mathrm{H} f(\mathrm{x})>a\} \leqslant \frac{C}{a}\|f\|_{L^{1}},
$$

for every $a \in \mathbb{R}$ with $a>0$, where $\mathfrak{m}$ is the Lebesgue measure and where $C>0$ is some absolute constant.

2.28. Pointwise convergence of Fourier series. The boundedness of the Hilbert transform in $L^{\mathrm{p}}$ has a long history, closely related to the problem of pointwise convergence of Fourier series. In 1913, before M. Riesz proved the estimates (2.27), using complex function theory and the Riesz-Fischer theorem, Luzin showed that $\mathrm{H}$ is bounded in $L^{2}$ and formulated the celebrated conjecture that Fourier series of $L^{2}$ functions converge pointwise almost everywhere. This "hypothetical theorem" was established by Carleson ([Ca1966]) in 1966 and slightly later by Hunt ([ $\left[\right.$ 1966]) in $L^{\mathrm{p}}$ for $1<\mathrm{p}<\infty$. A complete self-contained restitution of these results is available in [DR2002]. Let us survey the main theorem. 
The $n$-th partial sum of the Fourier series of a function $f$ on $\partial \Delta$ is given by:

$$
\mathrm{F}_{n} f\left(e^{i \theta}\right)=\frac{1}{2 \pi} \int_{-\pi}^{\pi} D_{n}(t) f\left(e^{i(\theta-t)}\right) d t,
$$

where

$$
D_{n}(t):=\frac{\sin (n+1 / 2) t}{\sin t / 2}
$$

is the Dirichlet kernel, having unbounded $L^{1}$ norm $\left\|D_{n}\right\|_{L^{1}} \sim \frac{4}{\pi^{2}} \log n$. It is elementary to show that the behaviour of this convolution integral, as $n \rightarrow \infty$, is equivalent to the behaviour of the integral:

$$
\int_{-\pi}^{\pi} \frac{\sin n t}{t} f\left(e^{i(\theta-t)}\right) d t
$$

Without loss of generality, $f$ is assumed to be real-valued, so that the above integral is the imaginary part of the Carleson integral:

$$
\mathrm{C}_{n}\left(f, e^{i \theta}\right):=\text { p.v. } \int_{-\pi}^{\pi} \frac{e^{i n t}}{t} f\left(e^{i(\theta-t)}\right) d t .
$$

In, chapters 4, 5, 6, 7, 8, 9 and 10 of [DR2002], the main proposition is to prove that the Carleson maximal sublinear operator:

$$
\mathrm{C}^{*} f\left(e^{i \theta}\right):=\sup _{n \in \mathbb{N}}\left|\mathrm{C}_{n}\left(f, e^{i \theta}\right)\right|
$$

is bounded from $L^{\mathrm{p}}$ to $L^{\mathrm{p}}$. The proof involves dyadic partitions, changes of frequency, microscopic Fourier analysis of $f$, choices of allowed pairs and seven exceptional sets. By an elementary argument, one deduces that the maximal Fourier series sublinear operator:

$$
\mathbf{F}^{*} f\left(e^{i \theta}\right):=\sup _{n \in \mathbb{N}}\left|\mathbf{F}_{n} f\left(e^{i \theta}\right)\right|
$$

is bounded from $L^{\mathrm{p}}$ to $L^{\mathrm{p}}$.

Theorem 2.29. ([Ca1966, Hu1966, DR2002]]) If $f \in L^{\mathrm{p}}$ with $1<\mathrm{p}<\infty$, there exists an absolute constant $C>1$ such that:

$$
\left\|\mathrm{F}^{*} f\right\|_{L^{\mathrm{p}}} \leqslant C \frac{\mathrm{p}^{4}}{(\mathrm{p}-1)^{3}}\|f\|_{L^{\mathrm{p}}} .
$$

Then by a standard argument, $\lim _{n \rightarrow \infty} \mathrm{F}_{n} f\left(e^{i \theta}\right)=f\left(e^{i \theta}\right)$ almost everywhere.

2.30. Transition. Since the grounding article [HiTa1978], the nice behaviour of the Hilbert transform in the Hölder classes (Theorem 2.24) is the main reason why Bishop analytic discs have been constructed in the category of $\mathcal{C}^{\kappa, \alpha}$ generic submanifolds of $\mathbb{C}^{n}$ ([BPo1982, BPi1985, Tu1990, Trp1990, Bo1991, BRT1994, Tu1994a, Me1994, Trp1996, Jö1996, BER1999]). Perhaps it is also interesting to construct Bishop analytic discs in the Sobolev classes. 


\section{§3. SOLVING A LOCAL PARAMETRIZED BISHOP EQUATION WITH OPTIMAL LOSS OF SMOOTHNESS}

3.1. Analytic discs attached to a generic submanifold of $\mathbb{C}^{n}$. As in Theorem 4.2(III), let $M$ be a $\mathcal{C}^{\kappa, \alpha}$ local graphed generic submanifold of equation $v=\varphi(x, y, u)$, where $\varphi$ is defined for $|x+i y|<\rho_{1},|u|<\rho_{1}$, for some $\rho_{1}>0$ and where $\varphi(0)=0, d \varphi(0)=0$ and $|\varphi|<\rho_{1}$.

Definition 3.2. An analytic disc is a map

$$
\bar{\Delta} \ni \zeta \longmapsto A(\zeta)=(Z(\zeta), W(\zeta)) \in \mathbb{C}^{m} \times \mathbb{C}^{d}
$$

which is holomorphic in the unit disc $\Delta$ and at least $\mathcal{C}^{0,0}$ in $\bar{\Delta}$. It is attached to $M$ if it sends $\partial \Delta$ into $M$.

Thus, suppose that $(Z(\zeta), W(\zeta))$ is attached to $M$ and sufficiently small, namely $\left|[X+i Y]\left(e^{i \theta}\right)\right|<\rho_{1},\left|U\left(e^{i \theta}\right)\right|<\rho_{1}$ and $\left|V\left(e^{i \theta}\right)\right|<\rho_{1}$ on $\partial \Delta$, where $Z(\zeta)=X(\zeta)+i Y(\zeta)$ and $W(\zeta)=U(\zeta)+i V(\zeta)$. Then clearly, the disc sends $\partial \Delta$ to $M$ if and only if

$$
V\left(e^{i \theta}\right)=\varphi\left(X\left(e^{i \theta}\right), Y\left(e^{i \theta}\right), U\left(e^{i \theta}\right)\right),
$$

for every $e^{i \theta} \in \partial \Delta$. Thanks to the Hilbert transform, we claim that we may express analytically the fact that the disc is attached to $M$.

At first, in order to guarantee the applicability of the harmonic conjugation operator $\mathrm{T}$, all our analytic discs will $\mathcal{C}^{\kappa, \alpha}$ on $\bar{\Delta}$, with $\kappa \in \mathbb{N}$ and $0<\alpha<1$. We let $\mathrm{T}$ act componentwise on maps $U=\left(U^{1}, \ldots, U^{d}\right) \in \mathcal{C}^{\kappa, \alpha}\left(\partial \Delta, \mathbb{R}^{d}\right)$, namely $\mathrm{T} U:=\left(\mathrm{T} U^{1}, \ldots, \mathrm{T} U^{d}\right)$. We set $\|\mathrm{T} U\|_{\kappa, \alpha}:=\max _{1 \leqslant j \leqslant d}\left\|\mathrm{~T} U^{j}\right\|_{\kappa, \alpha}$. With a slight change of notation, instead of $\mathrm{P} U(0)$, we denote by $\mathrm{P}_{0} U:=$ $\frac{1}{2 \pi} \int_{-\pi}^{\pi} U\left(e^{i \theta}\right) d \theta$ the value at the origin of the Poisson extension PU. Equivalently, $\mathrm{P}_{0} U=\widehat{U}_{0}$ is the mean value of $U$ on $\partial \Delta$. Here is a summary of the most useful properties of $\mathrm{T}$.

Lemma 3.3. The $\mathbb{R}^{d}$-valued Hilbert transform $\mathrm{T}$ is a bounded linear endomorphism of $\mathcal{C}^{\kappa, \alpha}\left(\partial \Delta, \mathbb{R}^{d}\right)$ with $\frac{1 / C_{1}}{\alpha(1-\alpha)} \leqslant\|\mathrm{T}\|_{\kappa, \alpha} \leqslant \frac{C_{1}}{\alpha(1-\alpha)}$ satisfying $\mathrm{T}(\mathrm{cst})=0$ and

$$
\mathrm{T}(\mathrm{T} U)=-U+\mathrm{P}_{0} U .
$$

In the sequel, we shall rather use the mild modification $T_{1}$ of $T$ defined by:

$$
\mathrm{T}_{1} U\left(e^{i \theta}\right):=\mathrm{T} U\left(e^{i \theta}\right)-\mathrm{T} U(1) .
$$

In fact, $T_{1}$ is uniquely determined by the normalizing condition $T_{1} U(1)=0$. Then $\mathrm{T}_{1}$ is also bounded: $\frac{1 / C_{1}}{\alpha(1-\alpha)} \leqslant\left\|\mathrm{T}_{1}\right\|_{\kappa, \alpha} \leqslant \frac{C_{1}}{\alpha(1-\alpha)}$, also annihilates constants: $\mathrm{T}_{1}(\mathrm{cst})=0$ and

$$
\mathrm{T}_{1}\left(\mathrm{~T}_{1} U\right)=-U+U(1) .
$$

Furthermore, most importantly: 
Lemma 3.4. If $U \in \mathcal{C}^{\kappa, \alpha}\left(\partial \Delta, \mathbb{R}^{d}\right)$, then $U\left(e^{i \theta}\right)+i \mathrm{~T}_{1} U\left(e^{i \theta}\right)$ extends as a holomorphic map $\Delta \rightarrow \mathbb{C}^{d}$ which is $\mathcal{C}^{\kappa, \alpha}$ in the closed disc $\bar{\Delta}$.

To check that the extension is $\mathcal{C}^{\kappa, \alpha}$ in $\bar{\Delta}$, one may introduce the Poisson integral formula and apply Lemma 2.21 (ii).

If $A=(Z, W)$ is an analytic disc attached to $M$, we set $U_{0}:=U(1)$ and $V_{0}:=$ $V(1)$. Since $W$ is holomorphic, necessarily $V\left(e^{i \theta}\right)=\mathrm{T}_{1} U\left(e^{i \theta}\right)+V_{0}$. Applying $\mathrm{T}_{1}$ to both sides, we get $\mathrm{T}_{1} V\left(e^{i \theta}\right)=-U\left(e^{i \theta}\right)+U_{0}$ (the left and the right hand sides vanish at $\left.e^{i \theta}=1\right)$. Applying $\mathrm{T}_{1}$ to $V\left(e^{i \theta}\right)=\varphi\left(X\left(e^{i \theta}\right), Y\left(e^{i \theta}\right), U\left(e^{i \theta}\right)\right)$ above and reorganizing, we obtain that $U$ satisfies a functional equation ${ }^{7}$ involving the Hilbert transform:

$$
U\left(e^{i \theta}\right)=-\mathrm{T}_{1}[\varphi(X(\cdot), Y(\cdot), U(\cdot))]\left(e^{i \theta}\right)+U_{0} .
$$

Here, the map $U: \partial \Delta \rightarrow \mathbb{R}^{d}$ is the unknown, whereas the holomorphic map $Z=X+i Y: \partial \Delta \rightarrow \mathbb{C}^{m}$ and the constant vector $U_{0}$ are given data.

Conversely, given $X+i Y$ and $U_{0}$, assume that $U \in \mathcal{C}^{\kappa, \alpha}$ satisfies the above functional equation. Set $V\left(e^{i \theta}\right):=\mathrm{T}_{1} U\left(e^{i \theta}\right)+V_{0}$, where $V_{0}:=$ $\varphi(X(1), Y(1), U(1))$. Then $U\left(e^{i \theta}\right)+i V\left(e^{i \theta}\right)$ extends as a $\mathcal{C}^{\kappa, \alpha}$ map $\bar{\Delta} \ni \zeta \mapsto$ $W(\zeta) \in \mathbb{C}^{d}$ which is holomorphic in $\Delta$. If $\left|[X+i Y]\left(e^{i \theta}\right)\right|<\rho_{1},\left|U\left(e^{i \theta}\right)\right|<\rho_{1}$ and $\left|V\left(e^{i \theta}\right)\right|<\rho_{1}$, the $\operatorname{disc} A:=(Z, W)$ is attached to $M$.

Bishop (1965) in the $\mathcal{C}^{\kappa, 0}$ classes and then Hill-Taiani (1978), Boggess-Pitts (1985) in the Hölder classes $\mathcal{C}^{\kappa, \alpha}$ established existence and uniqueness of the solution $U$ to the fundamental functional equation (3.5).

Theorem 3.6. ([Bi1965, HiTa1978, BPi1985]) If $M$ is at least $\mathcal{C}^{1, \alpha}$, shrinking $\rho_{1}$ if necessary, there exists $\rho_{2}$ with $0<\rho_{2}<\rho_{1}$ such that whenever the data $Z \in \mathcal{C}^{0, \alpha}\left(\bar{\Delta}, \mathbb{C}^{m}\right) \cap \mathcal{O}\left(\Delta, \mathbb{C}^{m}\right)$ and $U_{0} \in \mathbb{R}^{d}$ satisfy $\left|Z\left(e^{i \theta}\right)\right|<\rho_{2}$ on $\partial \Delta$ and $\left|U_{0}\right|<\rho_{2}$, there exists a unique solution $U \in \mathcal{C}^{0, \beta}\left(\partial \Delta, \mathbb{R}^{d}\right), 0<\beta<\alpha^{2}$, to the Bishop-type functional equation (3.5) above such that $\left|U\left(e^{i \theta}\right)\right|<\rho_{1}$ on $\partial \Delta$ and such that in addition $\left|V\left(e^{i \theta}\right)\right|<\rho_{1}$ on $\partial \Delta$, where

$$
V\left(e^{i \theta}\right):=\mathrm{T}_{1} U\left(e^{i \theta}\right)+\varphi(X(1), Y(1), U(1)) .
$$

Consequently, the disc $(Z, U+i V)$ is attached to $M$.

\footnotetext{
${ }^{7}$ The origin of this equation may be found in the seminal article [Bi1965] of Bishop. Since then, it has been further exploited in [Pi1974a, Pi1974b, HiTa1978, BeFo1978, We1982, BG1983, BPo1982, KW1982, R1983, HiTa1984, KW1984, BPi1985, FR1985. Trp1986, Fo1986, Tu1988, Ai1989, Tu1990, Trp1990| Bo1991, DH1992, Tu1994a Tu1994b, BRT1994| CR1994, G11994, Me1994, HuKr1995, Jö1995, Trp1996, Tu1996. Jö1996, [ö1997, Me1997, Po1997, MP1998, Tu1998, Hu1998, CR1998, Jö1999a Jö1999b MP1999, BER1999, Po2000, MP2000, Tu2001, Da2001, DS2001, MP2002, HM2002, Po2003, [JS2004, Me2004d].
} 
Notice the (substantial) loss of smoothness, occuring also in [BRT1994, BER1999], which is due to an application of a general implicit function theorem in Banach spaces. The main theorem of this chapter ([Tu1990, Tu1996]) refines the preceding result with a negligible loss of smoothness, provided the graphing map $\varphi$ belongs to the Hölder space $\mathcal{C}^{\kappa, \alpha}$. In the geometric applications (Parts V and VI), it is advantageous to be able to solve a Bishop equation like (3.5) which involves supplementary parameters. Thus, instead of $\varphi$, we shall consider an $\mathbb{R}^{d}$-valued $\mathcal{C}^{\kappa, \alpha}$ map $\Phi=\Phi\left(u, e^{i \theta}, s\right)$, where $s$ is a parameter. For fixed $s$, we shall denote by $\left.\Phi\right|_{s}$ the map $\square_{\rho_{1}}^{d} \times \partial \Delta \ni\left(u, e^{i \theta}\right) \longmapsto \Phi\left(u, e^{i \theta}, s\right) \in \mathbb{R}^{d}$. In accordance with Section 1, we set:

$$
\left\|\Phi_{u}\right\|_{0,0}:=\max _{1 \leqslant j \leqslant d}\left(\sum_{1 \leqslant l \leqslant d}\left\|\Phi_{u_{l}}^{j}\right\|_{0,0}\right),
$$

and similarly $\left\|\Phi_{\theta}\right\|_{0,0}=\max _{1 \leqslant j \leqslant d}\left\|\Phi_{\theta}^{j}\right\|_{0,0}$.

Theorem 3.7. ([Tu1990, Tu1996], [*]) Let $\Phi=\Phi\left(u, e^{i \theta}, s\right)$ be an $\mathbb{R}^{d}$-valued map of class $\mathcal{C}^{\kappa, \alpha}, \kappa \geqslant 1,0<\alpha<1$, defined for $u \in \mathbb{R}^{d},|u|<\rho_{1}, \theta \in \mathbb{R}$ and $s \in \mathbb{R}^{b},|s|<\sigma_{1}$, where $0<\rho_{1}<1$ and $0<\sigma_{1}<1$. Assume that on its domain of definition $\square_{\rho_{1}}^{d} \times \partial \Delta \times \square_{\sigma_{1}}^{b}$, the map $\Phi$ and its derivatives with respect to $u$ and to $\theta$ satisfy the inequalities (nothing is required about $\Phi_{s}$ ):

$$
\|\Phi\|_{0,0} \leqslant c_{1}, \quad\left\|\Phi_{u}\right\|_{0,0} \leqslant c_{2}, \quad\left\|\Phi_{\theta}\right\|_{0,0} \leqslant c_{3},
$$

for some small positive constants $\mathrm{c}_{1}, \mathrm{c}_{2}$ and $\mathrm{c}_{3}$ such that

$$
\mathrm{c}_{1} \leqslant C \alpha \rho_{1}, \quad \mathrm{c}_{2} \leqslant C^{2} \alpha^{2}\left[1+\sup _{|s|<\sigma_{1}}\left\|\left.\Phi\right|_{s}\right\|_{1, \alpha}\right]^{-2}, \quad \mathrm{c}_{3} \leqslant \rho_{1}^{2} \mathrm{c}_{2},
$$

where $0<C<1$ is an absolute constant. Then for every fixed $U_{0}$ satisfying $\left|U_{0}\right|<\rho_{1} / 16$ and every fixed $s \in \square_{\sigma_{1}}^{b}$, the parameterized local Bishop-type functional equation:

$$
U\left(e^{i \theta}\right)=-\mathrm{T}_{1}[\Phi(U(\cdot), \cdot, s)]\left(e^{i \theta}\right)+U_{0}
$$

has a unique solution:

$$
\partial \Delta \ni e^{i \theta} \longmapsto U\left(e^{i \theta}, s, U_{0}\right) \in \mathbb{R}^{d},
$$

with $\|U\|_{0,0} \leqslant \rho_{1} / 4$ which is of class $\mathcal{C}^{\kappa, \alpha}$ on $\partial \Delta$. Furthermore, this solution is of class $\mathcal{C}^{\kappa, \alpha-0}=\bigcap_{\beta<\alpha} \mathcal{C}^{\kappa, \beta}$ with respect to all the variables, including parameters, namely the complete map

$$
\partial \Delta \times \square_{\sigma_{1}}^{b} \times \square_{\rho_{1} / 16}^{d} \ni\left(e^{i \theta}, s, U_{0}\right) \longmapsto U\left(e^{i \theta}, s, U_{0}\right) \in \mathbb{R}^{d}
$$

is $\mathcal{C}^{\kappa, \alpha-0}$. 
Since the assumptions involve only the $\mathcal{C}^{1, \alpha}$ norm of $\Phi$, we notice that the theorem is also true with $\Phi \in \mathcal{C}^{\kappa, \alpha-0}$, provided $\kappa \geqslant 2$, except that the solution will only be $\mathcal{C}^{\kappa, \alpha-0}$ with respect to $e^{i \theta}$ : it suffices to apply the theorem by considering that $\Phi \in \mathcal{C}^{\kappa, \beta}$, with $\beta<\alpha$ arbitrary, getting a solution that is $\mathcal{C}^{\kappa, \beta-0}$ with respect to all variables and concluding from $\bigcap_{\beta<\alpha} \mathcal{C}^{\kappa, \beta-0}=\mathcal{C}^{\kappa, \alpha-0}$.

The main purpose of this section is to provide a thorough proof of the theorem. In the sequel, $C, C_{1}, C_{2}, C_{3}$ and $C_{4}$ will denote positive absolute constants. We may assure that they all will be $\geqslant 10^{-5}$ and $\leqslant 10^{5}$.

The smallness of $\|\Phi\|_{0,0}$, of $\left\|\Phi_{u}\right\|_{0,0}$ and of $\left\|\Phi_{\theta}\right\|_{0,0}$ guarantee the smallness of $\|\Phi \mid s\|_{1, \alpha / 2}$ by virtue of an elementary observation.

Lemma 3.9. ([*]) Let $\mathrm{x} \in \square_{\rho}^{n}, n \in \mathbb{N}$, $n \geqslant 1$, where $0<\rho_{i} \leqslant \infty$, and let $f=f(\mathrm{x})$ be $\mathcal{C}^{0, \alpha}$ function with values in $\mathbb{R}^{d}, d \geqslant 1$. If $\|f\|_{0,0} \leqslant \mathrm{c}$, for some quantity $\mathrm{c}>0$, then:

$$
\|f\|_{\widehat{0, \alpha / 2}} \leqslant c^{1 / 2}\left[2+\|f\|_{\widehat{0, \alpha}}\right] .
$$

We apply this inequality to $\left.\Phi_{u}\right|_{s}$ and to $\left.\Phi_{\theta}\right|_{s}$, pointing out that for any $\beta$ with $0<\beta \leqslant \alpha$, by definition:

$$
\begin{aligned}
\left\|\left.\Phi_{u}\right|_{s}\right\|_{\widehat{0, \beta}} & =\max _{1 \leqslant j \leqslant d}\left(\sum_{l=1}^{d} \frac{\left|\Phi_{u_{l}}^{j}\left(u^{\prime \prime}, e^{i \theta^{\prime \prime}}, s\right)-\Phi_{u_{l}}^{j}\left(u^{\prime}, e^{i \theta^{\prime}}, s\right)\right|}{\left|\left(u^{\prime \prime}, \theta^{\prime \prime}\right)-\left(u^{\prime}, \theta^{\prime}\right)\right|^{\beta}}\right), \\
\left\|\left.\Phi_{\theta}\right|_{s}\right\|_{\widehat{0, \beta}} & =\max _{1 \leqslant j \leqslant d} \frac{\left|\Phi_{\theta}^{j}\left(u^{\prime \prime}, e^{i \theta^{\prime \prime}}, s\right)-\Phi_{\theta}^{j}\left(u^{\prime}, e^{i \theta^{\prime}}, s\right)\right|}{\left|\left(u^{\prime \prime}, \theta^{\prime \prime}\right)-\left(u^{\prime}, \theta^{\prime}\right)\right|^{\beta}} .
\end{aligned}
$$

Lemma 3.10. ([*]) Independently of $s$, we have:

$$
\begin{aligned}
& \left\|\left.\Phi_{u}\right|_{s}\right\|_{0, \alpha / 2} \leqslant c_{2}^{1 / 2}\left[2+\left\|\left.\Phi\right|_{s}\right\|_{1, \alpha}\right], \\
& \left\|\left.\Phi_{\theta}\right|_{s}\right\|_{\widehat{0, \alpha / 2}} \leqslant c_{3}^{1 / 2}\left[2+\left\|\left.\Phi\right|_{s}\right\|_{1, \alpha}\right], \\
& \left\|\left.\Phi\right|_{s}\right\|_{1, \alpha / 2} \leqslant c_{1}+c_{2}+c_{3}+\left(c_{2}^{1 / 2}+c_{3}^{1 / 2}\right)\left[2+\left\|\left.\Phi\right|_{s}\right\|_{1, \alpha}\right] .
\end{aligned}
$$

The presence of the squares in the inequalities of Theorem 3.7 anticipates the roots $c_{2}^{1 / 2}$ and $c_{3}^{1 / 2}$ above. These two lemmas and the next involve dry computations with Hölder norms. The detailed proofs are postponed to Section 4.

Lemma 3.11. ([*]) If $U \in \mathcal{C}^{1, \beta}\left(\partial \Delta, \mathbb{R}^{d}\right)$ with $0<\beta \leqslant \alpha$ satisfies $\left|U\left(e^{i \theta}\right)\right|<\rho_{1}$ on $\partial \Delta$, then for every fixed $s \in \square_{\sigma_{1}}^{b}$, we have:

$$
\begin{aligned}
\|\Phi(U(\cdot), \cdot, s)\|_{\mathcal{C}^{1, \beta}(\partial \Delta)} & \leqslant\|\Phi\|_{0,0}+\left\|\Phi_{\theta}\right\|_{0,0}+\left\|\left.\Phi_{\theta}\right|_{s}\right\|_{\widehat{0, \beta}}\left[1+\left(\|U\|_{\widehat{1,0}}\right)^{\beta}\right]+ \\
& +\left\|\Phi_{u}\right\|_{0,0}\|U\|_{1, \beta}+\left\|\left.\Phi_{u}\right|_{s}\right\|_{\widehat{0, \beta}}\left[\|U\|_{\widehat{1,0}}+\left(\|U\|_{\widehat{1,0}}\right)^{1+\beta}\right] .
\end{aligned}
$$


Remind $\|U\|_{\widehat{1,0}}=\sup _{\theta}\left|U_{\theta}\left(e^{i \theta}\right)\right|$. We then introduce the map:

$$
U \longmapsto \mathfrak{F}(U):=U_{0}-\mathrm{T}_{1}[\Phi(U(\cdot), \cdot, s)]\left(e^{i \theta}\right) .
$$

To construct the solution $U$, we endeavour a Picard iteration process, setting $\left.U_{k}\right|_{k=0}:=U_{0}$ with $\left|U_{0}\right|<\rho_{1} / 16$ and $U_{k+1}:=\mathfrak{F}\left(U_{k}\right)$, for $k \in \mathbb{N}$, whenever $\mathfrak{F}\left(U_{k}\right)$ may be defined, i.e. whenever $\left\|U_{k}\right\|_{0,0}<\rho_{1}$. We shall first work in $\mathcal{C}^{1, \alpha / 2} \subset \mathcal{C}^{\kappa, \alpha}$.

Lemma 3.12. If we choose the absolute constant $C<1$ appearing in the theorem sufficiently small, then independently of $s$, the sequence $U_{k}$ satisfies the uniform boundedness estimate:

$$
\left\|U_{k}\right\|_{1, \alpha / 2} \leqslant \rho_{1} / 4<\rho_{1},
$$

hence it is defined for every $k \in \mathbb{N}$ and each $U_{k}$ belongs to $\mathcal{C}^{1, \alpha / 2}(\partial \Delta)$.

Proof. By Theorem 2.24, there exists an absolute constant $C_{1}>1$ (not exactly the same), such that

$$
\left\|\mathrm{T}_{1}\right\|_{1, \alpha / 2} \leqslant C_{1} / \alpha
$$

Majorating by means of the $\mathcal{C}^{0, \alpha / 2}$-norm:

$$
\left\|\mathfrak{F}\left(U_{k}\right)\right\|_{1, \alpha / 2} \leqslant\left|U_{0}\right|+\left\|\mathbf{T}_{1}\right\|_{1, \alpha / 2}\left\|\Phi\left(U_{k}(\cdot), \cdot, s\right)\right\|_{\mathcal{C}^{1, \alpha / 2}(\partial \Delta)} .
$$

Assume by induction that $U_{k}$ is $\mathcal{C}^{1, \alpha / 2}$ and satisfies $\left\|U_{k}\right\|_{1, \alpha / 2} \leqslant \rho_{1} / 4$ (this holds for $k=0)$. Clearly $U_{k+1}=\mathfrak{F}\left(U_{k}\right)$ is $\mathcal{C}^{1, \alpha / 2}$. Thanks to Lemma 3.11, and to the trivial majoration $\left(\left\|U_{k}\right\|_{\widehat{1,0}}\right)^{\alpha / 2} \leqslant\left(\rho_{1} / 4\right)^{\alpha / 2}<1$ :

$$
\begin{aligned}
\left\|\Phi\left(U_{k}(\cdot), \cdot, s\right)\right\|_{\mathcal{C}^{1, \alpha / 2}(\partial \Delta)} \leqslant & \|\Phi\|_{0,0}+\left\|\Phi_{\theta}\right\|_{0,0}+2\left\|\left.\Phi_{\theta}\right|_{s}\right\|_{\widehat{0, \alpha / 2}}+ \\
& +\left\|\Phi_{u}\right\|_{0,0}\left\|U_{k}\right\|_{1, \alpha / 2}+2\left\|\left.\Phi_{u}\right|_{s}\right\|_{\widehat{0, \alpha / 2}}\left\|U_{k}\right\|_{1, \alpha / 2} .
\end{aligned}
$$

Using then the assumptions (3.8) of the theorem together with Lemma 3.10:

$$
\begin{aligned}
\left\|U_{k+1}\right\|_{1, \alpha / 2} & \leqslant \rho_{1} / 16+C_{1} \alpha^{-1}\left[\mathrm{c}_{1}+\mathrm{c}_{3}+4 \mathrm{c}_{3}^{1 / 2}\left(1+\left\|\left.\Phi\right|_{s}\right\|_{1, \alpha}\right)+\right. \\
& \left.+\left\|U_{k}\right\|_{1, \alpha / 2}\left(\mathrm{c}_{2}+4 \mathrm{c}_{2}^{1 / 2}\left(1+\left\|\left.\Phi\right|_{s}\right\|_{1, \alpha}\right)\right)\right] .
\end{aligned}
$$

Using the two trivial majorations $\mathrm{c}_{3} \leqslant C \alpha \rho_{1}$ and $\mathrm{c}_{2} \leqslant C \alpha$ together with the main assumptions (3.8) to majorate $c_{2}^{1 / 2}$ and $c_{3}^{1 / 2}$, we get:

$$
\left\|U_{k+1}\right\|_{1, \alpha / 2} \leqslant \rho_{1} / 16+C_{1} \rho_{1} 6 C+\left\|U_{k}\right\|_{1, \alpha / 2} C_{1} 5 C .
$$

Choosing $C \leqslant \frac{1}{16 C_{1} 6}$ (whence $C \leqslant \frac{1}{2 C_{1} 5}$ ), we finally get:

$$
\left\|U_{k+1}\right\|_{1, \alpha / 2} \leqslant \rho_{1} / 8+(1 / 2)\left\|U_{k}\right\|_{1, \alpha / 2} .
$$

By immediate induction, the assumption $\left|U_{0}\right|<\rho_{1} / 16$ and these (strict) inequalities entail that $\left\|U_{k}\right\|_{1, \alpha / 2} \leqslant \rho_{1} / 4$ for every $k \in \mathbb{N}$, as claimed. 
Lemma 3.13. ([Tu1990], [*]) For every $\beta$ with $0<\beta \leqslant \alpha$ and every fixed $s \in \square_{\rho_{1}}^{b}$, if two maps $U^{j} \in \mathcal{C}^{1,0}\left(\partial \Delta, \mathbb{R}^{d}\right)$ with $\left\|U^{j}\right\|_{0,0}<\rho_{1} / 3$ for $j=1,2$ are given, the following inequality holds:

$$
\left\|\Phi\left(U^{2}(\cdot), \cdot, s\right)-\Phi\left(U^{1}(\cdot), \cdot, s\right)\right\|_{\mathcal{C}^{0, \beta}(\partial \Delta)} \leqslant \mathrm{C}\left\|U^{2}-U^{1}\right\|_{\mathcal{C}^{0, \beta}(\partial \Delta)},
$$

where

$$
\mathbf{C}=\left\|\Phi_{\mid s}\right\|_{1, \beta} 2\left[1+\left(\left\|U^{1}\right\|_{\widehat{1,0}}\right)^{\beta}+\left(\left\|U^{2}\right\|_{\widehat{1,0}}\right)^{\beta}\right] .
$$

Again, the (latexnically lengthy) proof is postponed to Section 4.

Lemma 3.14. If we choose the absolute constant $C$ of the theorem sufficiently small, then independently of $s$, the map:

$$
U \longmapsto \mathfrak{F}(U):=U_{0}-\mathrm{T}_{1}[\Phi(U(\cdot), \cdot, s)]\left(e^{i \theta}\right),
$$

restricted to the set of those $U \in \mathcal{C}^{1, \alpha / 2}\left(\partial \Delta, \mathbb{R}^{d}\right)$ that satisfy $\|U\|_{1, \alpha / 2} \leqslant \rho_{1} / 4$, is a contraction:

$$
\left\|\mathfrak{F}\left(U^{2}\right)-\mathfrak{F}\left(U^{1}\right)\right\|_{0, \alpha / 2} \leqslant \frac{1}{2}\left\|U^{2}-U^{1}\right\|_{0, \alpha / 2} .
$$

Proof. Let $U^{j} \in \mathcal{C}^{1, \alpha / 2}$ with $\left\|U^{j}\right\|_{1, \alpha / 2} \leqslant \rho_{1} / 4$ for $j=1,2$. In particular, $\left\|U^{j}\right\|_{0,0}<\rho_{1} / 3$, so Lemma 3.13 applies. In the majorations below, to pass to the fourth line, we use the assumption $\rho_{1}<1$, which enables us to majorate simply by 3 the three terms in the brackets of the third line:

$$
\begin{aligned}
& \left\|\mathfrak{F}\left(U^{2}\right)-\mathfrak{F}\left(U^{1}\right)\right\|_{0, \alpha / 2}=\left\|\mathrm{T}_{1}\left[\Phi\left(U^{2}(\cdot), \cdot, s\right)-\Phi\left(U^{1}(\cdot), \cdot, s\right)\right]\right\|_{0, \alpha / 2} \\
& \leqslant\left\|\mathrm{~T}_{1}\right\|_{0, \alpha / 2}\left\|\Phi\left(U^{2}(\cdot), \cdot, s\right)-\Phi\left(U^{1}(\cdot), \cdot, s\right)\right\|_{0, \alpha / 2} \\
& \leqslant \frac{C_{1}}{\alpha}\left\|\Phi_{\mid s}\right\|_{1, \alpha / 2} 2\left[1+\left(\left\|U^{1}\right\|_{1, \alpha / 2}\right)^{\alpha / 2}+\left(\left\|U^{2}\right\|_{1, \alpha / 2}\right)^{\alpha / 2}\right]\left\|U^{2}-U^{1}\right\|_{0, \alpha / 2} \\
& \leqslant \frac{C_{2}}{\alpha}\left\|\Phi_{\mid s}\right\|_{1, \alpha / 2}\left\|U^{2}-U^{1}\right\|_{0, \alpha / 2},
\end{aligned}
$$

where $C_{2}>1$ is absolute. Then we apply Lemma 3.10 to majorate $\left\|\left.\Phi\right|_{s}\right\|_{1, \alpha / 2}$, we use the three trivial majorations $\mathrm{c}_{1}, \mathrm{c}_{2}, \mathrm{c}_{3} \leqslant C \alpha$ and we majorate $\mathrm{c}_{2}^{1 / 2}, \mathrm{c}_{3}^{1 / 2}$ by means of (3.8), dropping $\rho_{1}<1$ in $\mathrm{c}_{3}^{1 / 2}$, which yields:

$$
\begin{aligned}
\left\|\left.\Phi\right|_{s}\right\|_{1, \alpha / 2} & \leqslant c_{1}+c_{2}+c_{3}+\left(c_{2}^{1 / 2}+c_{3}^{1 / 2}\right)\left[2+\left\|\Phi_{\mid s}\right\|_{1, \alpha}\right] \\
& \leqslant 3 C \alpha+2 C \alpha+4 C \alpha=9 C \alpha .
\end{aligned}
$$

Then we conclude that

$$
\left\|\mathfrak{F}\left(U^{2}\right)-\mathfrak{F}\left(U^{1}\right)\right\|_{0, \alpha / 2} \leqslant C C_{3}\left\|U^{2}-U^{1}\right\|_{0, \alpha / 2} .
$$

Choosing the absolute constant $C$ of the theorem $\leqslant \frac{1}{2 C_{3}}$ yields the desired contracting factor $\frac{1}{2}$. 
The fixed point theorem then entails that our sequence $U_{k}$ converges in $\mathcal{C}^{0, \alpha / 2}$ norm towards some map $U \in \mathcal{C}^{0, \alpha / 2}\left(\partial \Delta, \mathbb{R}^{d}\right)$. More is true:

Lemma 3.15. For every fixed parameters $\left(s, U^{0}\right)$, this solution $U=U\left(e^{i \theta}, s, U_{0}\right)=\lim _{k \rightarrow \infty} U_{k}$ belongs in fact to $\mathcal{C}^{1, \alpha / 2}\left(\partial \Delta, \mathbb{R}^{d}\right)$ and satisfies $\|U\|_{1, \alpha / 2} \leqslant \rho_{1} / 4$.

Proof. Indeed, since $\left\|U_{k}\right\|_{1, \alpha / 2} \leqslant \rho_{1} / 4$ is bounded, it is possible thanks to the Arzelà-Ascoli lemma to extract some subsequence converging in $\mathcal{C}^{1,0}(\partial \Delta)$ to a map, still denoted by $U=U\left(e^{i \theta}, s, U_{0}\right)$, which is $\mathcal{C}^{1,0}$ on $\partial \Delta$. Still denoting by $U_{k}$ such a subsequence, we observe that the uniform convergence $\left\|U_{k}-U\right\|_{1,0} \rightarrow$ 0 plus the boundedness $\left\|U_{k}\right\|_{1, \alpha / 2} \leqslant \rho_{1} / 4$ entail immediately that the following majoration holds:

$$
\frac{\left|U_{\theta}\left(e^{i \theta^{\prime \prime}}\right)-U_{\theta}\left(e^{i \theta^{\prime}}\right)\right|}{\left|\theta^{\prime \prime}-\theta^{\prime}\right|^{\alpha / 2}}=\lim _{k \rightarrow \infty} \frac{\left|U_{k, \theta}\left(e^{i \theta^{\prime \prime}}\right)-U_{k, \theta}\left(e^{i \theta^{\prime}}\right)\right|}{\left|\theta^{\prime \prime}-\theta^{\prime}\right|^{\alpha / 2}} \leqslant \frac{\rho_{1}}{4},
$$

for arbitrary $0<\left|\theta^{\prime \prime}-\theta^{\prime}\right| \leqslant \pi$. Consequently, $U$ belongs to $\mathcal{C}^{1, \alpha / 2}$. Passing to the limit in $\left\|U_{k}\right\|_{1, \alpha / 2} \leqslant \rho_{1} / 4$, we also deduce $\|U\|_{1, \alpha / 2} \leqslant \rho_{1} / 4$.

The next crucial step is to study the regularity of the solution $U=U\left(e^{i \theta}, s, U_{0}\right)$ with respect to $\left(s, U_{0}\right)$.

Lemma 3.16. The solution $U=U\left(e^{i \theta}, s, U_{0}\right)$ satisfies a Lipschitz condition with respect to the parameters $s$ and $U_{0}$.

Proof. Consider two parameters $s^{1}, s^{2} \in \square_{\sigma_{1}}^{b}$ and define $U^{j}:=U\left(e^{i \theta}, s^{j}, U_{0}\right)$ for $j=1,2$. Then substract the two corresponding Bishop equations, insert two innocuous opposite terms and majorate:

$$
\begin{aligned}
\left\|U^{2}-U^{1}\right\|_{0, \alpha / 2} \leqslant\left\|T_{1}\right\|_{0, \alpha / 2}[ & \left\|\Phi\left(U^{2}(\cdot), \cdot, s^{2}\right)-\Phi\left(U^{2}(\cdot), \cdot, s^{1}\right)\right\|_{0, \alpha / 2}+ \\
& \left.+\left\|\Phi\left(U^{2}(\cdot), \cdot, s^{1}\right)-\Phi\left(U^{1}(\cdot), \cdot, s^{1}\right)\right\|_{0, \alpha / 2}\right] .
\end{aligned}
$$

To majorate the difference in the second line, we again apply Lemma 3.13. To majorate the difference in the first line, we apply:

Lemma 3.17. ([*]) Let $\beta$ with $0<\beta \leqslant \alpha$, let $U \in \mathcal{C}^{1,0}\left(\partial \Delta, \mathbb{R}^{d}\right)$ with $\|U\|_{0,0}<$ $\rho_{1}$ and let two parameters $s^{1}, s^{2} \in \square_{\sigma_{1}}^{b}$. Then

$$
\left\|\Phi\left(U(\cdot), \cdot, s^{2}\right)-\Phi\left(U(\cdot), \cdot, s^{1}\right)\right\|_{0, \beta} \leqslant\left|s^{2}-s^{1}\right|\left(\|\Phi\|_{1,0}+\|\Phi\|_{1, \beta}\left[1+\left(\|U\|_{\widehat{1,0}}\right)^{\beta}\right]\right) .
$$


With $\beta:=\frac{\alpha}{2}$, we thus obtain:

$$
\begin{aligned}
\left\|U^{2}-U^{1}\right\|_{0, \alpha / 2} & \leqslant \frac{C_{1}}{\alpha}\left[\left|s^{2}-s^{1}\right|\left(\|\Phi\|_{1,0}+\|\Phi\|_{1, \alpha / 2}\left[1+\left(\left\|U^{2}\right\|_{1,0}\right)^{\frac{\alpha}{2}}\right]\right)+\right. \\
& \left.+\sup _{|s|<\sigma_{1}}\left\|\Phi_{\mid s}\right\|_{1, \alpha / 2} 2\left[1+\left(\left\|U^{1}\right\|_{\widehat{1,0}}\right)^{\frac{\alpha}{2}}+\left(\left\|U^{2}\right\|_{\widehat{1,0}}\right)^{\frac{\alpha}{2}}\right]\right]\left\|U^{2}-U^{1}\right\|_{0, \alpha / 2} .
\end{aligned}
$$

Then we apply the majoration of Lemma 3.10 to $\left\|\Phi_{\mid s}\right\|_{1, \alpha / 2}$ and we use $\rho_{1}<1$ to majorate by 1 the terms $\left\|U^{j}\right\|_{\widehat{1,0}} \leqslant \rho_{1} / 4$ :

$$
\left\|U^{2}-U^{1}\right\|_{0, \alpha / 2} \leqslant C_{1} \alpha^{-1}\left|s^{2}-s^{1}\right| 3\|\Phi\|_{1, \alpha / 2}+C C_{2}\left\|U^{2}-U^{1}\right\|_{0, \alpha / 2} .
$$

Setting $\mathrm{K}:=C_{1} \alpha^{-1} 3\|\Phi\|_{1, \alpha / 2}$, requiring $C \leqslant \frac{1}{2 C_{2}}$ and reorganizing we obtain that $U\left(e^{i \theta}, s, U_{0}\right)$ is Lipschitzian with respect to $s$ :

$$
\left\|U^{2}-U^{1}\right\|_{0,0} \leqslant\left\|U^{2}-U^{1}\right\|_{0, \alpha / 2} \leqslant 2 \mathrm{~K}\left|s^{2}-s^{1}\right| .
$$

The proof that $U\left(e^{i \theta}, s, U_{0}\right)$ is Lipschitzian with respect to $U_{0}$ is similar and even simpler.

In summary, the solution $U=U\left(e^{i \theta}, s, U_{0}\right)$ is $\mathcal{C}^{1, \alpha / 2}$ with respect to $e^{i \theta}$ and Lipschitzian with respect to all the variables $\left(e^{i \theta}, s, U_{0}\right)$.

Consequently, according to a theorem due to Rademacher ([Ra1919, Fe1969]), the partial derivatives $U_{s_{k}}, k=1, \ldots, b$ and $U_{U_{0}^{m}}, m=1, \ldots, d$ exist in $L^{\infty}$. We then have to differentiate the Bishop-type equation of Theorem 3.7 with respect to $\theta$, to $s_{k}$ and to $U_{0}^{m}$. However, the linear operator $\mathrm{T}_{1}$ is not bounded in $L^{\infty}$; in fact, according to (2.27), $\|T\|_{L^{\mathrm{p}}} \sim \mathrm{p}$ as $\mathrm{p} \rightarrow \infty$. So we need more information.

Lemma 3.18. There exists a null-measure subset $\mathfrak{N} \subset \square_{\sigma_{1}}^{b} \times \square_{\rho_{1} / 16}^{d}$ and there exists a quantity $\mathrm{K}>0$ such that at every $\left(s, U_{0}\right) \notin \mathfrak{N}$, for every $k=1, \ldots, b$ and for every $m=1, \ldots, d$ :

(i) the partial derivatives $U_{s_{k}}\left(e^{i \theta}, s, U_{0}\right)$ and $U_{U_{0}^{m}}\left(e^{i \theta}, s, U_{0}\right)$ exist for every $e^{i \theta} \in \partial \Delta$

(ii) the maps $e^{i \theta} \mapsto U_{s_{k}}\left(e^{i \theta}, s, U_{0}\right)$ and $e^{i \theta} \mapsto U_{U_{0}^{m}}\left(e^{i \theta}, s, U_{0}\right)$ are $\mathcal{C}^{0, \alpha / 2}$ on $\partial \Delta$ and satisfy the uniform inequality

$$
\left\|U_{s_{k}}\left(\cdot, s, U_{0}\right)\right\|_{\mathcal{C}^{0, \alpha / 2}(\partial \Delta)} \leqslant \mathrm{K} \quad \text { and } \quad\left\|U_{U_{0}^{m}}\left(\cdot, s, U_{0}\right)\right\|_{\mathcal{C}^{0, \alpha / 2}(\partial \Delta)} \leqslant \mathrm{K} .
$$

Proof. Since $U$ is almost everywhere differentiable with respect to all its arguments, there exist a subset $\mathfrak{F} \subset \square_{\sigma_{1}}^{b} \times \square_{\rho_{1} / 16}^{d} \times \partial \Delta$ having full measure, namely its complement has null measure, such that for every $\left(e^{i \theta}, s, U_{0}\right) \in \mathfrak{F}$, all partial 
derivatives $U_{\theta}, U_{s_{k}}, U_{U_{0}^{m}}$ exist at $\left(e^{i \theta}, s, U_{0}\right)$. Since $\mathfrak{F}$ has full measure, there exists a null measure subset $\mathfrak{N} \subset \square_{\sigma_{1}}^{b} \times \square_{\rho_{1} / 16}^{d}$ such that for every $\left(s, U_{0}\right) \notin \mathfrak{N}$, the slice

$$
\mathfrak{F}_{s, U_{0}}:=\left(\partial \Delta \times\{s\} \times\left\{U_{0}\right\}\right) \cap \mathfrak{F}
$$

is a subset of $\partial \Delta$ having full measure, so that $U_{\theta}, U_{s_{k}}, U_{U_{0}^{m}}$ exist at $\left(e^{i \theta}, s, U_{0}\right)$ with $e^{i \theta} \in \mathfrak{F}_{s, U_{0}}$.

Fix $\left(s, U_{0}\right) \notin \mathfrak{N}$. We will treat only the partial derivatives with respect to the $s_{k}$, arguments being similar for the $U_{U_{0}^{m}}$. In the end of the proof of Lemma 3.17, we have shown:

$$
\left\|U^{2}-U^{1}\right\|_{0, \alpha / 2} \leqslant \mathrm{~K}\left|s^{2}-s^{1}\right|,
$$

for some (not the same) quantity $\mathrm{K}>0$. Fix $k \in\{1,2, \ldots, b\}$, take $s^{2}$ and $s^{1}$ with $s_{k}^{2} \neq s_{k}^{1}$ but $s_{l}^{2}=s_{l}^{1}$ for $l \neq k$. The inequality above says that for every $e^{i \theta}, e^{i \theta^{\prime}}, e^{i \theta^{\prime \prime}} \in \partial \Delta$ with $0<\left|\theta^{\prime \prime}-\theta^{\prime}\right| \leqslant \pi$, we have

$$
\begin{aligned}
& \left|\frac{U\left(e^{i \theta}, s^{2}, U_{0}\right)-U\left(e^{i \theta}, s^{1}, U_{0}\right)}{s_{k}^{2}-s_{k}^{1}}\right|+ \\
& +\mid \frac{U\left(e^{i \theta^{\prime \prime}}, s^{2}, U_{0}\right)-U\left(e^{i \theta^{\prime \prime}}, s^{1}, U_{0}\right)}{s_{k}^{2}-s_{k}^{1}}- \\
& \quad-\frac{U\left(e^{i \theta^{\prime}}, s^{2}, U_{0}\right)-U\left(e^{i \theta^{\prime}}, s^{1}, U_{0}\right)}{s_{k}^{2}-s_{k}^{1}}|/| \theta^{\prime \prime}-\left.\theta^{\prime}\right|^{\alpha / 2} \leqslant \mathrm{~K} .
\end{aligned}
$$

Assume $e^{i \theta}, e^{i \theta^{\prime}}, e^{i \theta^{\prime \prime}} \in \mathfrak{F}_{s^{1}, U_{0}}$, let $s_{k}^{2} \rightarrow s_{k}^{1}$ (the limits of the quotients above exist) and rename $s^{1}$ by $s$ :

$$
\left|U_{s_{k}}\left(e^{i \theta}, s, U_{0}\right)\right|+\frac{\left|U_{s_{k}}\left(e^{i \theta^{\prime \prime}}, s, U_{0}\right)-U_{s_{k}}\left(e^{i \theta^{\prime}}, s, U_{0}\right)\right|}{\left|\theta^{\prime \prime}-\theta^{\prime}\right|^{\alpha / 2}} \leqslant \mathrm{~K} .
$$

This inequality says that $U_{s_{k}}\left(\cdot, s, U_{0}\right)$ is $\mathcal{C}^{0, \alpha / 2}$ almost everywhere on $\partial \Delta$. The next extension lemma concludes the proof.

Lemma 3.19. Let $n \geqslant 1$, let $\mathrm{x} \in \mathbb{R}^{n}$, let $m \geqslant 1$, let $\mathrm{y} \in \mathbb{R}^{m}$, let $\rho>0$, let $\sigma>0$, and let $f=f(\mathrm{x}, \mathrm{y})$ be a function defined (only) in a full-measure subset $\mathfrak{F} \subset \square_{\rho}^{n} \times \square_{\sigma}^{m}$, so that there exists a null-measure subset $\mathfrak{N} \subset \square_{\sigma}^{m}$ with the property that for every $\mathrm{y} \notin \mathfrak{N}$, the slice $\mathfrak{F}_{\mathrm{y}}:=\left(\square_{\rho}^{n} \times\{\mathrm{y}\}\right) \times \mathfrak{F}$ has full measure in $\square_{\rho}^{n}$. Assume that for every $\mathrm{y} \notin \mathfrak{N}$, every $\mathrm{x}, \mathrm{x}^{\prime}, \mathrm{x}^{\prime \prime} \in \mathfrak{F}_{\mathrm{y}}$, we have

$$
|f(\mathrm{x}, \mathrm{y})|+\frac{\left|f\left(\mathrm{x}^{\prime \prime}, \mathrm{y}\right)-f\left(\mathrm{x}^{\prime}, \mathrm{y}\right)\right|}{\left|\mathrm{x}^{\prime \prime}-\mathrm{x}^{\prime}\right|^{\beta}} \leqslant \mathrm{K},
$$

for some $\beta$ with $0<\beta \leq 1$ and some quantity $\mathrm{K}>0$. Then for every $\mathrm{y} \notin \mathfrak{N}$, the function $\mathrm{x} \mapsto f(\mathrm{x}, \mathrm{y})$ admits a unique continuous prolongation to $\square_{\rho}^{n}$, still 
denoted by $f(\cdot, \mathrm{y})$, that is $\mathcal{C}^{0, \beta}$ in $\square_{\rho}^{n}$ with

$$
\|f(\cdot, \mathrm{y})\|_{\mathcal{C}^{0, \beta}\left(\square_{\rho}^{n}\right)} \leqslant \mathrm{K} .
$$

Thus, for every $\left(s, U_{0}\right) \notin \mathfrak{N}$, the partial derivatives $U_{s_{k}}, U_{U_{0}^{m}}$ belong to $\mathcal{C}^{0, \alpha / 2}\left(\partial \Delta, \mathbb{R}^{d}\right)$. Since the operator $\mathrm{T}_{1}$ is linear and bounded in $\mathcal{C}^{0, \alpha / 2}$, we may differentiate the $d$ scalar Bishop-type equations of Theorem 3.7 with respect to $\theta$, to $s_{k}, k=1, \ldots, b$ and to $U_{0}^{m}, m=1, \ldots, d$, which yields, for $j=1, \ldots, d$ :

$$
\left\{\begin{aligned}
& U_{\theta}^{j}\left(e^{i \theta}\right)=-\mathrm{T}_{1} {\left[\sum_{1 \leqslant l \leqslant d} \Phi_{u_{l}}^{j}(U(\cdot), \cdot, s) U_{\theta}^{l}(\cdot)+\Phi_{\theta}^{j}(U(\cdot), \cdot, s)\right]\left(e^{i \theta}\right), } \\
& U_{s_{k}}^{j}\left(e^{i \theta}\right)=-\mathrm{T}_{1}\left[\sum_{1 \leqslant l \leqslant d} \Phi_{u_{l}}^{j}(U(\cdot), \cdot, s) U_{s_{k}}^{l}(\cdot)+\Phi_{s_{k}}^{j}(U(\cdot), \cdot, s)\right]\left(e^{i \theta}\right), \\
& U_{U_{0}^{m}}^{j}\left(e^{i \theta}\right)=\delta_{m}^{j}-\mathrm{T}_{1}\left[\sum_{1 \leqslant l \leqslant d} \Phi_{u_{l}}^{j}(U(\cdot), \cdot, s) U_{U_{0}^{m}}^{l}(\cdot)\right]\left(e^{i \theta}\right),
\end{aligned}\right.
$$

for every $e^{i \theta} \in \partial \Delta$, provided $\left(s, U_{0}\right) \notin \mathfrak{N}$. In the first line, we noticed that $\left(\mathrm{T}_{1} V\right)_{\theta}=\mathbf{T}\left(V_{\theta}\right)$. We observe that as $U$ is Lipschitzian, as $\Phi \in \mathcal{C}^{\kappa, \alpha}$ and as $\kappa \geqslant 1$, the composite functions $\Phi_{u_{l}}^{j}, \Phi_{\theta}^{j}, \Phi_{s_{i}}^{j}\left(U\left(e^{i \theta}, s, U_{0}\right), e^{i \theta}, s\right)$ are of class $\mathcal{C}^{0, \alpha}$ with respect to all variables.

We notice that in each of the three linear systems of Bishop-type equations (3.20) above, $t:=\left(s, U_{0}\right)$ is a parameter and there appears the same matrix coefficients:

$$
p_{l}^{j}\left(e^{i \theta}, t\right):=\Phi_{u_{l}}^{j}\left(U\left(e^{i \theta}, s, U_{0}\right), e^{i \theta}, s\right),
$$

for $j, l=1, \ldots, d$. For any $\beta$ with $0<\beta \leqslant \alpha$, in order to be coherent with the definition of $\left\|\left.\Phi_{u}\right|_{s}\right\|_{\widehat{0, \beta}}$ given after Lemma 3.9, we set:

$$
\left\|\left.p\right|_{t}\right\|_{\widehat{0, \beta}}:=\max _{1 \leqslant j \leqslant d}\left(\sum_{1 \leqslant l \leqslant d}\left\|\left.p_{l}^{j}\right|_{t}\right\|_{\widehat{0, \beta}}\right) .
$$

We also set:

$$
\|p\|_{0,0}:=\max _{1 \leqslant j \leqslant d}\left(\sum_{1 \leqslant l \leqslant d}\left\|p_{l}^{j}\right\|_{0,0}\right)=\left\|\Phi_{u}\right\|_{0,0} .
$$

With these definitions, it is easy to check the inequality:

$$
\left\|\left.p\right|_{t}\right\|_{0, \beta} \leqslant\left\|\left.\Phi_{u}\right|_{s}\right\|_{0, \beta}\left[1+\left(\|U\|_{1,0}\right)^{\beta}\right] .
$$

As $\|U\|_{1,0} \leqslant \rho_{1} / 4<1$, with $\beta:=\alpha$, we deduce:

$$
\left\|\left.p\right|_{t}\right\|_{0, \alpha} \leqslant 2\left\|\left.\Phi_{u}\right|_{s}\right\|_{0, \alpha} \leqslant 2\left\|\left.\Phi\right|_{s}\right\|_{1, \alpha} .
$$


Taking $\sup _{s}$ and then $\sup _{U_{0}}$, adding 1 , squaring and inverting:

$$
\left[1+\sup _{s}\left\|\left.\Phi\right|_{s}\right\|_{1, \alpha}\right]^{-2} \leqslant 4\left[1+\sup _{s, U_{0}}\left\|\left.p\right|_{t}\right\|_{0, \alpha}\right]^{-2} .
$$

Consequently, the main assumption of the next Proposition 3.21, according to which:

$$
\|p\|_{0,0} \leqslant C^{2} \alpha^{2}\left[1+\sup _{t}\left\|\left.p\right|_{t}\right\|_{0, \alpha}\right]^{-2}
$$

for some positive absolute constant $C<1$, is superseded by one of the main assumptions, made in the theorem, according to which:

$$
\left\|\Phi_{u}\right\|_{0,0} \leqslant C^{2} \alpha^{2}\left[1+\sup _{s}\left\|\left.\Phi\right|_{s}\right\|_{0, \alpha}\right]^{-2}
$$

for some (a priori distinct) positive absolute constant $C<1$.

The following proposition applies to the three systems (3.20) and suffices to conclude the proof of Theorem 3.7 in the case $\kappa=1$. The case $\kappa \geqslant 2$ shall be discussed afterwards.

Proposition 3.21. ([[Tu1996], [*]) Let $c \in \mathbb{N}$ with $c \geqslant 1$, let $\tau_{1}=\left(\tau_{1,1}, \ldots, \tau_{1, c}\right) \in \mathbb{R}^{c}$ with $0<\tau_{1, i} \leqslant \infty, i=1, \ldots, c$, and denote by $\square_{\tau_{1}}^{c}$ the polycube $\left\{t \in \mathbb{R}^{c}:\left|t_{i}\right|<\tau_{1, i}\right\}$. Consider vector-valued and matrix-valued Hölder data:

$$
\begin{aligned}
& q=\left(q^{j}\left(e^{i \theta}, t\right)\right)^{1 \leqslant j \leqslant d} \in \mathcal{C}^{0, \alpha}\left(\partial \Delta \times \square_{\tau_{1}}^{c}, \mathbb{R}^{d}\right), \\
& p=\left(p_{l}^{j}\left(e^{i \theta}, t\right)\right)_{1 \leqslant l \leqslant d}^{1 \leqslant j \leqslant d} \in \mathcal{C}^{0, \alpha}\left(\partial \Delta \times \square_{\tau_{1}}^{c}, \mathbb{R}^{d \times d}\right),
\end{aligned}
$$

with $0<\alpha<1$. Suppose that a bounded measurable map:

$$
u=\left(u^{j}\left(e^{i \theta}, t\right)\right)^{1 \leqslant j \leqslant d} \in L^{\infty}\left(\partial \Delta \times \square_{\tau_{1}}^{c}, \mathbb{R}^{d}\right),
$$

is $\mathcal{C}^{0, \alpha / 2}$ on $\partial \Delta$ for every fixed $t$ not belonging to some null-measure subset $\mathfrak{N}$ of $\square_{\tau_{1}}^{c}$ and suppose that it satisfies the system of linear Bishop-type equations in $\mathcal{C}^{0, \alpha / 2}\left(\partial \Delta, \mathbb{R}^{d}\right)$ :

$$
u^{j}=\mathrm{T}_{*}\left(\sum_{1 \leqslant l \leqslant d} p_{l}^{j} u^{l}\right)+q^{j},
$$

for $j=1, \ldots, d$, where $\mathrm{T}_{*}=\mathrm{T}$ or $\mathrm{T}_{*}=\mathrm{T}_{1}$. Assume that the norm of the matrix p satisfies:

$$
\|p\|_{0,0}=\max _{1 \leqslant j \leqslant d}\left(\sum_{1 \leqslant l \leqslant d}\left\|p_{l}^{j}\right\|_{0,0}\right) \leqslant \mathrm{c}_{4},
$$


for some small positive constant $\mathrm{c}_{4}<1 / 16$ such that

$$
\mathrm{c}_{4} \leqslant C^{2} \alpha^{2}\left[1+\sup _{|t|<\tau_{1}}\left\|\left.p\right|_{t}\right\|_{0, \alpha}\right]^{-2},
$$

where $C<1$ is a positive absolute constant. Then, after a correction of $u$ on $\mathfrak{N}$ :

(i) on its full domain of definition $\partial \Delta \times \square_{\tau_{1}}^{c}$, the corrected map $u\left(e^{i \theta}, t\right)$ is $\mathcal{C}^{0, \alpha-0}=\bigcap_{0<\beta<\alpha} \mathcal{C}^{0, \beta}$ and furthermore:

(ii) for every fixed the map $e^{i \theta} \mapsto u\left(e^{i \theta}, t\right)$ is $\mathcal{C}^{0, \alpha}$ on $\partial \Delta$.

In general, the Hilbert transform $\mathrm{T}$ does not preserve $\mathcal{C}^{0, \alpha}$ smoothness with respect to parameters, so that the above solution $u=u\left(e^{i \theta}, t\right)$ is not better than $\mathcal{C}^{0, \alpha-0}$.

Example 3.24. ([Tu1996]) If $s \in \mathbb{R}$ with $|s|<1$ is a parameter, the function:

$$
\begin{aligned}
u\left(e^{i \theta}, s\right) & :=|s|^{\alpha} \text { if }-\pi \leqslant \theta \leqslant-|s|^{1 / 2}, \\
& :=\theta^{2 \alpha} \quad \text { if }-|s|^{1 / 2} \leqslant \theta \leqslant 0, \\
& :=\theta^{\alpha} \quad \text { if } 0 \leqslant \theta \leqslant|s|, \\
& :=|s|^{\alpha} \text { if }|s| \leqslant \theta \leqslant \pi,
\end{aligned}
$$

is $2 \pi$-periodic with respect to $\theta$ and $\mathcal{C}^{0, \alpha}$ with respect to $\left(e^{i \theta}, s\right)$. As the function $\cot (t / 2)-2 / t$ is $\mathcal{C}^{0,0}$ on $[-\pi, \pi]$, the regularity properties of the singular integral $\mathrm{T} u\left(e^{i \theta}\right)=$ p.v. $\frac{1}{\pi} \int_{-\pi}^{\pi} \frac{u\left(e^{i(\theta-t)}\right)}{\tan (t / 2)} d t$ are the same as those of:

$$
\widetilde{\mathrm{T}} u\left(e^{i \theta}\right):=\text { p.v. } \frac{1}{\pi} \int_{-\pi}^{\pi} \frac{u\left(e^{i(\theta-t)}\right)}{t} d t .
$$

However $\widetilde{T} u(1)$ involves the term $|s|^{\alpha} \log |s|$ which is $\mathcal{C}^{0, \alpha-0}$ but not $\mathcal{C}^{0, \alpha}$ :

$$
\begin{aligned}
\widetilde{\mathrm{T}} u(1) & =\frac{1}{\pi}\left(\int_{-|s|^{1 / 2}}^{-|s|} \frac{|s|^{\alpha}}{t} d t+\int_{-|s|}^{0} \frac{(-t)^{\alpha}}{t} d t+\int_{0}^{|s|^{1 / 2}} \frac{t^{2 \alpha}}{t} d t\right) \\
& =\frac{1}{2 \pi}\left(|s|^{\alpha} \log |s|-\frac{|s|^{\alpha}}{\alpha}\right) .
\end{aligned}
$$

Proof of the proposition. We shall drop the indices, writing $u, p$ and $q$, without arguments. Assume that $t \notin \mathfrak{N}$. For future majorations, it is necessary to have $\mathrm{P}_{0} u=0$. If this is not the case, we set $u^{\prime}:=u-\mathrm{P}_{0} u$ in order that $\mathrm{P}_{0} u^{\prime}=0$. Since $u$ satisfies either $u=\mathrm{T}(p u)+q$ or $u=\mathrm{T}(p u)-\mathrm{T}[p u](1)+q$, it follows that $u^{\prime}$ satisfies similar equations: either $u^{\prime}=\mathrm{T}\left(p u^{\prime}\right)+q^{\prime}$, with $q^{\prime}:=q-\mathrm{P}_{0} u$ or $u^{\prime}=\mathrm{T}\left(p u^{\prime}\right)+q^{\prime}$, with $q^{\prime}:=q-\mathrm{P}_{0} u-\mathrm{T}(p u)(1)$. Notice that $p$ is unchanged. It then suffices to establish the improvements of smoothness (i) and (ii) for $u^{\prime}$. Equivalently, we may assume that $\widehat{u}_{0}=\mathrm{P}_{0} u=0$ in the proposition. 
For $t \notin \mathfrak{N}$, the function $\phi$ is $\mathcal{C}^{0, \alpha / 2}$ on $\partial \Delta$. Applying $T$ either to the equation $u=\mathrm{T}(p u)+q$ or to the equation $u=\mathrm{T}(p u)-\mathrm{T}[p u](1)+q$, we get the same equation for both:

$$
\mathrm{T} u=-p u+\mathrm{P}_{0}(p u)+\mathrm{T} q .
$$

As $\widehat{u}_{0}=0$, we may write $u\left(e^{i \theta}\right)=\sum_{k<0} \widehat{u}_{k} e^{i k \theta}+\sum_{k>0} \widehat{u}_{k} e^{i k \theta}=: \bar{\phi}+\phi$, where $\phi$ extends holomorphically to $\Delta$. In fact, $\phi$ is determined up to a imaginary constant $i A$ and we choose $A:=-\mathrm{P}_{0}(p u) / 2$, so that:

$$
\phi=\frac{u+i \mathrm{~T} u-i \mathrm{P}_{0}(p u)}{2} .
$$

Equivalently:

$$
\left\{\begin{aligned}
u & =\phi+\bar{\phi}, \\
\mathrm{T} u & =\mathrm{P}_{0}(p u)-i(\phi-\bar{\phi}) .
\end{aligned}\right.
$$

Substituting, we rewrite (3.25) as:

$$
-i(\phi-\bar{\phi})=-p(\phi+\bar{\phi})+\mathrm{T} q
$$

or under the more convenient form:

$$
\phi=\bar{\phi}+P \bar{\phi}+Q,
$$

where the $d \times d$-matrix $P:=-2 i p(I+i p)^{-1}$ and the $d$-vector $Q:=i(I+$ $i p)^{-1} \mathrm{~T} q$ both belong to $\mathcal{C}^{0, \alpha}$.

First of all, we establish (ii) before any correction of $u$.

Lemma 3.26. For $t \notin \mathfrak{N}$, the map $e^{i \theta} \mapsto u\left(e^{i \theta}, t\right)$ is $\mathcal{C}^{0, \alpha}$ on $\partial \Delta$.

Proof. By assumption, the map $e^{i \theta} \mapsto \phi\left(e^{i \theta}, t\right)$ is $\mathcal{C}^{0, \alpha / 2}$ on $\partial \Delta$. Since $\mathrm{C}^{+}$is bounded in $\mathcal{C}^{0, \alpha / 2}$, we may apply $\mathrm{C}^{+}$to the vectorial equation $\phi=\bar{\phi}+P \bar{\phi}+Q \bar{\phi}$, noticing that $\mathrm{C}^{+}(\phi)=\phi$ and that $\mathrm{C}^{+}(\bar{\phi})=\mathrm{P}_{0}(\bar{\phi})$, since, by construction, $\phi$ extends holomorphically to $\Delta$. We thus get:

$$
\phi=\mathrm{P}_{0} \bar{\phi}+\mathrm{C}^{+}[P \bar{\phi}+Q] .
$$

Remind that $\mathrm{P}_{0} \psi=\frac{1}{2 \pi} \int_{-\pi}^{\pi} \psi\left(e^{i \theta}\right) d \theta$, so that $\left\|\mathrm{P}_{0} \psi\right\|_{0,0} \leqslant\|\psi\|_{0,0}$. Notice ${ }^{8}$ that $\|\phi(\cdot, t)\|_{0,0}<\infty$ for $t \notin \mathfrak{N}$. Taking the $\mathcal{C}^{0, \alpha}$ norm to (3.27) and applying crucially Theorem 2.18 in its $\mathbb{R}^{d}$-valued version, as in [Tu1996], we get:

$$
\begin{aligned}
\|\phi\|_{0, \alpha} & \leqslant\left\|\mathrm{P}_{0} \bar{\phi}\right\|_{0,0}+\left\|\mathrm{C}^{+}[P \bar{\phi}+Q]\right\|_{0, \alpha} \\
& \leqslant\|\phi\|_{0,0}+\frac{C_{1}}{\alpha(1-\alpha)}\left(\|P\|_{0, \alpha}\|\phi\|_{0,0}+\|Q\|_{0, \alpha}\right) \\
& <\infty
\end{aligned}
$$

\footnotetext{
${ }^{8}$ In Lemma 3.15 above, for $t \notin \mathfrak{N}$, the map $e^{i \theta} \mapsto u\left(e^{i \theta}, t\right)$ was shown to be $\mathcal{C}^{0, \alpha / 2}$ on $\partial \Delta$ in order to insure that $\mathrm{T} u(\cdot, t)$ and $\phi(\cdot, t)$ are both bounded on $\partial \Delta$, so that Theorem 2.24 may be applied in the next phrase. In [Tu1996], $u(\cdot, t)$ is only shown to be in $L^{\infty}(\partial \Delta)$, but then $T u(\cdot, t)$ and $\phi$ are not necessarily bounded.
} 
whence $\phi=\phi(\cdot, t)$ is $\mathcal{C}^{0, \alpha}$ on $\partial \Delta$, as claimed.

Next, we shall establish (i) before any correction of $u$. To this aim, let $t^{1}, t^{2} \notin$ $\mathfrak{N}$ and simply denote by $u^{1}, u^{2}$, by $\phi^{1}, \phi^{2}$, by $P^{1}, P^{2}$ and by $Q^{1}, Q^{2}$ the functions on $\partial \Delta$ with these two values of $t$. In the theorem, to establish that $u$ is $\mathcal{C}^{0, \alpha-0}$, it is natural to show that $u$ is $\mathcal{C}^{0, \beta}$ for every $\beta<\alpha$ arbitrarily close to $\alpha$.

Lemma 3.28. For every $\beta$ with $\frac{\alpha}{2}<\beta<\alpha$, every two $t^{1}, t^{2} \notin \mathfrak{N}$, we have $\left\|u^{2}-u^{1}\right\|_{0,0} \leqslant 4 \mathrm{~K}_{\alpha, \beta}\left|t^{2}-t^{1}\right|^{\beta}$, for some positive quantity $\mathrm{K}_{\alpha, \beta}<\infty$.

We shall obtain $\mathrm{K}_{\alpha, \beta}$ involving a nonremovable factor $1 /(\alpha-\beta)$. This will confirm the optimality of $\mathcal{C}^{0, \alpha-0}$-smoothness of $u$ with respect to the parameter $t$.

Proof. Since $\mathrm{P}_{0} u=\mathrm{P}_{0}(\mathrm{~T} u)=0$, applying $\mathrm{P}_{0}$ to the conjugate of (3.25), we get $\mathrm{P}_{0} \bar{\phi}=\frac{i}{2} \mathrm{P}_{0}(p u)$, so that we may rewrite (3.27) under the form:

$$
\phi=\frac{i}{2} \mathrm{P}_{0}(p u)+\mathrm{C}^{+}[P \bar{\phi}+Q] .
$$

We may then organize the difference $\phi^{2}-\phi^{1}$ as follows:

$$
\begin{aligned}
\phi^{2}-\phi^{1}= & \frac{i}{2} \mathrm{P}_{0}\left(p^{2}\left(u^{2}-u^{1}\right)\right)+\frac{i}{2} \mathrm{P}_{0}\left(\left(p^{2}-p^{1}\right) u^{1}\right)+ \\
& +\mathrm{C}^{+}\left(\left(P^{2}-P^{1}\right) \bar{\phi}^{2}+Q^{2}-Q^{1}\right)+\mathrm{C}^{+}\left(P^{1}\left(\bar{\phi}^{2}-\bar{\phi}^{1}\right)\right) \\
= & : E_{1}+E_{2}+E_{3}+E_{4} .
\end{aligned}
$$

To majorate these four $E_{i}$ 's, we shall need various preliminaries.

Firstly, to majorate $E_{1}$, we first observe the elementary inequality:

$$
\left\|u^{2}-u^{1}\right\|_{0,0} \leqslant 2\left\|\phi^{2}-\phi^{1}\right\|_{0,0} .
$$

Also, we notice that:

$$
\left\|p^{2}\right\|_{0,0}=\left\|p\left(\cdot, t^{2}\right)\right\|_{\mathcal{C}^{0,0}(\partial \Delta)} \leqslant\|p\|_{0,0} \leqslant \mathrm{c}_{4} .
$$

The majoration of $E_{1}$ is then easy:

$$
\left\|E_{1}\right\|_{0,0} \leqslant \frac{1}{2}\left\|p^{2}\right\|_{0,0}\left\|u^{2}-u^{1}\right\|_{0,0} \leqslant 4 c_{4}\left\|\phi^{2}-\phi^{1}\right\|_{0,0} .
$$

Secondly, to majorate $E_{2}$, let again $\beta$ with $\frac{\alpha}{2}<\beta<\alpha$. Using the inequality $\|p\|_{0, \beta} \leqslant 3\|p\|_{0, \alpha}$ proved in the beginning of Section 1 , we may majorate $E_{2}$ :

$$
\left\|E_{2}\right\|_{0,0} \leqslant \frac{1}{2}\left\|u^{1}\right\|_{0,0}\left\|p^{2}-p^{1}\right\|_{0,0} \leqslant \frac{3}{2}\left\|u^{1}\right\|_{0,0}\|p\|_{0, \alpha}\left|t^{2}-t^{1}\right|^{\beta} .
$$

Thirdly, to majorate $E_{3}$, we need: 
Lemma 3.30. Let $f=f(\mathrm{x}, \mathrm{y})$ be a $\mathcal{C}^{0, \alpha}$ function, defined in the product open cube $\square_{\rho}^{n} \times \square_{\rho}^{m}$, where $0<\alpha<1$ and $\rho>0$. Let $\beta$ with $0<\beta<\alpha$. For $\mathrm{x}^{\prime}, \mathrm{x}^{\prime \prime} \in \square_{\rho}^{n}$ arbitrary, the $\mathcal{C}^{0, \alpha-\beta}$-norm of the function $\square_{\rho}^{m} \ni \mathrm{y} \longmapsto f\left(\mathrm{x}^{\prime \prime}, \mathrm{y}\right)-f\left(\mathrm{x}^{\prime}, \mathrm{y}\right) \in \mathbb{R}$ satisfies:

$$
\left\|f\left(\mathrm{x}^{\prime \prime}, \cdot\right)-f\left(\mathrm{x}^{\prime}, \cdot\right)\right\|_{0, \alpha-\beta} \leqslant 4\|f\|_{0, \alpha}\left|\mathrm{x}^{\prime \prime}-\mathrm{x}^{\prime}\right|^{\beta} .
$$

Again, assume $\frac{\alpha}{2}<\beta<\alpha$. Thanks to Theorem 2.18 and to the lemma above, we may majorate $E_{3}$ :

$$
\begin{aligned}
\left\|E_{3}\right\|_{0,0} \leqslant\left\|E_{3}\right\|_{0, \alpha-\beta} & \leqslant \frac{C_{1}}{\alpha-\beta}\left(\left\|P^{2}-P^{1}\right\|_{0, \alpha-\beta}\left\|\phi^{2}\right\|_{0,0}+\left\|Q^{2}-Q^{1}\right\|_{0, \alpha-\beta}\right) \\
& \leqslant \frac{C_{2}}{\alpha-\beta}\left(\|P\|_{0, \alpha}\left\|\phi^{2}\right\|_{0,0}+\|Q\|_{0, \alpha}\right)\left|t^{2}-t^{1}\right|^{\beta} .
\end{aligned}
$$

Fourthly, to majorate $E_{4}$, we need a statement whose proof is similar to that of Lemma 3.10.

Lemma 3.31. As $\|p\|_{0,0} \leqslant \mathrm{c}_{4}$, then independently of $t \notin \mathfrak{N}$, we have:

$$
\left\|p_{\mid t}\right\|_{0, \alpha / 2} \leqslant \mathrm{c}_{4}+\mathrm{c}_{4}^{1 / 2}\left[2+\sup _{t}\left\|\left.p\right|_{t}\right\|_{0, \alpha}\right] .
$$

Reminding the main assumption $c_{4} \leqslant C^{2} \alpha^{2}\left[1+\sup _{t}\left\|\left.p\right|_{t}\right\|_{0, \alpha}\right]^{-2}$, whence $c_{4} \leqslant$ $C \alpha$, we deduce:

$$
\left\|p_{\mid t}\right\|_{0, \alpha / 2} \leqslant 3 C \alpha \text {. }
$$

Choosing then $C<1 / 6$, we may insure that $\left\|p_{\left.\right|_{t}}\right\|_{0, \alpha / 2} \leqslant 1 / 2$ for every fixed $t$. It follows in particular that we may develope $P=-2 i p \sum_{k \in \mathbb{N}}(-i p)^{k}$ and deduce the norm inequality:

$$
\left\|P_{\left.\right|_{t}}\right\|_{0, \alpha / 2} \leqslant \frac{2\left\|p_{\left.\right|_{t}}\right\|_{0, \alpha / 2}}{1-\left\|p_{\mid t}\right\|_{0, \alpha / 2}} \leqslant 4\left\|p_{\left.\right|_{t}}\right\|_{0, \alpha / 2},
$$

valid for every fixed $t$. Then again thanks to Theorem 2.18 and thanks to the previous inequalities:

$$
\begin{aligned}
\left\|E_{4}\right\|_{0,0} \leqslant\left\|E_{4}\right\|_{0, \alpha / 2} & \leqslant C_{1} \alpha^{-1}\left\|P^{1}\right\|_{0, \alpha / 2}\left\|\phi^{2}-\phi^{1}\right\|_{0,0} \\
& \leqslant C_{2} \alpha^{-1}\left\|\left.p\right|_{t^{1}}\right\|_{0, \alpha / 2}\left\|\phi^{2}-\phi^{1}\right\|_{0,0} \\
& \leqslant C_{3} C\left\|\phi^{2}-\phi^{1}\right\|_{0,0} \\
& \leqslant 4^{-1}\left\|\phi^{2}-\phi^{1}\right\|_{0,0},
\end{aligned}
$$

provided $C<\frac{1}{4 C_{3}}$. We then insert these four majorations in the inequality

$$
\left\|\phi^{2}-\phi^{1}\right\|_{0,0} \leqslant\left\|E_{1}\right\|_{0,0}+\left\|E_{2}\right\|_{0,0}+\left\|E_{3}\right\|_{0,0}+\left\|E_{4}\right\|_{0,0},
$$

and we get after reorganization:

$$
\left\|\phi^{2}-\phi^{1}\right\|_{0,0}\left(1-4 \mathrm{c}_{4}-4^{-1}\right) \leqslant \mathrm{K}_{\alpha, \beta}\left|t^{2}-t^{1}\right|^{\beta},
$$


for some quantity $\mathrm{K}_{\alpha, \beta}<\infty$ whose precise expression is:

$$
K_{\alpha, \beta}:=\frac{C_{2}}{\alpha-\beta}\left(\|P\|_{0, \alpha}\left\|\phi^{2}\right\|_{0,0}+\|Q\|_{0, \alpha}\right)+\frac{3}{2}\left\|u^{1}\right\|_{0,0}\|p\|_{0, \alpha} .
$$

It suffices then to remind that $c_{4}<1 / 16$ in the assumptions of the theorem to insure the uniform Hölder condition:

$$
\left\|\phi^{2}-\phi^{1}\right\|_{0,0} \leqslant 2 \mathrm{~K}_{\alpha, \beta}\left|t^{2}-t^{1}\right|^{\beta}
$$

valid for $t^{1}, t^{2} \notin \mathfrak{N}$. From (3.29), we conclude that $\left\|u^{2}-u^{1}\right\|_{0,0} \leqslant$ $4 \mathrm{~K}_{\alpha, \beta}\left|t^{2}-t^{1}\right|^{\beta}$. The proof of Lemma 3.28 is complete.

Then the correction of $u$ is provided by the following statement.

Lemma 3.32. ([*]) Let $f=f(\mathrm{x}, \mathrm{y}): \square_{\rho}^{n} \times\left(\square_{\rho}^{m} \backslash \mathfrak{N}\right) \rightarrow \mathbb{R}$ be a measurable $L^{\infty}$ map defined only for y not belonging to some null-measure subset $\mathfrak{N} \subset \square_{\rho}^{m}$ and let $\beta$ with $0<\beta<\alpha$. If the map $\mathrm{x} \mapsto f(\mathrm{x}, \mathrm{y})$ is $\mathcal{C}^{0, \beta}$ for every $\mathrm{y} \notin \mathfrak{N}$ and if there exists $\mathrm{K}<\infty$ such that:

$$
\sup _{\mathrm{x}}\left|f\left(\mathrm{x}, \mathrm{y}^{2}\right)-f\left(\mathrm{x}, \mathrm{y}^{1}\right)\right| \leqslant \mathrm{K}\left|\mathrm{y}^{2}-\mathrm{y}^{1}\right|^{\beta},
$$

for every two $\mathrm{y}^{1}, \mathrm{y}^{2} \notin \mathfrak{N}$, then $f$ may be extended as a $\mathcal{C}^{0, \beta}$ map in the full domain $\square_{\rho}^{n} \times \square_{\rho}^{m}$.

In sum, still denoting by $u$ the $\mathcal{C}^{0, \alpha-0}$ extension of $u$ through $\mathfrak{N}$, property (i) of the proposition is proved. To obtain that $u$ is $\mathcal{C}^{0, \alpha}$ with respect to $e^{i \theta}$, we re-apply the reasoning of Lemma 3.26 to this extension.

The proof of Proposition 3.21 is complete.

In conclusion, the functions $U_{\theta}^{j}, U_{s_{k}}^{j}$ and $U_{U_{0}^{m}}^{j}$ are $\mathcal{C}^{0, \alpha-0}$ with respect to $\left(e^{i \theta}, s, U_{0}\right)$ and $\mathcal{C}^{\alpha, 0}$ with respect to $e^{i \theta}$. Thus, the theorem is achieved if $\kappa=1$.

If $\kappa=2$, the composite functions $\Phi_{u_{l}}^{j}, \Phi_{\theta}^{j}, \Phi_{s_{i}}^{j}\left(U\left(e^{i \theta}, s, U_{0}\right), e^{i \theta}, s\right)$ are then of class $\mathcal{C}^{1, \alpha-0}$ with respect to $\left(e^{i \theta}, s, U_{0}\right)$ and of class $\mathcal{C}^{1, \alpha}$ with respect to $e^{i \theta}$. We then apply the next lemma to the three families of Bishop-type vector equations (3.20).

Lemma 3.33. Let $t \in \square_{\tau_{1}}^{c}$ be a parameter with $c \in \mathbb{N}, 0<\tau_{1, i} \leqslant$ $\infty, i=1, \ldots, c$, and consider vector-valued and matrix-valued Hölder data $q=\left(q^{j}\left(e^{i \theta}, t\right)\right)^{1 \leqslant j \leqslant d}$ and $p=\left(p_{l}^{j}\left(e^{i \theta}, t\right)\right)_{1 \leqslant l \leqslant d}^{1 \leqslant j \leqslant d}$ that are $\mathcal{C}^{1, \alpha-0}$ with respect to $\left(e^{i \theta}, t\right)$ and $\mathcal{C}^{1, \alpha}$ with respect to $e^{i \theta}$. Suppose that a given map $u=$ $\left(u^{j}\left(e^{i \theta}, t\right)\right)^{1 \leqslant j \leqslant d}$ which is $\mathcal{C}^{0, \alpha-0}$ with respect to $\left(e^{i \theta}, t\right)$ and $\mathcal{C}^{0, \alpha}$ with respect to $e^{i \theta}$ satisfies the linear Bishop-type equation $u=\mathrm{T}_{*}(p u)+q$, where $\mathrm{T}_{*}=\mathrm{T}$ or $\mathrm{T}_{*}=\mathrm{T}_{1}$. Assume that the norm of the matrix $p$ satisfies the same inequality as in the proposition: $\|p\|_{0,0} \leqslant \mathrm{c}_{4}$, for some small positive constant 
$\mathrm{c}_{4} \leqslant C^{2} \alpha^{2}\left[1+\sup _{t}\left\|\left.p\right|_{t}\right\|_{0, \alpha}\right]^{-2}$, where $0<C<1$ is some absolute constant. Then $u$ is $\mathcal{C}^{1, \alpha}$ with respect to $e^{i \theta}$ and satisfies the Lipschitz condition

$$
\left\|u\left(\cdot, t^{2}\right)-u\left(\cdot, t^{1}\right)\right\|_{0, \alpha / 2} \leqslant \mathrm{~K}\left|t^{2}-t^{1}\right|
$$

for some quantity $\mathrm{K}<\infty$. Furthermore, there exists a null-measure subset $\mathfrak{N} \subset$ $\square_{\tau_{1}}^{c} \times \square_{\rho_{1} / 16}^{d}$ such that at every $t \notin \mathfrak{N}$, for every $l=1, \ldots, c$ :

(i) the partial derivative $u_{t_{l}}\left(e^{i \theta}, t\right)$ exists for every $e^{i \theta} \in \partial \Delta$;

(ii) the map $e^{i \theta} \mapsto u_{t_{l}}\left(e^{i \theta}, t\right)$ is $\mathcal{C}^{0, \alpha / 2}$ on $\partial \Delta$.

Proof. The fact that $u$ is $\mathcal{C}^{1, \alpha}$ with respect to $e^{i \theta}$ is proved as in Lemma 3.26. For the Lipschitz condition, the reasoning is simpler than Lemma 3.17, due to the linearity of $u=\mathrm{T}_{*}(p u)+q$. Indeed, for two parameters $t^{1}, t^{2} \in \square_{\tau_{1}}^{c}$, if we take the $\mathcal{C}^{0, \alpha / 2}$-norm of the difference:

$$
u^{2}-u^{1}=\mathrm{T}_{*}\left(p^{2}\left(u^{2}-u^{1}\right)\right)+\mathrm{T}_{*}\left(\left(p^{2}-p^{1}\right) u^{1}\right)+q^{2}-q^{1},
$$

we get:

$$
\left\|u^{2}-u^{1}\right\|_{0, \alpha / 2} \leqslant C_{1} \alpha^{-1}\left\|p^{2}\right\|_{0, \alpha / 2}\left\|u^{2}-u^{1}\right\|_{0, \alpha / 2}+\mathrm{K}\left|t^{2}-t^{1}\right|,
$$

and after substraction, taking account of Lemma 3.31:

$$
\left\|u^{2}-u^{1}\right\|_{0, \alpha / 2} \leqslant 2 \mathrm{~K}\left|t^{2}-t^{1}\right| .
$$

Then the sequel of the reasoning is already known.

So for $l=1, \ldots, c$, the partial derivatives $u_{t_{l}}$ exist almost everywhere and they satisfy:

$$
u_{t_{l}}=\mathrm{T}_{*}\left(p u_{t_{l}}\right)+q_{l},
$$

with the same matrix $p$, where $q_{l}:=\mathrm{T}_{*}\left(p_{t_{l}} u\right)+q_{t_{l}}$ is $\mathcal{C}^{0, \alpha-0}$ with respect to $\left(e^{i \theta}, t\right)$ and $\mathcal{C}^{0, \alpha}$ with respect to $e^{i \theta}$.

Lemma 3.34. Proposition 3.21 holds true if more generally, $q$ is only assumed to be $\mathcal{C}^{0, \alpha-0}$ with respect to $\left(e^{i \theta}, t\right)$ and $\mathcal{C}^{0, \alpha}$ with respect to $e^{i \theta}$, with the same conclusion.

(It suffices only to inspect the majoration of $E_{3}$.) Consequently, with $u=$ $U_{\theta}, U_{s_{k}}, U_{U_{0}^{m}}$ in the three equations (3.20), we have verified that the partial derivatives $u_{\theta}, u_{s_{k}}$ and $u_{U_{0}^{m}}$ exist everywhere, are $\mathcal{C}^{0, \alpha-0}$ with respect to $\left(e^{i \theta}, s, U_{0}\right)$ and are $\mathcal{C}^{0, \alpha}$ with respect to $e^{i \theta}$. In summary, the theorem is achieved if $\kappa=2$.

Needless to say, we have clarified how to cover the case of general $\kappa \geqslant 2$ by pure logical induction. In conclusion, the proof of Theorem 3.7 is complete.

Open problem 3.35. Solve parametrized Bishop-type equations in Sobolev spaces. 


\section{§4. APPENDIX: PROOFS OF SOME LEMMAS}

4.1. Proofs of Lemmas 3.9 and 3.10. Let $x \in \mathbb{R}^{n}, n \geqslant 1$, with $|x|<\rho$, where $0<\rho \leqslant \infty$. Assuming $\|f\|_{0,0} \leqslant \mathrm{c}$, we estimate:

$$
\|f\|_{\widehat{0, \alpha / 2}} \leqslant \sup _{\mathrm{x}^{\prime \prime} \neq \mathrm{x}^{\prime}} \frac{\left|f\left(\mathrm{x}^{\prime \prime}\right)-f\left(\mathrm{x}^{\prime}\right)\right|}{\left|\mathrm{x}^{\prime \prime}-\mathrm{x}^{\prime}\right|^{\alpha / 2}}=\max (\mathrm{A}, \mathrm{B}) \leqslant \mathrm{A}+\mathrm{B},
$$

where $A:=\sup _{0<\left|x^{\prime \prime}-x^{\prime}\right|<c^{1 / \alpha}}$ and $B:=\sup _{\left|x^{\prime \prime}-x^{\prime}\right| \geqslant c^{1 / \alpha}}$ satisfy:

$$
\begin{aligned}
& \mathrm{A}=\sup _{0<\left|\mathrm{x}^{\prime \prime}-\mathrm{x}^{\prime}\right|<\mathrm{c}^{1 / \alpha}}\left(\frac{\left|f\left(\mathrm{x}^{\prime \prime}\right)-f\left(\mathrm{x}^{\prime}\right)\right|}{\left|\mathrm{x}^{\prime \prime}-\mathrm{x}^{\prime}\right|^{\alpha}}\left|\mathrm{x}^{\prime \prime}-\mathrm{x}^{\prime}\right|^{\alpha / 2}\right) \leqslant\|f\|_{\widehat{0, \alpha}} \mathrm{c}^{1 / 2}, \\
& \mathrm{~B}=\sup _{\left|\mathrm{x}^{\prime \prime}-\mathrm{x}^{\prime}\right| \geqslant \mathrm{c}^{1 / \alpha}} \frac{\left|f\left(\mathrm{x}^{\prime \prime}\right)-f\left(\mathrm{x}^{\prime}\right)\right|}{\left|\mathrm{x}^{\prime \prime}-\mathrm{x}^{\prime}\right|^{\alpha / 2}} \leqslant \frac{2\|f\|_{0,0}}{\mathrm{c}^{1 / 2}} \leqslant 2 \mathrm{c}^{1 / 2} .
\end{aligned}
$$

Lemma 3.9 is proved.

Applying this to $\mathrm{x}=(u, \theta)$, we deduce:

$$
\begin{aligned}
& \left\|\left.\Phi_{u}\right|_{s}\right\|_{\widehat{0, \alpha / 2}} \leqslant c_{2}^{1 / 2}\left[2+\left\|\left.\Phi_{u}\right|_{s}\right\|_{\widehat{0, \alpha}}\right] \leqslant c_{2}^{1 / 2}\left[2+\left\|\left.\Phi\right|_{s}\right\|_{1, \alpha}\right], \\
& \left\|\left.\Phi_{\theta}\right|_{s}\right\|_{\widehat{0, \alpha / 2}} \leqslant c_{3}^{1 / 2}\left[2+\left\|\left.\Phi_{\theta}\right|_{s}\right\|_{\widehat{0, \alpha}}\right] \leqslant c_{3}^{1 / 2}\left[2+\left\|\left.\Phi\right|_{s}\right\|_{1, \alpha}\right] .
\end{aligned}
$$

Consequently:

$$
\begin{aligned}
\left\|\left.\Phi\right|_{s}\right\|_{1, \alpha / 2} & =\left\|\left.\Phi\right|_{s}\right\|_{0,0}+\left\|\left.\Phi_{u}\right|_{s}\right\|_{0,0}+\left\|\left.\Phi_{\theta}\right|_{s}\right\|_{0,0}+\left\|\left.\Phi_{u}\right|_{s}\right\| \widehat{0, \alpha / 2}+\left\|\left.\Phi_{\theta}\right|_{s}\right\|_{\widehat{0, \alpha / 2}} \\
& \leqslant c_{1}+c_{2}+c_{3}+\left(c_{2}^{1 / 2}+c_{3}^{1 / 2}\right)\left[2+\|\Phi\|_{1, \alpha}\right] .
\end{aligned}
$$

Lemma 3.10 is proved.

4.2. Proof of Lemma 3.11. We shall abbreviate $\sup _{0<\left|\theta^{\prime \prime}-\theta^{\prime}\right| \leqslant \pi}$ by $\sup _{\theta^{\prime \prime} \neq \theta^{\prime}}$. By definition:

$$
\begin{aligned}
& \|\Phi(U(\cdot), \cdot, s)\|_{\mathcal{C}^{1, \beta}(\partial \Delta)}= \\
& =\sup _{\theta}\left|\Phi\left(U\left(e^{i \theta}\right), e^{i \theta}, s\right)\right|+ \\
& +\sup _{\theta}\left|\sum_{1 \leqslant l \leqslant d} \Phi_{u_{l}}\left(U\left(e^{i \theta}\right), e^{i \theta}, s\right) U_{\theta}^{l}\left(e^{i \theta}\right)+\Phi_{\theta}\left(U\left(e^{i \theta}\right), e^{i \theta}, s\right)\right|+ \\
& +\sup _{\theta^{\prime \prime} \neq \theta^{\prime}} \mid \sum_{1 \leqslant l \leqslant d} \Phi_{u_{l}}\left(U\left(e^{i \theta^{\prime \prime}}\right), e^{i \theta^{\prime \prime}}, s\right) U_{\theta}^{l}\left(e^{i \theta^{\prime \prime}}\right)+\Phi_{\theta}\left(U\left(e^{i \theta^{\prime \prime}}\right), e^{i \theta^{\prime \prime}}, s\right)- \\
& -\sum_{1 \leqslant l \leqslant d} \Phi_{u_{l}}\left(U\left(e^{i \theta^{\prime}}\right), e^{i \theta^{\prime}}, s\right) U_{\theta}^{l}\left(e^{i \theta^{\prime}}\right)-\Phi_{\theta}\left(U\left(e^{i \theta^{\prime}}\right), e^{i \theta^{\prime}}, s\right)|| \theta^{\prime \prime}-\left.\theta^{\prime}\right|^{-\beta}
\end{aligned}
$$


Majorating and inserting some appropriate new terms whose sum is zero:

$$
\begin{aligned}
\leqslant & \|\Phi\|_{0,0}+\left\|\Phi_{u}\right\|_{0,0}\|U\|_{1,0}+\left\|\Phi_{\theta}\right\|_{0,0}+ \\
& +\sup _{\theta^{\prime \prime} \neq \theta^{\prime}}\left|\sum_{1 \leqslant l \leqslant d} \Phi_{u_{l}}\left(U\left(e^{i \theta^{\prime \prime}}\right), e^{i \theta^{\prime \prime}}, s\right)\left[U_{\theta}^{l}\left(e^{i \theta^{\prime \prime}}\right)-U_{\theta}^{l}\left(e^{i \theta^{\prime}}\right)\right]\right|\left|\theta^{\prime \prime}-\theta^{\prime}\right|^{-\beta}+ \\
+ & \sup _{\theta^{\prime \prime} \neq \theta^{\prime}}\left|\sum_{1 \leqslant l \leqslant d}\left[\Phi_{u_{l}}\left(U\left(e^{i \theta^{\prime \prime}}\right), e^{i \theta^{\prime \prime}}, s\right)-\Phi_{u_{l}}\left(U\left(e^{i \theta^{\prime \prime}}\right), e^{i \theta^{\prime}}, s\right)\right] U_{\theta}^{l}\left(e^{i \theta^{\prime}}\right)\right|\left|\theta^{\prime \prime}-\theta^{\prime}\right|^{-\beta}+ \\
+ & \sup _{\theta^{\prime \prime} \neq \theta^{\prime}}\left|\sum_{1 \leqslant l \leqslant d}\left[\Phi_{u_{l}}\left(U\left(e^{i \theta^{\prime \prime}}\right), e^{i \theta^{\prime}}, s\right)-\Phi_{u_{l}}\left(U\left(e^{i \theta^{\prime}}\right), e^{i \theta^{\prime}}, s\right)\right] U_{\theta}^{l}\left(e^{i \theta^{\prime}}\right)\right|\left|\theta^{\prime \prime}-\theta^{\prime}\right|^{-\beta}+ \\
+ & \sup _{\theta^{\prime \prime} \neq \theta^{\prime}}\left|\Phi_{\theta}\left(U\left(e^{i \theta^{\prime \prime}}\right), e^{i \theta^{\prime \prime}}, s\right)-\Phi_{\theta}\left(U\left(e^{i \theta^{\prime \prime}}\right), e^{i \theta^{\prime}}, s\right)\right|\left|\theta^{\prime \prime}-\theta^{\prime}\right|^{-\beta}+ \\
+ & \sup _{\theta^{\prime \prime} \neq \theta^{\prime}}\left|\Phi_{\theta}\left(U\left(e^{i \theta^{\prime \prime}}\right), e^{i \theta^{\prime}}, s\right)-\Phi_{\theta}\left(U\left(e^{i \theta^{\prime}}\right), e^{i \theta^{\prime}}, s\right)\right|\left|\theta^{\prime \prime}-\theta^{\prime}\right|^{-\beta} .
\end{aligned}
$$

Majorating:

$$
\begin{aligned}
\leqslant & \|\Phi\|_{0,0}+\left\|\Phi_{u}\right\|_{0,0}\|U\|_{\widehat{1,0}}+\left\|\Phi_{\theta}\right\|_{0,0}+ \\
& +\left\|\Phi_{u}\right\|_{0,0}\|U\|_{\widehat{1, \beta}}+\left\|\left.\Phi_{u}\right|_{s}\right\|_{\widehat{0, \beta}}\|U\|_{\widehat{1,0}}+\left\|\left.\Phi_{u}\right|_{s}\right\|_{\widehat{0, \beta}}\left(\|U\|_{\widehat{1,0}}\right)^{\beta}\|U\|_{\widehat{1,0}}+ \\
& +\left\|\left.\Phi_{\theta}\right|_{s}\right\|_{\widehat{0, \beta}}+\left\|\left.\Phi_{\theta}\right|_{s}\right\|_{\widehat{0, \beta}}\left(\|U\|_{\widehat{1,0}}\right)^{\beta},
\end{aligned}
$$

which yields the lemma, noticing that $\left\|\Phi_{u}\right\|_{0,0}\left(\|U\|_{\widehat{1,0}}+\|U\|_{\widehat{1, \beta}}\right) \leqslant$ $\left\|\Phi_{u}\right\|_{0,0}\|U\|_{1, \beta}$.

4.3. Proof of Lemma 3.13. We need two preparatory lemmas.

Lemma 4.4. Let $n \geqslant 1$, let $\mathrm{x} \in \mathbb{R}^{n}$, let $m \geqslant 1$, let $\mathrm{y} \in \mathbb{R}^{m}$, let $\rho>0$ and let $f=f(\mathrm{x}, \mathrm{y})$ be $a \in \mathcal{C}^{1, \alpha}$ map, with $0<\alpha \leqslant 1$, defined in the strip $\{(\mathrm{x}, \mathrm{y}) \in$ $\left.\mathbb{R}^{m} \times \mathbb{R}^{n}:|\mathrm{x}+\mathrm{y}|<\rho\right\}$ and valued in $\mathbb{R}^{d}, d \geqslant 1$. If four vertices $\left(\mathrm{x}^{\prime}, \mathrm{y}^{\prime}\right),\left(\mathrm{x}^{\prime \prime}, \mathrm{y}^{\prime}\right)$, $\left(\mathrm{x}^{\prime}, \mathrm{y}^{\prime \prime}\right)$ and $\left(\mathrm{x}^{\prime \prime}, \mathrm{y}^{\prime \prime}\right)$ of a rectangle belong to the strip, then:

$$
\left|f\left(\mathrm{x}^{\prime \prime}, \mathrm{y}^{\prime \prime}\right)-f\left(\mathrm{x}^{\prime}, \mathrm{y}^{\prime \prime}\right)-f\left(\mathrm{x}^{\prime \prime}, \mathrm{y}^{\prime}\right)+f\left(\mathrm{x}^{\prime}, \mathrm{y}^{\prime}\right)\right| \leqslant\|f\|_{\widehat{1, \alpha}}\left|\mathrm{x}^{\prime \prime}-\mathrm{x}^{\prime}\right|\left|\mathrm{y}^{\prime \prime}-\mathrm{y}^{\prime}\right|^{\alpha} .
$$

A similar inequality holds by reversing the rôles of the variables $\mathrm{x}$ and $\mathrm{y}$.

Proof. We apply twice the Taylor integral formula (1.2) with respect to the variable $\mathrm{x}$ and we majorate:

$$
\begin{aligned}
& \left|\left(f\left(\mathrm{x}^{\prime \prime}, \mathrm{y}^{\prime \prime}\right)-f\left(\mathrm{x}^{\prime}, \mathrm{y}^{\prime \prime}\right)\right)-\left(f\left(\mathrm{x}^{\prime \prime}, \mathrm{y}^{\prime}\right)-f\left(\mathrm{x}^{\prime}, \mathrm{y}^{\prime}\right)\right)\right| \leqslant \\
& \quad \leqslant \int_{0}^{1} \sum_{1 \leqslant i \leqslant n}\left|\frac{\partial f}{\partial \mathrm{x}_{i}}\left(\mathrm{x}^{\prime}+\mathrm{s}\left(\mathrm{x}^{\prime \prime}-\mathrm{x}^{\prime}\right), \mathrm{y}^{\prime \prime}\right)-\frac{\partial f}{\partial \mathrm{x}_{i}}\left(\mathrm{x}^{\prime}+\mathrm{s}\left(\mathrm{x}^{\prime \prime}-\mathrm{x}^{\prime}\right), \mathrm{y}^{\prime}\right)\right|\left|\mathrm{x}_{i}^{\prime \prime}-\mathrm{x}_{i}^{\prime}\right| d \mathrm{~s} \\
& \quad \leqslant\|f\|_{\widehat{1, \alpha}}\left|\mathrm{y}^{\prime \prime}-\mathrm{y}^{\prime}\right|^{\alpha}\left|\mathrm{x}^{\prime \prime}-\mathrm{x}^{\prime}\right| . \quad \square
\end{aligned}
$$


Lemma 4.5. Let $n \geqslant 1$, let $\mathrm{x} \in \mathbb{R}^{n}$, let $\rho>0$ and let $H=H(\mathrm{t})$ be a $\mathcal{C}^{1, \alpha}$ map, with $0<\alpha \leqslant 1$, defined in the open cube $\square_{\rho}^{n}=\{|t|<\rho\}$ and valued in $\mathbb{R}^{d}$. Let $\mathrm{x}, \mathrm{y}, \mathrm{z}$ with $|\mathrm{x}|,|\mathrm{y}|,|\mathrm{z}| \leqslant \rho / 3$, so that the four points $\mathrm{x}, \mathrm{y}, \mathrm{z}$ and $\mathrm{x}+\mathrm{y}-\mathrm{z}$ constitute the vertices of a parallelogram which is contained in $\square_{\rho}^{n}$. Then:

$$
|H(\mathrm{x}+\mathrm{y}-\mathrm{z})-H(\mathrm{x})-H(\mathrm{y})+H(\mathrm{z})| \leqslant 4\|H\|_{\widehat{1, \alpha}}|\mathrm{y}-\mathrm{z}||\mathrm{x}-\mathrm{z}|^{\alpha} .
$$

A similar inequality holds after exchanging $\mathrm{x}$ and $\mathrm{y}$.

Proof. To estimate the second difference of $H$, we introduce a new map

$$
f(\mathrm{x}, \mathrm{y}):=H(\mathrm{x}+\mathrm{y}),
$$

of $(x, y) \in \mathbb{R}^{n} \times \mathbb{R}^{n}$, whose domain is the strip $\{|x+y|<\rho\}$. Let $x, y$, $z$ with $|x|,|y|,|z| \leqslant \rho / 3$. Fixing $x^{\prime} \in \mathbb{R}^{n}$ arbitrary, there exist unique $y^{\prime}, x^{\prime \prime}$ and $y^{\prime \prime}$ solving the linear system:

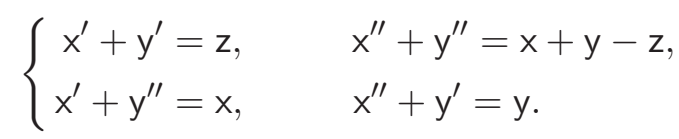

In fact, $y^{\prime}=z-x^{\prime}, x^{\prime \prime}=y-z+x^{\prime}$ and $y^{\prime \prime}=x-x^{\prime \prime}$. Taking the norms $|\cdot|$ of the four equations above, we see that the rectangle $\left(x^{\prime}, y^{\prime}\right),\left(x^{\prime \prime}, y^{\prime}\right),\left(x^{\prime}, y^{\prime \prime}\right),\left(x^{\prime \prime}, y^{\prime \prime}\right)$ is contained in the strip $\{|\mathrm{x}+\mathrm{y}|<\rho\}$. Applying then Lemma 4.4 (with $m=n$ ), we get:

$$
\begin{aligned}
& |H(\mathrm{x}+\mathrm{y}-\mathrm{z})-H(\mathrm{x})-H(\mathrm{y})+H(\mathrm{z})|= \\
& \quad=\left|f\left(\mathrm{x}^{\prime \prime}, \mathrm{y}^{\prime \prime}\right)-f\left(\mathrm{x}^{\prime}, \mathrm{y}^{\prime \prime}\right)-f\left(\mathrm{x}^{\prime \prime}, \mathrm{y}^{\prime}\right)+f\left(\mathrm{x}^{\prime}, \mathrm{y}^{\prime}\right)\right| \\
& \quad \leqslant\|f\|_{\widehat{1, \alpha}}\left|\mathrm{x}^{\prime \prime}-\mathrm{x}^{\prime}\right|\left|\mathrm{y}^{\prime \prime}-\mathrm{y}^{\prime}\right|^{\alpha} \\
& \quad=\|f\|_{\widehat{1, \alpha}}|\mathrm{y}-\mathrm{z}||\mathrm{x}-\mathrm{z}|^{\alpha} .
\end{aligned}
$$

We claim that $\|f\|_{\widehat{1, \alpha}} \leqslant 4\|H\|_{\widehat{1, \alpha}}$, which will conclude. Carefulness and rigor are required. In fact, to estimate:

$$
\|f\|_{\widehat{1, \alpha}}=\sum_{i=1}^{n} \sup _{\left(\mathrm{x}^{\prime \prime}, \mathrm{y}^{\prime \prime}\right) \neq\left(\mathrm{x}^{\prime}, \mathrm{y}^{\prime}\right)} \frac{\left|f_{\mathrm{x}_{i}}\left(\mathrm{x}^{\prime \prime}, \mathrm{y}^{\prime \prime}\right)-f_{\mathrm{x}_{i}}\left(\mathrm{x}^{\prime}, \mathrm{y}^{\prime}\right)\right|+\left|f_{\mathrm{y}_{i}}\left(\mathrm{x}^{\prime \prime}, \mathrm{y}^{\prime \prime}\right)-f_{\mathrm{y}_{i}}\left(\mathrm{x}^{\prime}, \mathrm{y}^{\prime}\right)\right|}{\left|\left(\mathrm{x}^{\prime \prime}, \mathrm{y}^{\prime \prime}\right)-\left(\mathrm{x}^{\prime}, \mathrm{y}^{\prime}\right)\right|^{\alpha}}
$$

we shall first transform the denominator. By definition:

$$
\left|\left(x^{\prime \prime}, y^{\prime \prime}\right)-\left(x^{\prime}, y^{\prime}\right)\right|=\max \left(\left|x^{\prime \prime}-x^{\prime}\right|,\left|y^{\prime \prime}-y^{\prime}\right|\right) .
$$

If we set $a:=\left|\mathrm{x}^{\prime \prime}-\mathrm{x}^{\prime}\right|$ and $b:=\left|\mathrm{y}^{\prime \prime}-\mathrm{y}^{\prime}\right|$ and if we invert the inequality $(a+$ $b)^{\alpha} \leqslant 2 \max \left(a^{\alpha}, b^{\alpha}\right)$, noticing $2^{\alpha} \leqslant 2$, we obtain:

$$
\begin{aligned}
\frac{1}{\left|\left(\mathrm{x}^{\prime \prime}, \mathrm{y}^{\prime \prime}\right)-\left(\mathrm{x}^{\prime}, \mathrm{y}^{\prime}\right)\right|^{\alpha}}=\frac{1}{\max \left(a^{\alpha}, b^{\alpha}\right)} \leqslant \frac{2}{(a+b)^{\alpha}} & =\frac{2}{\left(\left|\mathrm{x}^{\prime \prime}-\mathrm{x}^{\prime}\right|+\left|\mathrm{y}^{\prime \prime}-\mathrm{y}^{\prime}\right|\right)^{\alpha}} \\
& \leqslant \frac{2}{\left|\mathrm{x}^{\prime \prime}+\mathrm{y}^{\prime \prime}-\mathrm{x}^{\prime}-\mathrm{y}^{\prime}\right|^{\alpha}} .
\end{aligned}
$$


Replacing the denominator above, we then transform and majorate the numerator:

$$
\begin{aligned}
\|f\|_{\widehat{1, \alpha}} & \leqslant 2 \sum_{i=1}^{n} \sup _{\left(\mathrm{x}^{\prime \prime}, \mathrm{y}^{\prime \prime}\right) \neq\left(\mathrm{x}^{\prime}, \mathrm{y}^{\prime}\right)}\left(\frac{\left|H_{\mathrm{t}_{i}}\left(\mathrm{x}^{\prime \prime}+\mathrm{y}^{\prime \prime}\right)-H_{\mathrm{t}_{i}}\left(\mathrm{x}^{\prime}+\mathrm{y}^{\prime}\right)\right|+\left|H_{\mathrm{t}_{i}}\left(\mathrm{x}^{\prime \prime}+\mathrm{y}^{\prime \prime}\right)-H_{\mathrm{t}_{i}}\left(\mathrm{x}^{\prime}+\mathrm{y}^{\prime}\right)\right|}{\left|\mathrm{x}^{\prime \prime}+\mathrm{y}^{\prime \prime}-\mathrm{x}^{\prime}-\mathrm{y}^{\prime}\right|^{\alpha}}\right) \\
& \leqslant 4 \sum_{i=1}^{n} \sup _{\mathrm{t}^{\prime \prime} \neq \mathrm{t}^{\prime}}\left(\frac{\left|H_{\mathrm{t}_{i}}\left(\mathrm{t}^{\prime \prime}\right)-H_{\mathrm{t}_{i}}\left(\mathrm{t}^{\prime}\right)\right|}{\left|\mathrm{t}^{\prime \prime}-\mathrm{t}^{\prime}\right|^{\alpha}}\right) \\
& =4\|H\|_{\widehat{1, \alpha} .} .
\end{aligned}
$$

This completes the proof of Lemma 4.5.

We can now state a slightly simplified version of Lemma 3.13.

Lemma 4.6. ([Гu1990], [*]) Let $u \in \mathbb{R}^{d}, d \geqslant 1$, let $\rho_{1}>0$ and let $\Psi=\Psi(u)$ be $a \mathcal{C}^{1, \alpha}$ map, with $0<\alpha \leqslant 1, u \in \mathbb{R}^{d}$, defined in the cube $\left\{|u|<\rho_{1}\right\}$ and valued in $\mathbb{R}^{d}$. Let $U^{1}, U^{2} \in \mathcal{C}^{1,0}\left(\partial \Delta, \mathbb{R}^{d}\right)$ with $\left|U^{j}\left(e^{i \theta}\right)\right|<\rho_{1} / 3$ on $\partial \Delta$, for $j=1,2$. For every $\beta$ with $0<\beta \leqslant \alpha$ the following inequality holds:

$$
\left\|\Psi\left(U^{2}(\cdot)\right)-\Psi\left(U^{1}(\cdot)\right)\right\|_{\mathcal{C}^{0, \beta}(\partial \Delta)} \leqslant \mathrm{D}\left\|U^{2}-U^{1}\right\|_{0, \beta},
$$

with

$$
\mathrm{D}=\|\Psi\|_{1, \beta}\left[1+2\left(\left\|U^{1}\right\|_{\widehat{1,0}}\right)^{\beta}+2\left(\left\|U^{2}\right\|_{\widehat{1,0}}\right)^{\beta}\right] .
$$

Proof. Firstly and obviously:

$$
\left\|\Psi\left(U^{2}\right)-\Psi\left(U^{1}\right)\right\|_{0,0} \leqslant\|\Psi\|_{\widehat{1,0}}\left\|U^{2}-U^{1}\right\|_{0,0} .
$$

Secondly, we have $\left\|\Psi\left(U^{2}\right)-\Psi\left(U^{1}\right)\right\|_{\widehat{0, \beta}}=\sup _{0<\left|\theta^{\prime \prime}-\theta^{\prime}\right| \leqslant \pi}\left(\mathrm{Q} /\left|\theta^{\prime \prime}-\theta^{\prime}\right|^{\beta}\right)$, where:

$$
\mathrm{Q}:=\left|\Psi\left(U^{2}\left(e^{i \theta^{\prime \prime}}\right)\right)-\Psi\left(U^{1}\left(e^{i \theta^{\prime \prime}}\right)\right)-\Psi\left(U^{2}\left(e^{i \theta^{\prime}}\right)\right)+\Psi\left(U^{1}\left(e^{i \theta^{\prime}}\right)\right)\right| .
$$

To majorate $\mathrm{Q}$, we start by inserting the term $\Psi\left[U^{1}\left(e^{i \theta^{\prime \prime}}\right)+U^{2}\left(e^{i \theta^{\prime}}\right)-U^{1}\left(e^{i \theta^{\prime}}\right)\right]$, well-defined, thanks to the assumption $\left\|U^{j}\right\|_{0,0}<\rho_{1} / 3$ :

$$
\begin{array}{r}
Q \leqslant\left|\Psi\left(U^{2}\left(e^{i \theta^{\prime \prime}}\right)\right)-\Psi\left[U^{1}\left(e^{i \theta^{\prime \prime}}\right)+U^{2}\left(e^{i \theta^{\prime}}\right)-U^{1}\left(e^{i \theta^{\prime}}\right)\right]\right|+ \\
+\mid \Psi\left[U^{1}\left(e^{i \theta^{\prime \prime}}\right)+U^{2}\left(e^{i \theta^{\prime}}\right)-U^{1}\left(e^{i \theta^{\prime}}\right)\right]-\Psi\left(U^{1}\left(e^{i \theta^{\prime \prime}}\right)\right)- \\
-\Psi\left(U^{2}\left(e^{i \theta^{\prime}}\right)\right)+\Psi\left(U^{1}\left(e^{i \theta^{\prime}}\right)\right) \mid .
\end{array}
$$

To estimate the second absolute value, we apply Lemma 4.5 with $\mathrm{x}=U^{1}\left(e^{i \theta^{\prime \prime}}\right)$, with $\mathrm{y}=U^{2}\left(e^{i \theta^{\prime}}\right)$ and with $\mathrm{z}=U^{1}\left(e^{i \theta^{\prime}}\right)$ :

$$
\begin{aligned}
\mathrm{Q} \leqslant & \|\Psi\|_{\widehat{1,0}}\left|\left[U^{2}-U^{1}\right]\left(e^{i \theta^{\prime \prime}}\right)-\left[U^{2}-U^{1}\right]\left(e^{i \theta^{\prime}}\right)\right|+ \\
& +4\|\Psi\|_{\widehat{1, \beta}}\left|U^{2}\left(e^{i \theta^{\prime}}\right)-U^{1}\left(e^{i \theta^{\prime}}\right)\right|\left|U^{1}\left(e^{i \theta^{\prime \prime}}\right)-U^{1}\left(e^{i \theta^{\prime}}\right)\right|^{\beta} .
\end{aligned}
$$


We then achieve the remaining majorations:

$$
\begin{aligned}
Q \leqslant & \|\Psi\|_{\widehat{1,0}}\left\|U^{2}-U^{1}\right\|_{\widehat{0, \beta}}\left|\theta^{\prime \prime}-\theta^{\prime}\right|^{\beta}+ \\
& +4\|\Psi\|_{\widehat{1, \beta}}\left\|U^{2}-U^{1}\right\|_{0,0}\left(\left\|U^{1}\right\|_{\widehat{1,0}}\right)^{\beta}\left|\theta^{\prime \prime}-\theta^{\prime}\right|^{\beta} .
\end{aligned}
$$

Reminding that $\left\|U^{2}-U^{1}\right\|_{0,0}+\left\|U^{2}-U^{1}\right\|_{\widehat{0, \beta}}=\left\|U^{2}-U^{1}\right\|_{0, \beta}$ and summing, we obtain:

$$
\left\|\Psi\left(U^{2}\right)-\Psi\left(U^{1}\right)\right\|_{0, \beta} \leqslant\|\Psi\|_{1, \beta}\left[1+4\left(\left\|U^{1}\right\|_{\widehat{1,0}}\right)^{\beta}\right]\left\|U^{2}-U^{1}\right\|_{0, \beta} .
$$

A similar inequality holds with $\left(\left\|U^{2}\right\|_{\widehat{1,0}}\right)^{\beta}$ instead of $\left(\left\|U^{1}\right\|_{\widehat{1,0}}\right)^{\beta}$. Taking the arithmetic mean, we find the symmetric quantity $\mathrm{D}$ of the lemma. The proof is complete.

Proof of Lemma 3.13. By definition:

$$
\begin{aligned}
& \mathrm{R}:=\left\|\Phi\left(U^{2}(\cdot), \cdot, s\right)-\Phi\left(U^{1}(\cdot), \cdot, s\right)\right\|_{\mathcal{C}^{0, \beta}(\partial \Delta)} \\
&= \sup _{\theta}\left|\Phi\left(U^{2}\left(e^{i \theta}\right), e^{i \theta}, s\right)-\Phi\left(U^{1}\left(e^{i \theta}\right), e^{i \theta}, s\right)\right|+ \\
&+\sup _{\theta^{\prime \prime} \neq \theta^{\prime}} \mid \Phi\left(U^{2}\left(e^{i \theta^{\prime \prime}}\right), e^{i \theta^{\prime \prime}}, s\right)-\Phi\left(U^{1}\left(e^{i \theta^{\prime \prime}}\right), e^{i \theta^{\prime \prime}}, s\right)- \\
& \quad-\Phi\left(U^{2}\left(e^{i \theta^{\prime}}\right), e^{i \theta^{\prime}}, s\right)+\Phi\left(U^{1}\left(e^{i \theta^{\prime}}\right), e^{i \theta^{\prime}}, s\right)|| \theta^{\prime \prime}-\left.\theta^{\prime}\right|^{-\beta} .
\end{aligned}
$$

In the numerator, we insert $-\Phi\left(U^{2}\left(e^{i \theta^{\prime \prime}}\right), e^{i \theta^{\prime}}, s\right)+\Phi\left(U^{1}\left(e^{i \theta^{\prime \prime}}\right), e^{i \theta^{\prime}}, s\right)$ plus its opposite:

$$
\begin{aligned}
\mathrm{R} \leqslant\|\Phi\|_{\widehat{1,0}}\left\|U^{2}-U^{1}\right\|_{0,0}+ & \\
+\sup _{\theta^{\prime \prime} \neq \theta^{\prime}} \mid & \Phi\left(U^{2}\left(e^{i \theta^{\prime \prime}}\right), e^{i \theta^{\prime \prime}}, s\right)-\Phi\left(U^{1}\left(e^{i \theta^{\prime \prime}}\right), e^{i \theta^{\prime \prime}}, s\right)- \\
& -\Phi\left(U^{2}\left(e^{i \theta^{\prime \prime}}\right), e^{i \theta^{\prime}}, s\right)+\Phi\left(U^{1}\left(e^{i \theta^{\prime \prime}}\right), e^{i \theta^{\prime}}, s\right)|| \theta^{\prime \prime}-\left.\theta^{\prime}\right|^{-\beta}+ \\
+\sup _{\theta^{\prime \prime} \neq \theta^{\prime}} \mid & \Phi\left(U^{2}\left(e^{i \theta^{\prime \prime}}\right), e^{i \theta^{\prime}}, s\right)-\Phi\left(U^{1}\left(e^{i \theta^{\prime \prime}}\right), e^{i \theta^{\prime}}, s\right)- \\
& -\Phi\left(U^{2}\left(e^{i \theta^{\prime}}\right), e^{i \theta^{\prime}}, s\right)+\Phi\left(U^{1}\left(e^{i \theta^{\prime}}\right), e^{i \theta^{\prime}}, s\right)|| \theta^{\prime \prime}-\left.\theta^{\prime}\right|^{-\beta} .
\end{aligned}
$$

To majorate the first $\sup _{\theta^{\prime \prime} \neq \theta^{\prime}}$, we apply Lemma 4.4 with $\mathrm{x}^{\prime}=U^{1}\left(e^{i \theta^{\prime \prime}}\right), \mathrm{y}^{\prime}=$ $e^{i \theta^{\prime}}, \mathrm{x}^{\prime \prime}=U^{2}\left(e^{i \theta^{\prime \prime}}\right)$ and $\mathrm{y}^{\prime \prime}=e^{i \theta^{\prime}}$, where $s$ is considered as a dumb parameter. To majorate the second $\sup _{\theta^{\prime \prime} \neq \theta^{\prime}}$, we apply Lemma 4.6 to $\Psi(u):=\Phi\left(u, e^{i \theta}, s\right)$, 
where $\left(e^{i \theta}, s\right)$ are considered as dumb parameters. We get:

$$
\begin{aligned}
\mathrm{R} \leqslant & \|\Phi\|_{\widehat{1,0}}\left\|U^{2}-U^{1}\right\|_{0,0}+ \\
& +\|\Phi\|_{\widehat{1, \beta},} \sup _{\theta}\left|U^{2}\left(e^{i \theta}\right)-U^{1}\left(e^{i \theta}\right)\right|+ \\
& +\|\Phi\|_{1, \beta}\left[1+2\left(\left\|U^{1}\right\|_{\widehat{1,0}}\right)^{\beta}+2\left(\left\|U^{2}\right\|_{\widehat{1,0}}\right)^{\beta}\right]\left\|U^{2}-U^{1}\right\|_{0, \beta} .
\end{aligned}
$$

To conclude, we use $\|\Phi\|_{\widehat{1,0}}+\|\Phi\|_{\widehat{1, \beta}} \leqslant\|\Phi\|_{1, \beta}$ and we get the term $\mathrm{C}$ of Lemma 3.13.

With these techniques, the proofs of Lemmas 3.17, 3.19, 3.30, 3.31 and 3.32 are easily guessed and even simpler. 


\title{
V: Holomorphic extension of CR functions
}

\author{
Table of contents \\ 1. Hartogs theorem, jump formula and domains having the holomorphic extension

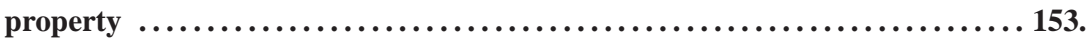 \\ 2. Trépreau's theorem, deformations of Bishop discs and propagation on hyper-

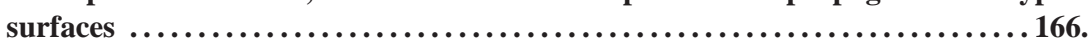 \\ 3. Tumanov's theorem, deformations of Bishop discs and propagation on generic

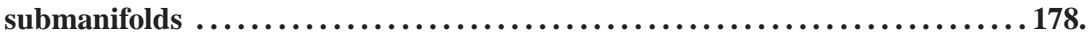 \\ 4. Holomorphic extension on globally minimal generic submanifolds .......192. \\ [19 diagrams]
}

The method of analytic discs is rooted in the very birth of the theory of functions of several complex variables. The discovery by Hurwitz and Hartogs of the compulsory extension of holomorphic functions relied upon an application of Cauchy's integral formula along a family of analytic discs surrounding an illusory singularity. Since H. Cartan, Thullen, Behnke and Sommer, various versions of this argument were coined "Kontinuitättsatz" or "Continuity principle".

The removal of compact singularities culminated in the so-called HartogsBochner theorem, usually proved by means of integral formulas or thanks to the resolution of a $\bar{\partial}$ problem with compact support. Contradicting all expectations, a subtle example due to Fornaess (1998), shows that on a non-pseudoconvex domain, the disc method may fail to fill in the domain, if the discs are required to stay inside the domain.

Nevertheless, it is of the highest prize to build constructive methods in order to describe significant parts of the envelope of holomorphy of a domain, of a CR manifold, as well as the polynomial hull of certain compact sets. In such problems, analytic discs with boundary in the domain, the CR manifold or the compact set remain the most adequate tools.

The precise existence Theorem 3.7(IV) for the solutions of Bishop's equation that was established in the previous Part IV may now be applied systematically to a variety of geometric situations. In this respect, we just followed Bishop's genuine philosophy that required to ensure an explicit control of the size of solutions in terms of the size of data, instead of appealing to some general, imprecise version of the implicit function theorem.

Thanks to the jump theorem, holomorphic extension of CR functions defined on a hypersurface $M$ is equivalent to the extension of the functions that are holomorphic in one of the two sides to the other side. Trépreau's original theorem (1986) states that such an extension holds at a point $p$ if and only if there does not exist a local complex hypersurface $\Sigma$ of $\mathbb{C}^{n}$ with $p \in \Sigma \subset M$. A deeper 
phenomenon of propagation (Trépreau, 1990) holds: if CR functions extend holomorphically to one side at a point $p$, a similar extension holds at every point of the CR orbit of $p$ in $M$. By means of deformations of attached Bishop discs, there is an elementary (and folklore) proof that contains both the local and the global extension theorems on hypersurfaces, yielding a satisfactory understanding of the phenomenon.

On a generic submanifold $M$ of $\mathbb{C}^{n}$ of higher codimension, the celebrated $T u$ manov extension theorem (1988) states that CR functions defined on $M$ extend holomorphically to a local wedge of edge $M$ at a point $p$ if the local CR orbit of $p$ contains a neighborhood of $p$ in $M$. A globalization of this statement, obtained independently by Jöricke and the first author in 1994, states that the same extension phenomenon holds if $M$ consists of a single CR orbit, i.e. is globally minimal. Both proofs heavily relied on the local Tumanov theorem and on a precise control of the propagation of directions of extension.

A clever proof that treats both locally minimal and globally minimal generic submanifolds on the same footing constitutes the main Theorem 4.12 of the present Part V: if $M$ is a globally minimal $\mathcal{C}^{2, \alpha}(0<\alpha<1)$ generic submanifold of $\mathbb{C}^{n}$ of codimension $\geqslant 1$ and of $C R$ dimension $\geqslant 1$, there exists a wedgelike domain $\mathcal{W}$ attached to $M$ such that every continuous $C R$ function $f \in \mathcal{C}_{C R}^{0}(M)$ possesses a holomorphic extension $F \in \mathcal{O}(\mathcal{W}) \cap \mathcal{C}^{0}(M \cup \mathcal{W})$ with $\left.F\right|_{M}=f$. This basic statement as well as the techniques underlying its proof will be the very starting point of the study of removable singularities in Parts VI and in [MP2006.

\section{$\S 1$. HARTOGS THEOREM, JUMP FORMULA AND DOMAINS HAVING THE EXTENSION PROPERTY}

1.1. Hartogs extension theorem: brief history. ${ }^{9}$ In 1897 , Hurwitz showed that a function holomorphic in $\mathbb{C}^{2} \backslash\{0\}$ extends holomorphically through the origin. In his thesis (1906), Hartogs generalized this discovery, emphasizing the compulsory holomorphic extendability of functions that are defined on the nowadays celebrated Hartogs skeleton (diagram below). The main argument is to apply Cauchy's integral formula along families of analytic discs having their boundary inside the domain and whose interior goes outside the domain. In fact, the thinness of an embedded circle in $\mathbb{C}^{n}(n \geqslant 2)$ offers much freedom to include illusory singularities inside a disc.

In 1924, Osgood stated the ultimate generalization of the discovery of Hurwitz and Hartogs: if $\Omega \subset \mathbb{C}^{n}(n \geqslant 2)$ is a domain and if $K \subset \Omega$ is any compact such that $\Omega \backslash K$ connected, then $\mathcal{O}(\Omega \backslash K)=\left.\mathcal{O}(\Omega)\right|_{\Omega \backslash K}$. This statement is nowadays called the Hartogs-Bochner theorem. Although the proof of Osgood was correct for geometrically simple complements $\Omega \backslash K$, as for instance spherical shells, it was incomplete for general $\Omega \backslash K$. In fact, unpleasant topological and monodromy

\footnotetext{
${ }^{9}$ Further historical information may be found in Ra1986, Str1988, Fi1991, Ra2002. HM2002.
} 
obstructions occur for general $\Omega \backslash K$ when pushing analytic discs. In 1998, Fornaess exhibited certain domains in which discs are forced to first leave some intermediate domain $\Omega \backslash K_{1}, K_{1} \subset K$, before $\Omega$ may be filled in.

In the late 1930's, a rigorous proof of Osgood's general statement was obtained by Fueter, by means of a generalization of the classical Cauchy-Green-Pompeiu integral formula to several variables, in the context of complex and quaternionic functions (Moisil 1931, Fueter 1935). In 1943, Martinelli simplified the formal treatment of Fueter. Then Bochner observed that the same result holds more generally if one assumes given on $\partial \Omega$ just a CR function.

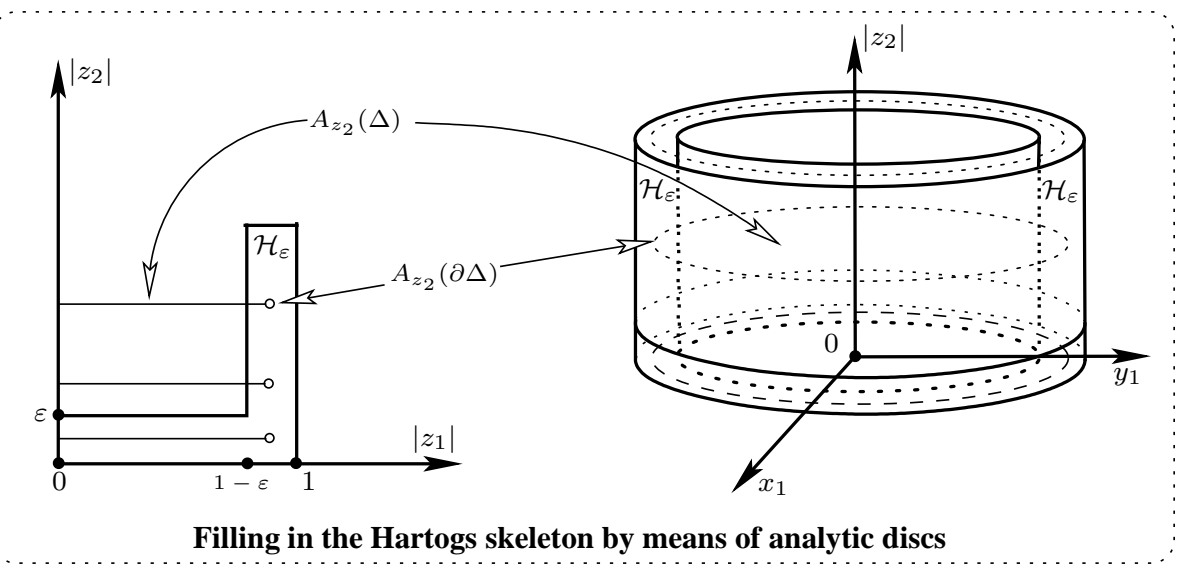

1.2. Hartogs domain. Consider the $\varepsilon$-Hartogs skeleton (pot-looking) domain:

$$
\mathcal{H}_{\varepsilon}:=\left\{\left(z_{1}, z_{2}\right) \in \mathbb{C}^{2}:\left|z_{1}\right|<1,\left|z_{2}\right|<\varepsilon\right\} \bigcup\left\{1-\varepsilon<\left|z_{1}\right|<1,\left|z_{2}\right|<1\right\} .
$$

We draw two diagrams: in $\left(\left|z_{1}\right|,\left|z_{2}\right|\right)$ and in $\left(x_{1}, y_{1},\left|z_{2}\right|\right)$ coordinates.

Lemma 1.3. Every holomorphic function $f \in \mathcal{O}\left(\mathcal{H}_{\varepsilon}\right)$ extends holomorphically to the bidisc $\Delta \times \Delta$, the convex hull of $\mathcal{H}_{\varepsilon}$.

Proof. Letting $\delta$ with $0<\delta<\varepsilon$, for every $z_{2} \in \mathbb{C}$ with $\left|z_{2}\right|<1$, the analytic disc

$$
\Delta \ni \zeta \longmapsto A_{z_{2}}(\zeta):=\left([1-\delta] \zeta, z_{2}\right) \in \mathbb{C}^{2}
$$

has its boundary $A_{z_{2}}(\partial \Delta)$ contained in $\mathcal{H}_{\varepsilon}$, the domain where the function $f$ is defined. Thus, we may compute the Cauchy integral

$$
F\left(z_{1}, z_{2}\right):=\frac{1}{2 \pi i} \int_{\partial \Delta} \frac{f\left(A_{z_{2}}(\zeta)\right)}{\zeta-z_{1}} d \zeta .
$$

Differentiating under the sum, this extension $F$ is seen to be holomorphic. In addition, for $\left|z_{2}\right|<\varepsilon$, it coincides with $f$. Obviously, the discs $A_{z_{2}}(\Delta)$ fill in the hole of the domain $\mathcal{H}_{\varepsilon}$. 
1.4. Bounded domains in $\mathbb{C}^{n}$ and Hartogs-Bochner extension phenomenon. Let $\Omega$ be a connected open subset of $\mathbb{C}^{n}$, a domain. We assume it to be bounded, i.e. $\bar{\Omega}$ is compact and that its boundary $\partial \Omega:=\bar{\Omega} \backslash \Omega$ is a hypersurface of $\mathbb{C}^{n}$ of class at least $\mathcal{C}^{1}$. By means of a partition of unity, one can construct a real-valued function $r$ defined on $\mathbb{C}^{n}$ such that $\Omega=\{z: r(z)<0\}$ and $\partial \Omega=\{z: r(z)=0\}$, with $d r(z) \neq 0$ for every $z \in \partial \Omega$. Then $\partial \Omega$ is orientable.

Extensions of the above disc argument led to the most general ${ }^{10}$ form of the Hartogs theorem: if $\Omega$ is a bounded domain in $\mathbb{C}^{n}(n \geqslant 2)$ having connected boundary $\partial \Omega$, then every function holomorphic in a neighborhood of $\partial \Omega$ uniquely extends as a function holomorphic in $\Omega$. There are three classical methods of proof:

- using the Bochner-Martinelli kernel;

- using solutions of $\bar{\partial} u=v$ having compact support;

- pushing analytic discs, in successive Hartogs skeletons.

The first two are rigorously established and we shall review the first in a while. For almost one hundred years, it has been a folklore belief that the third method could be accomplished somehow. Let us be precise.

1.5. Fornaess' counterexample and a disc theorem. Thus, let $\Omega$ be a bounded domain of $\mathbb{C}^{2}$ having connected $\mathcal{C}^{1}$ boundary. For $\delta>0$ small, consider the one-sided neighborhood of $\partial \Omega$ defined by:

$$
\widetilde{\Omega}_{\delta}:=\{z \in \Omega: \operatorname{dist}(z, \partial \Omega)<\delta\} .
$$

The complement $\Omega \backslash \widetilde{\Omega}_{\delta}$ is a compact hole. Remind that the bidisc $\Delta^{2}$ is the convex hull of the Hartogs skeleton $\mathcal{H}_{\varepsilon}$. Following [F1998], we say that $\Omega$ can be filled in by analytic discs if for every $\delta>0$, there exist a finite sequence of subdomains of $\Omega$ having $\mathcal{C}^{1}$ boundary, $\widetilde{\Omega}_{\delta}=\Omega_{1} \subset \Omega_{2} \subset \cdots \subset \Omega_{k}=\Omega$ and for each $j=1, \ldots, k-1$, an $\varepsilon_{j}>0$ and a univalent holomorphic map $\Phi_{j}$ defined in a neighborhood of $\bar{\Delta}^{2}$ such that:

(1) $\Omega_{j+1} \subset \Omega_{j} \cup \Phi_{j}\left(\Delta^{2}\right) \subset \Omega$;

(2) $\Phi_{j}\left(\mathcal{H}_{\varepsilon}\right) \subset \Omega_{j}$;

(3) $\Omega_{j} \cap \Phi_{j}\left(\Delta^{2}\right)$ is connected;

(4) $\Omega_{j+1} \cap \Phi_{j}\left(\Delta^{2}\right)$ is connected.

For such domains, by pushing analytic discs in the embedded Hartogs figure, taking account of connectedness, we have $\left.\mathcal{O}\left(\Omega_{j+1}\right)\right|_{\Omega_{j}}=\mathcal{O}\left(\Omega_{j}\right)$. Then by induction, uniquely determined holomorphic extension holds from $\Omega_{1}$ up to $\Omega$. Importantly, the intermediate domains are required to be all contained in $\Omega$.

\footnotetext{
${ }^{10}$ Often, some authors consider instead a compact $K \subset \Omega$ with $\Omega \backslash K$ connected and state that $\mathcal{O}(\Omega \backslash K)=\left.\mathcal{O}(\Omega)\right|_{\Omega \backslash K}$; a technical check shows that the two statements are equivalent.
} 
In 1998, Fornaess [F1998] constructed a topologically strange domain $\Omega \subset \mathbb{C}^{2}$ that cannot be filled in this way. This example shows that the requirement that $\Omega_{j} \subset \Omega_{j+1} \subset \Omega$ is too stringent.

Nevertheless, taking care of monodromy and working in the envelope of holomorphy of $\Omega$, one may push analytic discs by allowing them to wander in the outside, in order to get the general Hartogs theorem stated above. As a preliminary, one perturbs and smoothes out the boundary. Denote by $\|z\|:=$ $\left(\left|z_{1}\right|^{2}+\cdots+\left|z_{n}\right|^{2}\right)^{1 / 2}$ the Euclidean norm of $z=\left(z_{1}, \ldots, z_{n}\right) \in \mathbb{C}^{n}$ and by $\mathbb{B}^{n}(p, \delta):=\{\|z-p\|<\delta\}$ the open ball of radius $\delta>0$ centered at a point $p$.

Theorem 1.6. ([MP2006] $)$ Let $M \Subset \mathbb{C}^{n}(n \geqslant 2)$ be a connected $\mathcal{C}^{\infty}$ hypersurface bounding a domain $\Omega_{M} \Subset \mathbb{C}^{n}$. Suppose to fix ideas that $2 \leqslant \operatorname{dist}\left(0, \bar{\Omega}_{M}\right) \leqslant$ 5 and assume that the restriction $r_{M}:=\left.r\right|_{M}$ of the distance function $r(z)=\|z\|$ to $M$ is a Morse function having only a finite number $\kappa$ of critical points $\widehat{p}_{\lambda} \in M$, $1 \leqslant \lambda \leqslant \kappa$, located on different sphere levels:

$$
2 \leqslant \widehat{r}_{1}:=r\left(\widehat{p}_{1}\right)<\cdots<\widehat{r}_{\kappa}:=r\left(\widehat{p}_{\kappa}\right) \leqslant 5+\operatorname{diam}\left(\bar{\Omega}_{M}\right) .
$$

Then there exists $\delta_{1}>0$ such that for every $\delta$ with $0<\delta<\delta_{1}$, the (tubular) neighborhood

$$
\mathcal{V}_{\delta}(M):=\cup_{p \in M} \mathbb{B}^{n}(p, \delta)
$$

enjoys the global Hartogs extension property into $\Omega_{M}$ :

$$
\mathcal{O}\left(\mathcal{V}_{\delta}(M)\right)=\left.\mathcal{O}\left(\Omega_{M} \cup \mathcal{V}_{\delta}(M)\right)\right|_{\mathcal{V}_{\delta}(M)},
$$

by "pushing" analytic discs inside a finite number of Hartogs figures, without using neither the Bochner-Martinelli kernel, nor solutions of some auxiliary $\bar{\partial}$ problem.

1.7. Hartogs-Bochner theorem via the Bochner-Martinelli kernel. By $\mathcal{O}(C)$, where $C \subset \mathbb{C}^{n}$ is closed, we mean $\mathcal{O}(\mathcal{V}(C))$ for some open neighborhood $\mathcal{V}(C)$ of $C$. Here is the general statement.

Theorem 1.8. ([HeLe1984, He1985, Ra1986]) Let $\Omega$ be a bounded domain in $\mathbb{C}^{n}$ having connected boundary. Then for every neighborhood $U$ of $\partial \Omega$ in $\mathbb{C}^{n}$ and every holomorphic function $f \in \mathcal{O}(U)$, there exists a function $F \in \mathcal{O}(\bar{\Omega})$ with $\left.F\right|_{\partial \Omega}=\left.f\right|_{\partial \Omega}$

In the thin neighborhood $U$ of the not necessarily smooth boundary $\partial \Omega$, by means of a partion of unity, one may construct a connected boundary $\partial \Omega_{1} \subset$ $U$ close to $\partial \Omega$ which is $\mathcal{C}^{1}$, or $\mathcal{C}^{\infty}$, or even $\mathcal{C}^{\omega}$, using Whitney approximation ([Hi1976]; in addition, one may assure that $\left.r(z)\right|_{\partial \Omega_{1}}$ is as in Theorem 1.6, whence both statements are equivalent). Then the restriction $\left.F\right|_{\partial \Omega_{1}}$ is CR on $\partial \Omega_{1}$ and the previous theorem is a consequence of the next. 
Theorem 1.9. ([Ra1986, He1985]) Let $\Omega$ be a bounded domain in $\mathbb{C}^{n}(n \geqslant 2)$ having connected $\mathcal{C}^{\kappa, \alpha}$ boundary, with $1 \leqslant \kappa \leqslant \infty, 0 \leqslant \alpha \leqslant 1$. Then for every $C R$ function $f: \partial \Omega \rightarrow \mathbb{C}$ of class $\mathcal{C}^{\kappa, \alpha}$, there exists a function $F \in \mathcal{O}(\Omega) \cap$ $\mathcal{C}^{\kappa, \alpha}(\bar{\Omega})$ with $\left.F\right|_{\partial \Omega}=f$.

Some words about the proof. With $\zeta, z \in \mathbb{C}^{n}$, consider the Bochner-Martinelli kernel:

$$
\operatorname{BM}(\zeta, z):=\frac{(n-1) !}{(2 \pi i)^{n}}|\zeta-z|^{-2 n} \sum_{j=1}^{n}\left(\bar{\zeta}_{j}-\bar{z}_{j}\right) d \zeta_{j} \underset{k \neq j}{\wedge} d \bar{\zeta}_{k} \wedge d \zeta_{k} .
$$

This is a $(n, n-1)$-form which is $\mathcal{C}^{\omega}$ off the diagonal $\{\zeta=z\}$. For $n=2$, it coincides with the Cauchy kernel $\frac{1}{2 \pi i} \frac{1}{\zeta-z}$. If $f$ and $\partial \Omega$ are $\mathcal{C}^{1}$, the integral formula:

$$
F(z):=\int_{\partial \Omega} f(\zeta) \operatorname{BM}(\zeta, z)
$$

provides the holomorphic extension $F$.

1.10. Hypersurfaces of $\mathbb{C}^{n}$ and jump theorem for CR functions. Let $M$ be a real hypersurface of $\mathbb{C}^{n}$ without boundary. In the sequel, we shall mainly deal with three geometric situations.

- Local: $M$ is defined in a small open polydisc centered at one point $p \in$ $M$.

- Global: $M$ is a connected orientable embedded submanifold of $\mathbb{C}^{n}$.

- Boundary: $\mathbb{C}^{n} \backslash M$ consists of two open sets $\Omega^{+}$, bounded and $\Omega^{-}$, unbounded.

Then there exists some appropriate neighborhood $\mathcal{M}$ of $M$ in $\mathbb{C}^{n}$ in which $M$ is relatively closed, in the sense that $\bar{M} \cap \mathcal{M}=M$.

More generally, let $\mathcal{M}$ be an arbitrary complex manifold of dimension $n \geqslant 1$ and let $M \subset \mathcal{M}$ be a hypersurface of class at least $\mathcal{C}^{1}$ which is relatively closed in $\mathcal{M}$ and oriented. The complement $\mathcal{M} \backslash M$ then consists of two connected components $\Omega^{+}$and $\Omega^{-}$, where $\Omega^{+}$is located on the positive side to $M$. Also, let $f: M \rightarrow \mathbb{C}$ be a CR function of class at least $\mathcal{C}^{0}$. By definition, $f$ is $\mathrm{CR}$ if the current of integration on $M$ of bidegree $(0,1)$ defined by ${ }^{11}$ :

$$
f_{M}(\omega):=\int_{M} f \omega, \quad \omega \in \mathcal{D}^{n, n-1}
$$

satisfies $\int_{M} f \bar{\partial} \varpi=0$ for every $\varpi \in \mathcal{D}^{n, n-2}$. Equivalently, $\bar{\partial} f_{M}=0$ in the sense of currents, where $f_{M}$ is interpreted as a $(0,1)$-form having measure coefficients.

\footnotetext{
${ }^{11}$ Here, $\mathcal{D}^{p, q}$ is the space of $\mathcal{C}^{\infty}$ forms of bidegree $(p, q)$ having compact support; fundamental notions about currents may be found in [Ch1989].
} 
To formulate the jump theorem in arbitrary complex manifolds, we shall mainly assume that the Dolbeault $\bar{\partial}$-complex on $\mathcal{M}$ is exact in bidegree $(0,1)$, namely $H_{\bar{\partial}}^{0,1}(\mathcal{M})=0$. This assumption holds for instance when $\mathcal{M}=\Delta^{n}, \mathbb{C}^{n}$ or $P_{n}(\mathbb{C})$. It means that the equation $\bar{\partial} u=v$, where $v$ is a $\bar{\partial}$-closed $(0,1)$-form on $\mathcal{M}$ having $\mathcal{C}^{\infty}, L^{2}$ or distributional coefficients has a $\mathcal{C}^{\infty}, L^{2}$ or distributional solution $u$ on $\mathcal{M}$.

Consequently, there exists a distribution $F$ on $\mathcal{M}$ with $\bar{\partial} F=f_{M}$. As supp $f_{M} \subset M$, such a function $F$ is holomorphic in $\mathcal{M} \backslash M$. The difference $F_{2}-F_{1}$ of two solutions to $\bar{\partial} F=f_{M}$ is holomorphic in $\mathcal{M}$. In the case where $\mathcal{M}=\mathbb{C}^{n}$, a solution to $\bar{\partial} F=f_{M}$ may be represented ([Ch1975, Ra1986]) by means of the Bochner-Martinelli kernel as $F(z):=\int_{M} f(\zeta) \operatorname{BM}(\zeta, z)$. In complex dimension $n=1$, such a solution coincides with the classical Cauchy transform.

In 1975, after previous work of Andreotti-Hill ([AH1972b] $)$, Chirka obtained a several complex variables version of the Sokhotskiî-Plemelj Theorem 2.7(IV).

Theorem 1.11. (Ch1975]) Assume that $H_{\bar{\partial}}^{0,1}(\mathcal{M})=0$ and that the hypersurface $M \subset \mathcal{M}$ is orientable and relatively closed, i.e. $\bar{M} \cap \mathcal{M}=M$. Assume $\operatorname{dim} \mathcal{M}=$ $n \geqslant 1$ and let $(\kappa, \alpha)$ with $0 \leqslant \kappa \leqslant \infty, 0<\alpha<1$. If $M$ is $\mathcal{C}^{\kappa+1, \alpha}$ and if the current $f_{M}$ associated to a $\mathcal{C}^{\kappa, \alpha}$ function $f: M \rightarrow \mathbb{C}$ is $C R$, then every distributional solution $F \in \mathcal{O}(\mathcal{M} \backslash M)$ to $\bar{\partial} F=f_{M}$ extends to be $\mathcal{C}^{\kappa, \alpha}$ in the two closures $\overline{\Omega^{ \pm}}=\Omega^{ \pm} \cup M$, yielding two functions $F^{ \pm} \in \mathcal{O}\left(\Omega^{ \pm}\right) \cap \mathcal{C}^{\kappa, \alpha}\left(\Omega^{ \pm} \cup M\right)$ whose jump across $M$ equals $f$ :

$$
F^{+}(z)-F^{-}(z)=f(z), \quad \forall z \in M .
$$

A similar jump formula holds for $f \in L_{\text {loc,CR }}^{\mathrm{p}}(M)$, with $M$ at least $\mathcal{C}^{1}$ (or a Lipschitz graph) and for $f \in \mathcal{D}_{C R}^{\prime}(M)$, with $M \in \mathcal{C}^{\infty}$.

When $\mathcal{M}=\mathbb{C}$, the conditions that $f$ is $\mathrm{CR}$ and that $H_{\bar{\partial}}^{0,1}(\mathcal{M})=0$ are automatically satisfied and we recover the Sokhotskiî-Plemelj jump formula. However, we mention that in several complex variables $(n \geqslant 2)$, there is no analog of the second formula $\frac{1}{2}\left[F^{+}\left(\zeta_{0}\right)+F^{-}\left(\zeta_{0}\right)\right]=$ p.v. $\frac{1}{2 \pi i} \int_{\Gamma} \frac{f(\zeta)}{\zeta-\zeta_{0}} d \zeta$. The reason is the inexistence of a universal integral formula solving $\bar{\partial} F=f_{M}$. Nevertheless, there should exist generalized principal value integrals which depend on the kernel.

If $M$ is only $\mathcal{C}^{1}$ and $f$ is only $\mathcal{C}^{0}$, it is in general untrue that $F^{-}$and $F^{+}$ extend continuously to $M$. Fortunately, there is a useful substitute result, analog to Theorem 2.9(IV). Consider a open subset $M^{\prime} \subset M$ having compact closure $\overline{M^{\prime}}$ not meeting $\partial M=\bar{M} \backslash M$. We may embedd $M^{\prime}$ in a one-parameter family $\left(M_{\varepsilon}^{\prime}\right)_{|\varepsilon|<\varepsilon_{0}}, \varepsilon_{0}>0$, of hypersurfaces that foliates a strip thickening of $M^{\prime}$.

Theorem 1.12. (Ch1975]) If $f$ is $C R$ and $\mathcal{C}^{\kappa}$ on a $\mathcal{C}^{\kappa+1}$ hypersurface $M$, then

$$
\lim _{\varepsilon \rightarrow 0}\left\|\left.F\right|_{M_{\varepsilon}^{\prime}}-\left.F\right|_{M_{-\varepsilon}^{\prime}}-f\right\|_{\kappa}=0 .
$$


1.13. CR extension in the projective space. Unlike in $\mathbb{C}^{n}$, there is no privileged "interior" side of an orientable connected hypersurface in the projective space $P_{n}(\mathbb{C}), n \geqslant 2$. Nevertheless, a version of the Hartogs-Bochner theorem holds. The proof is an illustration of the use of the jump theorem.

Theorem 1.14. ( $n \geqslant 3$ : HL1975]; $n=2$ : [Sa1999, DM2002]) Let $M$ be a compact orientable connected $\mathcal{C}^{2}$ real hypersurface of $P_{n}(\mathbb{C})$ that divides the projective space into two domains $\Omega^{-}$and $\Omega^{+}$. Then:

(i) there exists a side, $\Omega^{-}$or $\Omega^{+}$, to which every function holomorphic in some neighborhood of $M$ extends holomorphically ${ }^{12}$;

(ii) every function that is holomorphic in the union of the other side of $M$ together with a neighborhood of $M$ must be constant.

Let us summarize the proof. Let $f$ be holomorphic in some neighborhood $\mathcal{V}(M)$ of $M$ in $P_{n}(\mathbb{C})$. As the Dolbeault cohomology group $H^{0,1}\left(P_{n}(\mathbb{C})\right)$ vanishes for $n \geqslant 2$ ([[HeLe1984, He1985]]), thanks to Theorem 1.11 above, the CR function $\left.f\right|_{M}$ on $M$ can be decomposed as the jump $f=F^{+}-F^{-}$between two functions $F^{ \pm}$holomorphic in $\Omega^{ \pm}$which are (at least) continuous up to $M$. It suffices then to show that either $F^{+}$or $F^{-}$is constant, since clearly, if $F^{+}$(resp. $F^{-}$) is constant equal to $c^{+}$(resp. $c^{-}$), then $f$ extends holomorphically to $\Omega^{-}$ (resp. to $\Omega^{+}$) as $c^{+}-F^{-}$(resp. as $F^{+}-c^{-}$).

By contradiction, assume that both $F^{+}$and $F^{-}$are nonconstant. We choose two domains $U^{+}$and $U^{-}$with $\mathcal{V}(M) \cup \Omega^{ \pm} \supset U^{ \pm} \supset M \cup \Omega^{ \pm}$. By a preliminary (technical) deformation argument, we may assume that $F^{ \pm}$is holomorphic in $U^{ \pm}$. According to a theorem due to Takeuchi [Ta1964, holomorphic functions in an arbitrary domain of $P_{n}(\mathbb{C})(n \geqslant 2)$, either are constant or separate points. Since $F^{-}$is nonconstant, $\mathcal{O}\left(U^{-}\right)$separates points. Conjugating with elements of the group $\operatorname{PGL}(n, \mathbb{C})$ of projective automorphisms of $P_{n}(\mathbb{C})$, shrinking $\mathcal{V}(M)$ and $U^{-}$slightly if necessary, we may verify ([DM2002] that $\mathcal{O}\left(U^{-}\right)$separates points and provides local system of holomorphic coordinates at every point. By standard techniques of Stein theory ([Hö1973] $)$, it follows that $U^{-}$is embeddable in some $\mathbb{C}^{N}$, with $N$ large. The image of $M$ under such an embedding $\Phi$ is a compact CR submanifold of $\mathbb{C}^{N}$ that is filled by the relatively compact complex manifold $\Sigma^{-}=\Phi\left(U^{-}\right)$with boundary $\Phi(M)$. Two applications of the maximum principle to the nonconstant holomorphic function $F^{+} \circ \Phi^{-1}$ say that it must decrease inside $\Sigma^{-}$, since $\Sigma^{-}$is interior to $\Phi(M)$ in $\mathbb{C}^{N}$, and that it must increase, since the one-sided neighborhood $U^{-} \cap \mathcal{V}(M)$ is exterior to $U^{+}$. This is the desired contradiction.

\footnotetext{
${ }^{12}$ Using propagation techniques of Section 3, the theorem holds assuming that $M$ is globally minimal and considering continuous CR functions on $M$.
} 
1.15. Levi extension theorem. A $\mathcal{C}^{2}$ hypersurface $M \subset \mathbb{C}^{n}$ may always be represented as $M=\{z \in U: r(z)=0\}$, where $U$ is some open neighborhood of $M$ in $\mathbb{C}^{n}$, and where $r: U \rightarrow \mathbb{R}$ is a $\mathcal{C}^{2}$ implicit defining function that satisfies $d r(q) \neq 0$ at every point $q$ of $M$. Two defining functions $r^{1}, r^{2}$ are nonzero multiple of each other in some neighborhood $V \subset U$ of $M$ : there exists $\lambda: V \rightarrow \mathbb{R}$ nowhere vanishing with $r_{2}=\lambda r_{1}$.

At a point $p \in M$, the Levi form of $r$ :

$$
\mathfrak{L}_{p} r\left(L_{p}, \bar{L}_{p}\right):=\sum_{1 \leqslant j, k \leqslant n} \frac{\partial^{2} r}{\partial z_{j} \partial \bar{z}_{k}}(p) L_{p}^{j} \bar{L}_{p}^{k}, \quad L_{p} \in T_{p}^{1,0} M,
$$

is a Hermitian form that may be diagonalized. Its signature at $p$ :

$$
\left(a_{p}, b_{p}\right):=\text { (\# positive eigenvalues, \# negative eigenvalues) }
$$

is the same for $r_{1}$ and $r_{2}$ if they are positive multiples of each other. It is also invariant through local biholomorphic changes of coordinates $z \mapsto \widetilde{z}(z)$ that do not reverse the orientation of $M$. Reversing the orientation or taking a negative factor $\lambda$ corresponds to the transposition $\left(a_{p}, b_{p}\right) \mapsto\left(b_{p}, a_{p}\right)$.

The Levi form may be read off a graphed equation $v=\varphi(x, y, u)$ for $M$.

Lemma 1.16. There exist local holomorphic coordinates $(z, u+i v) \in \mathbb{C}^{n-1} \times \mathbb{C}$ centered at $p$ in which $M$ is represented as a graph of the form:

$$
v=\varphi(z, u)=\sum_{1 \leqslant k \leqslant a_{p}+b_{p}} \varepsilon_{k} z_{k} \bar{z}_{k}+\mathrm{o}\left(|z|^{2}\right)+\mathrm{O}(|z||u|)+\mathrm{O}\left(|u|^{2}\right),
$$

where $\varepsilon_{k}=+1$ for $1 \leqslant k \leqslant a_{p}, \varepsilon_{k}=-1$ for $a_{p}+1 \leqslant k \leqslant a_{p}+b_{p}$. If $a_{p}=n-1$, the open set $\{v>\varphi\}$ is strongly convex (in the real sense) in a neighborhood of p.

Assuming that $M$ is orientable, it is surrounded by two open sides. By an open side of $M$, we mean a connected component of $\mathcal{V} \backslash M$ for a (thin) neighborhood $\mathcal{V}$ of $M$ which is divided by $M$ in two components. As germs of open sets along $M$, there exist two open sides (if $M$ were not orientable, there would exist only one).

Assuming that $M$ is represented either by an implicit equation $r=0$ or as a graph $-v+\varphi(x, y, u)=0$, we adopt the convention of denoting:

$$
\begin{aligned}
& \Omega^{+}:=\{r<0\} \quad \text { or } \quad \Omega^{+}:=\{v>\varphi(x, y, u)\}, \\
& \Omega^{-}:=\{r>0\} \quad \text { or } \quad \Omega^{-}:=\{v<\varphi(x, y, u)\} .
\end{aligned}
$$

Once a local side $\Omega$ of $M$ has been fixed, $M$ is oriented and the indetermination $r \leftrightarrow-r$ disappears. By convention, we will always represent $\Omega=\{r<0\}$. Then the number of positive and of negative eigenvalues of the Levi-form of $r$ at a point $p \in \partial \Omega$ is an invariant. By common abuse of language, we speak of the Levi form of $\partial \Omega$. 
At one of its points $p$, a boundary $\partial \Omega$ is called strongly pseudoconvex (resp. strongly pseudoconcave) if its Levi form has all its eigenvalues $>0$ (resp. $<0)$ at $p$. It is called weakly pseudoconvex (resp. weakly pseudoconcave) at $p$ if all eigenvalues are $\geqslant 0$ (resp. $\leqslant 0$ ). Often, the term "weakly" is dropped in common use.

Definition 1.17. If $\Omega$ is an open side of $M$, we say that $\Omega$ is holomorphically extendable at $p$ if for every open ${ }^{13}$ polydisc $U_{p}$ centered at $p$, there exists an open polydisc $V_{p}$ centered at $p$ such that for every $f \in \mathcal{O}\left(\Omega \cap U_{p}\right)$, there exists $F \in$ $\mathcal{O}\left(V_{p}\right)$ with $\left.F\right|_{\Omega \cap V_{p}}=\left.f\right|_{\Omega \cap V_{p}}$.

In 1910, Levi localized the Hartogs extension phenomenon.

Theorem 1.18. ([Bo1991, Trp1996, Tu1998, BER1999]) If the Levi form of $M \subset$ $\partial \Omega$ has one negative eigenvalue at a point $p$, then $\Omega$ is holomorphically extendable at $p$.

Proof. As $\Omega$ is given by $\left\{v>-z_{1} \bar{z}_{1}+\cdots\right\}$, for $\varepsilon>0$ small, the disc $A_{\varepsilon}(\zeta):=$ $(\varepsilon \zeta, 0, \ldots, 0)$ has its boundary $A_{\varepsilon}(\partial \Delta)$ contained in $\Omega$ near $p$.

Lemma 1.19. Assume $M$ is $\mathcal{C}^{1}$, let $p \in M$ and $\Omega$ be an open side of $M$ at $p$. Suppose that for every open polydisc $U_{p}$ centered at $p$, there exists an analytic disc $A: \Delta \rightarrow U_{p}$ continuous in $\bar{\Delta}$ with $A(0)=p$ and $A(\partial \Delta) \subset \Omega$. Then $\Omega$ is holomorphically extendable at $p$.

To draw $A(\Delta)$, decreasing by 1 its dimension, we represent it as a curve. The cusp illustrates the fact that $A(\Delta)$ is not assumed to be embedded.

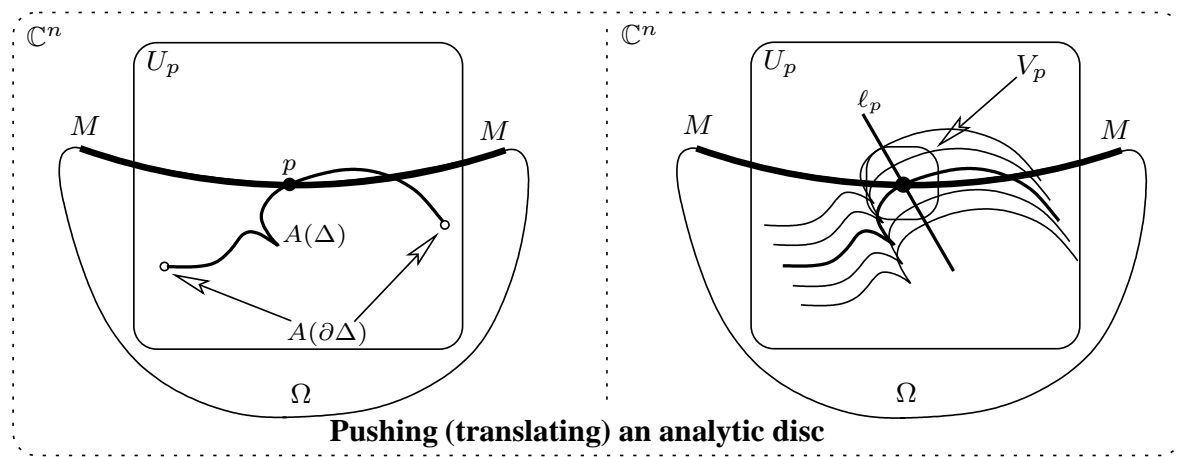

To prove the lemma, we may assume that $p=0$. Since $A(\partial \Delta)$ is contained in $\Omega$, for $z \in \mathbb{C}^{n}$ very small, say $|z|<\delta$, the translates $z+A(\partial \Delta)$ of the disc boundary are also contained in $\Omega$.

\footnotetext{
${ }^{13}$ Although the shape of polydiscs is not invariant by local biholomorphisms, their topology is. To avoid dealing implicitly with possibly wild open sets, we prefer to speak of neighborhoods $U_{p}, V_{p}, W_{p}$ of $p$ that are polydiscs.
} 
Consequently, the Cauchy integral:

$$
F(z):=\frac{1}{2 \pi i} \int_{\partial \Delta} f(z+A(\zeta)) \frac{d \zeta}{\zeta}
$$

is meaningful and it defines a holomorphic function of $z$ in the polydisc $V_{p}:=$ $\{|z|<\delta\}$.

Does it coincide with $f$ in $V_{p} \cap \Omega$ ? The assumption that $M$ is $\mathcal{C}^{1}$ yields a local real segment $\ell_{p}$ transversal to $M$ at $p$. If $U_{p}$ is sufficiently small and if $z \in \ell_{p} \cap \Omega$ goes sufficiently deep in $\Omega$, the disc $z+A(\bar{\Delta})$ is contained in $\Omega$, so that the Cauchy integral $F(z)$ coincides with $f(z)$ for those $z$.

1.20. Contact of weakly pseudoconvex domains with complex hypersurfaces. The domain $\Omega$ is said to admit a support complex hypersurface at $p \in \partial \Omega$ if there exists a local (possibly singular) complex hypersurface $\Sigma$ passing through $p$ that does not intersect $\Omega$. In this situation, if $\Sigma=\{h(z)=0\}$ with $h$ holomorphic, the function $1 / h$ does not extend holomorphically at $p$, being unbounded. With $\alpha>0$ not integer, one may define branches $h^{\alpha}$ which are uniform in $\Omega$ and continuous up to $\partial \Omega$, but whose extension through $p$ would be ramified around $\Sigma$. Consequently, the existence of a support complex hypersurface prevents $\mathcal{O}(\Omega)$ to be holomorphically extendable at $p$. Is it the right obstruction? For instance, at a strongly pseudoconvex boundary point, the complex tangent plane is support.

Nevertheless, in 1973, Kohn-Nirenberg constructed a special pseudoconvex domain $\Omega_{\mathrm{KN}}^{+}$in $\mathbb{C}^{2}$ showing that:

- not every weakly pseudoconvex smoothly bounded domain is locally biholomorphically equivalent to a domain which is weakly convex in the real sense;

- the holomorphic non-extendability of $\mathcal{O}(\Omega)$ at $p$ is totally independent from the existence of a local supporting complex hypersurface at $p$.

The boundary of this domain

$$
M_{\mathrm{KN}}:=\left\{(z, w) \in \mathbb{C}^{2}: \operatorname{Im} w=|z w|^{2}+z^{4} \bar{z}^{4}+15\left(z^{7} \bar{z}+\bar{z}^{7} z\right) / 14\right\}
$$

may be checked to be strongly pseudoconvex at every point except the origin, where it is weakly pseudoconvex. Hence $\mathcal{O}\left(\Omega_{\mathrm{KN}}^{+}\right)$is not holomorphically extendable at the origin. However, $M_{\mathrm{KN}}$ has the striking property that every local (possibly singular) complex hypersurface $\Sigma$ passing through the origin meets both sides of $M_{\mathrm{KN}}$ in every neighborhood of the origin. By means of a Puiseux parametrization ([ [aPf2000] $)$, such a complex curve $\Sigma$ is always the image of a certain holomorphic disc $\lambda: \Delta \rightarrow \mathbb{C}^{2}$ with $\lambda(0)=0$.

Theorem 1.21. ([KN1973]) Whatever the holomorphic disc $\lambda$, for every $\varepsilon>0$, there are $\zeta^{-}$and $\zeta^{+}$in $\Delta$ with $\left|\zeta^{ \pm}\right|<\varepsilon$ such that $\lambda\left(\zeta^{ \pm}\right) \in \Omega_{\mathrm{KN}}^{ \pm}$.

Clearly, $\Omega_{\mathrm{KN}}^{+}$is not locally convexifiable at the origin (otherwise, the biholomorphic image of the complex tangent line would be support). 
Often for technical reasons, certain results in several complex variables require boundaries to be convex in the real sense. Although this condition is not biholomorphically invariant, it is certainly meaningful to characterize the class of convexifiable domains, at least locally: does there exist an analytico-geometric criterion enabling to recognize local convexifiability by reading a defining equation?

1.22. Holomorphic extendability across finite type hypersurfaces. Let $M$ be a $\mathcal{C}^{\omega}$ hypersurface and let $p \in M$. Then $M$ is of type $m$ at $p$, in the sense of Definition 4.22(III), if and only if there exists a local graphed equation centered at $p$ of the form:

$$
v=\varphi_{m}(z, \bar{z})+\mathrm{O}\left(|z|^{m+1}\right)+\mathrm{O}(|z||u|)+\mathrm{O}\left(|u|^{2}\right),
$$

where $\varphi_{m} \in \mathbb{C}[z, \bar{z}]$ is a nonzero homogeneous real-valued polynomial of degree $m$ having no pluriharmonic term, namely $0 \equiv \varphi_{m}(0, \bar{z}) \equiv \varphi_{m}(z, 0)$. The restriction $\varphi_{m}(\ell(\zeta), \overline{\ell(\zeta)})$ of $\varphi_{m}$ to a complex line $\mathbb{C} \ni \zeta \mapsto \ell(\zeta) \in \mathbb{C}^{n-1}, \ell(0)=0$, is a polynomial in $\mathbb{C}[\zeta, \bar{\zeta}]$. For almost every choice of $\ell$, this polynomial is nonzero, homogeneous of the same degree $m$ and contains no harmonic term. After a rotation, such a line is the complex $z_{1}$-axis. Denoting $z^{\prime}=\left(z_{2}, \ldots, z_{n-1}\right)$, we obtain:

$$
v=\varphi_{m}\left(z_{1}, \bar{z}_{1}\right)+\mathrm{O}\left(\left|z_{1}\right|^{m+1}\right)+O\left(\left|z^{\prime}\right|\right)+\mathrm{O}(|z||u|)+\mathrm{O}\left(|u|^{2}\right) .
$$

Theorem 1.24. ([BeFo1978, R1983, BT1984]) If $m$ is even, at least one side $\Omega^{+}$or $\Omega^{-}$is holomorphically extendable at $p$. If $m$ is odd, both sides have this property.

Proof. Let $\varepsilon>0$ arbitrarily small, let $a \in \mathbb{C}$ with $|a|<1$, let $\zeta$ be in the closed unit disc $\bar{\Delta}$, and introduce a $\mathbb{C}^{n}$-valued analytic disc:

$$
A_{\varepsilon}(\zeta):=\left(\varepsilon(a+\zeta), 0, \ldots, 0, \varepsilon^{m} W(\zeta)\right)
$$

having zero $z^{\prime}$-component and $z_{1}$-component being a disc of radius $\varepsilon$ centered at $-a$. We assume its $w$-component $W(\zeta)$ be defined by requiring that the $\mathbb{C}^{2}$-valued disc

$$
B_{\varepsilon}(\zeta):=\left(\varepsilon(a+\zeta), \varepsilon^{m} W(\zeta)\right)
$$

has its boundary $B_{\varepsilon}(\partial \Delta)$ attached to $v=\varphi_{m}\left(z_{1}, \bar{z}_{1}\right)$. By homogeneity, $\varepsilon^{m}$ drops and it is equivalent to require that $B_{1}$ is attached to $v=\varphi_{m}\left(z_{1}, \bar{z}_{1}\right)$. Equivalently, the imaginary part $V(\zeta)$ of $W=U+i V$ should satisfy:

$$
V\left(e^{i \theta}\right)=\varphi_{m}\left(a+e^{i \theta}, \bar{a}+e^{-i \theta}\right)
$$

for all $e^{i \theta} \in \partial \Delta$. To obtain a harmonic extension to $\Delta$ of the function $V$ thus defined on $\partial \Delta$, no Bishop equation is needed. It suffices to take the harmonic prolongation by means of Poisson's formula, as in $\S 2.20(\mathrm{IV})$ :

$$
V(\eta)=\mathrm{P} V(\eta)=\frac{1}{2 \pi i} \int_{\partial \Delta} \varphi_{m}(a+\zeta, \bar{a}+\bar{\zeta}) \frac{1-|\eta|^{2}}{|\zeta-\eta|^{2}} \frac{d \zeta}{\zeta} .
$$


Since $\varphi_{m}$ has no harmonic term, it may be factored as $\varphi_{m}=z_{1} \bar{z}_{1} \psi_{1}\left(z_{1}, \bar{z}_{1}\right)$, with $\psi_{1} \in \mathbb{C}\left[z_{1}, \bar{z}_{1}\right]$ homogeneous of degree $(m-2)$ and nonzero. In the integral above, we put $\eta:=-a$ and we replace $\varphi_{m}=z_{1} \bar{z}_{1} \psi_{1}$ to get the value of $V$ at $-a$ :

$$
\begin{aligned}
V(-a) & =\frac{1}{2 \pi i} \int_{\partial \Delta} \varphi_{m}(a+\zeta, \bar{a}+\bar{\zeta}) \frac{1-|a|^{2}}{|\zeta+a|^{2}} \frac{d \zeta}{\zeta} \\
& =\frac{1-|a|^{2}}{2 \pi} \int_{-\pi}^{\pi} \psi_{1}\left(a+e^{i \theta}, \bar{a}+e^{-i \theta}\right) d \theta .
\end{aligned}
$$

As a function of $a \in \Delta$, the last integral is identically zero if and only if the polynomial $\psi_{1}$ is zero. Thus, there exists $a$ such that $V(-a) \neq 0$. (However, we have no information about the possible signs of $V(-a)$ in terms of $\varphi_{m}$.) Then we define $U(\zeta)$ to be the harmonic prolongation of $-\mathrm{T} V$ that vanishes at $-a$ and $B_{\varepsilon}(\zeta):=\left(\varepsilon(a+\zeta), \varepsilon^{m} W(\zeta)\right)$.

The positivity (resp. negativity) of the sign of $V(-a)$ means that $B_{\varepsilon}(-a)=$ $(0, i V(-a))$ is in $\Omega^{+}$(resp. $\Omega^{-}$). Then after translating slightly $B_{\varepsilon}$ in the right direction along the $v$-axis, Lemma 1.19 applies to deduce that $\Omega_{1}^{-} \subset \mathbb{C}^{2}$ (resp. $\left.\Omega_{1}^{+} \subset \mathbb{C}^{2}\right)$ is holomorphically extendable at the origin. Since $\varphi_{m}\left(-z_{1},-\bar{z}_{1}\right)=$ $(-1)^{m} \varphi_{m}\left(z_{1}, \bar{z}_{1}\right)$, in the case where $m$ is odd, the disc $-B_{\varepsilon}$ will also be attached to $M_{1}$ and will provide extendability of the other side.

Thanks to basic majorations of the "O" remainders in the equation (1.23) of $M$, if $\varepsilon>0$ is sufficiently small, then $\Omega^{-} \subset \mathbb{C}^{n}$ (resp. $\Omega^{+} \subset \mathbb{C}^{n}$ ) has the same extendability property.

If $M$ is a real analytic hypersurface, it is easily seen, by inspecting the Taylor series of its graphing function, that $M$ is not of finite type at a point $p$ if and only if it may be represented by $v=u \widetilde{\varphi}(z, u)$, with $\widetilde{\varphi} \in \mathcal{C}^{\omega}$. Then the local complex hypersurface $\{v=u=0\}$ is contained in $M$.

Corollary 1.25. ([BeFo1978, R1983, BT1984]) If $M$ is $\mathcal{C}^{\omega}$ and if $p \in M$, the following properties are equivalent:

- $M$ has finite type at $p$;

- $M$ does not contain any local complex analytic hypersurface passing through $p$;

- $\Omega^{+}$or $\Omega^{-}$is holomorphically extendable at $p$.

1.26. Which side is holomorphically extendable? We claim that it suffices to study osculating domains in $\mathbb{C}^{2}$ of the form:

$$
\Omega_{\varphi_{m}}^{+}:=\left\{-v+\varphi_{m}(z, \bar{z})<0\right\}, \quad z \in \mathbb{C}, w=u+i v \in \mathbb{C},
$$

where $\varphi_{m} \neq 0$ is real, homogeneous of degree $m \geqslant 2$ and has no harmonic term. Indeed, extendability properties of such domains transfer to perturbations (1.23). Also, extendability properties of $\Omega_{\varphi_{m}}^{-}$are just the same, via $\varphi_{m} \leftrightarrow-\varphi_{m}$. For this reason, if $m$ is odd, both $\Omega_{\varphi_{m}}^{+}$and $\Omega_{\varphi_{m}}^{-}$are holomorphically extendable at $p$. 
The local complex line $\{(z, 0)\}$ intersects the closure $\overline{\Omega_{\varphi_{m}}^{+}}$in regions that are closed angular sectors (cones), due to homogeneity. We call these regions interior. The complement $\mathbb{C}^{2} \backslash \Omega_{\varphi_{m}}^{+}$intersects $\{(z, 0)\}$ in open, exterior sectors.

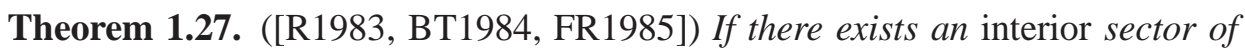
angular width $>\frac{\pi}{m}$, then $\Omega_{\varphi_{m}}^{+}$is holomorphically extendable at $p$.

The proof consists in choosing an appropriate truncated angular sector as the $z$-component of a disc attached to $\partial \Omega_{\varphi_{m}}^{+}$, instead of the round disc $\zeta \mapsto \varepsilon(a+\zeta)$.

Example 1.28. Every homogeneous quartic $v=\varphi_{4}(z, \bar{z})$ in $\mathbb{C}^{2}$ is biholomorphically equivalent to a model

$$
0=r_{a}:=-v+z^{2} \bar{z}^{2}+a z \bar{z}\left(z^{2}+\bar{z}^{2}\right),
$$

for some $a \in \mathbb{R}$. Such a hypersurface bounds two open sides $\Omega_{a}^{+}=\left\{r_{a}<0\right\}$ and $\Omega_{a}^{-}=\left\{r_{a}>0\right\}$ which enjoy the following properties (R1983, BT1984]):

- $\Omega_{a}^{-}$is holomorphically extendable at $p$, for every $a$;

- $|a|<2 / 3$ if and only if $\Omega_{a}^{+}$is everywhere strongly pseudoconvex;

- $|a| \leqslant 1 / \sqrt{2}$ if and only if $\Omega_{a}^{+}$is not holomorphically extendable at $p$;

- $|a|>1 / \sqrt{2}$ if and only if $\Omega_{a}^{+}$is holomorphically extendable at $p$;

- the above extendability property holds true for any perturbation of $\partial \Omega$ by higher order terms.

Finer results, strictly more general than the above theorem that apply to sixtics, were obtained in [FR1985]. If we remove all exterior sectors of angular width $\geqslant \frac{\pi}{m}$, the rest of the complex line $\{(z, 0)\}$ is formed by disjoint closed sectors, which are called supersectors of order $m$ of $\Omega_{\varphi_{m}}^{+}$at $p$. A supersector is proper if it contains points of $\Omega_{\varphi_{m}}^{+}$.

Theorem 1.29. ([FR1985])

(i) If $\Omega_{\varphi_{m}}^{+}$has a proper supersector of angular width $>\frac{\pi}{m}$, then $\Omega_{\varphi_{m}}^{+}$is holomorphically extendable at $p$.

(ii) If all supersectors of $\Omega_{\varphi_{m}}^{+}$have angular width $<\frac{\pi}{m}$, then there exists $f \in \mathcal{O}\left(\Omega_{\varphi_{m}}^{+}\right) \cap \mathcal{C}^{0}\left(\overline{\Omega_{\varphi_{m}}^{+}}\right)$that does not extend holomorphically at $p$.

Even in the case $m=6$, some cases in this theorem are left open. Examples may be found in [FR1985].

Open problem 1.30. In the case where $m$ is even, find a necessary and sufficient condition for $\Omega_{\varphi_{m}}^{+}=\left\{v>\varphi_{m}(z, \bar{z})\right\}$ to be holomorphically extendable at $p$, or show that the problem is undecidable.

One could generalize this (already wide open) question to a not necessarily finite type boundary, $\mathcal{C}^{\omega}, \mathcal{C}^{\infty}, \mathcal{C}^{2}$ or even $\mathcal{C}^{0}$ graph. 


\section{§2. TRÉPREAU'S THEOREM, DEFORMATIONS OF BISHOP DISCS AND PROPAGATION ON HYPERSURFACES}

2.1. Holomorphic extension of $\mathbf{C R}$ functions via jump. Let $M$ be a hypersurface in $\mathbb{C}^{n}$ of class at least $\mathcal{C}^{1, \alpha}$ with $0<\alpha<1$ and let $f$ be a continuous CR function on $M$. At each point $p$ of $M$, we may restrict $f$ to a small open ball (or polydisc) $\Omega_{p}$ centered at $p$. Applying the jump Theorem 1.11, we may represent $f=F^{+}-F^{-}$, with $F^{ \pm} \in \mathcal{O}\left(\Omega_{p}^{ \pm}\right) \cap \mathcal{C}^{0}\left(\overline{\Omega_{p}^{ \pm}}\right)$. If $\Omega_{p}^{+}$(resp. $\Omega_{p}^{-}$) is holomorphically extendable at $p$, then $F^{+}$(resp. $F^{-}$) extends to a neighborhood $\omega_{p}$ of $p$ in $\mathbb{C}^{n}$ as $G \in \mathcal{O}\left(\omega_{p}\right)$ (resp. $H \in \mathcal{O}\left(\omega_{p}\right)$ ). Then $f$ extends holomorphically to the small one-sided neighborhood $\omega_{p}^{-}$(resp. to $\left.\omega_{p}^{+}\right)$as $G-F^{-}$(resp. as $\left.F^{+}-H\right)$.

Lemma 2.2. On hypersurfaces, at a given point, local holomorphic extendability of CR functions to one side is equivalent to holomorphic extendability to the same side of the holomorphic functions defined in the opposite side.

Consequently, the theorems of $\S 1.22$ yield gratuitously extension results about CR functions. For instance:

Corollary 2.3. ([BeFo1978, R1983, BT1984]) On a real analytic hypersurface $M$, at a given point $p$, continuous $C R$ functions extend holomorphically to one side if and only if $M$ does not contain any local complex hypersurface passing through $p$.

The assumption of real analyticity, or the assumption of finite typeness in case $M$ is $\mathcal{C}^{\infty}$, both consume much smoothness. The removal of these assumptions was accomplished by Trépreau in 1986.

Theorem 2.4. ([TTrp1986]) Let $M$ be a $\mathcal{C}^{2}$ hypersurface of $\mathbb{C}^{n}, n \geqslant 2$ and let $p \in M$. The following two conditions are equivalent:

- $M$ does not contain any local complex hypersurface passing through $p$.

- for every open subset $U_{p} \subset M$ containing $p$, there exists a one-sided neighborhood $\omega_{p}^{ \pm}$of $M$ at $p$ with $\overline{\omega_{p}^{ \pm}} \cap M \Subset U_{p}$ such that for every $f \in \mathcal{C}_{C R}^{0}\left(U_{p}\right)$, there exists $F \in \mathcal{O}\left(\omega_{p}^{ \pm}\right) \cap \mathcal{C}^{0}\left(\omega^{ \pm} \cup U_{p}\right)$ with $\left.F\right|_{U_{p}}=f$.

We have seen that characterizing the side of extension is an open question, even for rigid polynomial hypersurfaces $v=\varphi_{m}(z, \bar{z})$ and even for $m=6$. Although the above theorem constitutes a neat answer for holomorphic extension to some imprecise side, it does not provide any control of the side of extension.

Let $M$ be a $\mathcal{C}^{2}$ orientable connected hypersurface and let $\Omega_{M}^{+}$be an open side of $M$. One could hope to characterize holomorphic extension to the other side at every point of $M$, since weak pseudoconvexity characterizes holomorphic nonextendability at every point of $M$, by Oka's theorem. 
Example 2.5. ([Trp1992]) In $\mathbb{C}^{3}$, let $\Omega_{M}^{+}$be $\left\{v>\varphi_{m}\left(z_{1}, \bar{z}_{1}\right)-\left|z_{2}\right|^{2}\left|z_{1}\right|^{2 N}\right\}$ where $\varphi_{m} \not \equiv 0$, of degree $m$ with $3 \leqslant m<N$ is as in Open problem 1.30. One verifies that holomorphic extension at every point of $M$ entails a characterization of holomorphic extension at the origin for the domain $\left\{v>\varphi_{m}(z, \bar{z})-\varepsilon|z|^{2 N}\right\}$.

In the sequel, we shall abandon definitely the difficult, still open question of how to control sides of holomorphic extension.

Although Theorem 2.4 is well known in Several Complex Variables, there is a more general formulation with a simpler proof than the original one. The remainder of this section will expose such a proof.

By a global one-sided neighborhood of a connected (not necessarily orientable) hypersurface $M \subset \mathbb{C}^{n}$, we mean a domain $\Omega_{M}$ with $\bar{\Omega}_{M} \supset M$ such that for every point $q \in M$, at least one open side $\omega_{q}^{ \pm}$of $M$ at $q$ is contained in $\Omega_{M}$. In fact, to insure connectedness, $\Omega_{M}$ is the interior of the closure of the union $\cup_{q \in M} \omega_{q}^{ \pm}$of all (possibly shrunk) one-sided neighborhoods.

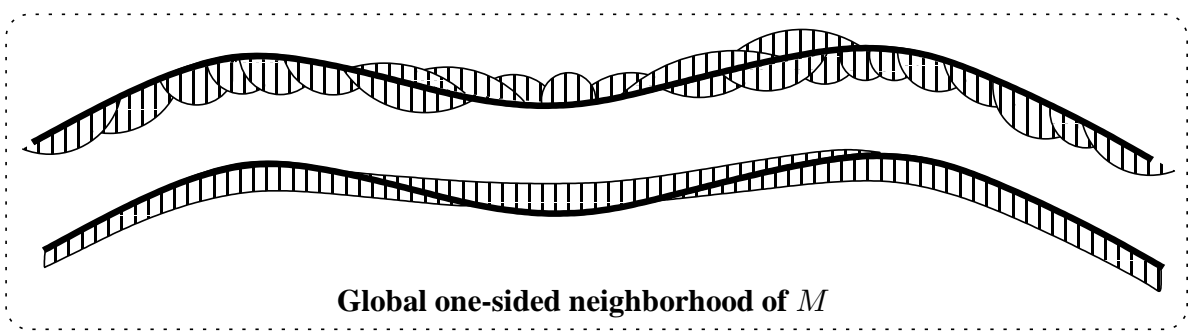

Then $\Omega_{M}$ contains a neighborhood in $\mathbb{C}^{n}$ of every point $r \in M$ which belongs to at least two one-sided neighborhoods that are opposite. The classical Morera theorem insures holomorphicity in a neighborhood of such points $r$.

Remind that $M$ is called globally minimal if it consists of a single CR orbit. The assumption that $M$ does not contain any complex hypersurface at any point means that for every $p \in M$, every open $U_{p} \ni p$, the CR orbit $\mathcal{O}_{C R}\left(U_{p}, p\right)$ contains a neighborhood of $p$ in $M$. This implies that $M$ is globally minimal and hence, Theorem 2.4 is less general than the following.

Theorem 2.6. ([Trp1990, Tu1994a $]$ Let $M$ be $e^{14}$ a connected $\mathcal{C}^{2, \alpha}(0<\alpha<1)$ hypersurface of $\mathbb{C}^{n}(n \geqslant 2)$. If $M$ is globally minimal, then there exists a global one-sided neighborhood $\Omega_{M}$ of $M$ such that for every continuous $C R$ function $f \in \mathcal{C}_{C R}^{0}(M)$, there exists $F \in \mathcal{O}\left(\Omega_{M}\right) \cap \mathcal{C}^{0}\left(\Omega_{M} \cup M\right)$ with $\left.F\right|_{M}=f$.

\footnotetext{
${ }^{14}$ This theorem also holds true with $M \in \mathcal{C}^{2}$ and even with $M \in \mathcal{C}^{1, \alpha}(0<\alpha<1)$, provided one redefines the notion of $\mathrm{CR}$ orbit in terms of boundaries of small attached analytic discs.
} 
It will appear that $\Omega_{M}$ is contructed by gluing discs to $M$ and to subsequent open sets $\Omega^{\prime} \subset \Omega_{M}$ which are all contained in the polynomial hull of $M$ :

$$
\widehat{M}:=\left\{z \in \mathbb{C}^{n}:|P(z)| \leqslant \sup _{w \in M}|P(w)|, \forall P \in \mathbb{C}[z]\right\} .
$$

Let us summarize the proof. Although the assumption of global minimality is so weak that $M$ may incorporate large open Levi-flat regions, there exists at least one point $p \in M$ in a neighborhood of which

$$
T_{q} M=T_{q}^{c} M+\left[T_{q}^{c} M, T_{q}^{c} M\right], \quad q \in U_{p} .
$$

Otherwise, the distribution $p \mapsto T_{p}^{c} M$ would be Frobenius-integrable and all CR orbits would be complex hypersurfaces! At such a point $p$, the classical Lewy extension theorem ( $\S 2.10$ below) insures that $\mathcal{C}_{C R}^{0}(M)$ extends holomorphically to (at least) one side at $p$.

Theorem 2.7. ([Trp1990, Tu1994] ]) Let $M$ be a connected $\mathcal{C}^{2, \alpha}$ hypersurface, not necessarily globally minimal. If $\mathcal{C}_{C R}^{0}(M)$ extends holomorphically to a onesided neighborhood at some point $p \in M$, then holomorphic extension to one side $\omega_{q}^{ \pm}$holds at every point $q \in \mathcal{O}_{C R}(M, p)$.

When $\mathcal{O}_{C R}(M, p)=M$ as in Theorem 2.6, the global one-sided neighborhood $\Omega_{M}$ will be the interior of the closure of the union $\cup_{q \in M} \omega_{q}^{ \pm}$of all (possibly shrunk) one-sided neighborhoods.

The next paragraphs are devoted to expose a detailed proof of both the Lewy theorem and of the above propagation theorem.

2.8. Approximation theorem and maximum principle. According to the approximation Theorem 5.2(III), for every $p \in M$, there exist a neighborhood $U_{p}$ of $p$ in $M$ and a sequence $\left(P_{\nu}(z)\right)_{\nu \in \mathbb{N}}$ of holomorphic polynomials with $\lim _{\nu \rightarrow \infty}\left\|P_{\nu}-f\right\|_{\mathcal{C}^{0}\left(U_{p}\right)}=0$.

Lemma 2.9. For every analytic disc $A \in \mathcal{O}(\Delta) \cap \mathcal{C}^{0}(\bar{\Delta})$ with $A(\partial \Delta) \subset U_{p}$, the sequence $P_{\nu}$ also converges uniformly on the closed disc $A(\bar{\Delta})$, even if $A(\bar{\Delta})$ goes outside $U_{p}$.

Proof. By assumption, $\lim _{\nu, \mu \rightarrow \infty}\left\|P_{\nu}-P_{\mu}\right\|_{\mathcal{C}^{0}\left(U_{p}\right)}=0$. Let $\eta \in \bar{\Delta}$ arbitrary. Thanks to the maximum principle and to $A(\partial \Delta) \subset U_{p}$ :

$$
\begin{aligned}
\left\|P_{\nu}(A(\eta))-P_{\mu}(A(\eta))\right\| & \leqslant \max _{\zeta \in \partial \Delta}\left\|P_{\nu}(A(\zeta))-P_{\mu}(A(\zeta))\right\| \\
& \leqslant \sup _{z \in U_{p}}\left\|P_{\nu}(z)-P_{\mu}(z)\right\| \longrightarrow 0 .
\end{aligned}
$$

The same argument shows that $P_{\nu}$ converges uniformly in the polynomial hull of $U_{p}$ (we shall not need this). 
Next, suppose that we have some family of analytic discs $A_{s}$, with $s$ a small parameter, such that $\cup_{s} A_{s}(\Delta)$ contains an open set in $\mathbb{C}^{n}$, for instance a onesided neighborhood at $p \in M$. Then $\left(P_{\nu}\right)_{\nu \in \mathbb{N}}$ converges uniformly on $\cup_{s} A_{s}(\Delta)$ and a theorem due to Cauchy assures that the limit is holomorphic in the interior of $\cup_{s} A_{s}(\Delta)$. It then follows that $f$ extends holomorphically to the interior of $\cup_{s} A_{s}(\Delta)$.

Remarkably, this short argument based on an application of the approximation Theorem 5.2(III) shows that ${ }^{15}$ :

\section{In order to establish local holomorphic extension of CR functions, it suffices to glue appropriate families of analytic discs to CR manifolds.}

In the sequel, the geometry of glued discs will be studied for itself; thus, it will be understood that statements about holomorphic or CR extension follow immediately; elementary details about continuity of the obtained extensions will be skipped.

2.10. Lewy extension. Since $M$ is globally minimal, there exists a point $p$ at which $T_{p} M=T_{p}^{c} M+\left[T_{p}^{c} M, T_{p}^{c} M\right]$. Granted Lemma 2.2, holomorphic extension to one side at such a point $p$ has already been proved in Theorem 1.18. Nevertheless, we want to present a geometrically different proof that will produce preliminaries and motivations for the sequel.

Since $T^{c} M=\operatorname{Re} T^{1,0} M=\operatorname{Re} T^{0,1} M$, we have equivalently $\left[T^{1,0} M, T^{0,1} M\right](p) \not \subset \mathbb{C} \otimes T_{p}^{c} M$, namely the intrinsic Levi form of $M$ at $p$ is nonzero. In other words, there exists a local section $L$ of $T^{1,0} M$ with $L(p) \neq 0$ and $[L, \bar{L}](p) \notin \mathbb{C} \otimes T_{p}^{c} M$. After a complex linear transformation of $T_{p}^{c} M$, we may assume that $L(p)=\frac{\partial}{\partial z_{1}}$. After removing the second order pluriharmonic terms, there exist local coordinates $\left(z_{1}, z^{\prime}, w\right)$ vanishing at $p$ such that $M$ is represented by

$$
v=-z \bar{z}_{1}+\mathrm{O}\left(\left|z_{1}\right|^{2+\alpha}\right)+\mathrm{O}\left(\left|z^{\prime}\right|\right)+\mathrm{O}(|z||u|)+\mathrm{O}\left(|u|^{2}\right) .
$$

The minus sign is set for clarity in the diagram of $\S 2.12$ below. We denote by $\varphi\left(z_{1}, z^{\prime}, u\right)$ the right hand side. Let $\varepsilon_{1}>0$ be small. For $\varepsilon$ satisfying $0<\varepsilon \leqslant \varepsilon_{1}$, we introduce the analytic disc

$$
A_{\varepsilon}(\zeta):=\left(\varepsilon(1-\zeta), 0^{\prime}, U_{\varepsilon}(\zeta)+i V_{\varepsilon}(\zeta)\right)
$$

with zero $z^{\prime}$-component, with $z_{1}$-component equal to a (reverse) round disc of radius $\varepsilon$ centered at $1 \in \mathbb{C}$ and with $u$-component $U_{\varepsilon}$ satisfying the Bishop-type equation:

$$
U_{\varepsilon}\left(e^{i \theta}\right)=-\mathrm{T}_{1}\left[\varphi\left(\varepsilon(1-\cdot), 0^{\prime}, U_{\varepsilon}(\cdot)\right)\right]\left(e^{i \theta}\right) .
$$

\footnotetext{
${ }^{15}$ This is the so-called Method of analytic discs ; $\bar{\partial}$ techniques are also powerful.
} 
Acoording to Theorem 3.7(IV), a unique solution $U_{\varepsilon}\left(e^{i \theta}\right)$ exists and is $\mathcal{C}^{2, \alpha-0}$ with respect to $\left(e^{i \theta}, \varepsilon\right)$. Since $\mathrm{T}_{1}(\psi)(1)=0$ by definition, we have $U_{\varepsilon}(1)=0$ and then $V_{\varepsilon}:=\mathrm{T}_{1}\left(U_{\varepsilon}\right)$ also satisfies $V_{\varepsilon}(1)=0$. Consequently, $A_{\varepsilon}(1)=0$. By applying $T_{1}$ to both sides of the above equation, we see that the disc is attached to $M$ :

$$
V_{\varepsilon}\left(e^{i \theta}\right)=\varphi\left(\varepsilon\left(1-e^{i \theta}\right), 0^{\prime}, U_{\varepsilon}\left(e^{i \theta}\right)\right) .
$$

We shall prove that for $\varepsilon_{1}$ sufficiently small, every disc $A_{\varepsilon}(\Delta)$ with $0<\varepsilon \leqslant \varepsilon_{1}$ is not tangent to $M$ at $p$. We draw two diagrams: a 2-dimensional and a 3dimensional view. In both, the $v$-axis is vertical, oriented down.

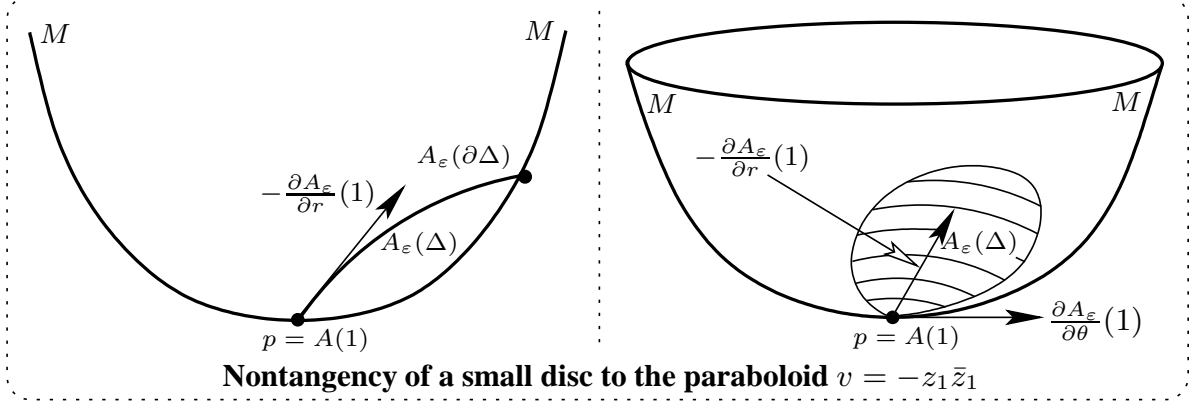

Just now, we need a geometrical remark. Let $A \in \mathcal{O}(\Delta) \cap \mathcal{C}^{1}(\bar{\Delta})$ be an arbitrary but small analytic disc attached to $M$ with $A(1)=0$. We use polar coordinates to denote $\zeta=r e^{i \theta}$.

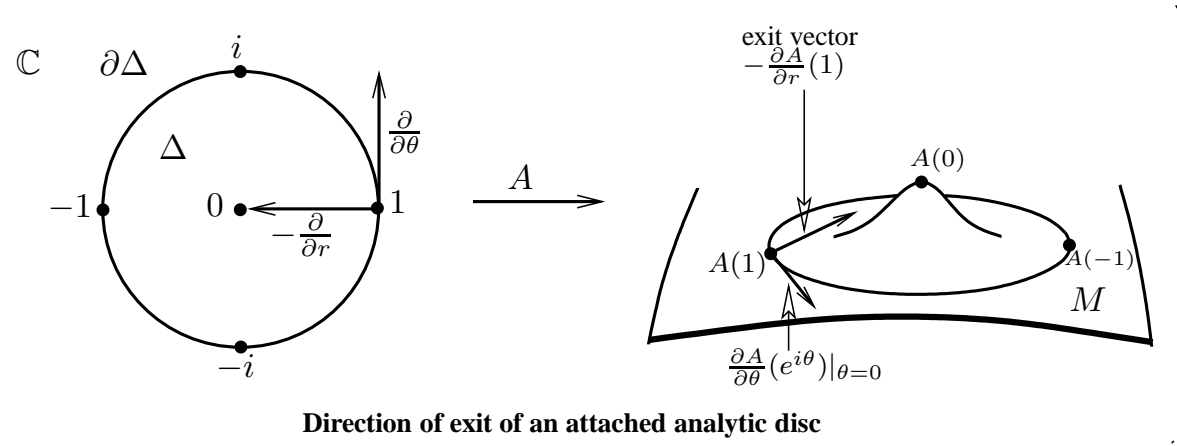

The holomorphicity of $A$ yields the following identities between vectors in $T_{p} \mathbb{C}^{n}$ :

$$
\left.i \frac{\partial A}{\partial \theta}\left(e^{i \theta}\right)\right|_{\theta=0}=-\left.\frac{\partial A}{\partial r}(r)\right|_{r=1}=-\left.\frac{\partial A}{\partial \zeta}(\zeta)\right|_{\zeta=1} .
$$

The multiplication by $i$ (or equivalently the complex structure $J$ ) provides an isomorphism $T_{p} \mathbb{C}^{n} / T_{p} M \rightarrow T_{p} M / T_{p}^{c} M$; in coordinates, $T_{p} \mathbb{C}^{n} / T_{p} M \simeq \mathbb{R}_{v}$, $T_{p} M / T_{p}^{c} M \simeq \mathbb{R}_{u}$ and $J$ sends $\mathbb{R}_{u}$ to $\mathbb{R}_{v}$. It follows that $\frac{\partial A}{\partial r}(1)$ is not tangent to $M$ at $p$ if and only if $\frac{\partial A}{\partial \theta}(1)$ is not complex tangent to $M$ at $p$. 
Coming back to $A_{\varepsilon}$, we call the vector

$$
-\frac{\partial A_{\varepsilon}}{\partial r}(1) \bmod T_{p} M=-\frac{\partial W_{\varepsilon}}{\partial r}(1) \bmod T_{p} M
$$

the exit vector of $A_{\varepsilon}$. By differentiating $V_{\varepsilon}=\varphi$ at $\theta=0$, taking account of $d \varphi(0)=0$, we get $\frac{\partial V_{\varepsilon}}{\partial \theta}(1)=0$. So only the real part $\frac{\partial U_{\varepsilon}}{\partial \theta}(1)$ of $\frac{\partial W_{\varepsilon}}{\partial \theta}(1)$ may be nonzero.

Lemma 2.11. Shrinking $\varepsilon_{1}$ if necessary, the exit vector of every disc $A_{\varepsilon}$ with $0<\varepsilon \leqslant \varepsilon_{1}$ is nonzero:

$$
-\frac{\partial W_{\varepsilon}}{\partial r}(1)=i \frac{\partial W_{\varepsilon}}{\partial \theta}(1)=i \frac{\partial U_{\varepsilon}}{\partial \theta}(1) \neq 0 .
$$

Proof. The principal term of $\varphi$ is $-z_{1} \bar{z}_{1}$. We compute first:

$$
\begin{aligned}
\mathrm{T}_{1}\left[-Z_{1}(\zeta) \bar{Z}_{1}(\zeta)\right] & =\mathrm{T}_{1}\left[\varepsilon^{2}\left(e^{-i \theta}-2+e^{i \theta}\right)\right] \\
& =\frac{1}{i} \varepsilon^{2}\left(-e^{-i \theta}+e^{i \theta}\right) .
\end{aligned}
$$

Proceeding as carefully as in Section 3(IV), we may verify that

$$
\begin{aligned}
U_{\varepsilon}\left(e^{i \theta}\right) & =-\mathrm{T}_{1}\left[-Z_{1}(\zeta) \bar{Z}_{1}(\zeta)+\text { Remainder }\right]\left(e^{i \theta}\right) \\
& =-2 \varepsilon^{2} \sin \theta+\widetilde{U}_{\varepsilon}\left(e^{i \theta}\right)
\end{aligned}
$$

with a $\mathcal{C}^{2, \alpha-0}$ remainder satisfying $\left\|\widetilde{U}_{\varepsilon}\right\|_{1,0} \leqslant \mathrm{~K} \varepsilon^{2+\alpha}$, for some quantity $\mathrm{K}>0$. So $\frac{\partial U_{\varepsilon}}{\partial \theta}(1)=-2 \varepsilon^{2}+\mathrm{O}\left(\varepsilon^{2+\alpha}\right) \neq 0$.

2.12. Translations of a nontangent analytic disc. We now fix $\varepsilon$ with $0<\varepsilon \leqslant \varepsilon_{1}$ and we denote simply by $A$ the disc $A_{\varepsilon}$. So the vector

$$
\frac{\partial A}{\partial \theta}(1)=\left(-i \varepsilon, 0^{\prime},-2 \varepsilon^{2}+\mathrm{O}\left(\varepsilon^{2+\alpha}\right)\right)
$$

is not tangent to $T_{p}^{c} M=\{v=u=0\}$ at the origin. Furthermore, it is not tangent to the $(2 n-2)$-dimensional sub-plane $\left\{y_{1}=v=0\right\}$ of $T_{p} M=\{v=0\}$.

We now introduce parameters of translation $x_{1}^{0} \in \mathbb{R}, z_{0}^{\prime} \in \mathbb{C}^{n-2}$ and $u_{0} \in \mathbb{R}$ with $\left|x_{1}^{0}\right|,\left|z_{0}^{\prime}\right|,\left|u_{0}\right|<\delta_{1}$, where $0<\delta_{1}<<\varepsilon$. The points in $M$ of coordinates

$$
\left(x_{1}^{0}, z_{0}^{\prime}, u_{0}+i \varphi\left(x_{1}^{0}, z_{0}^{\prime}, u_{0}\right)\right)
$$

cover a small $(2 n-2)$-dimensional submanifold $K_{p}$ with $T_{p} K_{p}=\left\{y_{1}=v=0\right\}$ transverse to the disc boundary $A_{\varepsilon}(\partial \Delta)$ at $p$ that we draw below. 


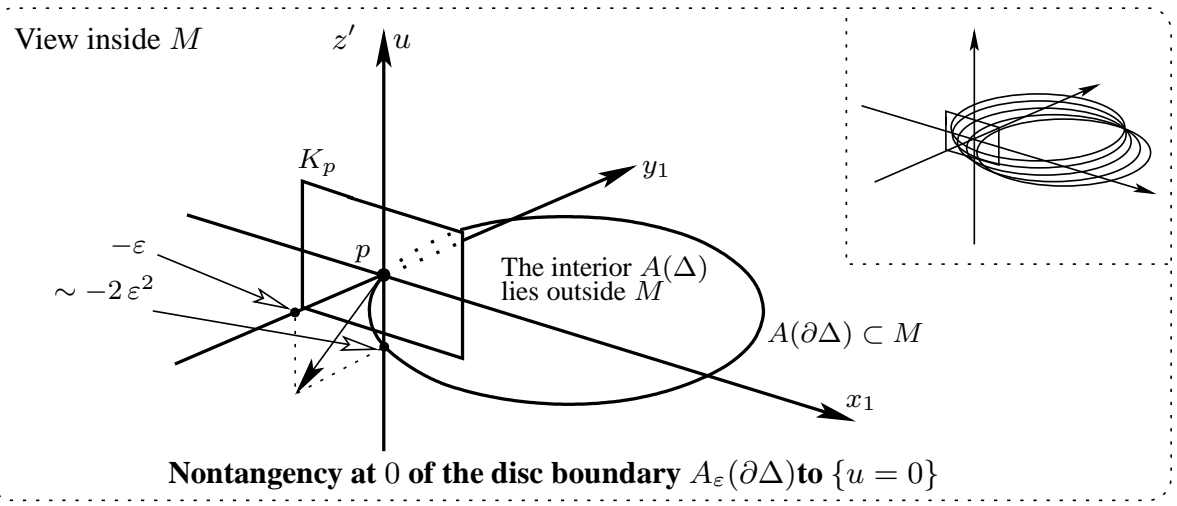

To conclude the proof of one-sided holomorphic extension at the Levi nondegenerate point $p$, it suffices to deform the disc $A_{x_{1}^{0}, z_{0}^{\prime}, u_{0}}$ so that its distinguished point $A_{x_{1}^{0}, z_{0}^{\prime}, u_{0}}(1)$ covers the submanifold $K_{p}$, namely

$$
A_{x_{1}^{0}, z_{0}^{\prime}, u_{0}}(1)=\left(x_{1}^{0}, z_{0}^{\prime}, u_{0}+i \varphi\left(x_{1}^{0}, z_{0}^{\prime}, u_{0}\right)\right) .
$$

This may be achieved easily by defining

$$
\left(Z_{1, x_{1}^{0}}(\zeta), Z_{z_{0}^{\prime}}^{\prime}(\zeta)\right):=\left(\varepsilon_{1}(1-\zeta)+x_{1}^{0}, z_{0}^{\prime}\right)
$$

and by solving the Bishop-type equation:

$$
U_{x_{1}^{0}, z_{0}^{\prime}, u_{0}}\left(e^{i \theta}\right)=u_{0}-\mathrm{T}_{1}\left[\varphi\left(Z_{1 ; x_{1}^{0}}(\cdot), Z_{z_{0}^{\prime}}^{\prime}(\cdot), U_{x_{1}^{0}, z_{0}^{\prime}, u_{0}}(\cdot)\right)\right]\left(e^{i \theta}\right)
$$

for the $u$-component of the sought disc $A_{x_{1}^{0}, z_{0}^{\prime}, u_{0}}$. Thanks to Theorem 3.7(IV), the solution exists and is $\mathcal{C}^{2, \alpha-0}$ with respect to all the variables. We finally define the $v$-component of $A_{x_{1}^{0}, z_{0}^{\prime}, u_{0}}$ :

$$
V_{x_{1}^{0}, z_{0}^{\prime}, u_{0}}\left(e^{i \theta}\right):=\mathrm{T}_{1}\left[U_{x_{1}^{0}, z_{0}^{\prime}, u_{0}}(\cdot)\right]\left(e^{i \theta}\right)+\varphi\left(x_{1}^{0}, z_{0}^{\prime}, u_{0}\right) .
$$

Applying $\mathrm{T}_{1}$ to (2.14), we see that this disc is attached to $M$; also, putting $e^{i \theta}:=1$ in (2.14) and in (2.15), we see that (2.13) holds. Geometrically, the $(2 n-2)$ added parameters $\left(x_{1}^{0}, z_{0}^{\prime}, u_{0}\right)$ correspond to translations in $M$ of the original disc $A_{\varepsilon_{1}}$.

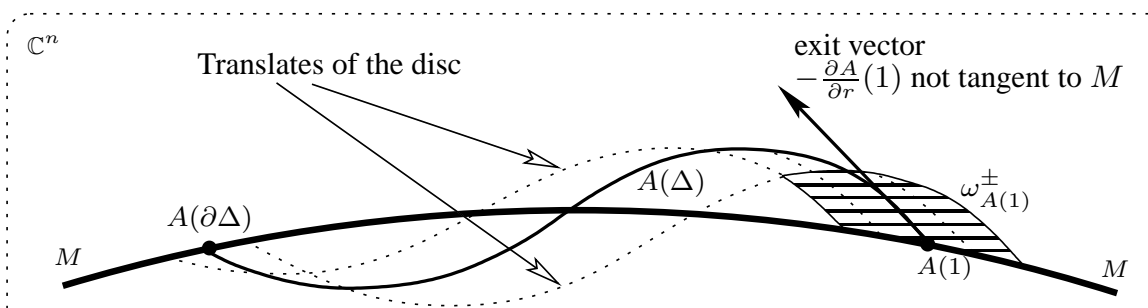

Translations of an attached analytic disc 
Define the open circular region $\Delta_{1}:=\left\{\zeta \in \Delta:|\zeta-1|<\delta_{1}\right\}$ around 1 in the unit disc. Then we claim that shrinking $\delta_{1}>0$ if necessary, the set

$$
\left\{A_{x_{1}^{0}, z_{0}^{\prime}, u_{0}}(\zeta): \zeta \in \Delta_{1},\left|x_{1}^{0}\right|<\delta_{1},\left|z_{0}^{\prime}\right|<\delta_{1},\left|u_{0}\right|<\delta_{1}\right\}
$$

contains a one-sided neighborhood of $M$ at $p=A_{0,0,0}(1)$. Indeed, by computation, one may check that the $2 n$ vectors of $T_{p} \mathbb{C}^{n}$

$\frac{\partial A_{0,0,0}}{\partial x_{1}}(1), \frac{\partial A_{0,0,0}}{\partial \theta}(1), \frac{\partial A_{0,0,0}}{\partial x_{k}^{\prime}}(1), \frac{\partial A_{0,0,0}}{\partial y_{k}^{\prime}}(1), \frac{\partial A_{0,0,0}}{\partial u}(1),-\frac{\partial A_{0,0,0}}{\partial r}(1)$,

are linearly independent; geometrically and by construction, the first $(2 n-1)$ of these vectors span $T_{p} M$ and the last one is linearly independent, since by construction the exit vector of $A_{\varepsilon_{1}}$ is nontangent to $M$ at $p$.

Incidentally, we have proved an elementary but crucial statement: by "translating" (through a suitable Bishop-type equation) any small attached disc whose exit vector is nonzero, we may always cover a one-sided neighborhood.

Lemma 2.16. If a small disc $A$ attached to a hypersurface $M$ satisfies $\frac{\partial A}{\partial \theta}(1) \notin$ $T_{A(1)}^{c} M$, or equivalently $-\frac{\partial A}{\partial r}(1) \notin T_{A(1)} M$, then continuous CR functions on $M$ extend holomorphically at $A(1)$ to the side in which points the nonzero exit vector $i \frac{\partial A}{\partial \theta}(1)=-\frac{\partial A}{\partial r}(1)$.

Of course, the choice of the point $1 \in \partial \Delta$ is no restriction at all, since after a Möbius reparametrization, any given point $\zeta_{0} \in \partial \Delta$ becomes $1 \in \partial \Delta$.

2.17. Propagation of holomorphic extension. The Levi form assumption $T_{p} M=T_{p}^{c} M+\left[T_{p}^{c} M, T_{p}^{c} M\right]$ was strongly used to insure the existence of a disc having a nonzero exit vector at $p$. But if a disc $A$ is attached to a highly degenerate part of $M$, for instance to a region where the Levi form is nearly flat, the disc $A$ might well satisfy $\frac{\partial A}{\partial \theta}\left(\zeta_{0}\right) \in T_{A\left(\zeta_{0}\right)}^{c} M$ (or equivalently, $\left.-\frac{\partial A}{\partial r}\left(\zeta_{0}\right) \in T_{A\left(\zeta_{0}\right)} M\right)$, for every $\zeta_{0} \in \partial \Delta$. Then we are stuck.

To go through, two strategies are known in the literature.

- Devise refined pointwise "finite type" assumptions insuring the existence of small discs having nonzero exit vector at a given central point.

- Devise deformation arguments that propagate holomorphic extension from Levi nondegenerate regions up to highly degenerate regions.

Unfortunately, the first, more developed strategy is unable to provide any proof of Theorem 2.6. Indeed, a smooth globally minimal hypersurface may well contain large Levi-flat regions, as for instance $\left\{(z, w) \in \mathbb{C}^{2}: v=\varpi(x)\right\}$ with a $\mathcal{C}^{\infty}$ function $\varpi$ satisfying $\varpi(x) \equiv 0$ for $x \leqslant 0$ and $\varpi(x)>0$ for $x>0$ (to check global minimality, proceed as in Example 3.10); Theorem 4.8(III) shows that a 
Levi-flat portion $M_{\mathrm{LF}}$ of a hypersurface $M$ is locally foliated by complex $(n-1)$ dimensional submanifolds; the uniqueness in Bishop's equation ${ }^{16}$ then entails that every small analytic disc $A \in \mathcal{O}(\Delta) \cap \mathcal{C}^{1}(\bar{\Delta})$ with $A(\partial \Delta) \subset M_{\mathrm{LF}}$ must satisfy $A(\partial \Delta) \subset \Sigma$, where $\Sigma \subset M_{\mathrm{LF}}$ is the unique local complex connected $(n-1)$ dimensional submanifold of the foliation that contains $A(1)$; then the uniqueness principle for holomorphic maps between complex manifolds yields $A(\bar{\Delta}) \subset \Sigma$; finally, $-\frac{\partial A}{\partial r}\left(\zeta_{0}\right) \in T_{A\left(\zeta_{0}\right)} \Sigma=T_{A\left(\zeta_{0}\right)}^{c} M$ has exit vector tangential to $M$ at every $\zeta_{0} \in \partial \Delta$.

For this reason, we will focus our attention only on the second, most powerful strategy, starting with a review.

After works of Sjöstrand ([HS1982, Sj1982a, Sj1982b]) on propagation of singularities for certain classes of partial differential operators, of BaouendiChang-Treves [BCT1983], and of Hanges-Treves [HT1983], Trépreau [Trp1990] showed that the hypoanalytic wave-front set of a CR function or distribution propagates along complex-tangential curves. The microlocal technique involves deforming $T^{*} M$ inside conic sets and controlling a certain oscillatory integral called Fourier-Bros-Iagolnitzer (FBI) transform. In 1994, Baouendi-RothschildTrépreau [BRT1994 showed how to deform analytic discs attached to a hypersurface in order to get some propagation results (however, Theorem 2.7 which appears in [Trp1990] is not formulated in [BRT1994]). Then Tumanov [Tu1994a] showed how to deformation discs attached to generic submanifolds of arbitrary codimension and provided extension results that cannot be obtained by means of the usual microlocal analysis.

Until the end of Section 4, our goal will be to describe and to exploit this technique of propagation. The geometric idea is as follows.

As in Theorem 2.7, assume that holomorphic extension is already known to hold in a one-sided neighborhood $\omega_{q}^{ \pm}$at some point $q \in M$. Referring to the diagram after the main Proposition 2.21 below, we may pick a disc $A$ with $A(-1)=q$. Then a small part of its boundary, namely for $e^{i \theta}$ near -1 , lies in $\overline{\omega_{q}^{ \pm}}$. If the vector $\frac{\partial A}{\partial \theta}(1)$ is not complex tangential at the opposite point $p=A(1)$, it suffices to apply Lemma 2.16 just above to get holomorphic extension at $p$, almost gratuitously. On the contrary, if $\frac{\partial A}{\partial \theta}(1)$ is complex tangential at $p$, we may well hope that by slightly deforming $M$ as a hypersurface $M^{\mathrm{d}}$ which goes inside $\omega_{q}^{ \pm}$ a bit, there exists a deformed disc $A^{\mathrm{d}}$ attached to $M^{\mathrm{d}}$ with again $A^{\mathrm{d}}(1)=p$ that will be not tangential: $-\frac{\partial A^{\mathrm{d}}}{\partial r}(1) \notin T_{A^{\mathrm{d}}(1)} M$. Then a translation of the $\operatorname{disc} A^{\mathrm{d}}$ as in Lemma 2.16 will provide holomorphic extension at $p$.

\footnotetext{
${ }^{16}$ A more general property holds true (see [Trp1990, Tu1994a, MP2006b): every small attached disc is necessarily attached to a single (local or global) CR orbit; here, $\Sigma$ is a local orbit, whence $A(\partial \Delta) \subset \Sigma$.
} 
2.18. Approximation theorem and chains of analytic discs. To prove Theorem 2.7, we first formulate a version of the approximation theorem which is apppropriate for our purposes.

Lemma 2.19. ([Tu1994a $]$ ) For every $p \in M$, there exists a neighborhood $U_{p}$ of $p$ in $M$ such that for every $q \in U_{p}$, for every one-sided neighborhood $\Omega_{q}^{ \pm}$of $U_{p}$ at $q$, there exists a smaller one-sided neighborhood $\omega_{q}^{ \pm} \subset \Omega_{q}^{ \pm}$of $U_{p}$ at $q$ such that the following approximation property holds:

- for every $F \in \mathcal{C}^{0}\left(M \cup \Omega_{q}^{ \pm}\right)$which is $C R$ on $M$ and holomorphic in $\Omega_{q}^{ \pm}$, there exists a sequence of holomorphic polynomials $\left(P_{\nu}(z)\right)_{\nu \in \mathbb{N}}$ such that $0=\lim _{\nu \rightarrow \infty}\left\|P_{\nu}-f\right\|_{\mathcal{C}^{0}\left(U_{p} \cup \omega_{q}^{ \pm}\right)}$.

The proof is an adaptation of Theorem 5.2(III). It suffices to allow the maximally real submanifolds $\Lambda_{u} \subset M$ be slightly deformed in $\Omega_{q}^{ \pm}$. With a control of the smallness of their $\mathcal{C}^{1}$ norm, one may insure that they cover not only $U_{p}$ but also $\omega_{q}^{ \pm}$. Further details will not be provided.

To establish local holomorphic extension of CR functions, it is allowed to glue discs not only to $M$ but also to previously constructed one-sided neighborhoods.

Pursuing, we formulate a lemma and a main proposition.

Lemma 2.20. ([Tu1994a]) Let $p \in M$ and let $U_{p}$ be a neighborhood of $p$ in $M$, arbitrarily small. For every $q \in \mathcal{O}_{C R}(M, p)$ and every small $\varepsilon>0$, there exist $\ell \in \mathbb{N}$ with $\ell=\mathrm{O}(1 / \varepsilon)$ and a chain of $\mathcal{C}^{2, \alpha-0}$ analytic discs $A^{1}, A^{2}, \ldots, A^{\ell-1}, A^{\ell}$ attached to $M$ with the properties:

- $A^{1}(-1) \in U_{p}$, i.e. this point is arbitrarily close to $p$;

- $A^{1}(1)=A^{2}(-1), A^{2}(1)=A^{3}(-1), \ldots, A^{\ell-1}(1)=A^{\ell}(-1)$;

- $A^{\ell}(1)=q$;

- $\left\|A^{k}\right\|_{\mathcal{C}^{1,0}(\bar{\Delta})} \leqslant \varepsilon$, for $k=1,2, \ldots, \ell$;

- each $A^{k}$ is an embedding $\bar{\Delta} \rightarrow \mathbb{C}^{n}$.
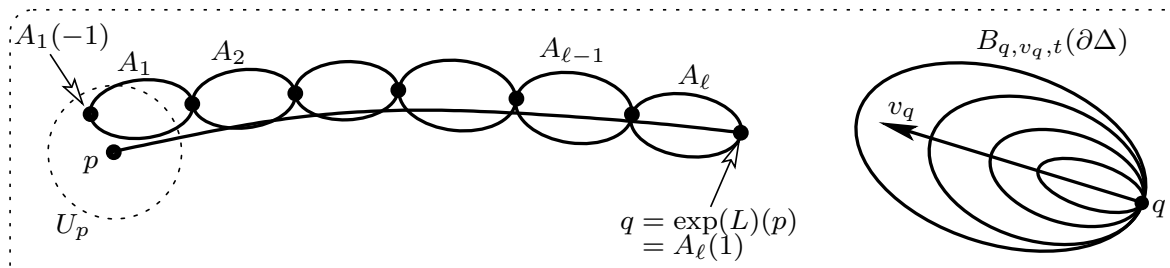

String of analytic discs approximating a CR curve 
Such a chain of analytic discs will be constructed by approximating a complextangential curve that goes from $q$ to $p$, using families of discs $B_{q, v_{q}, t}(\zeta)$ to be introduced in a while. The above lemma is essentially obvious, whereas the next proposition constitutes the very core of the argument.

Proposition 2.21. (BRT1994, Tu1994a]) (Propagation along a disc) Let $A$ be a small $\mathcal{C}^{2, \alpha-0}$ analytic disc attached to $M$ which is an embedding $\bar{\Delta} \rightarrow \mathbb{C}^{n}$. If $\mathcal{C}_{C R}^{0}(M)$ extends holomorphically to a one-sided neighborhood $\omega_{A(-1)}^{ \pm}$at the point $A(-1)$, then it also extends holomorphically to a one-sided neighborhood at $A(1)$. With more precisions:

- if the exit vector $-\frac{\partial A}{\partial r}(1)$ is not tangent to $M$ at $A(1)$, extension holds to the side in which points $-\frac{\partial A}{\partial r}(1)$ : this is already known, by Lemma 2.16;

- if the exit vector $-\frac{\partial A}{\partial r}(1)$ is tangent to $M$ at $A(1)$, there exists an arbitrarily small deformation $A^{\mathrm{d}}$ of $A$ with $A^{\mathrm{d}}(1)=A(1)$ having boundary $A^{\mathrm{d}}(\partial \Delta)$ contained in $M \cup \omega_{A(-1)}^{ \pm}$such that the new exit vector $-\frac{\partial A^{\mathrm{d}}}{\partial r}(1)$ is not tangent to $M$ at $A^{\mathrm{d}}(1)$; then by translating $A^{\mathrm{d}}$ as in Lemma 2.16, holomorphic extension holds at $A(1)$.

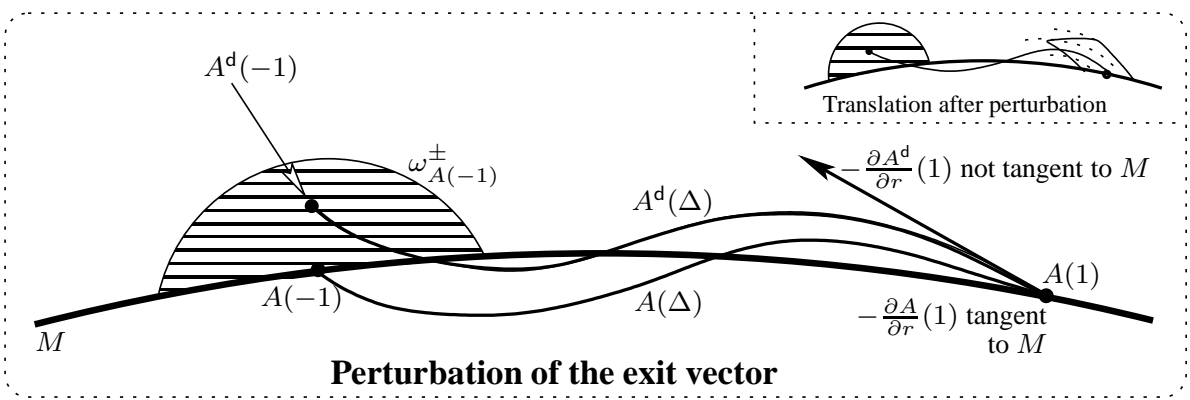

Indeed, thanks to the flexibility of the solutions to the parametrized Bishop equation provided by Theorem 3.7(IV), we can easily, as in Lemma 2.16, add translation parameters $\left(x_{1}^{0}, z_{0}^{\prime}, u_{0}\right)$ to a slightly deformed disc $A^{\mathrm{d}}$ attached to $M \cup$ $\omega_{A(-1)}^{ \pm}$and then $A_{x_{1}^{0}, z_{0}^{\prime}, u_{0}}^{\mathrm{d}}\left(\Delta_{1}\right)$ covers a small one-sided neighborhood of $M$ at $A(1)=A^{\mathrm{d}}(1)$, thanks to the crucial condition $-\frac{\partial A^{\mathrm{d}}}{\partial r}(1) \neq 0$. We shall not copy the details.

We claim that the proposition ends the proof of Theorem 2.7. By assumption, $\mathcal{C}_{C R}^{0}(M)$ extends holomorphically to a one-sided neighborhood $\omega_{p}^{ \pm}$at $p$. The closure $\overline{\omega_{p}^{ \pm}}$contains an open neighborhood $U_{p}$ of $p$. Let $q \in \mathcal{O}_{C R}(M, p)$ and construct a chain of analytic discs from $q$ up to a point $p^{\prime} \in U_{p}$. The endpoint $p^{\prime}=A^{1}(-1)$ of the chain of analytic discs being arbitrarily close to $p$, hence in $U_{p}$, holomorphic extension holds at $A^{1}(-1)$. We then apply the proposition successively to the discs $A^{1}, A^{2}, \ldots, A^{\ell}$ and deduce holomorphic extension at $q$. 
We now explain Lemma 2.20. To approximate a complex-tangential curve, it suffices to construct families of analytic discs that are essentially directed along given vectors $v_{q} \in T_{q}^{c} M$.

Lemma 2.22. For every point $q \in M$ and every nonzero complex tangent vector $v_{q} \in T_{q}^{c} M \backslash\{0\}$, there exists a family of $\mathcal{C}^{2, \alpha-0}$ analytic discs $B_{q, v_{q}, t}(\zeta)$ parametrized by $t \in \mathbb{R}$ with $|t|<t_{1}$, for some $t_{1}>0$, that satisfies:

- $B_{q, v_{q}, t}(\partial \Delta) \subset M$;

- $q=B_{q, v_{q}, t}(1)$;

- $v_{q}=\frac{\partial B_{q, v_{q}, 0}}{\partial t}(-1)$;

- $\left\|B_{q, v_{q}, t}\right\|_{\mathcal{C}^{1,0}(\bar{\Delta})} \leqslant \mathrm{K} t$, for some $\mathrm{K}>0$.

Proof. In coordinates centered at $q$, represent $M$ by $v=\varphi(z, u)$ with $\varphi(0)=0$ and $d \varphi(0)=0$. The vector $v_{q} \in T_{p}^{c} M=\{w=0\}$ has coordinates $\left(\dot{z}_{q}, 0\right)$ for some nonzero $\dot{z}_{q} \in \mathbb{C}^{n-1}$. Introduce the family of analytic discs

$$
B_{q, v_{q}, t}(\zeta):=\left(t \dot{z}_{q}(1-\zeta) / 2, W_{t}(\zeta)\right),
$$

where the real part $U_{t}$ of $W_{t}$ is the unique $\mathcal{C}^{2, \alpha-0}$ solution of the Bishop-type equation:

$$
U_{t}\left(e^{i \theta}\right)=-\mathrm{T}_{1}\left[\varphi\left(t \dot{z}_{q}(1-\cdot) / 2, U_{t}(\cdot)\right)\right]\left(e^{i \theta}\right) .
$$

Proceeding as carefully as in Section 3(IV), we may verify that the assumption $d \varphi(0)=0$ implies that $\left\|W_{t}\right\|_{1,0}=\mathrm{O}\left(|t|^{2}\right)$. Then it is obvious that $v_{q}=\left(\dot{z}_{q}, 0\right)=$ $\frac{\partial B_{q, v_{q}, 0}}{\partial t}(-1)$.

We now complete the proof of Lemma 2.20. Any point $q \in \mathcal{O}_{C R}(M, p)$ is the endpoint of a finite concatenation of integral curves of sections $L$ of $T^{c} M$. It suffices to construct the chain of discs for a single such curve $\exp (t L)(p)$. After multiplying $L$ by a suitable function, we may assume that $q$ is the time-one endpoint $q=\exp (L)(p)$.

Moving backwards, we start from $q_{\ell}:=q$, we define $A^{\ell}(\zeta):=$ $B_{q_{\ell},-L\left(q_{\ell}\right), 1 / \ell}(\zeta)$ and we set $q_{\ell-1}:=B_{q_{\ell},-L\left(q_{\ell}\right), 1 / \ell}(-1)$. Clearly, $q_{\ell-1}=q_{\ell}-\frac{1}{\ell} L\left(q_{\ell}\right)+\mathrm{O}\left(\frac{1}{\ell^{2}}\right)$. Starting again from $q_{\ell-1}$, we again move backwards and so on, i.e. we define by descending induction:

- $A^{k}(\zeta):=B_{q_{k},-L\left(q_{k}\right), 1 / \ell}(\zeta)$;

- $q_{k-1}:=B_{q_{k},-L\left(q_{k}\right), 1 / \ell}(-1)$,

until $k=1$. Since $q_{k-1}=q_{k}-\frac{1}{\ell} L\left(q_{k}\right)+\mathrm{O}\left(\frac{1}{\ell^{2}}\right)$ for $k=1, \ldots, \ell$, the sequence of points $q_{k}$ is a discrete approximation of the integral curve of $L$, hence the endpoint $q_{0}=A^{1}(-1)$ is arbitrarily close to $p$, provided $\ell$ is large enough. Finally, by construction $\left\|A^{k}\right\|_{1,0}=\mathrm{O}\left(\frac{1}{\ell}\right)$. 
The proof of the main Proposition 2.21 does not use special features of hypersurfaces. For this reason, we will directly deal with generic submanifolds of arbitrary codimension, passing to a new section.

\section{§3. TUMANOV'S THEOREM, DEFORMATIONS OF BISHOP DISCS AND PROPAGATION ON GENERIC MANIFOLDS}

3.1. Wedges and CR-wedges. Assume now that $M$ is a connected generic submanifold in $\mathbb{C}^{n}$ of codimension $d \geqslant 1$ and of CR dimension $m=d-n \geqslant 1$. The case $d=1$ corresponds to a hypersurface. The notion of local wedge at a point $p$ generalizes to codimension $d \geqslant 2$ the notion of one-sided neighborhood at a point of a hypersurface.

More briefly that was has been done in Section 4(III), a wedge may be defined as follows. Choose a $d$-dimensional real subspace $H_{p}$ of $T_{p} \mathbb{C}^{n}$ satisfying $T_{p} \mathbb{C}^{n}=$ $T_{p} H_{p} \oplus T_{p} M$ and a small convex open salient truncated cone $C_{p} \subset H_{p}$ with vertex $p$. Then a local wedge of edge $M$ at $p$ is:

$$
\mathcal{W}\left(U_{p}, C_{p}\right):=\left\{q+\mathrm{c}: q \in U_{p}, \mathrm{c} \in C_{p}\right\} .
$$

This is not yet the most effective definition. Up to shrinking open sets and parameter spaces, all definitions of local wedges will coincide. Concretely, the wedges we shall construct will always been obtained as unions of small pieces of families of analytic discs partly attached to $M$. So we formulate all the technical conditions that will insure that such pieces of discs cover a wedge.

Definition 3.2. A local wedge of edge $M$ at $p$ is a set of the form:

$$
\mathcal{W}_{p}:=\left\{A_{t, s}\left(r e^{i \theta}\right):|t|<t_{1},|s|<s_{1},|\theta|<\theta_{1}, r_{1}<r<1\right\}
$$

where, $t \in \mathbb{R}^{d-1}$ is a rotation parameter, $t_{1}>0$ is small, $s \in \mathbb{R}^{2 m+d-1}$ is a translation parameter, $s_{1}>0$ is small, $\theta_{1}>0$ is small, $r_{1}<1$ is close to 1 and $A_{t, s}(\zeta)$, with $\zeta \in \bar{\Delta}$, is a parametrized family of $\mathcal{C}^{2, \alpha-0}$ analytic discs satisfying:

- $A_{t, 0}(1)=p$ for every $t$

- the boundaries $A_{t, s}(\partial \Delta)$ are partly (sometimes completely) attached to $M$, namely $A_{t, s}\left(e^{i \theta}\right) \in M$, at least for $|\theta| \leqslant \frac{3 \pi}{2}$;

- for every fixed $t$, the mapping $\left(s, e^{i \theta}\right) \mapsto A_{t, s}\left(e^{i \theta}\right)$ is a diffeomorphism from $\left\{|s|<s_{1}\right\} \times\left\{|\theta|<\theta_{1}\right\}$ onto a neighborhood of $p$ in $M$;

- the exit vector $-\frac{\partial A_{0,0}}{\partial r}(1)$ is not tangent to $M$ at $p$, namely it has nonzero projection proj $T_{p} \mathbb{C}^{n} / T_{p} M\left(-\partial A_{t, 0} / \partial r(1)\right)$ onto the normal space $T_{p} \mathbb{C}^{n} / T_{p} M$ to $M$ at $p$; 
- choose any linear subspace $H_{p}$ of $T_{p} \mathbb{C}^{n}$ satisfying $T_{p} H_{p} \oplus T_{p} M=T_{p} \mathbb{C}^{n}$, so that $H_{p} \simeq T_{p} \mathbb{C}^{n} / T_{p} M$, denote by $\operatorname{proj}_{H_{p}}: T_{p} \mathbb{C}^{n} \rightarrow H_{p}$ the projection onto $H_{p}$ parallel to $T_{p} M$, define the associated exit vector

$$
\operatorname{ex}\left(A_{t, 0}\right):=\operatorname{proj}_{H_{p}}\left(-\frac{\partial A_{t, 0}}{\partial r}(1)\right) \in H_{p}
$$

and the associated normalized exit vector $\mathrm{n}-\operatorname{ex}\left(A_{t, 0}\right) \quad:=$ $\operatorname{ex}\left(A_{t, 0}\right) /\left|\operatorname{ex}\left(A_{t, 0}\right)\right|$; then the rank at $t=0$ of the mapping

$$
\mathbb{R}^{d-1} \ni t \longmapsto \mathrm{n}-\operatorname{ex}\left(A_{t, 0}\right) \in S^{d-1} \subset \mathbb{R}^{d}
$$

should be maximal equal to $d-1$.

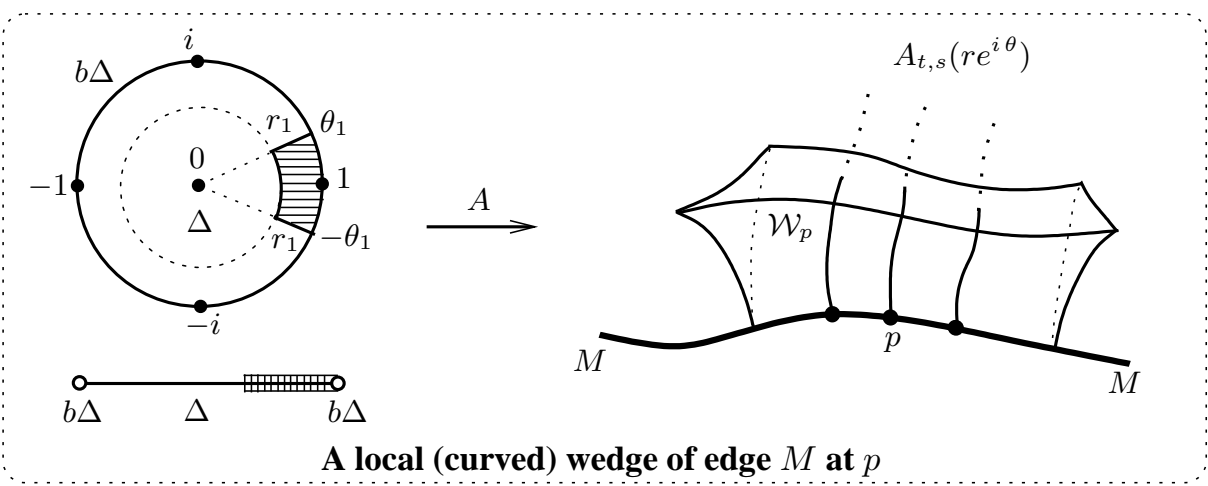

The last, most significant condition means that $\mathrm{n}-\operatorname{ex}\left(A_{t, 0}\right)$ describes an open neighborhood of n-ex $\left(A_{0,0}\right)$ in the unit sphere $S^{d-1} \subset \mathbb{R}^{d}$. This is of course independent of the choice of $H_{p}$. Then, fixing $s=0$ and $\theta=0$, as the rotation parameter $t \in \mathbb{R}^{d-1}$ varies with $|t|<t_{1}$, and as the radius $r$ with $r_{1}<r<1$ varies, the curves $A_{t, 0}(r)$ generate an open truncated (curved) cone in some $d$ dimensional local submanifold transverse to $M$ at $p$. Finally, as the translation parameter $s$ varies, the points $A_{t, s}\left(r e^{i \theta}\right)$ describe a (curved) local wedge of edge $M$ at $p$.

Lemma 3.3. Shrinking $t_{1}>0, s_{1}>0, \theta_{1}>0$ and $1-r_{1}>0$ if necessary, the points of $\mathcal{W}_{p}$ are covered injectively: $A_{t, s}\left(r e^{i \theta}\right)=A_{t^{\prime}, s^{\prime}}\left(r^{\prime} e^{i \theta^{\prime}}\right)$ if and only if $t=t^{\prime}, s=s^{\prime}, r=r^{\prime}$ and $\theta=\theta^{\prime}$.

This property follows directly from all the rank conditions. It will be useful to insure uniqueness of holomorphic extension (monodromy).

Definition 3.4. ([Tu1990, Trp1990]) A local CR-wedge of edge $M$ at $p$ of dimension $2 m+d+e$, with $1 \leqslant e \leqslant d$, is a set $\mathcal{W}_{p}^{C R, e}$ defined similarly as a local wedge, but assuming that the rotation parameter $t$ belongs to $\mathbb{R}^{e-1}$ and that the rank of the normalized exit vector mapping

$$
\mathbb{R}^{e-1} \ni t \longmapsto \mathrm{n}-\operatorname{ex}\left(A_{t, 0}\right) \in S^{d-1} \subset \mathbb{R}^{d}
$$


is equal to $e-1$.

Then, fixing $s=0$ and $\theta=0$, as the rotation parameter $t \in \mathbb{R}^{e-1}$ with $|t|<t_{1}$ varies, and as the radius $r$ with $r_{1}<r<1$ varies, the curves $A_{t, 0}(r)$ describe an open truncated (curved) cone in some $e$-dimensional local submanifold transverse to $M$ at $p$. These intermediate wedges of smaller dimension will play a crucial technical rôle in the sequel.

The case $e=1$ deserves special attention. A CR-wedge is then just a manifold with boundary $M_{p}^{1}$ with $\operatorname{dim} M_{p}^{1}=1+\operatorname{dim} M$ that is attached to $M$ at $p$, namely there exists an open neighborhood $U_{p}$ of $p$ in $M$ with $U_{p} \subset \partial M_{p}^{1}$. If in addition $M$ has codimension $d=1$, we recover the notion of one-sided neighborhood. It is clear that after a possible shrinking, every $\mathcal{C}^{2, \alpha-0}$ manifold with boundary $M_{p}^{1}$ attached to $M$ at $p$ may be prolonged as a local $\mathcal{C}^{2, \alpha-0}$ generic submanifold $\mathcal{M}_{p}^{1} \equiv$ $\mathcal{W}_{p}^{C R, 1}$ containing a neighborhood of $p$ in $M$ (as shown in the right diagram).

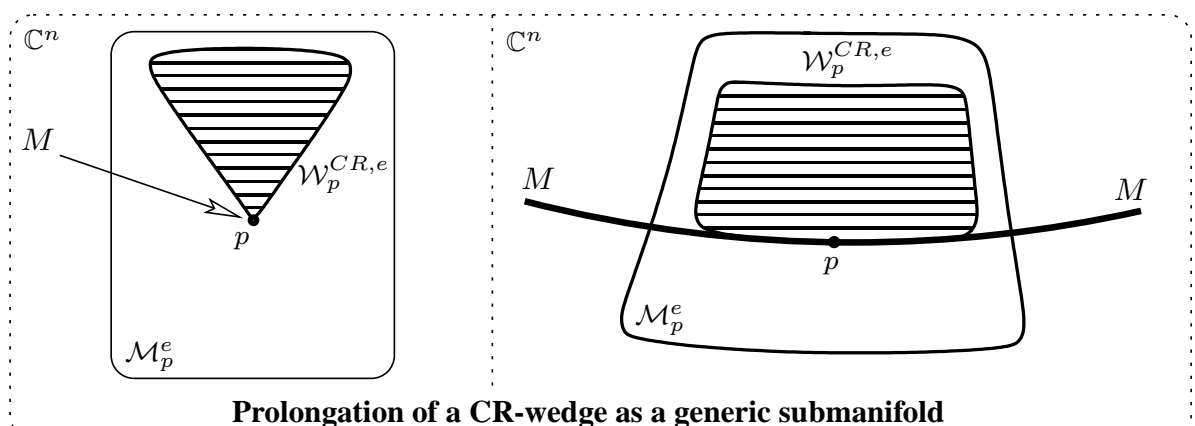

By elementary differential geometry, for $e \geqslant 2$, it may be verified that a local CR-wedge $\mathcal{W}_{p}^{C R, e}$ of edge $M$ at $p$ defined by means of a $\mathcal{C}^{2, \alpha-0}$ family of discs, namely

$$
\mathcal{W}_{p}^{C R, e}:=\left\{A_{t, s}\left(r e^{i \theta}\right):|t|<t_{1},|s|<s_{1},|\theta|<\theta_{1}, r_{1}<r<1\right\},
$$

may also be prolonged as a local generic submanifold $\mathcal{M}_{p}^{e}$ of dimension $2 m+d+e$ containing a neighborhood of $p$ in $M$. The left diagram is an illustration; in it, $e=d=2$, so that $M$ of codimension 2 is (unfortunately for intuition) collapsed to $p$.

However, the smoothness of $\mathcal{M}_{p}^{e}$ can decrease to $\mathcal{C}^{1, \alpha-0}$, because as in a standard local blowing down $\left(z_{1}, z_{2}\right) \mapsto\left(z_{1}, z_{1} z_{2}\right)$, the rank of the map $(r, \theta, s, t) \longmapsto A_{t, s}\left(r e^{i \theta}\right)$ degenerates when $r=1$, since the discs (partial) boundaries $\left\{A_{t, s}\left(e^{i \theta}\right):|\theta| \leqslant \frac{3 \pi}{2}\right\}$ are constrained to stay in $M$. For technical reasons, we will need in the sequel the existence of a prolongation $\mathcal{M}_{p}^{e}$ that is $\mathcal{C}^{2, \alpha-0}$ also when $e \geqslant 2$. The following modification of the definition of $\mathcal{W}_{p}^{C R, e}$ insures the existence of a $\mathcal{C}^{2, \alpha-0}$ prolongation $\mathcal{M}_{p}^{e}$. It will be applied implicitly in the sequel without further mention. 
So, assume $e \geqslant 2$, let $A_{t, s}$ be a family of discs as in Definition 3.4 with $\operatorname{ex}\left(A_{0,0}\right) \neq 0$ in $T_{p} \mathbb{C}^{n} / T_{p} M$ and $t \mapsto \mathrm{n}-\operatorname{ex}\left(A_{t, 0}\right)$ of rank $e-1$ at $t=0$. Fix $t:=0$ and define firstly

$$
\mathcal{W}_{p}^{C R, 1}:=\left\{A_{0, s}\left(r e^{i \theta}\right):|s|<s_{1},|\theta|<\theta_{1}, r_{1}<r<1\right\} .
$$

This is a manifold with boundary attached to $M$ at $p$. So there is a small $\mathcal{C}^{2, \alpha-0}$ prolongation $\mathcal{M}_{p}^{1} \supset \mathcal{W}_{p}^{C R, 1}$.

Choose $t \neq 0$ small with $A_{t, 0}$ having exit vector nontangent to $\mathcal{M}_{p}^{1}$ at $p$. Introduce a one-parameter family $M_{\sigma}, \sigma \in \mathbb{R},|\sigma|<\sigma_{1}, \sigma_{1}>0$, of generic submanifolds obtained by deforming slightly $M$ inside $\mathcal{M}_{p}^{1}$ near $p$, with $M_{\sigma} \subset M \cup \mathcal{W}_{p}^{C R, 1}$ for $\sigma \geqslant 0$. The $M_{\sigma}$ are "translates" of $M$ in $\mathcal{M}_{p}^{1}$ near $p$. To understand the process, we draw two diagrams in different dimensions.

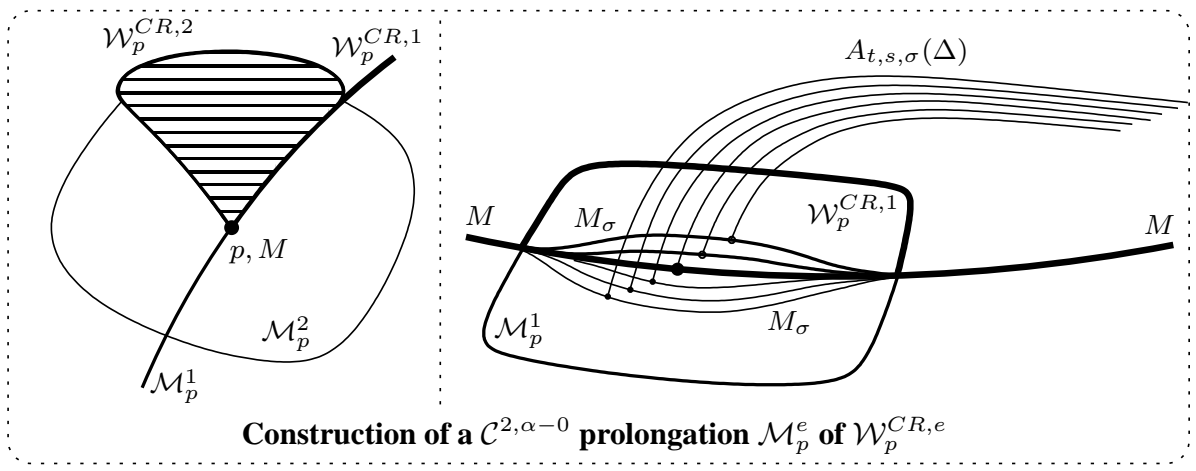

Thanks to the flexibility of Bishop's equation (Theorem 3.7(IV)), the $A_{t, s}$ may be deformed as a $\mathcal{C}^{2, \alpha-0}$ family $A_{t, s, \sigma}$ and we define secondly

$$
\mathcal{W}_{p}^{C R, 2}:=\left\{A_{t, s, \sigma}\left(r e^{i \theta}\right):|s|<s_{1}, 0<\sigma<\sigma_{1},|\theta|<\theta_{1}, r_{1}<r<1\right\} .
$$

Then this set constitutes a local CR-wedge of dimension $2 m+d+2$ with edge $M$ at $p$. Letting $\sigma$ run in $\left(-\sigma_{1}, \sigma_{1}\right)$ above, we get instead a certain manifold with boundary attached to $\mathcal{M}_{p}^{1}$ that may be extended as a $\mathcal{C}^{2, \alpha-0}$ generic submanifold $\mathcal{M}_{p}^{2}$ of dimension $2 m+d+2$. Then $\mathcal{W}_{p}^{C R, 2}$ is essentially one quarter of $\mathcal{M}_{p}^{2}$. We neither draw $\mathcal{W}_{p}^{C R, 2}$ nor $\mathcal{W}_{p}^{2}$ in the right diagram above, but the reader sees them. By induction, using that $t \mapsto \mathrm{n}-\operatorname{ex}\left(A_{t, 0}\right)$ has rank $e-1$ at $t=0$, we get the following.

Lemma 3.5. After a possible shrinking, a suitably constructed local $\mathcal{C}^{2, \alpha-0} C R$ wedge $\mathcal{W}_{p}^{C R, e}$ of edge $M$ at $p$ may be prolonged as a local $\mathcal{C}^{2, \alpha-0}$ generic submanifold $\mathcal{M}_{p}^{e}$ of dimension $2 m+d+e$ containing a neighborhood of $p$ in $M$.

In the sequel, similar technical constructions will be applied to insure the existence of $\mathcal{C}^{2, \alpha-0}$ prolongations $\mathcal{M}_{p}^{e} \supset \mathcal{W}_{p}^{C R, e}$ without further mention. 
3.6. Holomorphic extension of CR functions in higher codimension. In 1988, Tumanov [Tu1988] established a theorem that is nowadays celebrated in Several Complex Variables. Recall that by definition, $M$ is locally minimal at $p$ if the local CR orbit $\mathcal{O}_{C R}^{l o c}(M, p)$ contains a neighborhood of $p$ in $M$. Equivalently, $M$ does not contain any local submanifold $N$ passing through $p$ with $\operatorname{CRdim~} N=$ CRdim $M$ and $\operatorname{dim} N<\operatorname{dim} M$.

Theorem 3.7. ([Tu1988, BRT1994, Trp1996, Tu1998, BER1999]) Let $M$ be a local $\mathcal{C}^{2, \alpha}$ generic submanifold of $\mathbb{C}^{n}$ and let $p \in M$. If $M$ is locally minimal at $p$, then there exists a local wedge $\mathcal{W}_{p}$ of edge $M$ at p such that every $f \in \mathcal{C}_{C R}^{0}(M)$ possesses a holomorphic extension $F \in \mathcal{O}\left(\mathcal{W}_{p}\right) \cap \mathcal{C}^{0}\left(M \cup \mathcal{W}_{p}\right)$ with $\left.F\right|_{M}=f$.

Conversely, recall that according to Theorem 4.41(III), if $M$ is not locally minimal at $p$, there exists a local continuous CR function that is not holomorphically extendable to any local wedge at $p$.

Since the literature already contains abundant restitutions ${ }^{17}$, we will focus instead on propagation phenomena that are less known.

In 1994, as an answer to a conjecture formulated by Trépreau in [Trp1990], it was shown simultaneously by Jöricke and by the first author that Tumanov's theorem generalizes to globally minimal $M$. The preceding statement is a direct corollary of the next. Its proof given in [Me1994, ]ö1996] used techniques and ideas of Tumanov [Tu1988, Tu1994a] and of Trépreau [Trp1990].

Theorem 3.8. ([Me1994, Jö1996]) Let $M$ be a connected $\mathcal{C}^{2, \alpha}$ generic submanifold of $\mathbb{C}^{n}$. If $M$ is globally minimal then at every point $p \in M$, there exists a local wedge $\mathcal{W}_{p}$ of edge $M$ at $p$ such that every continuous $C R$ function $f \in \mathcal{C}_{C R}^{0}(M)$ possesses a holomorphic extension $F \in \mathcal{O}\left(\mathcal{W}_{p}\right) \cap \mathcal{C}^{0}\left(M \cup \mathcal{W}_{p}\right)$ with $\left.F\right|_{M}=f$.

With this statement, the extension theorem for CR function has reached a final, most general form. Philosophically, the main reason why it is true lies in the propagation of holomorphic extendability along complex-tangential curves. This was developed by Trépreau in 1990, using microlocal analysis.

Theorem 3.9. ([Trp1990]]) Let $M$ be a connected $\mathcal{C}^{\infty}$ generic submanifold of $\mathbb{C}^{n}$. If $\mathcal{C}_{C R}^{0}(M)$ extends holomorphically to a local wedge at some point $p \in M$, then at every point $q \in \mathcal{O}_{C R}(M, p)$, there exists a local wedge $\mathcal{W}_{q}$ of edge $M$ at $q$ such that every $f \in \mathcal{C}_{C R}^{0}(M)$ possesses a holomorphic extension $F \in \mathcal{O}\left(\mathcal{W}_{q}\right) \cap$ $\mathcal{C}^{0}\left(M \cup \mathcal{W}_{q}\right)$ with $\left.F\right|_{M}=f$.

\footnotetext{
${ }^{17}$ We recommend mostly the two elegant presentations [Trp1996] and [Tu1998]; other references are: [BRT1994, BER1999]. Excepting a conceptual abstraction involving the implicit function theorem in Banach spaces and the conormal bundle to $M$, the major arguments: differentiation of Bishop's equation and a crucial correspondence between an exit vector mapping and an evaluation mapping defined on the space of discs attached to $M$, the geometric structure of the proof is exactly the same in the original article Tu1988. as in the restitutions.
} 
Before surveying the original proof ([Me1994, एö1996]) of this theorem in Section 5, we shall expose in length a substantially simpler proof of Theorem 3.8 that was devised by the second author in Po2004]. This neat proof treats locally and globally minimal generic submanifolds on the same footing. It relies partly upon a natural deformation proposition due to Tumanov in [Tu1994a], but without any notion of defect of an analytic disc, without any needs to control the variation of the direction of CR-extendability, and without any partial connection, as in [Trp1990, Tu1994a, Me1994]. The next paragraphs and Section 4 are devoted to the proof of this most general Theorem 3.8.

Example 3.10. A globally minimal manifold may well be not locally minimal at any point.

Indeed, let $\chi: \mathbb{R} \rightarrow \mathbb{R}^{+}$be $\mathcal{C}^{\infty}$ with $\chi=0$ on $(-\infty, 1]$, with $\chi>0$ on $(1,+\infty)$ and with second derivative $\chi_{x x}>0$ on $(1,+\infty)$. Consider the generic manifold $M$ of $\mathbb{C}^{3}$ defined by the two equations

$$
v_{1}=\chi(x), \quad v_{2}=\chi(-x),
$$

in coordinates $\left(x+i y, u_{1}+i v_{1}, u_{2}+i v_{2}\right)$. Then $T^{1,0} M$ is generated by

$$
L=\frac{\partial}{\partial z}+i \chi_{x}(x) \frac{\partial}{\partial w_{1}}-i \chi_{x}(-x) \frac{\partial}{\partial w_{2}} .
$$

In terms of the four coordinates $\left(x, y, u_{1}, u_{2}\right)$ on $M$, the two vector fields generating $T^{c} M$ are

$$
\begin{aligned}
& L^{1}:=2 \operatorname{Re} L=\frac{\partial}{\partial x}, \\
& L^{2}:=2 \operatorname{Im} L=\frac{\partial}{\partial y}+\chi_{x}(x) \frac{\partial}{\partial u_{1}}-\chi_{x}(-x) \frac{\partial}{\partial u_{2}}
\end{aligned}
$$

(we have dropped $\chi_{x}(x) \frac{\partial}{\partial v_{1}}-\chi_{x}(-x) \frac{\partial}{\partial v_{2}}$ in $2 \operatorname{Re} L$ ). Denote by $\mathbb{L}^{0}$ the system of these two vector fields $\left\{L^{1}, L^{2}\right\}$ on $\mathbb{R}^{4} \simeq M$ and by $\mathbb{L}$ the $\mathcal{C}^{\infty}\left(\mathbb{R}^{4}\right)$-hull of $\mathbb{L}^{0}$. Observe that the Lie bracket

$$
\left[L^{1}, L^{2}\right]=\chi_{x x}(x) \frac{\partial}{\partial u_{1}}+\chi_{x x}(-x) \frac{\partial}{\partial u_{2}}
$$

is zero at points $p=\left(x_{p}, y_{p}, u_{1}^{p}, u_{2}^{p}\right)$ with $-1<x_{p}<1$, has non-zero $\frac{\partial}{\partial u_{2}}$ component at points $p$ with $x_{p}<-1$ and has non-zero $\frac{\partial}{\partial u_{1}}$-component at points $p$ with $x_{p}>1$. It follows that the local $\mathbb{L}$-orbit of a point $p$ with $x_{p}<-1$ is $\left\{u_{1}=u_{1}^{p}\right\}$, of a point $p$ with $-1<x_{p}<1$ is $\left\{u_{1}=u_{1}^{p}, u_{2}=u_{2}^{p}\right\}$ and of a point $x_{p}$ with $x_{p}>1$ is $\left\{u_{2}=u_{2}^{p}\right\}$. Also, observe that since the vector field $L^{1}=\frac{\partial}{\partial x}$ belongs to $\mathbb{L}$, the local $\mathbb{L}$-orbit of any point $p=\left(x_{p}, y_{p}, u_{1}^{p}, u_{2}^{p}\right)$ contains points of coordinates $\left(x_{p}+t, y_{p}, u_{1}^{p}, u_{2}^{p}\right)$, with $t$ small. We deduce that the local $\mathbb{L}$-orbit of 
points $p$ with $x_{p}=-1$ or $x_{p}=1$ are three-dimensional, hence in conclusion:

$$
\mathcal{O}_{\mathbb{L}}^{l o c}\left(\mathbb{R}^{4}, p\right)= \begin{cases}U_{p} \cap\left\{u_{1}=u_{1}^{p}\right\} & \text { if } x_{p} \leqslant-1, \\ U_{p} \cap\left\{u_{1}=u_{1}^{p}, u_{2}=u_{2}^{p}\right\} & \text { if }-1<x_{p}<1, \\ U_{p} \cap\left\{u_{2}=u_{2}^{p}\right\} & \text { if } x_{p} \geqslant 1,\end{cases}
$$

where $U_{p}$ is a neighborhood of $p$ in $M$. So $\mathbb{L}$ is nowhere locally minimal.

Lemma 3.11. The system $\mathbb{L}$ is globally minimal.

Proof. We check that any two points $p, q \in \mathbb{R}^{4}$ are in the same $\mathbb{L}$-orbit. Using the flow of $L^{1}=\frac{\partial}{\partial x}$ and then the flow of $L^{2}$ on $\{x=0\}$, the original two points $p$ and $q$ may be joined to points, still denoted by $p=\left(0,0, u_{1}^{p}, u_{2}^{p}\right)$ and $q=\left(0,0, u_{1}^{q}, u_{2}^{q}\right)$, having zero $x$-component and zero $y$-component.

We claim that the global $\mathbb{L}$-orbit $\mathcal{O}_{\mathbb{L}}\left(\mathbb{R}^{4}, p\right)$ of every point $p=\left(0,0, u_{1}^{p}, u_{2}^{p}\right)$ contains a neighborhood of $p$ in $\mathbb{R}^{4}$. Since the two-dimensional plane $\{x=y=$ $0\}$ is connected, this will assure that any two points $p=\left(0,0, u_{1}^{p}, u_{2}^{p}\right)$ and $q=$ $\left(0,0, u_{1}^{q}, u_{2}^{q}\right)$ are in the same $\mathbb{L}$-orbit.

Indeed, by means of $\frac{\partial}{\partial x}$, every point $p=\left(0,0, u_{1}^{p}, u_{2}^{p}\right)$ is joined to the two points $p^{-}:=\left(-1,0, u_{1}^{p}, u_{2}^{p}\right)$ and $p^{+}:=\left(1,0, u_{1}^{p}, u_{2}^{p}\right)$. Let $U_{p^{-}}$and $U_{p^{+}}$be small neighborhoods of $p^{-}$and of $p^{+}$. Denote by $H^{-}:=\left\{u_{1}=u_{1}^{p}\right\} \cap U_{p^{-}}$and by $H^{+}:=\left\{u_{2}=u_{2}^{p}\right\} \cap U_{p^{+}}$small pieces of the three-dimensional local $\mathbb{L}$-orbits of $p^{-}$and of $p^{+}$.

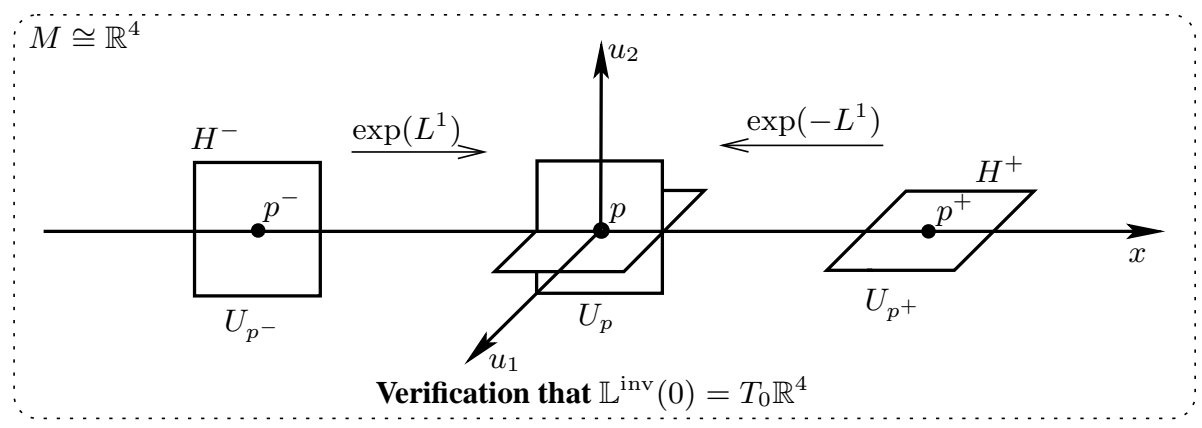

The flow of $L^{1}=\frac{\partial}{\partial x}$ being a translation, we deduce:

$$
\begin{aligned}
\exp \left(L^{1}\right)\left(H^{-}\right) & =\left\{u_{1}=u_{1}^{p}\right\} \cap U_{p}, \\
\exp \left(-L^{1}\right)\left(H^{+}\right) & =\left\{u_{2}=u_{2}^{p}\right\} \cap U_{p},
\end{aligned}
$$

where $U_{p}$ is a small neighborhood of $p$ in $M \simeq \mathbb{R}^{4}$. Observe that the two 3dimensional planes are transversal in $T_{p} \mathbb{R}^{4}$. Lemma 1.28(III) yields:

$$
\begin{aligned}
& \mathbb{L}^{\text {inv }}\left(p^{-}\right) \supset T_{p^{-}} \mathcal{O}_{\mathbb{L}}^{\text {loc }}\left(p^{-}\right)=\left\{u_{1}=u_{1}^{p}\right\}, \\
& \mathbb{L}^{\text {inv }}\left(p^{+}\right) \supset T_{p^{+}} \mathcal{O}_{\mathbb{L}}^{\text {loc }}\left(p^{+}\right)=\left\{u_{2}=u_{2}^{p}\right\} .
\end{aligned}
$$


By the very definition of $\mathbb{L}^{\text {inv }}$, we necessarily have:

$$
\begin{aligned}
\mathbb{L}^{\text {inv }}(p) & \supset \exp \left(L^{1}\right)_{*}\left(\mathbb{L}^{\text {inv }}\left(p^{-}\right)\right)+\exp \left(-L^{1}\right)_{*}\left(\mathbb{L}^{\text {inv }}\left(p^{+}\right)\right) \\
& =\left\{u_{1}=u_{1}^{p}\right\}+\left\{u_{2}=u_{2}\right\} \\
& =T_{p} \mathbb{R}^{4}
\end{aligned}
$$

so $\mathbb{L}^{\text {inv }}(p)=T_{p} \mathbb{R}^{4}$. Consequently, $\mathcal{O}_{\mathbb{L}}\left(\mathbb{R}^{4}, p\right)$ contains a neighborhood of $\left(0,0, u_{1}^{p}, u_{2}^{p}\right)$ in $\mathbb{R}^{4}$.

3.12. Setup for propagation. Let $M$ be connected, generic and $\mathcal{C}^{2, \alpha}$, let $q \in M$ and let $\mathcal{W}_{q}^{C R, e}$ be a CR-wedge of dimension $2 m+d+e$ at $q$, with $1 \leqslant e \leqslant d$. For short, we will say that $\mathcal{C}_{C R}^{0}(M)$ extends to be $C R$ on $\mathcal{W}_{q}^{C R, e}$ if for every $f \in \mathcal{C}_{C R}^{0}$, there exists $F \in \mathcal{C}_{C R}^{0}\left(M \cup \mathcal{W}_{q}^{C R, e}\right)$ with $\left.F\right|_{M}=f$.

Theorem 3.13. Let $e \in \mathbb{N}$ with $1 \leqslant e \leqslant d$. Assume that $\mathcal{C}_{C R}^{0}(M)$ extends to be CR on a $C R$-wedge $\mathcal{W}_{p}^{C R, e}$ of dimension $2 m+d+$ e at some point $p \in M$. Then for every $q \in \mathcal{O}_{C R}(M, p)$, there exists a CR-wedge $\mathcal{W}_{q}^{C R, e}$ at $q$ of the same dimension $2 m+d+e$ to which $\mathcal{C}_{C R}^{0}(M)$ extends to be $C R$.

In the case $e=d$, we recover ${ }^{18}$ Trépreau's Theorem 3.9, since continuous CR functions on an open set of $\mathbb{C}^{n}$ (here a usual wedge) are just holomorphic. If $M$ is globally minimal, then extension holds at every $q \in M$. Notice that this statement covers the propagation Theorem 2.7, stated previously in the hypersurface case $d=e=1$.

Let us start the proof. Through a chain of small analytic discs, every $q \in$ $\mathcal{O}_{C R}(M, p)$ is joined to a point $p^{\prime}$ arbitrarily close to $p$ : indeed, Lemma 2.20 and its proof remain the same in arbitrary codimension $d \geqslant 1$. At $p^{\prime}, \mathrm{CR}$ extension holds, because the edge of $\mathcal{W}_{p}^{C R, e}$ contains a small open neighborhood $U_{p}$ of $p$ in $M$. To deduce $\mathrm{CR}$ extension at $q$, it suffices therefore to propagate $\mathrm{CR}$ extension along a single disc, as stated in the next main proposition.

\footnotetext{
${ }^{18}$ Classical microlocal analysis was devised to measure the analytic wave front set of a distribution in terms of the exponential decay ot the Fourier transform restricted to open, conic submanifolds of the cotangent bundle. We suspect that there might exist higher generalizations of microlocal analysis in which one takes account of the good decay of the Fourier transform on submanifolds of positive codimension in the cotangent bundle.
} 


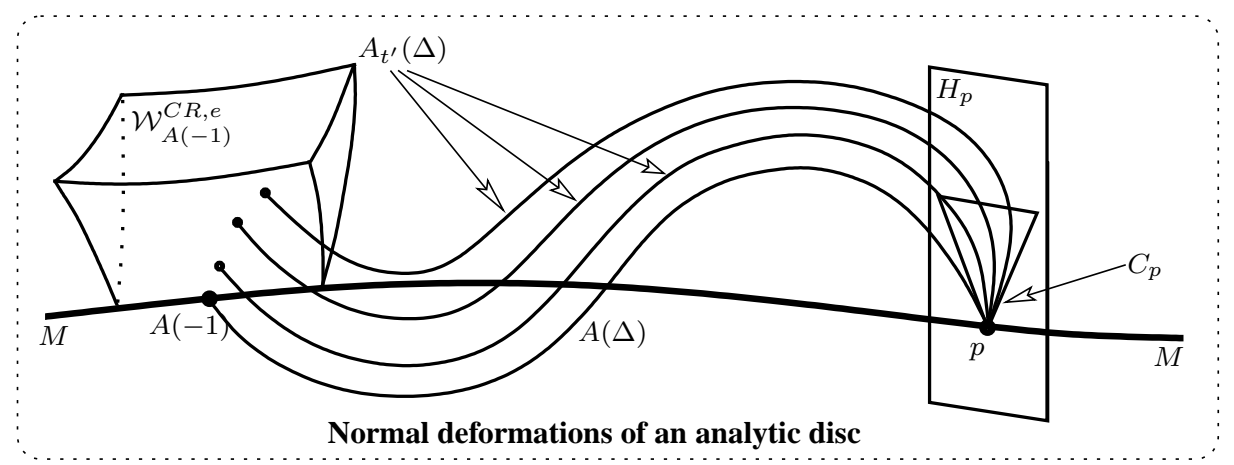

Proposition 3.14. (Propagation along a disc) ([Tu1994a, MP1999], [*]) Let A be a small $\mathcal{C}^{2, \alpha-0}$ analytic disc attached to $M$ which is an embedding $\bar{\Delta} \rightarrow \mathbb{C}^{n}$. Let $e \in \mathbb{N}$ with $1 \leqslant e \leqslant d$. Assume that there exists a $\mathcal{C}^{2, \alpha-0}$ CR-wedge $\mathcal{W}_{A(-1)}^{C R, e}$ at $A(-1)$ of dimension $2 m+d+e$ to which $\mathcal{C}_{C R}^{0}(M)$ extends to be $C R$. Then there exists a $\mathcal{C}^{2, \alpha-0} C R$-wedge $\mathcal{W}_{A(1)}^{C R, e}$ at $A(1)$ of the same dimension $2 m+d+e$ to which $\mathcal{C}_{C R}^{0}(M)$ extends to be $C R$.

With more precisions, the CR-wedge $\mathcal{W}_{A(1)}^{C R, e}$ is constructed by translating a certain family of analytic discs $A_{t^{\prime}}$ having the following properties. Setting $p:=$ $A(1)$, there exists a $\mathcal{C}^{2, \alpha-0}$ family $A_{t^{\prime}}$ of analytic discs, $t^{\prime} \in \mathbb{R}^{e},\left|t^{\prime}\right|<t_{1}^{\prime}, t_{1}^{\prime}>0$, with $\left.A_{t^{\prime}}\right|_{t^{\prime}=0}=A$, with $A_{t^{\prime}}(1)=p$, satisfying $A_{t^{\prime}}\left(e^{i \theta}\right) \in M$ for $|\theta| \leqslant \frac{3 \pi}{2}$ and having their boundaries $A_{t^{\prime}}(\partial \Delta) \subset M \cup \mathcal{W}_{A(-1)}^{C R, e}$ for $t^{\prime}$ belonging to some open truncated cone $\mathrm{C}^{\prime} \subset \mathbb{R}^{e}$, such that the exit vector mapping:

$$
\mathbb{R}^{e} \ni t^{\prime} \longmapsto \operatorname{ex}\left(A_{t^{\prime}}\right)=\operatorname{proj}_{H_{p}}\left(-\frac{\partial A_{t^{\prime}}}{\partial r}(1)\right) \in \mathbb{R}^{d}
$$

is of maximal rank equal to e at $t^{\prime}=0$, where $H_{p} \simeq \mathbb{R}^{d}$ is any linear subspace of $T_{p} \mathbb{C}^{n}$ such that $H_{p} \oplus T_{p} M=T_{p} \mathbb{C}^{n}$, and where proj $_{H_{p}}$ is the linear projection parallel to $T_{p} M$.

Geometrically, as $t^{\prime}$ varies, the exit vectors $\operatorname{ex}\left(A_{t^{\prime}}\right)$ describe an open cone $C_{p} \subset$ $H_{p}$, drawn in the diagram.

We claim that this statement covers the second, delicate case of Proposition 2.21. Indeed assuming that $e=d=1$ and that the exit vector $-\frac{\partial A}{\partial r}(1)$ is tangent to $M$ at $A(1)$, the above proposition includes $A$ in a one-parameter family $A_{t^{\prime}}$ whose direction of exit in the normal bundle has nonzero derivative with respect to $t^{\prime}$. Hence for every nonzero $t^{\prime}$, the direction of exit of $A_{t^{\prime}}$ is not tangent to $M$ at $p$. Thus, a non-tangential deformed disc $A^{\mathrm{d}}$ as in Proposition 2.21 may be chosen to be any $A_{t^{\prime}}$, with $t^{\prime} \neq 0$.

Proof of Proposition 3.14. We first explain how to get CR extension at $p$ from the family $A_{t^{\prime}}$, taking for granted its existence. 
(I) Suppose firstly that the exit vector of $A=A_{0}$ is non-tangential to $M$ at $p$. We have to restrict the parameter space $t^{\prime} \in \mathbb{R}^{e}$ to some parameter space $t \in \mathbb{R}^{e-1}$ so as to reach Definition 3.4.

Let us take for granted the fact that the exit vector mapping has rank $e$ at $t^{\prime}=0$. Then the normalized exit vector mapping

$$
\mathbb{R}^{e} \ni t^{\prime} \longmapsto \mathrm{n}-\operatorname{ex}\left(A_{t^{\prime}}\right)=\operatorname{ex}\left(A_{t^{\prime}}\right) /\left|\operatorname{ex}\left(A_{t^{\prime}}\right)\right| \in S^{d-1}
$$

has rank $\geqslant e-1$ at $t^{\prime}=0$. So there exists a small piece of an $(e-1)$-dimensional linear subspace $\Lambda_{0}$ of $\mathbb{R}^{d}$, parameterized as $t^{\prime}=\phi(t)$ for some linear map $\phi$, with $t \in \mathbb{R}^{e-1}$ small, namely $|t|<t_{1}$, for some $t_{1}>0$, such that $t \mapsto \mathrm{n}-\operatorname{ex}\left(A_{\phi(t)}\right)$ has rank $(e-1)$ at $t=0$.

Setting $A_{t}:=A_{\phi(t)}$, we thus reach Definition 3.4, without the translation parameter $s$.

But proceeding exactly as in the hypersurface case, it is easy to include some translation parameter getting a family $\left(A_{t^{\prime}}\right)_{s}=A_{t^{\prime}, s}$. The proof is postponed to the end $\$ 3.24$. Then the desired family $A_{t, s}$ of the proposition is just $A_{\phi(t), s}$, shrinking $t_{1}>0$ and $s_{1}>0$ if necessary.

Lemma 3.15. There exists a deformation $A_{t^{\prime}, s}$ of $A_{t^{\prime}}$, with $s \in \mathbb{R}^{2 m+d-1},|s|<$ $s_{1}$, such that:

- the boundaries $A_{t^{\prime}, s}(\partial \Delta)$ are contained in $M \cup \mathcal{W}_{A(-1)}^{C R, e}$ and $A_{t^{\prime}}\left(e^{i \theta}\right) \in$ $M$ for $|\theta| \leqslant \frac{3 \pi}{2}$

- for every fixed $t^{\prime}$, the mapping $\left(s, e^{i \theta}\right) \longmapsto A_{t^{\prime}, s}\left(e^{i \theta}\right)$ is a diffeomorphism from $\left\{|s|<s_{1}\right\} \times\left\{|\theta|<\theta_{1}\right\}$ onto a neighborhood of $p$ in $M$.

Therefore, the final family $A_{t, s}$ yields a CR-wedge $\mathcal{W}_{p}^{C R, e}$ at $p=A(1)$, as in Definition 3.4. The mild generalization of the approximation Theorem 5.2(III) stated as Lemma 2.19 above in the case $d=1$ holds in the general case $d \geqslant 1$ without modification. Consequently, $\mathcal{C}_{C R}^{0}(M)$ extends to be $\mathrm{CR}$ on $\mathcal{W}_{p}^{C R, e}$.

(II) Suppose secondly that the exit vector of $A=A_{0}$ is tangential to $M$ at $p$. Thanks to the fact that the exit vector mapping has rank $e$ at $t^{\prime}=0$, for every nonzero $t_{0}^{\prime}$, the disc $A_{t_{0}^{\prime}}$ is nontangential to $M$ at $p$. In this case, we fix a small $t_{0}^{\prime} \neq 0$ and we proceed with $A_{t^{\prime}+t_{0}^{\prime}}$ just as above.

In summary, it remains only to construct the family $A_{t^{\prime}}$ having the crucial property that the exit vector mapping has rank $e$ at $t^{\prime}=0$.

3.16. Normal deformations of analytic discs. Thus, we now expose how to construct $A_{t^{\prime}}$. We shall introduce a parameterized family $M_{t^{\prime}}$ of $\mathcal{C}^{2, \alpha-0}$ generic submanifolds by pushing $M$ near $A(-1)$ inside $\mathcal{W}_{A(-1)}^{C R, e}$ in $e$ independent normal directions, $e$ being the number of degrees of freedom offered by $\mathcal{W}_{A(-1)}^{C R, e}$. Outside a neighborhood of $A(-1)$, each $M_{t^{\prime}}$ shall coincides with $M$ and also $\left.M_{t^{\prime}}\right|_{t^{\prime}=0}=M$. 
We may assume that the point $p:=A(1)$ is the origin in coordinates $(z, u+$ $i v) \in \mathbb{C}^{m} \times \mathbb{C}^{d}$ in which $M$ is represented by $v=\varphi(z, u)$, where $\varphi$ satisfies $\varphi(0)=0$ and $d \varphi(0)=0$. Let $t^{\prime} \in \mathbb{R}^{e}$ be small, namely $\left|t^{\prime}\right|<t_{1}^{\prime}$, with $t_{1}^{\prime}>0$.

In terms of graphing equations, the deformation $M_{t^{\prime}}$ may be represented by

$$
v=\Phi\left(z, u, t^{\prime}\right),
$$

with $\Phi \in \mathcal{C}^{2, \alpha-0}$ defined for $\left|t^{\prime}\right|<t_{1}^{\prime}$ satisfying $\Phi(z, u, 0) \equiv \varphi(z, u)$. The point $A(-1)$ has small coordinates $\left(z_{-1}, u_{-1}+i \varphi\left(z_{-1}, u_{-1}\right)\right)$. We require that the $e$ vectors

$$
\Phi_{t_{k}^{\prime}}\left(z_{-1}, u_{-1}, 0\right), \quad k=1, \ldots, e,
$$

are linearly independent. There exists a truncated open cone $C^{\prime} \subset \mathbb{R}^{e}$ with the property that

$$
M_{t^{\prime}} \subset M \cup \mathcal{W}_{A(-1)}^{C R, e},
$$

whenever $t^{\prime} \in \mathrm{C}^{\prime}$. In fact, we implicitly assume in Proposition 3.14 that the CRwedge based at $A(-1)$ may be extended as a $\mathcal{C}^{2, \alpha-0}$ generic submanifold $\mathcal{M}_{A(-1)}^{e}$ of dimension $2 m+d+e$ passing through $A(-1)$ so that $M_{t^{\prime}}$ is contained in $M \cup \mathcal{M}_{A(-1)}^{e}$, for every $\left|t^{\prime}\right|<t_{1}^{\prime}$. The original CR-wedge $\mathcal{W}_{A(-1)}^{C R, e}$ may then be viewed as a curved real wedge of edge $M$ which is contained inside $\mathcal{M}_{A(-1)}^{C R, e}$.

The starting $\mathcal{C}^{2, \alpha} \operatorname{disc} A(\zeta)=(Z(\zeta), W(\zeta))$ with $W(\zeta)=(U(\zeta)+i V(\zeta))$ is attached to $M$ with $A(1)=0$. Equivalently:

$$
\left\{\begin{array}{l}
V\left(e^{i \theta}\right)=\varphi\left(Z\left(e^{i \theta}\right), U\left(e^{i \theta}\right)\right) \\
U\left(e^{i \theta}\right)=-\mathrm{T}_{1}[\varphi(Z(\cdot), U(\cdot))]\left(e^{i \theta}\right)
\end{array}\right.
$$

for every $e^{i \theta} \in \partial \Delta$. Thanks to the existence Theorem 3.7(IV), there exists a $\mathcal{C}^{2, \alpha-0}$ deformation $A_{t^{\prime}}$ of $A$, where each $A_{t^{\prime}}(\zeta):=\left(Z(\zeta), W\left(\zeta, t^{\prime}\right)\right)$ with $A_{t^{\prime}}(1)=p$ has the same $z$-component ${ }^{19}$ as $A$ and is attached to $M_{t^{\prime}}$, namely:

$$
\left\{\begin{array}{l}
V\left(e^{i \theta}, t^{\prime}\right)=\Phi\left(Z\left(e^{i \theta}\right), U\left(e^{i \theta}, t^{\prime}\right), t^{\prime}\right), \\
U\left(e^{i \theta}, t^{\prime}\right)=-\mathrm{T}_{1}\left[\Phi\left(Z(\cdot), U\left(\cdot, t^{\prime}\right), t^{\prime}\right)\right]\left(e^{i \theta}\right),
\end{array}\right.
$$

for every $e^{i \theta} \in \partial \Delta$. Observe that $W\left(e^{i \theta}, 0\right) \equiv W\left(e^{i \theta}\right)$. We then differentiate the first line above with respect to $t_{k}^{\prime}$ at $t^{\prime}=0$, for $k=1, \ldots, e$, which yields in matrix notation:

$$
V_{t_{k}^{\prime}}\left(e^{i \theta}, 0\right)=\Phi_{u}\left(Z\left(e^{i \theta}\right), U\left(e^{i \theta}\right), 0\right) U_{t_{k}^{\prime}}\left(e^{i \theta}, 0\right)+\Phi_{t_{k}^{\prime}}\left(Z\left(e^{i \theta}\right), U\left(e^{i \theta}\right), 0\right) .
$$

\footnotetext{
${ }^{19}$ Since first order partial derivatives $W_{t_{k}^{\prime}}\left(\zeta, t^{\prime}\right), k=1, \ldots, e$, will appear in a while, we do not write the parameter $t^{\prime}$ as a lower index in $U\left(\zeta, t^{\prime}\right)+i V\left(\zeta, t^{\prime}\right)$.
} 
Also, the $\mathcal{C}^{1, \alpha-0} \operatorname{discs} A_{t_{k}^{\prime}}(\zeta, 0)$ satisfy the linear Bishop-type equation

$$
U_{t_{k}^{\prime}}\left(e^{i \theta}, 0\right)=-\mathrm{T}_{1}\left[\Phi_{u}(Z(\cdot), U(\cdot), 0) U_{t_{k}^{\prime}}(\cdot, 0)+\Phi_{t_{k}^{\prime}}(Z(\cdot), U(\cdot), 0)\right]\left(e^{i \theta}\right) .
$$

As a supplementary space to $T_{p} M$ in $T_{p} \mathbb{C}^{n}$, we choose $H_{p}:=\{0\} \times i \mathbb{R}^{d}=$ $\{w=0, u=0\}$. Then $\operatorname{proj}_{H_{p}}\left(-\partial A_{t^{\prime}}(1) / \partial r\right)=-\partial V\left(1, t^{\prime}\right) / \partial r$, which yields after differentiating with respect to $t_{k}^{\prime}$ at $t^{\prime}=0$ :

$$
\left.\frac{\partial}{\partial t_{k}^{\prime}}\right|_{t^{\prime}=0} \operatorname{proj}_{H_{p}}\left(-\frac{\partial A_{t^{\prime}}}{\partial r}(1)\right)=-\frac{\partial V_{t_{k}^{\prime}}^{\prime}}{\partial r}(1,0),
$$

for $k=1, \ldots, e$. We will establish that if the local deformations $M_{t^{\prime}}$ of $M$ inside the CR-wedge $\mathcal{W}_{A(-1)}^{C R, e}$ are concentrated in a sufficiently thin neighborhood of $A(-1)$, then the above $e$ vectors $-\partial V_{t_{k}^{\prime}} / \partial r(1,0), k=1, \ldots, e$, are linearly independent. This will complete the proof of the proposition.

There is a singular integral operator $\mathcal{J}$ which yields the interior normal derivative at $1 \in \partial \Delta$ of any $\mathcal{C}^{1, \alpha-0}$ mapping $v=\bar{\Delta} \rightarrow \mathbb{R}^{d}$ which is harmonic in $\Delta$ and vanishes at $1 \in \partial \Delta$ :

$$
\mathcal{J}(v):=\operatorname{p.v} \cdot \frac{1}{\pi} \int_{-\pi}^{\pi} \frac{v\left(e^{i \theta}\right)}{\left|e^{i \theta}-1\right|^{2}} d \theta=-\frac{\partial v}{\partial r}(1) .
$$

The proof is postponed to Lemma 3.25 below. If $h: \bar{\Delta} \rightarrow \mathbb{C}^{d}$ is $\mathcal{C}^{1, \alpha-0}$ and holomorphic in $\Delta$, we have in addition

$$
\mathcal{J}(h)=-\frac{\partial h}{\partial r}(1)=i \frac{\partial h}{\partial \theta}(1) .
$$

With the singular integral $\mathcal{J}$, we may thus reformulate (3.19):

$$
\operatorname{proj}_{H_{p}}\left(-\frac{\partial^{2} A_{0}}{\partial t_{k}^{\prime} \partial r}(1)\right)=\mathcal{J}\left(V_{t_{k}^{\prime}}\right) \text {. }
$$

Lemma 3.21. Let $u, v \in \mathcal{C}^{1, \alpha-0}\left(\bar{\Delta}, \mathbb{R}^{d}\right)$ be harmonic in $\Delta$ and vanish at $1 \in \partial \Delta$. Then:

$$
0=\mathcal{J}\left(u v-\mathrm{T}_{1} u \mathrm{~T}_{1} v\right)
$$

In addition, $u$ (and also $v$ ) satisfies the two equations:

$$
\mathcal{J}(u)=-\frac{\partial\left(\mathrm{T}_{1} u\right)}{\partial \theta}(1) \quad \text { and } \quad \mathcal{J}\left(\mathrm{T}_{1} u\right)=\frac{\partial u}{\partial \theta}(1) .
$$

Proof. The holomorphic product $w:=\left(u+i \mathrm{~T}_{1} u\right)\left(v+i \mathrm{~T}_{1} v\right)$ vanishes to second order at $1 \in \partial \Delta$, so $\mathcal{J}(w)=0$, hence

$$
0=\operatorname{Re} \mathcal{J}(w)=\mathcal{J}\left(u v-\mathrm{T}_{1} u \mathrm{~T}_{1} v\right) .
$$

The pair of equations satisfied by $u$ is obtained by identifying the real and imaginary parts of $\mathcal{J}(h)=i \frac{\partial h}{\partial \theta}(1)$, where $h:=u+i \mathrm{~T}_{1} u$. 
Following [Tu1994a], we now introduce a $d \times d$ matrix $G$ of $\mathcal{C}^{1, \alpha}$ functions on $\partial \Delta$ defined by the functional equation

$$
G\left(e^{i \theta}\right)=I+\mathrm{T}_{1}\left[G(\cdot) \Phi_{u}(Z(\cdot), U(\cdot), 0)\right]\left(e^{i \theta}\right) .
$$

Here $\Phi_{u}=\left(\Phi_{u_{l}}^{j}\right)_{1 \leqslant l \leqslant d}^{1 \leqslant j \leqslant d}$ is a $d \times d$ matrix. Since $\Phi_{u}(z, u, 0) \equiv \varphi_{u}(z, u)$ is small, the solution $G$ exists and is unique, by an application of Proposition 3.21(IV). Notice that $G(1)=I$. Applying $\mathrm{T}_{1}$ to both sides, we get $\mathrm{T}_{1} G=-G \Phi_{u}+$ cst., without writing the arguments. In fact, the constant vanishes, since $\Phi_{u}(0,0,0)=$ $\varphi_{u}(0,0)=0$. So we get:

$$
\mathrm{T}_{1} G=-G \Phi_{u}
$$

We also notice that $V_{t_{k}^{\prime}}=\mathrm{T}_{1} U_{t_{k}^{\prime}}$ and $U_{t_{k}^{\prime}}=-\mathrm{T}_{1} V_{t_{k}^{\prime}}$.

Next, we rewrite (3.18) without arguments: $\Phi_{t_{k}^{\prime}}=V_{t_{k}^{\prime}}-\Phi_{u} U_{t_{k}^{\prime}}, k=1, \ldots, e$, we apply the matrix $G$ to both sides, we replace $G \Phi_{u}$ by $-\mathrm{T}_{1} G$ as well as $U_{t_{k}^{\prime}}$ by $-\mathrm{T}_{1} V_{t_{k}^{\prime}}$ and we let appear a term $u v-\mathrm{T}_{1} u \mathrm{~T}_{1} v$ :

$$
\begin{aligned}
G \Phi_{t_{k}^{\prime}} & =G V_{t_{k}^{\prime}}-G \Phi_{u} U_{t_{k}^{\prime}} \\
& =G V_{t_{k}^{\prime}}-\left(\mathrm{T}_{1} G\right)\left(\mathrm{T}_{1} V_{t_{k}^{\prime}}\right) \\
& =V_{t_{k}^{\prime}}+(G-I) V_{t_{k}^{\prime}}-\mathrm{T}_{1}(G-I) \mathrm{T}_{1} V_{t_{k}^{\prime}} .
\end{aligned}
$$

Finally ${ }^{20}$, applying the singular operator $\mathcal{J}$ and remembering Lemma 3.21, we obtain:

$$
\mathcal{J}\left(G \Phi_{t_{k}^{\prime}}\right)=\mathcal{J}\left(V_{t_{k}^{\prime}}\right) .
$$

We claim that if the support of the deformation $M_{t^{\prime}}$ is sufficiently concentrated near $A(-1)$, the $e$ vectors $\mathcal{J}\left(V_{t_{k}^{\prime}}\right)=\mathcal{J}\left(G \Phi_{t_{k}^{\prime}}\right) \in \mathbb{R}^{d}$ are linearly independent.

Indeed, since the deformations $M_{t^{\prime}}$ are localized near $A(-1)$, we have $\Phi_{t_{k}^{\prime}}\left(Z\left(e^{i \theta}\right), U\left(e^{i \theta}\right), 0\right) \equiv 0$, except for $|\theta+\pi|<\theta_{2}$, with $\theta_{2}>0$ small. We deduce:

$$
\begin{aligned}
\mathcal{J}\left(G \Phi_{t_{k}^{\prime}}\right) & =\frac{1}{\pi} \int_{|\theta+\pi|<\theta_{2}} \frac{G\left(e^{i \theta}\right) \Phi_{t_{k}^{\prime}}\left(Z\left(e^{i \theta}\right), U\left(e^{i \theta}\right), 0\right)}{\left|e^{i \theta}-1\right|^{2}} d \theta \\
& \approx \frac{1}{\pi} \frac{G(-1)}{4} \int_{|\theta+\pi|<\theta_{2}} \Phi_{t_{k}^{\prime}}\left(Z\left(e^{i \theta}\right), U\left(e^{i \theta}\right), 0\right) d \theta .
\end{aligned}
$$

Since, by assumption, the $e$ vectors $\Phi_{t_{k}^{\prime}}\left(z_{-1}, u_{-1}, 0\right)$ are linearly independent, the linear independence of the above (concentrated) vector-valued integrals follows.

The proofs of Proposition 3.14 and of Theorem 3.13 are complete.

\footnotetext{
${ }^{20}$ We can also check that $\mathcal{J}\left(U_{t_{k}^{\prime}}\right)=-\mathcal{J}\left(\mathrm{T}_{1} V_{t_{k}^{\prime}}\right)=\partial V_{t_{k}^{\prime}}(1,0) / \partial \theta=0$. Indeed, it suffices to differentiate (3.18) with respect to $\theta$ at $\theta=0$, noticing that $\Phi_{u}(0,0,0)=$ $\varphi_{u}(0,0)=0$, that $U_{t_{k}^{\prime}}(1,0)=0$ and that $\Phi_{t_{k}^{\prime}}(z, u, 0)=0$ for $(z, u)$ near $(0,0)$.
} 
3.24. Proofs of two lemmas. Firstly, we check formula (3.20).

Lemma 3.25. Let $u \in \mathcal{C}^{1, \beta}(\bar{\Delta})(0<\beta<1)$ be harmonic in $\Delta$, real-valued and satisfying $u(1)=0$. Then the interior normal derivative of $u$ at $1 \in \partial \Delta$ is given by:

$$
-\frac{\partial u}{\partial r}(1)=\text { p.v. } \frac{1}{\pi} \int_{-\pi}^{\pi} \frac{u\left(e^{i \theta}\right)}{\left|e^{i \theta}-1\right|^{2}} d \theta=\text { p.v. } \frac{i}{\pi} \int_{\partial \Delta} \frac{u(\zeta)}{(\zeta-1)^{2}} d \zeta .
$$

Proof. The function $h:=u+i \mathrm{~T} u$ is holomorphic in $\Delta$ and $\mathcal{C}^{1, \beta}$ in $\bar{\Delta}$. Since $\mathrm{T} u$ is also harmonic in $\Delta$, since $\frac{\partial h}{\partial r}(1)=\frac{\partial u}{\partial r}(1)+i \frac{\partial \mathrm{T} u}{\partial r}(1)$, and since the kernel $\left|e^{i \theta}-1\right|^{-2}$ is real, we may prove the lemma with $u$ replaced by $h \in \mathcal{O}(\Delta) \cap$ $\mathcal{C}^{1, \beta}(\bar{\Delta})$.

Let $\zeta=r e^{i \theta}$ and denote $h_{1}:=\frac{\partial h}{\partial \zeta}(1)=\frac{\partial h}{\partial r}(1)$, so that $h(\zeta)=(\zeta-1) h_{1}+$ $\mathrm{O}\left(|\zeta-1|^{1+\beta}\right)$. We remind that, for any $\zeta_{0} \in \partial \Delta$, by an elementary modification of Cauchy's formula, we have p.v. $\frac{1}{2 \pi i} \int_{\partial \Delta} \frac{d \zeta}{\zeta-\zeta_{0}}=\frac{1}{2}$. We deduce that the linear term $(\zeta-1) h_{1}$ provides the main contribution:

$$
\text { p.v. } \frac{i}{\pi} \int_{\partial \Delta} \frac{(\zeta-1) h_{1}}{(\zeta-1)^{2}} d \zeta=-2 h_{1} \text { p.v. } \frac{1}{2 \pi i} \int_{\partial \Delta} \frac{d \zeta}{\zeta-1}=-h_{1} .
$$

Thus, we have to prove that the remainder $r(\zeta):=h(\zeta)-(\zeta-1) h_{1}$, which belongs to $\mathcal{O}(\Delta) \cap \mathcal{C}^{1, \beta}(\bar{\Delta})$, gives no contribution, namely satisfies $\int_{\partial \Delta} \frac{r(\zeta)}{(\zeta-1)^{2}} d \zeta=0$.

Set $s(\zeta):=\frac{r(\zeta)}{(\zeta-1)^{2}}$. Then $s \in \mathcal{O}(\Delta)$ is continuous on $\bar{\Delta} \backslash\{1\}$ and satisfies $|s(\zeta)| \leqslant \mathrm{K}|\zeta-1|^{\beta-1}$ for some $\mathrm{K}>0$. We claim that by an application of Cauchy's theorem, the integral $\int_{\partial \Delta} s(\zeta) d \zeta$, which exists without principal value, vanishes.

Indeed, let $\varepsilon$ with $0<\varepsilon<<1$ and consider the open disc $\Delta(1, \varepsilon)$ of radius $\varepsilon$ centered at 1 . The drawing of this disc delineates three $\operatorname{arcs}$ of $\bar{\Delta}$ :

(i) the open $\operatorname{arc} \partial \Delta \backslash \overline{\Delta(1, \varepsilon)}$, of length $\approx 2 \pi-2 \varepsilon$;

(ii) the closed arc $\partial \Delta \cap \overline{\Delta(1, \varepsilon)}$, of length $\approx 2 \varepsilon$;

(iii) the closed arc $\partial \Delta(1, \varepsilon) \cap \bar{\Delta}$, of length is $\approx \pi \varepsilon$.

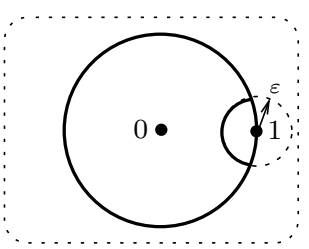

We then decompose the integral of $s$ on $\partial \Delta$ as integrals on the first two arcs:

$$
\int_{\partial \Delta} s(\zeta) d \zeta=\int_{\partial \Delta \backslash \overline{\Delta(1, \varepsilon)}} s(\zeta) d \zeta+\int_{\partial \Delta \cap \overline{\Delta(1, \varepsilon)}} s(\zeta) d \zeta .
$$

The estimate $|s(\zeta)| \leqslant \mathrm{K}|\zeta-1|^{\beta-1}$ insures the smallness of the second integral:

$$
\left|\int_{\partial \Delta \cap \overline{\Delta(1, \varepsilon)}} s(\zeta) d \zeta\right| \leqslant C_{1} \varepsilon^{\beta} .
$$


To transform the first integral, we observe that Cauchy's theorem entails that integration of $s(\zeta) d \zeta$ on the closed contour $[\partial \Delta \backslash \overline{\Delta(1, \varepsilon)}] \cup[\partial \Delta(1, \varepsilon) \cap \bar{\Delta}]$ vanishes:

$$
0=\int_{\partial \Delta \backslash \overline{\Delta(1, \varepsilon)}} s(\zeta) d \zeta+\int_{\partial \Delta(1, \varepsilon) \cap \bar{\Delta}} s(\zeta) d \zeta
$$

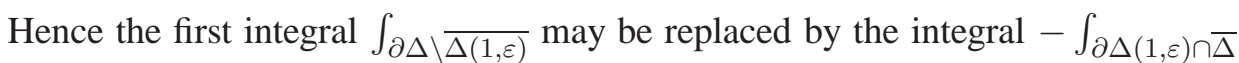
on the third arc. The estimate $|s(\zeta)| \leqslant \mathrm{K}|\zeta-1|^{\beta-1}$ again insures that this second integral is bounded by $C_{2} \varepsilon^{\beta}$. In conclusion $\left|\int_{\partial \Delta} s(\zeta) d \zeta\right| \leqslant\left(C_{1}+C_{2}\right) \varepsilon^{\beta}$.

Proof of Lemma 3.15. Secondly, we provide the details for the translation of the family $A_{t^{\prime}}$. Let $v=\varphi(z, u)$ represent $M$ in a neighborhood of $p$. By assumption, $A_{t^{\prime}}(\zeta)=\left(Z(\zeta), W\left(\zeta, t^{\prime}\right)\right)$ is attached to $M_{t^{\prime}}$, with $A_{t^{\prime}}(1)=p$. Equivalently, the two equations (3.17) hold. Since $A=\left.A_{t^{\prime}}\right|_{t^{\prime}=0}$ is an embedding, the vector $v_{p}:=\frac{\partial A}{\partial \theta}(1) \in T_{p} M$ is nonzero. As in $\S 2.12$, we choose a small $(2 m+d-1)$ dimensional submanifold $K_{p}$ passing through $p$ with $\mathbb{R} v_{p} \oplus T_{p} K_{p}=T_{p} M$ and we parametrize it by $s \mapsto(z(s), u(s)+i \varphi(z(s), u(s)))$, where $s \in \mathbb{R}^{2 m+d-1}$ is small, $|s|<s_{1}, s_{1}>0$. Then the translation

$$
A_{t^{\prime}, s}(\zeta)=\left(Z(\zeta)+z(s), W\left(\zeta, t^{\prime}, s\right)\right)
$$

is constructed by perturbing the two equations (3.17), requiring only that

$$
A_{t^{\prime}, s}(1)=(z(s), u(s)+i \varphi(z(s), u(s))) .
$$

This is easily done:

$$
\left\{\begin{array}{l}
V\left(e^{i \theta}, t^{\prime}, s\right)=\Phi\left(Z\left(e^{i \theta}\right)+z(s), U\left(e^{i \theta}, t^{\prime}, s\right), t^{\prime}\right), \\
U\left(e^{i \theta}, t^{\prime}, s\right)=u(s)-\mathrm{T}_{1}\left[\Phi\left(Z(\cdot)+z(s), U\left(\cdot, t^{\prime}, s\right), t^{\prime}\right)\right]\left(e^{i \theta}\right) .
\end{array}\right.
$$

The non-tangency of $v_{p}$ with $K_{p}$ at $p$ then insures that for every small fixed $t^{\prime}$, the mapping $(\theta, s) \mapsto A_{t^{\prime}, s}\left(e^{i \theta}\right)$ is a diffeomorphism onto a neighborhood of $p$ in $M$.

\section{$\S 4$. HOLOMORPHIC EXTENSION \\ ON GLOBALLY MINIMAL GENERIC SUBMANIFOLDS}

4.1. Structure of the proof of Theorem 3.8. Let $M$ be a $\mathcal{C}^{2, \alpha}$ globally minimal generic submanifold of $\mathbb{C}^{n}$. For clarity, we begin by a summary of the main steps of the proof of Theorem 3.8.

(a) Since $M$ is globally minimal, the distribution $q \mapsto T_{q}^{c} M$ must be somewhere not involutive, namely there must exist a point $p \in M$ and a section $L$ of $T^{1,0} M$ defined in an open neighborhood $U_{p}$ of $p$ in $M$ with $L(p) \neq 0$ such that $[L, \bar{L}](p) \notin T_{p}^{1,0} M \oplus T_{p}^{0,1} M$. 
(b) Thanks to an easy generalization of the Lewy extension theorem ( $§ 2.10)$, there exists a manifold $M_{p}^{1}$ attached to $M$ at $p$ with $\operatorname{dim} M_{p}^{1}=1+\operatorname{dim} M$ to which $\mathcal{C}_{C R}^{0}(M)$ extends to be $\mathrm{CR}$.

(c) Thanks to the main propagation Proposition 3.14, CR extension to a similar manifold $M_{q}^{1}$ attached to $M$ holds at every point $q \in M=$ $\mathcal{O}_{C R}(M, p)$.

(d) Since there are as many manifolds with boundary as points in $M$, it may well happen that at some point $p \in M$ which belongs to the edge of two different manifolds $M_{p^{\prime}}^{1}$ and $M_{p^{\prime \prime}}^{1}$, the tangent spaces $T_{p} M_{p^{\prime}}^{1}$ and $T_{p} M_{p^{\prime \prime}}^{1}$ are distinct. Refering to the diagram of $\S 4.5$ below, we may then immediately profit of such a situation, if it occurs.

(e) Indeed, in this case, an appropriate version of the edge-of-the-wedge theorem guarantees that $\mathcal{C}_{C R}^{0}(M)$ extends to be $\mathrm{CR}$ on a $\mathcal{C}^{2, \alpha-0} \mathrm{CR}$-wedge $\mathcal{W}_{p}^{C R, e}$ at $p$ whose dimension $e$ is $\geqslant 1+1=2$.

(f) To reason abstractly, let $e_{\max }$ be the maximal integer $e$ with $1 \leqslant e \leqslant d$ such that there exists a point $p \in M$ and a $\mathcal{C}^{2, \alpha-0} \mathrm{CR}$-wedge $\mathcal{W}_{p}^{C R, e}$ at $p$ of dimension $2 m+d+e$ to which $\mathcal{C}_{C R}^{0}(M)$ extends to be CR. Thanks to the main propagation Proposition 3.14, CR extension to a $\mathcal{C}^{2, \alpha-0} \mathrm{CR}$ wedge $\mathcal{W}_{q}^{C R, e_{\max }}$ holds at every point $q \in M=\mathcal{O}_{C R}(M, p)$.

(g) If $e_{\max }=d$, we are done, Theorem 3.8 is proved. Assuming $e_{\max } \leqslant$ $d-1$, we must construct a contradiction in order to complete the proof.

(h) Since $e_{\max }$ is maximal, again because of the edge-of-the-wedge theorem, the transversal situation (d) cannot occur; in other words, every point $p \in M$ that belongs to the edges of two different CR-wedges $\mathcal{W}_{p^{\prime}}^{C R, e_{\max }}$ and $\mathcal{W}_{p^{\prime \prime}}^{C R, e_{\max }}$ has the property that $T_{p} \mathcal{W}_{p^{\prime}}^{C R, e_{\max }}=T_{p} \mathcal{W}_{p^{\prime \prime}}^{C R, e_{\max }}$.

(i) It follows that, as $p$ runs in $M$, the $(2 m+d+e)$-dimensional tangent planes $T_{p} \mathcal{W}_{p}^{C R, e_{\max }} \cap T_{p} M$ glue together and they define a $\mathcal{C}^{1, \alpha-0}$ subdistribution $K M$ of the tangent bundle $T M$, of dimension $2 m+e_{\max }$, which contains $T^{c} M$.

(j) Since $M$ is globally minimal, such a distribution $p \mapsto K M(p)$ must be somewhere not involutive, namely there must exist a point $p \in M$ such that $[K M, K M](p) \not \subset K M(p)$.

(k) The $\mathcal{C}^{2, \alpha-0} \mathrm{CR}$-wedge $\mathcal{W}_{p}^{C R, e_{\max }}$ may be included in some $\mathcal{C}^{2, \alpha-0}$ local generic submanifold $\mathcal{M}_{p}^{e_{\max }}$ passing through $p$ and containing $M$ in a neighborhood of $p$.

(l) Multiplication by $i$ gives $T_{p}^{c} \mathcal{M}_{p}^{e_{\max }}=K M(p)+i K M(p)$ and the nondegeneracy $[K M, K M](p) \not \subset K M(p)$ implies that the Levi-form of $\mathcal{M}_{p}^{e_{\max }}$ is not identically zero at $p$, namely $\left[T_{p}^{c} \mathcal{M}_{p}^{e_{\max }}, T_{p}^{c} \mathcal{M}_{p}^{e_{\max }}\right](p) \not \subset$ $T_{p}^{c} \mathcal{M}_{p}^{e_{\max }}$. 
(m) Then a version of the Lewy-extension theorem on conic generic manifolds having a generic edge guarantees that $\mathcal{C}_{C R}^{0}(M)$ extends to be $\mathrm{CR}$ on a CR-wedge $\mathcal{W}_{p}^{C R, 1+e_{\max }}$ of dimension $2 m+d+1+e_{\max }$ at $p$. This new

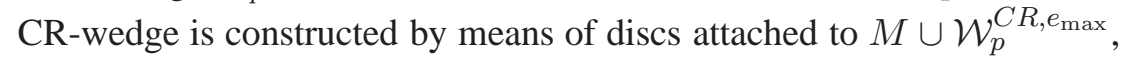
exploiting the nondegeneracy of the Levi form of $\mathcal{M}_{p}^{e_{\max }}$. This contradicts the assumption that $e_{\max } \leqslant d-1$ was maximal, hence completes the proof of Theorem 3.8.

The remainder of Section 4 is devoted to provide all the details of the proof.

4.2. Lewy extension in arbitrary codimension. As observed in (a) above, there exists a point $p \in M$ and a local section $L$ of $T^{1,0} M$ with $L(p) \neq 0$ such that $[L, \bar{L}](p) \not \subset \mathbb{C} \otimes T_{p}^{c} M$.

Lemma 4.3. ([[We1982, BPo1982] There exists a manifold with boundary $M_{p}^{1}$ attached to a neighborhood of $p$ in $M$ with $\operatorname{dim} M_{p}^{1}=1+\operatorname{dim} M$ to which $\mathcal{C}_{C R}^{0}(M)$ extends to be $C R$.

We shall content ourselves with only one direction of extension, since this will be sufficient for the sequel. Nevertheless, we mention that finer results expressed in terms of the Levi-cone of $M$ at $p$ may be found in [BPo1982, Bo1991]. Anyway, all the extension results that are based on pointwise nondegeneracy conditions as the openness of Levi-cone or the finite typeness of $M$ at a point are by far less general than Theorem 3.8, in which propagational aspects are involved.

Proof. The arguments are an almost straightforward generalization of the proof of the Lewy extension theorem (hypersurface case), already exposed in $\S 2.10$ above. Here is a summary.

By linear algebra reasonings, we may find local coordinates $(z, w) \in \mathbb{C}^{m} \times \mathbb{C}^{d}$ vanishing at $p$ with $L(p)=\left.\frac{\partial}{\partial z_{1}}\right|_{p}$, with $M$ given by $v=\varphi(z, u)$, where $\varphi(0)=0$, $d \varphi(0)=0$, and with first equation given by

$$
v_{1}=\varphi_{1}=z_{1} \bar{z}_{1}+\mathrm{O}\left(\left|z_{1}\right|^{2+\alpha}\right)+\mathrm{O}(|\widetilde{z}|)+\mathrm{O}(|z||u|)+\mathrm{O}\left(|u|^{2}\right),
$$

where we have split further the coordinates as $\left(z_{1}, \widetilde{z}, w_{1}, \widetilde{w}\right)$, with $\widetilde{z} \in \mathbb{C}^{m-1}$ and $\widetilde{w} \in \mathbb{C}^{d-1}$. For $\varepsilon>0$ small, we introduce the disc defined by

$$
A_{\varepsilon}(\zeta):=\left(\varepsilon(1-\zeta), \widetilde{0}, W_{\varepsilon}^{1}(\zeta), \widetilde{W}_{\varepsilon}(\zeta)\right)
$$

where $W_{\varepsilon}(\zeta)=U_{\varepsilon}(\zeta)+i V_{\varepsilon}(\zeta)$ is uniquely defined by requiring that $A_{\varepsilon}$ is attached to $M$ and satisfies $A_{\varepsilon}(1)=p$. As in $\S 2.10$, one verifies that

$$
-\frac{\partial V_{\varepsilon}^{1}}{\partial r}(1)=2 \varepsilon^{2}+\mathrm{O}\left(\varepsilon^{2+\alpha}\right) .
$$

Hence the exit vector of $A_{\varepsilon}$ at $1 \in \partial \Delta$ is nontangential to $M$ at $p$, provided $\varepsilon>0$ is small enough and fixed. By translating $A_{\varepsilon}$, we construct the desired manifold with boundary $M_{p}^{1}$. 
4.4. Maximal dimension for CR extension. As in $\S 4.1(\mathbf{f})$, let $e_{\max }$ be the maximal integer $e \leqslant d$ such that there exists a point $p \in M$ and a $\mathcal{C}^{2, \alpha-0} \mathrm{CR}$-wedge $\mathcal{W}_{p}^{C R, e}$ at $p$ of dimension $2 m+d+e$ to which $\mathcal{C}_{C R}^{0}(M)$ extends to be CR. By the above Lewy extension, we have $e_{\max } \geqslant 1$. Thanks to the main propagation Proposition 3.14, it immediately follows that CR extension to a $\mathcal{C}^{2, \alpha-0} \mathrm{CR}$-wedge $\mathcal{W}_{q}^{C R, e_{\max }}$ holds at every point $q \in M=\mathcal{O}_{C R}(M, p)$. If $e_{\max }=d$, Theorem 3.8 is proved, gratuitously.

Assuming that $1 \leqslant e_{\max } \leqslant d-1$, in order to establish Theorem 3.8, we must construct a contradiction. In the sequel, we shall simply denote $e_{\max }$ by $e$.

To proceed further, we must reformulate with high precision how were constructed all the CR-wedges obtained by the propagation Proposition 3.14.

For every point $p \in M$, there exists a local CR-wedge $\mathcal{W}_{p}^{C R, e}$ attached to a neighborhood of $p$ in $M$ which is described by means of a family of analytic discs $A_{p, t, s}(\zeta)$, where $t$ and $s$ are parameters. Here, the subscript $p$ is not a parameter, it indicates only that $p$ is the base point of $A_{p, t, s}$, namely $A_{p, t, 0}(1)=p$. The family $A_{p, t, s}$ enjoys properties that are listed below. In this list, the conditions are more uniform than those formulated in Definition 3.4, but one immediately verifies that both formulations are equivalent, up to a shrinking of $t_{1}(p)>0$, of $s_{1}(p)>0$, of $\theta_{1}(p)>0$ and of $1-r_{1}(p)>0$.

- The rotation parameter $t \in \mathbb{R}^{e-1}$ runs in $\left\{|t|<t_{1}(p)\right\}$, for some small $t_{1}(p)>0$.

- The translation parameter $s \in \mathbb{R}^{2 m+d-1}$ runs in $\left\{|s|<s_{1}(p)\right\}$, for some small $s_{1}(p)>0$.

- The point $q(p):=A_{p, 0,0}(-1) \in M$ is close to $p$.

- At $q(p)$, there is a CR-wedge $\mathcal{W}_{q(p)}^{C R, e}$.

- The family $A_{p, t, s}$ satisfies $A_{p, t, s}(\partial \Delta) \subset M \cup \mathcal{W}_{q(p)}^{C R, e}$.

- A small angle $\theta_{1}(p)>0$ and a radius $r_{1}(p)>0$ close to 1 are chosen.

- A family $H_{p^{\prime}}$ of linear subspaces of $T_{p^{\prime}} \mathbb{C}^{n}$ satisfying $T_{p^{\prime}} H_{p^{\prime}} \oplus T_{p^{\prime}} M=$ $T_{p^{\prime}} \mathbb{C}^{n}$ for all $p^{\prime} \in M$ in a neighborhood of $p$ is chosen.

- For every $t$ with $|t|<t_{1}(p)$, every $s$ with $|s|<s_{1}(p)$ and every $\theta$ with $|\theta|<\theta_{1}(p)$, the exit vector of $A_{p, t, s}\left(e^{i \theta}\right)$ at $e^{i \theta}$ is not tangent to $M$ :

$$
\operatorname{ex}\left(A_{p, t, s}\right)\left(e^{i \theta}\right):=\operatorname{proj}_{H_{A_{p, t, s}\left(e^{i \theta}\right)}}\left(i \frac{\partial A_{p, t, s}}{\partial \theta}\left(e^{i \theta}\right)\right) \neq 0 .
$$

- For every fixed $s$ with $|s|<s_{1}(p)$ and every fixed $\theta$ with $|\theta|<\theta_{1}(p)$, the normalized exit vector mapping

$$
\mathbb{R}^{e-1} \ni t \longmapsto \mathrm{n}-\operatorname{ex}\left(A_{p, t, s}\right)\left(e^{i \theta}\right) \in S^{d-1}
$$

is of rank $(e-1)$ at every $t \in\left\{|t|<t_{1}(p)\right\}$. 
- For some $t_{2}(p), s_{2}(p), \theta_{2}(p)$ and $r_{2}(p)$ satisfying $0<t_{2}(p)<t_{1}(p), 0<$ $s_{2}(p)<s_{1}(p), 0<\theta_{2}(p)<\theta_{1}(p)$ and $0<1-r_{2}(p)<1-r_{1}(p)<1$, the CR-wedge is precisely defined as:

$\mathcal{W}_{p}^{C R, e}:=\left\{A_{p, t, s}\left(r e^{i \theta}\right):|t|<t_{2}(p),|s|<s_{2}(p),|\theta|<\theta_{2}(p), r_{2}(p)<r<1\right\}$.

- Finally, the CR-wedge $\mathcal{W}_{p}^{C R, e}$ is contained in a $\mathcal{C}^{2, \alpha-0}$ local generic submanifold $\mathcal{M}_{p}^{e}$ of the same dimension $2 m+d+e$ that contains a neighborhood of $p$ in $M$. At a point $p^{\prime}=A_{p, t^{\prime}, s^{\prime}}\left(e^{i \theta^{\prime}}\right) \in M$ of the edge of $\mathcal{W}_{p}^{C R, e}$, the tangent space of $\mathcal{M}_{p}^{e}$ is:

$$
T_{p^{\prime}} \mathcal{M}_{p}^{e}=T_{p} M \oplus \mathbb{R}\left(i \frac{\partial A_{p, t^{\prime}, s^{\prime}}}{\partial \theta}\left(e^{i \theta^{\prime}}\right)\right) \bigoplus_{1 \leqslant k \leqslant e-1} \mathbb{R}\left(i \frac{\partial^{2} A_{p, t^{\prime}, s^{\prime}}}{\partial \theta \partial t_{k}}\left(e^{i \theta^{\prime}}\right)\right) .
$$

4.5. An edge-of-the-wedge theorem. There are as many generic submanifolds $\mathcal{M}_{p^{\prime}}^{C R, e}$ of codimension $d-e$ as points $p^{\prime} \in M$. At a point $p=A_{p^{\prime}, t^{\prime}, s^{\prime}}\left(e^{i \theta^{\prime}}\right)$ that belongs to the edge of such an $\mathcal{M}_{p^{\prime}}^{C R, e}$, we may define a linear subspace of $T_{p} M$ by

$$
K M_{p^{\prime}}(p):=T_{p}^{c} \mathcal{M}_{p^{\prime}}^{e} \cap T_{p} M .
$$

Since $\mathcal{M}_{p^{\prime}}^{e}$ is generic and contains $M$ in a neighborhood of $p$, this space $K M_{p^{\prime}}(p)$ contains $T_{p}^{c} M$ and is $(2 m+e)$-dimensional. Also, multiplication by $i$ induces an isomorphism $K M_{p^{\prime}}(p) / T_{p}^{c} M \simeq T_{p} \mathcal{M}_{p^{\prime}}^{e} / T_{p} M$.

In general, two different $K M_{p^{\prime}}(p)$ and $K M_{p^{\prime \prime}}(p)$ need not coincide, or equivalently, two different tangent spaces $T_{p} \mathcal{M}_{p^{\prime}}^{e}$ and $T_{p} \mathcal{M}_{p^{\prime \prime}}^{e}$ are unequal.

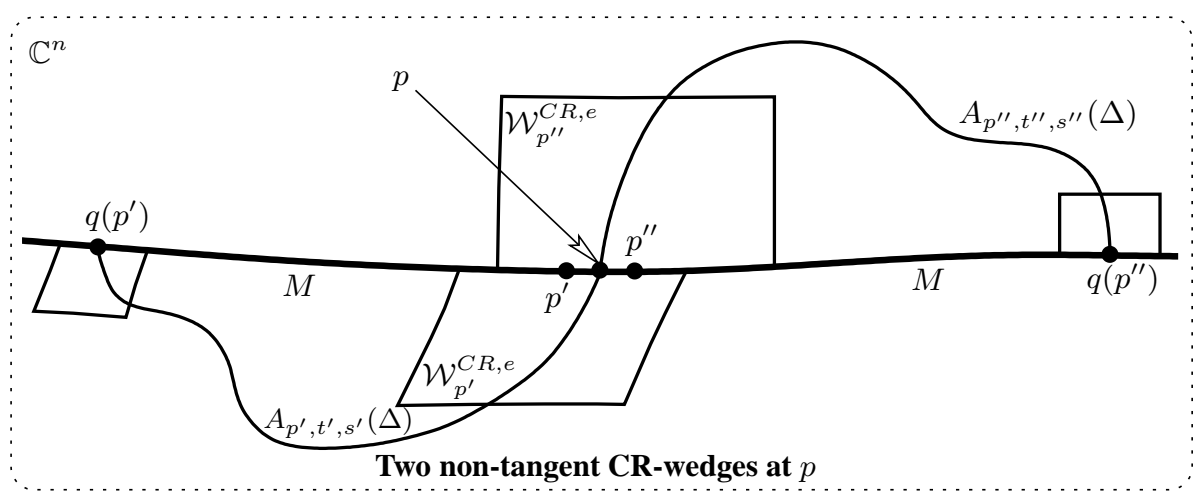

More precisely, there is a dichotomy.

(I) Either for every two points $p^{\prime}, p^{\prime \prime} \in M$ such that there exists a point $p$ belonging to the intersection of the edges of the two CR-wedges $\mathcal{W}_{p^{\prime}}^{C R, e}$ 
and $\mathcal{W}_{p^{\prime \prime}}^{C R, e}$, namely of the form:

$$
p=A_{p^{\prime}, t^{\prime}, s^{\prime}}\left(e^{i \theta^{\prime}}\right)=A_{p^{\prime \prime}, t^{\prime \prime}, s^{\prime \prime}}\left(e^{i \theta^{\prime \prime}}\right),
$$

for some values

$$
\begin{array}{lll}
\left|t^{\prime}\right|<t_{2}\left(p^{\prime}\right), & \left|s^{\prime}\right|<s_{2}\left(p^{\prime}\right), & \left|\theta^{\prime}\right|<\theta_{2}\left(p^{\prime}\right), \\
\left|t^{\prime \prime}\right|<t_{2}\left(p^{\prime \prime}\right), & \left|s^{\prime \prime}\right|<s_{2}\left(p^{\prime \prime}\right), & \left|\theta^{\prime \prime}\right|<\theta_{2}\left(p^{\prime \prime}\right),
\end{array}
$$

the two spaces $T_{p} \mathcal{M}_{p^{\prime}}^{e}$ and $T_{p} \mathcal{M}_{p^{\prime \prime}}^{e}$ coincide. Equivalently, $K M_{p^{\prime}}(p)=$ $K M_{p^{\prime \prime}}(p)$.

(II) Or there exist two points $p^{\prime}, p^{\prime \prime} \in M$ and a point $p=A_{p^{\prime}, t^{\prime}, s^{\prime}}\left(e^{i \theta^{\prime}}\right)=$ $A_{p^{\prime \prime}, t^{\prime \prime}, s^{\prime \prime}}\left(e^{i \theta^{\prime \prime}}\right)$ in the intersection of the edges of the two CR-wedges $\mathcal{W}_{p^{\prime}}^{C R, e}$ and $\mathcal{W}_{p^{\prime \prime}}^{C R, e}$ such that

$$
T_{p} \mathcal{M}_{p^{\prime}}^{e} \neq T_{p} \mathcal{M}_{p^{\prime \prime}}^{e}
$$

Lemma 4.6. The case $T_{p} \mathcal{M}_{p^{\prime}}^{e} \neq T_{p} \mathcal{M}_{p^{\prime \prime}}^{e}$ implies that $\mathcal{C}_{C R}^{0}(M)$ extends to be CR on a CR-wedge $\widetilde{\mathcal{W}}_{p}^{C R, 1+e}$ at $p$ whose dimension equals $2 m+d+1+e$, contradicting the maximality of $e=e_{\max }$.

Of course, this lemma follows by a known CR version of the edge-of-the-wedge theorem (Ai1989]), but for completeness, we summarize a shorter proof that exploits the existence of the discs $A_{p^{\prime}, t, s}$, as in Po2004.

Proof. By construction, the family $A_{p^{\prime}, t, s}(\zeta)$ covers the CR-wedge $\mathcal{W}_{p^{\prime}}^{C R, e}$. The point $p$ belongs to the edge of $\mathcal{W}_{p^{\prime}}^{C R, e}$.

Since $T_{p} \mathcal{M}_{p^{\prime}}^{e} \neq T_{p} \mathcal{M}_{p^{\prime \prime}}^{e}$, there exists a manifold $M_{p}^{1} \subset \mathcal{W}_{p^{\prime \prime}}^{C R, e}$ attached to $M$ at $p$ with $\operatorname{dim} M_{p}^{1}=1+\operatorname{dim} M$ such that

$$
1+e=\operatorname{dim}\left(\left[T_{p} M_{p}^{1}+T_{p} \mathcal{W}_{p^{\prime}}^{C R, e}\right] / T_{p} M\right) .
$$

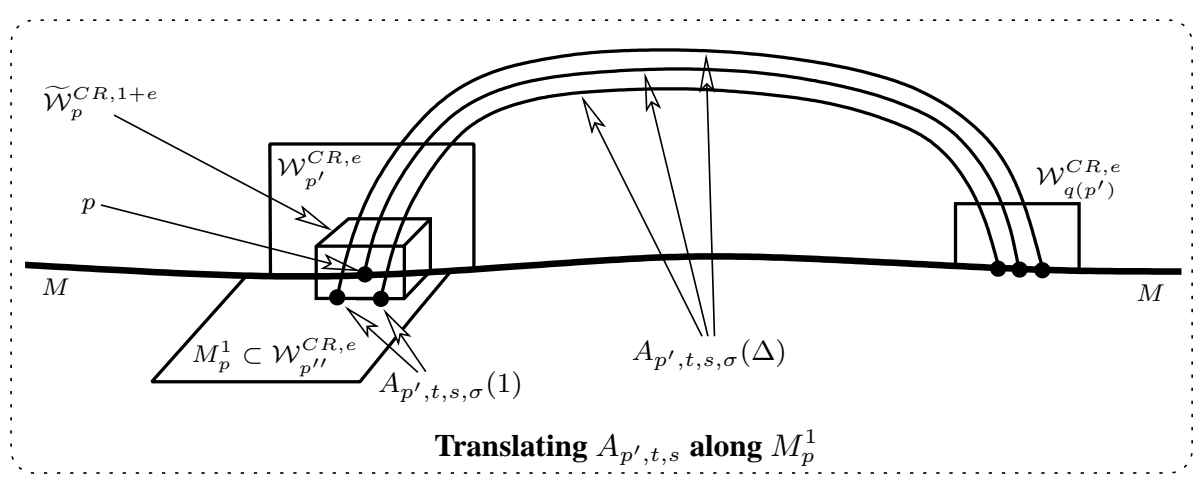


We may deform the family $A_{p^{\prime}, t, s}$ by translating it along $M_{p}^{1}$, as in the diagram. So we introduce a supplementary parameter $\sigma>0$ and we require that the point $A_{p^{\prime}, t, s, \sigma}(1)$ should cover a one-sided neighborhood of $p$ in $M_{p}^{1}$ as $\sigma$ runs in $\left(0, \sigma_{1}\right)$, for some small $\sigma_{1}>0$, and as the previous translation parameter $s \in \mathbb{R}^{2 m+d-1}$ runs in $\left\{|s|<s_{2}\left(p^{\prime}\right)\right\}$. Thanks to Theorem 3.7(IV), the corresponding Bishoptype equation has $\mathcal{C}^{2, \alpha-0}$ solutions.

If we choose $t_{3}>0$ with $\left|t^{\prime}\right|+t_{3}<t_{2}\left(p^{\prime}\right), s_{3}>0$ with $\left|s^{\prime}\right|+s_{3}<s_{2}\left(p^{\prime}\right)$, $\theta_{3}>0$ with $\left|\theta^{\prime}\right|+\theta_{3}<\theta_{2}\left(p^{\prime}\right), \sigma_{3}>0$ with $\sigma_{3}<\sigma_{1}$ and $r_{3}<1$ with $r_{2}\left(p^{\prime}\right)<$ $r_{3}<1$, the set:

$$
\begin{aligned}
\widetilde{W}_{p}^{C R, 1+e}:=\left\{A_{p^{\prime}, t, s, \sigma}\left(r e^{i \theta}\right):\right. & \left|t-t^{\prime}\right|<t_{3},\left|s-s^{\prime}\right|<s_{3}, \\
& \left.\left|\theta-\theta^{\prime}\right|<\theta_{3}, r_{3}<r<1,0<\sigma<\sigma_{3}\right\}
\end{aligned}
$$

will constitute a CR-wedge of dimension $2 m+d+1+e$ at $p$. By a technical adaptation of the approximation Theorem 5.2(III) (cf. Lemma 2.19), $\mathcal{C}_{C R}^{0}(M)$ extends to be CR on $\widetilde{W}_{p}^{C R, 1+e}$.

4.7. Definition of the (non-integrable) subbundle $K M \subset T M$. Consequently, case (II) cannot occur, because of the definition of $e=e_{\max }$. Thus, case (I) holds. In other words, as $p^{\prime}$ runs in $M$, the $\mathcal{C}^{1, \alpha-0}$ distributions $p \mapsto K M_{p^{\prime}}(p)$ defined for $p$ in the edge of $\mathcal{W}_{p^{\prime}}^{C R, e}$ (a neighborhood of $p^{\prime}$ in $M$ ) glue together in a welldefined $\mathcal{C}^{1, \alpha-0}$ vector subbundle of $T M$. Observe that $T^{c} M$ is a subbundle of $K M$ of codimension $e$. For every point $p \in M$, we have:

$$
T_{p}^{c} M \subset K M(p)=T_{p}^{c} \mathcal{M}_{p}^{e} \cap T_{p} M .
$$

As in $\S 4.1(\mathbf{j})$, since $M$ is globally minimal and since $K M$ is of codimension $d-e \geqslant 1$ in $T M$, there must exist a point $p \in M$ such that $[K M, K M](p) \not \subset$ $K M(p)$.

Lemma 4.8. At such a point $p$, the Levi form of $\mathcal{M}_{p}^{e}$ does not vanish identically:

$$
\left[T^{c} \mathcal{M}_{p}^{e}, T^{c} \mathcal{M}_{p}^{e}\right](p) \not \subset T_{p}^{c} \mathcal{M}_{p}^{e}
$$

Proof. We reason by contradiction, assuming that $\left[T^{c} \mathcal{M}_{p}^{e}, T^{c} \mathcal{M}_{p}^{e}\right](p) \subset T_{p}^{c} \mathcal{M}_{p}^{e}$. Let $K^{1}$ and $K^{2}$ be two arbitrary $\mathcal{C}^{1, \alpha-0}$ sections of $K M$ defined in a small neighborhood $U_{p}$ of $p$ in $M$. Since $\left.K M\right|_{U_{p}}$ is a subbundle of $\left.T M\right|_{U_{p}}$, we have

$$
\left[K^{1}, K^{2}\right](p) \in T_{p} M .
$$

We may extend $K^{1}$ and $K^{2}$ to a neighborhood $\mathcal{U}_{p}$ of $p$ in $\mathcal{M}_{p}^{e}$ that contains $U_{p}$ as sections $\mathcal{K}^{1}$ and $\mathcal{K}^{2}$ of $T^{c} \mathcal{M}_{p}^{e} \mid \mathcal{U}_{p}$. Since $K^{1}$ and $K^{2}$ are tangent to $M \cap U_{p}$, one verifies that, independently of the extension:

$$
\left[K^{1}, K^{2}\right](p)=\left[\mathcal{K}^{1}, \mathcal{K}^{2}\right](p) \in T_{p}^{c} \mathcal{M}_{p}^{e}
$$


where the second Lie bracket belongs to $T_{p}^{c} \mathcal{M}_{p}^{e}$, because we assumed that the Levi form of $\mathcal{M}_{p}^{e}$ vanishes at $p$. We deduce

$$
\left[K^{1}, K^{2}\right](p) \in T_{p}^{c} \mathcal{M}_{p}^{e} \cap T_{p} M=K M(p) .
$$

This contradicts $[K M, K M](p) \not \subset K M(p)$.

4.9. Lewy extension on CR-wedges. To contradict the maximality of $e=e_{\max }$ at a point $p$ at which $[K M, K M](p) \not \subset K M(p)$, we formulate a Lewy extension theorem on the conic manifold with edge $\mathcal{W}_{p}^{C R, e}$.

Proposition 4.10. Let $p \in M$ and assume that $\left[T_{p}^{c} \mathcal{M}_{p}^{e}, T_{p}^{c} \mathcal{M}_{p}^{e}\right](p) \not \subset T_{p}^{c} \mathcal{M}_{p}^{e}$. Then there exists a $(2 m+d+1+e)$-dimensional local $C R$-wedge $\widetilde{W}_{p}^{C R, 1+e}$ of edge $M$ at $p$ to which $\mathcal{C}_{C R}^{0}\left(M \cup \mathcal{W}_{p}^{C R, e}\right)$ extends to be $C R$.

Thus, this proposition concludes the proof of Theorem 3.8.

Proof. There exists a local section $L$ of $T^{1,0} \mathcal{M}_{p}^{e}$ with $L(p) \neq 0$ such that $[L, \bar{L}](p) \notin T_{p}^{1,0} \mathcal{M}_{p}^{e} \oplus T_{p}^{0,1} \mathcal{M}_{p}^{e}$. It is appropriate to distinguish two cases.

Firstly, assume that $L(p) \in T^{1,0} M$. Then as in $\S 4.2$, we may construct a small analytic disc $A_{\varepsilon}$ attached to $M$ in a neighborhood of $p$ having exit vector $-\frac{\partial A_{\varepsilon}}{\partial r}(1)$ approximately directed by $[L, \bar{L}](p) \notin \mathbb{C} \otimes T_{p} \mathcal{M}_{p}^{e}$. So this disc has exit vector nontangential to $\mathcal{M}_{p}^{e}$ at $p$. By translating it along $M$ and along the $e$ supplementary directions offered by $\mathcal{W}_{p}^{C R, e}$, we deduce CR extension to a $(2 m+$ $d+1+e$ )-dimensional CR wedge $\widetilde{W}_{p}^{C R, 1+e}$.

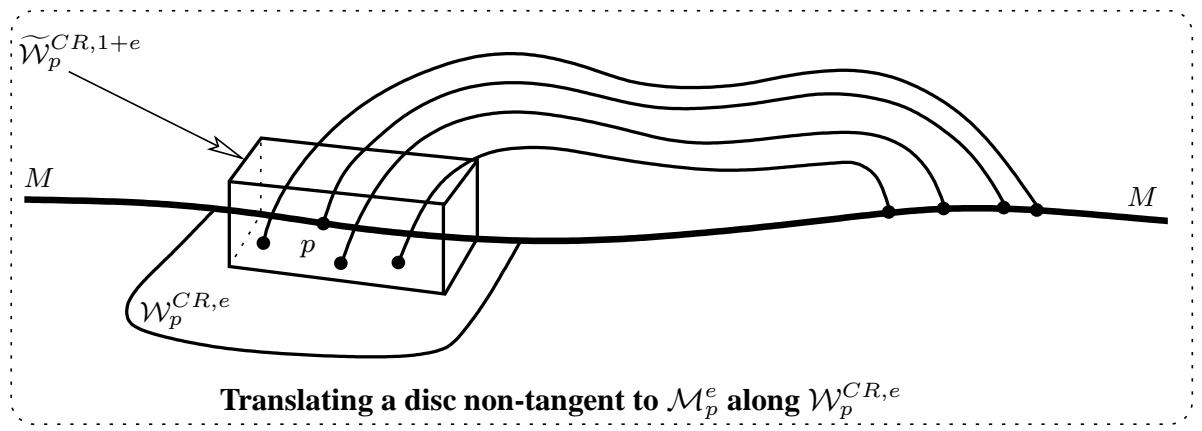

Secondly, assume that $L(p) \notin T_{p}^{1,0} M$ for every local section $L$ of $T^{1,0} M$ such that $[L, \bar{L}](p) \notin T_{p}^{1,0} \mathcal{M}_{p}^{e} \oplus T_{p}^{0,1} \mathcal{M}_{p}^{e}$.

We explain the case $d=2, e=1$ first, since this case is easier to understand. Under this assumption, $\mathcal{M}_{p}^{1}$ is a hypersurface of $\mathbb{C}^{n}$ divided in two parts by $M$, one part being $\mathcal{W}_{p}^{C R, 1}$. We draw a diagram. 


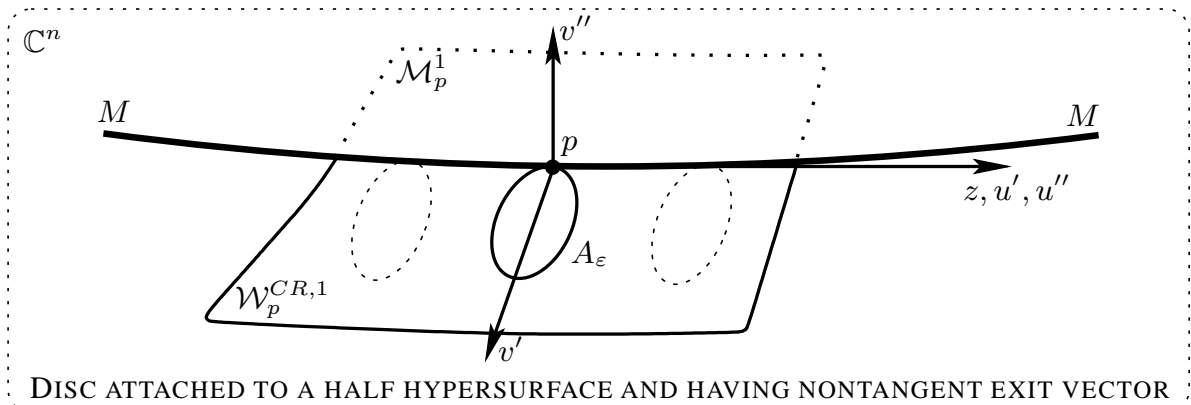

There exist coordinates $\left(z, w^{\prime}, w^{\prime \prime}\right) \in \mathbb{C}^{n-2} \times \mathbb{C} \times \mathbb{C}$ centered at $p$ in which $\mathcal{M}_{p}^{1}$ is given by $v^{\prime \prime}=\psi\left(z, w^{\prime}, u^{\prime \prime}\right)$, with $\psi(0)=0$ and $d \psi(0)=0$ and in which $M$ is given by a supplementary equation $v^{\prime}=\varphi^{\prime}\left(z, u^{\prime}, u^{\prime \prime}\right)$ with $\varphi^{\prime}(0)=0$ and $d \varphi^{\prime}(0)=0$. Changing the orientation of the $v^{\prime}$-axis if necessary, it follows $\mathcal{W}_{p}^{C R, 1}$ is given by the equation $v^{\prime \prime}=\psi\left(z, w^{\prime}, u^{\prime \prime}\right)$ and the inequation $v^{\prime}>\varphi^{\prime}\left(z, u^{\prime}, u^{\prime \prime}\right)$, with $\varphi^{\prime}(0)=0$ and $d \varphi^{\prime}(0)=0$. In the diagram, $T_{p}^{c} \mathcal{M}_{p}^{1}$ is the direct sum of the $z$-coordinate space with the $u^{\prime}+i v^{\prime}$-coordinate axis.

The Levi form of $\mathcal{M}_{p}^{1}$ is represented by a scalar Hermitian form $H\left(z, w^{\prime}, \bar{z}, \bar{w}^{\prime}\right)$. By assumption, its restriction to $T_{p}^{c} M$ vanishes (otherwise, the first case holds), so $H(z, 0, \bar{z}, 0) \equiv 0$. The assumption that the Levi form of $\mathcal{M}_{p}^{1}$ does not vanish identically insures that $H$ is nonzero. To proceed further, we need $H\left(0, w^{\prime}, 0, \bar{w}^{\prime}\right) \not \equiv 0$. If $H\left(0, w^{\prime}, 0, \bar{w}^{\prime}\right) \equiv 0$, since $H$ is nonzero, by a linear coordinate change of the form $\widetilde{w}^{\prime}=w^{\prime}, \widetilde{z}_{k}=z_{k}+a_{k} w^{\prime}$, $k=1, \ldots, n-2, \widetilde{w}^{\prime \prime}=w^{\prime \prime}$, we may insure that $H\left(0, w^{\prime}, 0, \bar{w}^{\prime}\right) \not \equiv 0$. Observe that such a change of coordinates stabilizes both $T_{p} M$ and $T_{p} \mathcal{M}_{p}^{1}$. After a real dilation, we can assume that the equation of $\mathcal{M}_{p}^{1}$ is of the form:

$$
v^{\prime \prime}=w^{\prime} \bar{w}^{\prime}+\mathrm{O}\left(\left|w^{\prime}\right|^{2+\alpha-0}\right)+\mathrm{O}\left(|z|\left|\left(z, w^{\prime}\right)\right|\right)+\mathrm{O}\left(\left|u^{\prime \prime}\right|\left|\left(z, w^{\prime}\right)\right|\right)+\mathrm{O}\left(\left|u^{\prime \prime}\right|^{2}\right) \text {. }
$$

To the hypersurface $\mathcal{M}_{p}^{1}$, we attach a disc $A_{\varepsilon}(\zeta)$ with zero $z$-component, with $w^{\prime}$ component equal to $i \varepsilon(1-\zeta)$ and with $w^{\prime \prime}$-component $\left(U_{\varepsilon}^{\prime \prime}(\zeta)+i V_{\varepsilon}^{\prime \prime}(\zeta)\right)$ of class $\mathcal{C}^{2, \alpha-0}$ satisfying the corresponding Bishop-type equation. Exactly as in the Lewy extension theorem ( $\$ 2.10)$, for $\varepsilon>0$ small enough and fixed, the exit vector of $A_{\varepsilon}$ at $p$ is nontangent to $\mathcal{M}_{p}^{1}$ (this is uneasy to draw in the diagram above, but imagine that the disc drawn in $\S 2.10$ is attached to a half-paraboloid). Furthermore, using the inequality $v^{\prime}\left(e^{i \theta}\right)=\varepsilon(1-\cos \theta) \geqslant \varepsilon \frac{\theta^{2}}{\pi}$ for $|\theta| \leqslant \pi$ together with the property $d \varphi^{\prime}(0)=0$, it is elementary to verify that $A_{\varepsilon}(\partial \Delta \backslash\{1\})$ is contained in the open half-hypersurface $\left\{v^{\prime}>\varphi^{\prime}\right\}$, as shown in the diagram.

Since the exit vector of $A_{\varepsilon}$ is nontangent to $\mathcal{M}_{p}^{1}$, in order to get holomorphic extension to a wedge at $p$, it suffices to translate the disc $A_{\varepsilon}$ in the half-hypersurface $\mathcal{W}_{p}^{C R, 1}$. 
However, if we translate $A_{\varepsilon}$ as usual by requiring that the base point $A_{\varepsilon, s}(1)=$ $p_{s}$, with $s \in \mathbb{R}^{2 n-2}$ small, covers a neighborhood of $p$ in $M$, it may well happen that, due to the curvature of $M$ in a neighborhood of $p$, the boundary of the translated disc enters slightly the other side of $\mathcal{M}_{p}^{1}$, which is forbidden.

To remedy this imperfection, two equally good options present themselves. The first option would be to rotate slightly the translated disc $A_{\varepsilon, s}$ in order that it becomes tangent to $M$ at the point $p_{s}=A_{\varepsilon, s}(1)$. Then adding a small parameter $\sigma>0$, we would translate it slightly in the positive direction of $\mathcal{W}_{p}^{C R, 1}$, essentially along the positive $v^{\prime}$-direction.

The second option is to introduce a family of complex affine biholomorphisms $\Psi_{s}$ that transfer $p_{s} \in M$ to the origin and transfer the tangent spaces at $p_{s}$ of $\mathcal{M}_{p}^{1}$ and of $M$ to $\left\{v^{\prime \prime}=0\right\}$ and to $\left\{v^{\prime \prime}=v^{\prime}=0\right\}$. So $\Psi_{s}\left(\mathcal{M}_{p}^{1}\right)$ is given by $v^{\prime \prime}=\psi^{\prime \prime}\left(z, w^{\prime}, u^{\prime \prime}: s\right)$ with $\psi$ of class $\mathcal{C}^{2, \alpha-0}$ with respect to all variables and with the map $\left(z, w^{\prime}, u^{\prime \prime}\right) \mapsto \psi^{\prime \prime}\left(z, w^{\prime}, u^{\prime \prime}: s\right)$ vanishing to second order at the origin for every $s \in \mathbb{R}^{2 n-2}$ small. Also, $\Psi_{s}\left(\mathcal{W}_{p}^{C R, 1}\right)$ is given by a supplementary inequation $v^{\prime}>\varphi^{\prime}\left(z, u^{\prime}, u^{\prime \prime}: s\right)$, with $\varphi^{\prime}$ of class $\mathcal{C}^{2, \alpha}$ (the smoothness of $M$ ) with respect to all variables and with $\left(z, u^{\prime}, u^{\prime \prime}\right) \mapsto \varphi\left(z, u^{\prime}, u^{\prime \prime}: s\right)$ vanishing to second order at the origin.

To the hypersurface $\Psi_{s}\left(\mathcal{M}_{p}^{1}\right)$, we attach the family of discs

$$
\widetilde{A}_{\varepsilon, s, \sigma}(\zeta)=\left(0, i \sigma+i \varepsilon(1-\zeta), \widetilde{U}_{\varepsilon, s, \sigma}^{\prime \prime}(\zeta)+i \widetilde{V}_{\varepsilon, s, \sigma}^{\prime \prime}(\zeta)\right)
$$

having zero $z$-component and $w^{\prime}$-component equal to $i \sigma+i \varepsilon(1-\zeta)$, where $\sigma \in \mathbb{R}$ with $|\sigma|<\sigma_{1}, \sigma_{1}>0$, is a small parameter of translation along the $v^{\prime}$-axis. Of course:

$$
\left\{\begin{array}{l}
\widetilde{U}_{\varepsilon, s, \sigma}^{\prime \prime}\left(e^{i \theta}\right)=-\mathrm{T}_{1}\left[\psi\left(0, i \sigma+i \varepsilon(1-\cdot), \widetilde{U}_{\varepsilon, s, \sigma}^{\prime \prime}(\cdot): s\right)\right]\left(e^{i \theta}\right), \\
\widetilde{V}_{\varepsilon, s, \sigma}^{\prime \prime}\left(e^{i \theta}\right)=\mathrm{T}_{1}\left[\widetilde{U}_{\varepsilon, s, \sigma}^{\prime \prime}\right]\left(e^{i \theta}\right) .
\end{array}\right.
$$

By means of elementary computations involving Taylor's formula, we verify two facts.

- If $\varepsilon>0$ is sufficiently small and fixed, $\widetilde{A}_{\varepsilon, s, 0}(\partial \Delta \backslash\{1\})$ is contained in the open half-hypersurface $\left\{v^{\prime}>\varphi^{\prime}\left(z, u^{\prime}, u^{\prime \prime}: s\right)\right\}$, for all $s \in \mathbb{R}^{2 n-2}$ with $|s|<s_{1}, s_{1}>0$ small.

- Furthermore, for all $\sigma$ with $0<\sigma \leqslant \sigma_{1}$, and all $s$ with $|s|<s_{1}$, the disc boundary $\widetilde{A}_{\varepsilon, s, \sigma}(\partial \Delta)$ is contained in the open half-hypersurface $\left\{v^{\prime}>\right.$ $\left.\varphi^{\prime}\left(z, u^{\prime}, u^{\prime \prime}: s\right)\right\}$.

Coming back to the old system of coordinates, it follows that the family of discs $A_{\varepsilon, s, \sigma}:=\Psi_{s}^{-1} \circ \widetilde{A}_{\varepsilon, s, \sigma}$ has base point $A_{\varepsilon, s, \sigma}(1)$ covering a neighborhood of $p$ in the half-hypersurface $\mathcal{W}_{p}^{C R, 1}$, as $s$ and $\sigma$ vary. Since the exit vector of $A_{\varepsilon}$ is not tangent to $\mathcal{M}_{p}^{1}$ at $p$, this family of discs covers a $2 n$-dimensional wedge $\widetilde{W}_{p}^{C R, 2 n}$ 
of edge $M$ at $p$. This completes the proof of the second case of the proposition when $e=1$ and $d=2$.

Based on these explanations, we may now summarize the general case. There exist coordinates $\left(z, w^{\prime}, w^{\prime \prime}\right) \in \mathbb{C}^{m} \times \mathbb{C}^{e} \times \mathbb{C}^{d-e}$ vanishing at $p$ in which the $\mathcal{C}^{2, \alpha-0}$ generic submanifold $\mathcal{M}_{p}^{e}$ is represented by $v^{\prime \prime}=\psi\left(z, w^{\prime}, u^{\prime \prime}\right)$, with $\psi(0)=0$ and $d \psi(0)=0$. After killing the second order pluriharmonic quadratic terms in every right hand side $\psi_{j^{\prime \prime}}\left(z, w^{\prime}, 0\right), j^{\prime \prime}=1, \ldots, d-e$, we may assume that the quadratic terms are Hermitian forms $H_{j^{\prime \prime}}\left(z, w^{\prime}, \bar{z}, \bar{w}^{\prime}\right)$.

After a linear change of coordinates in the $w^{\prime}$-space, $T_{p} M=\left\{v^{\prime}=v^{\prime \prime}=0\right\}$, the $\mathcal{C}^{2, \alpha}$ generic edge $M$ is defined by $v=\varphi(z, u)$ with $\varphi(0)=0, d \varphi(0)=0$ and the conic open submanifold $\mathcal{W}_{p}^{C R, e}$ of $\mathcal{M}_{p}^{e}$ is defined by $v^{\prime \prime}=\psi\left(z, w^{\prime}, u^{\prime \prime}\right)$ together with the inequations

$$
v_{j^{\prime}}^{\prime}>\varphi_{j^{\prime}}^{\prime}\left(z, u^{\prime}, u^{\prime \prime}\right), \quad j^{\prime}=1, \ldots, e,
$$

where $\varphi=\left(\varphi^{\prime}, \varphi^{\prime \prime}\right)$. In fact, we may assume that the cone defining the CR-wedge on the tangent space is slightly larger than the salient cone $v_{j^{\prime}}^{\prime}>0, j^{\prime}=1, \ldots, e$.

The nonvanishing of the Levi form of $\mathcal{M}_{p}^{e}$ at $p$ entails that at least one Hermitian form $H_{j^{\prime \prime}}\left(z, w^{\prime}, \bar{z}, \bar{w}^{\prime}\right)$ is nonzero. After renumbering, $H_{1}$ is nonzero. Also, since $T_{p}^{c} M$ is the $z$-coordinate space, we have $H_{1}(z, 0, \bar{z}, 0) \equiv 0$ (otherwise, the first case holds). After a complex linear coordinate change of the form $\widetilde{w}^{\prime}=w^{\prime}, \widetilde{z}_{k}=z_{k}+\sum_{j^{\prime}=1}^{e} a_{k}^{j^{\prime}} w_{j^{\prime}}^{\prime}, \widetilde{w}^{\prime \prime}=w^{\prime \prime}$, we may insure that $H_{1}\left(0, w^{\prime}, 0, \bar{w}^{\prime}\right) \not \equiv 0$. Then the set of vectors $\left(0, w^{\prime}\right)$ on which $H_{1}$ vanishes is a proper real quadratic cone of $\mathbb{C}^{e}$. Consequently, for almost every real vector $\left(0, i v^{\prime}\right)$, the quadratic form $H_{1}$ is nonzero on the complex line $\mathbb{C}\left(0, i v^{\prime}\right)$. Since the cone defining $\mathcal{W}_{p}^{C R, e}$ is open and may be slightly shrunk, we can assume that $H_{1}$ does not vanish on $\mathbb{C}\left(0, i v_{1}^{\prime}\right)$, with $v_{1}^{\prime}=(1, \ldots, 1) \in \mathbb{R}^{e}$. It follows that the disc $A_{\varepsilon}$ attached to $\mathcal{M}_{p}^{e}$ having zero $z$-component and $w^{\prime}$-component equal to $(i \varepsilon(1-\zeta), \ldots, i \varepsilon(1-\zeta))$ is nontangent to $\mathcal{M}_{p}^{e}$ at $p$.

Furthermore, letting a point $p_{s} \in M$ of coordinates $s:=(z, u)$ vary in a small neighborhood of $p$ in $M$, we may construct a family of biholomorphisms $\Psi_{s}$ sending $p_{s}$ to the origin and normalizing the equations of $M$, of $\mathcal{M}_{p}^{e}$ and of $\mathcal{W}_{p}^{C R, e}$ under the form $v=\varphi(z, u: s), v^{\prime \prime}=\psi\left(z, w^{\prime}, u^{\prime \prime}: s\right)$ and $v_{j^{\prime}}^{\prime}>\varphi_{j^{\prime}}^{\prime}\left(z, u^{\prime}, u^{\prime \prime}: s\right)$, with $\varphi$ being $\mathcal{C}^{2, \alpha}$ and with $\psi$ being $\mathcal{C}^{2, \alpha-0}$ with respect to all variables and both vanishing to second order at the origin.

Let $\sigma \in \mathbb{R}^{e},|\sigma|<\sigma_{1}$, be a small parameter of translation along the $v^{\prime}$ coordinate space. To the generic submanifold $\Psi_{s}\left(\mathcal{M}_{p}^{e}\right)$, we attach the family of discs

$$
\widetilde{A}_{\varepsilon, s, \sigma}(\zeta)=\left(0, W_{\varepsilon, \sigma}^{\prime}(\zeta), U_{\varepsilon, s, \sigma}^{\prime \prime}(\zeta)+i V_{\varepsilon, s, \sigma}^{\prime \prime}(\zeta)\right)
$$

where

$$
W_{\varepsilon, \sigma}^{\prime}(\zeta)=\left(i \sigma_{1}+i \varepsilon(1-\zeta), \ldots, i \sigma_{e}+i \varepsilon(1-\zeta)\right),
$$


and where

$$
\left\{\begin{array}{l}
\widetilde{U}_{\varepsilon, s, \sigma}^{\prime \prime}\left(e^{i \theta}\right)=-\mathrm{T}_{1}\left[\psi\left(0, i \sigma+i \varepsilon(1-\cdot), \widetilde{U}_{\varepsilon, s, \sigma}^{\prime \prime}(\cdot): s\right)\right]\left(e^{i \theta}\right), \\
\widetilde{V}_{\varepsilon, s, \sigma}^{\prime \prime}\left(e^{i \theta}\right)=\mathrm{T}_{1}\left[\widetilde{U}_{\varepsilon, s, \sigma}^{\prime \prime}\right]\left(e^{i \theta}\right) .
\end{array}\right.
$$

By means of elementary computations involving Taylor's formula, we may verify that for all $\sigma \in \mathbb{R}^{e}$ with $0<\sigma_{j^{\prime}} \leqslant \sigma_{1}, j^{\prime}=1, \ldots, e$, and all $s \in \mathbb{R}^{2 m+d}$, $|s|<s_{1}$, the disc boundary $\widetilde{A}_{\varepsilon, s, \sigma}(\partial \Delta)$ is contained in $\left\{v_{j^{\prime}}^{\prime}>\varphi_{j^{\prime}}^{\prime}\left(z, u^{\prime}, u^{\prime \prime}\right.\right.$ : $\left.s), j^{\prime}=1, \ldots, e\right\}$.

Coming back to the old system of coordinates, it follows that the family of $\operatorname{discs} A_{\varepsilon, s, \sigma}:=\Psi_{s}^{-1} \circ \widetilde{A}_{\varepsilon, s, \sigma}$ has base point $A_{\varepsilon, s, \sigma}(1)$ covering a neighborhood of $p$ in the CR-wedge $\mathcal{W}_{p}^{C R, e}$, as $s$ and $\sigma$ vary. Since its exit vector is not tangent to $\mathcal{M}_{p}^{1}$ at $p$, this family of discs covers a $(2 m+d+1+e)$-dimensional CR-wedge $\widetilde{W}_{p}^{C R, 1+e}$ of edge $M$ at $p$.

The proofs of the proposition and of Theorem 3.8 are complete.

4.11. Wedgelike domains. On a globally minimal $M$, at every point $p \in M$, we have constructed a local wedge $\mathcal{W}_{p}$ by gluing deformations of discs. It may well happen that at a point $p$ that belongs to the edges of two different wedges $\mathcal{W}_{q^{\prime}}$ and $\mathcal{W}_{q^{\prime \prime}}$, the wedges have empty intersection in $\mathbb{C}^{n}$ (imagine two thin opposite cones having vertex at $0 \in \mathbb{R}^{2}$ ). Fortunately, by means of the translation trick presented in $\S 4.5$ ( $c f$. the diagram), we can fill in the space in between. Achieving this systematically, by a sort of gluing-shrinking processus, we obtain some connected open set $\mathcal{W}$ attached to $M$ containing possibly smaller wedges $\mathcal{W}_{p}^{\prime} \subset \mathcal{W}_{p}$ at every point.

To set-up a useful definition, by a wedgelike domain $\mathcal{W}$ attached to $M$ we mean a connected open set that contains a local wedge of edge $M$ at every point. Geometrically speaking, the requirement of connectedness prevents jumps of the directions of local wedges in the normal bundle $\left.T \mathbb{C}^{n}\right|_{M} / T M$.

We may finally conclude this section by the formulation of a statement that is the very starting point of the study of removable singularities for CR functions ([MP1998, MP1999, MP2000, MP2002, MP2006]).

Theorem 4.12. (Me1997, MP1999]) If $M$ is a globally minimal $\mathcal{C}^{2, \alpha}$ generic submanifold of $\mathbb{C}^{n}$, there exists a wedgelike domain $\mathcal{W}$ attached to $M$ such that every continuous $C R$ function $f \in \mathcal{C}_{C R}^{0}(M)$ possesses a holomorphic extension $F \in \mathcal{O}(\mathcal{W}) \cap \mathcal{C}^{0}(M \cup \mathcal{W})$ with $\left.F\right|_{M}=f$.

Its $L^{\mathrm{p}}$ version deserves special attention. Let $\mathcal{W}$ be a wedgelike domain attached to $M$. A holomorphic function $F \in \mathcal{O}(\mathcal{W})$ is said to belong to the Hardy space $H_{l o c}^{\mathrm{p}}(\mathcal{W})$ if, for every $p \in M$, for every local coordinate system centered at $p$ in which $M$ is given by $v=\varphi(x, y, u)$, for every local wedge of edge $M$ at $p$ 
contained in $\mathcal{W}$ of the form

$$
\begin{gathered}
\mathcal{W}=\mathcal{W}(\rho, \sigma, C):=\left\{(x+i y, u+i v) \in \Delta_{\rho}^{m} \times \square_{\rho}^{d} \times i \square_{\sigma}^{d}:\right. \\
v-\varphi(x, y, u) \in C\},
\end{gathered}
$$

as defined in $\S 4.29$ (III), for every cone $C^{\prime} \subset \mathbb{R}^{d}$ with $C^{\prime} \cap S^{d-1} \subset \subset C \cap S^{d-1}$ and for every $\rho^{\prime}<\rho$, the supremum:

$$
\sup _{\theta^{\prime} \in C^{\prime}} \int_{\Delta_{\rho^{\prime}}^{m} \times \square_{\rho^{\prime}}^{d}}\left|F\left(x+i y, u+i \varphi(x, y, u)+i \theta^{\prime}\right)\right|^{\mathrm{p}} d x \wedge d y \wedge d u<\infty
$$

is finite. An adaptation of the proof of the preceding theorem yields its $L^{\mathrm{p}}$ version.

Theorem 4.13. ([Po1997, Po2000]) If $M$ is a globally minimal $\mathcal{C}^{2, \alpha}$ generic submanifold of $\mathbb{C}^{n}$, there exists a wedgelike domain $\mathcal{W}$ attached to $M$ such that every function $f \in L_{l o c, C R}^{\mathrm{p}}, 1 \leqslant \mathrm{p} \leqslant \infty$, possesses a Hardy space holomorphic extension $F \in \mathrm{H}_{l o c}^{\mathrm{p}}(\mathcal{W})$.

To conclude, we would like to mention that arguments similar to those of Theorem 4.12 yield a mild generalization, worth to be mentioned: the $\mathrm{CR}$ extension theory is valid for $\mathcal{C}^{2, \alpha} \mathrm{CR}$ manifolds that are only locally embeddable.

However, for concreteness reasons, we preferred to set up the theory in a globally embedded context. In the remainder of the memoir, not to enter superficial corollaries, we will formulate all our results under the paradigmatic assumption of global minimality. Thus, Theorems 4.12 and 4.13 will be our basic main starting point.

The two monographs [Trv1992, BCH2005] deal not only with embedded structures but also with locally integrable structures. Nevertheless, most topics exposed here are not yet embraced in a comprehensive theory ( $c f . \S 3.29$ (III)). So it is an open direction of research to transfer the theory of holomorphic extension of CR functions (including removable singularities) to locally integrable structures. 


\title{
VI: Removable singularities
}

\author{
Table of contents
}

1. Removable singularities for linear partial differential operators ........206.

2. Removable singularities for holomorphic functions of one or several complex

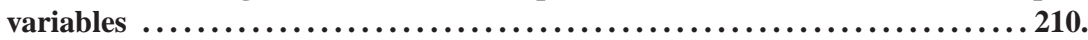

3. Hulls and removable singularities at the boundary $\ldots \ldots \ldots \ldots \ldots \ldots \ldots \ldots 227$.

4. Smooth and metrically thin removable singularities for CR functions ....221.

5. Removable singularities in CR dimension $1 \ldots \ldots \ldots \ldots \ldots \ldots \ldots \ldots \ldots . \ldots 252$.

[7 diagrams]

Removable singularities for general linear partial differential operators $P=$ $\sum_{\beta \in \mathbb{N}^{m}} a_{\beta}(x) \partial_{x}^{\beta}$ on domains $\Omega \subset \mathbb{R}^{n}$ having order $m \geqslant 1$ and $\mathcal{C}^{\infty}$ coefficients have been studied by Harvey and Polking (1970) in a general setting. Assumptions of metrical thinness of singularities, in the sense of Minkowski content or of Hausdorff measure, insure automatic removability. For instance, relatively closed sets $C \subset \Omega$ whose $(n-m)$-dimensional Hausdorff measure is null are $\left(P, L_{l o c}^{\infty}\right)$-removable. For structural reasons, these general results (valid whatever the structure of the operator) necessitate a control of growth when dealing with $L_{l o c}^{1}$-removability. In addition, when $P$ is an embedded complex-tangential operator, this approach does not convey to the adequate results, because removable singularities for holomorphic or CR functions must take advantage of automatic extension to larger sets.

Since almost two decades, thanks to the impulse of Stout, removable singularities have attracted much attention in several complex variables. A natural question is whether the Hartogs-Bochner extension Theorem 1.9(V) holds when considering CR functions that are defined only in the complement $\partial \Omega \backslash K$ of some compact set $K \subset \partial \Omega$ of a connected smooth boundary $\partial \Omega \subset \mathbb{C}^{n}$. In complex dimension $n=2$, Stout showed that the answer is positive if and only if $K$ is convex with respect to the space of functions that are holomorphic in a neighborhood of $\bar{\Omega}$. In complex dimension $n \geqslant 3$, a complete cohomological characterization of different nature was obtained by Lupacciolu (1994).

In another direction, by means of the above-cited global continuity principle, Jöricke (1995) generalized Stout's theorem to weakly pseudoconvex domains. Recently, Jöricke and the second author were able to remove the pseudoconvexity assumption by applying purely geometrical constructions without integral formulas, controlling uniqueness of the extension (monodromy) by fine arguments.

Within the general framework of CR extension theory (exposed in Part V), the study of removable singularities has been endeavoured by Jöricke in the hypersurface case since 1988, and after by the two authors in arbitrary codimension since 1995. The notions of CR-, $\mathcal{W}$ - and $L^{\mathrm{P}}$-removability, although different, may be 
shown to be essentially equivalent, thanks to technical deformation arguments. All the surveyed results hold in $L_{l o c}^{\mathrm{p}}$ with $1 \leqslant \mathrm{p} \leqslant \infty$, including $\mathrm{p}=1$ and without any growth assumption near the singularity. On a generic globally minimal $\mathcal{C}^{2, \alpha}$ generic submanifold $M$ of $\mathbb{C}^{n}$, closed sets $C \subset M$ having vanishing $(\operatorname{dim} M-2)$-dimensional Hausdorff measure are $\mathrm{CR}-, \mathcal{W}$ - and $L^{\mathrm{P}}$-removable. As an application, CR meromorphic functions defined on an everywhere locally minimal $M$ do extend meromorphically to a wedgelike domain attached to $M$.

In conjunction with the Harvey-Lawson complex Plateau theorem, singularities $C$ that are a priori contained in a 2-codimensional $\mathcal{C}^{2, \alpha}$ submanifold $N$ of a strongly pseudoconvex $\mathcal{C}^{2, \alpha}$ boundary $\partial \Omega \subset \mathbb{C}^{n}(n \geqslant 3)$ are shown by Jöricke to be not removable if and only if $N$ is a maximally complex cycle. The condition that $N$ be somewhere generic was shown by the two authors to be sufficient for its removability in arbitrary codimension.

Concerning more massive singularities, a compact subset $K$ of a onecodimensional submanifold $M^{1} \subset \partial \Omega \subset \mathbb{C}^{n}$ is CR-, $\mathcal{W}$ - and $L^{\mathrm{P}}$-removable provided the CR dimension of $\partial \Omega$ is $\geqslant 2$ (viz. $n \geqslant 3$ ) and provided $K$ does not contain any CR orbit of $M^{1}$ (Jöricke, 1999). The second author generalized this theorem to higher codimension, assuming that $M$ is globally minimal of $\mathrm{CR}$ dimension $m \geqslant 2$. The main geometric argument (called sweeping out by wedges) being available only in CR dimension $m \geqslant 2$, the more delicate case of CR dimension $m=1$ is studied extensively in the research article [MP2006, placed in direct continuation to this survey.

\section{§1. REMOVABLE SINGULARITIES FOR LINEAR PARTIAL DIFFERENTIAL OPERATORS}

1.1. Hausdorff measure. Let $M$ be a $\mathcal{C}^{1}$ abstract manifold of dimension $n \geqslant 1$ equipped with some Riemannian metric. For $\ell \in \mathbb{R}$ with $0 \leqslant \ell \leqslant n$, we remind ([Ch1989] $]$ the definition of the notion of $\ell$-dimensional Hausdorff measure $\mathrm{H}^{\ell}$ on $M$, that generalizes the notion of integer dimension of submanifolds.

If $C \subset M$ is an arbitrary subset and if $\delta>0$ is small, we define

$$
\mathrm{H}_{\delta}^{\ell}(C)=\inf \left\{\sum_{j=1}^{\infty} r_{j}^{\ell}: C \text { is covered by geodesic balls } B_{j} \text { of radius } r_{j} \leqslant \delta\right\} \text {. }
$$

Clearly, $\mathrm{H}_{\delta}^{\ell}(C) \leqslant \mathrm{H}_{\delta^{\prime}}^{\ell}(C)$, for $\delta^{\prime} \leqslant \delta$, so the limit $\mathrm{H}^{\ell}(C)=\lim _{\delta \rightarrow 0^{+}} \mathrm{H}_{\delta}^{\ell}(C)$ exists in $[0, \infty]$. This limit is called the $\ell$-dimensional Hausdorff measure of $C$. The value of $\mathrm{H}^{\ell}(C)$ depends on the choice of a metric, but the two properties $\mathrm{H}^{\ell}(C)=0$ and $\mathrm{H}^{\ell}(C)=\infty$ are independent. The most significant property is that there exists a critical exponent $\ell_{C} \geqslant 0$, called the Hausdorff dimension of $C$, such that $\mathrm{H}^{\ell}(C)=\infty$ for all $\ell<\ell_{C}$ and such that $\mathrm{H}^{\ell}(C)=0$ for all $\ell>\ell_{C}$. Then the value $\mathrm{H}^{\ell_{C}}(C)$ may be arbitrary in $[0, \infty]$. 
Proposition 1.2. ([Fe1969, Ch1989] $]$ ) The following properties hold true:

(1) $\mathrm{H}^{0}(C)=\operatorname{Card}(C)$;

(2) $\mathrm{H}^{n}(C)$ coincides with the outer Lebesgue measure of $C \subset M$;

(3) a $\mathcal{C}^{1}$ submanifold $N \subset M$ has Hausdorff dimension $\ell_{N}=\operatorname{dim} N$;

(4) if $\mathrm{H}^{n-1}(C)=0$, then $M \backslash C$ is locally connected;

(5) if $f: M \rightarrow N$ is a $\mathcal{C}^{1}$ map and if $C \subset M$ satisfies $\mathrm{H}^{\ell}(C)=0$ for some $\ell \geqslant \operatorname{dim} N$, then for almost every $q \in N$, it holds that $\mathrm{H}^{\ell-\operatorname{dim} N}(C \cap$ $\left.f^{-1}(q)\right)=0$.

(6) $\mathrm{H}^{\ell}(C)=0$ if and only if $\mathrm{H}^{\ell}(K)=0$ for each compact set $K \subset C$.

1.3. Metrically thin singularities of linear partial differential operators. Let $\Omega$ be a domain in $\mathbb{R}^{n}$, where $n \geqslant 1$. We shall denote the Lebesgue measure by $\mathrm{H}^{n}$. Consider a class of $\mathcal{F}(\Omega)$ of distributions defined on $\Omega$, for instance $L_{\mathrm{loc}}^{\mathrm{p}}(\Omega)$, $\mathcal{C}^{\kappa, \alpha}(\Omega)(\kappa \in \mathbb{N}, 0 \leqslant \alpha \leqslant 1)$ or $\mathcal{C}^{\infty}(\Omega)$. Consider a linear partial differential operator

$$
P=P\left(x, \partial_{x}\right)=\sum_{\beta \in \mathbb{N}^{n},|\beta| \leqslant m} a_{\beta}(x) \partial_{x}^{\beta}
$$

of order $m \geqslant 1$, defined in $\Omega$ and having $\mathcal{C}^{\infty}$ coefficients $a_{\beta}(x)$.

Definition 1.4. A relatively closed subset $C$ of $\Omega$ is called $(P, \mathcal{F})$-removable if every $f \in \mathcal{F}(\Omega)$ satisfying $P f=0$ in $\Omega \backslash C$ does satisfy $P f=0$ in all of $\Omega$, in the sense of distributions.

For instance, according to the classical Riemann removability theorem, discrete subsets $\left\{p_{k}\right\}_{k \in \mathbb{N}}$ of a domain $\Omega$ in $\mathbb{C}$ are $\left(\bar{\partial}, L^{\infty}\right)$-removable. In fact, since every distribution solution of $\bar{\partial}$ is holomorphic (hypoellipticity), functions extend to be true holomorphic functions in a neighborhood of each $p_{k}$. The Riemann removability theorem also holds under the weaker assumption that $f \in \mathcal{O}\left(\Omega \backslash\left\{p_{k}\right\}_{k \in \mathbb{N}}\right)$ satisfies $f\left(z-p_{k}\right)=\mathrm{o}\left(\left|z-p_{k}\right|^{-1}\right)$ as $z$ approaches $p_{k}$.

In several complex variables, the classical Riemann removability theorem may be stated as follows.

Theorem 1.5. ([Ch1989]) Let $\Sigma$ be a complex analytic subset of $\Omega$. Holomorphic functions in $\Omega \backslash \Sigma$ extend uniquely through $\Sigma$ either if $\operatorname{dim}_{\mathbb{C}} \Sigma \leqslant n-2$ or if $\operatorname{dim}_{\mathbb{C}} \Sigma=n-1$ and they belong to $L_{\text {loc }}^{\infty}(\Omega)$.

The second case also holds true for functions that belong to $L_{l o c}^{2}(\Omega)$. The proofs are elementary and short: in one or several complex variables, everything comes down to observing that $\frac{1}{z}$ is a true $\mathrm{O}\left(\frac{1}{|z|}\right)$ near $z=0$ and does not belong to $L_{l o c}^{2}$.

These preliminary statements are superseded by more general removability theorems, exposed in [HP1970], that we shall now restitute. Some of the (elementary) proofs will be surveyed to give the flavour of the arguments. 
In 1956, S. Bochner ([Bo1956, HP1970]) established remarkable removability theorems, valid for general linear differential operators $P$, in which the metrical conditions on the size of the singularity $C$ depend only on the order $m$ of $P$. Some preliminary material is needed.

Lemma 1.6. Let $K \subset \mathbb{R}^{n}$ be a compact set. For every $\varepsilon>0$, there exists a function $\varphi_{\varepsilon} \in \mathcal{C}_{c}^{\infty}\left(\mathbb{R}^{n}\right)$ with $\varphi_{\varepsilon} \equiv 1$ in a neighborhood of $K$ and with $\operatorname{supp} \varphi_{\varepsilon} \subset$ $K_{\varepsilon}$ such that $\left|\partial_{x}^{\beta} \varphi_{\varepsilon}(x)\right| \leqslant C_{\beta} \varepsilon^{-|\beta|}$ for all $x \in \mathbb{R}^{n}$ and all $\beta \in \mathbb{N}^{n}$.

Proof. Denote by $\mathbf{1}_{B}(\cdot)$ the characteristic function of a set $B \subset \mathbb{R}^{n}$. It suffices to define the (rescaled) convolution integral $\varphi_{\varepsilon}(x):=\varepsilon^{-n} \int_{\mathbb{R}^{n}} \mathbf{1}_{K_{\varepsilon / 2}}(y) \psi((x-$ $y) / \varepsilon) d y$, where $\psi \in \mathcal{C}_{c}^{\infty}\left(\mathbb{R}^{n}\right)$ has support contained in $\{|x| \leqslant 1 / 3\}$ and satisfies $\int \psi(y) d y=1$.

It may happen that $C$ is not $(P, \mathcal{F})$-removable, whereas $C$ is removable for some individual function $f \in \mathcal{F}(\Omega)$ satisfying certain supplementary conditions. In this case, we shall say that $C$ is an illusory singularity of $f$.

Theorem 1.7. ([Bo1956, HP1970]) Let $f \in L_{l o c}^{1}(\Omega)$. If, for each compact set $K \subset C$, we have

$$
\liminf _{\varepsilon \rightarrow 0^{+}}\left[\varepsilon^{-m}\left\|f \mathbf{1}_{K_{\varepsilon}}\right\|_{L^{1}}\right]=0,
$$

then $C$ is an illusory singularity of $f$.

Whenever the integral is meaningful, for instance if $f \in L_{\text {loc }}^{1}(\Omega)$ and $\varphi \in$ $\mathcal{C}_{c}^{\infty}(\Omega)$, we define $(f, \varphi):=\int_{\Omega} f \varphi$, where the integral is computed with respect to the Lebesgue measure. The formal adjoint of $P$, denoted by ${ }^{t} P$, satisfies the relations $(P \varphi, \psi)=\left(\varphi,{ }^{t} P \psi\right)$ for all $\varphi, \psi \in \mathcal{C}_{c}^{\infty}(\Omega)$, and these relations define it uniquely as

$$
{ }^{t} P(\varphi):=\sum_{|\beta| \leqslant m}(-1)^{|\beta|} \partial_{x}^{\beta}\left(a_{\beta} \varphi\right) .
$$

Proof of Theorem 1.7. Let $K:=(\operatorname{supp} \varphi) \cap C$ and let $\varphi_{\varepsilon}$ be the family of functions constructed in Lemma 1.6. Since supp $P f \subset C$, we have $(P f, \varphi)=$ $\left(P f, \varphi_{\varepsilon} \varphi\right)=\left(f,{ }^{t} P\left(\varphi_{\varepsilon} \varphi\right)\right)$. Lemma 1.6 entails that $\left\|{ }^{t} P\left(\varphi_{\varepsilon} \varphi\right)\right\|_{L^{\infty}} \leqslant C \varepsilon^{-m}$, for some quantity $C>0$ that is independent of $\varepsilon$. We deduce that $|(P f, \varphi)| \leqslant$ $C \varepsilon^{-m}\left\|f \mathbf{1}_{K_{\varepsilon}}\right\|_{L^{1}}$ for all $\varepsilon>0$. Thanks to the main assumption, this implies that $(P f, \varphi)=0$.

If $\mathrm{p} \in \mathbb{R}$ with $1 \leqslant \mathrm{p} \leqslant \infty$ is the exponent of an $L^{\mathrm{p}}$-space, we denote by $\mathrm{p}^{\prime}:=$ $\frac{\mathrm{p}}{\mathrm{p}-1} \in[1, \infty]$ the conjugate exponent, also defined by the relation $1=\frac{1}{\mathrm{p}}+\frac{1}{\mathrm{p}^{\prime}}$. By Hölder's inequality, we have $\left\|f \mathbf{1}_{K_{\varepsilon}}\right\|_{L^{1}} \leqslant\left(\mathrm{H}^{n}\left(K_{\varepsilon}\right)\right)^{1 / \mathrm{p}^{\prime}}\left\|f \mathbf{1}_{K_{\varepsilon}}\right\|_{L^{\mathrm{p}}}$.

Corollary 1.8. Let $f \in L_{l o c}^{\mathrm{p}}(\Omega)$, where $1 \leqslant \mathrm{p} \leqslant \infty$. If, for each compact set $K \subset C$,

$$
\liminf _{\varepsilon \rightarrow 0^{+}}\left[\left(\varepsilon^{-m \mathbf{p}^{\prime}} \mathbf{H}^{n}\left(K_{\varepsilon}\right)\right)^{1 / \mathbf{p}^{\prime}}\left\|f \mathbf{1}_{K_{\varepsilon}}\right\|_{L^{\mathrm{p}}}\right]=0,
$$


then $C$ is an illusory singularity of $f$.

The next theorem translates Corollary 1.9 in terms of Hausdorff measures, a finer concept than the Minkowski content.

Theorem 1.9. ([HP1970]) (i) Let $1<\mathrm{p}<\infty$ and assume that $n-m \mathrm{p}^{\prime} \geqslant 0$. If $\mathrm{H}^{n-m \mathrm{p}^{\prime}}(K)<\infty$ for every compact set $K \subset C$, then $C$ is $\left(P, L_{\text {loc }}^{\mathrm{p}}\right)$-removable.

(ii) Let $\mathrm{p}=\infty$ and assume that $n-m \geqslant 0$. If $\mathrm{H}^{n-m}(C)=0$, then $C$ is $\left(P, L_{\text {loc }}^{\infty}\right)$-removable.

(iii) Let $\mathrm{p}=\infty$ and assume that $n-m \geqslant 0$. If, $\mathrm{H}^{n-m}(K)<\infty$ for each compact set $K \subset C$, then $P f$ is a measure supported on $C$, for every $f \in L_{\text {loc }}^{\infty}$ satisfying $P f=0$ on $\Omega \backslash C$.

An application of (ii) to $P=\bar{\partial}$ in one or several complex variables yields the Riemann removability Theorem 2.31 below.

Proof. We survey only the proof of (i). Let $\varphi \in \mathcal{C}_{c}^{\infty}(\Omega)$ and set $K:=C \cap \operatorname{supp} \varphi$.

Lemma 1.10. ([HP1970]) Let $K \subset \mathbb{R}^{n}$ be a compact set. Let $\mathrm{p}^{\prime}$ with $1 \leqslant \mathrm{p}^{\prime}<\infty$ and assume $n-m p^{\prime} \geqslant 0$. For every $\varepsilon>0$, there exists $\varphi_{\varepsilon} \in \mathcal{C}_{c}^{\infty}\left(\mathbb{R}^{n}\right)$ with $\varphi_{\varepsilon} \equiv 1$ in a neighborhood of $K$ and with $\operatorname{supp} \varphi_{\varepsilon} \subset K_{\varepsilon}$ such that for all $\beta \in \mathbb{N}^{n}$ with $|\beta| \leqslant m$, we have

$$
\left\|\partial_{x}^{\beta} \varphi_{\varepsilon}\right\|_{L^{\mathrm{p}^{\prime}}} \leqslant C \varepsilon^{m-|\beta|}\left(\mathrm{H}^{n-m \mathrm{p}^{\prime}}(K)+\varepsilon\right)^{1 / \mathrm{p}^{\prime}}
$$

where $C>0$ is independent of $\varepsilon$.

With such cut-off functions $\varphi_{\varepsilon}$, since $\operatorname{supp} P f \subset C$, we have $(P f, \varphi)=$ $\left(P f, \varphi_{\varepsilon} \varphi\right)=\left(f,{ }^{t} P\left(\varphi_{\varepsilon} \varphi\right)\right)$. By Hölder's inequality and the preceding lemma:

$$
|(P f, \varphi)| \leqslant\left\|f \mathbf{1}_{K_{\varepsilon}}\right\|_{L^{\mathrm{p}}}\left\|t P\left(\varphi_{\varepsilon} \varphi\right)\right\|_{L^{\mathrm{p}^{\mathrm{p}}}} \leqslant C\left\|f \mathbf{1}_{K_{\varepsilon}}\right\|_{L^{\mathrm{p}}}\left(\mathrm{H}^{n-m \mathrm{p}^{\prime}}(K)+\varepsilon\right)^{1 / \mathrm{p}^{\prime}} .
$$

The theorem follows from

$$
\lim _{\varepsilon \rightarrow 0^{+}}\left\|f \mathbf{1}_{K_{\varepsilon}}\right\|_{L^{\mathrm{p}}}=0,
$$

since $\mathrm{H}^{n}\left(K_{\varepsilon}\right) \rightarrow 0$ (remind $\left.\mathrm{H}^{n-m \mathrm{p}^{\prime}}(K)<\infty\right)$.

It seems impossible to get $L^{1}$ removability without an assumption of growth. At the opposite, in a CR context, the techniques introduced in ["rö1999b, MP1999] that are developed in Section 5 and in [MP2006a] will exhibit $L^{1}$-removability of certain closed subsets of generic submanifolds with only metrico-geometric assumptions. 


\section{§2. REMOVABLE SINGULARITIES FOR HOLOMORPHIC FUNCTIONS OF ONE OR SEVERAL COMPLEX VARIABLES}

2.1. Painlevé problem, zero length and analytic capacity. The classical Painlevé problem ([Pa1888, Ah1947]) is to find metric or geometric characterizations of compact sets $K \subset \mathbb{C}$ that are $\left(\bar{\partial}, L^{\infty}\right)$-removable, i.e. such that every $f \in \mathcal{O}(\mathbb{C} \backslash K) \cap L^{\infty}(\mathbb{C} \backslash K)$ extends holomorphically through $K$.

Theorem 1.9(ii) says that $\mathrm{H}^{1}(K)=0$ suffices. It is also known ([Ma1984, Pa2005]) that if $\mathrm{H}^{1+\varepsilon}(K)>0$ for some $\varepsilon>0$, then $K$ has positive analytic capacity (definition below) and is never $\left(\bar{\partial}, L^{\infty}\right)$-removable. Furthermore, Garnett ([Gar1970]) constructed a self-similar Cantor compact set $K \subset \mathbb{C}$ with $0<\mathrm{H}^{1}(K)<+\infty$ which is $\left(\bar{\partial}, L^{\infty}\right)$-removable. Consequently, Hausdorff measure is not fine enough.

Under a geometric tameness assumption a converse to the sufficiency of $H^{1}(K)=0$ holds and is usually called the solution to Denjoy's conjecture.

Theorem 2.2. ([Cal1977, CMM1982]) A compact set $K \subset \mathbb{C}$ that is a priori contained in a Lipschitz curve is $\left(\bar{\partial}, L^{\infty}\right)$-removable if and only if it has zero 1-dimensional Hausdorff measure.

Classically, this statement is an application of the celebrated result of Calderón, Coifman, McIntosh and Meyer about the $L^{2}$-boundedness of the Cauchy integral on Lipschitz curves. Let us survey one of the simplified proofs ([MV1995] which involves Menger curvature, a concept useful in a recent answer to Painlevé's problem obtained in [To2003].

Let $\Gamma:=\left\{(x, y) \in \mathbb{R}^{2}: y=\varphi(x)\right\}$ be a (global) Lipschitz graph; here $\varphi \in \mathcal{C}^{0,1}$ is locally absolutely continuous and $\varphi^{\prime}$ exists almost everywhere (a.e.) with $\left\|\varphi^{\prime}\right\|_{L^{\infty}}<+\infty$.

Theorem 2.3. ([Cal1977, CMM1982, MV1995]) If $f \in L^{2}(\Gamma)$, the Cauchy principal value integral

$$
C^{0} f(z):=\lim _{\varepsilon \rightarrow 0} \frac{1}{2 \pi i} \int_{|\zeta-z|>\varepsilon} \frac{f(\zeta)}{\zeta-z} d \zeta
$$

exists for almost every $z \in \Gamma$ and defines a function $C^{0} f(z)$ on $\Gamma$, the Cauchy transform of $f$, which belongs to $L^{2}(\Gamma)$ and satisfies in addition

$$
\left\|C^{0} f\right\|_{L^{2}(\Gamma)} \leqslant C_{1}\|f\|_{L^{2}(\Gamma)},
$$

for some positive constant $C_{1}=C_{1}\left(\left\|\varphi^{\prime}\right\|_{L^{\infty}}\right)$.

Parametrizing $\Gamma$ by $\zeta(t)=t+i \varphi(t)$, dropping the innocuous factor $1+i \varphi^{\prime}(t)$ and setting $z:=x+i \varphi(x)$, one has to estimate the $L^{2}$-norm of the truncated integral

$$
C_{\varepsilon}^{\prime}(f)(x):=\int_{|t-x|>\varepsilon} \frac{f(t)}{\zeta(t)-\zeta(x)} d t
$$


with a constant independent of $\varepsilon$. Even more, interpolation arguments reduce the task to a single estimate of the form

$$
\int_{\mathbb{R}}\left|C_{\varepsilon}^{\prime}\left(\chi_{I}\right)\right|^{2} \leqslant C_{1}|I|,
$$

where $C_{1}=C_{1}\left(\left\|\varphi^{\prime}\right\|_{L^{\infty}}\right)$ and where $\chi_{I}$ is the characteristic function of an interval $I \subset \mathbb{R}$ of length $|I|$. Following [MV1995], a symmetrization of the (implicitely triple) integral $\int_{I} \mathrm{C}_{\varepsilon}^{\prime}\left(\chi_{I}\right) \overline{\mathrm{C}_{\varepsilon}^{\prime}\left(\chi_{I}\right)}$ provides

$$
\begin{array}{r}
6 \int_{I}\left|C_{\varepsilon}^{\prime}\left(\chi_{I}\right)\right|^{2}=\iiint_{S_{\varepsilon}}\left(\sum_{\sigma \in \mathfrak{S}_{3}} \frac{1}{\zeta\left(x_{\sigma(2)}\right)-\zeta\left(x_{\sigma(1)}\right)} \frac{1}{\overline{\zeta\left(x_{\sigma(3)}\right)-\zeta\left(x_{\sigma(1)}\right)}}\right) \\
\cdot d x_{1} d x_{2} d x_{3}+\mathrm{O}(|I|),
\end{array}
$$

where $S_{\varepsilon}:=\left\{(x, y, t) \in I^{3}:|y-x|>\varepsilon,|t-x|>\varepsilon,|t-y|>\varepsilon\right\}$ and where $\mathfrak{S}_{3}$ is the permutation group of $\{1,2,3\}$.

Then a "magic" ([Po2005]) formula enters the scene:

$$
\left(\frac{4 S\left(z_{1}, z_{2}, z_{3}\right)}{\left|z_{1}-z_{2}\right|\left|z_{1}-z_{3}\right|\left|z_{2}-z_{3}\right|}\right)^{2}=\sum_{\sigma \in \mathfrak{S}_{3}} \frac{1}{\zeta\left(x_{\sigma(2)}\right)-\zeta\left(x_{\sigma(1)}\right)} \frac{1}{\overline{\zeta\left(x_{\sigma(3)}\right)-\zeta\left(x_{\sigma(1)}\right)}},
$$

where $S\left(z_{1}, z_{2}, z_{3}\right)$ denotes the enclosed area; the left hand side measures the "flatness" of the triangle. This crucial formula enables one to link rectifiability properties to the Cauchy kernel.

Definition 2.4. The Menger curvature of the triple $\left\{z_{1}, z_{2}, z_{3}\right\}$ is the square root of the above

$$
c\left(z_{1}, z_{2}, z_{3}\right):=\frac{4 S\left(z_{1}, z_{2}, z_{3}\right)}{\left|z_{1}-z_{2}\right|\left|z_{1}-z_{3}\right|\left|z_{2}-z_{3}\right|}
$$

one sets $c:=0$ if the points are aligned. One also verifies that $c\left(z_{1}, z_{2}, z_{3}\right)=$ $1 / R\left(z_{1}, z_{2}, z_{3}\right)$, where $R$ is the radius of the circumbscribed circle.

Thanks to the nice formula and to the basic inequality

$$
c(\zeta(x), \zeta(y), \zeta(t)) \leqslant 2\left|\frac{\frac{\varphi(y)-\varphi(x)}{y-x}-\frac{\varphi(t)-\varphi(x)}{t-x}}{|t-y|}\right|
$$

the previous symmetric Cauchy triple integral is transformed to an integral involving geometric Lipschitz properties of $\Gamma$. After some computations (MV1995]), one gets $\int_{I}\left|C_{\varepsilon}^{\prime}\left(\chi_{I}\right)\right|^{2} \leqslant C_{1}\left(\left\|\varphi^{\prime}\right\|_{L^{\infty}}\right) \cdot|I|$.

Menger curvature also appears in a recent result, considered to be an answer to Painlevé's problem.

Theorem 2.5. ([To2003, Pa2005]]) A compact set $K \subset \mathbb{C}$ is not removable for $\mathcal{O}(\mathbb{C} \backslash K) \cap L^{\infty}(\mathbb{C} \backslash K)$ if and only if there exists a nonzero positive Radon measure $\mu$ with $\operatorname{supp} \mu \subset K$ such that 
- there exists $C_{1}>0$ with $\mu(\Delta(z, \rho)) \leqslant C_{1} \rho$ for every $z \in \mathbb{C}$ and $\rho>0$;

- $\iiint[c(x, y, z)]^{2} d \mu(x) d \mu(y) d \mu(z)<+\infty$.

The first condition concerns the size of $K$; the second one is of quantitativegeometric nature.

We conclude by mentioning a classical functional characterization due to Ahlfors, usually considered to be only a reformulation of Painlevé's problem, but which has already found generalizations in locally integrable structures $(\S 2.16$ below). The analytic capacity of a compact set $K \subset \mathbb{C}$ is ${ }^{21}$

$$
\operatorname{an}-\operatorname{cap}(K):=\sup \left\{\left|f^{\prime}(\infty)\right|: f \in H^{\infty}(\mathbb{C} \backslash K),\|f\|_{L^{\infty}} \leqslant 1\right\},
$$

where $H^{\infty}(\mathbb{C} \backslash K)$ denotes the space of bounded holomorphic functions defined in $\mathbb{C} \backslash K$ (or defined in the complement of $K$ in the Riemann sphere $\mathbb{C} \cup\{\infty\}$, because $\{\infty\}$ is removable).

Theorem 2.6. ([Ah1947, Ma1984, HT1997]) A compact set $K \subset \mathbb{C}$ is removable for $\mathcal{O}(\mathbb{C} \backslash K) \cap L^{\infty}(\mathbb{C} \backslash K)$ if and only if an-cap $(K)=0$.

2.7. Radó-type theorems. A classical theorem due to Radó (Ra1924, Stu1968, RS1989, Ch1994]) asserts that a continuous function $f$ defined in a domain $\Omega \subset \mathbb{C}$ that is holomorphic outside its zero-set $f^{-1}(0)$ is in fact holomorphic everywhere. By a separate holomorphicity argument, this statement extends directly to several complex variables. In [Stu1993], it is shown that $f^{-1}(0)$ may be replaced by $f^{-1}(E)$, where $E \subset \mathbb{C}$ is compact and has null analytic capacity. In [RS1989], it is shown that a continuous function defined in a strongly pseudoconvex $\mathcal{C}^{2}$ hypersurface $M \subset \mathbb{C}^{n}(n \geqslant 2)$ that is CR outside its zero-set is CR everywhere; a thin subset of weakly pseudoconvex points is allowed, but the case of general hypersurfaces is not covered. In [Al1993, it is shown that closed sets $f^{-1}(E)$ are removable in the same situation, wehere $E \subset \mathbb{C}$ is a closed polar set, viz. $E \subset\{u=-\infty\}$ for some subharmonic function $u \not \equiv-\infty$. Chirka strengthens these results in the following theorem, where no assumption is made on the geometry of the hypersurface.

Remind ([Ch1989, De1997]) that $E \subset \mathbb{C}^{m}$ is called complete pluripolar if $E=\{\varphi=-\infty\}$ for some plurisubharmonic function $\varphi \not \equiv-\infty$ on $\mathbb{C}^{m}$.

Theorem 2.8. ([Ch1994]) Let $M \subset \mathbb{C}^{n}(n \geqslant 2)$ be hypersurface that is a local Lipschitz $\left(\mathcal{C}^{0,1}\right)$ graph at every point, let $C$ be a closed subset of $M$ and let $f$ : $M \backslash C \rightarrow \mathbb{C}^{m} \backslash E$ be a continuous mapping satisfying $\|f\|_{\mathcal{C}^{0}(M \backslash C)}<\infty$ such that the set of limit values of $f$ from $M \backslash C$ up to $C$ is contained in a closed complete

\footnotetext{
${ }^{21}$ If $\|f\|_{L^{\infty}} \leqslant 1$, setting $g(z):=[f(\infty)-f(z)] /[1-\overline{f(\infty)} f(z)]$, we have $g(\infty)=0$, $\|g\|_{L^{\infty}} \leqslant 1$ and $\left|g^{\prime}(\infty)\right|=\left|f^{\prime}(\infty)\right| /\left(1-|f(\infty)|^{2}\right) \geqslant\left|f^{\prime}(\infty)\right|$, so that in the definition of analytic capacity, we may restrict to take the supremum over functions $g \in H^{\infty}(\mathbb{C} \backslash K)$ with $\|g\|_{L^{\infty}} \leqslant 1$ and $g(\infty)=0$.
} 
pluripolar set $E \subset \mathbb{C}^{m}(m \geqslant 1)$. Then the trivial extension $\tilde{f}$ of $f$ to $C$ defined by $\widetilde{f}:=0$ on $C$ is a CR mapping of class $L^{\infty}$ on the whole of $M$.

In higher codimension, nothing is known.

Open question 2.9. Let $M \subset \mathbb{C}^{n}(n \geqslant 3)$ be a generic submanifold of codimension $d \geqslant 2$ and of $C R$ dimension $m \geqslant 1$ that is at least $\mathcal{C}^{1}$. Let $f \in \mathcal{C}^{0}(M)$ that is CR outside its zero-set $f^{-1}(0)$. Is $f$ CR everywhere?

Remind that condition (P) (Definition 3.5(III)) for a linear partial differential operator $P$ of principal type assures local solvability of the equations $P f=g$. Remind also that nowhere vanishing vector fields are of principal type.

Theorem 2.10. ([HT1993]) Let $\Omega \subset \mathbb{R}^{n}(n \geqslant 2)$ be a domain and let $L$ be a nowhere vanishing vector field on $\Omega$ having $\mathcal{C}^{\infty}$ complex-valued coefficients and satisfying condition (P) of Nirenberg-Treves. If $f \in \mathcal{C}^{0}(\Omega)$ satisfies $L f=0$ in $\Omega \backslash f^{-1}(0)$ in the sense of distributions, then $f$ is a weak solution of $L f=0$ all over $\Omega$.

2.11. Capacity and partial differential operators having constant coefficients. The preceding results admit partial generalizations to vector field systems. Let $\Omega \subset \mathbb{R}^{n}$ be an open set and let $P=P\left(\partial_{x}\right)=\sum_{\beta \in \mathbb{N}^{n}} a_{\beta} \partial_{x}^{\beta}$ be a linear partial differential operator having constant coefficients $a_{\beta} \in \mathbb{C}$. By a theorem due to Malgrange, Ehrenpreis and Palamodov ([Hö1963]), such a $P$ always admits a fundamental solution, namely there exists a distribution $E \in \mathcal{D}^{\prime}\left(\mathbb{R}^{n}\right)$ such that $P\left(\partial_{x}\right) E=\delta_{0}$ is the Dirac measure at the origin.

Let $\mathcal{F} \subset \mathcal{D}^{\prime}(\Omega)$ be a Banach space, e.g. $\mathcal{F}=L^{\mathrm{p}}(\Omega)$ with $1 \leqslant \mathrm{p} \leqslant \infty$, or $\mathcal{F}=L^{\infty}(\Omega) \cap \mathcal{C}^{0}(\Omega)$, or $\mathcal{F}=\mathcal{C}^{0, \alpha}(\Omega)$ with $0<\alpha \leqslant 1$.

Definition 2.12. For each relatively closed set $C \subset \Omega$, the $\mathcal{F}$-capacity of $C$ with respect to $P$ is

$$
\mathcal{F}-\operatorname{cap}_{P}(C, \partial \Omega):=\sup \left\{\left|\left(P f, \mathbf{1}_{\Omega}\right)\right|: f \in \mathcal{F},\|f\|_{\mathcal{F}} \leqslant 1, \operatorname{supp}(P f) \Subset C\right\} .
$$

If a closed set $C \subset \Omega$ is $(P, \mathcal{F})$-removable, by definition $P f=0$ everywhere, hence $\mathcal{F}-\operatorname{Cap}_{P}(C, \Omega)=0$. The following theorem establishes the converse for a wide class of differential operators having constant coefficients. For $\beta \in \mathbb{N}^{n}$, denote by $Q^{(\beta)}(x):=\partial_{x}^{\beta} Q(x)$ the $\beta$-th partial derivative of a polynomial $Q(x) \in$ $\mathbb{R}[x]$.

Theorem 2.13. ([HP1972]) Assume that $P$ possesses a fundamental solution $E \in$ $\mathcal{D}^{\prime}\left(\mathbb{R}^{n}\right)$ such that $\overline{P^{(\beta)}\left(\partial_{x}\right)}$ E is a regular Borel measure on $\mathbb{R}^{n}$ for every $\beta \in \mathbb{N}^{n}$. Let $\Omega \Subset \mathbb{R}^{n}$ be a bounded domain and let $C \subset \Omega$ be a relatively closed subset. Then

- $C$ is $\left(P, L_{l o c}^{\mathrm{p}}\right)$-removable, $1<\mathrm{p} \leqslant \infty$, if and only if $L^{\mathrm{p}}-\operatorname{cap}_{P}(C, \Omega)=0$;

- $C$ is $\left(P, L^{\infty} \mathcal{C}^{0}\right)$-removable if and only if $L^{\infty} \mathcal{C}^{0}-\operatorname{cap}_{P}(C, \Omega)=0$; 
- $C$ is $\left(P, \mathcal{C}^{0, \alpha}\right)$-removable, $0<\alpha \leqslant 1$, if and only if $\mathcal{C}^{0, \alpha}{ }_{-} \operatorname{cap}_{P}(C, \Omega)=$ 0.

This hypothesis about $P$ is satisfied by elliptic, semi-elliptic, and parabolic operators and also by the wave operator in $\mathbb{R}^{2}$ ([HP1972] $)$. The theorem (whose proof is rather short) also holds true if $P=P\left(x, \partial_{x}\right)$ has real analytic coefficients and admits a fundamental solution $E$ such that $P^{(\beta)} E$ is a regular Borel measure for every $\beta \in \mathbb{N}^{n}$. But it is void in $L^{1}$.

Theorem 2.14. ([HP1972]) There is a unique function, called a capacitary extremal, $f^{\text {cap }} \in L^{\mathrm{p}}(\Omega)$ with $\left\|f^{\text {cap }}\right\|_{L^{\mathrm{p}}} \leqslant 1$ and $P f^{\text {cap }}=0$ in $\Omega \backslash K$ such that $\left(P f^{\text {cap }}, \mathbf{1}_{\Omega}\right)=L^{\mathrm{p}}-\operatorname{cap}_{P}(K, \Omega)$.

We observe that the definition of $L^{\mathrm{p}}-\operatorname{cap}_{P}(K, \Omega)$ is inspired from Ahlfors' notion of analytic capacity and we mention that the capacitary extremal $f^{\text {cap }}$ is linked to the Riemann uniformization theorem.

Example 2.15. In fact, with $\Omega=\mathbb{C}$ and $P=\partial / \partial \bar{z}=: \bar{\partial}$, the $L^{\infty}$-capacity of a compact set $K \subset \mathbb{C}$ with respect to $\bar{\partial}$ may be shown to be equal, up to the constant $\pi$, to the analytic capacity of $K$, namely

$$
L^{\infty}-\operatorname{cap}_{\bar{\partial}}(K, \mathbb{C})=\pi \operatorname{an}-\operatorname{cap}(K) .
$$

Indeed, letting $f \in L^{\infty}(\mathbb{C})$, assuming that $\bar{\partial} f$ is supported by $K$, choosing a big open disc $D \Subset \mathbb{C}$ containing $K$, integrating by parts (Riemann-Green) and performing the change of variables $w:=1 / z$, we may compute

$$
\left(\bar{\partial} f, \mathbf{1}_{\mathbb{C}}\right)=\left(\bar{\partial} f, \mathbf{1}_{D}\right)=\frac{1}{2 i} \iint_{D} \frac{\partial f}{\partial \bar{z}} d \bar{z} \wedge d z=\frac{1}{2 i} \int_{\partial D} f(z) d z=\pi f^{\prime}(\infty) .
$$

Remind (footnote) that in the definition of an-cap $(K)$ given in $\$ 2.1$, one may assume that $f(\infty)=0$. If in addition the complement of $K$ in the Riemann sphere $\mathbb{C} \cup\{\infty\}$ is simply connected, the unique solution $f^{\text {cap }}$ of $\left(\bar{\partial} f^{\text {cap }}, \mathbf{1}_{\mathbb{C}}\right)=$ $L^{\infty}$-cap $\overline{\bar{\partial}}(K, \mathbb{C})$ asserted by Theorem 2.14 , viz. the unique solution of the extremal problem

$$
\sup \left\{\left|f^{\prime}(\infty)\right|: f \in L^{\infty}(\mathbb{C}), \partial f / \partial \bar{z}=0 \text { in } \mathbb{C} \backslash K, f(\infty)=0 \text { and }\|f\|_{L^{\infty}} \leqslant 1\right\}
$$

is the (unique) Riemann uniformization map $f^{\text {cap }}:(\mathbb{C} \cup\{\infty\}) \backslash K \rightarrow \Delta$ satisfying $f^{\text {cap }}(\infty)=0$ and $\partial_{z} f^{\text {cap }}(\infty)>0$.

\subsection{Removable singularities of locally solvable vector fields. Let}

$$
\mathcal{S}\left(\mathbb{R}^{n}\right):=\left\{f \in \mathcal{C}^{\infty}\left(\mathbb{R}^{n}\right): \lim _{|x| \rightarrow \infty}\left|x^{\alpha} \partial_{x}^{\beta} f(x)\right|=0, \forall \alpha, \beta \in \mathbb{N}^{n}\right\}
$$

be the space of $\mathcal{C}^{\infty}$ functions defined in $\mathbb{R}^{n}$ and having tempered growth. As is known ([Hö1963]), the Fourier transform

$$
\mathrm{F} f(\xi):=\int_{\mathbb{R}^{n}} e^{-2 \pi i\langle x, \xi\rangle} f(x) d x, \quad f \in \mathcal{S}\left(\mathbb{R}^{n}\right),
$$


$\langle x, \xi\rangle:=\sum_{k=1}^{n} x_{k} \xi_{k}$, is an automorphism of $\mathcal{S}\left(\mathbb{R}^{n}\right)$ having as inverse

$$
\mathrm{F}^{-1} f(\xi):=\int_{\mathbb{R}^{n}} e^{2 \pi i\langle x, \xi\rangle} f(x) d x=\mathrm{F} f(-\xi)
$$

Equipping $\mathcal{S}\left(\mathbb{R}^{n}\right)$ with the countable family of semi-norms $p_{\alpha, \beta}(f):=$ $\sup _{x \in \mathbb{R}^{n}}\left|x^{\alpha} \partial_{x}^{\beta} f(x)\right|$, the space $\mathcal{S}^{\prime}\left(\mathbb{R}^{n}\right)$ of tempered distributions consists of linear functionals $\mathrm{T}$ on $\mathcal{S}\left(\mathbb{R}^{n}\right)$ that are continuous, viz. there exists $C>0$ and $\alpha, \beta \in \mathbb{N}^{n}$ such that $|\langle\mathrm{T}, f\rangle| \leqslant C p_{\alpha, \beta}(f)$ for every $f \in \mathcal{S}\left(\mathbb{R}^{n}\right)$.

For $\mathrm{p} \in \mathbb{R}$ with $1 \leqslant \mathrm{p} \leqslant \infty$ and for $\sigma \in \mathbb{R}$, we remind the definition of the Sobolev space

$$
L_{\sigma}^{\mathrm{p}}\left(\mathbb{R}^{n}\right):=\left\{\mathrm{T} \in \mathcal{S}^{\prime}\left(\mathbb{R}^{n}\right):\|\mathrm{T}\|_{L_{\sigma}^{\mathrm{p}}}:=\left\|\Lambda^{-\sigma} \mathrm{T}\right\|_{L^{\mathrm{p}}}<\infty\right\},
$$

where $\Lambda^{-\sigma} \mathrm{T}(x):=\mathrm{F}^{-1}\left[\left(1+|\xi|^{2}\right)^{-\sigma / 2} \mathrm{~F} \mathrm{~T}(\xi)\right](x)$. For $\sigma=\kappa \in \mathbb{N}$ and $\mathrm{p}$ in the range $1<\mathrm{p}<\infty$, the space $L_{\kappa}^{\mathrm{P}}\left(\mathbb{R}^{n}\right)$ is exactly the subspace of functions $u \in$ $L^{\mathrm{P}}\left(\mathbb{R}^{n}\right)$ whose partial derivatives of order $\leqslant \kappa$ (in the distributional sense) belong to $L^{\mathrm{p}}\left(\mathbb{R}^{n}\right)$. This space is equivalently normed by $\|u\|_{L_{\kappa}^{\mathrm{p}}}:=\sum_{|\beta| \leqslant \kappa}\left\|\partial_{x}^{\beta} u\right\|_{L^{\mathrm{p}}}$.

Let $\Omega \subset \mathbb{R}^{n}$ be a domain and let $P=P\left(x, \partial_{x}\right)=\sum_{|\beta| \leqslant m} a_{\beta}(x) \partial_{x}^{\beta}$ be a linear partial differential operator of order $m \geqslant 1$ defined in $\Omega$ and having $\mathcal{C}^{\infty}$ coefficients $a_{\beta}(x)$.

Definition 2.17. We say that $P$ is locally solvable in $L^{\mathrm{p}}$ with one loss of derivative if every point $p \in \Omega$ has an open neighborhood $U_{p} \subset \Omega$ such that for every compactly supported $\mathrm{T} \in L_{\sigma}^{\mathrm{p}}\left(U_{p}\right)$, the equation $\mathrm{PS}=\mathrm{T}$ has a solution $\mathrm{S} \in$ $L_{\sigma+m-1}^{\mathrm{p}}\left(U_{p}\right)$.

Theorem 2.18. ([BeFe1973, HP1996]) Let L be a nowhere vanishing vector field having $\mathcal{C}^{\infty}$ coefficients in a domain $\Omega \subset \mathbb{R}^{n}(n \geqslant 2)$ and assume that $L$ satisfies condition (P) of Nirenberg-Treves. Then for every $\mathrm{p} \in \mathbb{R}$ with $1<\mathrm{p}<\infty$, the operator $L$ is locally solvable in $L^{\mathrm{p}}$ with loss of one derivative.

Example 2.19. As discovered in [HT1996], local solvability fails to hold in $L^{\infty}$ for the (locally solvable) vector field $\frac{\partial}{\partial x}-\frac{i}{x^{2}} e^{-1 /|x|} \frac{\partial}{\partial y}$ satisfying (P) on $\mathbb{R}^{2}$.

Removable singularities for vector fields in $L^{\mathrm{P}}$ have been studied in [HT1996, HT1997]. Because of the example, results in $L^{\mathrm{p}}$ with $1<\mathrm{p}<\infty$ differ from results in $L^{\infty}$.

Definition 2.20. A relatively closed set $C \subset \Omega$ of an open set $\Omega \subset \mathbb{R}^{n}$ is everywhere $\left(P, L^{\mathrm{P}}\right)$-removable if for every open subset $U \subset \Omega$ and for every $f \in L^{\mathrm{P}}(U)$ satisfying $P f=0$ in $U \backslash C$, then $f$ also satisfies $P f=0$ in all of $U$.

Theorem 2.21. ([HT1996, HT1997]) Let L be a nowhere vanishing vector field having $\mathcal{C}^{\infty}$ coefficients in an open subset $\Omega \subset \mathbb{R}^{n}(n \geqslant 2)$ and assume that $L$ satisfies condition $(\mathbf{P})$. Let $\mathrm{p} \in \mathbb{R}$ with $1<\mathrm{p}<\infty$. Then a relatively closed set 
$C \subset \Omega$ is everywhere $\left(L, L^{\mathrm{p}}\right)$-removable if and only if there is an open covering of $C$ by open sets $\Omega_{j}, j \in J$, such that

$$
L^{\mathrm{P}}-\operatorname{cap}_{L}\left(C \cap \Omega_{j}, \Omega_{j}\right)=0,
$$

for every $j \in J$.

In $L^{\infty}$, when trying to perform the proof of this theorem, local solvability of positive multiples of $L$ is technically needed. Observing that $P f=0$ is equivalent to $e^{\psi} P f=0$, the following notion appeared to be appropriate to deal with $\left(L, L^{\infty}\right)$-removability.

Definition 2.22. ([HT1996, HT1997]) The full $L^{\infty}$-capacity of a relatively closed set $C \subset \Omega$ with respect to $L$ is

$$
\text { full- } L^{\infty}-\operatorname{cap}_{L}(C, \Omega):=\sup _{\widetilde{L}}\left\{L^{\infty}-\operatorname{cap}_{\widetilde{L}}(C, \Omega)\right\}
$$

where the supremum is taken over all vector fields $\widetilde{L}=e^{\psi} L$ with $\psi \in \mathcal{C}^{\infty}(\Omega)$ satisfying $\sup _{\Omega}\left|\partial_{x}^{\alpha} \psi(x)\right| \leqslant 1$ for $|\alpha| \leqslant 1$.

By a fine analysis of the degeneracies of $L$ and of the structure of the Sussmann orbits of $\{\operatorname{Re} L, \operatorname{Im} L\}$, Hounie-Tavares were able to substantially generalize Ahlfors' characterization.

Theorem 2.23. ([HT1996, HT1997]) A relatively closed set $C \subset \Omega$ is everywhere $\left(L, L^{\infty}\right)$-removable if and only if there is an open covering of $C$ by open sets $\Omega_{j}$, $j \in J$, such that

$$
\text { full- } L^{\infty}-\operatorname{cap}_{L}\left(C \cap \Omega_{j}, \Omega_{j}\right)=0
$$

for every $j \in J$.

On orbits of dimension one, $L$ behaves as a multiple of a real vector field (onedimensional behavior); on orbits of dimension two, $L$ has the behavior of $\bar{\partial}$ on a Riemann surface $\Sigma \subset \mathcal{O}$, but on the complement $\mathcal{O} \backslash \Sigma$ which is a union of curves with different endpoints along which $\operatorname{Re} L$ and $\operatorname{Im} L$ are both tangent (degeneracy), $L$ behaves again as a multiple of a real vector field (one-dimensional behavior). As shown in [HT1996] (main Theorem 7.3 there) a relatively closed set $C \subset \Omega$ is everywhere removable if and only if $C$ does not disconnect almost every curve on which $L$ has one-dimensional behavior and furthermore, the intersection of $C$ with almost every (reduced) orbit of dimension two has zero analytic capacity for its natural holomorphic structure.

Open problem 2.24. Study removability of a $\mathcal{C}^{\infty}$ locally integrable involutive structure of rank $\lambda \geqslant 2$ in terms of analytic capacity. 
2.25. Cartan-Thullen argument and a local continuity principle. The BehnkeSommer Kontinuitätssatz, alias Continuity Principle, states informally as follows ([Sh1990]). Let $\left(\Sigma_{\nu}\right)_{\nu \in \mathbb{N}}$ be a sequence of complex manifolds with boundary $\partial \Sigma_{\nu}$ contained in a domain $\Omega$ of $\mathbb{C}^{n}$. If $\Sigma_{\nu}$ converges to a set $\Sigma_{\infty} \subset \bar{\Omega}$ and if $\partial \Sigma_{\infty}$ is contained in $\Omega$, then every holomorphic function $f \in \mathcal{O}(\Omega)$ extends holomorphically to a neighborhood of the set $\Sigma_{\infty}$ in $\mathbb{C}^{n}$. The geometries of the $\Sigma_{\nu}$ and of $\Sigma_{\infty}$ have to satisfy certain assumptions in order that the statement be correct; in addition, monodromy questions have to be considered carefully. For applications to removable singularities in [MP2006a], the rigorous Theorem 2.27 below is formulated, with the $\Sigma_{\nu}$ being embedded analytic discs.

We denote by $z=\left(z_{1}, \ldots, z_{n}\right)$ the complex coordinates on $\mathbb{C}^{n}$ and by $|z|=$ $\max _{1 \leqslant i \leqslant n}\left|z_{i}\right|$ the polydisc norm. If $E \subset \mathbb{C}^{n}$ is an arbitrary subset, for $\rho>0$, we denote by

$$
\mathcal{V}_{\rho}(E):=\bigcup_{p \in E}\left\{z \in \mathbb{C}^{n}:|z-p|<\rho\right\}
$$

the union of all open polydiscs of radius $\rho$ centered at points of $E$.

Lemma 2.26. ([Me1997]) Let $\Omega$ be a nonempty domain of $\mathbb{C}^{n}$ and let $A: \bar{\Delta} \rightarrow \Omega$, $A \in \mathcal{O}(\Delta) \cap \mathcal{C}^{1}(\bar{\Delta})$, be an analytic disc contained in $\Omega$ having the property that there exist two constants $c$ and $C$ with $0<c<C$ such that

$$
c\left|\zeta_{2}-\zeta_{1}\right|<\left|A\left(\zeta_{2}\right)-A\left(\zeta_{1}\right)\right|<C\left|\zeta_{2}-\zeta_{1}\right|,
$$

for all distinct points $\zeta_{1}, \zeta_{2} \in \bar{\Delta}$. Set

$$
\rho:=\inf \{|z-A(\zeta)|: z \in \partial \Omega, \zeta \in \partial \Delta\},
$$

namely $\rho$ is the polydisc distance between $A(\partial \Delta)$ and $\partial \Omega$, and set $\sigma:=\rho c / 2 C$. Then for every holomorphic function $f \in \mathcal{O}(\Omega)$, there exists a holomorphic function $F \in \mathcal{O}\left(\mathcal{V}_{\sigma}(A(\bar{\Delta}))\right)$ such that $F=f$ on $\mathcal{V}_{\sigma}(A(\partial \Delta))$.

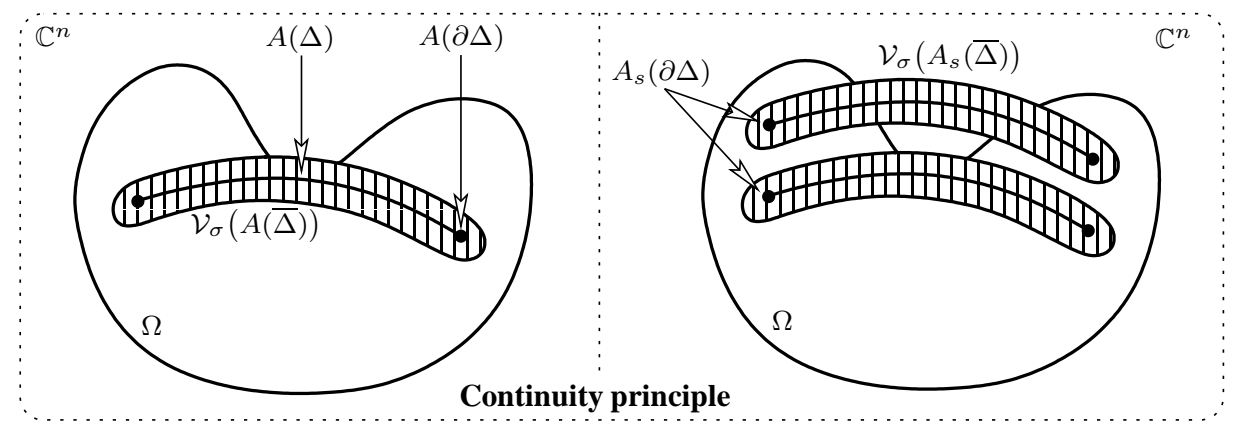

The inequalities involving $c$ and $C$ are satisfied for instance when $A$ is $\mathcal{C}^{1}$ embedding of $\bar{\Delta}$ into $\Omega$. Whereas $A(\bar{\Delta})$ is contained in $\Omega$, the neighborhood $\mathcal{V}_{\sigma}(A(\bar{\Delta}))$ is allowed to go beyond. We do not claim that the two functions $f \in \mathcal{O}(\Omega)$ and $F \in \mathcal{O}\left(\mathcal{V}_{\sigma}(A(\bar{\Delta}))\right)$ stick together as a holomorphic function 
globally defined in $\Omega \cup \mathcal{V}_{\sigma}(A(\bar{\Delta}))$. In fact, $\Omega \cap \mathcal{V}_{\sigma}(A(\bar{\Delta}))$ may have several connected components.

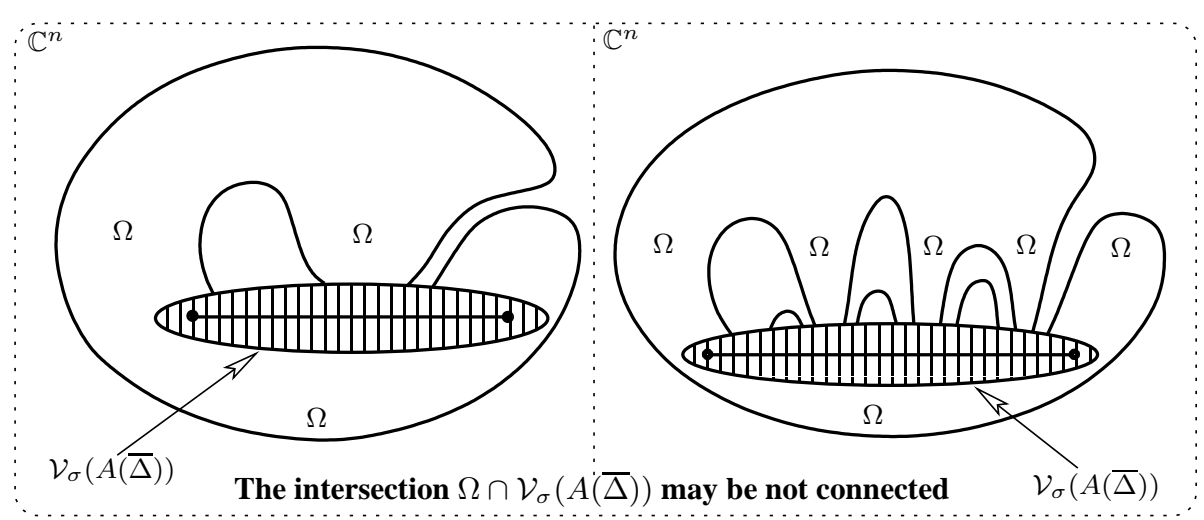

In the geometric situations we encounter in [Me1997, MP1999, MP2002, MP2006a, after shrinking $\Omega$ somehow slightly to some subdomain $\Omega^{\prime}$, we shall be able to insure that the intersection $\Omega^{\prime} \cap \mathcal{V}_{\sigma}(A(\bar{\Delta}))$ is connected and that the union $\Omega^{\prime} \cup \mathcal{V}_{\sigma}(A(\bar{\Delta}))$ is still significantly "bigger" than $\Omega$.

Proof of Lemma 2.26. Let $f \in \mathcal{O}(\Omega)$. For $\zeta \in \bar{\Delta}$ arbitrary, we consider the locally converging Taylor series $\sum_{\alpha \in \mathbb{N}^{n}} f_{\alpha}(z-A(\zeta))^{\alpha}$ of $f$ at $A(\zeta)$. For $\rho^{\prime}$ with $0<\rho^{\prime}<\rho$ arbitrarily close to $\rho$, since $\mathcal{V}_{\rho^{\prime}}(A(\partial \Delta)) \Subset \Omega$, the quantity

$$
M_{\rho^{\prime}}(f):=\sup \left\{|f(z)|: z \in \mathcal{V}_{\rho^{\prime}}(A(\partial \Delta))\right\}<\infty,
$$

is finite (it may explode as $\rho^{\prime} \rightarrow \rho$ ). Thus, Cauchy's inequality on a polydisc of radius $\rho^{\prime}$ centered at an arbitrary point $A\left(e^{i \theta}\right)$ of $\partial \Delta$ yields

$$
\frac{1}{\alpha !}\left|\frac{\partial^{|\alpha|} f}{\partial z^{\alpha}}\left(A\left(e^{i \theta}\right)\right)\right| \leqslant \frac{M_{\rho^{\prime}}(f)}{\rho^{\prime \alpha}},
$$

uniformly for all $e^{i \theta} \in \partial \Delta$. Then the maximum principle applied to the function $\zeta \mapsto \frac{\partial^{|\alpha|} f}{\partial z^{\alpha}}(A(\zeta))$ holomorphic in $\Delta$ provides the crucial inequalities (CartanThullen argument):

$$
\begin{aligned}
\left|f_{\alpha}\right|=\frac{1}{\alpha !}\left|\frac{\partial^{|\alpha|} f}{\partial z^{\alpha}}(A(\zeta))\right| & \leqslant \frac{1}{\alpha !} \sup _{e^{i \theta} \in \partial \Delta}\left|\frac{\partial^{|\alpha|} f}{\partial z^{\alpha}}\left(A\left(e^{i \theta}\right)\right)\right| \\
& \leqslant \frac{M_{\rho^{\prime}}(f)}{\rho^{\prime \alpha}} .
\end{aligned}
$$

Consequently, the Taylor series of $f$ converges normally in the polydisc $\Delta_{\rho}^{n}(A(\zeta))$ of center $A(\zeta)$ and of radius $\rho$, this being true for every $A(\zeta) \in A(\bar{\Delta})$. These series define a collection of holomorphic functions $F_{A(\zeta), \rho} \in \mathcal{O}\left(\Delta_{\rho}^{n}(A(\zeta))\right)$ parametrized by $\zeta \in \bar{\Delta}$. We claim that the restrictions of all these functions 
to the smaller polydiscs $\Delta_{\sigma}^{n}(A(\zeta))$ stick together in a well defined holomorphic function $F \in \mathcal{O}\left(\mathcal{V}_{\sigma}(A(\bar{\Delta}))\right)$.

Indeed, assume that two distinct points $\zeta_{1}, \zeta_{2} \in \bar{\Delta}$ are such that the intersection of the two small polydiscs $\Delta_{\sigma}^{n}\left(A\left(\zeta_{1}\right)\right) \cap \Delta_{\sigma}^{n}\left(A\left(\zeta_{2}\right)\right)$ is nonempty, so $\mid A\left(\zeta_{2}\right)-$ $A\left(\zeta_{1}\right) \mid<2 \sigma$. It follows that for every $\zeta$ belonging to the segment $\left[\zeta_{1}, \zeta_{2}\right]$, we have:

$$
\left|\zeta-\zeta_{1}\right| \leqslant\left|\zeta_{2}-\zeta_{1}\right|<\left|A\left(\zeta_{2}\right)-A\left(\zeta_{1}\right)\right| / c<2 \sigma / c
$$

whence

$$
\left|A(\zeta)-A\left(\zeta_{1}\right)\right|<C\left|\zeta-\zeta_{1}\right|<2 C \sigma / c=\rho .
$$

This means that the curved segment $A\left(\left[\zeta_{1}, \zeta_{2}\right]\right)$ is contained in the connected intersection of the two large polydiscs $\Delta_{\rho}^{n}\left(A\left(\zeta_{1}\right)\right) \cap \Delta_{\rho}^{n}\left(A\left(\zeta_{2}\right)\right)$. In a small neighborhood of $A\left(\zeta_{1}\right)$ and of $A\left(\zeta_{2}\right)$, the two holomorphic functions $F_{A\left(\zeta_{1}\right), \rho}$ and $F_{A\left(\zeta_{2}\right), \rho}$ coincide with $f$ by construction. Thanks to the principle of analytic continuation, it follows that they even coincide with $f$ in a thin connected neighborhood of the segment $A\left(\left[\zeta_{1}, \zeta_{2}\right]\right)$. Again thanks to the principle of analytic continuation, $F_{A\left(\zeta_{1}\right), \rho}$ and $F_{A\left(\zeta_{2}\right), \rho}$ coincide in the connected intersection $\Delta_{\rho}^{n}\left(A\left(\zeta_{1}\right)\right) \cap \Delta_{\rho}^{n}\left(A\left(\zeta_{2}\right)\right)$. It follows that they stick together to provide a well defined function $F_{A\left(\zeta_{1}\right), A\left(\zeta_{2}\right), \rho}$ that is holomorphic in $\Delta_{\rho}^{n}\left(A\left(\zeta_{1}\right)\right) \cup \Delta_{\rho}^{n}\left(A\left(\zeta_{2}\right)\right)$. In conclusion, the restriction $\left.F_{A\left(\zeta_{1}\right), A\left(\zeta_{2}\right), \rho}\right|_{\Delta_{\sigma}\left(A\left(\zeta_{1}\right)\right) \cup \Delta_{\sigma}\left(A\left(\zeta_{2}\right)\right)}$ is holomorphic in the union of the two small polydiscs $\Delta_{\sigma}\left(A\left(\zeta_{1}\right)\right) \cup \Delta_{\sigma}\left(A\left(\zeta_{2}\right)\right)$, whenever the intersection $\Delta_{\sigma}\left(A\left(\zeta_{1}\right)\right) \cap \Delta_{\sigma}\left(A\left(\zeta_{2}\right)\right)$ is nonempty. This proves that all the restrictions $\left.F_{A(\zeta), \rho}\right|_{\Delta_{\sigma}^{n}(A(\zeta))}$ stick together in a well defined holomorphic function $F \in \mathcal{O}\left(\mathcal{V}_{\sigma}(A(\bar{\Delta}))\right)$.

In the next theorem (a local continuity principle often used in Me1997, MP1999, MP2002, MP2006a $), A_{1}(\bar{\Delta}) \subset \Omega$, but contrary to Lemma 2.26, $A_{s}(\bar{\Delta})$ may well be not contained in $\Omega$ for $s<1$; nevertheless, the boundaries $A_{s}(\partial \Delta)$ must always stay in $\Omega$.

Theorem 2.27. ([Me1997]) Let $\Omega$ be a nonempty domain in $\mathbb{C}^{n}$ and let $A_{s}: \bar{\Delta} \rightarrow$ $\mathbb{C}^{n}, A_{s} \in \mathcal{O}(\Delta) \cap \mathcal{C}^{1}(\bar{\Delta})$, be a one-parameter family of analytic discs, where $s \in[0,1]$. Assume that there exist two constants $c_{s}$ and $C_{s}$ with $0<c_{s}<C_{s}$ such that

$$
c_{s}\left|\zeta_{2}-\zeta_{1}\right|<\left|A\left(\zeta_{2}\right)-A\left(\zeta_{1}\right)\right|<C_{s}\left|\zeta_{2}-\zeta_{1}\right|,
$$

for all distinct points $\zeta_{1}, \zeta_{2} \in \bar{\Delta}$. Assume that $A_{1}(\bar{\Delta}) \subset \Omega$, set

$$
\rho_{s}:=\inf \left\{\left|z-A_{s}(\zeta)\right|: z \in \partial \Omega, \zeta \in \partial \Delta\right\},
$$

namely $\rho_{s}$ is the polydisc distance between $A_{s}(\partial \Delta)$ and $\partial \Omega$, and set $\sigma_{s}:=$ $\rho_{s} c_{s} / 2 C_{s}$. Then for every holomorphic functions $f \in \mathcal{O}(\Omega)$, and for all $s \in[0,1]$, there exist holomorphic functions $F_{s} \in \mathcal{O}\left(\mathcal{V}_{\sigma_{s}}\left(A_{s}(\bar{\Delta})\right)\right)$ such that $F_{s}=f$ in $\mathcal{V}_{\sigma_{s}}\left(A_{s}(\partial \Delta)\right)$. 
Proof. Let $\mathcal{I} \subset[0,1]$ be the connected set of real $s_{0}$ such that the statement is true for all $s$ with $s_{0} \leqslant s \leqslant 1$. By Lemma 2.26, we already know that $1 \in \mathcal{I}$. We want to prove that $\mathcal{I}=[0,1]$. It suffices to prove that $\mathcal{I}$ is both open and closed.

The fact that $\mathcal{I}$ is closed follows by "abstract nonsense". We claim that $\mathcal{I}$ is also open. Indeed, let $s_{0} \in \mathcal{I}$ and let $s_{1}<s_{0}$ be such that $A_{s_{1}}(\bar{\Delta})$ is contained in $\mathcal{V}_{\sigma_{s_{0}}}\left(A_{s_{0}}(\bar{\Delta})\right)$. Since $F_{s_{0}}=f$ in $\mathcal{V}_{\sigma_{s_{0}}}\left(A_{s_{0}}(\partial \Delta)\right)$ and since the polydisc distance between $A_{s_{1}}(\partial \Delta)$ and $\partial \Omega$ is equal to $\rho_{s_{1}}$, it follows as in the first part of the proof of Lemma 2.26, that the Taylor series of $F_{s_{0}}$ at arbitrary points of the form $A_{s_{1}}(\zeta)$, $\zeta \in \bar{\Delta}$, converges in the polydisc $\Delta_{\rho_{s_{1}}}^{n}\left(A_{s_{1}}(\zeta)\right)$. This gives holomorphic functions $F_{A(\zeta), \rho_{s_{1}}} \in \mathcal{O}\left(\Delta_{\rho_{s_{1}}}^{n}\left(A_{s_{1}}(\zeta)\right)\right)$, for every $\zeta \in \bar{\Delta}$. Reasoning as in the second part of the proof of Lemma 2.26, we obtain a function $F_{s_{1}} \in \mathcal{O}\left(\mathcal{V}_{\sigma_{s_{1}}}\left(A_{s_{1}}(\bar{\Delta})\right)\right)$ with $F_{s_{1}}=f$ in $\mathcal{V}_{\sigma_{s_{1}}}\left(A_{s_{1}}(\partial \Delta)\right)$. This shows that $\mathcal{I}$ is open, as claimed and completes the proof.

2.28. Singularities as complex hypersurfaces. Let $\Omega \subset \mathbb{C}^{n}(n \geqslant 2)$ be a domain. A typical elementary singularity in $\Omega$ is just the zero set $Z_{f}:=\{f=0\}$ of a holomorphic function $f \in \mathcal{O}(\Omega)$ since for instance, the functions $1 / f^{k}, k \geqslant 1$, and $e^{1 / f}$ are holomorphic in $\Omega \backslash Z_{f}$ and singular along $Z_{f}$. Because $\mathbb{C}$ is algebraically closed, the closure in $\bar{\Omega}$ of such $Z_{f}$ 's necessarily intersects $\partial \Omega$. Early in the twentieth century, the italian mathematicians Levi, Severi and B. Segre ([Se1932] $]$ ) interpreted Hartogs' extension theorem as saying that compact sets $K \subset \Omega$ are removable, confirming the observation $\overline{Z_{f}} \cap \partial \Omega \neq \emptyset$.

Definition 2.29. (i) A relatively closed subset $C$ of a domain $\Omega \subset \mathbb{C}^{n}$ is called removable if the restriction map $\mathcal{O}(\Omega) \rightarrow \mathcal{O}(\Omega \backslash C)$ is surjective.

(ii) Such a set $C$ is called locally removable if for every $p \in C$, there exists an open neighborhood $U_{p}$ of $p$ in $\Omega$ such that the restriction map $\mathcal{O}\left(\left(U_{p} \cup \Omega\right) \backslash C\right) \rightarrow$ $\mathcal{O}(\Omega \backslash C)$ is surjective.

Under the assumption that $C$ is contained in a real submanifold of $\Omega$, the general philosophy of removable singularities is that a set too small to be a $Z_{f}$ (viz. a complex $(n-1)$-dimensional variety) is removable. The following theorem collects five statements saying that $C$ is removable provided it cannot contain any complex hypersurface of $\Omega$. Importantly, our submanifolds $N$ of $\Omega$ will always be assumed to be embedded, namely for every $p \in N$, there exist an open neighborhood $U_{p}$ of $p$ in $\Omega$ and a diffeomorphism $\psi_{p}: U_{p} \rightarrow \mathbb{R}^{2 n}$ such that $\psi_{p}\left(N \cap U_{p}\right)=\mathbb{R}^{\operatorname{dim} N} \times\{0\}$.

Theorem 2.30. Let $\Omega$ be a domain of $\mathbb{C}^{n}(n \geqslant 2)$ and let $C \subset \Omega$ be a relatively closed subset. The restriction map $\mathcal{O}(\Omega) \rightarrow \mathcal{O}(\Omega \backslash C)$ is surjective, namely $C$ is removable, under each one of the following five circumstances.

(rm1) $C$ is contained in a connected submanifold $N \subset \Omega$ of codimension $\geqslant 3$.

(rm2) $\mathrm{H}^{2 n-2}(C)=0$. 
(rm3) $C$ is a relatively closed proper subset of a connected $\mathcal{C}^{2}$ submanifold $N \subset \Omega$ of codimension 2.

(rm4) $C=N$ is a connected $\mathcal{C}^{2}$ submanifold $N \subset \Omega$ that is not a complex hypersurface of $\Omega$.

(rm5) $C$ is a closed subset of a connected $\mathcal{C}^{2}$ real hypersurface $M^{1} \subset \Omega$ that does not contain any CR orbit of $M^{1}$.

In (rm1) and in (rm2), $C$ is in fact locally removable. In (rm5), two kinds of CR orbits coexist: those of real dimension $(2 n-2)$, that are necessarily complex hypersurfaces, and those of real dimension $(2 n-1)$, that are open subsets of $M$. It is necessary to exclude them also. Indeed, if for instance $\Omega$ is divided in two connected components $\Omega^{ \pm}$by a globally minimal $M^{1}$, taking $C=M^{1}$, any locally constant function on $\Omega \backslash M^{1}$ equal to two distinct constants $c^{ \pm}$in $\Omega^{ \pm}$does not extend holomorphically through $M^{1}$. The proof of the theorem is elementary $^{22}$ and we will present it in $\S 2.34$ below, as a relevant preliminary to Theorems 4.9, 4.10, 4.31 and 4.32, and to the main Theorem 1.7 of [MP2006a]).

We mention that under the assumption of local boundedness, more massive singularities may be removed. An application of Theorem 1.9(ii) to several complex variables deserves to be emphasized.

Theorem 2.31. ([[HP1970] If $\Omega \subset \mathbb{C}^{n}$ is a domain and if $C \subset \Omega$ is a relatively closed subset satisfying $\mathrm{H}^{2 n-1}(C)=0$, then $C$ is removable for functions holomorphic in $\Omega \backslash C$ that are locally bounded in $\Omega$.

Following [Stu1989] and [Lu1990], we now provide variations on (rm2). Any global complex variety of codimension one in $\mathbb{C}^{n}$ is certainly of infinite $(2 n-2)$ dimensional area.

Theorem 2.32. ([Stu1989]) Every closed set $C \subset \mathbb{C}^{n}$ satisfying $\mathrm{H}^{2 n-2}(C)<\infty$ is removable for $\mathcal{O}\left(\mathbb{C}^{n}\right)$.

The result also holds true in the unit ball $\mathbb{B}_{n}$ of $\mathbb{C}^{n}$, provided one computes the $(2 n-2)$-dimensional Hausdorff measure with respect to the distance function derived from the Bergman metric ([Stu1989]). Also, if $\Sigma$ is an arbitrary complex $k$-dimensional closed submanifold of $\mathbb{C}^{n}$, every closed subset $C \subset \Sigma$ with $\mathrm{H}^{2 k-2}(C)<\infty$ is removable for $\mathcal{O}(\Sigma \backslash C)$ ([Stu1989]).

A finer variation on the theme requires that $\mathrm{H}^{2 n-2}\left(C \cap R \mathbb{B}_{n}\right)$ does not grow too rapidly as a function of the radius $R \rightarrow \infty$. For instance ([Stu1989]), a closed subset $C \subset \mathbb{C}^{2}$ that satisfies $\mathrm{H}^{2}\left(C \cap R \mathbb{B}_{n}\right)<c R^{2}$ for all large $R$ is removable, provided $c<\frac{\pi^{2}}{4 \sqrt{2}}$. It is expected that $c<\pi$ is optimal, since the line $L:=\left\{\left(z_{1}, 0\right)\right\}$ satisfies $\mathrm{H}^{2}\left(L \cap R B_{2}\right)=\pi R^{2}$.

\footnotetext{
${ }^{22}$ Some refinements of the statements may be formulated, for instance assuming in (rm3) and (rm4) that $N$ is $\mathcal{C}^{1}$ and has some metrically thin singularities ([CSt1994).
} 
Yet another variation, raised in [Stu1989], is as follows. Consider a closed set $C$ in the complex projective space $P_{n}(\mathbb{C})(n \geqslant 2)$ such that the Hausdorff $(2 n-2)$-dimensional measure (with respect to the Fubini-Study metric) of $C$ is strictly less than that of any complex algebraic hypersurface of $P_{n}(\mathbb{C})$. Is it true that $C$ is a removable singularity for meromorphic functions, in the sense that every meromorphic function on $P_{n}(\mathbb{C}) \backslash C$ extends meromorphically through $C$ ? This question was answered by Lupacciolu.

Let $d_{\mathrm{FS}}(z, w)$ denote the geodesic distance between two points $z, w \in P_{n}(\mathbb{C})$ relative to the Fubini-Study metric and let $\mathrm{H}_{\mathrm{FS}}^{\ell}$ denote the $\ell$-dimensional Hausdorff measure in $P_{n}(\mathbb{C})$ computed with $d_{\mathrm{FS}}$. Given a nonempty closed subset $C \subset P_{n}(\mathbb{C})$, define:

$$
\rho(C):=\frac{\max _{z \in P_{n}(\mathbb{C})} d_{\mathrm{FS}}(z, C)}{\max _{z, w \in P_{n}(\mathbb{C})} d_{\mathrm{FS}}(z, w)}=\frac{\max _{z \in P_{n}(\mathbb{C})} d_{\mathrm{FS}}(z, C)}{\operatorname{diam}_{\mathrm{FS}}\left(P_{n}(\mathbb{C})\right)} \leqslant 1 .
$$

If the Fubini-Study metric is normalized so that vol $P_{n}(\mathbb{C})=1$, the $(2 n-2)$ dimensional volume of an irreducible complex algebraic hypersurface $\Sigma \subset P_{n}(\mathbb{C})$ is equal to $\operatorname{deg} V$ and $\mathrm{H}_{\mathrm{FS}}^{2 n-2}(C)=(4 / \pi)^{n-1}(n-1) ! \operatorname{deg} V$. It follows that the minimum value of $\mathrm{H}^{2 n-2}(\Sigma)$ is equal to $(4 / \pi)^{n-1}(n-1)$ ! and is attained for $V$ equal to any hyperplane of $P_{n}(\mathbb{C})$. Let $\mathcal{M}$ denote the sheaf of meromorphic functions on $P_{n}(\mathbb{C})$.

Theorem 2.33. ([Lu1990]) Let $C \subset P_{n}(\mathbb{C})$ be a closed subset such that

$$
\mathrm{H}_{\mathrm{FS}}^{2 n-2}(C)<[\rho(C)]^{4 n-4}(4 / \pi)^{n-1}(n-1) !
$$

Then the restriction map $\mathcal{M}\left(P_{n}(\mathbb{C})\right) \longrightarrow \mathcal{M}\left(P_{n}(\mathbb{C}) \backslash C\right)$ is onto.

2.34. Proof of Theorem 2.30. We claim that we may focus our attention only on (rm2) and on (rm5). Indeed, since a submanifold $N \subset \Omega$ of codimension $\geqslant 3$ satisfies $\mathrm{H}^{2 n-2}(N)=0,(\mathbf{r m} \mathbf{1})$ is a corollary of $(\mathbf{r m} 2)$.

In both ( $\mathbf{r m 3})$ and $(\mathbf{r m 4})$, we may include $N$ in some $\mathcal{C}^{2}$ hypersurface $M^{1}$ of $\mathbb{C}^{n}$, looking like a thin strip elongated along $N$. We claim that $C$ then does not contain any CR orbit of any such $M^{1}$, so that (rm5) applies. Indeed, CR orbits of $M^{1}$ being of dimension $(2 n-2)$ or $(2 n-1)$ and $C$ being already contained in the $(2 n-2)$-dimensional $N \subset M^{1}$, it could only happen that $C=N=\Sigma$ identifies as a whole to a connected (CR orbit) complex hypersurface $\Sigma \subset M^{1}$. But this is excluded by the assumption that $C \neq N$ in $(\mathbf{r m 3})$ and by the existence of generic points in (rm4).

Firstly, we prove ( $\mathbf{r m 2}$ ). We show that $C$ is locally removable. Let $p \in C$ and let $B_{p} \subset \Omega$ be a small open ball centered at $p$. By a relevant application of Proposition 1.2(5), one may verify that for almost every complex line $\ell$ passing through $p$, the intersection $\ell \cap B_{p} \cap C$ is reduced to $\{p\}$. Choose such a line $\ell_{1}$. Centering coordinates at $p$ and rotating them if necessary, we may assume 
that $\ell_{1}=\left\{\left(z_{1}, 0, \ldots, 0\right)\right\}$, whence for $\varepsilon>0$ small and fixed, the disc $A_{\varepsilon}(\zeta):=$ $(\varepsilon \zeta, 0, \ldots, 0)$ satisfies $A_{\varepsilon}(\partial \Delta) \cap C=\emptyset$.

Fix such a small $\varepsilon_{0}>0$ and set $\rho_{0}:=\operatorname{dist}\left(A_{\varepsilon_{0}}(\partial \Delta), C\right)>0$. For $\tau=$ $\left(\tau_{2}, \ldots, \tau_{n}\right) \in \mathbb{C}^{n-1}$ satisfying $|\tau|<\frac{1}{2} \rho_{0}$, set $A_{\varepsilon_{0}, \tau}(\zeta):=\left(\varepsilon_{0} \zeta, \tau_{2}, \ldots, \tau_{n}\right)$. Letting $s \in[0,1]$, we interpolate between $A_{\varepsilon_{0}, 0}$ and $A_{\varepsilon_{0}, \tau}$ by defining

$$
A_{\varepsilon_{0}, \tau, s}(\zeta):=\left(\varepsilon_{0} \zeta, s \tau_{2}, \ldots, s \tau_{n}\right) \text {. }
$$

Since $|\tau|<\frac{1}{2} \rho_{0}$, these discs all satisfy dist $\left(A_{\varepsilon_{0}, \tau, s}(\partial \Delta), C\right) \geqslant \frac{1}{2} \rho_{0}$. Since the embedded disc $A_{\varepsilon_{0}, \tau, 1}(\bar{\Delta})$ is 2-dimensional and since $\mathrm{H}^{2 n-2}(C)=0$, Proposition 1.2(5) assures that for almost every $\tau$ with $|\tau|<\frac{1}{2} \rho_{0}$, its intersection with $C$ is empty. Thus, we may apply the continuity principle Theorem 2.27 , setting $c_{s}=\frac{1}{2} \varepsilon_{0}, C_{s}=2 \varepsilon_{0}$ and $\rho_{s}:=\frac{1}{2} \rho_{0}$ uniformly for every $s \in[0,1]$, whence $\sigma_{s}=\frac{1}{8} \rho_{0}$ independently of the smallness of $\tau$ : for every $f \in \mathcal{O}(\Omega \backslash C)$, there exists $F_{0} \in \mathcal{O}\left(\mathcal{V}_{\frac{\rho_{0}}{8}}\left(A_{\varepsilon_{0}, \tau, 0}(\bar{\Delta})\right)\right)$ with $F_{0}=f$ in $\mathcal{V}_{\frac{\rho_{0}}{8}}\left(A_{\varepsilon_{0}, \tau, 0}(\partial \Delta)\right)$. But since $H^{2 n-2}(C)=0$, for every connected open set $\mathcal{V} \subset \Omega$, the intersection $\mathcal{V} \cap(\Omega \backslash C)$ is also connected (Proposition 1.2(4)), so $F_{0}$ and $f$ stick together as a well defined function holomorphic in $\Omega \cup \mathcal{V}_{\frac{\rho_{0}}{8}}\left(A_{\varepsilon_{0}, \tau, 0}(\bar{\Delta})\right)$. If $\tau$ was chosen sufficiently small, it is clear that $p=0 \in \mathbb{C}^{n}$ is absorbed in $\mathcal{V}_{\frac{\rho_{0}}{8}}\left(A_{\varepsilon_{0}, \tau, 0}(\bar{\Delta})\right)$, hence removable.

Secondly, we prove (rm5). Let $C \subset M^{1}$ containing no CR orbit and define

$$
\mathcal{C}^{\prime}:=\left\{C^{\prime} \subset C \text { closed, } \forall f \in \mathcal{O}(\Omega \backslash C), \exists f^{\prime} \in \mathcal{O}\left(\Omega \backslash C^{\prime}\right) \text { with }\left.f^{\prime}\right|_{\Omega \backslash C}=f\right\} .
$$

Lemma 2.35. If $C_{1}^{\prime}, C_{2}^{\prime} \in \mathcal{C}^{\prime}$, then $C_{1}^{\prime} \cap C_{2}^{\prime} \in \mathcal{C}^{\prime}$.

Proof. Let $f_{j}^{\prime} \in \mathcal{O}\left(\Omega \backslash C_{j}^{\prime}\right), j=1,2$, with $\left.f_{j}^{\prime}\right|_{\Omega \backslash C}=f$. We claim that $f_{1}^{\prime}$ and $f_{2}^{\prime}$ match up on $C \backslash\left(C_{1}^{\prime} \cup C_{2}^{\prime}\right)$, hence define together a holomorphic function $f_{12}^{\prime} \in$ $\mathcal{O}\left(\Omega \backslash\left(C_{1}^{\prime} \cap C_{2}^{\prime}\right)\right)$ with $\left.f_{12}^{\prime}\right|_{\Omega \backslash C}=f$. Indeed, choose an arbitrary point $p \in$ $C \backslash\left(C_{1}^{\prime} \cup C_{2}^{\prime}\right)$. There exists a small open ball $B_{p}$ centered at $p$ with $B_{p} \cap\left(C_{1}^{\prime} \cup\right.$ $\left.C_{2}^{\prime}\right)=\emptyset$. Since $f_{1}^{\prime}=f_{2}^{\prime}=f$ at least in the dense subset $B_{p} \backslash M^{1}$ of $M^{1} \cap B_{p}$, by continuity of $f_{1}^{\prime}$ and of $f_{2}^{\prime}$ in $B_{p}$, necessarily $f_{1}^{\prime}(p)=f_{2}^{\prime}(p)$.

Next, we define

$$
\widetilde{C}:=\bigcap_{C^{\prime} \in \mathcal{C}^{\prime}} C^{\prime}
$$

Intuitively, $\widetilde{C}$ is the "nonremovable core" of $C$. By the lemma, for every $f \in \mathcal{O}(\Omega \backslash C)$, there exists $\widetilde{f} \in \mathcal{O}(\Omega \backslash \widetilde{C})$ with $\left.\widetilde{f}\right|_{\Omega \backslash C}=f$. To prove (rm5), we must establish that $\widetilde{C}=\emptyset$. Reasoning by contradiction, we assume that $\widetilde{C} \neq \emptyset$ and we apply to $C:=\widetilde{C}$ the lemma below, which is in fact a corollary of Trépreau's Theorem 2.4(V). Of course, $\widetilde{C}$ cannot contain any CR orbit of $M^{1}$. Remind ( $\left.\$ 1.27(\mathrm{III}), \S 4.9(\mathrm{III})\right)$ that for us, local CR orbits are not germs, but local CR submanifolds of a certain small size. 
Lemma 2.36. Let $M^{1} \subset \mathbb{C}^{n}(n \geqslant 2)$ be a $\mathcal{C}^{2}$ hypersurface and let $C \subset M^{1}$ be a closed subset containing no CR orbit of $M^{1}$. Then there exists at least one point $p \in C$ such that for every neighborhood $V_{p}^{1}$ of $p$ in $M^{1}$, we have:

$$
V_{p}^{1} \cap \mathcal{O}_{C R}^{\text {loc }}\left(M^{1}, p\right) \not \subset C,
$$

and in addition, all such points $p$ are locally removable.

Then $\widetilde{f}$ extends holomorphically to a neighborhood of all such points $p \in \widetilde{C}$, contradicting the definition of $\widetilde{C}$, hence completing the proof of (rm5).

Proof. If $\mathcal{O}_{C R}^{\text {loc }}\left(M^{1}, q\right) \subset C$ for every $q \in C$, then small complex-tangential curves issued from $q$ necessarily remain in $\mathcal{O}_{C R}^{l o c}\left(M^{1}, q\right)$, hence in $C$, and pursuing from point to point, global complex-tangential curves issued from $q$ remain in $C$, whence $\mathcal{O}_{C R}\left(M^{1}, q\right) \subset C$, contrary to the assumption.

So, let $p \in C$ with $\mathcal{O}_{C R}^{l o c}\left(M^{1}, p\right) \not \subset C$. To pursue, we need that $M^{1}$ is minimal at $p$. Since $M^{1}$ is a hypersurface, it might only happen that $\mathcal{O}_{C R}^{\text {loc }}\left(M^{1}, p\right)$ is a local complex hypersurface, a bad situation that has to be changed in advance.

Fortunately, without altering the conclusion of the lemma (and of (rm5)), we have the freedom of perturbing the auxiliary hypersurface $M^{1}$, leaving $C$ fixed of course. Thus, assuming that $\mathcal{O}_{C R}\left(M^{1}, p\right)$ is a complex hypersurface, we claim that there exists a small (in $\mathcal{C}^{2}$ norm) deformation $M_{d}^{1}$ of $M^{1}$ supported in a neighborhood of $p$ with $M_{d}^{1} \supset C$ such that $M_{d}^{1}$ is minimal at $p$.

Indeed, let $\left(q_{k}\right)_{k \in \mathbb{N}}$ be a sequence of points tending to $p$ in $\mathcal{O}_{C R}^{\text {loc }}\left(M^{1}, p\right)$ with $q_{k} \notin C$. To destroy the local complex hypersurface $\mathcal{O}_{C R}^{l o c}\left(M^{1}, p\right)$, it suffices to achieve, by means of cut-off functions, small bump-deformations of $M$ centered at all the $q_{k}$; it is easy to write the technical details in terms of a local graphed representation $v=\varphi^{1}(z, u)$ for $M^{1}$. Outside a small neighborhood of the union of the $q_{k}, M_{d}^{1}$ coincides with $M^{1}$. Then the resulting $M_{d}^{1}$ is necessarily minimal at $p$, since if it where not, the uniqueness principle ${ }^{23}$ for complex manifolds would force $\mathcal{O}_{C R}^{l o c}\left(M^{1}, p\right)=\mathcal{O}_{C R}^{l o c}\left(M_{d}^{1}, p\right)$, but $M_{d}^{1}$ does not contains the $q_{k}$.

So we can assume that $M^{1}$ is minimal at every point $p \in C$ at which $\mathcal{O}_{C R}^{\text {loc }}\left(M^{1}, p\right) \not \subset C$. Let $B_{p} \subset \mathbb{C}^{n}$ be a small open ball centered at $p$ with $B_{p} \cap M^{1} \subset \mathcal{O}_{C R}^{\text {loc }}\left(M^{1}, p\right)$. We will show that $\mathcal{O}(\Omega \backslash C)$ extends holomorphically to $B_{p}$. By assumption, $B_{p} \cap M^{1} \not \subset C$, hence $B_{p} \backslash C$ is connected, a fact that will insure monodromy.

Fixing $v_{p} \in T_{p} \mathbb{C}^{n} \backslash\{0\}$ with $v_{p} \notin T_{p} M^{1}$, we consider the global translations

$$
M_{s}^{1}:=M^{1}+s v_{p}, \quad s \in \mathbb{R},
$$

of $M^{1}$. Let $f \in \mathcal{O}(\Omega \backslash C)$ be arbitrary. For small $s \neq 0, M_{s}^{1} \cap B_{p}$ does not intersect $M^{1}$, hence the restriction $\left.f\right|_{M_{s}^{1} \cap B_{p}}$ is a $\mathcal{C}^{2} \mathrm{CR}$ function on $M_{s}^{1} \cap B_{p}$ (but $\left.f\right|_{M_{0}^{1} \cap B_{p}}$ has possible singularities at points of $C \cap B_{p}$ ).

\footnotetext{
${ }^{23}$ Minimalization at a point takes strong advantage of the rigidity of complex hypersurfaces in this argument.
} 
With $U_{p}:=M^{1} \cap B_{p}$, Theorem $2.4(\mathrm{~V})$ says that $\mathcal{C}_{C R}^{0}\left(U_{p}\right)$ extends holomorphically to some one-sided neighborhood $\omega_{p}^{ \pm}$of $M^{1}$ at $p$. Reorienting if necessary, we may assume that the extension side is $\omega_{p}^{-}$and that $p+s v_{p} \in B_{p}^{+}$for $s>0$ small. The statement and the proof of Theorem 2.4(V) are of course invariant by translation. Hence $\mathcal{C}_{C R}^{0}\left(U_{p}+s v_{p}\right)$ extends holomorphically to $\omega_{p}^{-}+s v_{p}$, for every $s>0$. It is geometrically clear that for $s>0$ small enough, $\omega_{p}^{-}+s v_{p}$ contains $p$. Thus $\left.f\right|_{M_{s}^{1} \cap B_{p}}$ extends holomorphically to a neighborhood of $p$ for such $s$. Monodromy of the extension follows from the fact that $B_{p}^{\prime} \backslash C$ is connected for every open ball $B_{p}^{\prime}$ centered at $p$. This completes the proof of the lemma.

2.37. Removability and extension of complex hypersurfaces. Let $\Omega \subset \mathbb{C}^{n}$ be a domain. Theorem 2.30 (rm4) shows that a connected 2-codimensional submanifold $N \subset \Omega$ is removable provided it is not a complex hypersurface, or equivalently, is generic somewhere. Conversely, assume that $\Omega$ is pseudoconvex and let $H \subset \Omega$ be a (not necessarily connected) closed complex hypersurface. Then $\Omega \backslash H$ is (obviously) locally pseudoconvex at every point, hence the characterization of domains of holomorphy yields a function $f \in \mathcal{O}(\Omega \backslash H)$ whose domain of existence is exactly $\Omega \backslash H$. Thus, $H$ is nonremovable. But in a nonpseudoconvex domain, closed complex hypersurfaces may be removable.

Example 2.38. For $\varepsilon>0$ small, consider the following nonpseudoconvex subdomain of $\mathbb{B}_{2}$, defined as the union of a spherical shell together with a thin rod of radius $\varepsilon$ directed by the $y_{2}$-axis:

$$
\Omega_{\varepsilon}:=\left\{1 / 2<\left|z_{1}\right|^{2}+\left|z_{2}\right|^{2}<1\right\} \bigcup\left(\mathbb{B}_{2} \cap\left\{x_{1}^{2}+y_{1}^{2}+x_{2}^{2}<\varepsilon^{2}\right\}\right) .
$$

Then the intersection of the $z_{1}$-axis with the spherical shell, namely

$$
H:=\left\{\left(z_{1}, 0\right): 1 / 2<\left|z_{1}\right|<1\right\}
$$

is a relatively closed complex hypersurface of $\Omega_{\varepsilon}$ homeomorphic to an open annulus. We claim that $H$ is removable.

Indeed, applying the continuity principle along discs parallel to the $z_{1}$-axis, $\mathcal{O}\left(\Omega_{\varepsilon}\right)$ extends holomorphically to $\mathbb{B}_{2} \backslash\left\{z_{2}=0\right\}$. Since the open small disc $\left\{\left(z_{1}, 0\right):\left|z_{1}\right|<\varepsilon\right\}$, considered as a subset of the closed complex hypersurface $\widetilde{H}$ of $\mathbb{B}_{2}$ defined by

$$
\widetilde{H}:=\left\{\left(z_{1}, 0\right):\left|z_{1}\right|<1\right\}
$$

is contained in the thin rod, hence in $\Omega_{\varepsilon}$, Theorem $2.30(\mathbf{r m 3})$ finishes to show that

$$
\mathrm{E}\left(\Omega_{\varepsilon} \backslash H\right)=\mathrm{E}\left(\Omega_{\varepsilon}\right)=\mathbb{B}_{2} .
$$

In such an example, we point out that the closed complex hypersurface $H \subset \Omega_{\varepsilon}$ extends as the closed complex hypersurface $\widetilde{H} \subset \mathrm{E}\left(\Omega_{\varepsilon} \backslash H\right)$ but that the intersection

$$
\widetilde{H} \cap \Omega_{\varepsilon}=\left\{\left(z_{1}, 0\right):\left|z_{1}\right|<\varepsilon\right\} \bigcup\left\{\left(z_{1}, 0\right): 1 / 2<\left|z_{1}\right|<1\right\}
$$


is strictly bigger than $H$.

Problem 2.39. Understand which relatively closed complex hypersurfaces of a general domain $\Omega \subset \mathbb{C}^{n}$ are removable.

We thus consider a (possibly singular and reducible) closed complex hypersurface $H$ of $\Omega$. Basic properties of complex analytic sets ([Ch1991]) insure that $H=\bigcup_{j \in J} H_{j}$ decomposes into at most countably many closed complex hypersurfaces $H_{j} \subset \Omega$ that are irreducible.

Definition 2.40. We say that $H_{j}$ allows an $H$-compatible extension to $\mathrm{E}(\Omega)$ if there exists an irreducible closed complex hypersurface $\widetilde{H}_{j}$ of $\mathrm{E}(\Omega)$ extending $H_{j}$ in the sense that $H_{j} \subset \widetilde{H}_{j} \cap \Omega$ whose intersection with $\Omega$ remains contained in $H$ :

$$
\widetilde{H}_{j} \cap \Omega \subset \bigcup_{j^{\prime} \in J} H_{j^{\prime}}
$$

The principle of analytic continuation for irreducible complex analytic sets ([Ch1991]) assures that $H_{j}$ has at most one $H$-compatible extension. On the other hand, $\widetilde{H}_{j}$ may be an $H$-compatible extension of several $H_{j^{\prime}}$. In the above example, the removable annulus $H$ had no $H$-compatible extension to $\mathrm{E}(\Omega)$.

Theorem 2.41. ([Dl1977, [aPf2000]) Let $\Omega \subset \mathbb{C}^{n}(n \geqslant 2)$ be a domain and let $H=\bigcup_{j \in J} H_{j}$ be a closed complex hypersurface of $\Omega$, decomposed into irreducible components $H_{j}$. Set

$$
J_{\text {comp }}:=\left\{j \in J: H_{j} \text { allows an } H \text {-compatible extension } \widetilde{H}_{j} \text { to } \mathrm{E}(\Omega)\right\} .
$$

Then

$$
\mathrm{E}(\Omega \backslash H)=\mathrm{E}(\Omega) \backslash \bigcup_{j \in J_{\text {comp }}} \widetilde{H}_{j} .
$$

In particular, $H$ is removable (resp. nonremovable) if and only if $J_{\text {comp }}=\emptyset$ (resp. $J_{\text {comp }} \neq \emptyset$ ).

This statement was obtained after a chain of generalizations originating from the classical results of Hartogs [Ha1909] and of Oka [Ok1934]. In [GR1956] it was proved for the case that $H$ is of the form $\Omega \cap \widetilde{H}, \widetilde{H} \subset \mathrm{E}(\Omega)$, and in [Nis1962] under the additional assumption that $\mathrm{E}(\Omega \backslash H)$ is a subset of $\mathrm{E}(\Omega)$ (a priori, it is only a set over $\mathrm{E}(\Omega)$ ). Actually Theorem 2.41 was stated in [D11977] even for Riemann domains $\Omega$. But it was remarked in JaPf2000 (p. 306) that the proof in D11977 is complete only if the functions of $\mathcal{O}(\Omega)$ separate the points of $\Omega$, i.e. if $\Omega$ can be regarded as a subdomain of $\mathrm{E}(\Omega)$. Actually the proof in D11977] starts from the special case where $\Omega$ is a Hartogs figure, which can be treated by a subtle geometric examination. Then a localization argument shows that extension of hypersurfaces which are singularity loci of holomorphic functions cannot stop when passing from $\Omega$ to $\mathrm{E}(\Omega)$. But in the nonseparated case, the global effect 
of identifying points of $\Omega$ interferes nastily, and it is unclear how to justify the localization argument. The final step for general Riemann domains was achieved by the second author by completely different methods.

Theorem 2.42. ([Po2002]) Let $\pi: X \rightarrow \mathbb{C}^{n}$ be an arbitrary Riemann domain, and let $H \subset X$ be a closed complex hypersurface. Denote by $\alpha: X \rightarrow \mathrm{E}(X)$ the canonical immersion of $X$ into $\mathrm{E}(X)$. Then there is a closed complex hypersurface $\widetilde{H}$ of $\mathrm{E}(X)$ with $\alpha^{-1}(\widetilde{H}) \subset H$ such that

$$
\mathrm{E}(X \backslash H)=\mathrm{E}(X) \backslash \widetilde{H} .
$$

Let us briefly sketch the main idea of the proof ([Po2002]). The essence of the argument is to reduce extension of hypersurfaces to that of meromorphic functions. For every pseudoconvex Riemann domain $\pi: X \rightarrow \mathbb{C}^{n}$, there exists $f \in \mathcal{O}(X) \cap L^{2}(X)$ having $X$ as domain of existence whose growth is controlled by some power of the polydisc distance to the abstract boundary $\breve{\partial} X$. At boundary points where $\breve{\partial} X$ can be locally identified with a complex hypersurface, $f$ has just a pole of positive order. One can deduce that those hypersurfaces $H$ of $X$ along which some holomorphic function on $X \backslash H$ becomes singular can be represented as the polar locus of some meromorphic function $g$ defined in $X$. But $g$ extends meromorphically to $\mathrm{E}(X)$, and the polar locus of the extension yields the desired extension of $H$.

\section{§3. HuLLS AND REMOVABLE SINGULARITIES AT THE BOUNDARY}

3.1. Motivations for removable singularities at the boundary. As already observed in Section 1, beyond the harmonious realm of pseudoconvexity, the general problem of understanding compulsory holomorphic (or CR) extension is intrinsically rich and open. Some elementary Baire category arguments show that most domains are not pseudoconvex, most CR manifolds have nontrivial disc-envelope, and most compact sets have nonempty essential polynomial hull. Hence, the Grail for the theory of holomorphic extension would comprise:

- a geometric and constructive view of the envelope of holomorphy of most domains, following the Behnke-Sommer Kontinuitätssatz and Bishop's philosophy;

- a clear correspondence between function-theoretic techniques, for instance those involving $\bar{\partial}$ arguments, and geometric techniques, for instance those involving families of complex analytic varieties.

Several applications of the study of envelopes of holomorphy appear, for instance in the study of boundary regularity of solutions of the $\bar{\partial}$-complex, in the complex Plateau problem, in the study of CR mappings, in the computation of polynomial hulls and in removable singularities, the topics of this Part VI and of [MP2006] ]. 
In the 1980's, rapid progress in the understanding of the boundary behavior of holomorphic functions led many authors to study the structure of singularities up to the boundary. In $\S 2.28$, we discussed removability of relatively closed subsets $C$ of domains $\Omega \subset \mathbb{C}^{n}$, i.e. the problem whether $\mathcal{O}(\Omega) \rightarrow \mathcal{O}(\Omega \backslash C)$ is surjective. Typically $C$ was supposed to be lower-dimensional and its geometry near $\partial \Omega$ was irrelevant. Now we assume $\Omega$ to be bounded in $\mathbb{C}^{n}(n \geqslant 2)$ and we consider compact subsets $K$ of $\bar{\Omega}$, possibly meeting $\partial \Omega$.

Problem 3.2. Find criteria of geometric, or of function-theoretic nature, assuring that the restriction map $\mathcal{O}(\Omega) \rightarrow \mathcal{O}(\Omega \backslash K)$ is surjective.

If $K \subset \bar{\Omega} \cap \partial \Omega=\emptyset$ and $\Omega \backslash K$ is connected, surjectivity follows from the Hartogs-Bochner extension Theorem 1.9(V). Since this theorem even gives extension of CR functions on $\partial \Omega$, it seems reasonable to ask for holomorphic extension of CR functions on $\partial \Omega \backslash K$, and then it is natural to assume that $K$ is contained in $\partial \Omega$. Hence the formulation of a second trend of questions ${ }^{24}$.

Problem 3.3. Let $K$ is a compact subset of $\partial \Omega$ such that $\partial \Omega \backslash K$ is a hypersurface of class at least $\mathcal{C}^{1}$. Understand under which circumstances $C R$ functions of class $\mathcal{C}^{0}$ or $L_{l o c}^{\mathrm{p}}$ on $\partial \Omega \backslash K$ extend holomorphically to $\Omega$.

A variant of these two problems consists in assuming that functions are holomorphic in some thin (one-sided) neighborhood of $\partial \Omega \backslash K$. In all the theorems that will be surveyed below, it appears that the thinness of the (one-sided) neighborhood of $\partial \Omega \backslash K$ has no influence on extension, as in the original Hartogs theorem. In this respect, it is of interest to immediately indicate the connection of these two problems with the problem of determining certain envelopes of holomorphy.

In the second problem, the hypersurface $\partial \Omega \backslash K$ is often globally minimal, a fact that has to be verified or might be one of the assumptions of a theorem. For instance, several contributions deal with the paradigmatic case where $\partial \Omega$ is at least $\mathcal{C}^{2}$ and strongly pseudoconvex (hence obviously globally minimal). Then thanks to the elementary Levi-Lewy extension theorem (Theorem 1.18(V), lemma 2.2(V) and $\S 2.10(\mathrm{~V}))$, there exists a one-sided neighborhood $\mathcal{V}(\partial \Omega \backslash K)$ of $\partial \Omega \backslash K$ contained in $\Omega$ to which both $\mathcal{C}_{C R}^{0}(\partial \Omega \backslash K)$ and $L_{l o c, C R}^{\mathrm{p}}(\partial \Omega \backslash K)$ extend holomorphically. The size of $\mathcal{V}(\partial \Omega \backslash K)$ depends only on the local geometry of $\partial \Omega$, because $\mathcal{V}(\partial \Omega \backslash K)$ is obtained by gluing small discs (Part V). In fact, an inspection of the proof of the Levi-Lewy extension theorem together with an application of the continuity principle shows also that the envelope of holomorphy of any thin one-sided neighborhood $\mathcal{V}^{\prime}(\partial \Omega \backslash K)$ (not necessarily contained in $\Omega$ !) contains a one-sided neighborhood $\mathcal{V}(\partial \Omega \backslash K)$ of $\partial \Omega \backslash K$ contained in the pseudoconvex domain $\Omega$ that has a fixed, incompressible size ${ }^{25}$.

\footnotetext{
${ }^{24} \mathrm{CR}$ distributions may also be considered, but in the sequel, we shall restrict considerations to continuous and integrable CR functions.

${ }^{25}$ To be rigorous: for every holomorphic function $f \in \mathcal{O}\left(\mathcal{V}^{\prime}(\partial \Omega \backslash K)\right)$, there exists a holomorphic function $F \in \mathcal{O}(\mathcal{V}(\partial \Omega \backslash K))$ that coincides with $f$ in a possibly smaller
} 
As they are formulated, the above two problems turn out to be slightly too restrictive. In fact, the final goal is to understand the envelope $\mathrm{E}(\mathcal{V}(\partial \Omega \backslash K))$, or at least to describe some significant part of $\mathrm{E}(\mathcal{V}(\partial \Omega \backslash K))$ lying above $\Omega$. Of course, the question to which extent is the geometry of $\mathrm{E}(\mathcal{V}(\partial \Omega \backslash K)$ ) accessible (constructively speaking) depends sensitively on the shape of $\Omega$. Surely, the strictly pseudoconvex case is the easiest and the best understood up to now. In what follows we will encounter situations where $\mathrm{E}(\mathcal{V}(\partial \Omega \backslash K))$ contains $\Omega \backslash \widehat{K}$, for some subset $\widehat{K} \subset \bar{\Omega}$ defined in function-theoretic terms and depending on $K \subset \partial \bar{\Omega}$. We will also encounter situations where $\mathrm{E}(\mathcal{V}(\Omega \backslash K))$ is necessarily multisheeted over $\mathbb{C}^{n}$. In this concern, we will see a very striking difference between the complex dimensions $n=2$ and $n \geqslant 3$.

In the last two decades, a considerable interest has been devoted to a subproblem of these two problems, especially with the objective of characterizing the singularities at the boundary that are removable.

Definition 3.4. In the second Problem 3.3, the compact subset $K \subset \partial \Omega$ is called CR-removable if for every CR function $f \in \mathcal{C}_{C R}^{0}(\partial \Omega \backslash K)$ (resp. $f \in$ $L_{l o c, C R}^{\mathrm{p}}(\partial \Omega \backslash K)$ ), there exists $F \in \mathcal{O}(\Omega) \cap \mathcal{C}^{0}(\bar{\Omega} \backslash K)$ (resp. $F \in \mathcal{O}(\Omega) \cap$ $H_{l o c}^{\mathrm{p}}(\bar{\Omega} \backslash K)$ ) with $\left.F\right|_{\partial \Omega \backslash K}=f$ (resp. locally at every point $p \in \partial \Omega \backslash K$, the $L_{l o c, C R}^{\mathrm{p}}$ boundary value of $F$ equals $f$ ).

Before exposing and surveying some major results we would like to mention that a complement of information and different approaches may be found in the two surveys [Stu1993, CSt1994], in the two monographs [Ky1995, Lt1997] and in the articles [Stu1981, LT1984, Lu1986, Lu1987, Jö1988, Lt1988, Stu1989, Ky1990, Ky1991, Stu1991, FS1991, Jö1992, KN1993, Du1993, LS1993, Lu1994, AC1994, 『ö1995, KR1995, 『ö1999a, 『ö1999b, 『S2000, 『P2002, 『S2004].

3.5. Characterization of removable sets contained in strongly pseudoconvex boundaries. Taking inspiration from the pivotal Oka theorem, one of the goals of the study of removable singularities ([Stu1993] $]$ ) is to characterize removability in function-theoretically significant terms, especially in terms of convexity with respect to certain spaces of functions. In the very beginnings of Several Complex Variables, polynomial convexity appeared in connexion with holomorphic approximation. According to the Oka-Weil theorem ([AW1998]), functions that are holomorphic in some neighborhood of a polynomially convex compact set $K \subset \mathbb{C}^{n}$ may be approximated uniformly by polynomials. Later on, holomorphic convexity appeared to be central in Stein theory ([Ḧ̈1973]), one of the seminal frequently used idea being to encircle convex compact sets by convenient analytic polyhedra.

one-sided neighborhood $\mathcal{V}^{\prime \prime}(\partial \Omega \backslash K) \subset \mathcal{V}^{\prime}(\partial \Omega \backslash K)$. Details of the proof (involving a deformation argument) will not be provided here (see [Me1997, Jö1999a]). 
The notion of convexity adapted to our pruposes is the following. By $\mathcal{O}(\bar{\Omega})$, we denote the ring of functions that are holomorphic in some neighborhood of the closure $\bar{\Omega}$ of a domain $\Omega \subset \mathbb{C}^{n}$. As in the concept of germs, the neighborhood may depend on the function.

Definition 3.6. Let $\Omega \Subset \mathbb{C}^{n}$ be a bounded domain and let $K \subset \bar{\Omega}$ be a compact set. The $\mathcal{O}(\bar{\Omega})$-convex hull of $K$ is

$$
\widehat{K}_{\mathcal{O}(\bar{\Omega})}:=\left\{z \in \bar{\Omega}:|g(z)| \leqslant \max _{w \in K}|g(w)| \text { for all } g \in \mathcal{O}(\bar{\Omega})\right\} .
$$

If $K=\widehat{K}_{\mathcal{O}(\bar{\Omega})}$, then $K$ is called $\mathcal{O}(\bar{\Omega})$-convex.

If $\Omega$ is strongly pseudoconvex, a generalization of the Oka-Weil theorem shows that every function which is holomorphic in a neighborhood of some $\mathcal{O}(\bar{\Omega})$ convex compact set $K \subset \bar{\Omega}$ may be approximated uniformly on $K$ by functions of $\mathcal{O}(\bar{\Omega})$ (nevertheless, for nonpseudoconvex domains, this approximation property fails ${ }^{26}$.

We may now begin with the formulation of a seminal theorem due to Stout that inspired several authors. We state the CR version, due to Lupacciolu ${ }^{27}$.

Theorem 3.7. ([Stu1981, Lu1986, Stu1993]) In complex dimension $n=2$, a compact subset $K$ of a $\mathcal{C}^{2}$ strongly pseudoconvex boundary $\partial \Omega \Subset \mathbb{C}^{2}$ is $C R$ removable if and only if it is $\mathcal{O}(\bar{\Omega})$-convex.

The "only if" part is the easiest, relies on a lemma due to Słodkowski ([RS1989, Stu1993) and will be presented after Lemma 3.11. Let us sketch the beautiful key idea of the "if" part ([Stu1981, Lu1986, Stu1993, Po1997]).

From $\S 1.7(\mathrm{~V})$, remind the expression of the Bochner-Martinelli kernel:

$$
\operatorname{BM}(\zeta, z)=\frac{1}{(2 \pi i)^{2}|\zeta-z|^{4}}\left[\overline{\left(\zeta_{2}-z_{2}\right)} d \bar{\zeta}_{1}-\overline{\left(\zeta_{1}-z_{1}\right)} d \bar{\zeta}_{2}\right] \wedge d \zeta_{1} \wedge d \zeta_{2}
$$

Let $\mathcal{M} \subset \mathbb{C}^{2}$ be a thin strongly pseudoconvex neighborhood of $\bar{\Omega}$. By means of a fixed function $g \in \mathcal{O}(\mathcal{M})$, it is possible to construct some explicit primitive of BM as follows. This idea goes back to Martinelli and has been exploited by Stout,

\footnotetext{
${ }^{26}$ Indeed, consider for instance the Hartogs figure $\Omega:=\left\{\left|z_{1}\right|<1,\left|z_{2}\right|<2\right\} \cup\{1 \leqslant$ $\left.\left|z_{1}\right|<2,1<\left|z_{2}\right|<2\right\}$ in $\mathbb{C}^{2}$. Then the annulus $\left.K=\left\{z_{1}=1,1 \leqslant\left|z_{2}\right| \leqslant 2\right)\right\} \subset \partial \Omega$ is $\mathcal{O}(\bar{\Omega})$-convex. We claim that the function $g:=1 / z_{2}$, holomorphic in a neighborhood of $K$, cannot be approximated on $K$ by functions $f \in \mathcal{O}(\bar{\Omega})$. Indeed, by Hartogs extension $\mathcal{O}(\bar{\Omega})=\mathcal{O}\left(\left\{\left|z_{1}\right| \leqslant 2,\left|z_{2}\right| \leqslant 2\right\}\right)$, which implies that every $f \in \mathcal{O}(\bar{\Omega})$ has to satisfy the maximum principle on the disc $\left.\left\{z_{1}=1,\left|z_{2}\right| \leqslant 2\right)\right\} \supset K$. Rounding off the corners, we get an example with $\partial \Omega \in \mathcal{C}^{\infty}$.

${ }^{27}$ Said differently, the envelope of holomorphy of an arbitrarily thin (interior) one-sided neighborhood of $\partial \Omega \backslash K$ is one-sheeted and identifies with $\Omega$.
} 
Lupacciolu, Leiterer, Laurent-Thiébaut, Kytmanov and others. By a classical result ([HeLe1984]), $g$ admits a Hefer decomposition

$$
g(\zeta)-g(z)=g_{1}(\zeta, z)\left[\zeta_{1}-z_{1}\right]+g_{2}(\zeta, z)\left[\zeta_{2}-z_{2}\right],
$$

with $g_{1}, g_{2} \in \mathcal{O}(\mathcal{M} \times \mathcal{M})$. Then a direct calculation shows that for $z \in \mathcal{M}$ fixed, the $(0,2)$-form

$$
\Theta_{g, z}(\zeta)=\frac{g_{2}(\zeta, z) \overline{\left(\zeta_{1}-z_{1}\right)}-g_{1}(\zeta, z) \overline{\left(\zeta_{2}-z_{2}\right)}}{(2 \pi i)^{2}|\zeta-z|^{2}[g(\zeta)-g(z)]} d \zeta_{1} \wedge d \zeta_{2}
$$

satisfies

$$
\bar{\partial}_{\zeta} \Theta_{g, z}(\zeta)=d_{\zeta} \Theta_{g, z}(\zeta)=\operatorname{BM}(\zeta, z),
$$

on $\{\zeta \in \mathcal{M}: g(\zeta) \neq g(z)\}$, i.e. provides a primitive of BM outside some thin set. In $\mathbb{C}^{n}$ for $n \geqslant 3$, there is also a similar explicit primitive.

Let $K \subset \partial \Omega$ be as in Theorem 3.7 and fix $z \in \bar{\Omega} \backslash K$. By $\mathcal{O}(\bar{\Omega})$-convexity of $K$, there exists $g \in \mathcal{O}(\bar{\Omega})$ with $g(z)=1$ and $\max _{w \in K}|g(w)|<1$. After a slight elementary modification of $g$ ([ण̈1995, Po1997]), one can insure that the set $\{w \in \mathcal{M}:|g(w)|=1\}$ is a geometrically smooth $\mathcal{C}^{\omega}$ Levi-flat hypersurface of $\mathcal{M}$ transverse to $\partial \Omega$. Then the region $\Omega_{g}:=\Omega \cap\{|g|>1\}$ has piecewise smooth connected boundary

$$
\partial \Omega_{g}=(\partial \Omega \cap\{|g|>1\}) \bigcup(\Omega \cap\{|g|=1\})
$$

and its closure $\bar{\Omega}_{g}$ in $\mathbb{C}^{2}$ does not intersect $K$.

Let $f$ be an arbitrary continuous CR function on $\partial \Omega \backslash K$. Supposing for a while that $f$ already enjoys a holomorphic extension $F \in \mathcal{O}(\Omega) \cap \mathcal{C}^{0}(\bar{\Omega} \backslash K)$, the Bochner-Martinelli representation formula then provides for every $z \in \Omega_{g}$ the value

$$
F(z)=\int_{\partial \Omega_{g}} f(\zeta) \operatorname{BM}(\zeta, z) .
$$

Decomposing $\partial \Omega_{g}$ as above and using the primitive $\Theta_{g, z}$, we may write

$$
F(z)=\int_{\partial \Omega \cap\{|g|>1\}} f(\zeta) \operatorname{BM}(\zeta, z)+\int_{\Omega \cap\{|g|=1\}} f(\zeta) d_{\zeta} \Theta_{g, z}(\zeta) .
$$

Supposing $f \in \mathcal{C}^{1}$ and applying Stokes' theorem ${ }^{28}$ to the (Levi-flat) hypersurface $\Omega \cap\{|g|=1\}$ with boundary equal to $\partial \Omega \cap\{|g|=1\}$, we get

$$
F(z)=\int_{\partial \Omega \cap\{|g|>1\}} f(\zeta) \operatorname{BM}(\zeta, z)+\int_{\partial \Omega \cap\{|g|=1\}} f(\zeta) \Theta_{g, z}(\zeta) .
$$

But the holomorphic extension $F$ of an arbitrary $f \in \mathcal{C}_{C R}^{0}(\partial \Omega \backslash K)$ is still unknown and in fact has to be constructed! Since the two integrations in the

\footnotetext{
${ }^{28}$ In the general case $f \in \mathcal{C}^{0}$, one shrinks slightly $\Omega_{g}$ inside $\Omega$, rounds off its corners and passes to the limit.
} 
above formula are performed on parts of $\partial \Omega \backslash K$ where $f$ is defined, we are led to set:

$$
F_{g}(z)=\int_{\partial \Omega \cap\{|g|>1\}} f(\zeta) \operatorname{BM}(\zeta, z)+\int_{\partial \Omega \cap\{|g|=1\}} f(\zeta) \Theta_{g, z}(\zeta),
$$

as a candidate extension of $f$ at every $z \in \Omega_{g}$. Since $K$ is $\mathcal{O}(\bar{\Omega})$-convex, $\Omega$ is the union of the regions $\Omega_{g}$ for $g$ running in $\mathcal{O}(\bar{\Omega})$, but these extensions $F_{g}(z)$ do depend on $g$, because $\Theta_{g, z}$ does. The remainder of the proof ([Stu1993, Po1997]) then consists in:

(a) verifying that $F_{g}$ is holomorphic (the kernels are not holomorphic with respect to $z$ );

(a) showing that two differente candidates $F_{g_{1}}$ and $F_{g_{2}}$ coincide in fact on $\Omega_{g_{1}} \cap \Omega_{g_{2}}$

(b) verifying that at least one candidate $F_{g}$ has boundary value equal to $f$ on some controlled piece of $\partial \Omega \backslash K$.

The reader is referred to [Stu1981, Lu1986, Stu1993 for complete arguments.

In the above construction, the strict pseudoconvexity of $\Omega$ insured the existence of a Stein (i.e. pseudoconvex) neighborhood basis $\left(\mathcal{M}_{j}\right)_{j \in J}$ of $\bar{\Omega}$ which guaranteed in turn the existence of a Hefer decomposition. It was pointed out by Ortega that Hefer decomposition (called Gleason decomposition in [Or1987]) holds on $\mathcal{C}^{\infty}$ pseudoconvex boundaries $\partial \Omega \Subset \mathbb{C}^{n}$, but may fail in the nonpseudoconvex realm. So, let $\Omega \Subset \mathbb{C}^{n}$ be a bounded domain having $\mathcal{C}^{\infty}$ boundary. Denote by $A^{\infty}(\Omega):=\mathcal{O}(\Omega) \cap \mathcal{C}^{\infty}(\bar{\Omega})$ the ring of holomorphic functions in $\Omega$ that are $\mathcal{C}^{\infty}$ up to the boundary.

Theorem 3.8. ([Or1987]) If $\partial \Omega \Subset \mathbb{C}^{n}(n \geqslant 1)$ is $\mathcal{C}^{\infty}$ and pseudoconvex, then every $g \in A^{\infty}(\Omega)$ has a decomposition

$$
g(z)-g(w)=\sum_{k=1}^{n} g_{k}(z, w)\left[z_{k}-w_{k}\right],
$$

with the $g_{k} \in A^{\infty}(\Omega \times \Omega)$.

This decomposition formula also holds under the assumption that $\Omega$ is a domain of holomorphy (having possibly nonsmooth boundary), but provided that $\bar{\Omega}$ has a basis of neighborhoods consisting of Stein domains. However, not every $\mathcal{C}^{\infty}$ weakly pseudoconvex boundary admits a Stein neighborhood basis, as is shown by the so-called worm domains ([DF1977, ES1987]).

Example 3.9. Furthermore, the above decomposition theorem fails to hold on general domains. Following [Or1987], consider the union $\Omega_{1} \cup \Omega_{2}$ in $\mathbb{C}^{2}$ of the two sets

$$
\begin{aligned}
& \Omega_{1}:=\left\{-4<x_{1}<0,\left|z_{2}\right|<e^{x_{1}}\right\} \quad \text { and } \\
& \Omega_{2}:=\left\{0 \leqslant x_{1}<4, e^{-1 / x_{1}}<\left|z_{2}\right|<1\right\} .
\end{aligned}
$$


The continuity principle along families of analytic discs parallel to the $z_{2}$-axis shows that the envelope of holomorphy of $\Omega_{1} \cup \Omega_{2}$ contains $\Omega_{1} \cup \Omega_{3}$, where $\Omega_{3}:=\left\{0 \leqslant x_{1}<4,\left|z_{2}\right|<1\right\}$.

The holomorphic mapping $R\left(z_{1}, z_{2}\right):=\left(e^{i z_{1}}, z_{2}\right)$ is one-to-one from $\Omega_{1} \cup \Omega_{2}$ onto its image $R\left(\Omega_{1} \cup \Omega_{2}\right)$. However, the extension of $R$ to $\Omega_{1} \cup \Omega_{3}$ is not injective, because $R$ takes the same value at the two points $\left( \pm \pi, e^{-2 \pi}\right) \in \Omega_{1} \cup \Omega_{3}$. If Theorem 3.8 were true on the domain $R\left(\Omega_{1} \cup \Omega_{2}\right)$, pulling the decomposition formula back to $\Omega_{1} \cup \Omega_{2}$, it would follow that every $g \in \mathcal{O}\left(\Omega_{1} \cup \Omega_{2}\right)$ has a decomposition

$$
\begin{aligned}
g(z)-g(w) & =\widetilde{g}_{1}\left(e^{i z}, w\right)\left[e^{i z_{1}}-e^{i z_{2}}\right]+\widetilde{g}_{2}\left(e^{i z}, w\right)\left[z_{2}-w_{2}\right] \\
& =g_{1}(z, w)\left[e^{i z_{1}}-e^{i z_{2}}\right]+g_{2}(z, w)\left[z_{2}-w_{2}\right] .
\end{aligned}
$$

Then the same decomposition would hold for every $g \in \mathcal{O}\left(\Omega_{1} \cup \Omega_{3}\right)$, by automatic holomorphic extension of $g, g_{1}, g_{2}$. Choosing $z=\left(-\pi, e^{-2 \pi}\right), w=\left(\pi, e^{-2 \pi}\right)$ and $g$ such that $g(z) \neq g(w)\left(g:=z_{1}\right.$ will do !), we reach a contradiction.

Corollary 3.10. ([Or1987, [P2003]) Every function holomorphic in a domain $\Omega \subset \mathbb{C}^{n}$ enjoys the Hefer division property precisely when the envelope of holomorphy of $\Omega$ is schlicht.

The above results mean that a direct application of the integral formula approach sketched above becomes impossible for domains having nonschlicht envelope. Nevertheless, in [Lt1988], using more general divison methods ([ HeLe1984]]), a Bochner-Martinelli kernel on an arbitrary Stein manifold was constructed that enabled to obtain Theorem 3.28 below, valid for nonpseudoconvex domains.

We conclude our presentation of Theorem 3.7 by exposing the "only if" of Theorem 3.7.

Lemma 3.11. ([RS1989, Stu1993]) Let $\partial \Omega \Subset \mathbb{C}^{2}$ be a $\mathcal{C}^{2}$ strongly pseudoconvex boundary and let $K \subset \partial \Omega$ be a compact set. Then $\Omega \backslash \widehat{K}_{\mathcal{O}(\bar{\Omega})}$ is pseudoconvex.

Taking for granted the lemma, by contraposition, suppose that $K \subset \partial \Omega$ is not $\mathcal{O}(\bar{\Omega})$-convex, viz. $K \varsubsetneqq \widehat{K}_{\mathcal{O}(\bar{\Omega})}$ and show that $K$ is not removable. It follows from strict pseudoconvexity of $\partial \Omega$ that $\Omega \cap \widehat{K}_{\mathcal{O}(\bar{\Omega})}$ is nonempty ([Stu1993]). Leaving $K$ fixed, by deforming $\partial \Omega$ away from $\Omega$, we may enlarge slightly $\Omega$ as a domain $\Omega^{\prime} \supset \Omega$ with $\partial \Omega^{\prime} \supset K$ and $\Omega^{\prime} \supset \partial \Omega \backslash K$, having $\mathcal{C}^{2}$ boundary $\partial \Omega^{\prime}$ close to $\partial \Omega$, as illustrated. Since by the lemma, $\Omega \backslash \widehat{K}_{\mathcal{O}(\bar{\Omega})}$ is pseudoconvex, it follows easily ([Stu1993] ) that $\Omega^{\prime} \backslash \widehat{K}_{\mathcal{O}(\bar{\Omega})}$ is also pseudoconvex. Consequently ([Hö1973]), there exists a holomorphic function $F^{\prime} \in \mathcal{O}\left(\Omega^{\prime} \backslash \widehat{K}_{\mathcal{O}(\bar{\Omega})}\right)$ that does not extend holomorphically at any point of the boundary of $\Omega^{\prime} \backslash \widehat{K}_{\mathcal{O}(\bar{\Omega})}$. The restriction of $F^{\prime}$ to $\partial \Omega \backslash K$ is a CR function on $\partial \Omega \backslash K$ for which $K$ is not removable, since $\Omega \cap \widehat{K}_{\mathcal{O}(\bar{\Omega})} \neq \emptyset$. 
3.12. Removability, polynomial hulls and Cantor sets. A generalization of Theorem 3.7, essentially with the same proof (excepting notational complications) holds in arbitrary complex dimension $n \geqslant 2$.

Theorem 3.13. ([Lu1986, Stu1993]) Let $\Omega \Subset \mathbb{C}^{n}, n \geqslant 2$, be a bounded pseudoconvex domain such that $\bar{\Omega}$ has a Stein neighborhood basis. If $K \subset \partial \Omega$ is compact and $\mathcal{O}(\bar{\Omega})$-convex, and if $\partial \Omega=K \cup M$, where $M$ is a connected $\mathcal{C}^{1}$ hypersurface of $\mathbb{C}^{n} \backslash K$, then $K$ is CR-removable.

Example 3.14. ([CSt1994, एö1999a]) Let $M$ be a connected compact orientable $(2 n-3)$-dimensional maximally complex (Definition 4.7 below) CR manifold of class $\mathcal{C}^{1}$ contained in the unit sphere $\partial \mathbb{B}_{n}(n \geqslant 2)$ with empty boundary in the sense of currents. Such an $M$ is called a maximally complex cycle. By a theorem due to Harvey-Lawson (reviewed as Theorem 4.16 below), if $M$ satisfies the moments' condition, then $M$ is the boundary of a unique complex $(n-1)$-dimensional complex subvariety $\Sigma \subset \mathbb{B}_{n}$. Since the cohomology group $H^{2}\left(\mathbb{B}_{n}, \mathbb{Z}\right)$ vanishes, by a standard Cousin problem, $\Sigma$ may be defined as the zeroset of some global holomorphic function $f \in \mathcal{O}\left(\mathbb{B}_{n}\right) \cap \mathcal{C}^{0}\left(\overline{\mathbb{B}}_{n}\right)$. The maximum principle yields that the compact set $K:=\Sigma \cup M=\bar{\Sigma}$ is $\mathcal{O}\left(\overline{\mathbb{B}}_{n}\right)$-convex. Consequently, the envelope of holomorphy of an arbitrarily thin one-sided neighborhood of $\partial \mathbb{B}_{n} \backslash M$ is equal to the pseudoconvex domain $\mathbb{B}_{n} \backslash(M \cup \Sigma)$.

If in addition $\Omega$ is Runge ([Hö1973] ) or if $\bar{\Omega}$ is polynomially convex, then every $f \in \mathcal{O}(\bar{\Omega})$ may be approximated uniformly by polynomials on some sufficiently small neighborhood of $\bar{\Omega}$ (whose size depends on $f$ ). It then follows that polynomial convexity and $\mathcal{O}(\bar{\Omega})$-convexity are equivalent. As a paradigmatic example, this holds when $\Omega=\mathbb{B}_{n}$ is the unit ball.

Corollary 3.15. ([Stu1993]) Let $\Omega$ and $K \subset \Omega$ be as in Theorem 3.13 and assume that $\Omega$ is Runge in $\mathbb{C}^{n}$, for instance $\Omega=\mathbb{B}_{n}$. If $K$ is polynomially convex, then $K$ is CR-removable. If $n=2$, the CR-removability of $K$ is equivalent to its polynomial convexity.

Although the last necessary and sufficient condition seems to be satisfactory, we must point out that concrete geometric characterizations of polynomial convexity usually are hard to provide. In $\S 5.14$ below, we shall describe a class of removable compact sets whose polynomial convexity may be established directly.

A compact subset $K$ of $\mathbb{R}^{n}(n \geqslant 1)$ is a Cantor set if it is perfect, viz. coincides with its first derived set $K^{\prime}$. It is called tame if there is a homeomorphism of $\mathbb{R}^{n}$ onto itself that carries $K$ onto the standard middle-third Cantor set contained in the coordinate line $\mathbb{R}_{x_{1}}$.

Tame Cantor sets $K$ in a $\mathcal{C}^{2}$ strongly pseudoconvex boundary $\partial \Omega \Subset \mathbb{C}^{2}$ were shown to be CR-removable in [FS1991], provided there exists a Stein neighborhood $\mathcal{D}$ of $K$ in $\mathbb{C}^{2}$ such that $K$ is $\mathcal{O}(\overline{\mathcal{D}})$-convex. By further analysis, this last assumption was shown later to be redundant and in general, tame Cantor sets are 
CR-removable. It was then suggested in [Stu1993] that all Cantor subsets of $\partial \mathbb{B}_{n}$ $(n \geqslant 2)$ are removable, or equivalently polynomially convex. Nevertheless, Rudin and then Vitushkin, Henkin and others had constructed Cantor sets $K \subset \mathbb{C}^{2}$ having large polynomial hull $\widehat{K}$, e.g. so that $\widehat{K}$ contains a complex curve, or even contains interior points. Recently, in a beautiful paper, Jöricke showed how to put such sets in the 3 -sphere $\partial \mathbb{B}_{2}$, thus solving the question in the negative.

Theorem 3.16. ([ذo2005]) For every positive number $r<1$, there exists a Cantor set $K \subset \partial \mathbb{B}_{2}$ whose polynomial hull $\widehat{K}$ contains the closed ball $r \overline{\mathbb{B}}_{2}$.

3.17. $L^{\mathrm{p}}$-removability and further results. In the definition of CR-removability, nothing is assumed about the behavior from $\bar{\Omega} \backslash K$ up to $K$ : the rate of growth may be arbitrarily high. If, differently, functions are assumed to be tame on $\partial \Omega$ (including $K$ ), better removability assertions hold.

Definition 3.18. A compact subset $K$ of a $\mathcal{C}^{1}$ boundary $\partial \Omega \Subset \mathbb{C}^{n}(n \geqslant 2)$ is called $L^{\mathrm{p}}$-removable $(1 \leqslant \mathrm{p} \leqslant \infty)$ if every function $f \in L^{\mathrm{p}}(\partial \Omega)$ which is $\mathrm{CR}$ on $\partial \Omega \backslash K$ is in fact CR on the whole boundary $\partial \Omega$.

Then by the Hartogs-Bochner theorem, $f$ admits a holomorphic extension to $\Omega$ that may be checked to belong to $H^{\mathrm{p}}(\Omega)$.

Theorem 3.19. ([AC1994]) Let $\Omega \Subset \mathbb{C}^{n}(n \geqslant 2)$ be a bounded domain having $\mathcal{C}^{2}$ boundary $\partial \Omega$ and let $M$ be a $\mathcal{C}^{2}$ totally real embedded submanifold of $\partial \Omega$. If $K \subset M$ is a polynomially convex compact subset, then $K$ is $L^{\mathrm{P}}$-removable.

In complex dimension $n \geqslant 3$, the two extension Theorems 3.13 and 3.19 are not optimal. In general, additional extension phenomena occur, which are principally overlooked by assumptions on the hull of the singularity. A more geometric point of view ( $\$ 3.23$ below) shows that these theorems may be established by means of holomorphic extension along one-parameter families of complex analytic hypersurfaces, whereas the (finer) Kontinuitätssatz holds along families of analytic discs, whose thinness offers more freedom to fill in maximal domains of extension.

Example 3.20. Let $\Omega:=\mathbb{B}_{3}$ be the unit ball in $\mathbb{C}^{3}$, and let

$$
K=\left\{\left(z_{1}, z_{2}, 0\right) \in \partial \mathbb{B}_{3}:\left|z_{1}\right| \geqslant 1 / 2\right\}
$$

be a 3-dimensional ring in the intersection of $\partial \mathbb{B}_{3}$ with the $\left(z_{1}, z_{2}\right)$-plane. The maximum principle along discs parallel to the $z_{2}$-axis yields:

$$
\widehat{K}_{\mathcal{O}\left(\overline{\mathbb{B}}_{3}\right)}=\left\{\left(z_{1}, z_{2}, 0\right) \in \overline{\mathbb{B}}_{3}:\left|z_{1}\right| \geqslant 1 / 2\right\} \neq K,
$$

so $K$ is not $\mathcal{O}\left(\overline{\mathbb{B}}_{3}\right)$-convex. Nevertheless, this $K$ is removable. Indeed, applying the continuity principle, we may first fill in $\mathbb{B}_{3} \backslash \widehat{K}$ by means of discs parallel to the $z_{2}$-axis and then fill in the complete ball $\mathbb{B}_{3}$, by means of discs parallel to the $z_{3}$-axis. 
In higher dimensions $n \geqslant 3$, the relevant characterizations of CR-removable compact sets contained in strongly pseudoconvex frontiers are of cohomological nature ( $\$ 3.33$ below). In another vein, the assumption that $\bar{\Omega}$ possesses a Stein neighborhood basis in Theorem 3.13 above inspired some authors to generalize Stout's theorem as follows.

Definition 3.21. Let $\Omega$ be a relatively compact domain of a Stein manifold $\mathcal{M}$ and let $K \subset \bar{\Omega}$ be a compact set. The $\mathcal{O}(\mathcal{M})$-convex hull of $K$ is

$$
\widehat{K}_{\mathcal{O}(\mathcal{M})}:=\left\{z \in \mathcal{M}:|g(z)| \leqslant \max _{w \in K}|g(w)| \text { for all } g \in \mathcal{O}(\mathcal{M})\right\} .
$$

If $K=\widehat{K}_{\mathcal{O}(\mathcal{M})}$, then $K$ is called $\mathcal{O}(\mathcal{M})$-convex.

In $\mathbb{C}^{n}$, the $\mathcal{O}(\mathcal{M})$-convex hull coincides with the polynomial hull. Notice that the next theorem is valid without pseudoconvexity assumption on $\Omega$.

Theorem 3.22. ([Stu1981, Lt1988, Ky1991, Stu1993, Jö1995]) Let $\mathcal{M}$ be a Stein manifold of dimension $n \geqslant 2$, let $\Omega \Subset \mathcal{M}$ be a relatively compact domain such that $\mathcal{M} \backslash \bar{\Omega}$ is connected and let $K \subset \bar{\Omega}$ be a compact set with $K=\widehat{K}_{\mathcal{O}(\mathcal{M})} \cap$ $\partial \Omega$. Then every $C R$ function $f$ defined on $\partial \Omega \backslash K$ extends holomorphically to $\Omega \backslash \widehat{K}_{\mathcal{O}(\mathcal{M})}$, i.e.:

- if $\partial \Omega \backslash K$ is a $\mathcal{C}^{\kappa, \alpha}$ hypersurface, with $\kappa \geqslant 1$ and $0 \leqslant \alpha \leqslant 1$, and if $f \in$ $\mathcal{C}_{C R}^{\kappa, \alpha}(\partial \Omega \backslash K)$, then the holomorphic extension $F \in \mathcal{O}(\Omega \backslash K)$ belongs to the class $\mathcal{C}^{\kappa, \alpha}\left(\bar{\Omega} \backslash \widehat{K}_{\mathcal{O}(\mathcal{M})}\right)$;

- if $\partial \Omega \backslash K$ is a $\mathcal{C}^{1}$ hypersurface and if $f \in L_{l o c}^{\mathrm{p}}(\partial \Omega \backslash K)$ with $1 \leqslant$ $\mathrm{p} \leqslant \infty$, then at every point $p \in \partial \Omega \backslash K$, the holomorphic extension $F \in \mathcal{O}\left(\bar{\Omega} \backslash \widehat{K}_{\mathcal{O}(\mathcal{M})}\right)$ belongs to the Hardy space $H_{\text {loc }}^{\mathrm{p}}\left(U_{p} \cap \Omega\right)$, for some small neighborhood $U_{p}$ of $p$ in $\mathcal{M}$.

3.23. $A(\Omega)$-hull and removal of singularities on pseudoconvex boundaries. Following [Jö1995, Po1997, [P2002], we now expose a geometric aspect of some of the preceding removability theorems. Let $\Omega \Subset \mathbb{C}^{n}$ with $n \geqslant 2$ be a bounded domain having frontier of class at least $\mathcal{C}^{1}$. By $A(\Omega)=\mathcal{O}(\Omega) \cap \mathcal{C}^{0}(\bar{\Omega})$, we denote the ring of holomorphic functions in $\Omega$ that are continuous up to the boundary. Let $K \subset \bar{\Omega}$ be a compact set.

Definition 3.24. The $A(\Omega)$-hull of $K$ is

$$
\widehat{K}_{A(\Omega)}:=\left\{z \in \bar{\Omega}:|g(z)| \leqslant \max _{w \in \bar{\Omega}}|g(w)| \text { for all } g \in \mathcal{A}(\bar{\Omega})\right\} .
$$

If $K=\widehat{K}_{A(\Omega)}$, then $K$ is called $A(\Omega)$-convex. If $K=\partial \Omega \cap \widehat{K}_{A(\Omega)}$, then $K$ is called $C R$-convex.

The next theorem is stronger than Theorem 3.13 in two aspects: 
- the inclusion $\widehat{K}_{A(\Omega)} \subset \widehat{K}_{\mathcal{O}(\bar{\Omega})}$ holds in general and may be strict;

- it is not assumed that the pseudoconvex domain $\Omega$ has a Stein neighborhood basis.

Theorem 3.25. ([एँ̈1995]]) Let $\Omega$ be a bounded weakly pseudoconvex domain in $\mathbb{C}^{2}$ having frontier of class $\mathcal{C}^{2}$ and let $K$ be a compact subset of $\partial \Omega$ with $K \neq \partial \Omega$ such that $K$ is CR-convex, namely $K=\partial \Omega \cap \widehat{K}_{A(\Omega)}$. Then the following are true.

1) Let $\mathcal{V}(\partial \Omega \backslash K)$ be an interior one-sided neighborhood of $\partial \Omega \backslash K$ with the property that each connected component of $\mathcal{V}(\partial \Omega \backslash K)$ contains in its boundary exactly one component of $\partial \Omega \backslash K$ and no other point of $\partial \Omega \backslash K$. Then for every holomorphic function $f \in \mathcal{O}(\mathcal{V}(\partial \Omega \backslash K))$, there exists a holomorphic function $F \in \mathcal{O}\left(\Omega \backslash \widehat{K}_{A(\Omega)}\right)$ with $F=f$ in $\mathcal{V}(\partial \Omega \backslash K)$.

2) ([AS1990]) There is a one-to-one correspondence between connected components of $\partial \Omega \backslash K$ and connected components of $\Omega \backslash \widehat{K}_{A(\Omega)}$, namely the boundary of each component of $\Omega \backslash \widehat{K}_{A(\Omega)}$ contains exactly one connected component of $\partial \Omega \backslash K$ and does not intersect any other component.

3) If the boundary $\partial \Omega$ is of class $\mathcal{C}^{\infty}$, then $\Omega \backslash \widehat{K}_{A(\Omega)}$ is pseudoconvex, hence it is the envelope of holomorphy of $\mathcal{V}(\partial \Omega \backslash K)$.

If $K$ is not CR-convex, the one-to-one correspondence between the connected components of $\partial \Omega \backslash K$ and those of $\partial \Omega \backslash \widehat{K}_{A(\Omega)}$ may fail.

Example 3.26. Indeed, let $\Omega:=\mathbb{B}_{2} \cap\left\{x_{1}<\frac{1}{2}\right\}$ be a truncation of the unit ball and let $K:=\partial \mathbb{B}_{2} \cap\left\{x_{1}=\frac{1}{2}\right\}$ be the intersection of the three-sphere $\partial \mathbb{B}_{2}$ with the real hyperplane $\left\{x_{1}=\frac{1}{2}\right\}$ (see only the left hand side of the diagram).

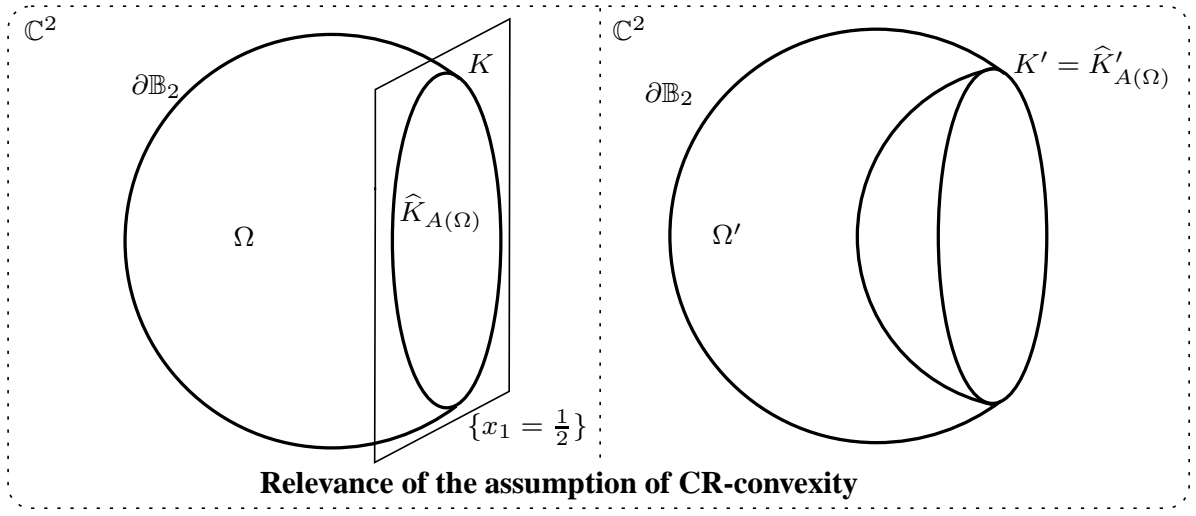

The Levi-flat 3-ball $\mathbb{B}_{2} \cap\left\{x_{1}=\frac{1}{2}\right\}$ being foliated by complex discs, the maximum principle entails that $\widehat{K}_{A(\Omega)}=\overline{\mathbb{B}}_{2} \cap\left\{x_{1}=\frac{1}{2}\right\}=\widehat{K}_{A(\Omega)} \cap \partial \Omega \neq K$, hence $K$ is not CR-convex. Also, $\partial \Omega \backslash K$ has two connected components $\partial \mathbb{B}_{2} \cap\left\{x_{1}<\frac{1}{2}\right\}$ 
and $\mathbb{B}_{2} \cap\left\{x_{1}=\frac{1}{2}\right\}$, whereas $\partial \Omega \backslash \widehat{K}_{A(\Omega)}=\partial \mathbb{B}_{2} \cap\left\{x_{1}<\frac{1}{2}\right\}$ is connected. Any function on $\partial \Omega \backslash K$ equal to two distinct constants on the two connected components of $\partial \Omega \backslash K$ is CR and not holomorphically extendable to $\Omega=\Omega \backslash \widehat{K}_{A(\Omega)}$. Finally, by smoothing out $\partial \Omega$ near the two-sphere $\partial \mathbb{B}_{2} \cap\left\{x_{1}=\frac{1}{2}\right\}$, we obtain an example with $\mathcal{C}^{\infty}$ boundary.

3.27. Hulls and holomorphic extension from nonpseudoconvex boundaries. Since the work [Lu1986] of Lupacciolu, the extension of Theorem 3.7 to nonpseudoconvex boundaries was a daring open problem (Stu1993).

Theorem 3.28. ([Po1997, JP2002, [LP2003]) Let $\Omega$ be a not necessarily pseudoconvex bounded domain in $\mathbb{C}^{n}(n \geqslant 2)$ having connected $\mathcal{C}^{2}$ frontier and let $K \subset \partial \Omega$ be a compact set with $\partial \Omega \backslash K$ connected such that $K=\partial \Omega \cap \widehat{K}_{A(\Omega)}$. Then for every continuous $C R$ function $f \in \mathcal{C}_{C R}^{0}(\partial \Omega \backslash K)$, there exists a holomorphic function $F \in \mathcal{O}\left(\Omega \backslash \widehat{K}_{A(\Omega)}\right) \cap \mathcal{C}^{0}\left(\left[\Omega \backslash \widehat{K}_{A(\Omega)}\right] \cup[\partial \Omega \backslash K]\right)$ such that $\left.F\right|_{\partial \Omega \backslash K}=f$.

A purely geometrical proof applying a global continuity principle together with a fine control of monodromy may be found in [Po1997, [P2002]; $c f$. also [MP2007]. By a topological device, a second proof ([LP2003]) derives the theorem from the following statement, established by means of $\bar{\partial}$ techniques.

Theorem 3.29. ([Lt1988]) Let $\mathcal{M}$ be a Stein manifold of complex dimension $n \geqslant$ 2 , let $K \subset \mathcal{M}$ be a compact set that is $\mathcal{O}(\mathcal{M})$-convex and let $\Omega \subset \mathcal{M}$ be a relatively compact not necessarily pseudoconvex domain such that $\partial \Omega \backslash K$ is a connected $\mathcal{C}^{1}$ hypersurface of $\mathcal{M} \backslash K$. Then for every continuous $C R$ function $f$ on $\partial \Omega \backslash K$, there exists a holomorphic function $F \in \mathcal{O}(\Omega \backslash K) \cap \mathcal{C}^{0}(\bar{\Omega} \backslash K)$ with $\left.F\right|_{\partial \Omega \backslash K}=f$.

Contrary to the case where $\partial \Omega$ is pseudoconvex (as in Theorem 3.25), even if $K$ is CR-convex, the one-to-one correspondence between the connected components of $\partial \Omega \backslash K$ and those of $\Omega \backslash \widehat{K}_{A(\Omega)}$ may fail to hold. For this reason, $\partial \Omega \backslash K$ is assumed to be connected in Theorem 3.29.

Example 3.30. ([LP2003]) We modify Example 3.26 so as to get a nonpseudoconvex boundary as follows (see the right hand side of the diagram above). Let $\Omega^{\prime}$ be the unit ball $\mathbb{B}_{2}$ from which we substract the closed ball $\overline{B(q, 1)}$ of radius 1 centered at the point $q$ of coordinates $(1,0)$. A computation with defining (in)equations shows that $\Omega^{\prime}$ is contained in $\left\{x_{1}<\frac{1}{2}\right\}$. Notice that $\Omega^{\prime}$ is not pseudoconvex and in fact, its envelope of holomorphy is single-sheeted and equal to the domain $\Omega=\mathbb{B}_{2} \cap\left\{x_{1}<\frac{1}{2}\right\}$ drawn in the left hand side. Let $K^{\prime}:=\mathbb{B}_{2} \cap\left\{x_{1}=\frac{1}{2}\right\} \subset \partial \Omega^{\prime}$ (this set is the same 2-sphere as the set $K$ of the preceding example). Then $K^{\prime}$ is CR-convex, since the candidate for its $A\left(\Omega^{\prime}\right)$ hull is the three-sphere $\overline{\mathbb{B}_{2}} \cap\left\{x_{1}=\frac{1}{2}\right\}$ that lies outside $\Omega^{\prime}$. However, $\partial \Omega^{\prime} \backslash K^{\prime}$ has two connected components, namely $\partial \mathbb{B}_{2} \cap\left\{x_{1}<\frac{1}{2}\right\}$ and $\partial B(q, 1) \cap\left\{x_{1}<\frac{1}{2}\right\}$, 
whereas $\Omega^{\prime} \backslash \widehat{K}_{A(\Omega)}^{\prime}=\Omega^{\prime} \backslash K^{\prime}=\Omega^{\prime}$ is connected. Hence any CR function equal to two distinct constants on these two components fails to extend holomorphically to $\Omega^{\prime}$. Finally, by smoothing out $\partial \Omega$ near the two-sphere $\partial \mathbb{B}_{2} \cap\left\{x_{1}=\frac{1}{2}\right\}$, we obtain an example with $\mathcal{C}^{\infty}$ boundary.

If we drop CR-convexity of $K$, viz. if $K \neq \widehat{K}_{A(\Omega)} \cap \partial \Omega$, then monodromy problems come on scene: the natural embedding of $\Omega \backslash \widehat{K}_{A(\Omega)}$ into the envelope of holomorphy of a one-sided neighborhood of $\partial \Omega \backslash K$ may fail to be one-to-one.

Example 3.31. (LP2003]) Consider the real four-dimensional open cube $C:=$ $(-1,1) \times i(-1,1) \times(-1,1) \times i(-1,1)$ in $\mathbb{C}^{2} \simeq \mathbb{R}^{4}$.

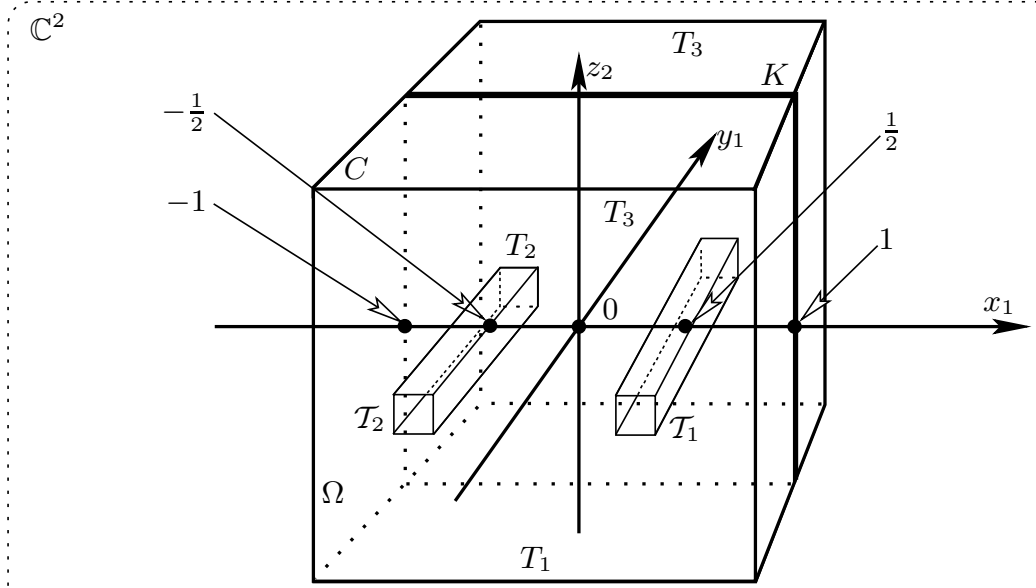

Multisheetedness if $K$ is not CR-convex

Choose $\varepsilon>0$ small and remove from this cube $C$ firstly the narrow tunnel $\mathcal{T}_{1}:=\left\{\left|z_{2}\right| \leqslant \varepsilon,\left|x_{1}-1 / 2\right| \leqslant \varepsilon\right\}$ having an entrance and an exit and secondly the (incomplete) narrow tunnel $\mathcal{T}_{2}:=\left\{\left|z_{2}\right| \leqslant \varepsilon,\left|x_{1}+1 / 2\right| \leqslant \varepsilon,-1<y_{1} \leqslant\right.$ $1 / 2\}$ having only an entrance, and call $\Omega$ the obtained domain. Let $K:=\partial C \cap$ $\left\{y_{1}=0\right\}$. The complete tunnel insures that $\partial \Omega \backslash K$ is connected. Moreover, the maximum principle along families of analytic discs parallel to the complex $z_{2}$-axis enables to verify that

$$
\widehat{K}_{A(\Omega)}=\left(\Omega \cap\left\{y_{1}=0\right\}\right) \bigcup K \bigcup\left(\partial \mathcal{T}_{1} \cap\left\{y_{1}=0\right\}\right) \bigcup\left(\partial \mathcal{T}_{2} \cap\left\{y_{1}=0\right\}\right) .
$$

It follows that $\partial \Omega \backslash \widehat{K}_{A(\Omega)}$ has three connected components, firstly the part $T_{1}$ of $\partial \Omega$ that lies in the half-space $\left\{y_{1}<0\right\}$; secondly the dead-lock part $T_{2}$ of the second tunnel that lies in $\left\{y_{1}>0\right\}$; and thirdly, the remainder $T_{3}$ of the boundary, that lies in $\left\{y_{1}>0\right\}$.

The branch of $\log z_{1}$ satisfying $\log 1=0$ is uniquely defined in $\mathbb{C}^{2} \backslash\left\{\left(x_{1}, z_{2}\right)\right.$ : $\left.x_{1} \leqslant 0\right\}$, hence $\log z_{1}$ is holomorphic in a neighborhood of $\partial \Omega \backslash \bar{T}_{2}$, where $\bar{T}_{2}:=\partial \mathcal{T}_{2} \cap\left\{y_{1} \geqslant 0\right\}$. In addition, $\log z_{1}$ extends from points near $\mathcal{T}_{2}$ in 
$\left\{y_{1}<0\right\}$ to a neighborhood of $\bar{T}_{2}$. In sum, it defines a single-valued function that is holomorphic in a neighborhood of $\partial \Omega$.

Observe that $\left(-\frac{1}{2}+\frac{i}{2}, 0\right) \in T_{2} \subset \partial \Omega$. The value of $\log z_{1}$ thus defined at this point is $\log \left(\frac{1}{\sqrt{2}} e^{-i 5 \pi / 4}\right)=\log \frac{1}{\sqrt{2}}-i \frac{5 \pi}{4}$. On the other hand, $\log z_{1}$ restricted to a neighborhood of $\partial C \cap\left\{y_{1}>0\right\} \subset \partial \Omega$ extends holomorphically to $C \cap\left\{y_{1}>0\right\}$ (by means of unit discs parallel to the $z_{2}$-axis) as $\log z_{1}$ itself! But the value of this extension at $\left(-\frac{1}{2}+\frac{i}{2}, 0\right)$ is different: $\log \left(\frac{1}{\sqrt{2}} e^{i 3 \pi / 4}\right)=\log \frac{1}{\sqrt{2}}+i \frac{3 \pi}{4}$.

To conclude this paragraph, before surveying the cohomological characterizations of removable singularities in dimension $n \geqslant 3$, we reformulate the obtained characterization in complex dimension $n=2$. It is known that a compact set $K \subset \mathbb{C}^{n}$ is polynomially convex if and only if the $\bar{\partial}$-cohomology group $H_{\bar{\partial}}^{0,1}(K)$ is trivial and holomorphic functions in a neighborhood of $K$ can be approximated by polynomials uniformly on $K$. Thus, we can state a complete formulation of Theorem 3.7, with the supplementary assumption that $\mathcal{O}(\bar{\Omega})$ may be approximated uniformly by polynomials. This insures that polynomial convexity coincides with $\mathcal{O}(\bar{\Omega})$-convexity. As a major example, the theorem holds for $\Omega$ equal to the unit ball $\mathbb{B}_{2}$ (Corollary 3.15).

Theorem 3.32. ([Stu1989, Stu1993, Lu1994, CSt1994]) The following four conditions for a compact subset $K$ of a $\mathcal{C}^{2}$ strongly pseudoconvex compact boundary $\partial \Omega \Subset \mathbb{C}^{2}$ with $\Omega$ Runge or $\bar{\Omega}$ polynomially convex are equivalent:

- $K$ is $\mathcal{O}(\bar{\Omega})$-convex.

- $K$ is polynomially convex.

- $H_{\bar{\partial}}^{0,1}(K)=0$ and holomorphic functions in a neighborhood of $K$ can be approximated by polynomials uniformly on $K$

- $K$ is removable.

Thus, in this situation, removability amounts to polynomial convexity. Nevertheless, the problem of characterizing geometrically the polynomial convexity of compact sets hides several fine questions. We shall come back to this topic in Section 5 .

3.33. Luppaciolu's characterizations. An outstanding theorem due to Lupacciolu provides complete cohomological characterizations of removable sets that are contained in strongly pseudoconvex boundaries, for general $n \geqslant 2$.

Let $\mathcal{M}$ be a Stein manifold of dimension $n \geqslant 2$ and let $\Omega \Subset \mathcal{M}$ be a relatively compact strongly pseudoconvex domain having $\mathcal{C}^{2}$ boundary.

Let $H_{\bar{\partial}}^{p, q}:=\mathcal{Z}_{\bar{\partial}}^{p, q} / \bar{\partial} \mathcal{E}^{p, q-1}$ denote the usual $(p, q)$-th Dolbeault cohomology group $^{29}$. We endow the space $\mathcal{Z}_{\bar{\partial}}^{n, n-2}(K)$ of $\bar{\partial}$-closed $(n, n-2)$-forms defined

\footnotetext{
${ }^{29}$ Appropriate background, further survey of Lupacciolu's results and additional material may be found in CSt1994.
} 
in a neighborhood of a compact set $K \subset \mathcal{M}$ with the standard locally convex inductive limit topology derived from the inductive system of the Fréchet-Schwartz spaces $\mathcal{Z}_{\bar{\partial}}^{n, n-2}(U)$, as $U$ ranges through a fundamental system of open neighborhoods of $K$ in $\mathcal{M}$.

Theorem 3.34. ([Lu1994, CSt1994]) Assume that $\bar{\Omega}$ is $\mathcal{O}(\mathcal{M})$-convex. A proper closed subset $K$ of $\partial \Omega$ is removable if and only if $H_{\bar{\partial}}^{n, n-1}(K)=0$ and the restriction map $\mathcal{Z}_{\bar{\partial}}^{n, n-2}(\mathcal{M}) \rightarrow \mathcal{Z}_{\bar{\partial}}^{n, n-2}(K)$ has dense image.

For $n=2$, the two conditions of the theorem reduce to the $\mathcal{O}(\mathcal{M})$-convexity of $K$ ([Lu1994, CSt1994]). For $n \geqslant 3$, the following improvement is valid. By $\sigma E$, we denote the separated space associated to a given topological vector space $E$, namely the quotient $E / \overline{0}$ of $E$ by the closure of 0 .

Theorem 3.35. ([Lu1994, CSt1994]]) Assume that $n \geqslant 3$. Without the assumption that $\bar{\Omega}$ is $\mathcal{O}(\mathcal{M})$-convex, the compact set $K \subset \partial \Omega$ is removable if and only if $H_{\bar{\partial}}^{n, n-1}(K)=0$ and $^{\sigma} H_{\bar{\partial}}^{n, n-2}(K)=0$.

Lupacciolu also obtains an extrinsic characterization as follows. Let $\Phi$ be the paracompactifying family of all closed subsets of $\mathcal{M} \backslash K$ that have compact closure in $\mathcal{M}$. Let $H_{\Phi}^{p, q}$ the Dolbeault cohomology groups with support in $\Phi$.

Theorem 3.36. ([Lu1994, CSt1994]) For $n \geqslant 3$, a compact subset $K$ of the boundary $\partial \Omega$ of a $\mathcal{C}^{2}$-bounded strongly pseudoconvex domain $\Omega \Subset \mathcal{M}$ is removable if and only if $H_{\Phi}^{0,1}(\mathcal{M} \backslash K)=0$.

Notice that, for $n \geqslant 3$, this theorem has the striking consequence that the condition that $K$ be removable in a strongly pseudoconvex boundary does not depend on the domain in question, but rather on the situation of $K$ itself in the ambient manifold. Also, Lupacciolu provides analogous characterizations for weak removability ([Lu1994, CSt1994]).

\section{§4. SMOOTH AND METRICALLY THIN REMOVABLE SINGULARITIES FOR CR FUNCTIONS}

4.1. Three notions of removability. We formulate the concerned notions of removability directly in arbitrary codimension. Let $M \subset \mathbb{C}^{n}$ be a $\mathcal{C}^{2, \alpha}$ generic submanifold of positive codimension $d \geqslant 1$ and of positive CR dimension $m \geqslant 1$. Such $M$ will always be supposed connected. In the sequel, not to mention superficial corollaries, we will systematically assume that $M$ is globally minimal.

Definition 4.2. ([Me1997, MP1998, Jö1999a, Jö1999b, MP1999, MP2002]) A closed subset $C$ of $M$ is said to be:

- $C R$-removable if there exists a wedgelike domain $\mathcal{W}$ attached to $M$ to which every continuous $\mathrm{CR}$ function $f \in \mathcal{C}_{C R}^{0}(M \backslash C)$ extends holomorphically; 
- $\mathcal{W}$-removable if for every wedgelike domain $\mathcal{W}_{1}$ attached to $M \backslash C$, there is a wedgelike domain $\mathcal{W}_{2}$ attached to $M$ and a wedgelike domain $\mathcal{W}_{3} \subset$ $\mathcal{W}_{1} \cap \mathcal{W}_{2}$ attached to $M \backslash C$ such that for every holomorphic function $f \in \mathcal{O}\left(\mathcal{W}_{1}\right)$, there exists a holomorphic function $F \in \mathcal{O}\left(\mathcal{W}_{2}\right)$ which coincides with $f$ in $\mathcal{W}_{3}$;

- $L^{\mathrm{p}}$-removable, where $1 \leqslant \mathrm{p} \leqslant \infty$, if every locally integrable function $f \in L_{l o c}^{\mathrm{p}}(M)$ which is CR in the distributional sense on $M \backslash C$ is in fact CR on all of $M$.

A few comments are welcome. CR-removability requires at least $M \backslash C$ to be globally minimal, in order that the main Theorem 4.12(V) applies, yielding a wedgelike domain $\mathcal{W}_{1}$ attached to $M \backslash C$. Then $\mathcal{W}$-removability of $C$ implies its CR-removability. In both CR- and $\mathcal{W}$-removabililty, after the removal of $C$, nothing is demanded about the growth of the holomorphic extension to a global wedgelike domain $\mathcal{W}_{2}$ attached to $M$. Such extensions might well have essential singularities at some points of $C$, although they are holomorphic in $\mathcal{W}_{2}$. On the contrary, for $L^{\mathrm{P}}$-removability of $C, \mathrm{CR}$ functions on $M \backslash C$ should really extend to be CR through $C$.

Notwithstanding this difference, the sequel will reveal that $L^{\mathrm{P}}$-removability is also a consequence of $\mathcal{W}$-removability, thanks to some Hardy-space control of the holomorphic extension $F \in \mathcal{O}\left(\mathcal{W}_{2}\right)$. In fact, functions are assumed to be $L_{l o c}^{\mathrm{p}}$ (a variant is to assume continuity on $M$ instead of integrability) even near points of $C$. This strong assumption enables to get a control of the growth of the wedge extension. Before providing more explanations, we assert in advance that $\mathcal{W}$-removability is the most general notion of removability, focusing the question on envelopes of holomorphy.

In codimension $d=1$, wedgelike domains identify to one-sided neighborhoods. Then $\mathcal{W}$-removability of $C$ means that the envelope of holomorphy of every (arbitrarily thin) one-sided neighborhood of $M \backslash C$ contains a complete onesided neighborhood of the hypersurface $M$ in $\mathbb{C}^{n}$. If $M=\partial \Omega$ is the boundary of a bounded domain $\Omega \subset \mathbb{C}^{n}$ (having connected boundary), then $\mathcal{W}$-removability of a compact set $K \subset \partial \Omega$ entails its removability in the sense of Problem 3.2, thanks to Hartogs Theorem 1.8(V).

As in [ö1999b, MP1999], we would like to emphasize that all the general theorems presented in Sections 3 and 4 are void for $L_{l o c}^{1}$ functions, or require a strong assumption of growth. On the contrary, the results that will be presented below hold in all spaces $L_{l o c}^{\mathrm{p}}$ with $1 \leqslant \mathrm{p} \leqslant \infty$, without any assumption of growth. The concept of $\mathcal{W}$-removability, interpreted as a result about envelopes of holomorphy, yields a (crucial) external drawing near the illusory singularity, an opportunity that is intrinsically attached to locally embeddable Cauchy-Riemann structures, but is of course absent for general linear partial differential operators. 
4.3. Removable singularities on hypersurfaces. In [LS1993], it is shown that if $\Omega \subset \mathbb{C}^{n}$ is a pseudoconvex bounded domain having $\mathcal{C}^{2}$ boundary, then every compact subset $K \subset \partial \Omega$ with $\mathrm{H}^{2 n-3}(K)=0$ is removable in the sense of Definition 3.4. In fact, Lemma 4.18(III) shows that $\partial \Omega$ is globally minimal and the next lemma shows that in codimension $d=1$, metrically thin singularities do not perturb global minimality.

Lemma 4.4. ([MP2002] If If $M \subset \mathbb{C}^{n}$ is a globally minimal $\mathcal{C}^{2}$ hypersurface, then for every closed set $C \subset M$ with $\mathrm{H}^{2 n-3}(C)=0$, the complement $M \backslash C$ is also globally minimal.

Example 4.5. However, this is untrue if $\mathrm{H}^{2 n-3}(C)>0$. Let $n \geqslant 2$ and $\varphi(z, u)$ be $\mathcal{C}^{2}$ defined for $|z|,|u|<1$ and satisfying $\varphi(z, 0) \equiv 0$ for $\operatorname{Re} z_{1} \leqslant 0$. Let $M \subset \mathbb{C}^{n}$ be the graph $v=\varphi(z, u)$ and define $C:=\left\{\left(i y_{1}, z_{2}, \ldots, z_{n-1}, 0\right)\right\}$. Clearly $\operatorname{dim} C=2 n-3, \mathrm{H}^{2 n-3}(C)>0$ and $\left\{(z, 0): \operatorname{Re} z_{1}<0\right\}$ is a single $\mathrm{CR}$ orbit $\mathcal{O}_{-}$of $M \backslash C$. Also, the function $\varphi$ may be chosen so that $M$ is of finite type at every point of $M \backslash \mathcal{O}_{-}$, whence $M \backslash C$ consists of exactly two CR orbits, namely $\mathcal{O}_{-}$and $M \backslash\left(\mathcal{O}_{-} \cup C\right)$. It follows that $M$ is globally minimal.

Theorem 4.6. ([LS1993, CSt1994, MP1998, MP2002]) If $M \subset \mathbb{C}^{n}$ is a globally minimal $\mathcal{C}^{2, \alpha}(0<\alpha<1)$ hypersurface, then every closed set $C \subset M$ with $\mathrm{H}^{2 n-3}(C)=\mathrm{H}^{\operatorname{dim} M-2}(C)=0$ is locally $C R$-, $\mathcal{W}$ - and $L^{\mathrm{P}}$-removable.

Sometimes, we shall say that $C$ is of codimension $2^{+0}$ in $M$. This is a version of (rm1) and of (rm2) of Theorem 2.30 for CR functions on general hypersurfaces. Except for $L^{\mathrm{P}}$-removability, refinements about smoothness assumptions may be found in [CSt1994].

The smallest (Hausdorff) dimension of $C \subset M \subset \mathbb{C}^{n}$ for which its removability may fail is equal to $2 n-3$. Indeed, if $C=M \cap \Sigma$ is equal to the intersection of $M$ with some local complex hypersurface $\Sigma=\{f=0\}$, the functions $1 / f^{k}$, $k \geqslant 1$ and $e^{1 / f}$ restrict to be CR on $M \backslash C$, but not holomorphically extendable to a one-sided neighborhood at points of $C$, since $\Sigma$ visits both sides of $M$. In such a situation, the real hypersurface $M \cap \Sigma$ of the complex hypersurface $\Sigma$ has dimension $(2 n-3)$ and $\mathrm{CR}$ dimension $(n-2)$.

Definition 4.7. A CR submanifold $N \subset \mathbb{C}^{n}$ is called maximally complex if it is of odd dimension satisfying $\operatorname{dim} N=1+2 \mathrm{CR} \operatorname{dim} N$.

Every real hypersurface of a complex manifold is maximally complex. The next step in to study singularities $C$ contained in $(2 n-3)$-dimensional submanifolds $N \subset M$.

Example 4.8. We show the necessity of assuming that $M \backslash C$ is also globally minimal (MP1999]). Take the complex hypersurface $\mathcal{O}_{-}$of the preceding example having boundary $\partial \mathcal{O}_{-}=C=N$. Applying Proposition 4.38(III) to $S:=\mathcal{O}_{-}$, we may construct a measure on $M \backslash C$ supported by $\mathcal{O}_{-}$that is CR on $M \backslash C$ but 
does not extend holomorphically to a wedge at any point of $\overline{\mathcal{O}}_{-}=\mathcal{O}_{-} \cup C$, for the same reason as in Corollary 4.39(III).

Because of this example, we shall systematically assume that $M \backslash C$ is also globally minimal, if this is not a consequence of other hypotheses. Here is a CR version of ( $\mathbf{r m 3})$ and of ( $\mathbf{r m} 4)$ of Theorem 2.30. It says that true singularities should be maximally complex. Before stating it, we point out that all submanifolds of given manifolds will constantly be assumed to be embedded submanifolds. Also, all subsets $C$ of a submanifold $N$ of manifold $M$ that are called closed are assumed to be closed both in $M$ and in $N$.

Theorem 4.9. ([ð̈1992, Me1997, 『ö1999a, Jö1999b]) Let $M \subset \mathbb{C}^{n}$ be a $\mathcal{C}^{2, \alpha}$ $(0<\alpha<1)$ globally minimal hypersurface and let $N \subset M$ be a connected $\mathcal{C}^{2, \alpha}$ embedded submanifold of dimension $(2 n-3)$, viz. of codimension 2 in $M$. A closed set $C \subset N$ such that $M \backslash C$ is also globally minimal is $C R$-, $\mathcal{W}$ - and $L^{\mathrm{P}}$-removable under each one of the following two circumstances:

(i) $n \geqslant 2$ and $C \neq N$;

(ii) $n \geqslant 3$ and $C=N$ is not maximally complex, viz. there exists at least one point $p \in N$ at which $N$ is generic.

One may verify ([ए̈̈1999a, MP1999]) that generic points of $N$ are locally removable and then after erasing them by deforming slightly $M$ inside the extensional wedge existing above, (ii) is seen to be a consequence of (i). For various smoothness refinements, the reader is referred to Jö1992, CSt1994, MP1998, Jö1999a, Jö1999b, MP1999]. One may also combine Theorem 4.6 and 4.9, assuming that the submanifold $N$ is smooth, except perhaps at all points of some metrically thin closed subset. The proof will not be restituted.

The study of more massive singularities contained in $(2 n-2)$-dimensional submanifolds has been initiated by Jöricke ("ö1988), having in mind some generalization of Denjoy's approach to Painlevé's problem.

Theorem 4.10. ([ð̈1999a, Jö1999b]) Let $M \subset \mathbb{C}^{n}$ be a $\mathcal{C}^{2, \alpha}(0<\alpha<1)$ globally minimal hypersurface and let $M^{1} \subset M$ be a connected $\mathcal{C}^{2, \alpha}$ embedded submanifold ${ }^{30}$ of dimension $(2 n-2)$, viz. of codimension 1 in $M$, that is generic in $\mathbb{C}^{n}$. If $n \geqslant 3$, a closed set $C \subset M^{1}$ is $C R$-, $\mathcal{W}$ - and $L^{\mathrm{P}}$-removable provided it does not contain any $C R$ orbit of $M^{1}$.

It may be established (see e.g. Lemma 3.3 in [MP2006a]) that $M^{1} \backslash C^{\prime}$ is also globally minimal for every closed $C^{\prime} \subset M^{1}$ containing no CR orbit of $M^{1}$.

\footnotetext{
${ }^{30}$ We believe that $\mathcal{C}^{2, \alpha}$-smoothness of $M^{1}$ is required in the proof built there, since the map $w \mapsto \widehat{h}(w)$ appearing in equation (3.12) of [\$̈1999a] (that corresponds essentially to the singular integral $\mathcal{J}(v)$ defined in $(3.20)(\mathrm{V}))$ already requires $M^{1}$ to be $\mathcal{C}^{1, \alpha}$ with $0<\alpha<1$ to exist; then to compute the differential of $w \mapsto \widehat{h}(w)$, one must require $M^{1}$ to be at least $\mathcal{C}^{2, \alpha}$.
} 
We would like to mention that the removability of two-codimensional singularities (Theorem 4.9) is not a consequence of the removability of the bigger onecodimensional singularities (Theorem 4.10). Indeed, it may happen that $T_{p} N$ contains $T_{p}^{c} M$ at several points $p \in N$ in Theorem 4.9, preventing the existence of a generic $M^{1} \subset M$ containing $N$. In addition, even if $T_{p} N \not \supset T_{p}^{c} M$ for every $p \in N$, Theorem 4.9 is not anymore a corollary of Theorem 4.10. Indeed, with $m=2$ and $d=1$, choosing a local hypersurface $M \subset \mathbb{C}^{3}$ containing a complex curve $\Sigma$, choosing $N \subset M$ of dimension 3 containing $\Sigma$ and being maximally real outside $\Sigma$, and choosing an arbitrary generic $M^{1} \subset M$ containing $N$ (some explicit local defining equations may easily be written), then $\Sigma$ is a CR orbit of $M^{1}$, so $N \supset \Sigma$ is not considered to be removable by Theorem 4.10, whereas Theorem 4.9(ii) asserts that $N$ is removable.

Although singularities are more massive in Theorem 4.10, the assumption $n \geqslant$ 3 in it entails that the CR dimension $(n-1)$ of $M$ is $\geqslant 2$, whence $M^{1}$ has positive $\mathrm{CR}$ dimension $\geqslant 1$. This insures the existence of small analytic discs with boundary in $M^{1}$. Section 5 below and [MP2006a] as a whole are devoted to the more delicate case where $M^{1}$ has null CR dimension.

Example 4.11. ([匹ö1999a]) In $\mathbb{C}^{3}$, let $M=\partial \mathbb{B}_{3}$ and let $M^{1}:=\left\{\left(z_{1}, z_{2}, z_{3}\right)\right.$ : $\left.0<x_{1}<1 / 2, y_{1}=0\right\}$. Clearly, $M^{1}$ is foliated by the 3 -spheres

$$
S_{x_{1}^{*}}^{3}:=\left\{z_{1}=x_{1}^{*},\left|z_{2}\right|^{2}+\left|z_{3}\right|^{2}=1-\left|x_{1}^{*}\right|^{2}\right\},
$$

$x_{1}^{*} \in(0,1 / 2)$, that are globally minimal compact 3-dimensional strongly pseudoconvex maximally complex CR submanifolds of CR dimension 1 bounding the 2-dimensional complex balls

$$
\mathbb{B}_{2, x_{1}^{*}}:=\left\{z_{1}=x_{1}^{*},\left|z_{2}\right|^{2}+\left|z_{3}\right|^{2}<1-\left|x_{1}^{*}\right|^{2}\right\} .
$$

Theorem 4.10 asserts that a compact set $K \subset M^{1}$ is removable if and only if it does not contain a whole sphere $S_{x_{1}^{*}}$, for some $x_{1}^{*} \in(0,1 / 2)$. If $K$ contains such a sphere $S_{x_{1}^{*}}^{3}$, the complex 2-ball $\mathbb{B}_{2, x_{1}^{*}}$ coincides with the $A(\Omega)$-hull of $S_{x_{1}^{*}}$ and is nonremovable. More generally, an application of both Theorems 4.10 and 3.25 yields the following.

Corollary 4.12. Let $K$ be a compact subset of $M^{1}$. For every (interior) one-sided neighborhood $\mathcal{V}^{-}\left(\partial \mathbb{B}_{3} \backslash K\right)$ that is contained in $\mathbb{B}_{3}$ and every function $f$ holomorphic in $\mathcal{V}^{-}\left(\partial \mathbb{B}_{3} \backslash K\right)$, there exists a function $F$ holomorphic in $\mathbb{B}_{3} \backslash \bigcup_{x_{1}^{*}: S_{x_{1}^{*}}^{3} \subset K} \mathbb{B}_{2, x_{1}^{*}}$ with $F=f$ in $\mathcal{V}^{-}\left(\partial \mathbb{B}_{3} \backslash K\right)$.

By means of the complex Plateau problem, the next paragraph discusses the necessity for $N$ not to be maximally complex in Theorem 4.9 and for $M^{1}$ not to contain any CR orbit in Theorem 4.10, in a more general context than $M=\partial \mathbb{B}_{n}$. 
4.13. Complex Plateau problem and nonremovable singularities contained in strongly pseudoconvex boundaries. Let $\mathcal{M}$ be a complex manifold of dimension $n \geqslant 2$. If $\Sigma \subset \mathcal{M}$ is a closed pure $k$-dimensional complex subvariety, we denote by $[\Sigma]$ the current of integration on $\Sigma$, whose existence was established by Lelong in 1957 ([Ch1989, De1997]).

Definition 4.14. ([HL1975, Ha1977]) A current $\mathrm{T}$ on $\mathcal{M}$ is called a holomorphic $k$-chain if it is of the form

$$
\mathrm{T}=\sum_{\text {finite }} n_{j}\left[\Sigma_{j}\right]
$$

where the $\Sigma_{j}$ denote the irreducible components of a pure $k$-dimensional complex subvariety $\Sigma$ of $\mathcal{M}$ and where the multiplicity $n_{j}$ of each $\Sigma_{j}$ is an integer.

The complex Plateau problem consists in filling boundaries $N$ by complex subvarieties $\Sigma$, or more generally by holomorphic chains T. Maximal complexity of the boundary $N$ is naturally required and since $N$ might encounter singular points of $\Sigma$, it should be allowed in advance to be "scarred" somehow. Also, the boundary $N$ inherits an orientation from $\Sigma$ and as the boundary of $\Sigma$, it should have empty boundary.

Definition 4.15. A scarred $\mathcal{C}^{\kappa}(1 \leqslant \kappa \leqslant \infty)$ maximally complex cycle of dimension $(2 m+1), m \geqslant 0$, is a compact subset $N \subset \mathcal{M}$ together with a thin compact scar set $\mathrm{sc}_{N} \subset N$ such that

- $\mathrm{H}^{2 m+1}\left(\mathbf{s c}_{N}\right)=0$;

- $N \backslash \mathrm{sc}_{N}$ is an oriented (2m+1)-dimensional embedded maximally complex $\mathcal{C}^{\kappa}$ submanifold of $\mathcal{M} \backslash \mathbf{s c}_{N}$ having finite $(2 m+1)$-dimensional Hausdorff measure;

- the current of integration over $N \backslash \mathbf{s c}_{N}$, denoted by [N], has no boundary: $d[N]=0$.

This definition was essentially devised by Harvey-Lawson and appears to be adequately large, but sufficiently stringent to maintain the possibility of filling a maximally complex cycle by a complex analytic set.

Theorem 4.16. (HL1975, Ha1977]) Suppose $N$ is a scarred $\mathcal{C}^{\kappa}(1 \leqslant \kappa \leqslant \infty)$ maximally complex cycle of dimension $(2 m+1), m \geqslant 0$, in a Stein manifold $\mathcal{M}$.

- If $m=0$, assume that $N$ satisfies the moment condition, viz. $\int_{N} \omega=0$ for every holomorphic 1-form $\omega=\sum_{k=1}^{n} \omega_{k}(z) d z_{k}$ having entire coefficients $\omega_{k} \in \mathcal{O}\left(\mathbb{C}^{n}\right)$.

- If $m \geqslant 1$, assume nothing, since the corresponding appropriate moment condition follows automatically from the assumption of maximal complexity ([HL1975]). 
Then there exists a unique holomorphic $(m+1)$-chain $\mathrm{T}$ in $\mathcal{M} \backslash N$ having compact support and finite mass in $\mathcal{M}$ such that

$$
d \mathrm{~T}=[N]
$$

in the sense of currents in $\mathcal{M}$. Furthermore, there is a compact subset $K$ of $N$ with $\mathrm{H}^{2 m+1}(K)=0$ such that every point of $N \backslash\left(K \cup \mathrm{sc}_{N}\right)$ possesses a neighborhood in which $(\operatorname{supp} T) \cup N$ is a regular $\mathcal{C}^{\kappa}$ complex manifold with boundary.

A paradigmatic example, much considered since Milnor studied it, consists in intersecting a complex algebraic subvariety of $\mathbb{C}^{n}$ passing through the origin with a spere centered at 0 ; topologists usually require that 0 is an isolated singularity and that the sphere is small or that the defining polynomial is homogeneous.

We apply this filling theorem in a specific situation. Let $\partial \Omega \Subset \mathbb{C}^{n}(n \geqslant 3)$ be a strongly pseudoconvex $\mathcal{C}^{2}$ boundary and let $M^{1} \subset \partial \Omega$ be an embedded $\mathcal{C}^{2}$ one-codimensional submanifold that is generic in $\mathbb{C}^{n}$. We assume that $M^{1}$ has no boundary and is closed, viz. is a compact submanifold. Since $M^{1}$ has CR dimension $(n-2)$, its CR orbits have dimension equal to either $(2 n-4)$, or to $(2 n-3)$ or to $(2 n-2)$. Because of Corollary 4.19(III), no CR orbit of $M^{1}$ can be an immersed complex 2-codimensional submanifold, of real dimension $(2 n-4)$, since its closure in $M^{1}$ would be a compact set laminated by complex manifolds. Nevertheless, there may exist $(2 n-3)$-dimensional CR orbits.

Proposition 4.17. ([एँ̈1999a]) Every $C R$ orbit $\mathcal{O}_{C R}^{1}$ of a connected $\mathcal{C}^{2}$ hypersurface $M^{1} \subset \partial \Omega$ of a $\mathcal{C}^{2}$ strongly pseudoconvex boundary $\partial \Omega \Subset \mathbb{C}^{n}$ is of the following types:

(i) $\mathcal{O}_{C R}^{1}$ is an open subset of $M$;

(ii) $\mathcal{O}_{C R}^{1}$ is a closed maximally complex $\mathcal{C}^{1}$ cycle embedded in $M^{1}$;

(iii) $\mathcal{O}_{C R}^{1}$ is a maximally complex $\mathcal{C}^{1}$ submanifold injectively immersed in $M^{1}$ whose closure $C$ consists of an uncountable union of similar CR orbits.

In the last situation, $C$ will be called a maximally complex exceptional minimal compact $C R$-invariant set. The intersection of $C$ with a local curve transversal to a piece CR orbit in $M^{1}$ may consist of either an open segment or of a Cantor (perfect) subset.

Here is the desired converse to both Theorems 4.9 and 4.10 in a situation where the Plateau complex filling works.

Corollary 4.18. ([ö1999a]) Suppose that $\partial \Omega \in \mathcal{C}^{2, \alpha}$ contains a compact embedded $(2 n-3)$-dimensional maximally complex submanifold $N$ (without boundary). Then $N$ is not removable.

Proof. Indeed, the scar set of $N$ is empty and the filling of $N$ by a holomorphic chain consists of an irreducible complex subvariety $\Sigma$ that is necessarily contained in $\Omega$, since $\partial \Omega$ is strongly pseudoconvex. Then the domain $\Omega \backslash \Sigma$ is seen to be 
pseudoconvex and $\widehat{N}_{A(\Omega)}=N \cup \Sigma$. Theorem 3.25 entails that CR functions on $\partial \Omega \backslash N$ extend holomorphicaly to $\Omega \backslash \Sigma$.

A very natural problem, raised in [Jö1999a] and inspired by a perturbation of Example 4.11, is to determine for which compact CR-invariant subsets $K$ of a strongly pseudoconvex boundary $\partial \Omega \subset \mathbb{C}^{n}$ the envelope of holomorphy of $\partial \Omega \backslash K$ is multi-sheeted.

Theorem 4.19. ([]S2004]) Let $M^{1} \subset \partial \mathbb{B}_{n}$ be an orientable $(2 n-2)$-dimensional generic $\mathcal{C}^{2, \alpha}$ submanifold of $\partial \mathbb{B}_{n}(n \geqslant 3)$ and let $K \subset M^{1}$ be a compact $C R$ invariant subset of $M^{1}$ such that

- the boundary of $K$ in $M^{1}$ is the disjoint union of finitely many connected compact maximally complex $C R$ manifolds $N_{1}, \ldots, N_{\ell}$ of dimension $(2 n-3)$ that are $\mathcal{C}^{2, \alpha-0} C R$ orbits of $M^{1}$;

- the interior of $K$ with respect to $M^{1}$ is globally minimal.

Then the envelope of holomorphy $\mathrm{E}\left(\mathcal{V}\left(\partial \mathbb{B}_{n} \backslash K\right)\right)$ is multi-sheeted in every neighborhood $\bar{U}_{p} \subset \overline{\mathbb{B}_{n}}$ of every point $p \in \operatorname{Int} K$.

We conclude these considerations by formulating a deeply open problem raised by Jöricke. The complex Plateau problem for laminated boundaries is a virgin mathematical landscape.

Open question 4.20. ([ð̈1999a]) Let $\partial \Omega \Subset \mathbb{C}^{n}, n \geqslant 3$, be a strongly pseudoconvex boundary of class at least $\mathcal{C}^{2}$. Suppose that $\partial \Omega$ contains a maximally complex exceptional minimal compact CR-invariant set $C$. Does $C$ bound a relatively compact subset $\Sigma \subset \Omega$ laminated by complex manifolds?

As observed in [DH1997, MP1998, Sa1999, DS2001], removable singularities have an unexpected interesting application to wedge extension of CRmeromorphic functions.

4.21. CR-meromorphic functions and metrically thin singularities. For $n \geqslant$ 2 , a local meromorphic map $f$ from a domain $\Omega \subset \mathbb{C}^{n}$ to the Riemann sphere $P_{1}(\mathbb{C})$ has an exceptional locus $I_{f} \subset \Omega$, at every point $p$ of which the value $f(p)$ is undefined. For instance the origin $(0,0) \in \mathbb{C}^{2}$ with $f=\frac{z_{1}}{z_{2}}$ (notice that every complex number in $\mathbb{C} \cup\{\infty\}$ is a limit of $\frac{z_{1}}{z_{2}}$ ). This exceptional set $I_{f}$ is a complex analytic subset of $\Omega$ having codimension $\geqslant 2$ ([De1997]). It is called the indeterminacy set of $f$.

A meromorphic function may be more conveniently defined as a $n$-dimensional irreducibe complex analytic subset $\Gamma_{f}$ of $\Omega \times P_{1}(\mathbb{C})$ having surjective projection onto $\Omega$, viz. $\pi_{\Omega}\left(\Gamma_{f}\right)=\Omega$. Here, $\Omega$ might be any complex manifold. Indeterminacy points correspond precisely to points $p \in \Omega$ satisfying $\pi_{\Omega}^{-1}(p) \cap \Gamma_{f}=$ $\{p\} \times P_{1}(\mathbb{C})$. So, the generalization of meromorphy to the CR category incorporates indeterminacy points. 
Definition 4.22. ([HL1975, DH1997, MP1998, Sa1999]) Let $M \subset \mathbb{C}^{n}$ be a scarred $\mathcal{C}^{1}$ generic submanifold of codimension $d \geqslant 1$ and of $\mathrm{CR}$ dimension $m=n-d \geqslant 1$. Then a $C R$ meromorphic function on $M$ with values in $P_{1}(\mathbb{C})$ consists of a triple $\left(f, \mathcal{D}_{f}, \Gamma_{f}\right)$ such that:

1) $\mathcal{D}_{f} \subset M$ is a dense open subset of $M$ and $f: \mathcal{D}_{f} \rightarrow P_{1}(\mathbb{C})$ is a $\mathcal{C}^{1}$ map;

2) the closure $\Gamma_{f}$ in $\mathbb{C}^{n} \times P_{1}(\mathbb{C})$ of the graph $\left\{(p, f(p)): p \in \mathcal{D}_{f}\right\}$ defines an oriented scarred $\mathcal{C}^{1} \mathrm{CR}$ submanifold of $\mathbb{C}^{n} \times P_{1}(\mathbb{C})$ of the same $\mathrm{CR}$ dimension as $M$ having empty boundary in the sense of currents.

The indeterminacy locus of $f$ is denoted by

$$
I_{f}:=\left\{p \in M:\{p\} \times P_{1}(\mathbb{C}) \subset \Gamma_{f}\right\} .
$$

In the CR category, $I_{f}$ is not as thin as in the holomorphic category (where it has real codimension $\geqslant 4$ ), but it is nevertheless thin enough for future purposes, as we shall see. A standard argument from geometric measure theory yields almost everywhere smoothness of almost every level set.

Lemma 4.23. ([Fe1969, HL1975, Ha1977]) Let $M \subset \mathbb{C}^{n}$ be a scarred $\mathcal{C}^{1}$ generic submanifold. Let $\left(f, \mathcal{D}_{f}, \Gamma_{f}\right)$ be a CR meromorphic function on $M$. Then for almost every $w \in P_{1}(\mathbb{C})$, the level set

$$
N_{f}(w):=\left\{p \in M:(p, w) \in \Gamma_{f}\right\}
$$

is a scarred 2-codimensional $\mathcal{C}^{1}$ submanifold of $M$.

Let $p \in I_{f}$. Since $(p, w) \in \Gamma_{f}$ for every $w \in P_{1}(\mathbb{C})$, it follows that $I_{f} \subset$ $N_{f}(w)$ for every $w$. Fixing such a $w \in P_{1}(\mathbb{C})$, we simply denote $N_{f}:=N_{f}(w)$. In particular, the scar set $\mathbf{s C}_{N_{f}}$ of $N_{f}$ is always of codimension $2^{+0}$ in $M$, namely $\mathrm{H}^{\operatorname{dim} M-2}\left(\mathrm{sc}_{N_{f}}\right)=0$.

So $I_{f} \subset N_{f}$ and by definition $I_{f} \times P_{1}(\mathbb{C}) \subset \Gamma_{f}$. We claim that, in addition, $I_{f}$ has empty interior in $N_{f} \backslash \mathbf{s c}_{N_{f}}$. Otherwise, there exist a point $p \in N_{f} \backslash \mathbf{s c}_{N_{f}}$ and a neighborhood $U_{p}$ of $p$ in $M$ with $U_{p} \cap \mathrm{sc}_{N_{f}}=\emptyset$ such that $I_{f}$ contains $U_{p} \cap N_{f}$, whence

$$
\left(U_{p} \cap N_{f}\right) \times P_{1}(\mathbb{C}) \subset \Gamma_{f} .
$$

Since $\left(U_{p} \cap N_{f}\right) \times P_{1}(\mathbb{C})$ has dimension equal to $\operatorname{dim} M=\operatorname{dim} \Gamma_{f}$, it follows that

$$
\Gamma_{f} \cap\left(U_{p} \times P_{1}(\mathbb{C})\right) \equiv\left(U_{p} \cap N_{f}\right) \times P_{1}(\mathbb{C}) .
$$

But $U_{p} \cap N_{f}$ having codimension two in $U_{p}$, this contradicts the assumption that $\Gamma_{f}$ is a (nonempty!) graph above the dense open subset $U_{p} \cap \mathcal{D}_{f}$ of $U_{p}$.

Lemma 4.24. (MP1998, Sa1999]) The indeterminacy set $I_{f}$ of $f$ is a closed set of empty interior contained in some 2-codimensional scarred $\mathcal{C}^{1}$ submanifold $N_{f}$ of $M$. Moreover, the scar set $\mathrm{sc}_{N_{f}}$ of $N_{f}$ is always of codimension $2^{+0}$ in $M$, viz. $\mathrm{H}^{2 m+d-2}\left(\mathbf{s c}_{N_{f}}\right)=0$. 
The statement below and its proof are clear if $\mathcal{D}_{f}=M$; in it, the condition $d\left[\Gamma_{f}\right]=0$ helps in an essential way to keep it true when the closure of $\Gamma_{f}$ possesses a nonempty scar set.

Proposition 4.25. (MP1998, Sa1999]) There exists a unique CR measure $\mathrm{T}_{f}$ on $M \backslash I_{f}$ with $\left.\mathrm{T}_{f}\right|_{\mathcal{D}_{f}}$ coinciding with the $\mathcal{C}^{1}$ CR function $f: \mathcal{D}_{f} \rightarrow P_{1}(\mathbb{C})$.

It is defined locally as follows. Let $p \in M \backslash I_{f}$ and let $U_{p}$ be an open neighborhood of $p$ in $M$. Since $p \notin I_{f}$, there exists $w_{p} \in P_{1}(\mathbb{C})$ with $\left(p, w_{p}\right) \notin \Gamma_{f}$. Composing with an automorphism of $P_{1}(\mathbb{C})$ and shrinking $U_{p}$, we may assume that $w_{p}=\infty$ and that $\left(U_{p} \times\{\infty\}\right) \cap \Gamma_{f}=\emptyset$. Letting $d \operatorname{Vol}_{U_{p}}$ be some $(2 m+d)$ dimensional volume form on $U_{p}$, letting $\pi_{\Gamma_{f}}: \Gamma_{f} \rightarrow M$ denote the natural projection, the CR measure $\left.\mathrm{T}_{f}\right|_{U_{p}}$ is defined by

$$
\left\langle\mathrm{T}_{f}, \varphi\right\rangle:=\int_{\Gamma_{f}} w \cdot \pi_{\Gamma_{f}}^{*}\left(\varphi d \operatorname{Vol}_{U_{p}}\right),
$$

for every $\varphi \in \mathcal{C}_{c}^{1}\left(U_{p}\right)$.

Thus, on $M \backslash I_{f}$, the CR-meromorphic function $\left(f, \mathcal{D}_{f}, \Gamma_{f}\right)$ behaves like an order zero CR distribution. With $\mathcal{C}_{C R}^{0}, L_{C R, l o c}^{\mathrm{p}}$, it therefore enjoys the extendability properties of Part $\mathrm{V}$ on $M \backslash I_{f}$, provided that $M$ is $\mathcal{C}^{2, \alpha}$. The next theorem should be applied to $C:=I_{f}$. Its final proof ([MP2002]) under the most general assumptions combines both the CR extension theory and the application of the Riemann-Hilbert problem to global discs attached to maximally real submanifolds ([G11994, Gl1996]). We cannot restitute the proof here.

Theorem 4.26. ("MP1998, DS2001, MP2002]) Suppose $M \subset \mathbb{C}^{n}$ is $\mathcal{C}^{2, \alpha}(0<$ $\alpha<1$ ) of codimension $d \geqslant 1$ and of $C R$ dimension $m \geqslant 1$. Then every closed subset $C$ of $M$ such that $M$ and $M \backslash C$ are globally minimal and such that $\mathrm{H}^{2 m+d-2}(C)=0$ is $C R$-, $\mathcal{W}$ - and $L^{\mathrm{P}}$-removable.

However, if $f$ is a CR-meromorphic function defined on such a $M$, with $\mathcal{C}^{1}$ replaced by $\mathcal{C}^{2, \alpha}$ in Definition 4.22, the complement $M \backslash I_{f}$ need not be globally minimal if $M$ is, and it is easy to construct manifolds $M$ and closed sets $C \subset M$ with $\mathrm{H}^{2 m-1}(C)<\infty$ which perturb global minimality, $c f$. Example 4.8. It is therefore natural to make the additional assumption that $M$ is locally minimal at every point. This assumption is the weakest one that insures that $M \backslash C$ is globally minimal, for arbitrary closed sets $C \subset M$.

Corollary 4.27. Assume that $M \in \mathcal{C}^{2, \alpha}$ is locally minimal at every point and let $f$ be a CR-meromorphic function. Then $I_{f}$ is $C R$-, $\mathcal{W}$ - and $L^{\mathrm{p}}$-removable.

Proof. Lemma 4.24 holds with $\mathcal{C}^{1}$ replaced by $\mathcal{C}^{2, \alpha}$. It says that $I_{f}$ is a closed subset with empty interior of some scarred $\mathcal{C}^{2, \alpha}$ submanifold $N_{f}$ of $M$. The removability of the portion of $I_{f}$ that is contained in the regular part of $N_{f}$ follows from Theorem 4.9(i). The removability of the remaining scar set $\mathrm{sc}_{N_{f}}$ follows from Theorem 4.26 above. 
Thus the CR measure $\mathrm{T}_{f}$ on $M \backslash I_{f}$ (Proposition 4.25) extends holomorphically to some wedgelike domain $\mathcal{W}_{1}$ attached to $M \backslash I_{f}$. The $\mathcal{W}$-removability of $I_{f}$ entails that the envelope of holomorphy of $\mathcal{W}_{1}$ contains a wedgelike domain $\mathcal{W}_{2}$ attached to $M$. Performing supplementary gluing of discs, the CR extension theory (Part $\mathrm{V}$ ) insures that such a $\mathcal{W}_{2}$ depends only on $M$, not on $f$. As envelopes of meromorphy and envelopes of holomorphy of domains in $\mathbb{C}^{n}$ coincide by a theorem going back to Levi ([KS1967, Iv1992] $)$, we may conclude.

Theorem 4.28. ([MP2002]) Suppose $M \subset \mathbb{C}^{n}$ is $\mathcal{C}^{2, \alpha}$ and locally minimal at every point. Then there exists a wedgelike domain $\mathcal{W}$ attached to $M$ to which every CR-meromorphic function on $M$ extends meromorphically.

4.29. Peak and smooth removable singularities in arbitrary codimension. A closed set $C \subset M$ is called a $\mathcal{C}^{0, \beta}$ peak set, $0<\beta<1$, if there exists a nonconstant function $\varpi \in \mathcal{C}_{C R}^{0, \beta}(M)$ such that $C=\{\varpi=1\}$ and $\max _{p \in M}|\varpi(p)| \leqslant 1$.

Theorem 4.30. ([KR1995, MP1999]) Let $M$ be $\mathcal{C}^{2, \alpha}(0<\alpha<1)$ globally minimal. Then every $\mathcal{C}^{0, \beta}$ peak set $C$ satisfies $\mathrm{H}^{\operatorname{dim} M}(C)=0$ and is $L^{\mathrm{P}}$-removable.

To conclude, we mention two precise generalizations of Theorems 4.9 and 4.10 to higher codimension. If $\Sigma=\{z: g(z)=0\}$ is a local complex hypersurface passing through a point $p$ of a generic submanifold $M \subset \mathbb{C}^{n}$ that is transverse to $M$ at $p$, viz. $T_{p} \Sigma+T_{p} M=T_{p} \mathbb{C}^{n}$, the intersection $\Sigma \cap M$ is a two-codimensional submanifold of $M$ that is nowhere generic in a neighborhood of $p$ and certainly not (locally) removable, since the CR function $\left.\frac{1}{g(z)}\right|_{M \backslash(\Sigma \cap M)}$ is not extendable to any local wedge at $p$.

Theorem 4.31. ([Me1997, MP1999] Let $M \subset \mathbb{C}^{n}$ be a $\mathcal{C}^{2, \alpha}(0<\alpha<1)$ globally minimal generic submanifold of positive codimension $d \geqslant 1$ and of positive $C R$ dimension $m=n-d \geqslant 1$. Let $N \subset M$ be a connected two-codimensional $\mathcal{C}^{2, \alpha}$ submanifold and assume that $M \backslash N$ is also globally minimal. A closed set $C \subset N$ is $C R$-, $\mathcal{W}$ - and $L^{\mathrm{p}}$-removable under each one of the following two circumstances:

(i) $m \geqslant 1$ and $C \neq N$;

(ii) $m \geqslant 2$ and there exists at least one point $p \in N$ at which $N$ is generic.

In (ii), the assumption that $m \geqslant 2$ is essential. Generally, if $m=1$, whence $d=n-1$ and $\operatorname{dim} M=n+1$, a local transverse intersection $C=\Sigma \cap M$ has dimension $n-1$, hence cannot be generic, and is not (locally) removable by construction. In the next statement, the similar assumption that $m \geqslant 2$ is strongly used in the proof: the one-codimensional submanifold $M^{1} \subset M$ has then CR dimension $m-1 \geqslant 1$, hence there exist small Bishop discs attached to $M^{1}$.

Theorem 4.32. ([יPo1997, Me1997, Po2000]]) Let $M \subset \mathbb{C}^{n}$ be a $\mathcal{C}^{2, \alpha}(0<\alpha<1)$ globally minimal generic submanifold of positive codimension $d \geqslant 1$. Assume that 
the CR dimension $m=n-d$ of $M$ satisfies $m \geqslant 2$. Let $M^{1} \subset M$ be a connected $\mathcal{C}^{2, \alpha}$ one-codimensional submanifold that is generic in $\mathbb{C}^{n}$. A closed set $C \subset M^{1}$ is $C R$-, $\mathcal{W}$ - and $L^{\mathrm{P}}$-removable provided it does not contain any $C R$ orbit of $M^{1}$.

Three geometrically different proofs of this theorem will be restituted in Section 10 of MP2006a. The next Section 5 and MP2006a are devoted to the study of the more delicate case where $m=1$ and where $C$ is contained in some one-codimensional submanifold $M^{1} \subset M$.

\section{§5. Removable SINGUlaRITIES IN CR DIMENSION 1}

5.1. Removability of totally real discs in strongly pseudoconvex boundaries. In 1988, applying a global version of the Kontinuitätssatz, Jöricke [Jö1988] established a remarkable theorem, opening the way to a purely geometric study of removable singularities.

Theorem 5.2. ([ण̈1988]) Let $\partial \Omega \Subset \mathbb{C}^{2}$ be a strongly pseudoconvex $\mathcal{C}^{2}$ boundary and let $D \subset \partial \Omega$ be a $\mathcal{C}^{2}$ one-codimensional submanifold that is diffeomorphic to the unit open 2-disc of $\mathbb{R}^{2}$ and maximally real at every point. Then every compact subset $K$ of $D$ is $C R$-, $L^{\infty}$ and $\mathcal{W}$-removable.

By maximal reality of $D$, the line distribution $D \ni p \mapsto \ell_{p}:=T_{p} D \cap T_{p}^{c} M$ is nowhere vanishing and may be integrated. This yields the characteristic foliation $\mathcal{F}_{D}^{c}$ on $D$. The compact set $K$ is contained in a slightly smaller disc $D^{\prime} \Subset D$ having $\mathcal{C}^{2}$ boundary $\partial D^{\prime}$. Poincaré-Bendixson's theorem on such a disc $D^{\prime}$ together with the inexistence of singularities of $\mathcal{F}_{D}^{c}$ entail that every characteristic curve that enters into $D^{\prime}$ must exit from $D^{\prime}$. Orienting then the real 2-disc $D$ and its characteristic foliation, we have the following topological observation (at the very core of the theorem) saying that there always exists a characteristic leaf that is not crossed by the removable compact set.

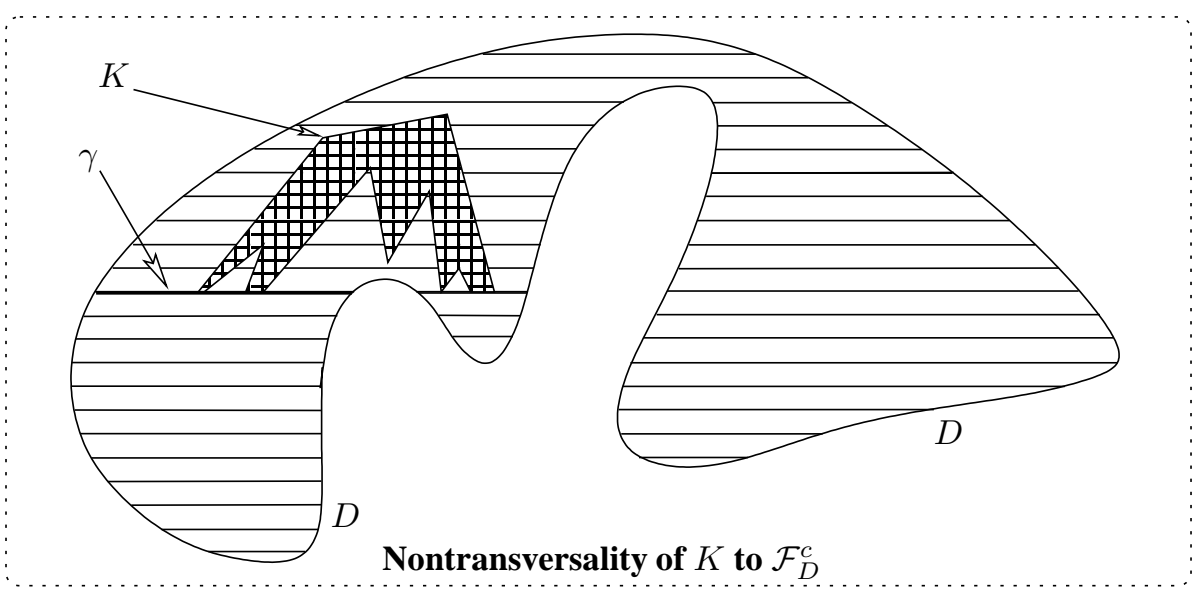


$\mathcal{F}_{D}^{c}\{K\}$ : For every compact subset $K^{\prime} \subset K$, there exists a Jordan curve $\gamma$ : $[-1,1] \rightarrow D$, whose range is contained in a single leaf of the characteristic foliation $\mathcal{F}_{D}^{c}$, with $\gamma(-1) \notin K^{\prime}, \gamma(0) \in K^{\prime}$ and $\gamma(1) \notin K^{\prime}$, such that $K^{\prime}$ lies completely in one closed side of $\gamma[-1,1]$ with respect to the topology of $D$ in a neighborhood of $\gamma[-1,1]$.

In the more general context of [MP2006a], we will argue that $\mathcal{F}_{D}^{c}\{K\}$ is the very reason why $K$ is removable. We will then remove locally a well chosen special point $p_{\mathrm{sp}}^{\prime} \in K^{\prime} \cap \gamma[-1,1]$. In fact, we shall establish removability of compact subsets $K$ of general surfaces $S$ that are not necessarily diffeomorphic to the unit 2-disc, provided that an analogous topological condition holds. Also, getting rid of strong pseudoconvexity, we shall work with a globally minimal $\mathcal{C}^{2, \alpha}$ hypersurface of $\mathbb{C}^{2}$. Finally, we shall relax slightly the assumption of total reality, admitting some complex tangencies.

Example 5.3. Let $\Omega=\mathbb{B}_{2}$ and let $P(z) \in \mathbb{C}[z]$ be a homogeneous polynomial of degree $\geqslant 2$ having 0 has its only singularity. The intersection $K:=\partial \mathbb{B}_{2} \cap\{P=$ $0\}$ is a finite union of closed real algebraic curves $\simeq S^{1}$ that are everywhere transverse to $T^{c} \partial \mathbb{B}_{2}$. We may enlarge each curve of $K$ as a thin $\mathcal{C}^{\omega}$ annulus. There is much freedom, but every such annulus is necessarily totally real. Denote by $S$ the union of all annuli, a surface in $\partial \mathbb{B}_{2}$. Clearly, no component of $K$ is removable. But the theorem does not apply: on each annulus, the characteristic foliation $\mathcal{F}_{S}^{c}$ is radial and $K$ crosses each characteristic leaf.

Example 5.4. The theorem may fail with the disc $D$ replaced by a surface $S$ having nontrivial fundamental group, even with $S$ compact without boundary. For instance, in $\partial \mathbb{B}_{2}=\left\{\left|z_{1}\right|^{2}+\left|z_{2}\right|^{2}=1\right\}$, the two-dimensional torus $T_{2}:=$ $\left\{\left(\frac{1}{\sqrt{2}} e^{i \theta_{1}}, \frac{1}{\sqrt{2}} e^{i \theta_{2}}\right): \theta_{1}, \theta_{2} \in \mathbb{R}\right\}$ is compact and $K:=T_{2}$ is not removable, since $\partial \mathbb{B}_{2} \backslash T_{2}$ has exactly two connected components.

Example 5.5. ([Ö1988]) In the same torus $T_{2}$, consider instead the proper compact subset $K:=\left\{\left(\frac{1}{\sqrt{2}} e^{i \theta_{1}}, \frac{1}{\sqrt{2}} e^{i \theta_{2}}\right):\left|\theta_{1}\right| \leqslant \frac{3 \pi}{2}, \theta_{2} \in \mathbb{R}\right\}$, diffeomorphic to a closed annulus. It is a set fibered by circles (contained in $\mathbb{C}_{z_{2}}$ ) over the curve $\widehat{\gamma}:=\left\{\frac{1}{\sqrt{2}} e^{i \theta_{1}}:\left|\theta_{1}\right| \leqslant \frac{3 \pi}{2}\right\}$ that is contained in $\mathbb{C}_{z_{1}}$. One may verify that the condition $\mathcal{F}_{T_{2}}^{c}\{K\}$ insuring removability does not hold. In fact, applying Theorem 2.2 (in the much simpler version due to Denjoy where the curve is real analytic), the curve $\widehat{\gamma}$ is not $\left(\bar{\partial}, L^{\infty}\right)$-removable in $\mathbb{C}_{z_{1}}$. So we may pick a holomorphic function $\widehat{f}\left(z_{1}\right) \in \mathcal{O}(\mathbb{C} \backslash \widehat{\gamma})$ that is bounded in $\mathbb{C} \cup\{\infty\}$ but does not extend holomorphically through $\widehat{\gamma}$. The restriction $\left.\widehat{f}\right|_{\partial \mathbb{B}_{2} \backslash K}$ belongs to $L^{\infty}\left(\partial \mathbb{B}_{2}\right)$, is $\mathrm{CR}$ on $\partial \mathbb{B}_{2} \backslash K$ but does not extend holomorphically to $\mathbb{B}_{2}$.

Before pursuing, we compare Theorem 5.2 and Theorem 4.10.

In codimension $\geqslant 2\left(e . g\right.$. for curves in $\left.\mathbb{R}^{3}\right)$, no satisfactory generalization of the Poincaré-Bendixson theory is known and perhaps is out of reach. This gap is 
caused by the complexity of the topology of phase diagrams, by the freedom that curves have to wind wildly around limit cycles, and by the intricate structure of singular points.

Nevertheless, in higher complex dimension $n \geqslant 3$, CR orbits are thicker than curves and often of codimension $\leqslant 1$. For triples $\left(M, M^{1}, C\right)$ as in Theorem 4.10 with $M=\partial \Omega$ being strongly pseudoconvex, one could expect that a statement analogous to Theorem 5.2 holds true, in which the assumption that $M^{1}$ has simple topology would imply automatic removability of every compact subset $K \subset M^{1}$.

To be precise, let $\partial \Omega \Subset \mathbb{C}^{n}(n \geqslant 3)$ be a $\mathcal{C}^{2, \alpha}$ strongly pseudoconvex boundary and let $M^{1} \subset \partial \Omega$ be a $\mathcal{C}^{2, \alpha}$ one-codimensional submanifold that is generic in $\mathbb{C}^{n}$. Strong pseudoconvexity of $\partial \Omega$ entails that CR orbits of $M^{1}$ are necessarily of codimension $\leqslant 1$ in $M^{1}$. Remind that Theorem 4.10 says that a compact subset $K$ of $M^{1}$ is removable provided it does not contain any CR orbit of $M^{1}$. Conversely, in the case where $M^{1}$ has no exceptional CR orbit, if $K$ contains a (then necessarily compact and maximally complex) CR orbit $N$ of $M^{1}$, then $K$ is not removable, since $N$ is fillable by some $(n-1)$-dimensional complex subvariety $\Sigma \subset \Omega$ with $\partial \Sigma=N$. Thus, while comparing the two Theorems 4.10 and 5.2, the true question is whether the assumption that $M^{1} \subset \partial \Omega=M$ be diffeomorphic to the real $(2 n-2)$-dimensional real ball $B^{2 n-2} \subset \mathbb{R}^{2 n-2}$ prevents the existence of compact $(2 n-3)$-dimensional CR orbits of $M^{1}$. This would yield a neat statement, valid in arbitrary complex dimension.

For instance, let $N:=\partial \mathbb{B}_{n} \cap H$ be the intersection of the sphere $\partial \mathbb{B}_{n} \simeq S^{2 n-1}$ with a complex linear hyperplane $H \subset \mathbb{C}^{n}$. With such a simple $N$ homeomorphic to a $(2 n-3)$-dimensional sphere, one may verify that every $\mathcal{C}^{\infty}$ submanifold $M^{1} \subset \partial \Omega$ containing $N$ which is diffeomorphic to $B^{2 n-2}$ must contain at least one nongeneric point. Nevertheless, admitting that $N$ has slightly more complicated topology, the expected generalization of Theorem 5.2 appears to fail, according to a discovery of Jöricke-Shcherbina. This confirms the strong differences between CR dimension $m=1$ and CR dimension $m \geqslant 2$.

Theorem 5.6. ([[S2000]) For $\varepsilon \in \mathbb{R}$ with $0<\varepsilon<1$ close to 1 , consider the intersection

$$
N_{\varepsilon}:=\left\{z_{1} z_{2} z_{3}=\varepsilon\right\} \cap \sqrt{3} \partial \mathbb{B}_{3}
$$

of the complex cubic $\left\{z_{1} z_{2} z_{3}=\varepsilon\right\}$ with the sphere $\sqrt{3} \partial \mathbb{B}_{3}=\left\{\left|z_{1}\right|^{2}+\left|z_{2}\right|^{2}+\right.$ $\left.\left|z_{3}\right|^{2}=3\right\}$. Then $N_{\varepsilon}$ is a maximally complex cycle diffeomorphic to $S^{1} \times S^{1} \times S^{1}$ bounding the (nonempty) complex surface $\Sigma_{\varepsilon}:=\left\{z_{1} z_{2} z_{3}=\varepsilon\right\} \cap \mathbb{B}_{3}$. Furthermore, there exists a suitably constructed $\mathcal{C}^{\infty}$ generic one-codimensional submanifold $M^{1} \subset \partial \mathbb{B}_{3}$ diffeomorphic to the real $(2 n-2)$-dimensional unit ball $B^{2 n-2}$ containing $N_{\varepsilon}$. Finally, since $N_{\varepsilon}$ bounds $\Sigma_{\varepsilon}$, every compact subset $K \subset M^{1}$ containing $N_{\varepsilon}$ is nonremovable. 
5.7. Elliptic isolated complex tangencies and Bishop discs. Coming back to complex dimension $n=2$, we survey known properties of isolated CR singularities of surfaces. So, let $S$ be a two-dimensional surface $S$ in $\mathbb{C}^{2}$ of class at least $\mathcal{C}^{2}$. At a point $p \in S$, the complex tangent plane $T_{p} S$ is either totally (and in fact maximally) real, viz. $T_{p} S \cap J T_{p} S=\{0\}$ or it is a complex line, viz. $T_{p} S=J T_{p} S=T_{p}^{c} S$. An appropriate application of the jet transversality theorem shows that after an arbitrarily small perturbation, the number of complex tangencies of $S$ is locally finite.

If $S$ has an isolated complex tangency at one of its points $p$, Bishop ([Bi1965]) showed that there exist local coordinates $(z, w)$ centered at $p$ in which $S$ may be represented by $w=z \bar{z}+\lambda\left(z^{2}+\bar{z}^{2}\right)+\mathrm{o}\left(|z|^{2}\right)$, where the real parameter $\lambda \in[0, \infty]$ is a biholomorphic invariant of $S$. The point $p$ is said to be elliptic if $\lambda \in\left[0, \frac{1}{2}\right)$, parabolic if $\lambda=\frac{1}{2}$ and hyperbolic if $\lambda \in\left(\frac{1}{2}, \infty\right]$. The case $\lambda=\infty$ should be understood as the surface $w=z^{2}+\bar{z}^{2}+\mathrm{o}\left(|z|^{2}\right)$. The shape of the projection of such a surface onto the real hyperplane $\{\operatorname{Im} w=0\} \simeq \mathbb{R}^{3}$ is essentially ellipsoidlike for $0<\lambda<1 / 2$ and essentially saddle-like for $\lambda>1 / 2$.

In the seminal article [Bi1965], Bishop introduced this terminology and showed that at an elliptic point, $S$ has a nontrivial polynomial hull $\widehat{S}$, foliated by a continuous one-parameter family of analytic discs attached to $M$. The geometric structure of this family has been explored further by Kenig and Webster.

Theorem 5.8. ([KW1982, BG1983, KW1984, Hu1998]) Let $S \subset \mathbb{C}^{2}$ be a $\mathcal{C}^{\kappa}$ $(\kappa \geqslant 7)$ surface having an elliptic complex tangency at one of its points $p$. Then there exists a $\mathcal{C}^{(\kappa-7) / 3}$ one-parameter family of disjoint regularly embdedded analytic discs attached to $S$ and converging to $p$. If $S$ is $\mathcal{C}^{5}$, then $\widehat{S}$ is $\mathcal{C}^{0,1}$. Furthermore, every small analytic disc attached to $M$ near $p$ is a reparametrization of one of the discs of the family.

For $\kappa=\infty$, the union of these discs form a $\mathcal{C}^{\infty}$ hypersurface $\widehat{S}$ with boundary $\partial \widehat{S}=S$ in a neighborhood of $p$. Furthermore, $\widehat{S}$ is the local hull of holomorphy of $S$ at $p$.

In the case where $S$ is real analytic, local normal forms may be found that provide a classification up to biholomorphic changes of coordinates.

Theorem 5.9. Let $S: w=z \bar{z}+\lambda\left(z^{2}+\bar{z}^{2}\right)+\mathrm{O}\left(|z|^{3}\right)$ be a local real analytic surface in $\mathbb{C}^{2}$ passing through the origin and having an elliptic complex tangency there.

- ([MW1983]) For every $\lambda$ satisfying $0<\lambda<1 / 2$, either $S$ is locally biholomorphic to the quadric $w=z \bar{z}+\lambda\left(z^{2}+\bar{z}^{2}\right)$ or there exists an integer $s \in \mathbb{N}, s \geqslant 1$, such that $S$ is locally biholomorphic to $w=$ $z \bar{z}+\left[\lambda+\delta u^{s}\right]\left(z^{2}+\bar{z}^{2}\right)$, where $u=\operatorname{Re} w$ and $\delta= \pm 1$. 
- ([Mo1985]) For $\lambda=0$, either $S$ is locally biholomorphic to $w=z \bar{z}+$ $z^{s}+\bar{z}^{s}+\mathrm{O}\left(|z|^{s+1}\right)$ for some integer $s \geqslant 3$ or $S$ is locally biholomorphic to $w=z \bar{z}$.

- ([HuKr1995]) For $\lambda=0$ and $s<\infty$, the surface $S$ is locally biholomorphic to the surface $w=z \bar{z}+z^{s}+\bar{z}^{s}+\sum_{j+k>s} a_{j k} z^{j} \bar{z}^{k}$, with $a_{j k}=\bar{a}_{k j}$.

In all cases, after the straightening, $S$ is contained in the real hyperplane $\{\operatorname{Im} w=0\}$.

In the third case $\lambda=0, s<\infty$, it is still unknown how many biholomorphic invariants $S$ can have.

5.10. Hyperbolic isolated complex tangencies. The existence of small Bishop discs attached to $S$ and growing at an elliptic complex tangency impedes local polynomial convexity. At the opposite, if $S$ is hyperbolic, Bishop's construction fails, discs are inexistent, and in fact $S$ is locally polynomially convex.

Theorem 5.11. ([FS1991] Let $S \subset \mathbb{C}^{2}$ be a $\mathcal{C}^{2}$ surface represented by $w=$ $z \bar{z}+\lambda\left(z^{2}+\bar{z}^{2}\right)+r(z, \bar{z})$, with a $\mathcal{C}^{2}$ remainder $r=\mathrm{o}\left(|z|^{2}\right)$. If $\lambda>1 / 2$, viz. if $S$ is hyperbolic at the origin, then for every $\rho_{1}>0$ sufficiently small, $S \cap\left(\rho_{1} \overline{\mathbb{B}_{2}}\right)$ is polynomially convex.

The Oka-Weil approximation theorem then assures that continuous functions in $S \cap\left(\rho_{1} \overline{\mathbb{B}}_{2}\right)$ are uniformly approximable by polynomials.

A local Bishop surface $S$ is called quadratic if it is locally biholomorphic to the quadric $w=z \bar{z}+\lambda\left(z^{2}+\bar{z}^{2}\right)$. An isolated complex point $p$ of $S$ is called holomorphically flat if there exist local coordinates centered at $p$ in which $S$ is locally contained in $\{\operatorname{Im} w=0\}$. Unlike elliptic points of $\mathcal{C}^{\omega}$ surfaces that are always flat, hyperbolic complex points of $\mathcal{C}^{\omega}$ surfaces may fail to be flat.

Example 5.12. (MW1983) The algebraic hyperbolic surface $(\lambda>1 / 2)$

$$
w=z \bar{z}+\lambda\left(z^{2}+\bar{z}^{2}\right)+\lambda z^{3} \bar{z}
$$

cannot be biholomorphically transformed into a real hyperplane.

Theorem 5.11 establishes local polynomial pseudoconvexity of surfaces at hyperbolic complex tangencies. By patching together local plurisubharmonic defining functions, one may easily construct a Stein neighborhood basis of every surface having only finitely many hyperbolic complex tangencies. Unfortunately, in this way one does not control well the topology of such neighborhoods. A finer result answering a question of Forstneric is as follows.

Theorem 5.13. ([S12004]]) Let $S$ be a compact real $\mathcal{C}^{\infty}$ surface embedded in a complex surface $\mathcal{X}$ having only finitely many complex points that are all hyperbolic and holomorphically flat. Then $S$ possesses a basis of open neighborhoods $\left(\mathcal{V}_{\varepsilon}\right)_{0<\varepsilon<\varepsilon_{1}}, \varepsilon_{1}>0$, such that: 
- $S=\bigcap_{\varepsilon>0} \mathcal{V}_{\varepsilon}$

- $\mathcal{V}_{\varepsilon}=\bigcup_{\varepsilon^{\prime}<\varepsilon} \mathcal{V}_{\varepsilon^{\prime}}$

- $\overline{\mathcal{V}}_{\varepsilon}=\bigcap_{\varepsilon^{\prime}>\varepsilon} \mathcal{V}_{\varepsilon^{\prime}}$;

- each $\mathcal{V}_{\varepsilon}$ has a $\mathcal{C}^{\infty}$ strongly pseudoconvex boundary $\partial \mathcal{V}_{\varepsilon}$;

- for every $\varepsilon$ with $0<\varepsilon<\varepsilon_{1}$, the surface $S$ is a strong deformation retract of $\mathcal{V}_{\varepsilon}$.

It is expected that the same statement remains true without the flatness assumption.

5.14. Real surfaces in strongly pseudoconvex boundaries. Coming back to removable singularities, let $\partial \Omega \Subset \mathbb{C}^{2}$ be a $\mathcal{C}^{2}$ strongly pseudoconvex boundary and let $S \subset \partial \Omega$ be a compact surface, with or without boundary. It will be no restriction to assume that $S$ is connected. Suppose that $S$ has a finite (possibly null) number of complex tangencies. These points then constitute the only singular points of the characteristic foliation of $S$. At an elliptic (resp. hyperbolic) complex tangency, the phase diagram simply looks like a focus (resp. saddle).

Theorem 5.15. ([FS1991] Let $\mathcal{M}$ be a two-dimensional Stein manifold, let $\partial \Omega \Subset$ $\mathcal{M}$ be a strongly pseudoconvex $\mathcal{C}^{2}$ boundary and let $D$ be a $\mathcal{C}^{2}$ one-codimensional submanifold that is diffeomorphic to the unit open 2-disc of $\mathbb{R}^{2}$ and is maximally complex, except at a finite number of hyperbolic complex tangencies. Then every compact subset $K$ of $D$ is $C R$-and $\mathcal{W}$-removable.

Indirectly, the characterizing Theorem 3.7 of Stout yields the following.

Corollary 5.16. Every compact subset $K \subset D \subset \partial \Omega$ is $\mathcal{O}(\bar{\Omega})$-convex. In particular, such a $K$ is polynomially convex if $\mathcal{M}=\mathbb{C}^{2}$ and if $\bar{\Omega}$ is Runge or polynomially convex, e.g. if $\Omega=\mathbb{B}_{2}$.

The (short) proof mainly relies upon the (very recent in 1991 and since then famous) works [BK1991] and [Kr1991] by Bedford-Klingenberg and by Kruzhilin about the hulls of two-dimensional spheres contained in such strictly pseudoconvex boundaries $\Omega \subset \mathcal{M}$, which may be filled by Levi-flat three-dimensional spheres after an arbitrarily small perturbation.

Theorem 5.17. ([BK1991, Kr1991] ) Let $\Omega \Subset \mathbb{C}^{2}$ be a $\mathcal{C}^{6}$ strongly pseudoconvex domain and let $S \subset \partial \Omega$ be a two-dimensional sphere of class $\mathcal{C}^{6}$ embdedded into $\partial \Omega$ that is totally real outside a finite subset consisting of $k$ hyperbolic and $k+2$ elliptic points. Then there exist:

1) a smooth domain $B \subset \mathbb{R}^{3}\left(x_{1}, x_{2}, x_{3}\right)$ with boundary $\partial B$ diffeomorphic to $S$ such that $x_{3}: B \rightarrow \mathbb{R}$ is a Morse function on $\partial B$ having $k+2$ extreme points and $k$ saddle points, whose level sets $\left\{x_{3}=\right.$ cst. $\} \cap B$ are unions of finite numbers of topological discs; and: 
1) a continuous injective map $\Phi: B \rightarrow \Omega$ sending $\partial B$ to $S$, the extreme and saddle points of $x_{3}$ on $\partial B$ to the elliptic and hyperbolic points of $S$ and the connected components of $\left\{x_{3}=\right.$ cst. $\} \cap B$ to geometrically smooth holomorphic discs.

The set $\Phi(B)$ is the envelope of holomorphy of $S$ as well as its $\mathcal{O}(\bar{\Omega})$-hull, i.e. its polynomial hull in case $\bar{\Omega}$ is polynomially convex.

In [Du1993], motivated by the problem of understanding polynomial convexity in geometric terms, the question of $\mathcal{O}(\bar{\Omega})$-convexity (instead of removability) of compact subsets of arbitrary surfaces $S \subset \partial \Omega$ (not necessarily diffeomorphic to a 2 -disc) is dealt with directly. If $K$ is a compact subset of a totally real surface $S \subset$ $\partial \Omega$, denote by $\widehat{K}_{\text {ess }}:=\widehat{K}_{\mathcal{O}(\bar{\Omega})} \backslash K$ the essential $\mathcal{O}(\bar{\Omega})$-hull of $K$. An application of Hopf's lemma shows that if $K=A(\partial \Delta)$ is the boundary of a $\mathcal{C}^{1}$ analytic disc $A \in \mathcal{O}(\Delta) \cap \mathcal{C}^{1}(\bar{\Delta})$ attached to the surface $S$, necessarily $K=\widehat{K}_{\text {ess }}$ is an immersed $\mathcal{C}^{1}$ curve that is everywhere transversal to the characteristic foliation of $S$. If $S$ has a hyperbolic complex tangency at one of its points $p$ and if $A(1)=p$, then $A(\partial \Delta)$ must cross at least one separatrix in every neighborhood of $p$. When $\widehat{K}_{\text {ess }}$ contains no analytic disc, similar transversality properties hold.

Theorem 5.18. ([Du1993]) Let $K \Subset S \subset \partial \Omega \Subset \mathbb{C}^{2}$ be as above, with $\partial \Omega \in \mathcal{C}^{2}$ strongly pseudoconvex and $S \in \mathcal{C}^{2}$ having finitely many hyperbolic complex tangencies. In the totally real part of $S$, the essential $\mathcal{O}(\bar{\Omega})$-hull $\widehat{K}_{\text {ess }}$ of $K$ crosses every characteristic curve that it meets. If $\widehat{K}_{\mathrm{ess}}$ meets a hyperbolic complex tangency, then it meets at least two hyperbolic sectors in every neighborhood of $p$.

As a consequence ([Du1993]), every compact subset $K$ of a two-dimensional disc $D \subset \partial \Omega$ that has only finitely many hyperbolic complex tangencies is $\mathcal{O}(\bar{\Omega})$ convex.

5.19. Totally real discs in nonpseudoconvex boundaries. All the above results heavily relied on strong pseudoconvexity, in contrast to the removability theorems presented in Section 6, where the adequate statements, based on general CR extension theory, are formulated in terms of CR orbits rather than in terms of Levi curvature. The first theorem for the non-pseudoconvex situation was established by the second author.

Theorem 5.20. ([[P02003]) Let $M$ be a $\mathcal{C}^{\infty}$ globally minimal hypersurface of $\mathbb{C}^{2}$ and let $D \subset M$ be a $\mathcal{C}^{\infty}$ one-codimensional submanifold that is diffeomorphic to the unit open 2-disc of $\mathbb{R}^{2}$ and maximally real at every point. Then every compact subset $K$ of $D$ is $C R$-, $L^{\mathrm{P}}$ - and $\mathcal{W}$-removable.

We would like to point out that, seeking theorems without any assumption of pseudoconvexity leads to substantial open problems, because one loses almost all of the strong interweavings between function-theoretic tools and geometric arguments which are valid in the pseudoconvex realm, for instance: Hopf 
Lemma, plurisubharmonic exhaustions, envelopes of function spaces, local maximum modulus principle, Stein neighborhood basis, etc.

We sketch the proof of the theorem. We first claim that $M \backslash K$ is (also) globally minimal. Indeed, if there were a lower-dimensional orbit $\mathcal{O}$ of $M \backslash K$, we would obtain a lower-dimensional orbit of $M$ by adding all characteristic arcs intersecting $\overline{\mathcal{O}}$ ([Po2003], Lemma 1; [MP2006a], Lemma 3.5). Then by Theorem 4.12(V), continuous CR functions on $M \backslash K$ extend holomorphically to a one-sided neighborhood $\mathcal{V}^{b}(M \backslash K)$.

For later application of the continuity principle, similarly as in MP2002, Po2003, MP2006a, we deform $M \backslash K$ in $\mathcal{V}^{b}(M \backslash K)$, so that the functions are holomorphic in some ambient neighborhood $\mathcal{U}$ of $M \backslash K$ in $\mathbb{C}^{2}$.

The first key idea is to construct an embedded 2-sphere containing a neighborhood of $K$ in $D$ and to apply the filling Theorem 5.17. This will give us a Levi flat 3-ball foliated by analytic discs, which by translations, will enable us to fill in a one-sided neighborhood of $K$.

In the case where $M=\partial \Omega$ is a strictly pseudoconvex boundary, the construction of the 2-sphere is quite direct: we pick an open 2-disc $D^{\prime}$ having $\mathcal{C}^{\infty}$ boundary $\partial D^{\prime} \simeq S^{1}$ with $K \subset D^{\prime} \Subset D$; translating it slightly and smoothly within $\partial \Omega$, we obtain an almost parallel copy $D^{\prime \prime} \subset \partial \Omega$; then we construct the 2-sphere $S^{\prime}$ by gluing (inside $\partial \Omega$ ) a thin closed strip $\simeq[-\varepsilon, \varepsilon] \times S^{1}$ to $\partial D^{\prime} \simeq S^{1}$ and to $\partial D^{\prime \prime} \simeq S^{1}$; finally, we perturb the strip part of $S^{\prime}$ in a generic way to assure that $S^{\prime}$ has only (a finite number of) isolated complex tangencies of elliptic or of hyperbolic type ${ }^{31}$. Then Theorem 5.17 yields a Levi-flat 3-ball $B^{\prime} \subset \Omega$ with $\partial B^{\prime}=S^{\prime}$.

If $M$ is not strongly pseudoconvex, the filling of $S^{\prime}$ by a Levi-flat ball $B^{\prime}$ may fail, because of a known counter-example [FM1995]. As a trick, we modify the construction. Using the fact that the squared distance function dist $\left(\cdot, D^{\prime}\right)^{2}$ is strictly plurisubharmonic in a neighborhood of $\bar{D}^{\prime}$ (by total reality), for $\varepsilon>0$ small, the sublevel sets

$$
\Omega_{\varepsilon}^{\prime}:=\left\{q \in \mathbb{C}^{2}: \operatorname{dist}\left(q, \overline{D^{\prime}}\right)<\varepsilon\right\}
$$

are strongly pseudoconvex neighborhoods of $\overline{D^{\prime}}$ intersecting $M$ transversally along the 2-spheres $\partial \Omega_{\epsilon}^{\prime} \cap M$.

\footnotetext{
${ }^{31}$ Observe that since $D$ is totally real, the last step can be done without changing $S^{\prime}$ along $D^{\prime}$.
} 

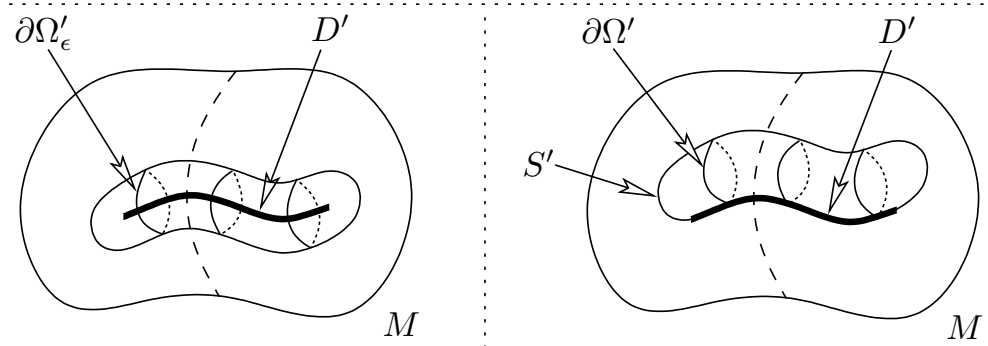

Construction of $S^{\prime}$

Furthermore, a given fixed $\Omega_{\epsilon}^{\prime}$ can be slightly isotoped (translated) to a domain $\Omega^{\prime}$ still strongly pseudoconvex and having boundary transverse to $M$ so that $D^{\prime}$ is precisely contained in the isotoped 2-sphere $\partial \Omega^{\prime} \cap M$. After a very slight generic perturbation, we may insure that $S^{\prime}$ has only elliptic or hyperbolic complex tangencies (a part of $\partial \Omega^{\prime}$ has also to be perturbed). In sum:

Lemma 5.21. ([Po2003]) There exists a bounded domain $\Omega^{\prime} \subset \mathbb{C}^{2}$ such that:

- $\partial \Omega^{\prime}$ is $\mathcal{C}^{\infty}$, strongly pseudoconvex and diffeomorphic to a 3-sphere;

- $\partial \Omega^{\prime}$ intersects $M$ transversally in a two-sphere $S^{\prime}:=\partial \Omega^{\prime} \cap M$;

- $S^{\prime}$ has $k$ hyperbolic and $k+2$ elliptic points;

- $\partial \Omega^{\prime}$ contains the open 2 -disc $D^{\prime} \supset K$.

Then Theorem 5.17 applies in the strongly pseudoconvex boundary $\partial \Omega^{\prime}$, yielding a Levi-flat 3 -sphere $B^{\prime} \subset \Omega^{\prime}$ with $\partial B^{\prime}=S^{\prime}$. However, the nonpseudoconvexity of $M$ obstructs further insights in the position of $B^{\prime}$ with respect to $M$. In fact, $B^{\prime}$ may change sides or even be partly contained in $M$.

In the (simpler) case where $M=\partial \Omega$ is a strongly pseudoconvex boundary, we introduce a foliation of a neighborhood of $S^{\prime}$ in $M$ by $\mathcal{C}^{\infty} 2$-spheres $S_{t}^{\prime}$ with $S_{0}^{\prime}=S^{\prime}$. By filling them, we get a family of Levi-flat 3-balls $B_{t}^{\prime}$ with $\partial B_{t}^{\prime}=S_{t}^{\prime}$. Denote $B_{t}^{\prime}=\cup_{s} \Delta_{t, s}^{\prime}$ the foliation of $B_{t}^{\prime}$ by holomorphic discs. For $t \neq 0$, each $\Delta_{t, s}^{\prime}$ has boundary $\partial \Delta_{t, s}^{\prime} \subset S_{t}^{\prime} \subset M \backslash K$. Thus, by means of the continuity principle, we may extend holomorphic functions in the neighborhood $\mathcal{U}$ of $M \backslash K$ to a neighborhood of $B_{t}^{\prime}$ in $\mathbb{C}^{n}$, for all small $t \neq 0$. A final simple check shows that Theorem 2.30 (rm5) applies to remove $B_{0}^{\prime}$, and we get holomorphic extension to the union $\cup_{t} B_{t}^{\prime}$, a set containing the strongly pseudoconvex open local side of $\Omega$ at every point of $K$.

Without pseudoconvexity assumption on $M$, we can still consider a foliation $S_{t}^{\prime}$, but now the global geometry of $B_{t}^{\prime}$ is no longer clear. If for instance $M$ is Levi-flat near $K$ and the $S_{t}^{\prime}$ are contained in the Levi-flat part, then the $B_{t}^{\prime}$ just form an increasing family whose union is just a subdomain of $M$. Therefore it seems necessary to deform $S^{\prime}$ once again in order to gain transversality of $B^{\prime}$ and $M$. Since the global behavior of $B^{\prime}$ is hard to control, a further localization is advisable. 
As in [Me1997], we consider the set $K_{\mathrm{nr}}$ of points $q \in K$ such that $\mathcal{O}(\mathcal{V}(M \backslash K))$ does not extend holomorphically to a one-sided neighborhood of $q$. So $\mathcal{O}(\mathcal{V}(M \backslash K))$ extends holomorphically to a one-sided neighorhood $\mathcal{V}^{b}\left(K \backslash K_{\mathrm{nr}}\right)$. By deforming $M$ at points of $K \backslash K_{\mathrm{nr}}$, we come down to the same situation with $K$ replaced with $K_{\mathrm{nr}}$, except that no point of $K_{\mathrm{nr}}$ should be removable. Assuming $K_{\mathrm{nr}} \neq \emptyset$, to conclude by contradiction, it then suffices to remove only one point of $K_{\mathrm{nr}}$.

To begin with, assume that $K_{\mathrm{nr}}$ is contained in finitely many of the disc boundaries $\partial \Delta_{0, s}^{\prime}$ which foliate $S^{\prime}=S_{0}^{\prime}$. Then we claim that no $\partial \Delta_{0, s}^{\prime}$ can be contained in $K_{\mathrm{nr}}$. Otherwise, $\partial \Delta_{0, s}^{\prime} \subset K_{\mathrm{nr}} \subset D^{\prime} \subset D$ and the 2-disc enclosed by $\partial \Delta_{0, s}$ in $S_{0}^{\prime}$ inside the totally real 2-disc $D^{\prime}$ contain no complex tangencies, but the filling provided by Theorem 7.17 excludes such a topological possibility. So $K_{\mathrm{nr}}$ is properly contained in a finite union of arcs, and hence removable by Theorem 4.9.

Therefore we may assume that $K_{\mathrm{nr}}$ has nonvoid intersection with infinitely many of $\partial \Delta_{0, s}^{\prime}$. Since there is only finitely many complex tangencies, there exists a $\partial \Delta_{0, s_{0}}^{\prime}$ with $\partial \Delta_{0, s_{0}}^{\prime} \cap K_{\mathrm{nr}} \neq \emptyset$ not encountering them. The same argument as above shows that $\partial \Delta_{0, s_{0}}^{\prime} \not \subset K_{\mathrm{nr}}$. Let $p_{0} \in \partial \Delta_{0, s_{0}}^{\prime} \cap K_{\mathrm{nr}}$.

If $\overline{\Delta_{0, s_{0}}^{\prime}}$ and $M$ meet transversally at $p_{0}^{\prime}$, holomorphic extension to a one-sided neighborhood at $p_{0}^{\prime}$ proceeds as in the strongly pseudoconvex case, by applying the continuity principle with discs $\Delta_{t, s}^{\prime} \subset B_{t}^{\prime}$ for $t \neq O$.

Assume now that $\overline{\Delta_{0, s_{0}}^{\prime}}$ is tangential to $M$ in $p_{0}^{\prime}$ or equivalently, that $\partial \Delta_{0, s_{0}}^{\prime}$ is tangential to the characteristic leaf in $p_{0}^{\prime}$. The idea is to change the angle of the discs close to $\Delta_{0, s_{0}}^{\prime}$, and to apply the above argument to the deformed disc passing through $p_{0}^{\prime}$. Since $\partial \Delta_{0, s_{0}}^{\prime} \not \subset K_{\mathrm{nr}}$, we may deform slightly $S^{\prime}$ near some point $q_{0}^{\prime} \in \partial \Delta_{0, s_{0}}^{\prime} \backslash K_{\mathrm{nr}}$ in the direction normal to $B^{\prime}$. More precisely, one deforms $S^{\prime}$ slightly, so that Theorem 5.17 still applies, and then picks up the disc of the deformed Levi-flat 3-ball that passes through $p_{0}^{\prime}$. In view of known results about normal deformations of small discs (Proposition 2.21(V); [Trp1990, BRT1994, Fu1994a]), the turning of the angle for large discs ([Fo1986, G11994]) may also be established in such a way (see [Po2003, Po2004]).

There is one final point to be handled carefully. We have to be sure that after turning the discs, the deformed disc boundary passing through the point $p_{0}^{\prime} \in K_{\mathrm{nr}}$ is not entirely contained in $K_{\mathrm{nr}}$. 


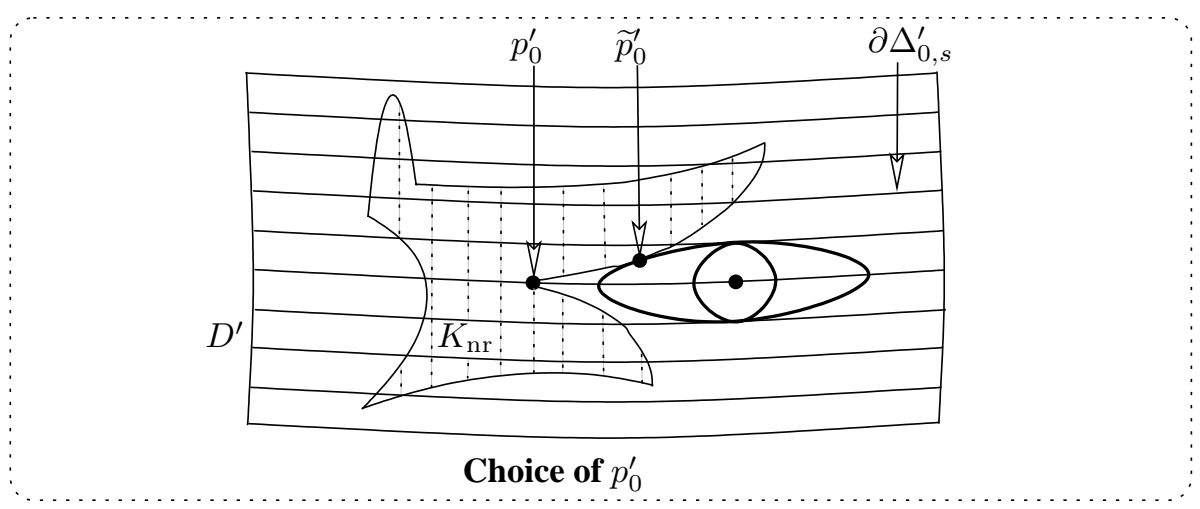

This can be assured by replacing $p_{0}^{\prime}$ by another special nearby point $\vec{p}_{0}^{\prime} \in K_{\mathrm{nr}}$ with a good transversality property as illustrated above.

Theorem 5.20 is not yet the complete generalization of Theorem 5.15 to nonpseudoconvex hypersurfaces, since $D$ is assumed to be totally real at every point. If $D$ has hyperbolic complex tangencies, it is not clear whether a sphere $S^{\prime}$ together with a strongly pseudoconvex boundary $\partial \Omega^{\prime} \supset S^{\prime}$ as in the above key lemma can be constructed. The recent Theorem 5.13 indicates that this is possible if hyperbolic complex tangencies are holomorphically flat, an assumption which would be rather ad hoc for the removal of compact sets $K \subset D$.

In fact, assuming generally that $M$ is an arbitrary globally minimal hypersurface, that a given surface $S \subset M$ has arbitrary topology (not necessarily diffeomorphic to an open 2-disc) and possesses complex tangencies, the reduction to the filling Theorem 5.17 seems to be impossible. Indeed, Fornæss-Ma ([FM1995]) constructed an unknotted nonfillable 2-sphere $S \subset \mathbb{C}^{2}$ having only two elliptic complex tangencies. To the authors' knowledge, the possibility of filling by Levi-flat 3 -spheres some 2-spheres lying in a nonpseudoconvex hypersurface is a delicate open problem. In addition, for the higher codimensional generalization of Theorem 1.2, the idea of global filling seems to be irrelevant at present times, because no analog of the filling Theorem 5.17 is known in dimension $n \geqslant 3$.

5.22. Beyond this survey. In the research article [MP2006a placed in direct continuation to this survey, we consider surfaces $S$ having arbitrary topology and we generalize Theorem 5.20 to arbitrary codimension, localizing the removability arguments and using only small analytic discs.

\section{REFERENCES}

[Ah1947] Ahlfors, L.V.: Bounded analytic functions, Duke Math. J. 14 (1947), 111.

[Ai1989] AĬRAPETIAN, R.A.: Extending CR functions from piecewise smooth $C R$ manifolds. Mat. Sbornik 134 (1987), 108-118. Trad. in english in Math. USSR Sbornik 62 (1989), 1, 111-120. 
[AH1981] Al̆RApetian, R.A.; Henkin, G.M.: Analytic continuation of CR-functions through the "edge of the wedge", Dokl. Akad. Nauk SSSR 259 (1981), 777781; English transl. in Soviet Math. Dokl. 24 (1981).

[AH1984] AĬRAPETIAN, R.A.; HENKIN, G.M.: Integral representations of differential forms on Cauchy-Riemann manifolds and the theory of CR-functions (Russian), Uspekhi Mat. Nauk 39 (1984), no. 3(237), 39-106. English translation: Russian Math. Surveys 39 (1984), no. 3, 41-118.

[Ak1987] AKAHORI, T.: A new approach to the local embedding theorem of CRstructures for $n \geqslant 4$, Memoirs Amer. Math. Soc., Number 366, Amer. Math. Soc., Providence, 1987.

[A11975] AlEKSANDROV, A.B.: The norm of Hilbert transform in the space of Hölder functions, Funkts. Anal. Prilozh. 9, 1-4 (1975). English tranls.: Funct. Anal. Appl. 9, 94-96 (1975).

[Al1972] Alexander, H.: A note on polynomially convex hulls, Proc. Amer. Math. Soc. 33 (1972), 389-391.

[A11993] AlEXANDER, H.: Removable sets for CR functions, Several complex variables (Stockholm, 1987/1988), 1-7, Math. Notes, 38, Princeton University Press, Princeton, NJ, 1993.

[AS1990] AleXAnder, H.; Stout, E.L.: A note on hulls, Bull. London Math. Soc. 22 (1990), no. 3, 258-260.

[AW1998] Alexander, H.; Wermer, J.: Several complex variables and Banach algebras, Third edition, Graduate texts in mathematics, 35, Springer-Verlag, Berlin-New York, xii+253 pp., 1998.

[ABR1994] AlinhaC, S.; BaOuendi, M.S.; Rothschild, L.P.: Flat analytic discs attached to real hypersurfaces of finite type, Math. Research Lett. 1 (1994), no. 3, 359-367.

[AC1994] Anderson, J.A.; CimA, J.T.: Removable singularities for $L^{p}$ CR functions, Mich. Math. J. 41 (1994), no. 1, 111-119.

[AH1972a] AndreotTi, A.; Hill, C.D.: Complex characteristic coordinates and tangential Cauchy-Riemann equations, Ann. Scuola Norm. Sup. Pisa, Sci. Fis. Mat. 26 (1972), 299-324.

[AH1972b] Andreotti, A.; Hill, C.D.: E. E. Levi convexity and the Hans Lewy problem, I and II, Ann. Scuola Norm. Sup. Pisa, Sci. Fis. Mat. 26 (1972), 325363, 747-806.

[Ar1978] ARNOL'D, V.I.: Ordinary differential equations. Translated from the Russian and edited by R.A. Silverman, MIT Press, Cambridge, Mass.-London, 1978.

[Ar1988] ARnOL'D, V.I.: Dynamical systems. I. Ordinary differential equations and smooth dynamical systems, Translated from the Russian. Edited by D. V. Anosov and V. I. Arnol'd. Encyclopaedia of Mathematical Sciences, 1. Springer-Verlag, Berlin, 1988. $\mathrm{x}+233$ pp.

[Ar1968] ARTIN, M.: On the solutions of analytic equations, Invent. Math. 5 (1968), 277-291.

[BCT1983] BAOUEnd, M.S.; CHAng, C.H.; Treves, F: Microlocal hypo-analyticity and extension of CR functions, J. Differential Geom. 18 (1983), no. 3, 331391. 
[BER1996] BAOUENDI, M.S.; EBENFELT, P.; RothSCHILD, L.P.: Algebraicity of holomorphic mappings between real algebraic sets in $\mathbb{C}^{n}$, Acta Math. 177 (1996), no. 2, 225-273.

[BER1999] BAOUENDI, M.S.; EBEnfelt, P.; RothsChiLD, L.P.: Real submanifolds in complex space and their mappings. Princeton Mathematical Series, 47, Princeton University Press, Princeton, NJ, 1999, xii+404 pp.

[BER2000] BaOuendi, M.S.; Ebenfelt, P.; Rothschild, L.P.: Local geometric properties of real submanifolds in complex space, Bull. Amer. Math. Soc. 37 (2000), no. 3, 309-336.

[BER2003] Baouendi, M.S.; Ebenfelt, P.; Rothschild, L.P.: Dynamics of the Segre varieties of a real submanifold in complex space, J. Algebraic Geom. 12 (2003), no. 1, 81-106.

[BMR2002] BAOUENDI, M.S.; MIR, N.; RoTHSCHILD, L.P.: Reflection ideals and mappings between generic submanifolds in complex space, J. Geom. Anal. 12 (2002), no. 4, 543-580.

[BJT1985] BAOUENDI, M.S.; JACOBOWITZ, H.; TREVES, F.: On the analyticity of $C R$ mappings, Ann. of Math. 122 (1985), no. 2, 365-400.

[BR1987] BAOUENDI, M.S.; ROTHSCHILD, L.P.: Normal forms for generic manifolds and holomorphic extension of CR functions, J. Differential Geom. 25 (1987), no. 3, 431-467.

[BR1988] BAOUENDI, M.S.; RothSCHILD, L.P.: Germs of CR maps between real analytic hypersurfaces, Invent. Math. 93 (1988), no. 3, 481-500.

[BR1990] BAOUENDI, M.S.; RotHSCHILD, L.P.: Geometric properties of mappings between hypersurfaces in complex space, J. Differential Geom. 31 (1990), 473-499.

[BR1993] BAOUENDI, M.S.; ROTHSCHILD, L.P.: Unique continuation and a Schwarz reflection principle for analytic sets, Commun. in partial differential equations 18 (1993), no. 11, 1961-1970.

[BRT1994] BAOUENDI, M.S.; RothSChILD, L.P.; TrÉPREAU, J.-M.: On the geometry of analytic discs attached to real manifolds, J. Diff. Geom. 39 (1994), 379405.

[BT1981] BAOUENDI, M.S.; TREVES, F: A property of the functions and distributions annihilated by a locally integrable system of complex vector fields, Ann. of Math. 113 (1981), no. 2, 387-421.

[BT1984] BAOUENDI, M.S.; TREVES, F: About the holomorphic extension of CR functions on real hypersurfaces in complex space, Duke Math. J. 51 (1984), no. 1, 77-107.

[BT1988] BAOUENDI, M.S.; TREVES, F: Unique continuation in CR manifolds and in hypo-analytic structures, Ark. Mat. 26 (1988), no. 1, 21-40.

[BeFe1973] Beals, R.; FefFerman, C.: On local solvability of partial differential equations, Ann. Math. 97 (1973), 552-571.

[BeFo1978] BEDFORD, E.; FORNÆSS, J.E.: Local extension of CR functions from weakly pseudoconvex boundaries, Michigan Math. J. 25 (1978), no. 3, 259-262.

[BG1983] Bedford, E.; Gaveau, B.: Envelopes of holomorphy of certain twospheres in $\mathbb{C}^{2}$, Amer. J. Math. 105 (1983), no. 4, 975-1009. 
[BK1991] BEDFORD, E.; KLIngEnBERG, W.: On the envelope of holomorphy of a 2-sphere in $\mathbb{C}^{2}$, J. Amer. Math. Soc. 4 (1991), no. 3, 623-646.

[BT1970] Behnke, H.; Thullen, P.: Theorie der Funktionen mehrerer komplexer Veränderlichen, Springer-verlag, Berlin-New York, xvi+225 pp., 1970.

[Bel1996] Bellaïche, A.: SubRiemannian Geometry, Progress in Mathematics 144, Birkhäuser Verlag, Basel/Switzerland, 1996, 1-78.

[Be1983] Bennequin, D.: Entrelacements et équations de Pfaff, Astérisque 107/108, 87-161, 1983.

[Be1957] Bers, L.: Riemann surfaces, Lecture Notes, Courant Inst. of Math. Sci., New York (1957-58).

[BM1997] BERHANU, S.; MENDOZA, G.A.: Orbits and global unique continuation for systems of vector fields, J. Geom. Anal. 7 (1997), no. 2, 173-194.

[BH2000] BERHANU, S.; HoUNIE, J.: Uniqueness for locally integrable solutions of overdetermined systems, Duke Math. J. 105 (2000), no. 3, 387-410.

[BH2003] Berhanu, S.; Hounie, J.: Traces and the F. and M. Riesz theorem for vector fields, Ann. Inst. Fourier (Grenoble) 53 (2003), no. 5, 1425-1460.

[BCH2005] Berhanu, S.; Cordaro, P.; Hounie, J.: An introduction to involutive structures, New Mathematical Monographs Series, Cambridge University Press, to appear.

[Bi1965] BISHOP, E.: Differentiable manifolds in complex Euclidean space, Duke Math. J. 32 (1965), no. 1, 1-22.

[Bo1938] Bochner, S.: A theorem on analytic continuation of functions in several variables, Ann. Math. 39 (1938), 14-19.

[Bo1956] BoCHNER, S.: Weak solutions of linear partial differential equations, J. Math. Pures Appl. 35 (1956), 193-202.

[BM1949] Bochner, S.; MARTin, W.: Several Complex Variables, Princeton Math. Ser. 10, Princeton Univ. Press, Princeton, NJ, 1949.

[Bo1991] Boggess, A.: CR manifolds and the tangential Cauchy-Riemann complex. Studies in Advanced Mathematics. CRC Press, Boca Raton, FL, 1991, xviii+364 pp.

[BPi1985] Boggess, A.; PITTS, J.: CR extension near a point of higher type, Duke Math. J. 52 (1985), no. 1, 67-102.

[BPo1982] Boggess, A.; Polking, J.C.: Holomorphic extension of CR functions, Duke Math. J. 49 (1982), no. 4, 757-784.

[Bo1972] BourbaKi, N.: Groupes at algèbres de Lie, chapitre 2, Hermann, Paris, 1972.

[Br1961] Browder, F.: Cohomology of maximal ideal spaces, Bull. Amer. Math. Soc. 67 (1961), 515-516.

[BSW1978] Burns, D.Jr.; Shnider, S.; Wells, R.O.Jr.: Deformations of strictly pseudoconvex domains Invent. Math. 46 (1978), no. 3, 237-253.

[Cal1977] CALDERón, A.P.: Cauchy integrals on Lipschitz curves and related operators, Proc. Nat. Acad. Sci. U.S.A. 74 (1977), 1324-1327.

[CLN1985] CAMACHO, C.; LINS NeTO, A.: Geometric theory of foliations, Birkhäuser, Boston, 1985.

[Ca1963] CARLESON, L.: Removable singularities of continuous harmonic functions in $\mathbb{R}^{m}$, Math. Scand. 12 (1963), 15-18. 
[Ca1966] CARLESON, L.: On convergence and growth of partial sums of Fourier series, Acta Math. 116 (1966), 135-157.

[Ca1932] CARTAN, É.: Sur la géométrie pseudo-conforme des hypersurfaces de l'espace de deux variables complexes, I, Ann. Math. Pura Appl. 11 (1932), $17-90$.

[Ca1831] CAUCHY, A.L.: Sur la mécanique céleste et sur un nouveau calcul appelé calcul des limites, Turin, 1831.

[Ch1955] CHERN, S.-S.: An elementary proof of the existence of isothermal parameters on a surface, Proc. Amer. Math. Soc. 6 (1955), 771-786.

[Ch1975] CHIRKA, E.M.: Analytic representation of CR-functions (Russian), Mat. Sb. (N.S.) 98(140) (1975), no. 4(12), 591-623, 640.

[Ch1982] CHIRKA, E.M.: Regularity of boundaries of analytic sets, (Russian) Mat. Sb (N.S.) 117 (159) (1982), 291-334. English translation in Mat. USSR Sb. 45 (1983), 291-336.

[Ch1989] ChIRKA, E.M.: Complex analytic sets, Mathematics and its applications (Soviet Series), 46. Kluwer Academic Publishers Group, Dordrecht, 1989. $\mathrm{xx}+372 \mathrm{pp}$.

[Ch1991] CHIRKA, E.M.: An introduction to the geometry of CR manifolds (Russian), Uspekhi Mat. Nauk 46 (1991), no. 1(277), 81-164, 240; translation in Russian Math. Surveys 46 (1991), no. 1, 95-197

[Ch1994] CHIRKA, E.M.: Radò's theorem for CR-mappings of hypersurfaces (Russian), Mat. Sb. 185 (1994), no. 6, 125-144; translation in Russian Acad. Sci. Sb. Math. 82 (1995), no. 1, 243-259.

[Ch2001] CHIRKA, E.M.: Levi and Trépreau theorems for continuous graphs. (Russian) Tr. Mat. Inst. Steklova 235 (2001), Anal. i Geom. Vopr. Kompleks. Analiza, 272-287; translation in Proc. Steklov Inst. Math. 2001, no. 4 (235), 261-276.

[CCS1999] ChiRKa, E.M.; Coupet, B.; Sukhov, A.: On boundary regularity of analytic discs. Michigan Math. J. 46 (1999), no. 2, 271-279.

[CR1994] CHIRKA, E.M.; REA, C: Normal and tangent ranks of CR mappings Duke Math. J. 76 (1994), no. 2, 417-431.

[CR1998] ChIRKA, E.M.; REA, C: Differentiable CR mappings and CR orbits, Duke Math. J. 94 (1998), no. 2, 325-340.

[CR2003] ChIRKA, E.M.; REA, C: The F. and M. Riesz theorem for CR functions, Math. Z. 250 (2005), no. 1, 1-6.

[CSh1995] Chirka, E.M.; ShCherbina, N. V.: Pseudoconvexity of rigid domains and foliations of hulls of graphs, Ann. Scuola Norm. Sup. Pisa Cl. Sci. (4) 22 (1995), no. 4, 707-735.

[CSt1994] Chirka, E.M.; Stout, E.L.: Removable singularities in the boundary. Contributions to complex analysis and analytic geometry, 43-104, Aspects Math., E26, Vieweg, Braunschweig, 1994.

[CMM1982] CoIfMAn, R.; McIntosh, A.; MeYer, Y.: L'opérateur de Cauchy définit un opérateur borné sur $L^{2}$ sur les courbes lipschitziennes, Ann. of Math. 116 (1982), 361-388.

[CMS1999] Coupet, B.; Meylan, F.; Sukhov, A.: Holomorphic maps of algebraic CR manifolds, Internat. Math. Res. Notices 1999, no. 1, 1-29. 
[Da2001] DAMOUR, S.: On the analyticity of smooth CR mappings between real analytic CR manifolds, Michigan Math. J. 49 (2001), no. 3, 583-603.

[De1996] Demailly, J.-P.: Analyse numérique et équations différentielles, Presses Universitaires de Grenoble, Grenoble, 1996.

[De1997] Demailly, J.-P.: Analytic and algebraic geometry, lecture notes available at: http://www-fourier.ujf-grenoble.fr/ demailly.

[DR2002] DE REYNA, J.A.: Pointwise convergence of Fourier Series, Lecture Notes in Mathematics, 1785, Springer-Verlag, Berlin, 2002, xviii+173 pp.

[DF1978] DIEDERICH, K.; FORNÆSS, J.E.: Pseudoconvex domains with real analytic boundary, Ann. of Math. 107 (1978), no. 2, 371-384.

[DF1977] DiEDERICH, K.; FORnÆSS, J.E.: Pseudoconvex domains: an example with nontrivial Nebenhülle, Math. Ann. 225 (1977), no. 3, 275-292.

[DF1988] DiEDERICH, K.; FORnÆSS, J.E.: Proper holomorphic mappings between real-analytic pseudoconvex domains in $\mathbb{C}^{n}$, Math. Ann 282 (1988), no. 4, 681-700.

[DP1993] DIEDERICH, K.; PINCHUK, S.: The inverse of a CR-homeomorphism is CR, Internat. J. Math. 4 (1993), no. 3, 379-394.

[DP1995] Diederich, K.; PinchuK, S.: Proper holomorphic maps in dimension 2 extend, Indiana Univ. Math. J. 44 (1995), no. 4, 1089-1126.

[DP1998] DIEDERICH, K.; PINCHUK, S.: Reflection principle in higher dimensions, Proceedings of the International Congress of Mathematicians, Vol II (Berlin 1998), Doc. Math. 1998, Extra Vol. II, 703-712.

[DP2003] DIEDERICH, K.; PINCHUK, S.: Regularity of continuous CR-maps in arbitrary dimension, Michigan Math. J. 51 (2003), 111-140. Erratum: ib., no. 3, 667-668.

[DW1980] DIEDERICH, K.; WEBSTER, S.M.: A reflection principle for degenerate real hypersurfaces, Duke Math. J. 47 (1980), no. 4, 835-843.

[Di1998] DinH, T.C.: Enveloppe polynomiale d'un compact de longueur finie et chaînes holomorphes à bord rectifiable, Acta Math. 180 (1998), no. 1, 3167.

[DS2001] DINH, T.C.; SARKIS, F.: Wedge removability of metrically thin sets and application to the CR meromorphic extension. Math. Z. 238 (2001), no. 3, 639-653.

[DP1980] DinI, G.; PARRINI, C.: Singolarità rimovibili per CR-distribuzioni su domini piatti, Boll. Un. Mat. Ital. B (5) 17 (1980), no. 1, 286-297.

[DP1982] DINI, G.; PARRINI, C.: Extending CR-distributions, Bull. Sci. Math. (2) 106 (1982), no. 1, 3-18.

[D11977] Dloussky, G.: Enveloppes d'holomorphie et prolongement d'hypersurfaces, Lecture Notes in Math. 578, Springer-Verlag 1977, 217-235.

[Do2000] Dolbeault, P.: Variétés et espaces analytiques complexes, Development of mathematics 1950-2000, 359-436, Birkhäuser, Basel, 2000, 359-436.

[DH1997] Dolbeault, P.; Henkin, G.M.: Chaînes holomorphes de bord donné dans $P_{n}(\mathbb{C})$, Bull. Soc. Math. France 125 (1997), 383-445. 
[Dy1991] DYN'KIN, E.M.: Methods of the theory of singular integrals: Hilbert transform and Calderón-Zygmund theory, in: Commutative Harmonic Analysis, I, Encyclopædia of mathematical sciences, Vol. 15, V.P. Khavin and N.K. Nikol'skij (Eds.), Springer-Verlag, 1991, pp. 167-259.

[Du1993] Duval, J.: Surfaces convexes dans un bord pseudoconvexe, Colloque d'Analyse Complexe et Géométrie, Marseille, 1992; Astérisque 217, Soc. Math. France, Montrouge, 1993, 6, 103-118.

[DH1992] DwilewiCZ, R.; Hill, C.D.: An analytic disc approach to the notion of type of points, Indiana Univ. Math. J. 41 (1992), no. 3, 713-739.

[DM2002] DWILEWICZ, R.; MERKER, J.: Hartogs-Bochner phenomenon and decomposition of CR functions, Proc. Amer. Math. Soc. 130 (2002), no. 7, 19751980.

[Eb2002] EBENFELT, P.: On the analyticity of CR mappings between nonminimal hypersurfaces, Math. Ann. 322 (2002), no. 3, 583-602.

[ER2004] EBENFELT, P.; RothSCHILD, L.P.: Transversality of CR mappings, to appear in Amer. Math. J.; e-print: arxiv/abs/math. CV/0410445.

[EDM1993] ENCYCLOPEDIC DictionARY OF MATHEMATICS (Itô, K. dir.), MIT Press, Massachusetts, Second Edition, 1993.

[ES1993] EgOROV, Y.V.; SHUBIN, M.A.: Partial differential equations. IV Microlocal analysis and hyperbolic equations, Encyclopædia of Mathematical Sciences, 33, Springer-Verlag, Berlin, 1993. vi+241 pp.

[El1989] Eliashberg, Y.: Classification of overtwisted contact structures on 3manifolds, Invent. Math. 98 (1989), no. 3, 623-637.

[EK2000] Estrada, R.; KAnwal, R.P.: Singular integral equations, Birkhäuser, Basel, 2000.

[Fa1906] Fatou, P.: Séries trigonométriques et séries de Taylor, Acta Math. 30 (1906), 335-400.

[Fe1969] FEDERER, H.: Geometric measure theory, Die Grundlehren der mathematischen Wissenschaften, Band 153 Springer-Verlag New York Inc., New York 1969, xiv+676.

[Fi1991] FicherA, G.: The Severi and Severi-Kneser thoerems for analytic functions of several complex variables and their further developments. Confer. Sem. Mat. Univ. Bari 1991, 237-244.

[FM1995] FornÆSS, J.E.; MA, D.: A 2-sphere in $\mathbb{C}^{2}$ that cannot be filled in with analytic discs, International Math. Res. Notices, 1995, no. 1, 17-22.

[FR1985] FORNÆSS, J.E.; REA, C.: Local holomorphic extendability and nonextendability of CR-functions on smooth boundaries, Ann. Scuola Norm. Sup. Pisa Cl. Sci. (4) 12 (1985), no. 3, 491-502.

[FS1987] FORnÆSs, J.E.; STENSøNES, B.: Lectures on counterexamples in several complex variables, Mathematical notes, 33, Princeton University Press, Princeton, 1987, 247 pp.

[F1998] FORNÆSS, J.E.: The disc method, Math. Z. 227 (1998), no. 4, 705-709.

[FS2001] FornÆSS, J.E.; SIBONY, N.: Some open problems in higher dimensional complex analysis and complex dynamics, Publ. Mat. 45 (2001), no. 2, 529547. 
[FoSt1987] FORnÆSs, J.E.; STEnsønes, B.: Lectures on counterexamples in several complex variables, Princeton university press, Princeton, N.J., University of Tokyo Press, Tokyo, viii+248 pp., 1987.

[Fo1986] FORSTNERIČ, F.; Analytic discs with boundaries in a maximal real submanifold of $\mathbb{C}^{2}$, Ann. Inst. Fourier (Grenoble), 37 (1987), no. 1, 1-44.

[Fo1993] ForSTnerič, F.: Proper holomorphic mappings: a survey, Several complex variables (Stockholm, 1987/1988), 297-363, Math. Notes, 38, Princeton Univ. Press, Princeton, NJ, 1993.

[FS1991] Forstnerič, F.; Stout, E.L.: A new class of polynomially convex sets, Ark. Mat. 29 (1991), no. 1, 52-62.

[Fo2004] FORSTNERIČ, F.; Most real analytic Cauchy-Riemann manifolds are nonalgebraizable, Manuscripta Math. 115 (2004), no. 4, 489-494.

[Fr1977] FreEman, M.: Real submanifolds with degenerate Levi form, Several complex variables (Proc. Sympos. Pure Math., Vol. XXX, Williams Coll., Williamstown, Mass., 1975), Part 1, pp. 141-147. Amer. Math. Soc., Providence, R.I., 1977.

[FG2002] FRITZSCHE, K.; GRAUERT, H.: From holomorphic functions to complex manifolds, Graduate Texts in Mathematics, 213, Springer-Verlag, BerlinNew York, xvi+392 pp., 2002.

[Fr1877] Frobenius, G.: Ueber das Pfaffsche Problem, J. Reine Angew. Math. 82 (1877), 230-315.

[Ga1966] GAKHOV, F.D.: Boudary value problems, Moscow, 1966. English translation editor I.N. Sneddon, Pergamon Press, Oxford, 1966, xix+561 pp.

[Gar1970] Garnett, J.B.: Positive length but zero analytic capacity, Proc. Amer. Math. Soc. 21 (1970), 696-699.

[GM2004] Gaussier, H.; Merker, J.: Nonalgebraizable real analytic tubes in $\mathbb{C}^{n}$, Math. Z. 247 (2004), no. 2, 337-383.

[Ge1988] GershKovich, V.Ya.: On normal forms of distribution jets. In: Topology and Geometry, Rokhlin Semin. 1984-1986, Lect. Notes Math. 1346 (Viro, O. Ya. (Ed.)), 1988, 77-98.

[GV1987] Gershrovich, V.Ya.; VershiK, A.M.: Nonholonomic dynamical systems. Geometry of distributions and variational problems. Dynamical Systems VII, Encyclopædia of mathematical sciences, Volume 16, V.I. Arnol'd and S.P. Novikov (Eds.), 1-81, Springer-Verlag, Berlin, 1994.

[G11994] Globevnik, J.: Perturbation by analytic discs along maximal real submanifolds of $\mathbb{C}^{N}$. Math. Z. 217 (1994), no. 2, 287-316.

[G11996] GlobeVnik, J.: Partial indices of analytic discs attached to lagrangian submanifolds of $\mathbb{C}^{n}$. Ann. Inst. Fourier, Grenoble 46 (1996), no. 5, 13071326.

[GR1956] GRAUERT, H.; REMMERT, R.: Konvexität in ther komplexen Analysis. Comment. Math. Helv. 31 (1956), 152-183.

[GPR1994] Grauert, H., Peternell, T., Remmert, R.: Several Complex Variables VII, Encyclopaedia of Mathematical Sciences 74, Springer-Verlag, Berlin 1994, xx+369 pp.

[Gr1968] GREENFIELD, S.J.: Cauchy-Riemann equations in several variables, Ann. Sc. Norm. Sup. Pisa 22 (1968), 275-314. 
[Gu1990] Gunning, R.: Introduction to Holomorphic Functions of Several Variables, 3 vol., Wadsworth \& Brooks/Cole, I: Function theory, xx+203 pp., II: Local theory, +218 pp, III: Homological theory, +194 pp., 1990.

[HS1982] Hanges, N; SJÖSTRAnd, J.: Propagation of analyticity for a class of nonmicrocharacteristic operators, Ann. of Math. 116 (1982), 559-577.

[HT1983] Hanges, N; Treves, F.: Propagation of holomorphic extendability of CR functions, Math. Ann. 263 (1983), no. 2, 157-177.

[HaTr1993] Hanges, N; Treves, F.: On the local holomorphic extension of CR functions, Colloque d'Analyse Complexe et Géométrie, Marseille, 1992; Astérisque 217, Soc. Math. France, Montrouge, 1993, 6, 119-137.

[Ha1885] HARNACK, A.: Beiträge zur Theorie des Cauchy'schen Integrals, 13, Bd 37 (1885), 379-398.

[Ha1982] HaRTMAn, P.: Ordinary Differential Equations. Birkhäuser, Boston 1982.

[Ha1906] HaRTogs, F.: Einige Folgerungen aus der Cauchyschen Integralformel bei Funktionen mehrerer Veränderlichen, Bayerische Akademie der Wissenschaften. Mathematish-Physikalisch Klasse 36 (1906), 223-292.

[Ha1909] HARTOGS, F.: Einige Folgerungen aus der Cauchyschen Integralformel bei Funktionen mehrerer Veränderlicher, Acta Math. 32 (1909), 57-79.

[Ha1977] HARVEY, F.R.: Holomorphic chains and their boundaries, Several complex variables (Proc. Sympos. Pure Math. Vol. XXX, Part 1, Williamstown, Mass. 1975), pp. 309-382, American Math. Soc. Providence, Rhode Island, 1977.

[HL1975] HARVEY, R.; LAWSON, B.: On boundaries of complex analytic varieties. Ann. of Math., I: 102 (1975), no. 2, 233-290; II: 106 (1977), no. 2, 213 238.

[HP1970] HARVEY, F.R.; POLKING, J.: Removable singularities of solutions of linear partial differential equations, Acta Math. 125 (1970), 39-56.

[HP1972] HARVEY, F.R.; POLKING, J.: A notion of capacity which characterizes removable singularities, Trans. Amer. Math. Soc. 169 (1972), 183-195.

[HW1975] HARVEY, R.; WELls, R.O.: Holomorphic approximation and hyperfunction theory on a $\mathcal{C}^{1}$ totally real submanifold of a complex manifold, Math. Ann. 197 (1972), 287-318.

[Hz1989] HatzIAFRATIS, T.: On certain integrals associated to CR-functions, Trans. Amer. Math. Soc. 314 (1989), no. 2, 781-802.

[Ha2003] HAUSER, H.: The Hironaka theorem on resolution of singularities (or: A proof we always wanted to understand), Bull. Amer. Math. Soc. (N.S.) 40 (2003), no. 3, 323-403.

[HH1983] HECTOR, G.; HIRSCH, U.: Introduction to the geometry of foliations, Part A \& B, Fr. Vieweg \& Sohn, Braunschweig/Wiesbaden, 1983.

[He1980] Henkin, G.M.: Analytic representation for CR-functions on manifolds of codimension 2 in $\mathbb{C}^{n}$, Soviet. Math. Dokl. 21 (1980), no. 1, 85-91.

[He1985] HENKIN, G.M.: The method of integral representations in complex analysis (Russian), Current problems in mathematics. Fundamental directions, Vol. 7, 23-124, 258, Itogi Nauki i Tekhniki, Akad. Nauk SSSR, Vsesoyuz. Inst. Nauchn. i Tekhn. Inform., Moscow, 1985. 
[HeLe1984] Henkin, G.M.; LeITERER, J.: Theory of functions on complex manifolds. Monographs in Mathematics, 79, Birkhäuser, Basel-Boston, Mass., 1984, 226 pp.

[HM2002] Henkin, G.M.; Michel, V.: Principe de Hartogs dans les variétés CR, J. Math. Pures Appl. 81 (2002), no. 12, 1313-1395.

[He1963] HeRMANN, R.: On the accessibility problem in control theory, Internat. Sympos. Nonlinear Differential Equations and Nonlinear Mechanics, Academic Press, New York, 1963, pp. 325-332.

[Hi1973] HILl, D.: A Kontinuitätssatz for $\bar{\partial}_{M}$ and Lewy extendibility, Indiana Univ. Math. J. 22 (1972/73), 339-353.

[HN2000] Hill, D.; NACINOVICH, M.: Conormal suspensions of differential complexes, J. Geom. Anal. 10 (2000), no. 3, 481-523.

[HiTa1978] HILl, D.; TAIAni, G.: Families of analytic discs in $\mathbb{C}^{n}$ with boundaries in a prescribed CR manifold, Ann. Scuola Norm. Sup. Pisa 5 (1978), 327-380.

[HiTa1984] Hill, D.; TAIANI, G.: On the Hans Lewy extension phenomenon in higher codimension, Proc. Amer. Math. Soc. 91 (1984), no. 4, 568-572.

[Hi1976] HiRsch, M.W.: Differential topology, Graduate Texts in Mathematics, 33, Springer-Verlag, Berlin, 1976, x+222 pp.

[HS1974] HiRSCH, M.W.; SMALE, S.: Differential equations, dynamical systems, and linear algebra, Pure and Applied Mathematics, Vol. 60, Academic Press, New York-London, 1974, xi+358 pp.

[Hö1963] HörmANDER, L.: Linear partial differential operators, Die Grundlehren der mathematischen Wissenschaften, Bd. 116 Academic Press, Inc., Publishers, New York; Springer-Verlag, Berlin-Göttingen-Heidelberg 1963 vii+287 pp.

[Hö1973] HÖRMANDER, L.: An introduction to complex analysis in several variables, Noth-Holland Publ. Co., $2^{\text {nd }}$ ed., Amsterdam, London, 1973 ( $1^{\text {st }}$ ed.: 1966).

[Hö1978] Hörmander, L.: Propagation of singularities and semi-global existence theorems for (pseudo)-differential operators of principal type, Ann. of Math. 108 (1978), 569-370.

[Hö1985] HÖRMANDER, L.: The analysis of linear partial differential operators, 4 vol., Springer Verlag, Berlin, 1983, 1985.

[Hö1994] Hörmander, L.: Notions of convexity, Progress in Mathematics, 127, Birkhäuser, Boston, Inc., Boston, MA, viii.+414 pp., 1994.

[HM1998] Hounie, J.; Malagutti, P.: On the convergence of the Baouendi-Treves approximation formula, Comm. P. D. E. 23 (1998), 1305-1347.

[HP1996] HounIE, J.; DE LEMOS, E.P.: On local solvability in $L^{\mathrm{p}}$ of first-order equations, J. Math. An. and Appl. 197 (1996), 42-53.

[HT1993] HOUNIE, J.; TAVARES, J.: Radó's theorem for locally solvable vector fields, Proc. Amer. Math. Soc. 119 (1993), 829-836.

[HT1996] HOUNIE, J.; TAVARES, J.: On removable singularities of locally solvable differential operators, Invent. Math. 126 (1996), 589-623.

[HT1997] HOUNIE, J.; TAVARES, J.: Removable singularities of vector fields and the Nirenberg-Treves property, Multidimensional complex analysis and partial differential equations (São Carlos, 1995), 127-139, Contemp. Math., 205, Amer. Math. Soc., Providence, RI, 1997, 127-139. 
[Hu1998] Huang, X.: On an n-manifold in $C^{n}$ near an elliptic complex tangent, J. Amer. Math. Soc. 11 (1998), no. 3, 669-692.

[Hu2001] HuANG, X.: On some problems in several complex variables and CR geometry, First International Congress of Chinese Mathematicians (Beijing, 1998), 383-396, AMS/IP Stud. Adv. Math., 20, Amer. Math. Soc., Providence, RI, 2001.

[HJY2001] HUANG, X.; JI, S.; YAU, S.T.: An example of a real analytic strongly pseudoconvex hypersurface which is not holomorphically equivalent to any algebraic hypersurface, Ark. Mat. 39 (2001), no.1, 75-93.

[HuKr1993] HUANG, X.; KRANTZ, S.G.: A unique contiunation problem for holomorphic mappings, Commun. in partial differential equations 18 (1993), no. 2, 241-253.

[HuKr1995] Huang, X.; Krantz, S.G.: On a problem of Moser, Duke Math. J. 78 (1995), no. 1, 213-228.

[Hu1966] HunT, R.A.: On the convergence of Fourier series. Orthogonal expansions and their continuous analogues, Proc. Conf. Edwardsville (1967), III, Southern Illinois Univ. Press, 235-255 (1966).

[Hur1897] HuRwITZ, A.: Über die Entwicklung der allgemeinen Theorie der analytischen Funktionen in neuerer Zeit, in: Proc. 1st International Congress of Mathematicians, Zürich, 1897, 1898, pp. 91-112.

[Iv1992] IVASHKOVITCH, S.M.; The Hartogs-type extension theorem for meromorphic maps into compact Kähler manifolds, Invent. Math., 109 (1992), no. 1, 47-54.

[Ja1990] JACOBOwITZ, An introduction to CR structures, Math. Surveys and Monographs, Amer. Math. Soc., Providence, 1990.

[JT1982] JaCobowitz, H.; Treves, F.: Nonrealizable CR structures, Invent. Math. 66 (1982), 231-249.

[JaPf2000] Jarnicki, J.; Pflug, P.: Extension of holomorphic functions, De Gruyter Expositions in Mathematics, 34, De Gruyter, Berlin, 2000, x+487 pp.

[Ji2002] JI, S.: Algebraicity of real analytic hypersurfaces with maximal rank, Amer. J. Math. 124 (2002), no. 6, 1083-1102.

[JoPf2000] DE Jong, T.; PFISTER, G.: Local analytic geometry. Basic theory and applications, Advanced Lectures in Mathematics. Friedr. Vieweg \& Sohn, Braunschweig, 2000. xii+382 pp.

[Jö1988] JÖRICKE, B.: Removable singularities of CR-functions, Ark. Mat. 26 (1988), $117-143$.

[Jö1992] JÖRICKE, B.: Envelopes of holomorphy and CR-invariant subsets of CRmanifolds, C. R. Acad. Sci. Paris Sér. I Math. 315 (1992), no. 4, 407-411.

[Jö1995] JÖRICKE, B.: Some remarks concerning holomorphically convex hulls and envelopes of holomorphy, Math. Z. 218 (1995), no. 1, 143-157.

[Jö1996] JöRICKE, B.: Deformation of CR-manifolds, minimal points and CRmanifolds with the microlocal analytic extension property, J. Geom. Anal. 6 (1996), no. 4, 555-611.

[Jö1997] JÖRICKE, B.: Local polynomial hulls of discs near isolated parabolic points, Indiana Univ. Math. J. 46 (1997), no. 3, 789-826. 
[Jö1999a] JÖRICKE, B.: Boundaries of singularity sets, removable singularities, and CR-invariant subsets of CR-manifolds, J. Geom. Anal. 9 (1999), no. 2, 257300.

[Jö1999b] JÖRICKE, B.: Removable singularities of $L^{\mathrm{p}}$ CR functions on hypersurfaces, J. Geom. Anal. 9 (1999), no. 3, 429-456.

[JP2002] JÖRICKE, B.; PORTEN, E.: Hulls and analytic extension from nonpseudoconvex boundaries, U.U.M.D. Report 2002:19, Uppsala University, 38 pp., 2002.

[JS2000] JÖRICKE, B.; SHCHERBINA, N.: A nonremovable generic 4-ball in the unit sphere of $\mathbb{C}^{3}$, Duke Math. J. 102 (2000), no. 1, 87-100.

[JS2004] JÖRICKE, B.; SHCHERBINA, N.: On some class of sets with multisheeted envelopes of holomorphy, Math. Z. 247 (2004), no. 4, 711-732.

[Jo2005] JÖRICKE, B.: A Cantor set in the unit sphere in $\mathbb{C}^{2}$ with large polynomial hull, Michigan Math. J. 53 (2005), no. 1, 189-207.

[KS1967] KAJIWARA, J.; SAKAI, E.: Generalization of Levi-Oka's theorem concerning meromorphic functions, Nagoya Math. J. 29 (1967), 75-84.

[Ka1968] Katznelson, Y.: An introduction to harmonic analysis, John Wiley \& Sons, Inc., New York-London-Sydney 1968 xiv+264 pp.

[KW1982] KENIG, C.E.; WEBSTER, S.M.: The local hull of holomorphy of a surface in the space of two complex variables, Invent. Math. 67 (1982), no. 1, 1-21.

[KW1984] KENIG, C.E.; WEBSTER, S.M.: On the hull of holomorphy of an n-manifold in $\mathbb{C}^{n}$, Ann. Scuola Norm. Sup. Pisa 11 (1984), no. 2, 261-280.

[KN1963] Kobayashi, S.; NomizU, K.m: Foundations of differential geometry, I, Interscience publishers, John Wiley \& Sons, New York, 1963. xi+329 pp.

[KN1973] KoHn, J.-J.: NiRENBERG, L.: A pseudoconvex domain not admitting a holomorphic support function, Math. Ann. 201 (1973), 265-268.

[Kn1936] KNESER, H.: Die Randwerte einer analytischen Funktion zweier Veränderlichen, Monatsh. Math. Phys. 43 (1936), 364-380.

[Kr1983] KRANTZ, S.G.: Lipschitz spaces, smoothness of functions, and approximation theory, Expo. Math. 3 (1983), 193-260.

[Kr1991] KrUZhilin, N.G.: Two-dimensional spheres on the boundaries of pseudoconvex domains in $\mathbb{C}^{2}$ (Russian), Izv. Akad. Nauk SSSR Ser. Mat. 55 (1991), no. 6, 1194-1237; translation in Math. USSR-Izv. 39 (1992), no. 3, 11511187.

[Ku1982] KURANISHI, M.: Strongly pseudo-convex CR structures over small balls, Part III, Ann. Math. 116 (1982), 249-330.

[Ky1990] KYTMAnOV, A.M.: Holomorphic continuation of integrable CR-functions from a part of the boundary of the domain, Mat. Notes 48 (1990), no. 1-2, 761-765.

[Ky1991] Kytmanov, A.M.: Holomorphic continuation of CR-functions with singularities on a hypersurface, Izv. Akad. Nauk SSSR 54 (1990), no. 6, 681-691; Englis tranls. Math. USSR-Izv 37 (1991), no. 3, 681-691.

[KN1993] Kytmanov, A.M.; NikitinA, T.N.: Removable singularities of CRfunctions given on generic manifolds, Russian Acad. Sci. Dokl. Math. 46 (1993), no. 2, 279-281. 
[Ky1995] Kytmanov, A.M.: The Bochner-Martinelli integral and its applications, tranlated from the Russian by H.P. Boas and revised by the author, Birkhäuser Verlag, Basel, 1995. xii+305 pp.

[KR1995] Kytmanov, A.M.; REA, C.: Elimination of $L^{1}$ singularities on Hölder peak sets for CR functions, Ann. Scuola Norm. Sup. Pisa, Classe di Scienze, 22 (1995), no. 2, 211-226.

[La1983] LAng, S.: Real analysis. Second edition, Addison-Wesley, xv+533 pp, 1983.

[Lt1988] LAURENT-ThIÉBAUT, C.: Sur l'extension des fonctions CR dans une variété de Stein, Ann. Mat. Pura Appl. 150 (1988), no. 4, 141-151.

[Lt1997] LAURENT-ThiÉBAUT, C.: Théorie des fonctions holomorphes de plusieurs variables, Savoirs Actuels, Mathématiques, InterEditions, Paris; Masson, Paris, 1997. xiv+245 pp.

[LL1989] Laurent-ThiéBaut, C.; Leiterer, J.: On the Hartogs-Bochner extension theorem for differential forms, Math. Ann. 284 (1989), no. 1, 103-119.

[LP2003] Laurent-Thiébaut, C.; Porten, E.: Analytic extension from nonpseudoconvex boundaries and $A(D)$-convexity, Ann. Inst. Fourier (Grenoble), 53 (2003), no. 3, 847-857.

[Lew1956] LEWY, H.: On the local character of solutions of an atypical differential equation in three variables and a related problem for regular functions of two complex variables, Ann. of Math. 64 (1956), 514-522.

[Lew1957] LEWy, H.: An example of a smooth linear partial differential equation without solution, Ann. Math. 66 (1957), 155-158.

[Lew1960] LEWy, H.: On hulls of holomorphy, Comm. Pure Appl. Math. 13 (1960), 587-591.

[Le1977] LEWY, H.: On the boundary behaviour of holomorphic mappings, Contrib. Centro Linceo Inter. Sc. Mat. e Loro Appl. No. 35, Accad. Naz. Lincei (1977), 1-8 ; reprint in the Hans Lewy Selecta, 2 vols, Contemporary mathematicians, Birkhäuser, Boston, 2002.

[Le1981] LEMPERT, L.: La métrique de Kobayashi et la représentation des domaines sur la boule, Bull. Soc. Math. France 109 (1981), no. 4, 427-474.

[Lo1970] Lobry, C.: Contôlabilité des systèmes linéaires, SIAM J. Control 8 (1970), 573-605.

[Lu1986] Lupacciolu, G.: A theorem on holomorphic extension of CR functions, Pacific Math. J. 124 (1986), no. 1, 177-191.

[Lu1987] Lupacciolu, G.: Holomorphic continuation in several complex variables, Pacific Math. J. 128 (1987), no. 1, 117-126.

[Lu1990] Lupacciolu, G.: Meromorphic continuation in projective space, Bolletino U. M. I. 4-A (1990), no. 7, 391-397.

[Lu1994] LupACCIOLU, G.: Characterization of removable sets in strongly pseudoconvex boundaries, Ark. Mat. 32 (1994), no. 2, 455-473.

[LS1993] Lupacciolu, G.; Stout, E.L.: Removable singularities for $\bar{\partial}_{b}$. Several Complex Variables (Stockholm, 1987/1988), 507-518, Math. Notes, 38, Princeton Univ. Press, Princeton, NJ, 1993.

[LT1984] Lupacciolu, G.; Tomassini, G.: Un teorema di estensione per le CRfunzioni, Ann. Mat. Pura Appl. 137 (1984), no. 4, 257-263. 
[Lu1913] LuZIN, N: Sur la convergence des séries trigonométriques de Fourier, C. R. Acad. Sci. Paris 156 (1913), 1655-1658.

[MM1994] MA, L.; MiCHEL, J.: Regularity of local embeddings of strictly pseudoconvex CR structures, J. reine angew. Math. 447 (1994), 147-164.

[MS1995] MCDUFF, D.; SAlamon, D.: Introduction to symplectic topology. Oxford Mathematical Monographs. Oxford Science Publications. The Clarendon Press, Oxford University Press, New York, 1995. viii+425 pp.

[Ma1984] MARShAlL, D.: Removable sets for bounded analytic functions, Lecture Notes in Math. 1043 485-490, Springer-Verlag, Berlin-Heidelberg-New York-Tokyo, 1984.

[Ma1992] MARSON, M.E.: Wedge extendability for hypo-analytic structures, Comm. Partial Differential Equations 17 (1992), 579-592.

[MV1995] Melnikov, M.; Verdera, J.: A geometric proof of the $L^{2}$ boundedness of the Cauchy integral on Lipschitz curves, Internat. Math. Res. Notices 7 (1995), 325-331.

[Me1994] MERKER, J.: Global minimality of generic manifolds and holomorphic extendibility of CR functions. Internat. Math. Res. Notices 1994, no. 8, 329342.

[Me1997] MERKER, J.: On removable singularities for CR functions in higher codimension, Internat. Math. Res. Notices 1997, no. 1, 21-56.

[Me1999] MERKER, J.: Vector field construction of Segre sets, Preprint 1998, augmented in 2000; downloadable at arXiv.org/abs/math.CV/9901010/.

[Me2001a] MERKER, J.: On the partial algebraicity of holomorphic mappings between two real algebraic sets, Bull. Soc. Math. France 129 (2001), no. 4, 547-591.

[Me2001b] MERKER, J.: Étude de la régularité analytique de l'application de symétrie CR formelle, C. R. Acad. Sci. Paris Sér. I Math. 333 (2001), no. 3, 165-168.

[Me2002] MERKER, J.: On envelopes of holomorphy of domains covered by Levi-flat hats and the reflection principle, Ann. Inst. Fourier Grenoble 52 (2002), no. $5,1443-1523$.

[Me2004a] MERKER, J.: On the local geometry of generic submanifolds of $\mathbb{C}^{n}$ and the analytic reflection principle, e-print: arXiv.org/abs/math.CV/0404249; Journal of Mathematical Sciences (N. Y.) 125 (2005), no. 6, 751-824.

[Me2004b] MERKER, J.: Four explicit formulas for the prolongations of an infinitesimal Lie symmetry and multivariate Faà di Bruno formulas, Preprint, University of Provence, december 2004; e-print: arXiv.org/abs/math.CV/0411650.

[Me2004c] Merker, J.: Propagation of analyticity for essentially finite $\mathcal{C}^{\infty}$-smooth CR mappings, Manuscripta Math., 115 (2004), no. 3, 313-338.

[Me2005] MERKER, J.: Étude de la régularité analytique de l'application de réflexion CR formelle, Annales Fac. Sci. Toulouse, XIV (2005), no. 2, 215-330.

[Me2006a] MERKER, J.: Lie symmetries of partial differential equations and CR geometry, Journal of Mathematical Sciences (N. Y.), to appear, $118 \mathrm{pp}$.

[Me2006b] MERKER, J.: Reflection principle and systems of analytic partial differential equations, preprint, $47 \mathrm{pp}$. 
[MP1998] MERKER, J.; Porten, E.: On the local meromorphic extension of CR meromorphic functions. Complex analysis and applications (Warsaw, 1997). Ann. Polon. Math. 70 (1998), no. 1, 163-193.

[MP1999] MERKER, J.; PORTEN, E.: On removable singularities for integrable CR functions, Indiana Univ. Math. J. 48 (1999), no. 3, 805-856.

[MP2000] MERKer, J.; PORTEN, E.: Metrically thin singularities of integrable CR functions. Internat. J. Math. 11 (2000), no.7, 857-872.

[MP2002] MERKER, J.; PORTEN, E.: On wedge extendability of CR meromorphic functions, Math. Z. 241 (2002) 485-512.

[MP2006a] MERKER, J.; PORTEN, E.: Characteristic foliations on maximally real submanifolds of $\mathbb{C}^{n}$ and removable singularities for $C R$ functions, Internat. Math. Research Publications, Volume 2006, Pages 1-131.

[MP2006b] MERKER, J.; PORTEN, E.: Holomorphic extension of CR functions: supplementary topics and open problems, WWW. cmi. univ-mrs. fr/ merker/.

[MP2006c] Merker, J.; PORTEN, E.: A geometrical proof of the Hartogs extension theorem, Preprint 06-10, november 2006, École Normale Supérieure, 36 pp., submitted to J. Geom. Anal.

[MP2007] MERKER, J.; PORTEN, E.: Envelopes of holomorphy and continuity principle: a survey, in preparation.

[MMZ2003a] Meylan, F.; MiR, N.; Zaitsev, D.: Holomorphic extension of smooth CR-mappings between real-analytic and real-algebraic CR-manifolds, Asian J. Math. 7 (2003), no. 4, 493-509.

[MMZ2003b] Meylan, F.; Mir, N.; Zaitsev, D.: Approximation and convergence of formal CR-mappings, Int. Math. Res. Not. 2003, no. 4, 211-242.

[Mi2002] MIR, N.: On the convergence of formal mappings, Comm. Anal. Geom. 10 (2002), no. 1, 23-59.

[Mo1985] Moser, J.: Analytic surfaces in $\mathbb{C}^{2}$ and their local hull of holomorphy, Ann. Acad. Sci. Fenn. Ser. A I Math. 10 (1985), 397-410.

[Mo1889] MorerA, G.: Intorno all'integrale di Cauchy, 8, Ser. II, Vol. XXII (1889), 191-200.

[MW1983] Moser, J.K.; WEBSTER, S.M.: Normal forms for real surfaces in $\mathbb{C}^{2}$ near complex tangents and hyperbolic surface transformations, Acta Math. 150 (1983), no. 3-4, 255-296.

[Mu1953] MushKelishVILI, N.I.: Singularintegral equations, P. Noordhoof, Groningen, Netherlands, 1953.

[Na1966] NAGANO, T.: Linear differential systems with singularities and applications to transitive Lie algebras, J. Math. Soc. Japan 18 (1966), 398-404.

[NR1989] NAGEL, A.; RosAy, J.-P.: Approximate local solutions of $\bar{\partial}_{b}$, but nonexistence of homotopy formula for $(0,1)$-forms on hypersurfaces in $\mathbb{C}^{3}$, Duke Math. J. 58 (1989), 823-827.

[NN1957] NEWlander, A.; NIRENBERG, L.: Complex analytic coordinates in almost complex manifolds, Ann. of Math. (2) 65 (1957), 391-404.

[NW1963] Nijenhuis, A.; Woolf, W.: Some integration problems in almost-complex manifolds, Ann. Math. (2) 77 (1963), 424-489. 
[Ni1973] NiREnberG, L.: Lectures on linear partial differential equations, Expository Lectures from the CBMS Regional Conference held at the Texas Technological University, Lubbock, Tex., May 22-26, 1972. Conference Board of the Mathematical Sciences Regional Conference Series in Mathematics, No. 17. American Mathematical Society, Providence, R.I., 1973. v+58 pp.

[Ni1974] Nirenberg, L.: On a question of Hans Lewy (Russian).Translated from the English by Ju. V. Egorov. Collection of articles dedicated to the memory of Ivan Georgievič Petrovskiǔ(1901-1973), I. Uspehi Mat. Nauk 29 (1974), no. 2(176), 241-251.

[NT1963] Nirenberg, L.; Treves, F.: Solvability of a first order linear differential equation, Comm. Pure Applied Math. 16 (1963), 331-351.

[NT1970] NiREnBERG, L.; TREVES, F.: On local solvability of linear partial differential equations. I. Necessary conditions. II. Sufficient conditions, Comm. Pure Appl. Math. 23 (1970), 1-38, 459-509.

[Nis1962] Nishino, T.: Sur les ensembles pseudoconcaves, J. Math. Kyoto Univ. 1-2 (1962), 225-245.

[Oh2002] OHSAwA, T.: Analysis of several complex variables. Transl. from the Japanese by Shu Gilbert Nakamura. Translations of Math. Monographs, 211, AMS, Providence, 2002, xviii+121 pp.

[Ok1934] OKA, K.: Note sur les familles de fonctions analytiques multiformes etc., J. Sci. Hiroshima Univ. 4 (1934), 93-98.

[O11995] Olver, P.J.: Equivalence, Invariance and Symmetries. Cambridge University Press, Cambridge, 1995, xvi+525 pp.

[Or1987] Ortega Aramburu, J.M.: On Gleason's decomposition for $A^{\infty}(\bar{D})$, Math. Z. 194 (1987), 565-571.

[Pa1888] PAInlEVÉ, P.: Sur les lignes singulières des fonctions analytiques, Ann. Fac. Sci. Toulouse 2 (1888), 1-130.

[Pa2002] PAJOT, H.: Analytic capacity, rectifiability, Menger curvature and the Cauchy integral, Lecture Notes in Mathematics, 1799. Springer-Verlag, Berlin, 2002. xii+119 pp.

[Pa2005] Pajot, H.: Capacité analytique et le problème de Painlevé, Séminaire Bourbaki. Vol. 2003/2004. Astérisque No. 299 (2005), Exp. No. 936, ix, 301-328.

[Pf2003] PFLug, P.: Extension of separately holomorphic functions - a survey 1899-2001. Proceedings of Conference on Complex Analysis (BielskoBiała, 2001). Ann. Polon. Math. 80 (2003), 21-36.

[Pi1972] PICHORIDES, S.K.: On the best values of constants in the theorems of M. Riesz, Zygmund and Kolmogorov, Stud. Math. 44 (1972), 165-179.

[Pi1974a] PINCHUK, S.: A boundary uniqueness theorem for holomorphic functions of several complex variables, Mat. Zametki 15 (1974), 205-212.

[Pi1974b] PINCHUK, S.: Bogoljubov's "edge of the wedge" theorem for generic manifolds (Russian) Mat. Sb. (N.S.) 94(136) (1974), 468-482, 496.

[Pi1975] PINCHUK, S.: On the analytic continuation of holomorphic mappings (Russian), Mat. Sb. (N.S.) 98(140) (1975) no.3(11), 375-392, 416-435, 495-496.

[Pi1978] PINCHUK, S.: Holomorphic mappings of real-analytic hypersurfaces (Russian), Mat. Sb. (N.S.) 105(147) (1978), no. 4 574-593, 640. 
[Pi1992] PINCHUK, S.: CR-transformations of real manifolds in $C^{n}$, Indiana Univ. Math. J. 41 (1992), no. 1, 1-16.

[P11908] PlemelJ, J.: Ein Ergänzungssatz zur Cauchyschen Integraldarstellung analytischer Funktionen, Randwerte betreffend. 18 XIX Jahrgang, 1908, 205210.

[Po1907] PoInCARÉ, H.: Les fonctions analytiques de deux variables et la représentation conforme, Rend. Circ. Mat. Palermo, II, Ser. 23, 185-220.

[Po1997] PORTEN, E.: Hebbare Singularitten von CR-Funktionen und analytische Fortsetzung von Teilen nicht-pseudokonvexer Ränder, Thesis, Humboldt Universität zu Berlin, 1997.

[Po2000] PORTEN, E.: Analytic extension and removable singularities of the integrable CR-functions, Preprint, Humboldt-Universität zu Berlin, 2000, no. 3, $18 \mathrm{pp}$.

[Po2002] PORTEN, E.: On the Hartogs-phenomenon and extension of analytic hypersurfaces in non-separated Riemann domains, Complex variables Theory Appl. 47 (2002), 325-332.

[Po2003] Porten, E.: Totally real discs in nonpseudoconvex boundaries, Ark. Mat. 41 (2003), no. 1, 133-150.

[Po2004] PoRTEn, E.: Habilitationsschrift, Humboldt Universität zu Berlin, November 2004.

[Po2005] Porten, E.: On Generalized Tube Domains over $\mathbb{C}^{n}$, Complex Variables Theory and Applications, 50 (2005), no. 1, 1-5.

[Pri1916] Privalov, I.I.: Sur les fonctions conjuguées, Bull. Soc. Math. France 44 (1916), 100-103.

[Pri1950] PRIVAlOV, I.I.: Boundary properties of analytic functions, 2nd ed., GITTL, Moscow, 1950, 336 pp. German transl. VEB Deutscher Verlag, Berlin, 1956.

[Pu1990] Pushnikov, Y.: Holomorphicity of CR-mappings into a space of large dimension, Mat. Zametki 48 (1990), no. 3, 147-149.

[Ra1919] RADEMACHER, H.: Über partielle und totale Differenzierbarkeit von Funktionen mehrerer Variabeln und über die Transformation der Doppelintegrale, Math. Ann. 79 (1919), 340-359.

[Ra1924] RADÓ, T.: Über eine nicht fortsetzbare Riemannsche Mannigfaltigkeit, Math. Z. 20 (1924), 1-6.

[Ra1986] RANGE, M.: Holomorphic functions and integral representations in several complex variables, Graduate Texts in Mathematics, 108, Springer-Verlag, New York, 1986. xx+386 pp.

[Ra2002] RANGE, M.: Extension phenomena in multidimensional complex analysis: correction of the historical record, Math. Intelligencer 24 (2002), no. 2, 412.

[R1972] REA, C.: Levi-flat submanifolds and holomorphic extension of foliations, Ann. Scuola Norm. Sup. Pisa (3) 26 (1972), 665-681.

[R1983] REA, C.: Prolongement holomorphe des fonctions CR, conditions suffisantes, C. R. Acad. Sci. Paris Sér I Math. 297 (1983), no. 3, 163-165.

[Re1993] ReutenAuer, C.: Free Lie algebras, London Mathematical Society Monograph, New Series, 7. Oxford Science Publications, The Clarendon Press, Oxford University Press, New York, 1993. xviii+269 pp. 
[Ro1986a] RosAY, J.-P.: À propos de "wedges" et d' "edges" et de prolongements holomorphes, Trans. Amer. Math. Soc. 297 (1986), no. 1, 63-72.

[Ro1986b] RosaY, J.-P.: Sur un problème d'unicité pour les fonctions CR, C. R. Acad. Sci. Sér. I Math. Paris 302 (1986), 9-11.

[Ro1993] Rosay, J.-P.: Trépreau's example, a pedestrian approach, L'enseignement Mathématique 39 (1993), 259-268.

[RS1989] Rosay, J.-P.; Stout, E.L.: Radò's theorem for CR functions, Proc. Amer. Math. Soc. 106 (1989), 1017-1026.

[Ro1960] Rossi, H.: The local maximum modulus principle, Ann. Math. 72 (1960), no. $1,1-11$.

[Ro1959] RothSteIn, W.: Bemerkungen zur Theorie komplexer Räume, Math. Ann. 137 (1959), 304-315.

[RoS1965] RothsteIn, W., SPERLING, H.: Einsetzen analytischer Flächenstücke in Zyklen auf komplexen Räumen, Festschrift zur Gedächtnisfeier für Karl Weierstrass 1815-1965 (Behnke and Kopfermann, eds.), Westdeutscher Verlag, Köln 1965, 531-554.

[Ru1980] Rudin, W.: Function Theory in the Unit Ball of $\mathbb{C}^{n}$, Grundlehren der Mathematischen Wissenschaften, 241, Springer-Verlag, New York-Berlin, xiii+436 pp., 1980.

[Ro2003] ROTHSCHILD, L.P.: Mappings between real submanifolds in complex space, Explorations in complex and Riemannian geometry, 253-266, Contemp. Math., 332, Amer. Math. Soc., Providence, RI, 2003.

[Sa1999] SARKIS, F.: CR-meromorphic extension and the non embeddability of the Andreotti-Rossi CR structure in the projective space, Int. J. Math. 10 (1999), no. 7, 897-915.

[Se1932] SEgRE, B.: Questioni geometriche legate colla teoria delle funzioni di due variabili complesse, Rendiconti del Seminario di Matematici di Roma, II, Ser. 7 (1932), no. 2, 59-107.

[Sh1990] ShABAT, B.; Introduction à l'analyse complexe, 2 vols, traduit du russe par Djilali Embarek, Mir, Moscou, 1990, 309 pp ; 420 pp.

[Sh2000] SHAFIKOV, R.: Analytic continuation of germs of holomorphic mappings between real hypersurfaces in $\mathbb{C}^{n}$, Michigan Math. J. 47 (2000), no. 1, 133149.

[Sh2003] SHAFIKOV, R.: Analytic continuation of holomorphic correspondences and equivalence of domains in $\mathbb{C}^{n}$, Invent. Math. 152 (2003), no. 3, 665-682.

[SS1996] SHARIPOV, R.; SUKHOV, A.: On CR mappings between algebraic CauchyRiemann manifolds and separate algebraicity for holomorphic functions, Trans. Amer. Math. Soc. 348 (1996), no. 2, 767-780.

[Sh1993] ShCHERBINA, N.V.; On the polynomial hull of a graph, Indiana Univ. Math. J. 42 (1993), no. 2, 477-503.

[ShTo1999] ShChERBINA, N.V.; TOMASSINI: The Dirichlet problem for Levi-flat graphs over unbounded domains, Internat. Math. Res. Notices 1999, no. 3 , 111-151.

[Si1969] SIEgEL, C.L.: Topics in Complex Function Theory, Wiley Classics Library, John Wiley \& Sons, Inc., New York, I: Elliptic functions and uniformization theory, xii+186 pp., II: Automorphic functions and abelian integrals, 
+193 pp., III: Abelian functions and modular functions of several variables, +244 pp., 1989.

[Si1974] SiU, Y.T.: Techniques of extension of analytic objects, Lecture Notes in Pure and Applied Mathematics, Vol. 8, Marcel Dekker, Inc., New York, 1974. iv +256 pp.

[Si2000] SIU, Y.T.: Nonexistence of smooth Levi-flat hypersurfaces in complex projective spaces of dimension $\geqslant 3$, Ann. of Math. (2) 151 (2000), no. 3, $1217-$ 1243.

[SiT1971] Siu, Y. T.; Trautmann, G.: Gap sheaves and extension of coherent analytic subsheaves, Lect. Notes Math. 172 (1971), Springer-Verlag.

[Sj1982a] SJÖstRAnd, Y.: Singulatités analytiques microlocales, Astérisque, t. 95, 1982.

[Sj1982b] SJÖstRand, Y.: The FBI transform for CR submanifolds of $\mathbb{C}^{n}$, Prépubl. Math. Orsay, Université Paris-Sud, 1982.

[S12004] SŁAPAR, M: On Stein neighborhood basis of real surfaces, Math. Z. 247 (2004), no. 4, 863-879.

[So1873] SокнотSкIц, Yu.V.: On definite integrals and functions employed in expansions into series (Ob opredelennykh i funtsiakh uprotrebljayemykh pri razlozhenii v ryady), St Petersburg, 1873.

[SME1988] Soviet Mathematical EnCyClopaedia (Vinogradov A. dir.) 10+1 vol., Kluwer, Dordrecht, 1988.

[Sp1970] SPIVAK, M.: Differential geometry, volumes one and two, Brandeis university, 1970.

[St1996] Stanton, N.: Infinitesimal CR automorphisms of real hypersurfaces, Amer. J. Math. 118 (1996), no. 1, 209-233.

[St1970] StEIN, E.: Singular integrals and differentiability properties of functions, Princetion University Press, Princeton, N.J., 1970.

[St1972] STEIN, E.: Boundary behavior of holomorphic functions of several complex variables. Mathematical Notes, No. 11. Princeton University Press, Princeton, N.J.; University of Tokyo Press, Tokyo, 1972. x+72 pp.

[Sto1963] StolzenberG, G.: A hull with no analytic structure, J. Math. Mech. 12 (1963), 103-112.

[Stk1982] Stormark, O.: On the theorem of Frobenius for complex vector fields, Ann. Scuola Norm. Sup. Pisa Cl. Sci. (4) 9 (1982), no. 1, 57-90.

[Stk2000] StORMARK, O.: Lie's structural approach to PDE systems, Encyclopædia of mathematics and its applications, vol. 80, Cambridge University Press, Cambridge, 2000, xv+572 pp.

[Stu1968] Stout, E.L.: A generalization of a theorem of Radó, Math. Ann. 177 (1968), 339-340.

[Stu1981] Stout, E.L.: Analytic continuation and boundary continuity of functions of several complex variables, Proc. Roy. Soc. Edinburgh Sect. A 89 (1981), no. 1-2, 63-74.

[Stu1989] Stout, E.L.: Removable sets for holomorphic functions of several complex variables, Publ. Mat. 33 (1989), no. 2, 345-362.

[Stu1991] Stout, E.L.: A note on removable singularities, Boll. Un. Mat. Ital. A. (7) 5 (1991), no. 2, 237-243. 
[Stu1993] Stout, E.L.: Removable singularities for the boundary values of holomorphic functions. Several Complex Variables (Stockholm, 1987/1988), 600629, Math. Notes, 38, Princeton Univ. Press, Princeton, NJ, 1993.

[Str1988] StRUPPA, D.: The first eighty years of Hartogs' theorem, in: Geometry Seminars, 1987-1988, Univ. Stud. Bologna, Bologna, 1988, 127-209.

[Su1973] Sussmann, H.J.: Orbits of families of vector fields and integrability of distributions, Trans. Amer. Math. Soc. 180 (1973), no. 1, 171-188.

[Su1976] Sussmann, H.J.: Some properties of vector field systems that are not altered by small perturbations, J. Differential Equations 20 (1976), no. 2, 292315.

[Ta1964] TAKeuchi, A.: Domaines psudoconvexes infinis et la métrique riemannienne dans un espace projectif, J. Math. Soc. Japan 16 (1964), 159-181.

[To2003] Tols A, X.: Painlevé's problem and the semiadditivity of analytic capacity, Acta Math. 190 (2003), no. 1, 105-149.

[Tr1984] TRÉPREAU, J.-M.: Sur l'hypoellipticité analytique microlocale des opérateurs de type principal, Comm. Partial Differential Equations 9 (1984), no. 11, 1119-1146.

[Trp1986] TrÉPREAU, J.-M.: Sur le prolongement holomorphe des fonctions CR définies sur une hypersurface réelle de classe $\mathbf{C}^{2}$, Invent. Math. 83 (1986), no. 3, 583-592.

[Trp1990] TrÉPREAU, J.-M.: Sur la propagation des singularités dans les variétés CR, Bull. Soc. Math. Fr. 118 (1990), no. 4, 403-450.

[Trp1992] TRÉPREAU, J.-M.: On the extension of holomorphic functions across a real hypersurface, Math. Z. 211 (1992), no. 1, 93-103.

[Trp1996] TrépreaU, J.-M.: Holomorphic extension of CR functions: a survey, Partial differential equations and mathematical physics (Copenhagen, 1995; Lund 1995), 333-355, Progr. Nonlinear Differential Equations Appl., 21, Birkhäuser Boston, Boston, MA, 1996.

[Trv1970] Treves, F.: On local solvability of linear partial differential equations, Bull. Amer. Math. Soc. 76 (1970), 552-571.

[Trv1981] TREVES, F.: Approximation and representation of functions and distributions annihilated by a system of complex vector fields, Palaiseau, École Polytechnique, Centre de Mathématiques, 1981.

[Trv1986] Treves, F.: Overdetermined systems defined by complex vector fields, Miniconference on geometry and partial differential equations (Canberra, 1985), 224-243, Proc. Centre Math. Anal. Austral. Nat. Univ., 10, Austral. Nat. Univ., Canberra, 1986.

[Trv1992] Treves, F.: Hypo-analytic structures: local theory. Princeton Mathematical Series, 40, Princeton University Press, Princeton, NJ, 1992, xvii+497 pp.

[Trv2000] TREVES, F.: A treasure trove of geometry and analysis: the hyperquadric, Notices Amer. Math. Soc. 47 (2000), no. 10, 1246-1256.

[Tu1988] TUMANOV, A.E.: Extending CR functions on a manifold of finite type over a wedge (Russian), Mat. Sb. (N.S.) 136(178) (1988), no.1, 128-139. English transl. in Math. USSR-Sb. 64 (1989) no. 1, 129-140. 
[Tu1990] Tumanov, A.E.: Extending CR-functions into a wedge, Mat. Sbornik 181 (1990), 951-964. Trad. in English in Math. USSR Sbornik 70 (1991), 2, 385 398.

[Tu1994a] Tumanov, A.E.: Connections and propagation of analyticity for CR functions, Duke Math. J. 73 (1994), no. 1, 1-24.

[Tu1994b] TUMANOV, A.E.: Analytic discs and the regularity of CR mappings in higher codimension, Duke Math. J. 76, no. 3, 793-807.

[Tu1996] Tumanov, A.E.: On the propagation of extendibility of CR functions. Complex analysis and geometry (Trento, 1993), 479-498, Lecture Notes in Pure and Appl. Math., 173, Dekker, New York, 1996.

[Tu1998] Tumanov, A.E.: Analytic discs and the extendibility of CR functions, Integral geometry, Radon transforms and complex analysis (Venice, 1996), 123141, Lecture Notes in Math., 1684, Springer, Berlin, 1998.

[Tu2001] Tumanov, A.E.: Extremal discs and the regularity of $C R$ mappings in higher codimension, Amer. J. Math. 123 (2001), no. 3, 445-473.

[Tu2002] Tumanov, A.E.: Foliations by complex curves and the geometry of real surfaces of finite type, Math. Z. 240 (2002), no. 2, 385-388.

[Ve1962] VEKUA, N.P.: Generalized analytic functions. Moscow, 1959. English translation editor I.N. Sneddon, Pergamon Press, Oxford, 1962.

[Ve1924] VESSIOT, E.: Sur une théorie nouvelle des problèmes généraux d'intégration, Bull. Soc. Math. France 52 (1924), 336-395.

[Vi1990] VitushKin, A.G.: Holomorphic mappings and the Geometry of Hypersurfaces, Encyclopædia of Mathematical Sciences, Volume 7, Several Complex Variables, I, Springer-Verlag, Berlin, 1990, pp. 159-214.

[We1977] WEBSTER, S.M.: On the mapping problem for algebraic real hypersurfaces, Invent. Math. 43 (1977), no.1, 53-68.

[We1978] WeBSTER, S.M.: On the reflection principle in several complex variables, Proc. Amer. Math. Soc. 71 (1978), no. 1, 26-28.

[We1982] WEBSTER, S.M.: Analytic discs and the regularity of $C$ - $R$ mappings of real submanifolds in $C^{n}$, Complex analysis of several variables (Madison, Wis., 1982), 199-208, Proc. Sympos. Pure Math., 41, Amer. Math. Soc., Providence, RI, 1984.

[We1985] WeBster, S.M.: The Euler and Pontrjagin numbers of an n-manifold in $\mathbb{C}^{n}$, Comment. Math. Helv. 60 (1985), no. 2, 193-216.

[We1989a] Webster, S.M.: On the local solution of the tangential Cauchy-Riemann equations, Ann. Inst. H. Poincaré Anal. Non Linéaire 6 (1989), no. 3, $167-$ 182.

[We1989b] WeBSTER, S.M.: On the proof of Kuranishi's embedding theorem, Ann. Inst. H. Poincaré Anal. Non Linéaire 6 (1989), no. 3, 183-207.

[We1989c] Webster, S.M.: A proof of the Newlander-Nirenberg theorem, Math. Z. 201 (1989), no. 3, 303-316.

[We1991] WEBSTER, S.M.: The integrability problem for CR vector bundles, Several complex variables and complex geometry, Part 3 (Santa Cruz, CA, 1989), 355-368.

[Za1999] ZAITSEV, D.: Algebraicity of local holomorphisms between real algebraic submanifolds in complex spaces, Acta Math. 183 (1999), 273-305. 
[Zy1959] Zygmund, A.: Trigonometric series, 2 vols. I and II, Cambridge University Press, Cambridge, 1959, 383 pp; 354 pp. 